\title{
WestVirginiaUniversity
}

THE RESEARCH REPOSITORY @ WVU

Graduate Theses, Dissertations, and Problem Reports

2003

\section{Flow characterization of the UL Smoke Box and spot-type smoke detectors}

Christopher P. Menchini

West Virginia University

Follow this and additional works at: https://researchrepository.wvu.edu/etd

\section{Recommended Citation}

Menchini, Christopher P., "Flow characterization of the UL Smoke Box and spot-type smoke detectors" (2003). Graduate Theses, Dissertations, and Problem Reports. 1388.

https://researchrepository.wvu.edu/etd/1388

This Thesis is protected by copyright and/or related rights. It has been brought to you by the The Research Repository @ WVU with permission from the rights-holder(s). You are free to use this Thesis in any way that is permitted by the copyright and related rights legislation that applies to your use. For other uses you must obtain permission from the rights-holder(s) directly, unless additional rights are indicated by a Creative Commons license in the record and/ or on the work itself. This Thesis has been accepted for inclusion in WVU Graduate Theses, Dissertations, and Problem Reports collection by an authorized administrator of The Research Repository @ WVU. For more information, please contact researchrepository@mail.wvu.edu. 


\title{
FLOW CHARACTERIZATION OF THE UL SMOKE BOX AND SPOT-TYPE SMOKE DETECTORS
}

\author{
by \\ Christopher P. Menchini \\ A Thesis Submitted to the \\ College of Engineering \& Mineral Resources \\ at \\ West Virginia University \\ in Partial Fulfillment of the Requirements \\ for the Degree of \\ Master of Science \\ in \\ Aerospace Engineering \\ Gary J. Morris, Ph.D., Chair \\ Wade W. Huebsch, Ph.D. \\ John M. Kuhlman, Ph.D. \\ Mehrdad Shahnam, Ph.D.
}

The Department of Mechanical \& Aerospace Engineering

Morgantown, West Virginia
2003

Keywords: CFD, Fluent ${ }^{\circledR}$, Fire Protection Engineering, Smoke Detector, Underwriters Laboratories, UL Smoke Box, Flow Characterization.

Copyright 2003 Christopher P. Menchini 


\section{ABSTRACT}

Flow Characterization of the UL Smoke Box and Spot-Type Smoke Detectors

By Christopher P. Menchini

The certification and testing of fire protection devices can be an expensive and arduous task. Although fire detection as an industry has rapidly evolved over the past quarter century, the engineering application based upon tools of a known degree of accuracy has seen little evolution in this field. Limited fluid mechanics tools have been involved in the design and improvement of smoke detecting devices. A third party firm responsible for certifying a number of consumer products, known as Underwriters Laboratories (UL) Inc., has dictated the standards by which smoke detectors have been brought to market. One key area of interest is the ability to track particles inside the sensing devices of the detector over time with respect to an external smoke source. The scope of this research involves the first known effort at designing a highly detailed computational fluid dynamics (CFD) model to characterize the flow fields with the UL Smoke Box and a spot type smoke detector within the test section of the smoke box. The proposed CFD model, designed to reflect the same measured conditions in the UL Smoke Box, was developed and validated through experimental velocity data using laser Doppler and hot-wire anemometry. With excellent qualitative comparisons between experimental and computational results, flow characterization using the laminar flow model available in the commercial software package Fluent ${ }^{\circledR}$ was sufficiently achieved. The computational model, therefore, shows excellent promise to expedite the evolution of new smoke detector designs. In industry, this design model has the potential to provide designers good preliminary insight into how a detector design will perform prior to physical construction. Although experimental testing will always be needed, CFD can be implemented as a virtual test bed to greatly reduce the time to market from product concept to a finished product. Also, there is the possibility that a more efficient, functional smoke detector may be developed. 


\section{ACKNOWLEDGEMENTS}

If I were to list everybody that has helped me to get to this point, I would need to add another 100 pages of text to this already quite pudgy thesis. However, there are a few notables that require their mention because without them, this document would not exist.

To start with, the author of this work would like to extend a formal thank you to BRK Brands, Inc. for sponsoring this research opportunity, greatly appreciating the suggestions and assistance provided by the Contract Monitor, Mr. Gene Brooks. Equally, I wish to thank Dr. Morris for giving me the opportunity to work on this project at West Virginia University. His offer was very swift and timely, and as it turned out, the best situation for me at the time. His guidance and supervision over this research and my studies helped to easily transcend the average student-advisor relationship, being an honest friend when sought and an instructor when warranted. Dr. Morris was always available when problems arose, constantly helping me find solutions in one form or another. My extended knowledge of fluid mechanics and exposure to quality engineering practice is due in large part to the personal strides he and other members on my committee took in providing me with personal instruction. His unending dedication to bringing out the best in students ultimately makes this report a success worth reading.

Two other committee members who serve as faculty members at WVU, Dr. Huebsch and Dr. Kuhlman, deserve a much needed thank you as well. My first advisor when first starting graduate school, Dr. Huebsch much like Dr. Morris offered guidance far beyond the academic spectrum. His background in CFD proved very useful in reaching the project's specific goals, offering awakening ideas and global observations that were often lost in the trenches of battle. Dr. Kuhlman, with his expert knowledge of fluid mechanics, is greatly appreciated for reviewing my final work offering professional, constructive criticism where needed. The final member of my committee, Dr. Shahnam, was very helpful in offering his expert knowledge of the CFD software package Fluent ${ }^{\circledR 18}$, and his keen advice in making sure the model was tailored to get the most use from the software package. He was extremely helpful in clarifying the capabilities of the program and how to implement some of the final modeling sequences. It would be extremely difficult to find a more capable group of advisors with as much positive guidance and wisdom as the ones present on my committee. 
Special thanks must also be given to Ms. Erika Echavarria and Mr. Gerald Angle for their prior assistance during the first, experimental phase of the project. Last but certainly not least, are my family and friends. I cannot extend a large enough thank you to my parents who have helped mold me into who I am today. My mother, especially, deserves the most thanks in guiding me on the lesser beaten path of not only leading a budding successful career, but personal one as well. My parents along with my sister and extended family (who are too many to list individually) have all had a genuine hand in supporting me in this venture - be it financially, physically, or emotionally. Without all of their support it is hard to tell where my unguided endeavors would have delivered me. My friends, who were encouraging in their own unique ways, cannot go un-thanked; and if mentioned specifically, egos would surely hang me for leaving one of them out of "print." 


\section{TABLE OF CONTENTS}

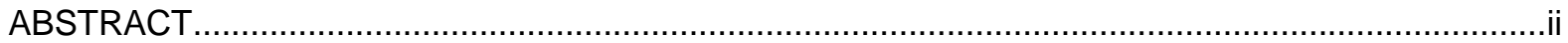

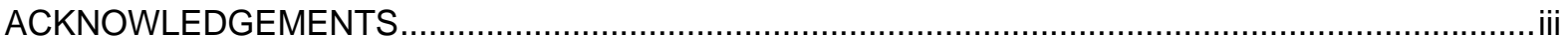

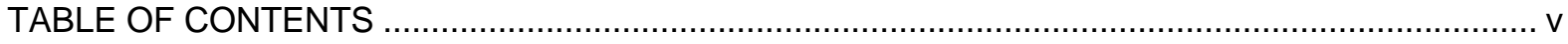

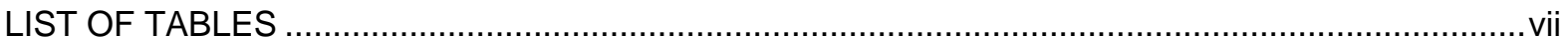

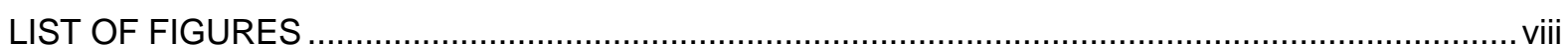

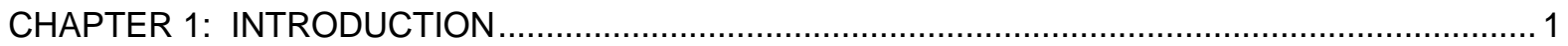

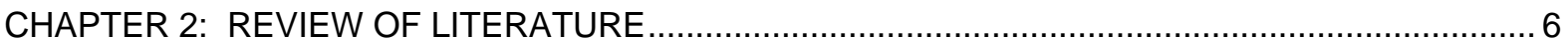

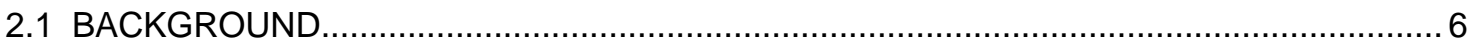

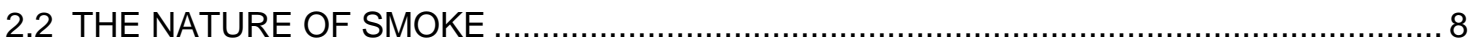

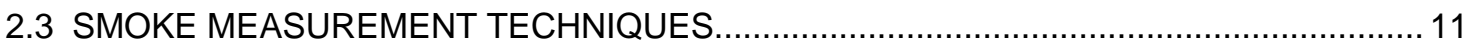

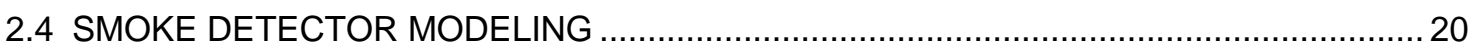

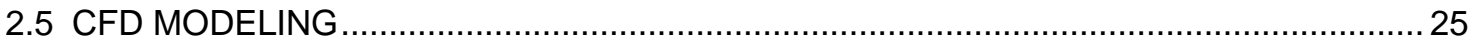

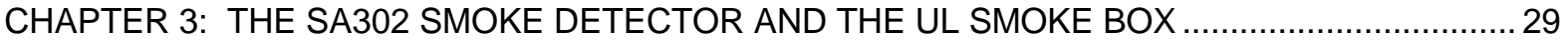

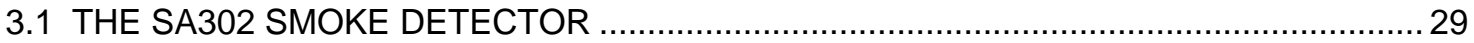

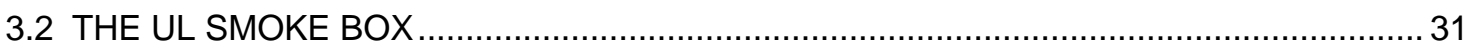

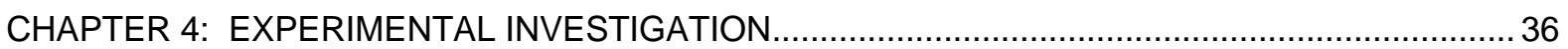

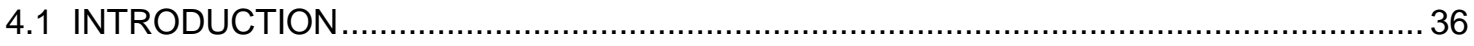

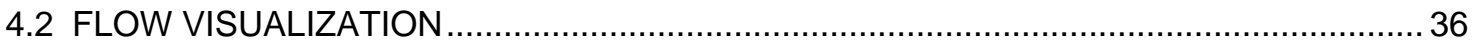

4.3 LDA VELOCITY MEASUREMENTS INSIDE THE UL SMOKE BOX …............................ 38

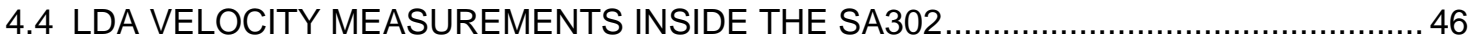

CHAPTER 5: COMPUTATIONAL FLUID DYNAMICS MODEL ..................................................... 49

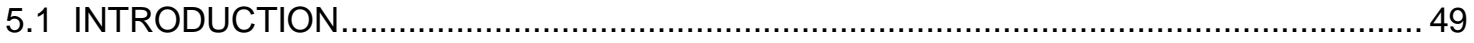

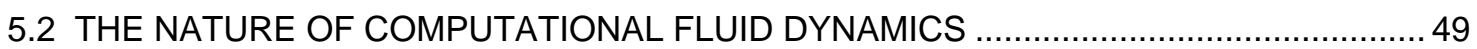

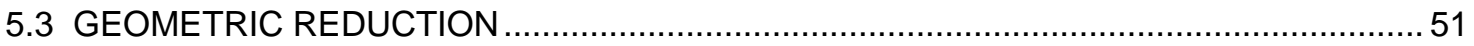

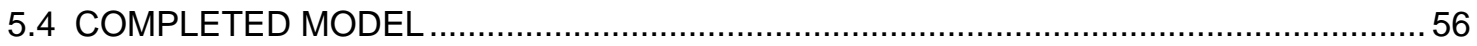

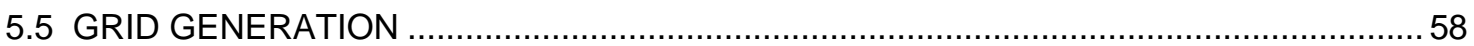

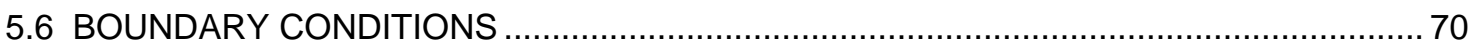

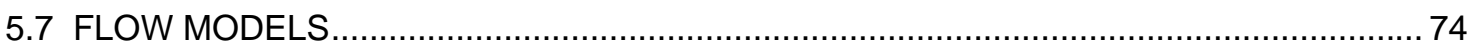

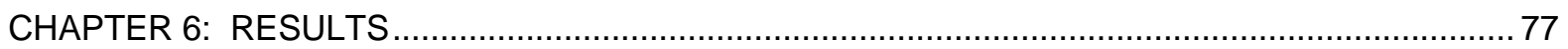

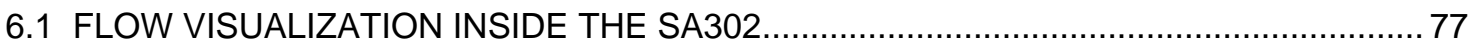

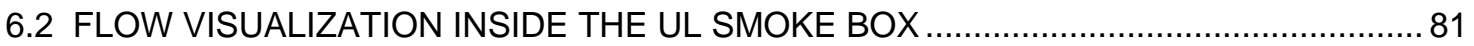

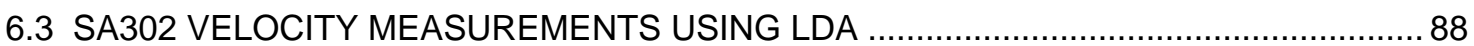

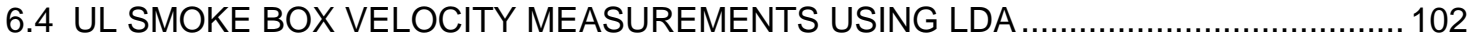

6.5 UL SMOKE BOX INLET PROFILE RESULTS USING HOT-WIRE ANEMOMETRY ....... 146

6.6 UL SMOKE BOX AND MODEL SA302 SMOKE DETECTOR VELOCITY PREDICTIONS USING CFD 


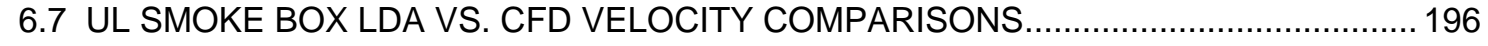

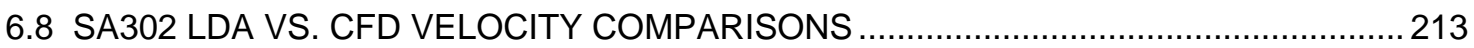

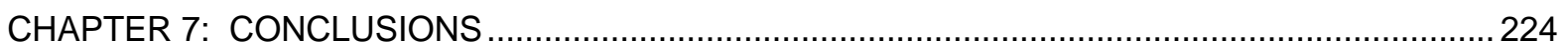

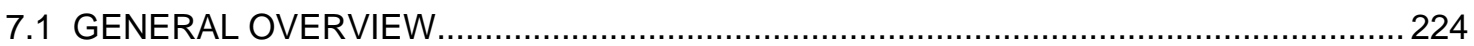

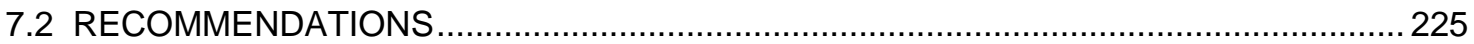

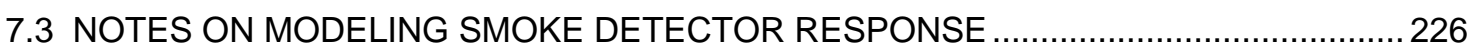

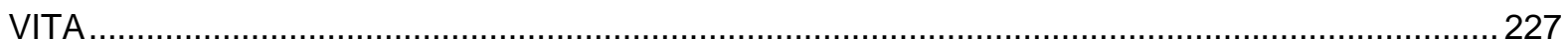

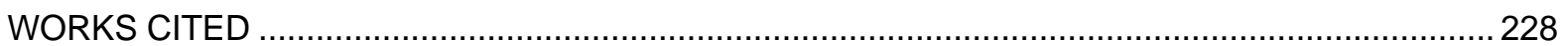

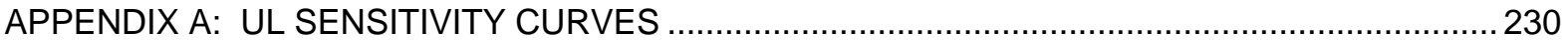

APPENDIX B: PRESSURE LOSS MEASUREMENTS ACROSS THE INSECT SCREEN .............. 232

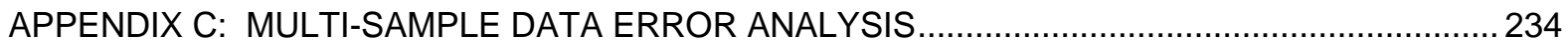




\section{LIST OF TABLES}

Table 2.2.1: Measured and derived parameters of smokes in the UL Smoke Box chamber. ${ }^{3} \ldots \ldots \ldots . . . .11$

Table 2.4.1: A summary of the range of characteristic $L$-numbers from multiple researchers. ${ }^{9} \ldots \ldots . . .24$

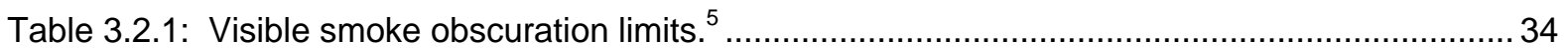




\section{LIST OF FIGURES}

Figure 2.2.1: Discrete size ranges as measured by an electrical aerosol analyzer. The dotted portion of the curve denotes an area of large uncertainty..$^{3}$

Figure 2.3.1: The basic layout of the light obscuration (projected beam) smoke detection system commonly known as a photometer. ${ }^{6}$

Figure 3.1.1: A three perspective view of the model SA302 detector.

Figure 3.1.2: An interior section slice showing the main components that make the SA302

function.

Figure 3.1.3: The component makeup of the photoelectric (light-scattering) sensor.

Figure 3.1.4: The component makeup of the ionization sensor.

Figure 3.2.1: Some still image photographs of a UL Smoke Box with its vertical hatch open exposing the interior.

Figure 3.2.2: A conceptual sketch of a UL test in progress. The red arrows indicate the direction of airflow.

Figure 3.2.3: The measuring ionization chamber (MIC).

Figure 3.2.4: The coordinate system in use for reporting all results in the UL Smoke Box test section.

Figure 4.2.1: A conceptual drawing of how images were captured inside the UL Smoke Box for flow visualization.

Figure 4.3.1: Laser Doppler anemometry and traversing bench positioned in front of the UL Smoke Box.

Figure 4.3.2: A close-up view of the UL Smoke Box test section using laser Doppler

Anemometry.

Figure 4.3.3: Close-up view of the laser Doppler anemometry measuring volume taking a data point just outside the realm of the detector.

Figure 4.3.4: The measuring volume of laser Doppler anemometer with frequency shifting to detect flow direction.

Figure 4.3.5: The optical components of the laser Doppler anemometry system.

Figure 4.3.6: The modified UL Smoke Box with the added optical access port used to take LDA measurements on the interior of the detector.

Figure 4.3.7: An oscilloscope trace of the laser Doppler anemometry signal processor as a fog particle passes through the measuring volume. 
Figure 4.3.8: The UL Smoke Box test section showing characteristic orthogonal planes of data where the laser Doppler anemometry measurements were taken

Figure 4.3.9: The location grid used in taking laser Doppler anemometry data. (a.) is for the $x-z$ plane, (b.) is for the $x-y$ plane, and (c.) is for the $y-z$ plane.

Figure 4.4.1: The bottom view of the SA302 model constructed for laser Doppler anemometry measurements inside of the detector housing and sensing chambers.

Figure 4.4.2: The grid defining the locations where axial and transverse ( $u$ and $w$ ) component flow velocities were measured in the SA302 model.

Figure 5.2.1: The general concept of the finite control volume method. 50

Figure 5.3.1: The original photoelectric sensor part compared to the de-featured part. 53

Figure 5.3.2: The original ion chamber sensor part compared to the de-featured part..................... 53

Figure 5.3.3: The original detector base plate compared to the de-featured assembly..... .54

Figure 5.3.4: The original SA302 smoke detector as compared to the de-featured assembly. 54

Figure 5.3.5: The original detector compared to the de-featured detector sliced along a central cutting plane.

Figure 5.3.6: A comparison of the originally modeled MIC to that of the de-featured, CFD model. ... 56

Figure 5.4.1: The total model with inlet and outlet boundary zones as developed in Solidworks ${ }^{\circledR 20} \ldots 57$

Figure 5.5.1: Mesh hierarchy starting with a node, and ending at a meshed volume. 59

Figure 5.5.2: The 4-node tetrahedral element employed in the computational model. 60

Figure 5.5.3: The surface mesh of the SA302 smoke detector.

Figure 5.5.4: The surface mesh of the detector's respective parts. Images of the (a.) base plate, (b.) ion chamber, and (c.) photoelectric sensor are portrayed.

Figure 5.5.5: The surface mesh of the MIC.

Figure 5.5.6: The (a.) test section walls with accompanying pressure outlet zone and (b.) hot-wire anemometer access hole.

Figure 5.5.7: The interior flow field at the $x=18$ in plane depicting the centerline cut of the SA302 smoke detector and an off-center slice of the MIC on the right......

Figure 5.5.8: The same cut as in Figure 5.6.10, but zoomed in on the SA302 smoke detector.

Figure 5.5.9: This figure shows the element size gradients established between the probe access hole, model SA302, and MIC.

Figure 5.5.10: A detector mesh slice at $y=0.50 \mathrm{in}$.

Figure 5.5.11: The ion sensor in the $y=0.50$ in plane. 
Figure 5.5.12: The photoelectric sensor in the $y=0.50$ in plane.

Figure 5.5.13: The lighted wall mesh of the major components in the test section.

Figure 5.5.14: The lighted wall mesh of the SA302, intricately defining the parts of (a.) the entire base plate, (b.) the ion chamber, along with the (c.) photoelectric chamber. 70

Figure 5.6.1: A simple diagram depicting the functionality of a flow straightener. 71

Figure 5.6.3: The photoelectric sensor and MIC with potential porous boundary conditions... .73

Figure 6.1.1: Flow visualization of the SA302 smoke detector in the UL Smoke Box at $32 \mathrm{ft} / \mathrm{min}$ and orientation angle $0^{\circ}$ (bottom view).

Figure 6.1.2: Flow visualization of the SA302 smoke detector in the UL Smoke Box at $32 \mathrm{ft} / \mathrm{min}$ and orientation angle $45^{\circ}$ (bottom view).

Figure 6.1.3: Flow visualization of the SA302 smoke detector in the UL Smoke Box at $32 \mathrm{ft} / \mathrm{min}$ and orientation angle $90^{\circ}$ (bottom view).

Figure 6.1.4: Flow visualization of the SA302 smoke detector in the UL Smoke Box at $32 \mathrm{ft} / \mathrm{min}$ and orientation angle $135^{\circ}$ (bottom view)........

Figure 6.1.5: Flow visualization of the SA302 smoke detector in the UL Smoke Box at $32 \mathrm{ft} / \mathrm{min}$ and orientation angle $180^{\circ}$ (bottom view).

Figure 6.1.6: Flow visualization of the SA302 smoke detector in the UL Smoke Box at $32 \mathrm{ft} / \mathrm{min}$ and orientation angle $225^{\circ}$ (bottom view)......

Figure 6.1.7: Flow visualization of the SA302 smoke detector in the UL Smoke Box at $32 \mathrm{ft} / \mathrm{min}$ and orientation angle $270^{\circ}$ (bottom view)

Figure 6.1.8: Flow visualization of the SA302 smoke detector in the UL Smoke Box at $32 \mathrm{ft} / \mathrm{min}$ and orientation angle $315^{\circ}$ (bottom view).

Figure 6.2.1: Flow visualization in the $y-z$ plane of the UL Smoke Box at $x=21$ in.

Figure 6.2.2: Flow visualization in the $y-z$ plane of the UL Smoke Box at $x=22$ in.

Figure 6.2.3: Flow visualization in the $y-z$ plane of the UL Smoke Box at $x=23$ in.

Figure 6.2.4: Flow visualization in the $x-z$ plane in the UL Smoke Box at $y=0.25$ in. 84

Figure 6.2.5: Flow visualization in the $x-z$ plane in the UL Smoke Box at $y=0.50$ in. 84

Figure 6.2.6: Flow visualization in the $x-z$ plane in the UL Smoke Box at $y=1.0$ in. 85

Figure 6.2.7: Flow visualization in the $x-z$ plane in the UL Smoke Box at $y=2.0 \mathrm{in}$. 85

Figure 6.2.8: Flow visualization in the wake of the SA302 Smoke Detector at $y=0.25$ in. 86

Figure 6.2.8: Flow visualization in the wake of the SA302 Smoke Detector at $y=0.50 \mathrm{in}$. 87

Figure 6.2.9: Flow visualization in the wake of the SA302 Smoke Detector at $y=1.0 \mathrm{in}$. 87 
Figure 6.2.10: Flow visualization in the wake of the SA302 Smoke Detector at $y=2.0 \mathrm{in}$.

Figure 6.3.1: Flow velocity vectors inside the SA302 Smoke Detector as measured by laser Doppler anemometry at an orientation of $0^{\circ}$ (bottom view)......

Figure 6.3.2: Flow velocity vectors inside the SA302 Smoke Detector as measured by laser

Doppler anemometry at an orientation of $90^{\circ}$ (bottom view).

Figure 6.3.3: Flow velocity vectors inside the SA302 Smoke Detector as measured by laser

Doppler anemometry at an orientation of $180^{\circ}$ (bottom view).

Figure 6.3.4: Flow velocity vectors inside the SA302 Smoke Detector as measured by laser Doppler anemometry at an orientation of $270^{\circ}$ (bottom view).

Figure 6.3.5: Flow velocity vectors inside the SA302 Smoke Detector as measured by laser Doppler anemometry at an orientation of $315^{\circ}$ (bottom view).

Figure 6.3.6: Air flow velocity vectors inside the SA302 smoke detector mid-plane as measured by LDA superposed on a flow visualization image at $0^{\circ}$ (bottom view)......

Figure 6.3.7: Air flow velocity vectors inside the SA302 smoke detector mid-plane as measured by LDA superposed on a flow visualization image at $90^{\circ}$ (bottom view).......

Figure 6.3.8: Air flow velocity vectors inside the SA302 smoke detector mid-plane as measured by LDA superposed on a flow visualization image at $180^{\circ}$ (bottom view).....

Figure 6.3.9: Air flow velocity vectors inside the SA302 smoke detector mid-plane as measured by LDA superposed on a flow visualization image at $270^{\circ}$ (bottom view).....

Figure 6.3.10: Air flow velocity vectors inside the SA302 smoke detector mid-plane as measured by LDA superposed on a flow visualization image at $315^{\circ}$ (bottom view).

Figure 6.3.11: The $u-v$ velocity vectors and streamlines in the SA302 smoke detector at the midplane of the detector flow channel at $0^{\circ}$ (bottom view) ( $a, b$, and $c$ denote the detector, photo, and ion chamber respectively.)

Figure 6.3.12: The $u-v$ velocity vectors and streamlines in the SA302 smoke detector at the midplane of the detector flow channel at $90^{\circ}$ (bottom view) ( $a, b$, and c denote the detector, photo, and ion chamber respectively.).

Figure 6.3.13: The $u-v$ velocity vectors and streamlines in the SA302 smoke detector at the midplane of the detector flow channel at $180^{\circ}$ (bottom view) (a, b, and c denote the detector, photo, and ion chamber respectively.)

Figure 6.3.14: The $u-v$ velocity vectors and streamlines in the SA302 smoke detector at the midplane of the detector flow channel at $270^{\circ}$ (bottom view) ( $a, b$, and c denote the detector, photo, and ion chamber respectively.)......

Figure 6.3.15: The $u-v$ velocity vectors and streamlines in the SA302 smoke detector at the midplane of the detector flow channel at $315^{\circ}$ (bottom view) (a, b, and c denote the detector, photo, and ion chamber respectively.)

Figure 6.4.1: The $u$ - $w$ velocity vectors in the UL Smoke Box test section at $y=0.25$ in. 103 
Figure 6.4.2: The $u$-w streamlines in the UL Smoke Box test section at $y=0.25 \mathrm{in}$. 104

Figure 6.4.3: The $u-w$ velocity vectors in the UL Smoke Box test section at $y=0.50$ in. 105

Figure 6.4.4: The $u$-w streamlines in the UL Smoke Box test section at $y=0.50 \mathrm{in}$. 106

Figure 6.4.5: The $u$ - $w$ velocity vectors in the UL Smoke Box test section at $y=1.0 \mathrm{in}$. 107

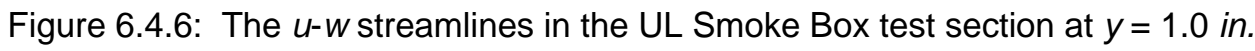
108

Figure 6.4.7: The $u$-w velocity vectors in the UL Smoke Box test section at $y=2.0 \mathrm{in}$. 109

Figure 6.4.8: The $u$-w streamlines in the UL Smoke Box test section at $y=2.0 \mathrm{in}$. 110

Figure 6.4.9: The $u$ - $w$ velocity vectors in the UL Smoke Box test section at $y=3.0 \mathrm{in}$. 111

Figure 6.4.10: The $u$-w streamlines in the UL Smoke Box test section at $y=3.0 \mathrm{in}$. 112

Figure 6.4.11: The $u$ - $w$ velocity vectors in the UL Smoke Box test section at $y=4.0$ in. 113

Figure 6.4.12: The $u-w$ streamlines in the UL Smoke Box test section at $y=4.0 \mathrm{in}$. 114

Figure 6.4.13: The $u-w$ velocity vectors in the UL Smoke Box test section at $y=5.0 \mathrm{in}$. 115

Figure 6.4.14: The $u$-w streamlines in the UL Smoke Box test section at $y=5.0 \mathrm{in}$. 116

Figure 6.4.15: The $u$-w velocity vectors in the UL Smoke Box test section at $y=6.0$ in. 117

Figure 6.4.16: The $u$-w streamlines in the UL Smoke Box test section at $y=6.0 \mathrm{in}$. 118

Figure 6.4.17: The $u-v$ velocity vectors and streamlines in the UL Smoke Box test section at $z=$ 3.0 in. 120

Figure 6.4.18: The $u-v$ velocity vectors and streamlines in the UL Smoke Box test section at $z=$ $4.0 \mathrm{in.}$

Figure 6.4.19: The $u-v$ velocity vectors and streamlines in the UL Smoke Box test section at $z=$ 5.0 in. 122

Figure 6.4.20: The $u-v$ velocity vectors and streamlines in the UL Smoke Box test section at $z=$ $6.0 \mathrm{in.}$

Figure 6.4.21: The $u-v$ velocity vectors and streamlines in the UL Smoke Box test section at $z=$ 7.0 in.

Figure 6.4.22: The $u-v$ velocity vectors and streamlines in the UL Smoke Box test section at $z=$ $8.0 \mathrm{in.}$ 125

Figure 6.4.23: The $u-v$ velocity vectors and streamlines in the UL Smoke Box test section at $z=$ 9.0 in. 126

Figure 6.4.24: The $u-v$ velocity vectors and streamlines in the UL Smoke Box test section at $z=$ 10.0 in (reverse angle view). 
Figure 6.4.25: The $u-v$ velocity vectors and streamlines in the UL Smoke Box test section at $z=$ 11.0 in (reverse angle view).

Figure 6.4.26: The $u-v$ velocity vectors and streamlines in the UL Smoke Box test section at $z=$ 12.0 in (reverse angle view).

Figure 6.4.27: The $u-v$ velocity vectors and streamlines in the UL Smoke Box test section at $z=$ 13.0 in (reverse angle view).

Figure 6.4.28: The $u-v$ velocity vectors and streamlines in the UL Smoke Box test section at $z=$ 14.0 in (reverse angle view).

Figure 6.4.29: The $u-v$ velocity vectors and streamlines in the UL Smoke Box test section at $z=$ 15.0 in (reverse angle view).

Figure 6.4.30: The $v$ - $w$ velocity vectors and streamlines in the UL Smoke Box test section at $x=$ $12.0 \mathrm{in}$

Figure 6.4.31: The $v$ - $w$ velocity vectors and streamlines in the UL Smoke Box test section at $x=$ 13.0 in.

Figure 6.4.32: The $v$ - $w$ velocity vectors and streamlines in the UL Smoke Box test section at $x=$ 14.0 in.

Figure 6.4.33: The $v-w$ velocity vectors and streamlines in the UL Smoke Box test section at $x=$ 15.0 in.

Figure 6.4.34: The $v$ - $w$ velocity vectors and streamlines in the UL Smoke Box test section at $x=$ 16.0 in.

Figure 6.4.35: The $v-w$ velocity vectors and streamlines in the UL Smoke Box test section at $x=$ $17.0 \mathrm{in.}$

Figure 6.4.36: The $v$ - $w$ velocity vectors and streamlines in the UL Smoke Box test section at $x=$ 18.0 in.

Figure 6.4.37: The $v$ - $w$ velocity vectors and streamlines in the UL Smoke Box test section at $x=$ 19.0 in (reverse angle view).

Figure 6.4.38: The $v$ - $w$ velocity vectors and streamlines in the UL Smoke Box test section at $x=$ 20.0 in (reverse angle view).

Figure 6.4.39: The $v$-w velocity vectors and streamlines in the UL Smoke Box test section at $x=$ 21.0 in (reverse angle view).

Figure 6.4.40: The $v-w$ velocity vectors and streamlines in the UL Smoke Box test section at $\mathrm{x}=$ 22.0 in (reverse angle view).

Figure 6.4.41: The $v$-w velocity vectors and streamlines in the UL Smoke Box test section at $x=$ 23.0 in (reverse angle view).

Figure 6.5.1: A surface plot of the inlet profile depicting the $x$, axial velocity as measured by hotwire anemometry.

Figure 6.6.1: A plot depicting grid independence for 5 computational models. 
Figure 6.6.2: A velocity contour comparison between the (a.) laminar and (b.) realizable $\kappa-\varepsilon$ turbulence models for the $x=12$ in approach plane.

Figure 6.6.3: A velocity contour comparison between the (a.) laminar and (b.) realizable $\kappa-\varepsilon$ turbulence models for the $x=15$ in approach plane.

Figure 6.6.4: A velocity contour comparison between the (a.) laminar and (b.) realizable $\kappa-\varepsilon$ turbulence models for the $y=0.50$ in plane (mid-plane of the detector's sensing elements).

Figure 6.6.5: A velocity contour comparison between the (a.) laminar and (b.) realizable $\kappa-\varepsilon$ turbulence models for the $y=0.50$ in plane (mid-plane of the detector's sensing elements).

Figure 6.6.6: A velocity contour comparison between the (a.) laminar and (b.) realizable $\kappa-\varepsilon$ turbulence models for the $x=18$ in plane, which is positioned at the center of the probe inlet.

Figure 6.6.7: A velocity contour comparison between the (a.) laminar and (b.) realizable $\kappa-\varepsilon$ turbulence models for the $z=9$ in plane detailing the velocity gradients induced by the probe inlet. 156

Figure 6.6.8: A velocity vector comparison between the (a.) laminar and (b.) realizable $\kappa-\varepsilon$ turbulence models for the $z=9$ in plane.

Figure 6.6.9: A comparison of velocity contours between the (a.) laminar and (b.) realizable $\kappa-\varepsilon$ turbulence models for the $x=18$ in plane visualizing the flow about the SA302.

Figure 6.6.10: A comparison between the (a.) laminar and (b.) realizable $\kappa-\varepsilon$ turbulence models for the $y=3.5$ in plane (mid-plane of the test section).

Figure 6.6.11: A velocity contour plot using the laminar model depicting the $y=0.50$ in plane for the $0^{\circ}$ orientation.

Figure 6.6.12: A velocity vector plot using the laminar model depicting the wake of the detector approaching the pressure outlet boundary in the $y=0.50$ in plane.

Figure 6.6.13: A velocity contour plot using the laminar model depicting the detector and its wake in the $y=0.50$ in plane for the $0^{\circ}$ orientation.

Figure 6.6.14: A velocity vector plot using the laminar model depicting the wake of the detector approaching the pressure outlet boundary in the $y=0.50$ in plane $\left(0^{\circ}\right.$ Orientation $) .$.

Figure 6.6.15: A velocity contour plot using the laminar model depicting the detector in the $y$ $=0.50$ in plane for the $0^{\circ}$ orientation.

Figure 6.6.16: A velocity vector plot using the laminar model depicting the detector in the $y=0.50$ in plane for the $0^{\circ}$ orientation.

Figure 6.6.17: A velocity contour plot using the laminar model depicting the ionization chamber in the $y=0.50$ in plane for the $0^{\circ}$ orientation.

Figure 6.6.18: A velocity vector plot using the laminar model depicting the ionization chamber in the $y=0.50$ in plane for the $0^{\circ}$ orientation. 
Figure 6.6.19: A velocity contour plot using the laminar model depicting the photoelectric chamber in the $y=0.50$ in plane for the $0^{\circ}$ orientation.

Figure 6.6.20: A velocity vector plot using the laminar model depicting the photoelectric chamber in the $y=0.50$ in plane for the $0^{\circ}$ orientation.

Figure 6.6.21: A velocity contour plot using the laminar model depicting the $x=12$ in approach plane.

Figure 6.6.22: A velocity contour plot using the laminar model depicting the $x=15$ in approach plane.

Figure 6.6.23: A velocity contour plot using the laminar model depicting the $x=18$ in plane (center cut of the SA302 detector and slightly off-center that of the MIC).......

Figure 6.6.24: A velocity contour plot using the laminar model depicting the $z=9$ in plane (center cut of the SA302 detector and probe inlet).

Figure 6.6.25: A velocity vector plot using the laminar model depicting the $z=9$ in plane (center cut of the SA302 detector and probe inlet).......

Figure 6.6.26: Test section flow visualization showing a pathline injection 1 in above the floor near the main inlet.

Figure 6.6.27: Test section flow visualization showing a pathline injection near the main inlet depicting the wake of the MIC.

Figure 6.6.28: Test section flow visualization showing the interaction using pathlines between the probe velocity inlet injection and the main inlet flow.

Figure 6.6.29: Test section flow visualization showing the combined interaction, using pathlines, of the presence of the MIC, SA302, and probe inlet.

Figure 6.6.30: Detector flow visualization showing the route of 100 pathlines injected 1 in above the floor near the entrance to the SA302 smoke detector ( $0^{\circ}$ orientation).

Figure 6.6.31: Detector flow visualization showing possible flow patterns inside the sensing elements of the SA302 ( $0^{\circ}$ orientation).

Figure 6.6.32: A velocity contour plot using the laminar model depicting the $y=0.50$ in plane for the $90^{\circ}$ orientation.

Figure 6.6.33: A velocity contour plot using the laminar model depicting the detector and its wake in the $y=0.50$ in plane for the $90^{\circ}$ orientation.

Figure 6.6.34: A velocity vector plot using the laminar model depicting the wake of the detector approaching the pressure outlet boundary in the $y=0.50$ in plane $\left(90^{\circ}\right.$ Orientation).

Figure 6.6.35: Detector flow visualization showing possible flow patterns inside the sensing elements of the SA302 $\left(90^{\circ}\right.$ orientation).

Figure 6.6.36: A velocity vector plot using the laminar model depicting the detector in the $y=0.50$ in plane for the $90^{\circ}$ orientation. 
Figure 6.6.37: A velocity contour plot using the laminar model depicting the ionization chamber in the $y=0.50$ in plane for the $90^{\circ}$ orientation.

Figure 6.6.38: A velocity vector plot using the laminar model depicting the ionization chamber in the $y=0.50$ in plane for the $90^{\circ}$ orientation.

Figure 6.6.39: A velocity contour plot using the laminar model depicting the photoelectric chamber in the $y=0.50$ in plane for the $90^{\circ}$ orientation.

Figure 6.6.40: A velocity vector plot using the laminar model depicting the photoelectric chamber in the $y=0.50$ in plane for the $90^{\circ}$ orientation.

Figure 6.6.41: Detector flow visualization showing the route of 100 pathlines injected 1 in above the floor near the entrance to the SA302 smoke detector ( $90^{\circ}$ orientation).

Figure 6.6.42: Detector flow visualization showing possible flow patterns inside the sensing elements of the SA302 ( $90^{\circ}$ orientation).

Figure 6.6.43: A velocity contour plot using the laminar model depicting the $y=0.50$ in plane for the $180^{\circ}$ orientation.

Figure 6.6.44: A velocity contour plot using the laminar model depicting the detector and its wake in the $y=0.50$ in plane for the $180^{\circ}$ orientation.

Figure 6.6.45: A velocity vector plot using the laminar model depicting the wake of the detector approaching the pressure outlet boundary in the $y=0.50$ in plane $\left(180^{\circ}\right.$ Orientation)........

Figure 6.6.46 Detector flow visualization showing possible flow patterns inside the sensing elements of the SA302 (180 orientation).

Figure 6.6.47 A velocity vector plot using the laminar model depicting the detector in the $y=0.50$ in plane for the $180^{\circ}$ orientation.

Figure 6.6.48 A velocity contour plot using the laminar model depicting the ionization chamber in the $y=0.50$ in plane for the $180^{\circ}$ orientation.

Figure 6.6.49 A velocity vector plot using the laminar model depicting the ionization chamber in the $y=0.50$ in plane for the $180^{\circ}$ orientation.

Figure 6.6.50 A velocity contour plot using the laminar model depicting the photoelectric chamber in the $y=0.50$ in plane for the $180^{\circ}$ orientation......

Figure 6.6.51 A velocity vector plot using the laminar model depicting the photoelectric chamber in the $y=0.50$ in plane for the $180^{\circ}$ orientation.

Figure 6.6.52 Detector flow visualization showing the route of 100 pathlines injected 1 in above the floor near the entrance to the SA302 smoke detector ( $180^{\circ}$ orientation).

Figure 6.6.53 Detector flow visualization showing possible flow patterns inside the sensing elements of the SA302 $\left(180^{\circ}\right.$ orientation).

Figure 6.6.54: A velocity contour plot using the laminar model depicting the $y=0.50$ in plane for the $270^{\circ}$ orientation. 
Figure 6.6.55: A velocity contour plot using the laminar model depicting the detector and its wake in the $y=0.50$ in plane for the $270^{\circ}$ orientation.

Figure 6.6.56: A velocity vector plot using the laminar model depicting the wake of the detector approaching the pressure outlet boundary in the $y=0.50$ in plane ( $270^{\circ}$ Orientation)............

Figure 6.6.57 Detector flow visualization showing possible flow patterns inside the sensing elements of the SA302 $\left(270^{\circ}\right.$ orientation).

Figure 6.6.58 A velocity vector plot using the laminar model depicting the detector in the $y=0.50$

in plane for the $270^{\circ}$ orientation.

Figure 6.6.59 A velocity contour plot using the laminar model depicting the ionization chamber in the $y=0.50$ in plane for the $270^{\circ}$ orientation.

Figure 6.6.60 A velocity vector plot using the laminar model depicting the ionization chamber in the $y=0.50$ in plane for the $270^{\circ}$ orientation.

Figure 6.6.61 A velocity contour plot using the laminar model depicting the photoelectric chamber in the $y=0.50$ in plane for the $270^{\circ}$ orientation.

Figure 6.6.62 A velocity vector plot using the laminar model depicting the photoelectric chamber in the $y=0.50$ in plane for the $270^{\circ}$ orientation.

Figure 6.6.63 Detector flow visualization showing possible flow patterns inside the sensing elements of the SA302 $\left(270^{\circ}\right.$ orientation).

Figure 6.6.64 Detector flow visualization showing the route of 100 pathlines injected 1 in above the floor near the entrance to the SA302 smoke detector (270 orientation).

Figure 6.6.65: A velocity contour plot using the laminar model depicting the $y=0.50$ in plane for the $315^{\circ}$ orientation.

Figure 6.6.66: A velocity contour plot using the laminar model depicting the detector and its wake in the $y=0.50$ in plane for the $315^{\circ}$ orientation.

Figure 6.6.67: A velocity vector plot using the laminar model depicting the wake of the detector approaching the pressure outlet boundary in the $y=0.50$ in plane ( $315^{\circ}$ Orientation).......

Figure 6.6.68 Detector flow visualization showing possible flow patterns inside the sensing elements of the SA302 (315 orientation).

Figure 6.6.69 A velocity vector plot using the laminar model depicting the detector in the $y=0.50$

in plane for the $315^{\circ}$ orientation.

Figure 6.6.70 A velocity contour plot using the laminar model depicting the ionization chamber in the $y=0.50$ in plane for the $315^{\circ}$ orientation.

Figure 6.6.71 A velocity vector plot using the laminar model depicting the ionization chamber in the $y=0.50$ in plane for the $315^{\circ}$ orientation.

Figure 6.6.72 A velocity contour plot using the laminar model depicting the photoelectric chamber in the $y=0.50$ in plane for the $315^{\circ}$ orientation. 
Figure 6.6.73 A velocity vector plot using the laminar model depicting the photoelectric chamber in the $y=0.50$ in plane for the $315^{\circ}$ orientation.

Figure 6.6.74 Detector flow visualization showing the route of 100 pathlines injected 1 in above the floor near the entrance to the SA302 smoke detector ( $315^{\circ}$ orientation)......

Figure 6.6.75 Detector flow visualization showing possible flow patterns inside the sensing elements of the SA302 ( $315^{\circ}$ orientation).

Figure 6.7.1: UL Smoke Box $u$ velocity comparisons between LDA and CFD at the $y=1$ in, $x=12$ in position spanning the transverse, $z$ direction.

Figure 6.7.2: UL Smoke Box $v$ velocity comparisons between LDA and CFD at the $y=1$ in, $x=12$ in position spanning the transverse, $z$ direction.

Figure 6.7.3: UL Smoke Box $w$ velocity comparisons between LDA and CFD at the $y=1$ in, $x=$ 12 in position spanning the transverse, $z$ direction.

Figure 6.7.4: UL Smoke Box $u$ velocity comparisons between LDA and CFD at the $y=1$ in, $x=13$ in position spanning the transverse, $z$ direction.

Figure 6.7.5: UL Smoke Box $v$ velocity comparisons between LDA and CFD at the $y=1$ in, $x=13$ in position spanning the transverse, $z$ direction.

Figure 6.7.6: UL Smoke Box $w$ velocity comparisons between LDA and CFD at the $y=1$ in, $x=$ 13 in position spanning the transverse, $z$ direction.

Figure 6.7.7: UL Smoke Box $u$ velocity comparisons between LDA and CFD at the $y=1$ in, $x=14$ in position spanning the transverse, $z$ direction.

Figure 6.7.8: UL Smoke Box $v$ velocity comparisons between LDA and CFD at the $y=1$ in, $x=14$ in position spanning the transverse, $z$ direction.

Figure 6.7.9: UL Smoke Box $w$ velocity comparisons between LDA and CFD at the $y=1$ in, $x=$ 14 in position spanning the transverse, $z$ direction.

Figure 6.7.10: UL Smoke Box $u$ velocity comparisons between LDA and CFD at the $y=1$ in, $x=$ 15 in position spanning the transverse, $z$ direction.

Figure 6.7.11: UL Smoke Box $v$ velocity comparisons between LDA and CFD at the $y=1 \mathrm{in}, x=$ 15 in position spanning the transverse, $z$ direction.

Figure 6.7.12: UL Smoke Box $w$ velocity comparisons between LDA and CFD at the $y=1$ in, $x=$ 15 in position spanning the transverse, $z$ direction.

Figure 6.7.13: UL Smoke Box $u$ velocity comparisons between LDA and CFD at the $x=18$ in, $y=$ 3 in position spanning the transverse, $z$ direction.

Figure 6.7.14: UL Smoke Box $v$ velocity comparisons between LDA and CFD at the $x=18$ in, $y=$ 3 in position spanning the transverse, $z$ direction.

Figure 6.7.15: UL Smoke Box $w$ velocity comparisons between LDA and CFD at the $x=18$ in, $y=$ 3 in position spanning the transverse, $z$ direction. 
Figure 6.7.16: UL Smoke Box $u$ velocity comparisons between LDA and CFD at the $x=18$ in, $y=$ 4 in position spanning the transverse, $z$ direction.

Figure 6.7.17: UL Smoke Box $v$ velocity comparisons between LDA and CFD at the $x=18$ in, $y=$ 4 in position spanning the transverse, $z$ direction.

Figure 6.7.18: UL Smoke Box $w$ velocity comparisons between LDA and CFD at the $x=18$ in, $y=$ 4 in position spanning the transverse, $z$ direction.

Figure 6.7.19: UL Smoke Box $u$ velocity comparisons between LDA and CFD at the $x=18$ in, $y=$ 5 in position spanning the transverse, $z$ direction.

Figure 6.7.20: UL Smoke Box $v$ velocity comparisons between LDA and CFD at the $x=18$ in, $y=$

5 in position spanning the transverse, $z$ direction.

Figure 6.7.21: UL Smoke Box $v$ velocity comparisons between LDA and CFD at the $x=18$ in, $y=$ 5 in position spanning the transverse, $z$ direction.

Figure 6.7.22: UL Smoke Box $u$ velocity comparisons between LDA and CFD at the $x=21$ in, $y=$ 1 in position spanning the transverse, $z$ direction.

Figure 6.7.23: UL Smoke Box $v$ velocity comparisons between LDA and CFD at the $x=21$ in, $y=$

1 in position spanning the transverse, $z$ direction.

Figure 6.7.24: UL Smoke Box $w$ velocity comparisons between LDA and CFD at the $x=21$ in, $y=$ 1 in position spanning the transverse, $z$ direction.

Figure 6.7.25: UL Smoke Box $u$ velocity comparisons between LDA and CFD at the $x=22$ in, $y=$

1 in position spanning the transverse, $z$ direction.

Figure 6.7.26: UL Smoke Box $v$ velocity comparisons between LDA and CFD at the $x=22$ in, $y=$ 1 in position spanning the transverse, $z$ direction.

Figure 6.7.27: UL Smoke Box $w$ velocity comparisons between LDA and CFD at the $x=22$ in, $y=$ 1 in position spanning the transverse, $z$ direction.

Figure 6.7.28: UL Smoke Box $u$ velocity comparisons between LDA and CFD at the $x=23$ in, $y=$ 1 in position spanning the transverse, $z$ direction.

Figure 6.7.29: UL Smoke Box $v$ velocity comparisons between LDA and CFD at the $x=23$ in, $y=$ 1 in position spanning the transverse, $z$ direction.

Figure 6.7.30: UL Smoke Box $v$ velocity comparisons between LDA and CFD at the $x=23$ in, $y=$ 1 in position spanning the transverse, $z$ direction.

Figure 6.8.1: SA302 smoke detector $u$ velocity comparisons between LDA and CFD at $z=9.75$ in, spanning the $x$, axial direction ( $0^{\circ}$ orientation).

Figure 6.8.2: SA302 smoke detector $w$ velocity comparisons between LDA and CFD at $z=9.75$

in, spanning the $x$, axial direction ( $0^{\circ}$ orientation).

Figure 6.8.3: SA302 smoke detector $u$ velocity comparisons between LDA and CFD at $z=8.50$ in, spanning the $x$, axial direction ( $0^{\circ}$ orientation). 
Figure 6.8.4: SA302 smoke detector $w$ velocity comparisons between LDA and CFD at $z=8.50$ in, spanning the $x$, axial direction ( $0^{\circ}$ orientation).....

Figure 6.8.5: SA302 smoke detector $u$ velocity comparisons between LDA and CFD at $z=8.00$ in, spanning the $x$, axial direction ( $0^{\circ}$ orientation).

Figure 6.8.6: SA302 smoke detector $w$ velocity comparisons between LDA and CFD at $z=8.00$ in, spanning the $x$, axial direction ( $0^{\circ}$ orientation)........

Figure 6.8.7: SA302 smoke detector $u$ velocity comparisons between LDA and CFD at $z=9.75$ in, spanning the $x$, axial direction ( $90^{\circ}$ orientation).

Figure 6.8.8: SA302 smoke detector $w$ velocity comparisons between LDA and CFD at $z=9.75$ in, spanning the $x$, axial direction ( $90^{\circ}$ orientation).

Figure 6.8.9: SA302 smoke detector $u$ velocity comparisons between LDA and CFD at $z=9.25$ in, spanning the $x$, axial direction ( $90^{\circ}$ orientation).

Figure 6.8.10: SA302 smoke detector $w$ velocity comparisons between LDA and CFD at $z=9.25$ in, spanning the $x$, axial direction $\left(90^{\circ}\right.$ orientation).......

Figure 6.8.11: SA302 smoke detector $u$ velocity comparisons between LDA and CFD at $z=8.50$ in, spanning the $x$, axial direction ( $90^{\circ}$ orientation)......

Figure 6.8.12: SA302 smoke detector $u$ velocity comparisons between LDA and CFD at $z=8.50$ in, spanning the $x$, axial direction $\left(90^{\circ}\right.$ orientation).

Figure 6.8.13: A velocity vector comparison between (a.) LDA and (b.) CFD ( $0^{\circ}$ Orientation)......... 221

Figure 6.8.14: A velocity vector comparison between (a.) LDA and (b.) CFD (90 Orientation)....... 221

Figure 6.8.15: A velocity vector comparison between (a.) LDA and (b.) CFD (180 Orientation)..... 222

Figure 6.8.16: A velocity vector comparison between (a.) LDA and (b.) CFD (270 Orientation)..... 222

Figure 6.8.17: A velocity vector comparison between (a.) LDA and (b.) CFD (315 Orientation) ..... 223

Figure B.1: Pressure loss across an insect screen as a function of constant flow velocity through a 0.475 in pipe.

Figure B.2: The screen porosity experiment in its basic form. The actual layout of the experiment consisted of 12 screens in series at various stations within the pipe. 


\section{LIST OF SYMBOLS}

\begin{tabular}{|c|c|c|c|}
\hline$\underline{\text { SYMBOL }}$ & DESCRIPTION & $\frac{\text { BRITISH }}{\underline{\text { UNITS }}}$ & SI UNITS \\
\hline$a$ & Characteristic Number Parameter & -- & -- \\
\hline$b$ & Characteristic Size Parameter & -- & -- \\
\hline$D$ & Diameter of Particle & $f t$ & $m$ \\
\hline DNS & Direct Numerical Simulation & -- & -- \\
\hline$F$ & Body Force & $l b_{f}$ & $N$ \\
\hline$G$ & Force of Gravity & $l b_{f}$ & $N$ \\
\hline$H W A$ & Hot-Wire Anemometry & -- & -- \\
\hline$I$ & Current & -- & -- \\
\hline$k$ & Iteration Parameter & -- & -- \\
\hline$L$ & Characteristic Detector Length & $f t$ & $m$ \\
\hline$L D A$ & Laser Doppler Anemometry & -- & -- \\
\hline MIC & Measuring Ionization Chamber & -- & -- \\
\hline \multirow[t]{2}{*}{$N$} & \multirow[t]{2}{*}{ Number Concentration } & \multirow[t]{2}{*}{ Particle No./ft ${ }^{3}$} & Particle \\
\hline & & & No. $/ m^{3}$ \\
\hline$O b$ & Obscuration & $1 / f t$ & $1 / m$ \\
\hline$O D$ & Optical Density & $1 / f t$ & $1 / m$ \\
\hline$p$ & Pressure & $l b_{f} / f^{2}$ & $N / m^{2}$ \\
\hline$Q$ & Number of Particles & -- & -- \\
\hline RANS & Reynolds Averaged Navier-Stokes & -- & -- \\
\hline $\operatorname{Re}$ & Reynolds Number & -- & -- \\
\hline$S$ & Signal & -- & -- \\
\hline$t$ & Time & sec & sec \\
\hline$u$ & Axial component Velocity & $\mathrm{ft} / \mathrm{sec}$ of $\mathrm{ft} / \mathrm{min}$ & $\mathrm{m} / \mathrm{sec}$ \\
\hline$u^{\prime}$ & Velocity Fluctuation & $\mathrm{ft} / \mathrm{sec}$ of $\mathrm{ft} / \mathrm{min}$ & $\mathrm{m} / \mathrm{sec}$ \\
\hline$U$ & Freestream Velocity & $\mathrm{ft} / \mathrm{sec}$ or $\mathrm{ft} / \mathrm{min}$ & $\mathrm{m} / \mathrm{sec}$ \\
\hline $\bar{U}$ & Average Mean Velocity & $\mathrm{ft} / \mathrm{sec}$ or $\mathrm{ft} / \mathrm{min}$ & $\mathrm{m} / \mathrm{sec}$ \\
\hline$U L$ & Underwriters Laboratories, Inc. & -- & -- \\
\hline
\end{tabular}




$\begin{array}{llll}v & \text { Vertical Component Velocity } & \mathrm{ft} / \mathrm{sec} \text { of ft/min } & \mathrm{m} / \mathrm{sec} \\ V & \text { Volume Concentration } & \mathrm{lb} / \mathrm{ft} & \mathrm{kg} / \mathrm{m}^{3} \\ V A R & \text { Variable } & -- & -- \\ w & \text { Transverse Component Velocity } & \mathrm{ft} / \mathrm{sec} \text { of ft/min } & \mathrm{m} / \mathrm{sec} \\ x & \text { Axial Coordinate } & \text { in or } \mathrm{ft} & \mathrm{m} \\ y & \text { Vertical Coordinate } & \text { in or } \mathrm{ft} & \mathrm{m} \\ z & \text { Transverse Coordinate } & \text { in or } \mathrm{ft} & \mathrm{m}\end{array}$

\section{GREEK}

\begin{tabular}{|c|c|}
\hline$\Delta$ & “Change in” \\
\hline$\sum$ & Riemann Sum \\
\hline$\psi$ & Shape Distribution $\mathrm{P}$ \\
\hline$\Gamma$ & Coagulation Coeffici \\
\hline$\varepsilon$ & Kinetic Energy Visce \\
\hline$\kappa$ & Turbulent Kinetic En \\
\hline$\rho$ & Density \\
\hline$\tau$ & Detector Time Const \\
\hline$\lambda$ & Attachment Coeffici \\
\hline$\sigma$ & Standard Deviation \\
\hline$\overline{\bar{\sigma}}$ & Stress Tensor \\
\hline$v$ & Standard Velocity \\
\hline$\in$ & Convergence Criteri \\
\hline \multicolumn{2}{|c|}{ SUBSCRIPTS } \\
\hline 0 & Initial \\
\hline$e$ & Exterior of Detector \\
\hline$g$ & Geometric Mean \\
\hline$i$ & Interior of Detector \\
\hline$P$ & Particle \\
\hline$r$ & Response \\
\hline rms & Root Mean Square \\
\hline
\end{tabular}


$x$

$\mathrm{y}$

$\mathrm{Z}$
Axial Vector

Vertical Vector

Transverse Vector
$-$

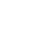




\section{CHAPTER 1: INTRODUCTION}

Fire protection engineering (FPE), compared to many other engineering disciplines, is still a fairly underdeveloped science. Although several methodologies have been developed to evaluate various fire protection devices, few published academic studies have resulted in direct industry application. The main objective of this study focuses on the application of modern fluids engineering tools to provide flow characterization and performance prediction capabilities for spot type smoke detectors operating within a test apparatus for the detectors. Often, the fire protection community is charged with the task of predicting the response time of spot-type smoke detectors. Normally the first indication of fire, early smoke detection is paramount in the quest to save the greatest number of lives in a fire emergency. This study examines the past attention brought to predicting detector response, along with an innovative technique that could accelerate detector prototype design iterations through the use of computational fluid dynamics (CFD). CFD, in short, is an emerging scientific tool that provides a numerical approach to solving fluid mechanics problems such as the complex flow characteristics entering a smoke detector.

The motivation for this project is the need for the development of a virtual test bed to become an eventual tool in forecasting the particle concentration levels both on the interior and surrounding flow regimes of a spot-type smoke detector. By tracking these concentration levels, analytical formulas can potentially be tailored to relate the calculated concentration to a specific sensing technique being used within the detector - ultimately predicting detector response results. This project is primarily concerned with initial stages of development in flow characterization. The major benefit of a virtual model is the potentially reduced time to market for detectors from the conceptual to manufacturing. It is hoped that the results presented in this study will further the application of CFD in the design process of smoke detectors.

The preliminary efforts carried out for this research center around examining the flow field characteristics associated with a spot-type smoke detector through experimentation. It has been theorized that, if the flow field was modeled computationally and reasonably validated with experimental results, numerical particle concentration studies from the same computational model could be used to potentially track the alarm performance of similar type 
detectors with moderately altered geometries. In general, particle concentration studies involving both the internal and external flow field are very important in that they help evaluate the sensitivity of the detector. A major interest lies in eventually comparing particle concentrations in the external, approaching flow field to the smoke concentrations measured within the detector's actual sensing elements at a particular instant in time. Ideally, one would want these two zones to have the same concentration of particles. It is understood that upon the buildup of smoke, however, the internal and external concentrations will never be identical. This is because the required settling conditions for the detector's sensors to alarm properly, resulting in a need for proper enclosure, and thus generating lag in the system. Over the past 30 years, mathematical models have been developed in an attempt to functionalize this offset relationship with respect to various sensor types, as well as for the entire detector itself to the free stream. Due to the chaotic nature of different types of fires, however, defining a well-posed analytical model can prove quite challenging. Parameters such as detector position and orientation with respect to the smoke source play a factor in varied concentration results as well, ultimately leading to a range of response times.

The deliverable for this investigation is a CFD model, validated and verified by experimental velocity measurements. Experimental results were gathered using a variety of data acquisition techniques in a test section developed by a non-profit organization called Underwriters Laboratories (UL) Inc. UL is an independent firm that is well known for its consumer product safety assessment, and over the years has developed a testing environment for spot-type smoke detectors - properly known as the UL Smoke Box. The UL Smoke Box, in essence, is a relatively small, very low-speed wind tunnel. Its design intentions revolved around creating a measurable, repeatable testing environment against which all spot-type smoke detectors can be formally evaluated. The smoke detector model being tested and modeled is the BRK Brands, Inc. SA302. The SA302 was chosen primarily due to the research sponsor's interest in its performance, as well as its unique dual sensor technology and innovative design. The SA302's two different types of sensing devices that will be analyzed individually are its ionization and photoelectric (light-scattering) sensors. The detector shares the UL Smoke Box with more elaborate and precise smoke sensing devices by which these two sensor outputs are compared to separately, namely the measuring ionization chamber (MIC) residing in the test section and the beam smoke detecting system. 
Tests are also carried out for different detector orientations, in a design attempt to make an alarm condition independent of the main flow direction's entry angle.

This entire study can be broken down into two main phases, the experimental phase along with the CFD modeling phase. As one will later see, confidence in the CFD model cannot exist without the experimental results because experimentation has been used to help condition and validate the model. However, the CFD model is the applicatory extension of this research ultimately giving this entire venture its purpose. During the experimental phase, laser Doppler anemometry (LDA) measurements were made recording velocities and turbulence intensities in all three dimensions around the near flow field of the detector. LDA data were also recorded in two dimensions on an imaginary plane passing through the flow passage within the detector including the inside of the two smoke sensing devices. Later on, hot-wire anemometry measurements were taken to develop the computational UL Smoke Box inlet profile upstream of the limiting point at which LDA measurements were physically possible. Other, less involved experimental work was conducted to study flow through porous elements inside the detector. 
The computational effort was spearheaded by computer-aided design (CAD) drawings of the detector supplied by the manufacturer using a powerful CAD package known as Solidworks $^{\circledR 20}$. The test section geometry was then meshed in a grid generation program entitled Gambit ${ }^{\circledR 18}$ where the test section environment was discretized using a variety of meshing procedures. Finally, the computational grid was exported to a robust, commercial CFD package called Fluent ${ }^{\circledR 18}$ where the model was numerically solved for its flow patterns. The computational model was calibrated using the boundary conditions defined experimentally through the LDA and hot-wire anemometry data. After flow characterization was fully achieved computationally using a variety of exploratory solution techniques, concentration studies using discrete phase models can eventually be employed for particle analysis. In an attempt to better understand smoke entry resistance, mass concentration results from the CFD model could potentially be analyzed with respect to the work of past researchers in order to close modeling gaps within reason, where possible. Although characterization of the air velocities were the main deliverables for this report, Fluent ${ }^{\circledR 18}$ is versatile in that it can model heat transfer and combustion along with a variety of other useful parameters where a complete fire model could take ultimate advantage.

The following literature review begins by providing information on the work done thus far on closely related topics, noting their successes as well as their shortcomings. Very little research has been published using computational fluid dynamics with regard to internally modeling spot-type smoke detectors. Thusly, the majority of past work reviewed studies the various types of models attempted and employed in the past (i.e. mathematical models, experimental systems, etc.). Afterwards, an overview of the science being explored from a classical fluid mechanics standpoint will be presented, covering topics such as the nature of smoke, and the analytical formulas that contribute an academic perspective to smoke detection. Basic technical information on different spot-type smoke detectors will then be addressed, with a detailed discussion describing the internal geometry and sensing elements of the SA302. Subsequently, the UL Smoke Box along with its components will be described in its entirety. After sufficient background has been established, the experimental procedure as well as the computational modeling sequence will be examined, offering indepth coverage where needed. The reporting of results and conclusions for all phases of the research will be discussed bringing the study to an eventual close. The material presented 
hereafter assumes the reader has a basic understanding of classical fluid dynamic principles, differential calculus with exposure to partial differential equations, and a working knowledge of personal computers - namely CAD. It is hoped that this venture not only provides an academic perspective to the science of smoke detection and CFD, but secondly, act as an initial stage in the conceptual development of future spot-type smoke detectors. 


\section{CHAPTER 2: REVIEW OF LITERATURE}

\subsection{BACKGROUND}

According to Pucci, ${ }^{1}$ although many papers have been written on spot-type smoke detector response, the basic reality still remains that such prediction methods are at best inexact. Because the life safety of building occupants is at stake, reviewers such as Underwriters Laboratories, Inc. (UL) need constant assurance that these prediction models are as reliable as possible. They are also scrutinized based upon how input conditions and safety margins can vary based upon the limitations of the prediction method used. Post-fire inspectors are equally concerned with whether or not detectors alarmed properly and swiftly in an attempt to deliver an advanced warning. ${ }^{1}$

Many specialists in this particular field oppose the theoretical modeling of smoke detector response entirely. Issues such as inconsistent measuring techniques between the detector mechanism and the prediction standard often warrant this valid concern. However, when a collection of studies has been used in concert with well-posed conditions as part of an overall analysis, these concerns can usually be allayed. Nonetheless, detector response is still far from refined due to the large interdependency of variables that come into play. Complex factors requiring accountability such as the nature of fire spread and growth, type and arrangement of combustibles, and size and geometry of not only the fire test room, but the detector itself can all lead to substantial modeling inaccuracies. Also, detector specific variables cannot be easily compared because different detectors respond to different types of fires. The two sensor types in the detector focused on in this study, for example, operate on two totally different principles. Internal geometries such as the arrangement of the sensors and flow deflectors all change the predicted response behavior as well. In general, the experimental data reported by manufacturers and testing laboratories are restricted in terms of characterizing the response of an individual detector to the many different types of fires that it may be exposed to during its lifetime. ${ }^{1}$

Currently, there are no straightforward methods for directly modeling the response of the two most common types of sensors in the spot-type smoke detector; namely, the ionization and photoelectric sensor. Although full-bodied theories exist, the lack of certainty in experimental data typically characterizes these methods as unusable. When dealt this 
predicament, the next best alternative lies in estimating their response by comparing the amount of smoke it takes at a detector location for that particular unit to go into alarm. These measurement systems are categorized as performance-based rather than prescriptive-based because standards are built around a detector's ability to alarm according to a certain smoke condition, relying specifically on the performance of its sensing elements. The most common method for measuring quantities of smoke is through a science known as obscuration (or optical density per unit length), which will be discussed in greater detail in Section 2.3, along with other accepted smoke sensing techniques. Even though the concept of obscuration is straightforward, neither the photoelectric nor the ionization sensors use this principle to detect smoke particles. ${ }^{1}$

An extension of the obscuration method takes a look at the somewhat complex phenomena known as smoke entry resistance, which was proposed by Heskestad ${ }^{1}$ and discussed in more detail in Section 2.4. This concept acknowledges the fact that spot-type smoke detectors undergo a time lag as the smoke inside the detector tries to reach the same smoke condition as that outside, upstream of the detector. Although several researchers have attempted to model this phenomenon, discrepancies over certainty and repeatability keep these ideas from becoming standards in performance and design. ${ }^{1}$

In summary, it is overwhelmingly clear that more information is needed on the optical characteristics produced by different fuels, specifically how the smoke produced from them reflects and refracts the light at the wavelength used by the detectors. Although the past attention brought to this subject matter should not go unacknowledged, Pucci states "merits of modeling [spot-type smoke detector] response quantitatively will see greater attention as performance-based requirements replace prescriptive ones.”"

The remainder of this review will delve deeper into the characteristic nature of smoke and how it affects the alarm sensitivity of spot-type smoke detectors by analyzing its physical characteristics. Afterwards, an overview of current smoke measurement technologies will be discussed, focusing mainly on the UL Smoke Box measurement standards, as well as the measuring schemes used inside the SA302 detector. The theory and advancements behind smoke entry resistance will then be fully introduced, along with some analytic insight on the present state of smoke detector modeling. Because the focus of this study is the development of a computationally-based prediction method, strides using CFD will be addressed as well. 


\subsection{THE NATURE OF SMOKE}

In order to understand how smoke detectors respond to an established presence of smoke particles, it is first necessary to investigate the physical nature of smoke. Simply stated by Oldweiler, ${ }^{2}$ smoke is made up of both small solid particles called an aerosol, and liquid droplets dispersed in the gaseous products of burning substances. Stoichiometric combustion always produces gaseous water vapor and carbon dioxide; however, carbon monoxide along with numerous other compounds can contribute to the anatomy of smoke due to incomplete combustion and the type of fuel being used. ${ }^{3}$

Smoke detection is chiefly concerned with the aerosol element of smoke. ${ }^{3}$ Because the UL Smoke Box test section is located well downstream of the smoke source, the smoke aerosols being modeled lie outside of the combustion zone. ${ }^{4}$ According to Lee and Mulholland, ${ }^{3}$ a very important area of smoke detector design technology is describing smoke in terms of the characteristics able to be sensed by the detector. Properties such as particle size distribution, mass concentration, number concentration, and the refraction index of smoke are significant because certain sensing mechanisms are highly dependent on these traits not only quantitatively, but qualitatively as well. Smoke aerosol can have a wide range of characteristics depending on the condition in which it was burned. Shapes can vary from spherical to chain agglomerates, and optical properties can change based upon whether the fire is more flaming than smoldering. The optical properties of smoke for most smoldering fires tend to be very light absorbing causing black, sooty smoke to form, with particle sizes on the order of 1 to 5 microns and larger. Flaming fires, on the other hand, will more likely create more transparent, light-absorbing smoke patterns with particle sizes much smaller ranging in diameters from 0.005 to 1 micron. Because detector sensitivity is a complicated function of all of the aforementioned properties, it is difficult to designate one characteristic of smoke as the optimum standard for measuring the presence of smoke. However, in comparing all properties of smoke, the most significant property of the aerosol is its size distribution. In general, most detectors will alarm to particle diameters between 0.005 and 5 micron. ${ }^{3}$

It should be noted here that there are a variety of analytical methods that can be employed in analyzing particle size distributions - namely the algebraic, log-normal, and Junge distributions as described in Lee and Mulholland. ${ }^{3}$ A mathematical way to commonly 
describe the geometric distribution of particles is through a log distribution, $\Delta N / \Delta \log D_{p}$ versus $D_{p}$, where $D_{p}$ and $\Delta N$ denote the particle diameter and the number of particles per $\mathrm{cm}^{3}$, respectively. The quantity $\Delta N$ represents the number of aerosol particles in the particle diameter size range from $\log D_{P}$ to $\log D_{P}+\Delta \log D_{P}$. The $\Delta$ term was implemented because the data obtained from the measuring devices were based upon an average within each discrete range. Figure 2.2.1 below depicts the size distribution for a typical smoke. ${ }^{3}$

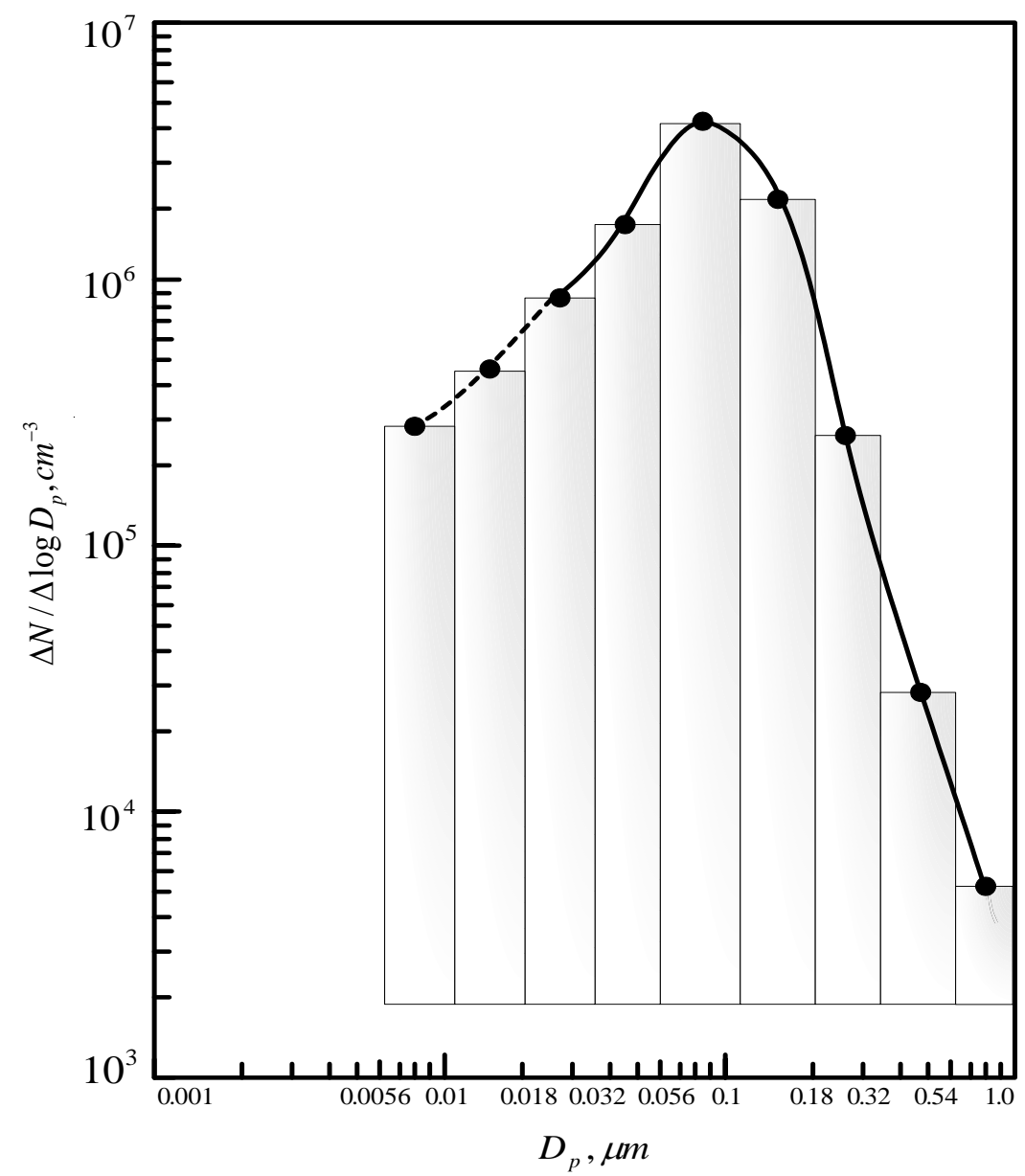

Figure 2.2.1: Discrete size ranges as measured by an electrical aerosol analyzer. The dotted portion of the curve denotes an area of large uncertainty. ${ }^{3}$

Without going into elaborate detail, it was observed that the algebraic distribution, which is a modified version of the Junge distribution, can represent the particle distribution measured in the UL Smoke Box the best for a variety of different atmospheric aerosols. Equation 2.2.1 depicts the algebraic relationship: ${ }^{3}$ 


$$
\frac{d N}{d \log D}=\frac{a D^{3}}{\left(b+D^{3}\right)^{2+\Psi}},
$$

where $\psi$ is a measure of the shape of the distribution through particle analysis, and parameters $a$ and $b$ can be related to two measurable characteristics in number concentration $N$, and volume concentration $V$ of the aerosol. One is able to provide a very good estimate of the size distribution for a particular smoke field by applying the above algebraic distribution. Because this study is primarily concerned with smoke from smoldering fires created in the UL Smoke Box, it has been found from previous experimental work that as $\varepsilon$ goes to zero, Equation 2.2.1 transforms given the measured variables, $N$ and $V$, into, ${ }^{3}$

$$
\frac{d N}{d \log D}=1.38 \frac{V D^{3}}{\left(D^{3}+0.2 \frac{V}{N}\right)^{2}}
$$

Because Equation 2.2.1 is integrated over an infinite amount of particle sizes, the size distribution function in Equation 2.2.2 breaks down for large particle sizes greater than about $5 \mu m^{3}$

Besides particle size distribution, mass and number concentration per unit volume are important measurable properties of smoke as well. For specific particle sizes, the response characteristics of both the ionization and photoelectric sensors are very linear functions of both the number and mass concentrations of the smoke in question up to a limiting quantity. Problems associated with measuring the number concentration of various smokes can create problems due to coagulation. ${ }^{3}$ Because smoke aerosols are dynamic with respect to their particle size distribution function, the particles tend to collide and stick together due to Brownian motion. The results of this behavior cause the number concentration to decrease while keeping the overall mass of the aerosol fixed. ${ }^{4}$ The basic parameter for describing the constant rate of coagulation is the coagulation coefficient $\Gamma$, defined as follows,

$$
\frac{d N}{d t}=-\Gamma N^{2}
$$

and $d N / d t$ represents the change in number concentration with respect to time.

Mass concentration, on the other hand, is a much more stable extrinsic property to measure that lends to more accuracy. The only major loss from measuring mass concentration is the wall loss effects as smoke tends to adhere to the interior walls of the 
measuring chamber over time. According to Lee and Mulholland ${ }^{3}$, losses comparing mass concentration and number concentrations are on the order of $25 \%$ over a 2 hour period compared to at minimum a factor of 10 in a $1.8 \mathrm{~m}^{3}$ cubical aerosol chamber. Both smoke properties were measured to determine particle diameter in comparison to other methods. For an in-depth look into the measuring methods used, the reader is referred to Section 2.3, and for further reading Lee and Mulholland. ${ }^{3}$

With regard to the UL Smoke Box, UL places no restrictions based on the absorption coefficient, particle size, and most importantly - concentrations, in the test chamber. Because it is highly evident that particle size as well as light absorption properties of the smoke strongly affects the sensitivity of the detector, the characteristics of the smoke in the UL Smoke Box is of critical importance. After conducting a standard test as dictated by the UL $217^{5}$ manual and waiting for adequate time for mixing and settling (30-35 seconds), the following mass and number concentration results were reported in Table 2.2.1. The $g$ term denotes maximum and minimum diameter ranges in deriving the geometric mean. The column denoting UL $217^{5}$ obscuration will be discussed in more detail in Section 2.3, when UL Smoke Box measurement techniques are fully introduced. The acronyms PMM, EAA, and CNM stand for particle mass monitor, electrical aerosol analyzer, and condensation nuclei monitor, respectively. $D_{g}$ is denoted as the geometric mean diameter. ${ }^{3}$

Table 2.2.1: Measured and derived parameters of smokes in the UL Smoke Box chamber. ${ }^{3}$

\begin{tabular}{|c|c|c|c|c|c|c|}
\hline Instrument & $\begin{array}{c}\text { UL 217 } \\
\text { Obscuration }\end{array}$ & PMM & EAA & EAA & CNM & D $_{\mathbf{g}}$ \\
\hline Source & $\mathbf{O D} / \mathbf{m}$ & \multicolumn{2}{|c|}{ Mass Conc. $\left(\mathrm{mg} / \mathrm{m}^{3}\right)$} & \multicolumn{2}{|c|}{ Number Conc. $10^{6} \mathrm{~cm}^{-3}$} & $\mu m$ \\
\hline Lamp Wick & 0.066 & 42 & 21 & 3.4 & 2.5 & 0.14 \\
\hline Lamp Wick & 0.015 & 10 & 6 & 1.4 & 1.0 & 0.12 \\
\hline Heptane & 0.017 & 5 & 5 & 0.9 & 0.3 & 0.16 \\
\hline
\end{tabular}

\subsection{SMOKE MEASUREMENT TECHNIQUES}

According to Schifiliti and Pucci, ${ }^{6}$ there are four principle types of smoke detectors available on the consumer market; specifically, light obscuration, light-scattering (photoelectric), ionization, and cloud chamber. The light-scattering and ionization detectors are normally associated with spot-type smoke detectors because the mechanics of such systems can be inexpensively produced and sized for easy residential employment. Obscuration type detectors are often associated with line type detectors, able to sense smoke 
anywhere along a line between a projector and a receiver. Cloud chamber detection systems use an aspirated air sampling system by analyzing air drawn through a series of input junctions. $^{6}$

There are two main branches of the light obscuration method; namely, the temperature approximation analysis and the optical density method. Problems associated with temperature approximation lie mainly in the added interdependency between the varying burn temperatures associated with specific fuels that cause fluctuations in obscuration measurements. According to Geimann and Gottuk, ${ }^{7}$ the temperature approximation method has been heavily criticized stating that numerical studies have shown that this relationship has little or no basis.

Optical density obscuration detectors, commonly known as photometers, work by projecting a beam of light from a source, across an open void where smoke can pass through, targeted at a receiver on the opposite end. Figure 2.3 .1 below depicts the basic setup.

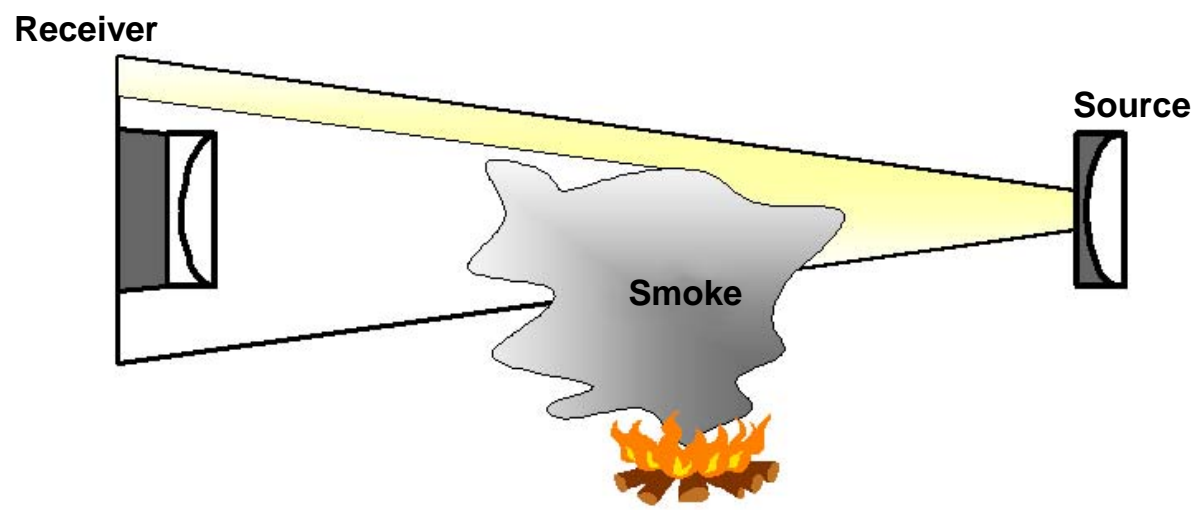

Figure 2.3.1: The basic layout of the light obscuration (projected beam) smoke detection system commonly known as a photometer.

By seeding the void space across the focused light beam with smoke particles, the light is obscured by the reflection, absorption, and refraction of light rays disrupting the transmitting light, and is measured through receiver current decline as a function of obscuration percentage. The system is said to have $0 \%$ obscuration when the receiver receives $100 \%$ of the source beam's light, and conversely $100 \%$ obscuration if enough smoke particles were to cloud the void space to where zero light from the source reaches the receiver. Schifiliti and Pucci ${ }^{6}$ mathematically define it as follows:

$$
\% \text { Obscuration }(O b)=\frac{100\left(I_{0}-I\right)}{I_{0}}
$$


where $I_{0}$ is the initial current flow with no smoke present, and $I$ is the current flow generated in the presence of smoke. When reporting results of obscuration, it is best to work in units of optical density, $O D$, which are represented by this log relationship,

$$
O D=\log _{10}\left(\frac{I_{0}}{I}\right)=-\log _{10}\left(\frac{I}{I_{0}}\right)
$$

These systems normally measure the rate of change of the received light, with slow reductions normally disregarded as dust buildup and major obscuration gradients characteristic of some real obstruction. This usually results more so in false alarms rather than true alarm signals. Although most systems are placed on opposite sides of a measuring space, mirrors can be used to change the orientation of the beam. Although a fairly direct measuring technique, spot-type obscuration detectors have been tried but have met little success. This is due in large part to the heavy loss of resolution in the signal due to the shrinking measuring space. Accuracy is lost because the obscuration signal decreases drastically, losing stability. A system like this finds a good home in a system such as the UL Smoke Box, where the system components can be spread on the order of feet apart as opposed to inches along a lengthy test chamber, where a much larger measuring zone can be used to produce reasonable results. ${ }^{6}$

Pucci $^{1}$ further expresses the limitations of the optical density method in citing that other major sources of error can be attributed to changing the fuel and smoke characteristics, which ultimately affect a detector's overall sensitivity ratings. The wavelength of light can also play a major role in affecting obscuration measurements as well. By altering the light's wavelength, the strength of the beam can fluctuate, ultimately increasing or decreasing the optical density measurement for the same smoke particle stream. ${ }^{1}$

The main objective of Lee and Mulholland ${ }^{3}$ was to use various aerosol analyzers to measure particle size distributions and mass concentrations by using the UL $217^{5}$ testing standard. UL provides no testing requirements based upon particle size, concentration, or absorption coefficient inside of the UL Smoke Box. From their tests with an electronic aerosol analyzer inside the UL Smoke Box briefly mentioned in Section 2.2, they were able to conclude consistent relationships between optical density mass concentration, number concentration, as well as particle size distribution of lamp wick and heptane generated aerosols. In the measurements using a smoldering lamp wick to create enough smoke to 
reach a desired obscuration level ranging from 0.005 to 0.08 optical density per meter, a 3035 second wait period ensured homogeneous mixing for each test. Mean rates of change in light attenuation during the concentration build-up period spanned 0.02 to 0.07 optical density per meter for about an 8-minute period. Large deviations existed in the generation rates are most likely due to the non-uniformity of the lamp wick geometry. The reader is referred to the work of Lee and Mulholland ${ }^{3}$ for in-depth coverage as to their measurement procedure, along with the precision and accuracy levels they achieved with their instrumentation. In summary, Lee and Mulholland ${ }^{3}$ state the limitations of the UL Smoke Box in the following statements:

1. Aerosols with the same light attenuation values in the UL Smoke Box can show significant concentration of size distribution differences which yield a varied effect on detector response.

2. Size distribution of smoke changes as smoke concentration increases in the UL Smoke Box chamber. The peak size increase is on the order of $20-40 \%$ as obscuration varies from 0.015 to 0.06 Optical Density per meter, with deficiencies removed by using a dependable steady-state aerosol generator.

Figure 2.3.2 below depicts the linear relationship witnessed when measuring mass concentration and obscuration percentage for two different types of fuels burned. Mass concentration will be important in the study conducted here because the CFD model being implemented will report concentration levels on a mass basis. Hence, one of the long term research goals is to develop a consistent relationship between experimental UL Smoke Box obscuration measurements with that of mass concentrations. The discrepancies in data points available is due to the complex data collecting nature of analyzing the particle concentrations because of the needed settling time for accurate measurements within the test chamber. ${ }^{3}$ 


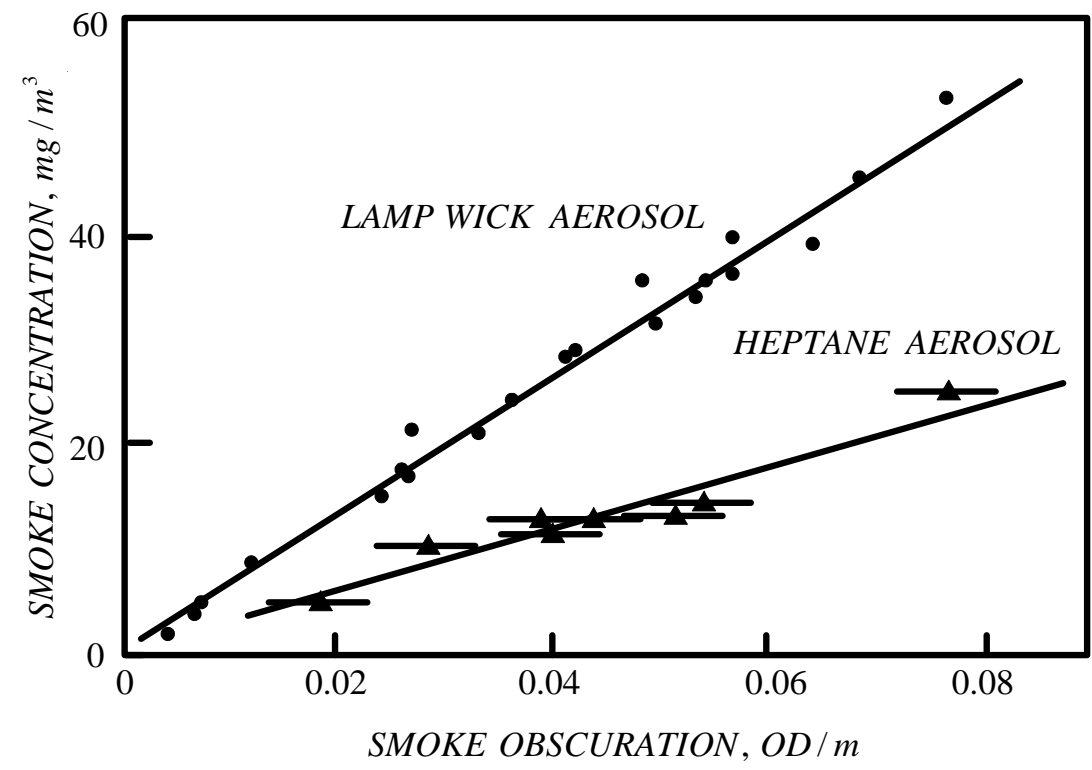

Figure 2.3.2: A plot showing the linear relationship between mass concentration and smoke obscuration per meter for two different fuels burned in the UL Smoke Box. ${ }^{3}$

The first sensor inside the SA302 smoke detector being modeled in this study is the photoelectric sensor. Light-scattering (photoelectric) smoke detecting devices operate by directing a light source into a control volume as shown in Figure 2.3.3.

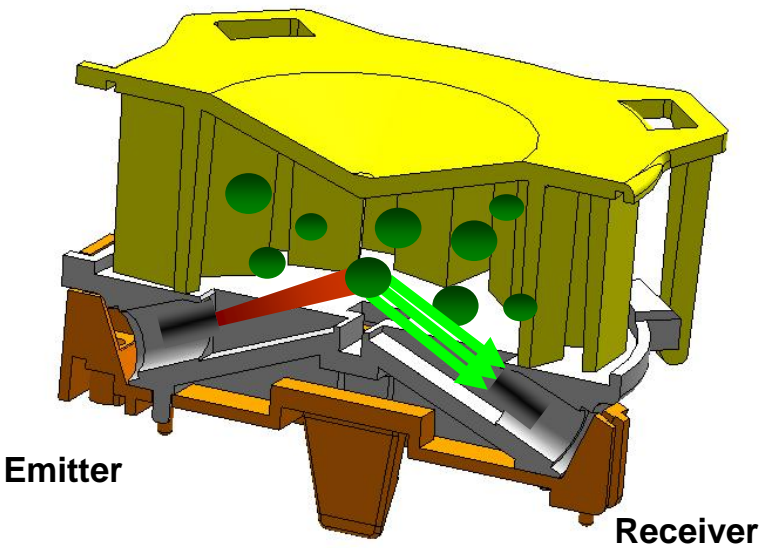

(a.)

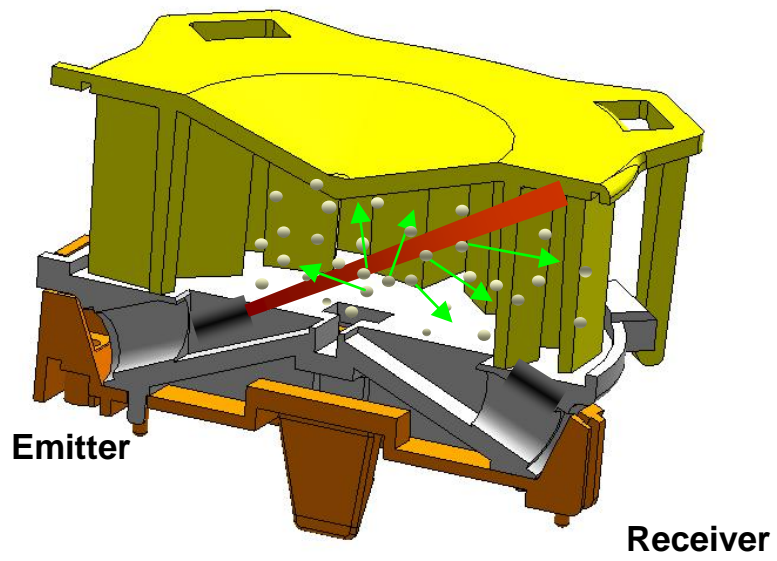

(b.)

Figure 2.3.3: A conceptual model of how the photoelectric sensor works comparing its effectiveness between (a.) larger, more reflective smoke particles and (b.) smaller, more absorbent smoke particles.

A light sensor is oriented such that it is at some relative angle to the infrared light source. If smoke is present in the chamber, light is scattered and refracted by the smoke, ultimately projecting light toward the receiver positioned at a similar angle opposing the measuring 
volume. Photoelectric sensors reach an "alarm condition" by monitoring a current increase due to the transmission of light between the source and receiver. The signal, $S$, produced by a light-scattering detector can be described by the following equation,

$$
S=\frac{\Delta I}{I_{0}}
$$

where $I$ and $I_{0}$ represent the change in output current and no smoke output current, respectively. These types of sensors normally run in a pulsed mode in a standard spot-type detector in order to save battery life. Although photoelectric sensors have come along way in the past few years, most still react, with some degree, to all wavelengths of light present. Thus, intricate labyrinths are often built around the sensing volumes as to halt external light from entering, but still allow smoke to enter. Aspirated sensors are also built with the principles of light-scattering detection in mind, but are not often implemented in the common residential spot-type detector due to additional cost and power requirements. With regard to Figures 2.3.3a and b, photoelectric sensors, much like other popular sensing devices, are sensitive to the size of the smoke particle it is sensing. As alluded to earlier in Section 2.2, photoelectric sensors are designed to alarm more accurately with smoldering fires in mind. In Figure 2.3.3a, larger (on the order of 1 to 5 micron), highly reflective smoke particles with greater surface area scatter incident light very readily, causing a generalized quicker alarm response. Conversely as shown in Figure 2.3.3b, smaller, more absorbent smoke particles tend to scatter less infrared light, rendering the sensor less sensitive to smaller particle sizes leading to slower response times. ${ }^{6}$

Even though ionization sensors are commonly found in spot-type smoke detectors as well, they work on a completely different operating principle then that of the obscuration and light-scattering detection methods. A low direct current voltage is supplied across two conducting plates separated by an air gap considered the measuring chamber. In normal circumstances, there is no electrical current flowing through the air between the plates. However, a minute radioactive source (usually Americium 241) emits a high energy alpha particle trace into the chamber. These high-speed alpha particles detach electrons off the air molecules passing through the sensor leaving the air molecules with a negative charge. The positive ions, in turn move towards the opposing negative plate across from the source alpha particles, while the negative ions propagate towards the positive plate, generating a small 
current flow on the order of pico-amps $(p A)$. As smoke particles enter the chamber, a few ions bond with smoke particles creating heavier molecules. The sizable increase in mass of the ion slows down the transfer of energy, allowing small air currents to transport the ionized particles out of the ionization chamber before charge can be transferred to the opposite plate. As more and more ionized particles are created, they are more apt to combine with one another, creating more and more of these type molecules. By exporting charge out of the chamber, a net loss in current flow is sensed between the two electrical plates that can then ultimately be sensed by the circuitry, creating an alarm condition based upon a minimum allowable voltage reading. The size of the smoke particle plays a major role in the ability for the charged ions to attach to them. Hence, smaller, less dense smoke particles from flaming fires (0.001 to 1 micron) are more apt to be sensed effectively using this operating principle. For ionization sensors, the signal produced by the chamber has been experimentally shown to be a product of particle diameter $D$ and the number of particles $Q$,

$$
S \propto Q D
$$

and for smoke with varying particle size,

$$
S \propto \sum_{k} Q_{k} D_{k}
$$

Exact signals produced by ionization sensors are represented by a more complex equation available in the literature referenced by Schifiliti and Pucci, ${ }^{6}$ requiring an additional parameter known as the chamber constant. Figure 2.3.4 characterizes the operating principles behind the ionization detector. ${ }^{6}$ 


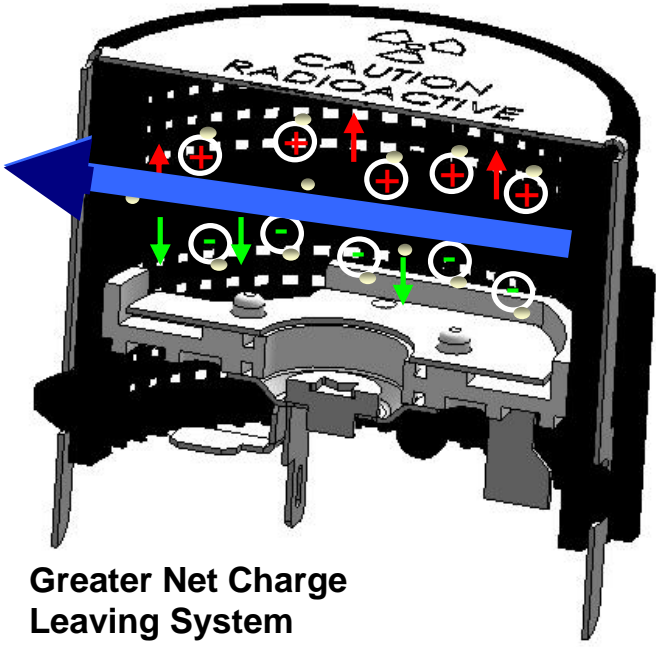

(a.)

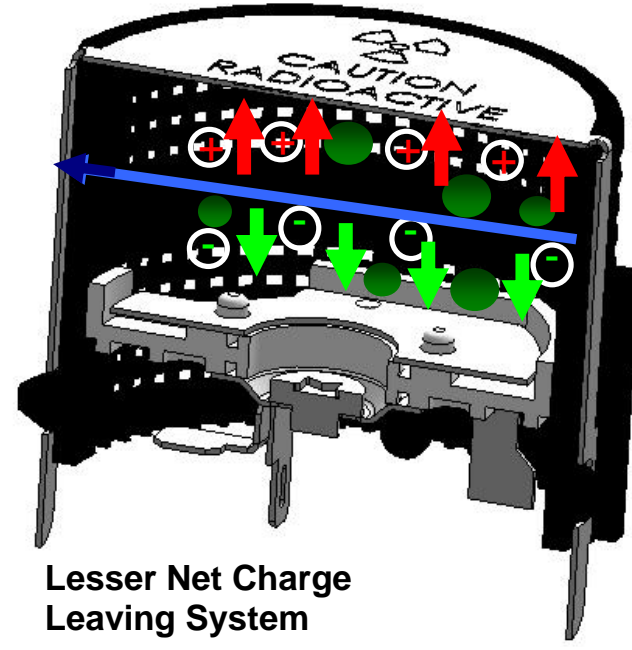

(b.)

Figure 2.3.4: A conceptual model of how an ionization sensor works comparing its effectiveness between (a.) smaller, more transparent smoke particles and (b.) larger, sootier particles. $^{6}$

It is of interest to show here after both the light-scattering and ionization sensor working principles have been introduced, how particle size experimentally affects each of their sensitivities. Reflected in the plot shown in Figure 2.3.5, commercial ionization type sensors can normally respond to particles as small as $0.05 \mu \mathrm{m}$, whereas light-scattering photoelectric-type detectors are virtually insensitive to sizes less than $0.1 \mu \mathrm{m}$. As one can see, as particle size increases, so does detector sensitivity for both type sensors. ${ }^{3}$ 


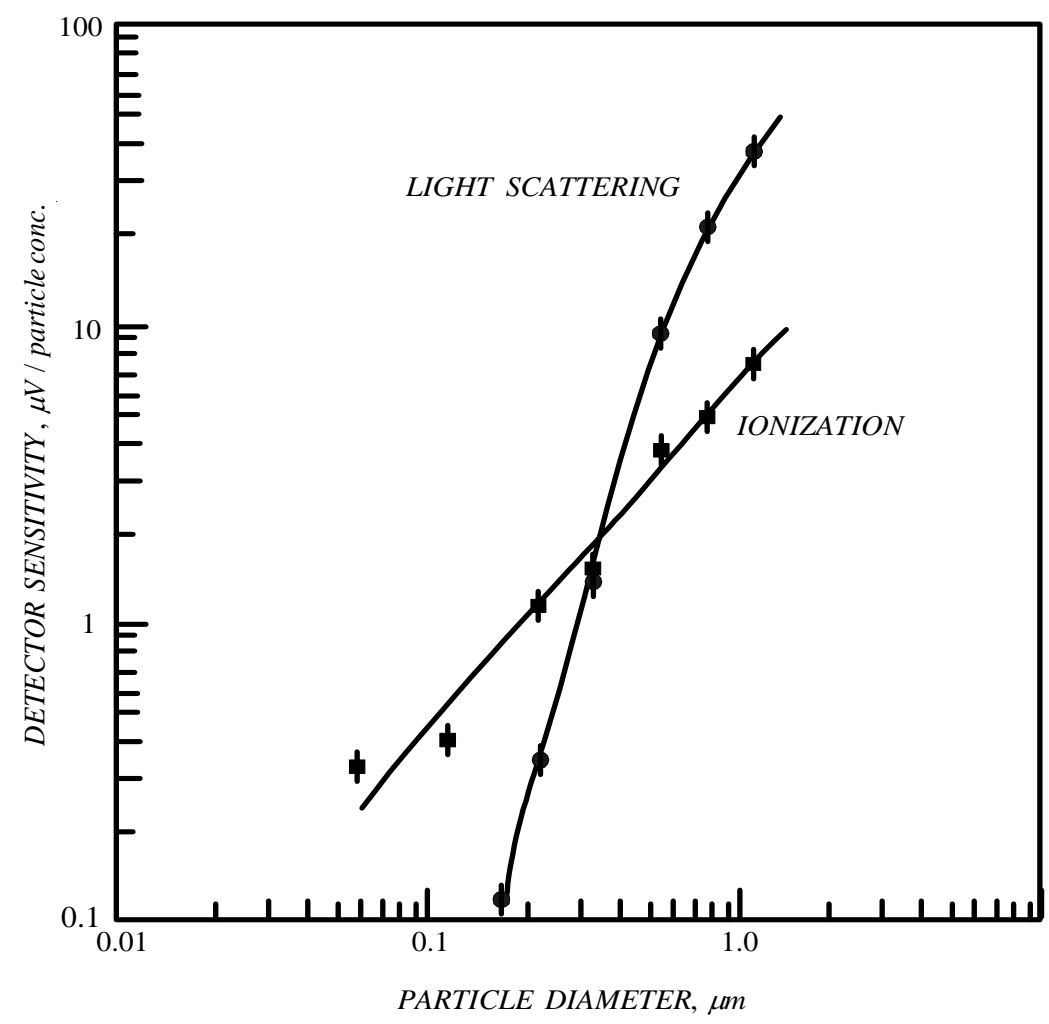

Figure 2.3.5: A logarithmic plot comparing detector sensitivity with respect to particle diameter for both the light-scattering (photoelectric) and ionization sensors. ${ }^{3}$

Cloud chamber smoke detectors, although not a primary focus of this study, make up another variety of spot-type smoke detectors extending on the principles of light obscuration. By sampling air conditioned by increasing the humidity close to the saturation point, the pressure can be lowered resulting in an overall cooling of the air sample in an aspirated setting, normally remotely located to the smoked area in question. This extended cooling causes excess moisture to condense on any smoke particles in the chamber, allowing a light obscuration device to be used to sense the presence of the water droplets. The resulting cloud formation present in the air sample yields nearly uniform water droplets, and when properly calibrated the optical density can eventually be translated into a particle concentration.

According to Cleary et al., ${ }^{8}$ the ability for a smoke detector to not respond to stimuli that are generated from non-threatening sources is equally as important as it is for it to respond to an actual fire situation. Ionization and photoelectric sensors react to virtually all particles entering and exiting their sensing chambers, and by themselves, cannot distinguish between true smoke particles from a nuisance aerosol. Because of this, a device known as 
the fire-emulator/detector-evaluator $(\mathrm{FE} / \mathrm{DE})$ is used to produce both real smoke and nuisance aerosols to measure the sensitivity and error of each sensor. The analog output of a multi-sensor detector similar to that of the SA302 used in this study is measured as a function of aerosol type, among the other popular methods to compare detector responses to a flaming fire. Comparisons were also made to the extinction of laser light in the FE/DE test section at optical densities up to 0.12 optical density per meter. Results from this study indicate that from the two fake aerosols used (peanut oil clouds and clay dust), that both the ionization and photoelectric sensors performed well with their predicted particle size ranges in question. However, there is a need to expand the number of nuisance aerosols beyond just two, and an ultimate need to expand aerosol characterization beyond size distribution to number and mass densities, as well as optical properties. ${ }^{8}$

\subsection{SMOKE DETECTOR MODELING}

There are several approaches that can be taken when trying to predict the response of various sensing devices. On one hand, researchers tend to take a more analytical route by mathematically modeling the operating principle at work. On the other, modelers try to take the detector geometry as a whole into account, leading to models based primarily upon entry resistance induced by the detector housing. This section will deal primarily with introducing a few mathematical models available in predicting the response of the sensors used in this study, with an in-depth look of what entry resistance really means, and how it can be described analytically.

In modeling light obscuration, manufacturer specifications normally refer to what levels of total obscuration or total optical density to which the detector would respond. A multitude of fire models estimate optical density in a uniform upper layer or volume, referred to as zone modeling. The optical density across the entire length of the measuring beam is then calculated by multiplying the optical density by the path length. This methodology assumes homogeneous distribution through the entire path, which is an assumption that can only be truly valid under settled conditions. Another way to model the response of projected beam obscuration is by calculating unit optical density, where discretized points in the smoke field between the source and receiver are measured. This form of modeling is known as field modeling, because the summation of each of the discrete points over the length of the beam denotes the entire optical density measurement. ${ }^{9}$ 
Over the past few years, there has been noticeable advancement in the design and optimization of ionization smoke detectors. According to Litton, ${ }^{10}$ numerical solutions of steady-state equations governing an ionization chamber's operation have provided a solid foundation for theoretical approximation, generally known as the reduced source approximation. The reduced source approximation, without going into excessive detail, explicitly defines the reduction in sensor current due to smoke particle entry as a function of chamber geometry, operating current, the physical properties of the smoke, along with sensor electrode voltages. Comparisons carried out by Litton using the approximation method were held in close agreement with experimental data done earlier by Hosemann, who is referenced through Litton. ${ }^{10}$ An analysis of Hosemann's results indicate that it is possible to design a detector having increased reliability without losing overall sensitivity. Litton ${ }^{10}$ goes on by presenting a theoretical comparison of his approximation algorithm with respect to a $4 \%$ obscuration per foot test. ${ }^{10}$

In its most fundamental form, the reduced source approximation method can be derived as,

$$
\Delta I / I_{0}=1.0-\frac{1.0}{\lambda N}\left(1-e^{-\lambda N}\right)
$$

where $N$ is the number of particles per $\mathrm{cm}^{-3}$, with $\lambda$ defined as a sensitivity parameter in terms of an attachment coefficient between the particles and ions. The reader is referred to Litton ${ }^{10}$ for in-depth coverage on the complex functionality involved in Equation 2.4.1. Conclusively, Litton states that noise considerations play a major role in the design optimization procedure for ionization sensors, and through reduced source approximation, ionization chambers can be made to not only be sensitive, but extremely reliable. ${ }^{10}$ In another paper by Litton ${ }^{11}$, the author delves into more of a rigorous mathematical model in creating a modeled electric field dominated by convective transport, providing exact solutions for different electrode geometries undergoing a diverse range of operating principles. He then goes on to further develop the reduced source approximation method, providing quantitative data using numerical solution techniques for predicting the performance of a detector in the presence of smoke particles. ${ }^{11}$

With regard to modeling light-scattering (photoelectric) sensors, theories are not as refined and developed as those compared to ionization theory. Photoelectric sensing theory 
is currently in need of more information on the burning characteristics of various fuels. Although researchers like Meacham ${ }^{9}$ have shown it is possible to model the response of photoelectric detectors, his recommended tests have not been further developed and incorporated into fire test programs. It has been cited time and time again that a lightscattering detector responds uniquely to different optical densities for different types of smoke. ${ }^{9}$ In essence, more research must be dedicated to conjoining generalized lightscattering (optical) theory with fire protection engineering.

Unlike other models presented thus far, particle entry resistance attempts to model the response characteristics of the entire smoke detector by employing a parameter known as the $L$-number. According to Oldweiler, ${ }^{2}$ with the progress of a fire near a smoke detector, the buildup of smoke within the sensing elements of the detector lags in comparison to the buildup of smoke on the outside of the detector because of the rate of convective diffusion present. Due to the particle entry induced by the presence of the detector, there will always be a concentration gradient as smoke is entrained into the sensing chamber(s). Figure 2.4.1 depicts this phenomenon.

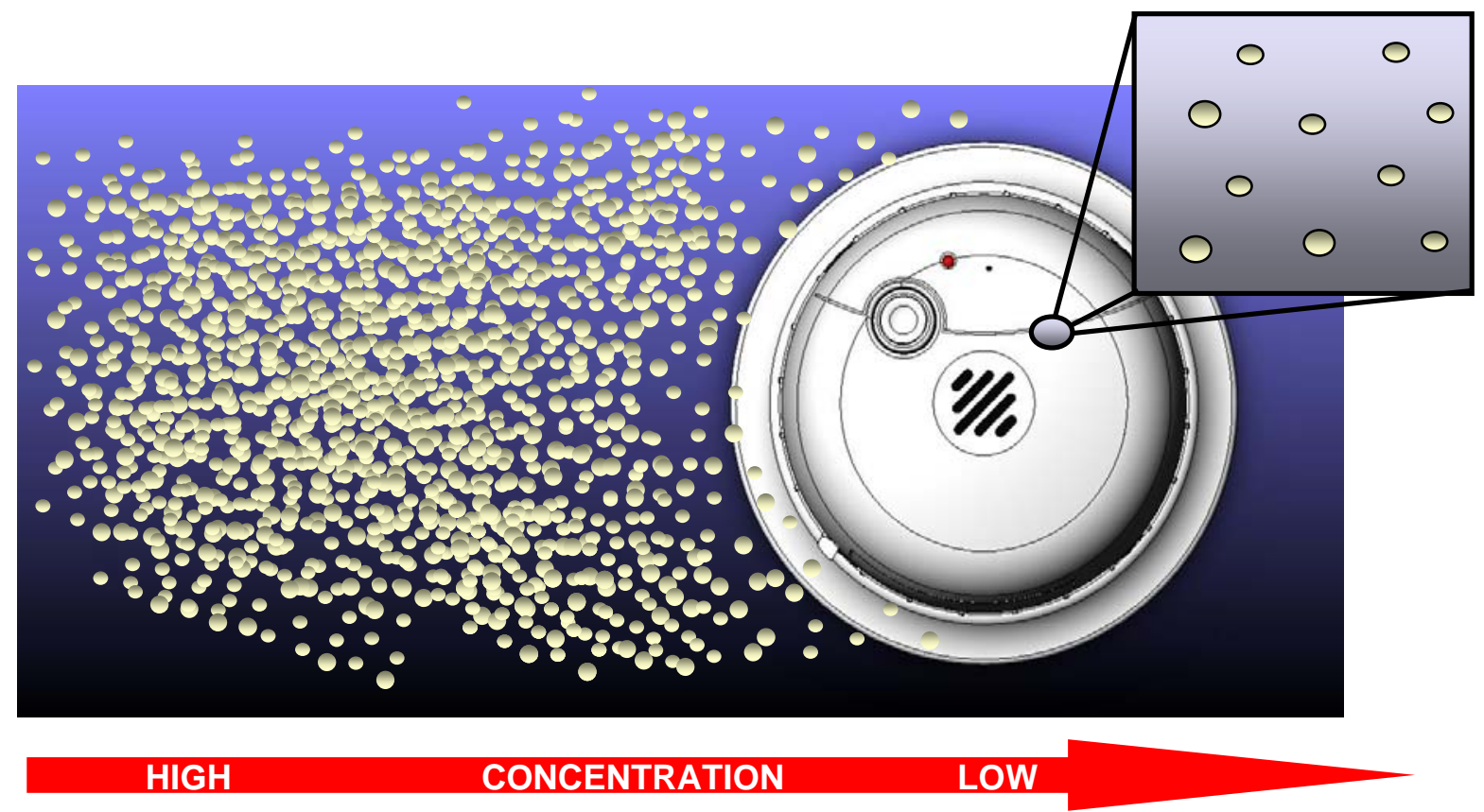

Figure 2.4.1: A simple sketch visualizing the concentration of smoke particles as they approach a detector upstream. 
The convective transport of smoke from a fire is the result of a momentum exchange created by a buoyant smoke plume introduced to a wall presence and natural convective currents. In 1975, Heskestad introduced the concept of lag time $\tau$, which is defined as the time it would take the concentration of smoke inside the sensing chamber of a detector to equal the concentration of smoke outside, upstream of the detector, denoted by the following equation, ${ }^{2}$

$$
\tau=\frac{L}{U}
$$

where $L$ is the detector's characteristic length, and $U$ is the free stream velocity. $L$ is a major function of geometry, and can be though of, in essence, as the theoretical distance the smoke would have to travel in order for the smoke concentrations to be the same inside as well as outside the detector. Employing the detector time constant $\tau$ and the measurable quantity of optical density per unit length, the following first order differential equation can be defined, ${ }^{9}$

$$
\frac{d\left(O D_{i}\right)}{d t}=\frac{1}{\tau}\left(O D_{e}-O D_{i}\right)
$$

where $\mathrm{OD}_{i}$ is the optical density per unit length inside the detector chamber and $\mathrm{OD}_{e}$ is the optical density per unit length outside of the detector. If the time constant and optical density outside of the detector are kept constant, Equation 2.4.3 can be solved. In combining the optical density required for alarm response inside the detector, the following response equation can be determined,

$$
O D_{r}=O D_{e, 0}+\tau\left(\frac{d\left(O D_{e}\right)}{d t}\right)\left\{1-\exp \left[-O D_{r} \frac{1}{\tau}\left(\frac{d\left(O D_{e}\right)}{d t}\right)\right]\right\}
$$

where $\mathrm{O} D_{r}$ is the optical density required for alarm response. Substituting Equation 2.4.2 into 2.4.4 finally yields the following,

$$
O D_{r}=O D_{e, 0}+\frac{L}{U}\left(\frac{d\left(O D_{e}\right)}{d t}\right)\left\{1-\exp \left[-O D_{r} \frac{1}{\tau}\left(\frac{d\left(O D_{e}\right)}{d t}\right)\right]\right\}
$$

In analyzing the components of Equation 2.4.5, the exponential term with respect to the other terms is negligible. Hence, the impact of entry resistance can be easily shown from the following,

$$
O D_{r}=O D_{e, 0}+\frac{L}{U}\left(\frac{d\left(O D_{e}\right)}{d t}\right)
$$


According to Equation 2.4.6, as optical density increases outside a detector, the optical density inside the detector will lag behind if entry resistance is present. According to Schifiliti $^{9}$ several past researchers have graphed test data to determine the $L$-number for a multitude of smoke detectors, namely Heskestad, Bjorkman, Marrion, and Oldweiler. ${ }^{2}$ The reader is referred to Schifiliti $^{9}$ for more detail into their work. For now, Table 2.4.1 summarizes the range of characteristic $L$-numbers from their research. ${ }^{9}$

Table 2.4.1: A summary of the range of characteristic $L$-numbers from multiple researchers. ${ }^{9}$

\begin{tabular}{|c|c|c|}
\hline Researcher & Ionization Detector $\boldsymbol{L}(\mathbf{m})$ & Photoelectric Detector $\boldsymbol{L}(\mathbf{m})$ \\
\hline Heskestad & 1.8 & $15(\mathrm{a})$ \\
\hline Bjorkman et al. & $3.2+/-0.2(\mathrm{~b})$ & $5.3+/-2.7(\mathrm{c})$ \\
\hline Marrion & Not Tested & $7.2(\mathrm{~d}), 11.0-13.0(\mathrm{e}), 18.4(\mathrm{f})$ \\
\hline Oldweiler & $4.0-9.5(\mathrm{~g}), 4.3-14.2(\mathrm{~h})$ & Not Tested \\
\hline
\end{tabular}

Notes: a.) Older style detector with an intricate labyrinth.

b.) L-number determined by best fit from 3 velocities.

c.) L-number based upon a single test velocity and a limited number of tests.

d.) Low L-number at low test velocity.

e.) Range of L-numbers for several fuel types and detector positions.

f.) L-number increased by adding a "fence" to further impede smoke entry.

g.) Range of L-numbers for a multitude of velocities using a simplified entry resistance equation.

h.) Range of L-numbers for a variety of velocities using full-fledged entry resistance equation.

The numbers listed in Table 2.4.1 obviously show that more work is needed on the study of velocity effects on the $L$-number. The large increase in the $L$-number at seemingly low velocities could possibly indicate entry resistance is largely a function of smoke particle size. It is also possible and has been theorized by Oldweiler, ${ }^{2}$ that L-number is a function of the smoke momentum at low speeds, meaning time lag would be inversely proportional to the velocity squared. Because validation of the $L$-number has yet to be established, researchers and test laboratories such as UL do not measure and report the $L$-number in their findings. ${ }^{9}$ 
A two parameter mixing chamber time response model proposed by Cleary and Chernovsky et al. ${ }^{12}$ has been recommended for situations in which the velocity is changing along with minor changes through proportionality constants based upon detector geometry and changes in ambient temperature and pressure. Using laser light to measure smoke extinction, flows were measured ranging from $0.03 \mathrm{~m} / \mathrm{sec}$ to $0.6 \mathrm{~m} / \mathrm{sec}$ with detector response times ranging from $5 \mathrm{sec}$ to $100 \mathrm{sec}$ for the slowest air velocities. In creating a model with measurable proportionality constants, based upon build-up smoke dwell time and mixing time within the sensing chamber, Cleary arrived at some plausible results that could be heavily expanded upon. The reader is referred to Cleary and Chernovsky et al. ${ }^{12}$ for an indepth look into the mathematical breakdown of their assumptions and detailed results. ${ }^{12}$

\subsection{CFD MODELING}

In short, there is very little literature publicly available on the use of computational fluid dynamics to model the complex geometry of present day spot-type smoke detectors. The few works that do try to address the issues of an actual spot-type smoke detector use detector geometries that are extremely simplified and do not offer the intricate, realistic structures of which real detectors are often composed. The majority of publicized CFD work focuses on simulations of smoke detection in rooms or buildings where external length scales are of much greater magnitude then the ones addressed in this study.

In a study done by Conte, ${ }^{13}$ a CFD simulation was constructed that attempted to model the transport of smoke through rooms with high ceilings in order to better design the ventilation system. Using the CFD software SOFIE and working in conjunction with experiments in a laboratory, the CFD software was fitted with experimental boundary conditions in order to model a proper temperature gradient for the high ceiling rooms. SOFIE is an acronym that stands for the Simulation Of Fires In Enclosures, which is a field model using CFD principles written in both Fortran and in C. SOFIE has the ability to solve the Reynolds-averaged Navier-Stokes (RANS) equations using a finite volume method similar to that of the software package used in this study, Fluent ${ }^{\circledR 18}$. The common $\kappa-\varepsilon$ turbulence model is used because of its excellent performance for many industrially relevant flows and easy input of simple boundary conditions. After a fairly large parametric study was conducted with over 60 ventilation inlets and outlet configurations, the simulation provided worthwhile insight as to the placement of various smoke detecting devices 
throughout a complicated room flow pattern. The study showed that there can be a definite problem detecting smoke when there is a temperature gradient within the room, causing the heat sensitive detectors to alarm at significantly different times in comparison to different detector placements. In all, the study was very beneficial in developing rules and advice on detector placement to facilitate early smoke detection in rooms with high ceilings. ${ }^{13}$

An actual study on the effects of a spot-type smoke detector can be found from the initial work of Ierardi and Barnett ${ }^{14}$ in characterizing the entry resistance of ionization smoke detectors. They recognized that low velocity particle flows are poorly understood when it comes to the entry resistance of spot-type smoke detectors, and attempted a CFD model to help fill the gaps in the knowledge base for smoke detector design and evaluation. Using a consumer grade ionization smoke detector and the commercially available CFD code called CFX-TASCflow (AEA Technology), a parametric study involving smoke entry into a smoke detector was conducted. Detector orientation with respect to the flow field was one of the primary varied parameters. ${ }^{14}$

They observed that the transport process of fire driven smoke fields are dominated by convective transport upstream of the detector, however, once smoke has been entrained in the ionization detector there is a greater dependence upon diffusion. This is due in large part to the intricate baffling of the chamber that is intended to settle the smoke cloud and prevent "ion drift" from strong convective flow patterns. The primary goal of the project was to characterize the entry resistance and to develop response criteria able to account for a multitude of fire signatures in terms of fire conditions and enclosures. They recognize that detector geometry plays a dominant role in the nature of entry resistance, and the influence of initial flow conditions along with the orientation of the detector with respect to the upstream flow field vector is very important on finalized results. However, investigators who have done pioneering CFD work in the past for spot-type smoke detectors such as Wood, ${ }^{15}$ Ierardi and Barnett ${ }^{14}$ state that Wood only modeled a square box with the only parameters being varied were the height of a slot opening and the free stream flow velocity. Ierardi and Barnett's proposed test matrix consisted of a series of 54 simulations involving three detector geometries, two different fuel sources, and six varying fire growth histories. This study is different than the one being proposed in this report because ceiling jet velocities are trying to be accounted for, whereas this study focuses on validating a known test standard laid out by 
the UL $217^{5}$ code for their test chamber. The preliminary work here shows that a three dimensional CFD model can be developed to study the non-reacting convective flows associated with entry into a spot-type smoke detector. ${ }^{14}$

Another study much closer to the work done in this current literature review topic was conducted by Ierardi and Barnett. ${ }^{16}$ Here, several of the same issues that have been addressed in this study have been dealt with previously, with their detector response broken down into five main categories: property generation, bulk property transport, local property transport, sensor modulation, and alarm condition. Ierardi and Barnett's ${ }^{16}$ were to provide a general reference for predicting smoke detector response that can be used by fire protection engineers to serve as a motivation for future research. They recognize that an appropriate combustion model must be implemented that include sub-models that account for aerosol dynamics in order to account for deposition, buoyancy effects, as well as a variety of other discrete phase circumstances presented due to a fire situation. Bulk property transport as well as local property transport was considered in formulating a model that not only realistically simulated smoke movement from an upstream source to the detector, but the local effects associated with a typical internal detector geometry. Unfortunately, although a multitude of fluid transport properties were accounted for in this study, the focus on a realistic, complicated detector geometry has yet to be seen. In summary, Ierardi and Barnett $^{16}$ profess that "the underlying phenomena of the overall smoke detection process are not fully addressed by such methods [mentioned earlier]. However, by treating the overall smoke detection process as a collection of related components for use with computational fluid dynamics modeling, it is possible to focus research efforts towards the development of improved predictive methods." The key areas of improvement they feel is necessary can be surmised in the following list:

1. Inclusion of an aerosol dynamics sub-model in a CFD code that includes the phenomena of agglomeration, sedimentation, and deposition.

2. Particle size and number concentration as additional variables in a CFD code.

3. Characterizing detection hazards as a source of energy and mass that is compatible with the aerosol sub-model of a CFD code.

4. Characterizing sensor modulation in terms of relevant combustion bi-products. 
In summary, Ierardi and Barnett's ${ }^{16}$ study is important because it points out a lot of the current issues the research done in this study has attempted to address. ${ }^{16}$

One of the final articles cited for review is Gobeau et al.'s ${ }^{17}$ assessment of CFD in complex enclosed spaces. Although Gobeau et al.'s study has little to do with the actual modeling of a complex spot-type smoke detector, the overall objectives and intentions of the study lay a solid foundation for the direction the study in this particular report wishes to take. A fundamental introduction of CFD is presented, denoting the need and scope for guidance, along with the evolution of completing a full CFD simulation. Similar to the other CFD simulations reviewed, the literature presented here expresses the need for physical submodels to be implemented such as combustion, turbulence, and radiation models in order to capture the true characteristics of the smoke field in which it tries to model. The study goes on to further address the importance of realistic boundary conditions inferred through experimentation, and the discretization process to create a well-posed, solvable grid. The important aspect of uncertainty in CFD problems is addressed in great detail, noting the main sources of error lie in (1) the level of detail represented by the geometry, (2) mesh resolution, (3) selection of physical sub-models, (4) selection of spatial and temporal discretization schemes, (5) specification of boundary conditions, (6) selection of convergence criteria, (7), user errors, (8) software errors, and last but not limited to (9) the experience of the CFD user. Lastly, the study reports that a CFD code is only as useful as the validation and verification that the model underwent. In other words, compared to experimental results, ensured that there is no fundamental mistakes, assessing the numerical behavior of the code, wrong definition of parameters, etc. Fortunately, due to the nature of the project in the study conducted here, the broad mechanics of the commercial code Fluent ${ }^{\circledR 18}$ have already been commercially tested and approved. ${ }^{17}$

The last piece of literature that proved quite helpful was the vast database of online tutorials and instruction Fluent ${ }^{\circledR 18}$ has available. The commercial software package offers a very extensive user guide, as well as a solid background on CFD fundamentals and modeling principles. Sub-models such as the discrete phase model discussed by Fluent ${ }^{\circledR 18}$ documentation ${ }^{18}$ proved quite helpful. 


\section{CHAPTER 3: THE SA302 SMOKE DETECTOR AND THE UL SMOKE BOX}

\subsection{THE SA302 SMOKE DETECTOR}

The spot-type smoke detector being used in this study is an advanced residential detector design, known as the BRK Brands, Inc. SA302. Designed with maximum protection in mind, the advanced technology employed by the SA302 uses a "smart" sensing internal microcontroller that helps distinguish between nuisance alarms such as kitchen smoke and smoke from a real emergency. The SA302 is outfitted with two smoke sensing devices; namely, the light-scattering (photoelectric) and ionization sensor. ${ }^{19}$ According to UL, its optical density sensitivity has been rated at $2.42 \pm 0.8 \% \mathrm{Ob} / \mathrm{ft}$. UL requires a sensitivity rating of at least $4 \% \mathrm{Ob} / \mathrm{ft} .^{5}$ Various detector orientations are shown as well as an interior view depicting major components in Figures 3.1.1 and 3.1.2, respectively. As one can see from those figures, the SA302 is shaped very similar to most other types of spot-type smoke detectors on the outside. However, due to the nature of the two-sensor scheme, the interior is somewhat more complicated than most. The main components are easily pointed out, with both sensors easily identifiable along with the circuitry and alarm horn.

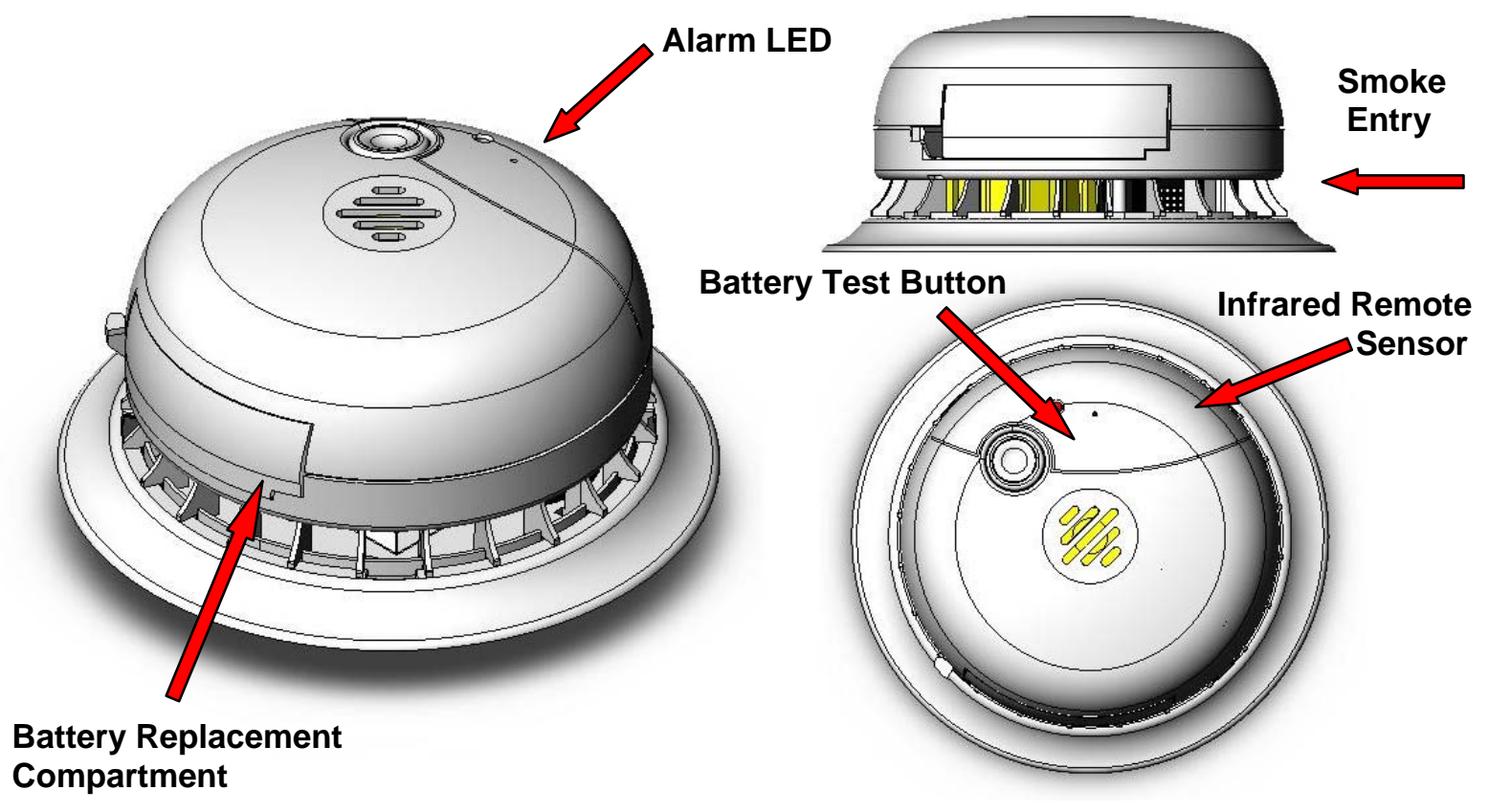

Figure 3.1.1: A three perspective view of the model SA302 detector. 


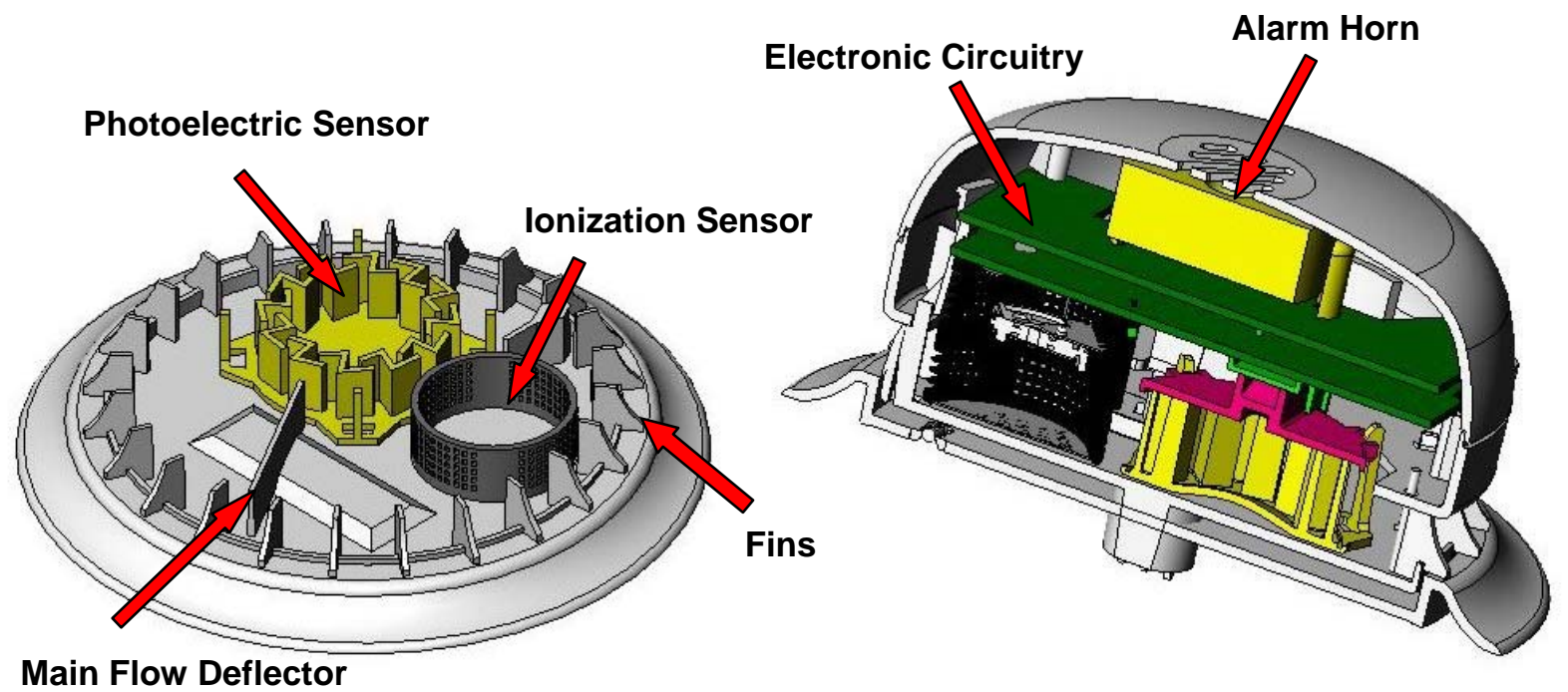

Figure 3.1.2: An interior section slice showing the main components that make the SA302 function.

With respect to past popular designs, the SA302's sensors are oriented in an innovative design orientation to provide smoke entry nearly flush with a room ceiling or wall mounting surface and to position the smoke sensing region away from non-sensing components. The unit's circuitry consists of stacked circuit boards above the sensors. The main flow deflector has been added by the manufacturer in an attempt direct flow fairly evenly to both sensors, regardless of detector orientation, with respect to the flow stream. One of the goals of this thesis is to interpret the amount of smoke each of the sensors experience dependent upon detector orientation. Thus, theoretically, results from the computational model should reflect a fairly uniform particle concentration within the sensors regardless of orientation.

Figures 3.1.3 and 3.1.4 show the photoelectric and ionization sensors in their entirety, respectively. Figure 3.1.3 shows the component breakdown between the infrared emitter and receiver retainer, connecting plate, along with the labyrinth with surrounding insect screen. The insect screen is a nylon screen with about $85 \%$ open area, which functions as a blockage for keeping various debris out of the measuring chamber as to not effect instrumentation. The ionization sensor broke down into its component parts in Figure 3.1.4 depicts its common components, denoting the charged connecting plate, radioactive source holder, along with the settling chamber. The ionization settling chamber is the most intricate 
element of the SA302, with over 70 plastic vertical ribs less than 0.04 in in width. Modeling issues associated with designing the computational model will be discussed in Section 5.3.

Cross-Sectional Assembly

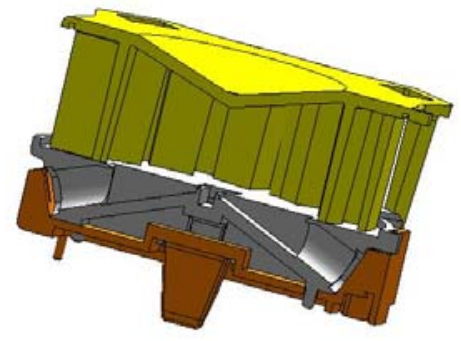

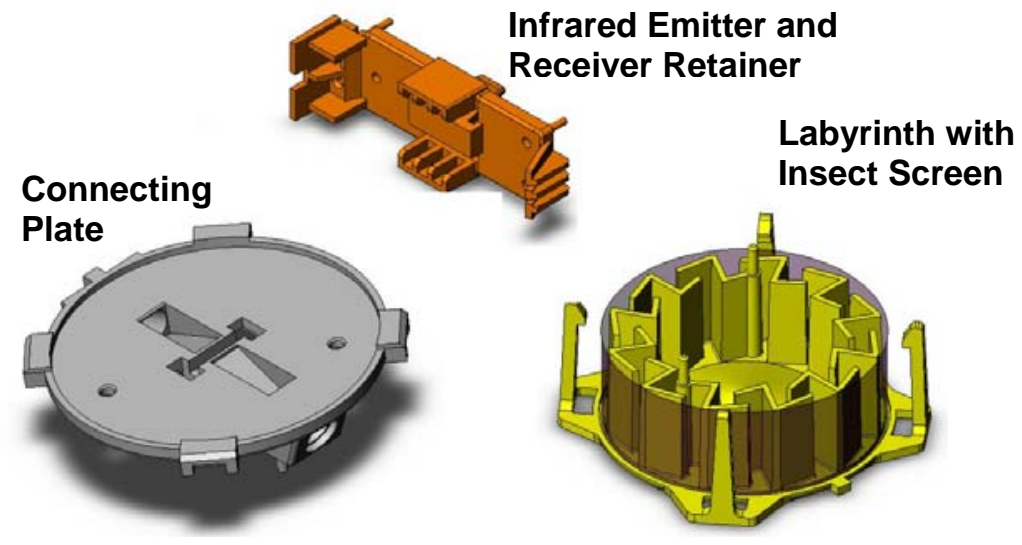

Figure 3.1.3: The component makeup of the photoelectric (light-scattering) sensor.

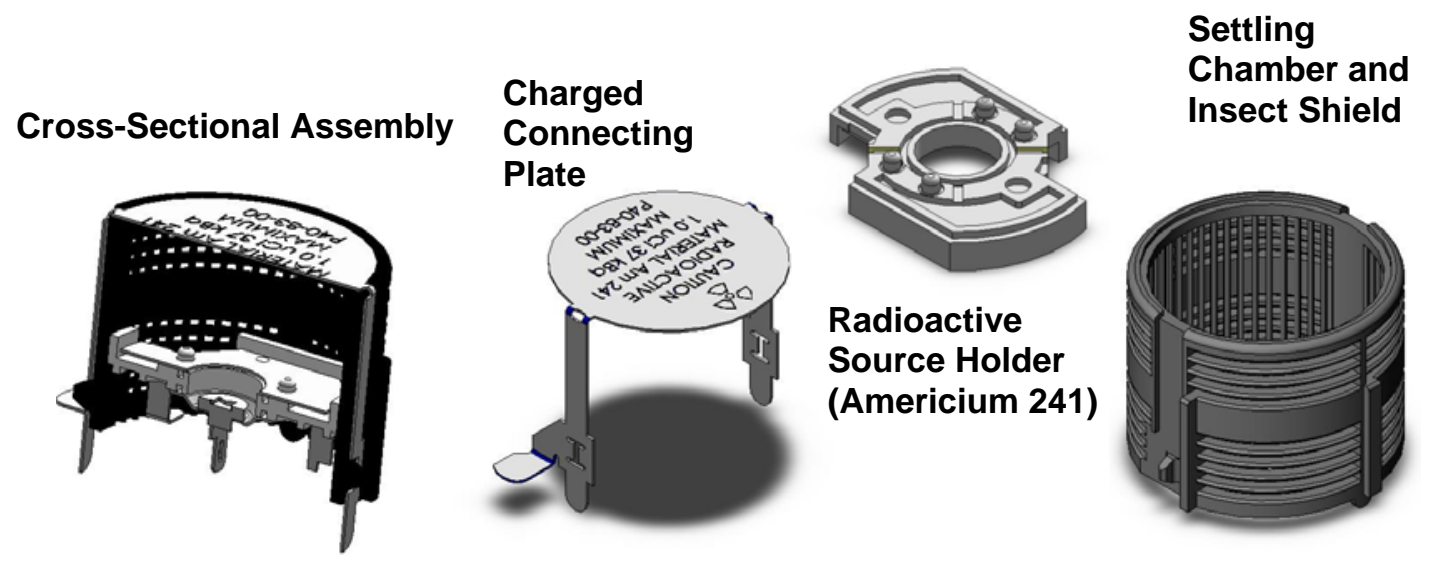

Figure 3.1.4: The component makeup of the ionization sensor.

\subsection{THE UL SMOKE BOX}

The testing of residential spot-type smoke detectors for safety approval in the United States is performed in large part by UL. The testing method used by UL to test these detectors is recorded in their UL $217^{5}$ standards manual where an entire procedural review can be found. ${ }^{3}$ The prime interest of the study conducted in this particular report revolves around understanding how a correct test is run, so the flow can be accurately characterized.

The UL Smoke Box is a detector evaluation chamber measuring $20 \times 20 \times 70$ in, essentially designed to act as a low-speed, closed-loop smoke tunnel. Figure 3.2.1 shows some photographs of the UL Smoke Box with its vertical hatch open exposing the interior, 
along with an oblique view of the real test chamber showing the instrumentation to read such properties as atmospheric pressure and relative humidity sitting atop the box. The detector is mounted at the center of the top surface as shown in the conceptual sketch in Figure 3.2.2, across from the measuring ionization chamber (MIC). The figure depicts what a normal test in progress would look like.
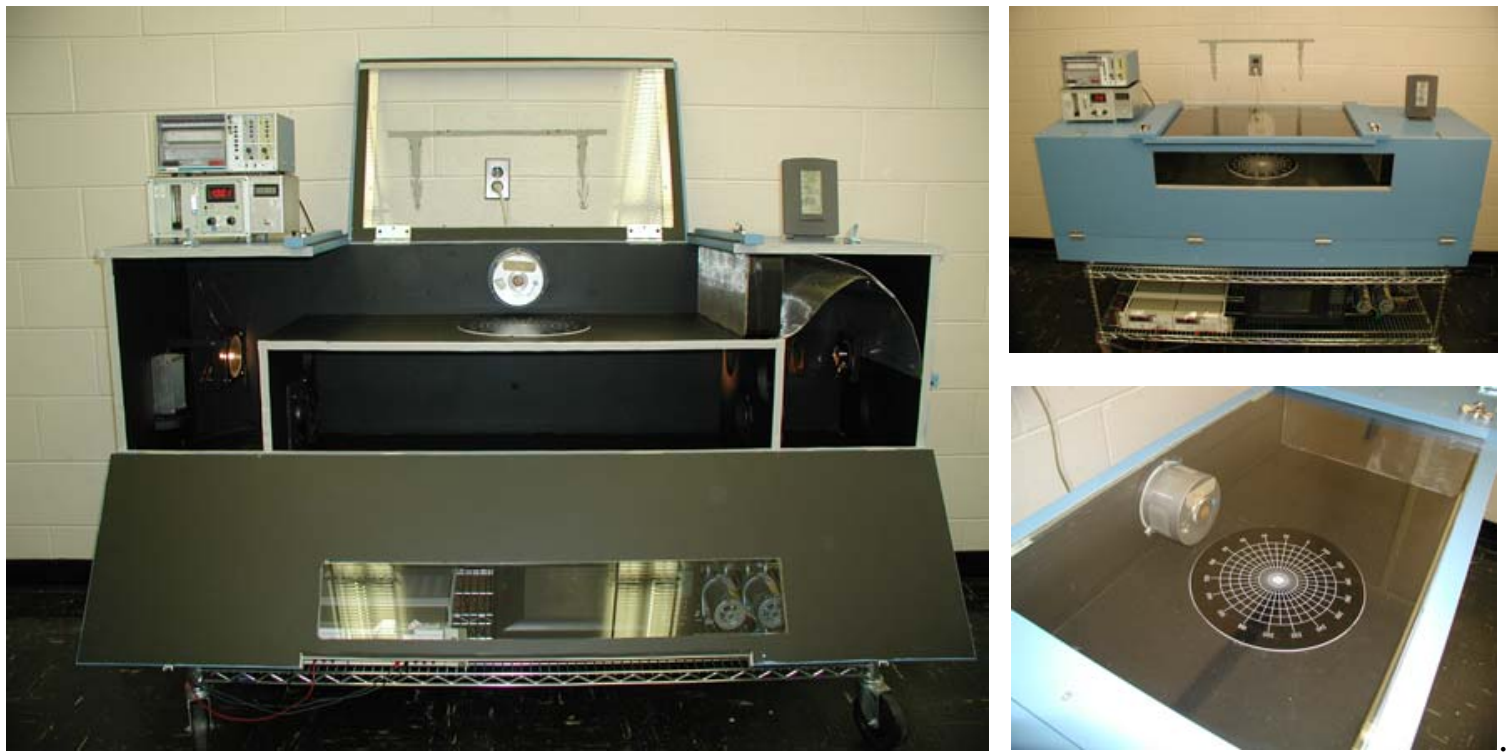

Figure 3.2.1: Some still image photographs of a UL Smoke Box with its vertical hatch open exposing the interior.

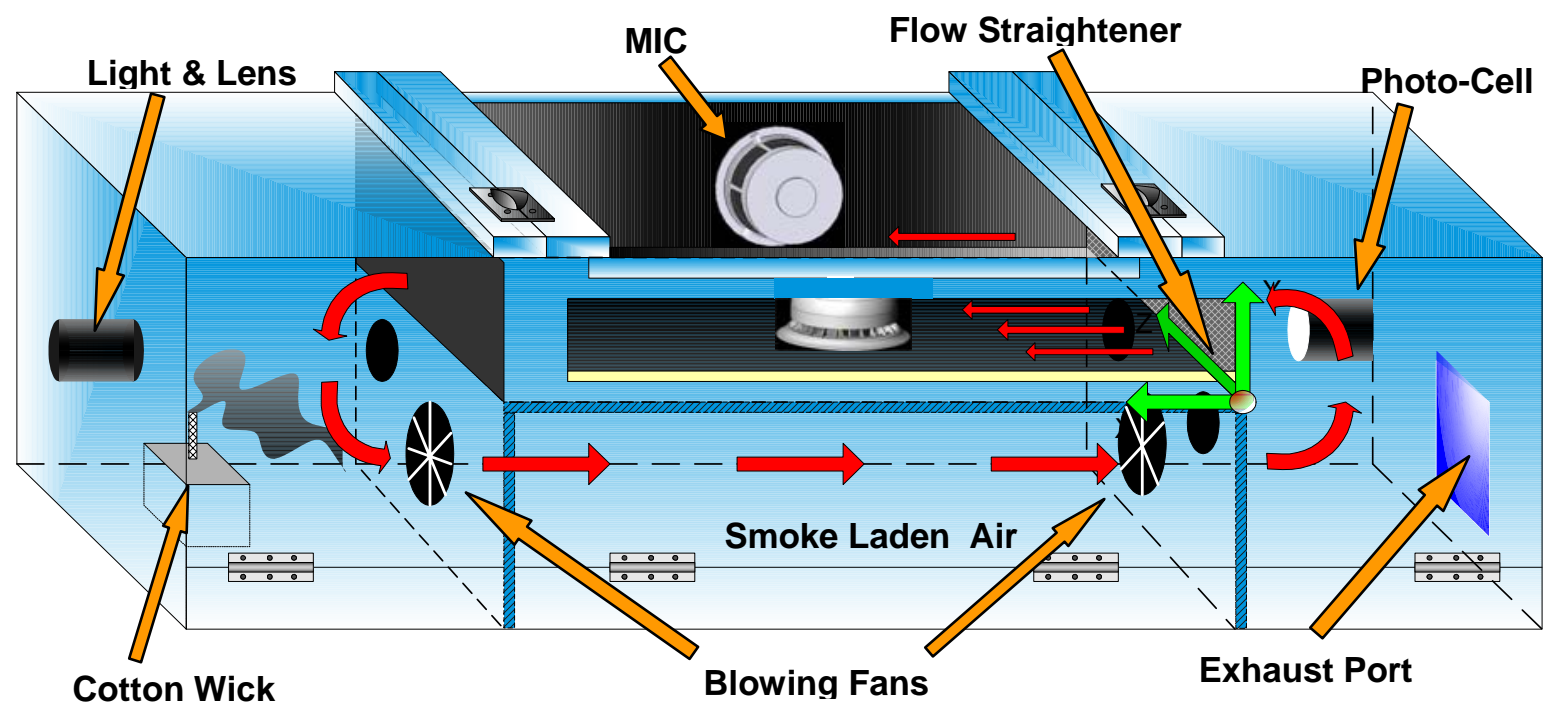

Figure 3.2.2: A conceptual sketch of a UL test in progress. The red arrows indicate the direction of airflow. 
The test chamber is divided into an upper and lower half. The top half, known as the test section, is a $36 \times 18 \times 7$ in chamber covered on the top and bottom by clear 0.25 in acrylic, met with a flow straightener at the forward end and an open outlet for the smoke to return.

For a typical test, smoke generated from a smoldering cotton lamp wick (or heptane flame) in a closed-loop chamber is allowed to build-up to the alarm threshold point of the smoke detector. As the smoke source constantly burns, the smoke concentration inside the box globally increases with time. The most common test uses a constant velocity of 32 $\mathrm{ft} / \mathrm{min}, 1$ in above of the test section floor where the detector would normally sit must be maintained. Flow is steadily maintained by a single muffin fan with a dc input of about 6.5 volts. ${ }^{3}$

The two UL Smoke Box measuring devices used to standardize the outputs of the photoelectric and ionization sensors, are the photometer and measuring ionization chamber (MIC), respectively. As a test is in progress, the photometer positioned underneath the test section (See Figure 3.2.2) measures the smoke accumulation in terms of percent obscuration as discussed in Section 2.3. Because greater resolution is met with obscuration systems the further the source and photo-cell are apart, the photometer spanning exactly $5 \mathrm{ft}$ with a beam width of $5 \mathrm{~cm}$ located on the underside of the test section maintains solid, repeatable accuracy. The photometer consists of an incandescent lamp (Ge-4515) source and a photovoltaic cell (Weston 594 RR) detector. For a more rigorous description of all of the UL Smoke Box components, the reader is referred to the UL $217^{5}$ standard manual. $^{5}$

The MIC, which fundamentally acts like an aspirated ionization sensor, measures smoke build-up by the decrease in current flow between the two charged plates in its settling chamber. Figure 3.2.3 below shows its basic external design.

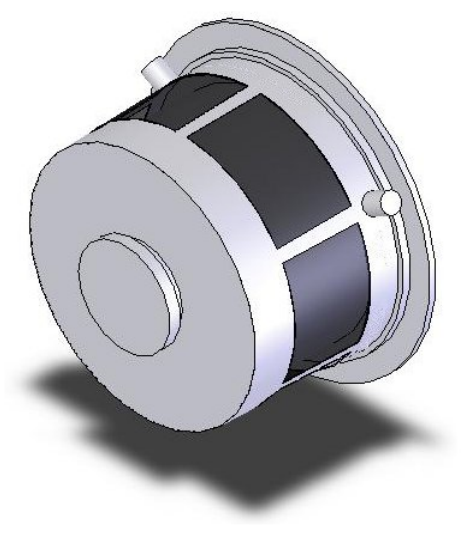

Figure 3.2.3: The measuring ionization chamber (MIC). 
For a test to be valid, air must be drawn through the MIC at a rate of $25 \pm 5 \mathrm{l} / \mathrm{min}$. For the tests conducted in this study, the volume flowrate was held at $30 \mathrm{l} / \mathrm{min}$. At this rate, the ingestion of flow by the MIC exchanges all of the air within the box in approximately 15 minutes - with an estimated inlet speed across each MIC screen of less than $4 \mathrm{~cm} / \mathrm{sec}$. Taking this into account, it is understood that the MIC's physical presence as opposed to its aspiration rate presents more of a challenge for the incoming flow to negotiate. The purpose of MIC aspiration is that theoretically, in real time, the MIC should be able to maintain a continuous measurement of the smoke concentration in the test section with minimal sampling time delay. Table 3.2.1 below describes the smoke obscuration threshold requirements for which a detector must alarm before it reaches the stated conditions.

Table 3.2.1: Visible smoke obscuration limits. ${ }^{5}$

\begin{tabular}{|c|c|c|}
\hline Percent by foot & Percent Obscuration per meter & Optical Density per meter \\
\hline 4.0 & 12.5 & 0.0581 maximum \\
\hline 0.5 & 1.6 & 0.0072 minimum \\
\hline
\end{tabular}

As for the MIC, a minimum allowable measurement of $93 \mathrm{pA}$ and a maximum measurement of $37.5 \mathrm{pA}$ sets the alarm standards for the ionization sensor. To view sensitivity plots of percent obscuration per foot versus the current output of the MIC, the reader is referred to Appendix A, adapted from UL $217 .^{5}$

Before addressing specific planes of data within the test section, the coordinate system is introduced that will be used to quantify the test chamber volume in Figure 3.2.4 for both experimental as well as computational data. 


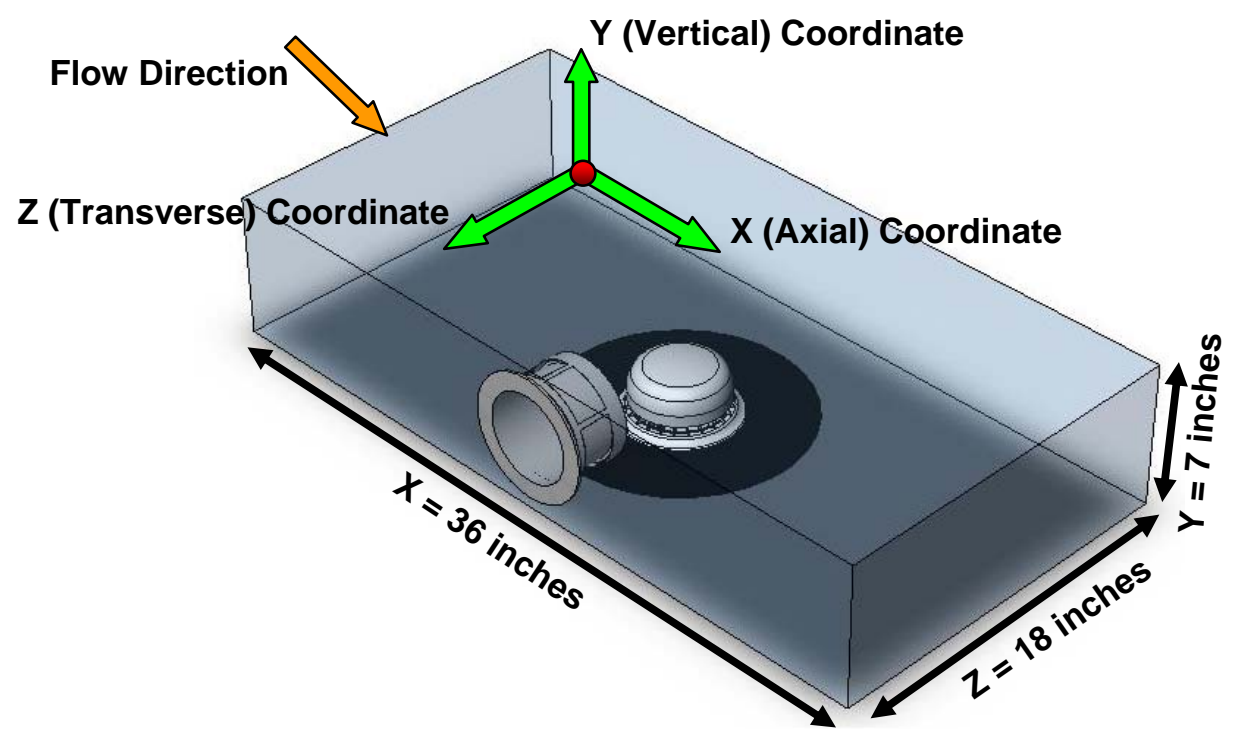

Figure 3.2.4: The coordinate system in use for reporting all results in the UL Smoke Box test section.

A three-dimensional coordinate system was established defining the origin (in green in Figures 3.2.2 \& 3.2.4) of measurement fixed at the bottom entrance of the test section, at the flow straightener screen, on the side front door of the UL Smoke Box. The positive $x$-axis, or axial coordinate, is defined as positive in the flow direction away from the screen. The positive $y$-axis, or vertical coordinate, is defined as positive upward away from the test section floor. The positive z-axis, or transverse coordinate, is defined as positive from the front door side of the UL Smoke Box to the MIC side of the test section. The positive $u, v$, and $w$ velocity components are defined to have the same directions and signs of the respective $x, y$, and $z$ axes.

The $y-z$ plane represents vertical, or "cross-flow" planes within the test section marching from $x=0$ to $36 \mathrm{in}$. Data presented in the $x-y$ plane are also vertical plane data, however these planes will march from $\mathrm{z}=0$ to $18 \mathrm{in}$. Horizontal plane data consists of values lying in the $x$-z plane, marching from $y=0$ to $7 \mathrm{in}$. Due to the ease of disorientation in reviewing data, legends will be produced on a need basis. This coordinate system is bodyfixed, meaning its orientation is maintained relative with the UL Smoke Box, no matter how the unit is rotated or translated. 


\section{CHAPTER 4: EXPERIMENTAL INVESTIGATION}

\subsection{INTRODUCTION}

The first main phase of this investigation focuses on experimental flow characterization of the UL Smoke Box test section. The experimental phase is comprised mainly of two stages: flow visualization and velocity measurements using laser Doppler anemometry. The flow visualization provides a view of the flow patterns of the smoke laden air flow approaching the detector, providing a rare view of the flow patterns inside not only the test section, but inside the detector as well. The flow visualization patterns were used to identify specific areas of interest where a laser-based measurement system was employed to acquire quantified, three-dimensional velocity measurements.

Flow characterization through experimental means is imperative for the long-term goal of a computational model for the purposes of verification and validation. The velocity measurements made will be used as boundary and initial conditions for the CFD model. The accurately measured flow velocities will serve to validate the utility of employing the CFD model to use in predicting the fluid dynamic behavior of smoked flow inside smoke detectors in the UL Smoke Box.

During construction of the virtual model, extra measurements needed to be taken at the inlet of the test chamber to account for new, unexpected boundary conditions that were originally immeasurable by LDA due to blockage. Because of this, the researchers took velocity measurements using a hand-held hot-wire anemometer as well. The remaining experimental endeavors consisted of pressure loss measurements across porous media due to some porous boundary zones present in the SA302 and the test chamber.

\subsection{FLOW VISUALIZATION}

The flow visualization inside of the SA302 smoke detector was performed with the detector positioned on the floor of the smoke box in the standard test position. In order to provide optical access to the inside of the smoke detector, the base plastic was modified to replace the white plastic flat base section, with a clear acrylic, circular disk approximately 0.09 in thick. Also, a 5 in hole was cut into the bottom of the smoke box test section and a 5 in acrylic disk 0.09 in thick as well was glued in place to seal the test section floor. By orienting a mirror $45^{\circ}$ in the UL Smoke Box return loop beneath the test section and by 
cutting another 5 in diameter hole (observation port) in the side door of the smoke box aligned with the mirror, the interior of the smoke detector was made clearly visible from the outside of the UL Smoke Box. Figure 4.2.1 below depicts the setup.

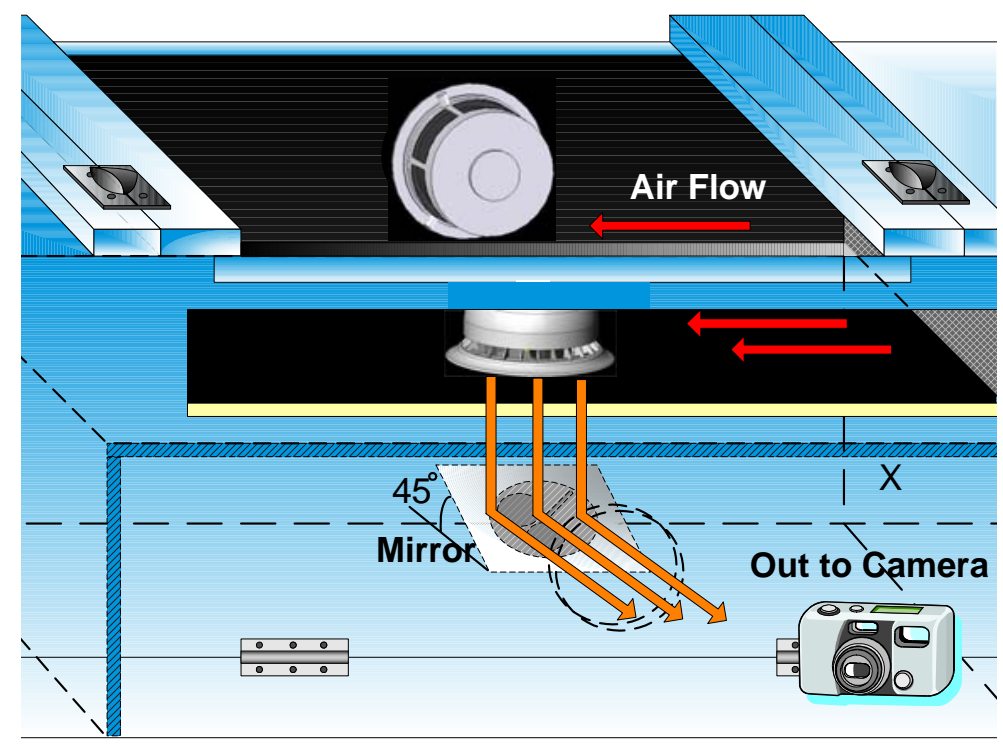

Figure 4.2.1: A conceptual drawing of how images were captured inside the UL Smoke Box for flow visualization.

A second 5 in diameter hole (illumination port not shown) was cut into the side door of the smoke box next to the first hole to permit a light source to illuminate the smoke detector interior by reflecting light on the mirror, without reflecting directly on to the observation port. Both the observation and illumination port were sealed with a 0.09 in acrylic sheet. A digital camcorder was used to record the flow patterns inside the smoke detector through the observation port.

Numerous attempts were made to produce the highest quality flow visualization records of smoke laden flow inside the SA302 smoke detector. A theatrical fog machine was employed to create a dense white fog (water and glycol mixture), however this fog was not easily directed since the fog machine operates under atmospheric pressure conditions. Other methods which did not prove as successful were fog reservoirs consisting of a several gallon plastic-sealed container with a small air pump used to convey the fog to a small nozzle near the smoke detector inlet. While met with some success, it did not produce a sufficiently dense enough fog for high quality flow visualization compared to other methods. 
The method that produced the best quality flow visualization consisted of using a 5 in long, approximately 0.25 in diameter cotton wick saturated in kerosene, and electrically heated by a Ni-Chrome wire wrapped several times about the wick. A DC power supply was used to provide the electrical power $(<4$ watts) to heat the wire-wrapped wick to produce kerosene smoke. The wick was positioned upstream of the detector and the current control on the power supply was positioned to provide a very dense, white cloud of smoke upstream of the detector that was swept into the detector interior and sensing (ion and photoelectric) chambers. Clear acrylic disks inserted into the top of the sensing chambers afforded optical access into the interior of these chambers. While this method worked very well to produce extremely optically dense clouds of kerosene smoke, there were a few limitations encountered. First, the smoke production had a life span of approximately 10 to $15 \mathrm{sec}$ maximum before the wick had to be reloaded with kerosene, with the wick having to be replaced after about 4 to 5 runs. Second, the optically dense kerosene smoke, while superb for flow visualization photography, tended to condense to kerosene liquid on the walls of the smoke detector rendering the optical windows partially obscured from condensed kerosene droplets after each test. This condensation tendency required that the smoke detector model be disassembled and the acrylic windows cleaned almost after each flow visualization test to maintain clear optical access into the detector's interior.

The results from this portion of the study can be found in Section 6.1 where unaltered flow visualization photographs were taken for various horizontal and vertical planes.

\subsection{LDA VELOCITY MEASUREMENTS INSIDE THE UL SMOKE BOX}

A laser-based velocity measurement system was used to quantify the speed and direction of the flow inside both the UL Smoke Box and SA302 smoke detector in the evaluation chamber's test section. A 2-watt Argon-Ion laser was used as the light source for the system, but was operated in the 0.25 to 1.0 watt range for this study.

A single channel laser Doppler anemometry (LDA) was used to measure the air velocity components inside the UL Smoke Box test section. The LDA system and the UL Smoke Box had to be oriented to measure each of the three orthogonal velocity components $(u, v$, and $w)$, and the laser had to be traversed from measuring location to measuring location to acquire three-dimensional velocity components at nearly 2500 different physical locations within the UL Smoke Box test section. The LDA measuring volume was positioned 
manually with the aid of a lead screw driven traversing bench with three degrees of freedom motion.

The LDA data acquisition and associated data reduction expanded into a very arduous task requiring approximately 300 man-hours to acquire the raw data and approximately 200 man-hours to reduce the nearly 7500 sets of data, each data set comprising 3000 to 5000 velocity and turbulence intensity data realizations to statistically analyze. The LDA system is shown in a photograph in Figure 4.3.1 in an orientation to measure the transverse, wcomponent velocity within the test section.

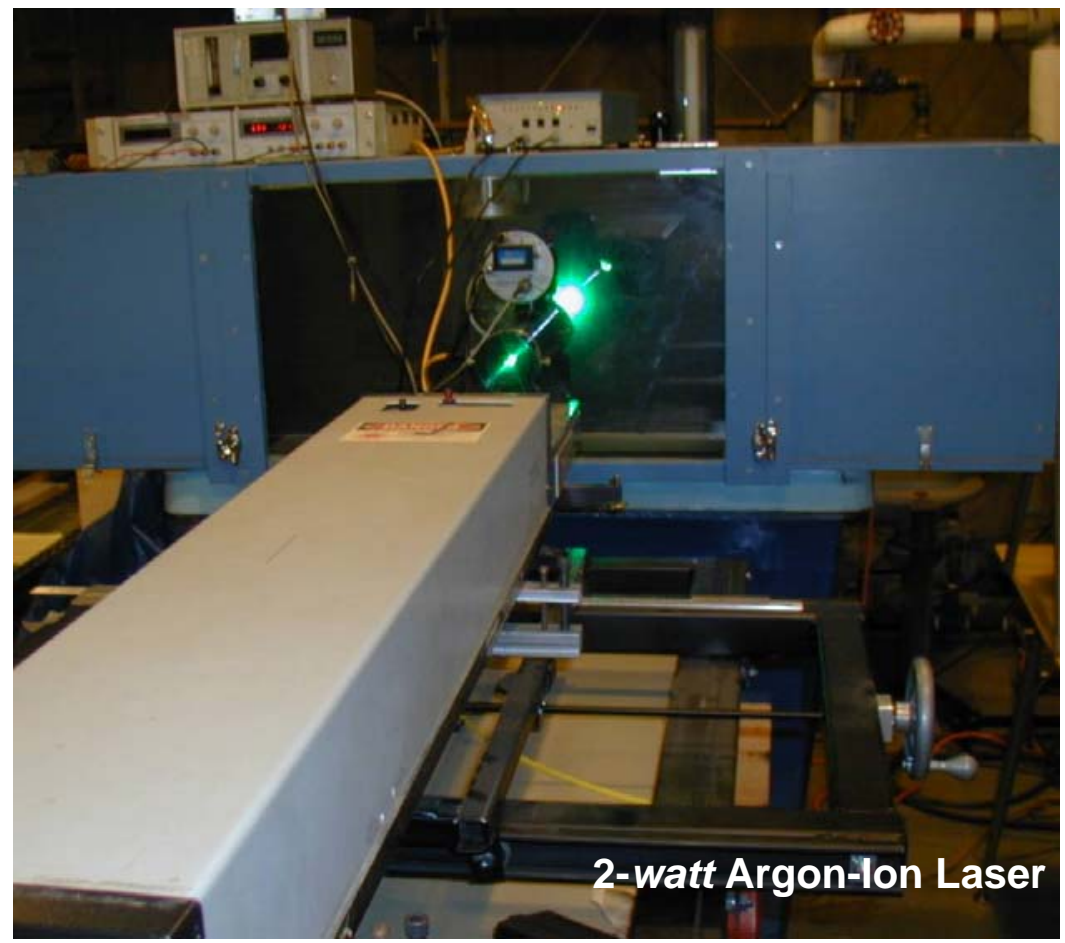

Figure 4.3.1: Laser Doppler anemometry and traversing bench positioned in front of the UL Smoke Box.

As shown in Figure 4.3.1, to facilitate the LDA velocity measurements in the test section, the smoke box was rotated so that the floor of the test section ( $y=0$ in plane) was vertical, and thus, perpendicular to the laser beam axis. This position was used to measure the axial, $u$-component velocity and the transverse, $w$-component velocity in the test section. These velocity components ( $u$ and $w$ ) were acquired separately with the single-channel LDA system. All of the axial, $u$-component velocities were acquired first, then the optics were rotated $90^{\circ}$ and all of the vertical, $v$-component velocities were acquired. The orientation of 
the UL Smoke Box has no effect on the aerodynamic flow behavior since the flow is essentially isothermal and is well mixed by the fan driving the air.

Figures 4.3.2 and 4.3.3 show zoomed in photographs of the laser entering the test section of the UL Smoke Box. The SA302 smoke detector was partially painted flat black to help reduce the laser light reflection. Figure 4.3.4 shows a close-up of the intersecting laser beams where the measuring volume is formed. One beam appears wider than the other due to its separation into several beams of various frequency shifting. The intentional frequency shift of one beam permits the measurement of flow reversals. Alignment and proper focusing of the LDA's photo-detector onto the proper intersecting beam pair is critical and required exceptionally tedious procedures. The wires seen in Figure 4.3.3 are reflections from the acrylic sheet.

The various components of the optics of the LDA system are shown in Figure 4.3.5. The optical arrangement is called a back-scatter configuration since both the transmitting optics and the receiving optics are on the same side of the test section. This configuration greatly facilitates traversing the measuring volume of the LDA, but requires significantly more laser power compared to a forward-scatter configuration.

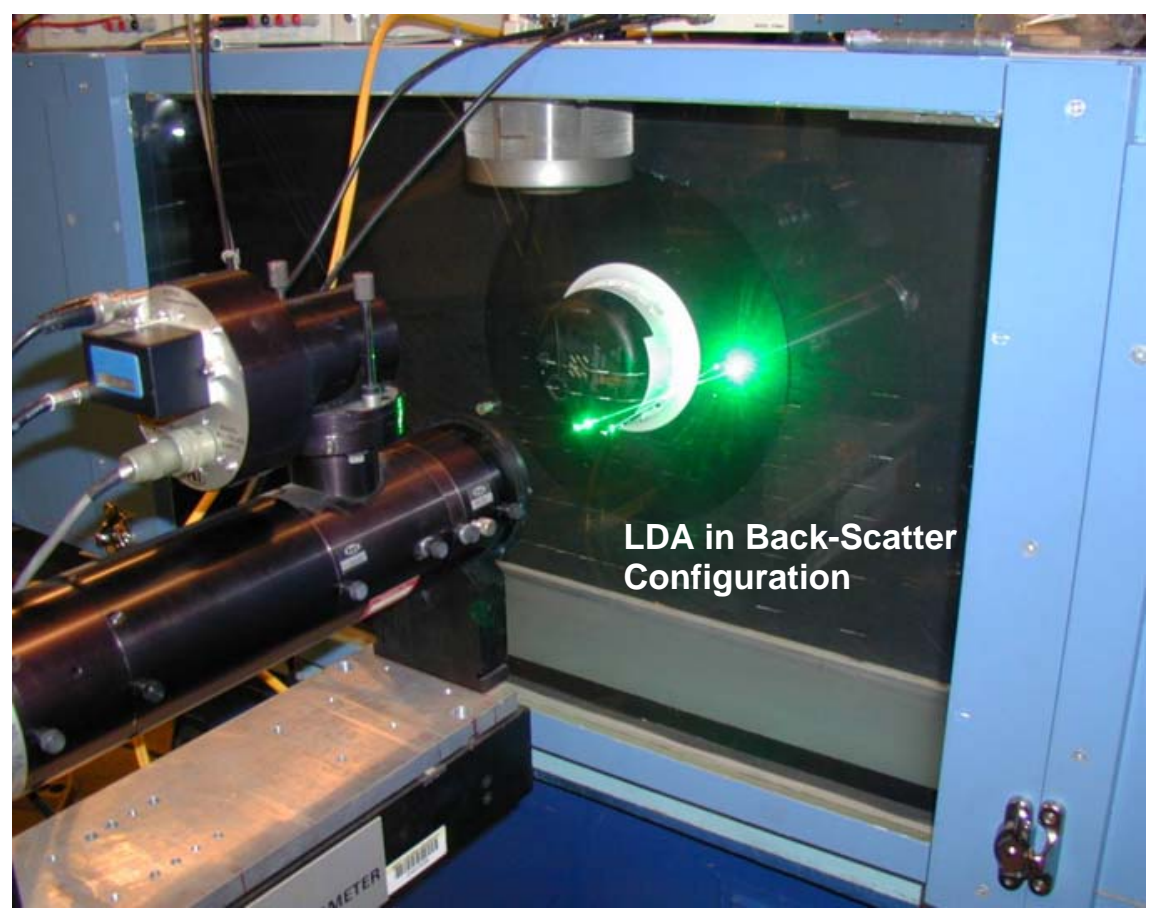

Figure 4.3.2: A close-up view of the UL Smoke Box test section using laser Doppler Anemometry. 


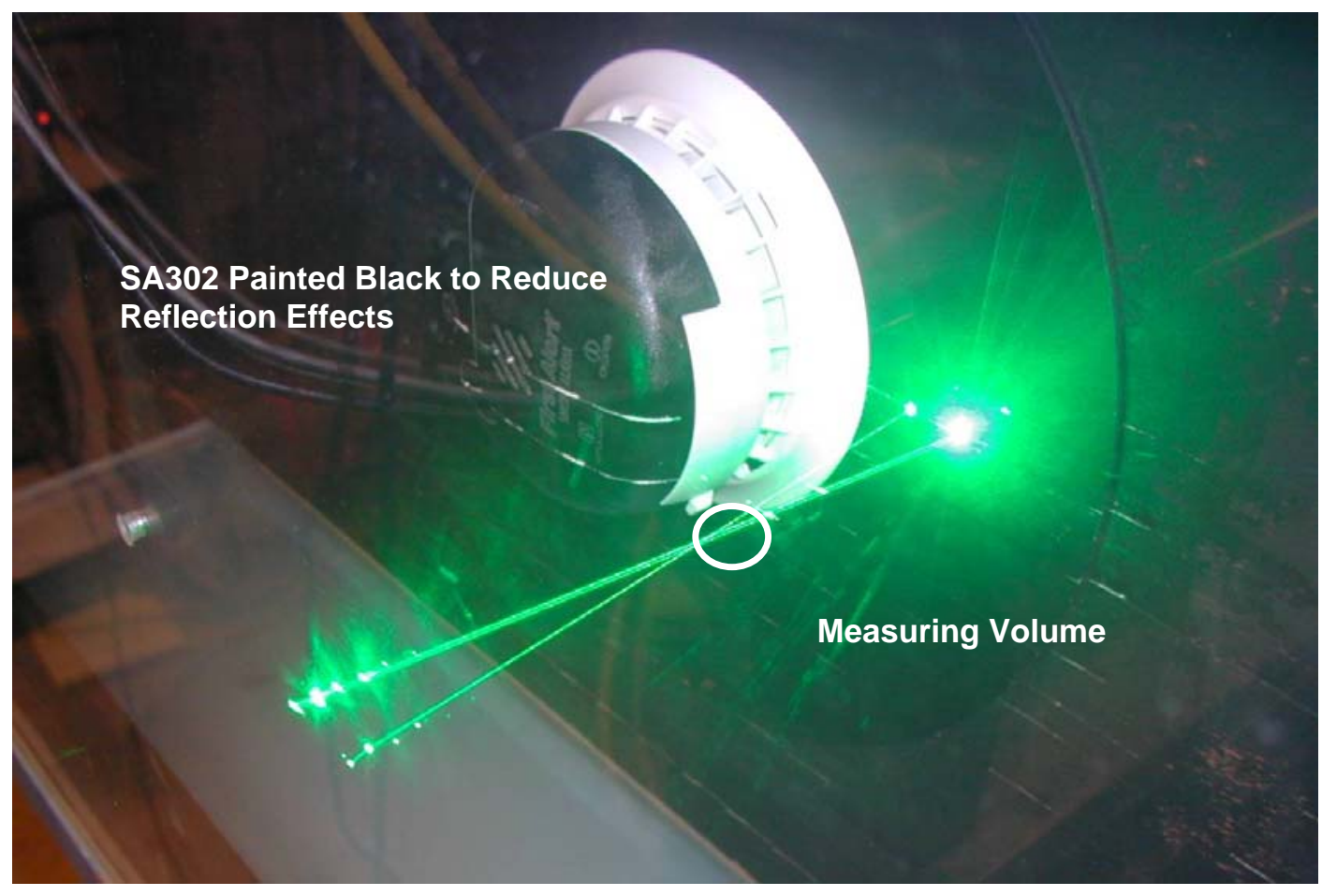

Figure 4.3.3: Close-up view of the laser Doppler anemometry measuring volume taking a data point just outside the realm of the detector.

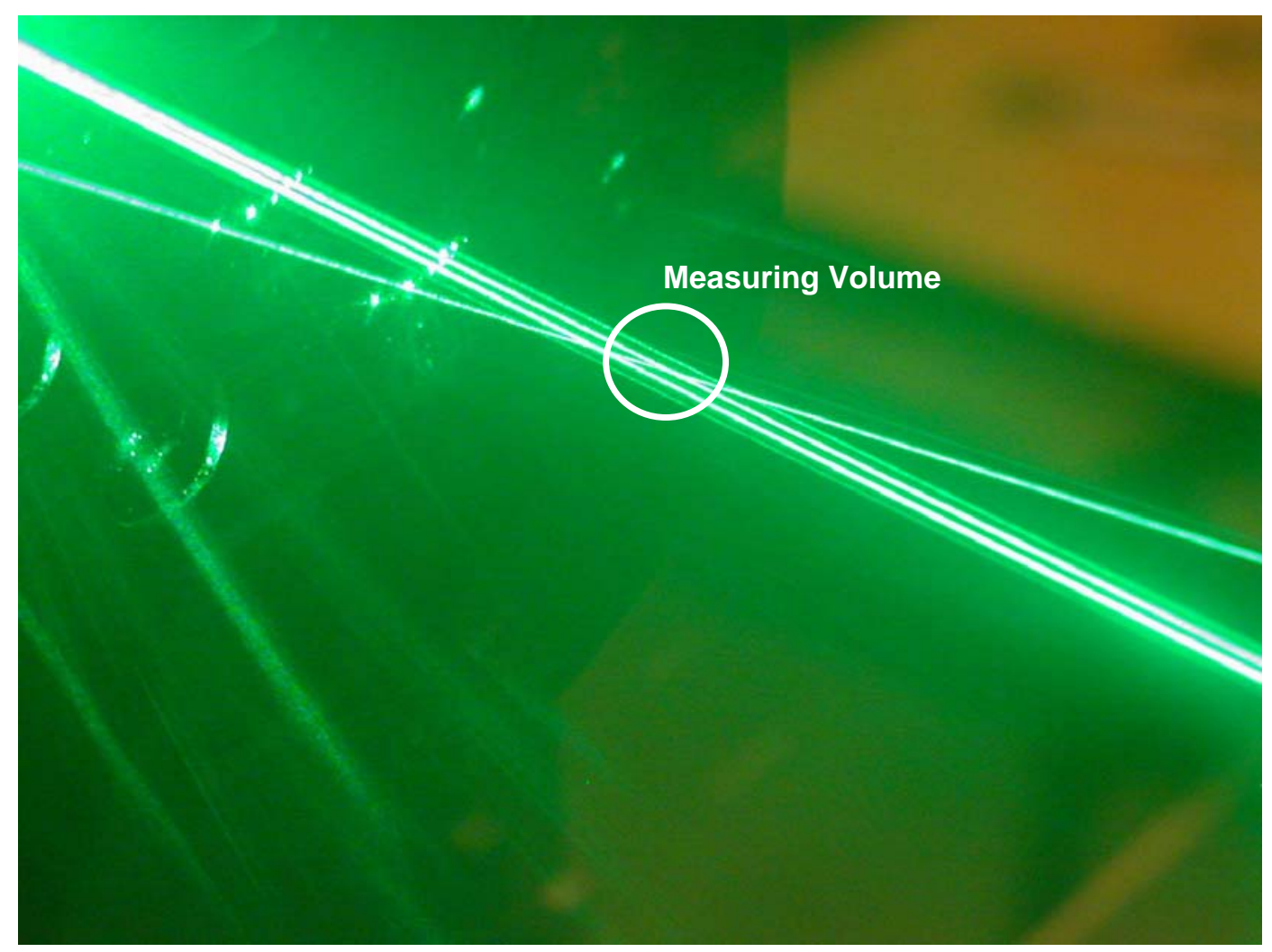

Figure 4.3.4: The measuring volume of laser Doppler anemometer with frequency shifting to detect flow direction. 


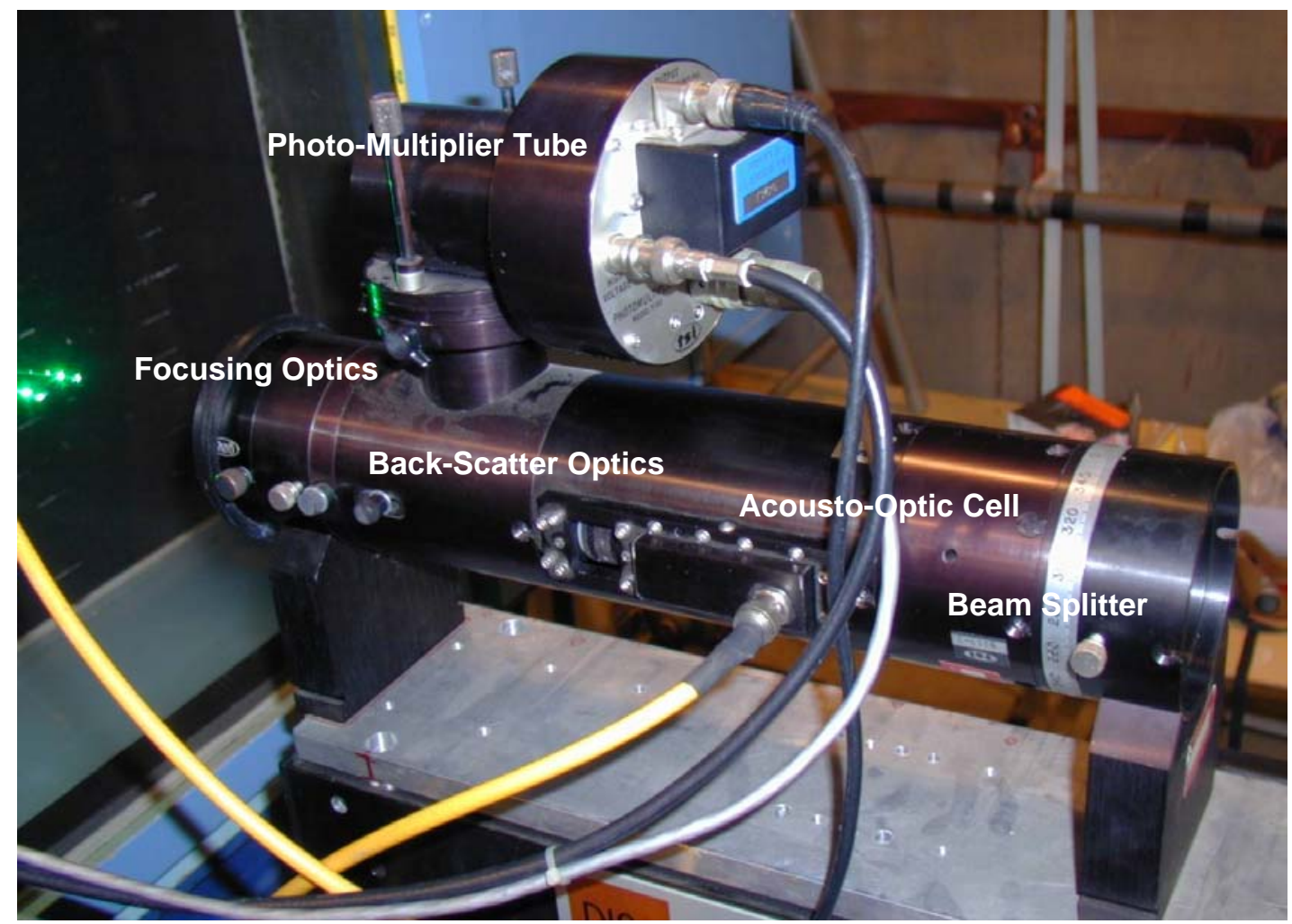

Figure 4.3.5: The optical components of the laser Doppler anemometry system.

An LDA system works by using a coherent laser light source to create two equally intense beams of light that intersect in space beyond the transmitting optics of the LDA system. Within the intersection region of the beams is created an ellipsoid-shaped envelope known as the measuring volume, filled with equally spaced interference fringes. As a "seed" particle (fog in this study) follows the flow streamlines and tracks the flow velocity moving through the measuring volume, the particle scatters light from the interference fringes such that the light-scattered signature is composed of light and dark periods, the length of which depends on the spacing of the fringes and the velocity component orthogonal to the fringe direction. By knowing the wavelength of light of the laser source, the focal length of the transmitting optics converging lens, and the parallel beam spacing approaching the converging lens, the interference fringe spacing is accurately known. By electronically measuring the time the seed particle takes to cross a prescribed number of fringes, the velocity of the particle is known. The scattered-light from the particle crossing the measuring volume fringes is received by a lens system focused on a photo-multiplier tube sensitive to the wavelength of the laser source. This fringe model is often used to simplify 
the more complex heterodyning theory of two scattered-light signatures each very slightly in frequency in opposite directions by the passing of a particle through two laser beams intersecting at an angle.

Unfortunately, the basic LDA velocity measurements are directionally ambiguous, meaning the system cannot discern the direction of the velocity component. In other words, the photo-multiplier tube experiences the same scattered-light signature if a particle traveled through the measuring volume at an angle of $0^{\circ}$ or $180^{\circ}$ with respect to some fixed reference plane. Also, it cannot measure zero velocity, since particles never cross any fringes. Frequency shifting helps to reduce "angle bias," so the component velocity is measured in equal probability no matter what trajectory through the measuring volume. To overcome this limitation, one of the two parallel laser beams is shifted in frequency prior to the formation of the measuring volume. This is accomplished through the use of an acousto-optic cell (known as a Bragg Cell) that is a transparent cell which oscillates at frequencies up to $40 \mathrm{Mhz}$. The fringe model effect of slightly shifting one of the laser beams in frequency with respect to the other is to create an interference fringe pattern in the measuring volume that moves with a fixed velocity. If the fringe velocity is greater than the most negative particle velocity, then the directional ambiguity of the particle is resolved through software by subtracting the effective fringe velocity from all velocity measurements. This way both positive and negative velocities of the seed particles (and thus the air) can be determined.

One of the main challenges of using LDA is obtaining optical access to the test article of interest and maintaining unblocked laser beams as the measuring volume is moved in space. To overcome this predicament, on the return loop of the UL Smoke Box, a diamond shaped entry hole wide enough for most of the optics to fit was fashioned, but shaped as to not disrupt the flow as much as a rectangular hole would. This hole permitted the laser optics to achieve close proximity to the detector so that a short focal length lens could be used. The short focal length afforded a short measuring within the confines of the flow passage inside the SA302 smoke detector. Figure 4.3.6 below visualizes the design. 

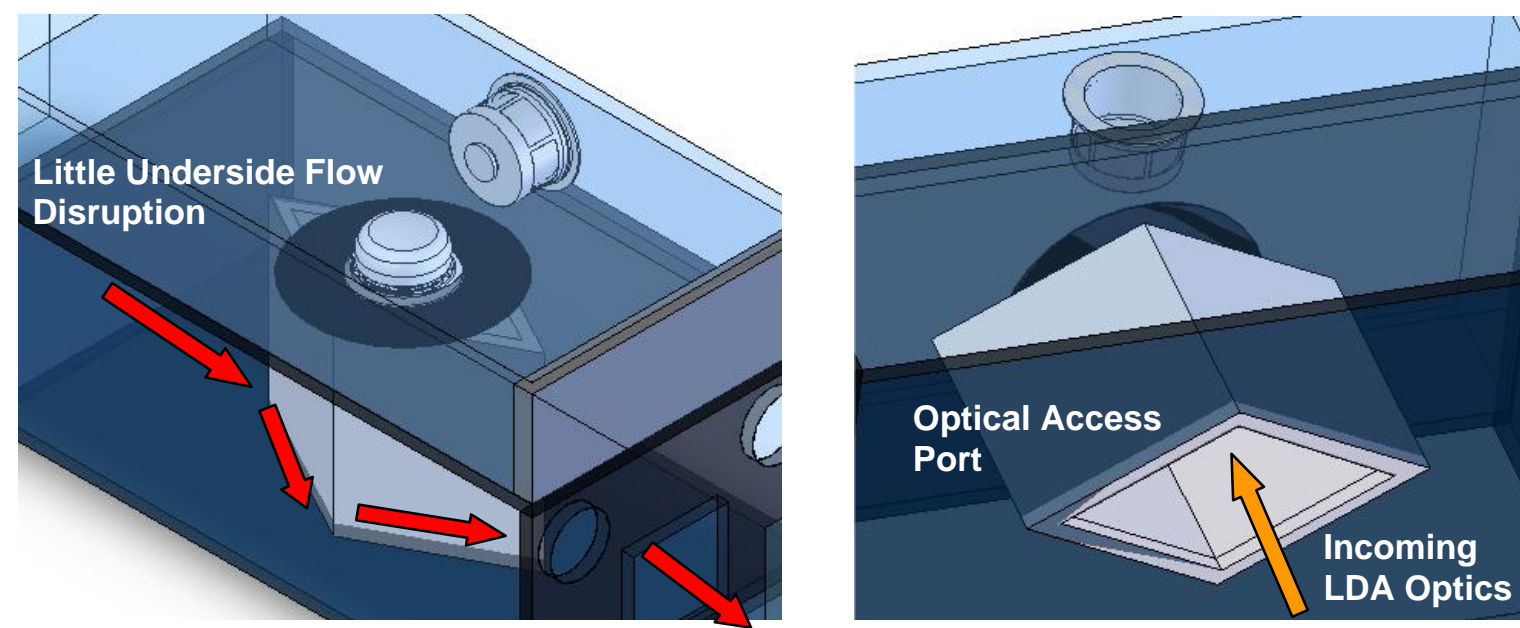

Figure 4.3.6: The modified UL Smoke Box with the added optical access port used to take LDA measurements on the interior of the detector.

Another problem arises when the measuring volume nears a perpendicular surface, opaque or transparent, because the signal-to-noise ratio rapidly diminishes due to the intensely reflected light from the surface reaching the photo-multiplier tube. In this study, velocity measurements as close as 0.125 in to a surface were achieved.

Figure 4.3.7 shows a typical signal conditioned oscilloscope trace from one fog particle passing through the measuring volume of the laser beams. Within the envelope of the "burst" is the high frequency Doppler signal, displaying an outstanding signal-to-noise ratio. The Doppler frequency here is on the order of $1 \mathrm{Mhz}$ with frequency shifting. The Doppler frequency times the interference fringe spacing in the measuring yields the velocity of the particle. The voltage scale is 0.2 volts/div here. The sample was taken with approximately 0.25 watts of laser power as well. The data acquisition rate was on the order of 1 to $2 \mathrm{kHz}$ while acquiring between 3000 to 5000 data points for each measurement location for each velocity component direction. Since the laser system responds to particles scattering light, the data rate is dependent upon the particle arrival rate into the measuring volume. 


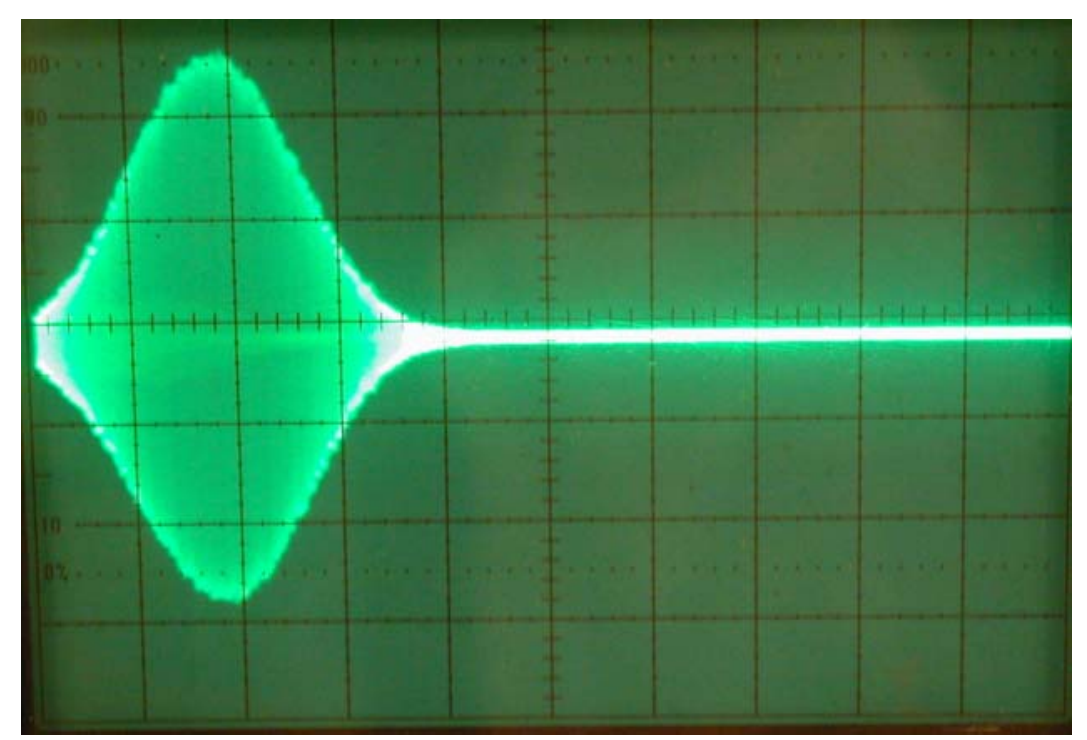

Figure 4.3.7: An oscilloscope trace of the laser Doppler anemometry signal processor as a fog particle passes through the measuring volume.

Figures 4.3.8 and 4.3.9 depict the grid spacing that was used in recording all of the data inside of the UL Smoke Box test section exterior to the detector. Figure 4.3.8 shows how each plane sits with respect to the test section. Grid spacing was reduced to 0.5 in increments as points approached the detector. In Figure 4.3.9, $x-z$ plane, velocity magnitude results range from $y=0.25$ to $6 \mathrm{in}$; for the $x-y$ plane, velocity magnitude data range from $z=3$ to 15 in; and finally, for the $y$-z plane, velocity magnitude results range from $x=12$ to 23 in.

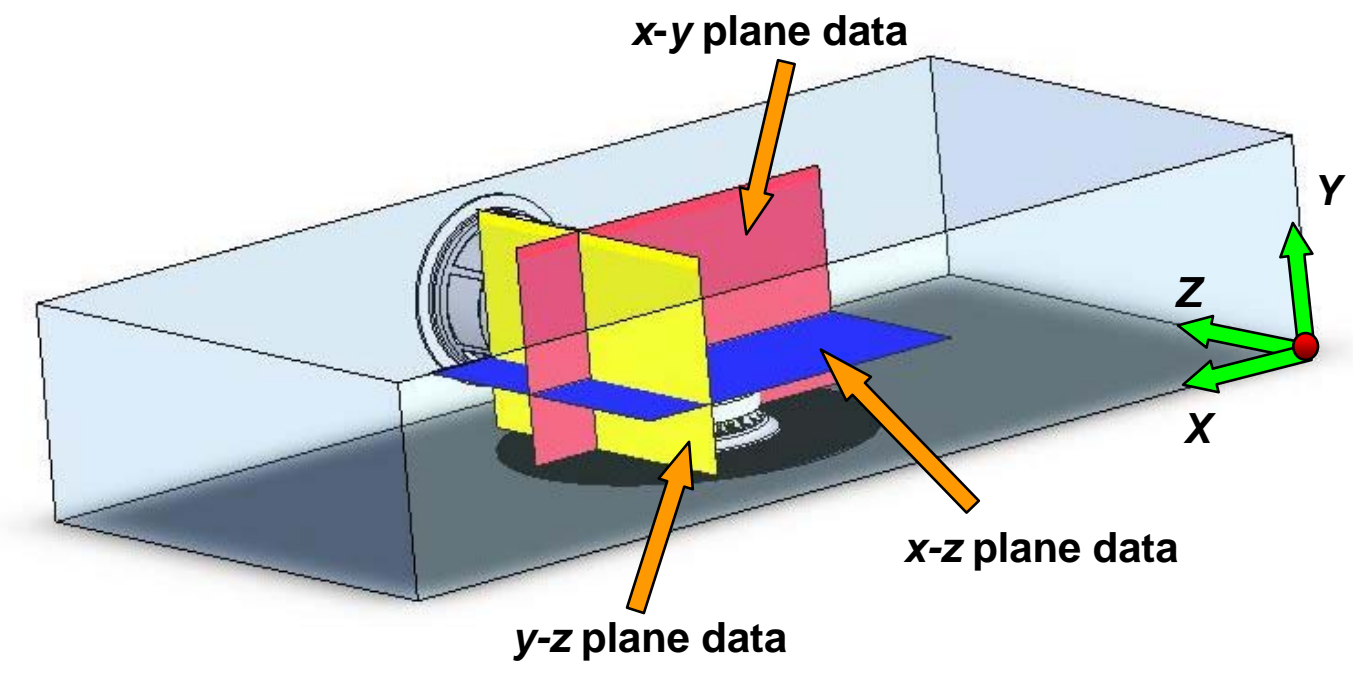

Figure 4.3.8: The UL Smoke Box test section showing characteristic orthogonal planes of data where the laser Doppler anemometry measurements were taken. 


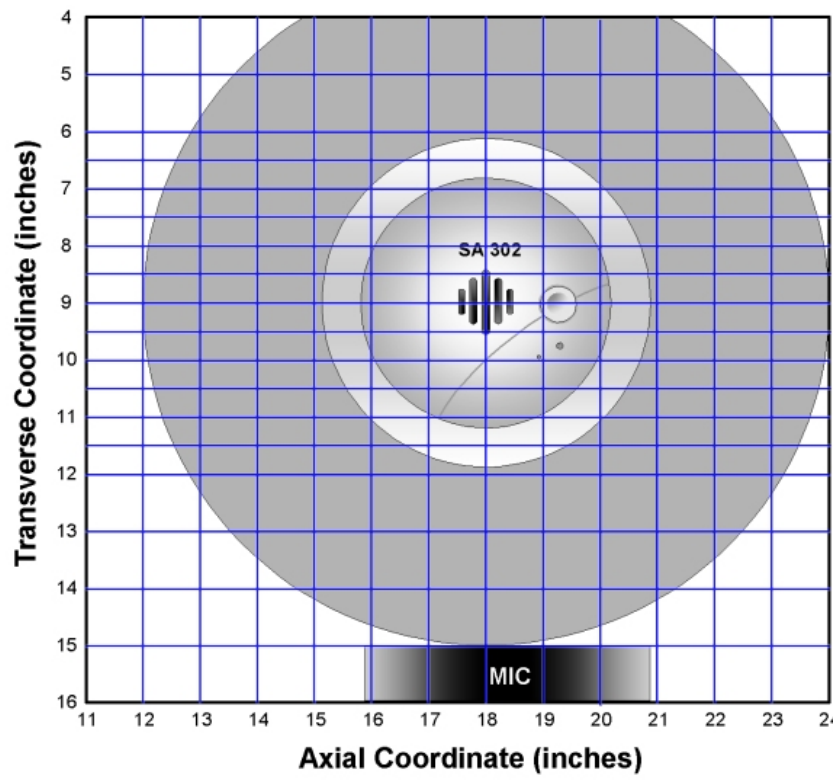

(a.)

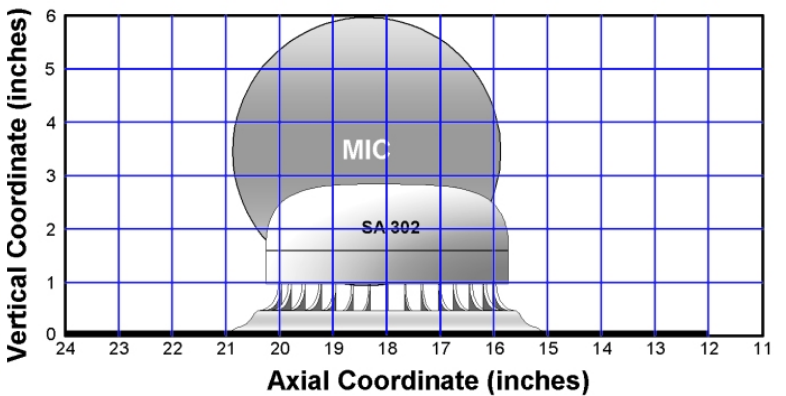

(b.)

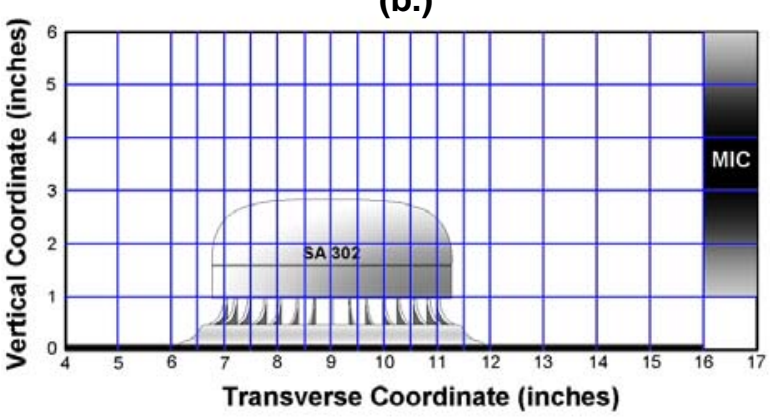

(c.)

Figure 4.3.9: The location grid used in taking laser Doppler anemometry data. (a.) is for the $x$ $z$ plane, (b.) is for the $x-y$ plane, and (c.) is for the $y-z$ plane.

\subsection{LDA VELOCITY MEASUREMENTS INSIDE THE SA302}

In order to gain optical access to the interior flow passages of the SA302 smoke detector, a model having transparent sections was constructed from SA302 translucent plastic components and clear acrylic sheeting. A circular acrylic sheet replaced the base plastic of the detector body and holes on the tops of the ion chamber and photo electric chamber where fitted with acrylic disks for optical access. Further, the upper body of a translucent model of the SA302 was used to complete the model to minimize laser light reflection. All electronic components were removed from the detector in the non-wetted areas. The ion chamber floor and radiation source holder were replaced with an identically shaped acrylic model and a circular hole was cut in the center of the photoelectric chamber floor and covered with a clear plastic film. Both of these modifications to the respective chambers significantly increased the LDA signal-to-noise ratio and permitted higher quality velocity data to be acquired inside these chambers. Figure 4.4.1 shows a photograph of the bottom view of the SA302 LDA model. 


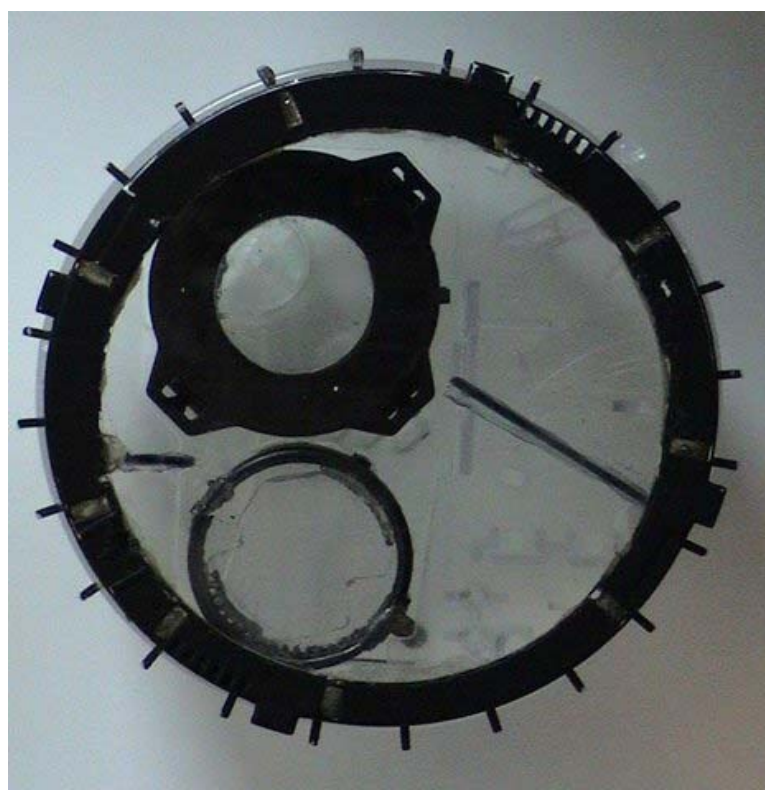

Figure 4.4.1: The bottom view of the SA302 model constructed for laser Doppler anemometry measurements inside of the detector housing and sensing chambers.

The LDA system was used to measure flow velocities inside the SA302 smoke detector model horizontal mid-plane with a grid resolution of $0.25 \mathrm{in}$. Figure 4.3.8 shows the grid pattern to define the physical locations of the LDA measuring volume at the mid-plane. Some detector orientations were more difficult to acquire data for than others due to the complex positioning of the optics inside of the UL Smoke Box.

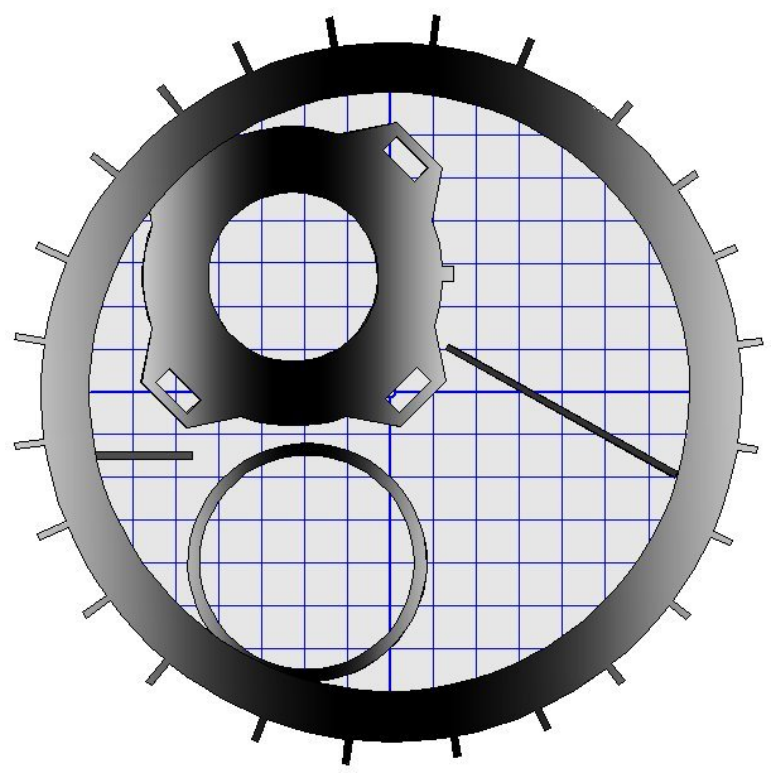

Figure 4.4.2: The grid defining the locations where axial and transverse ( $u$ and $w$ ) component flow velocities were measured in the SA302 model. 
Error analysis conducted on the LDA data in the resultant plots in Section 6.7 comparing experimental LDA data versus CFD data employed a technique known as multisample data error analysis. Appendix $\mathrm{C}$ explains the procedure in deriving the expressions presented in those particular figures for arriving at the standard deviation plotted. 


\section{CHAPTER 5: COMPUTATIONAL FLUID DYNAMICS MODEL}

\subsection{INTRODUCTION}

Once the majority of experimental work was completed, the next phase took on the primary objective of creating a validated computational model that would accurately reflect the experimental results recorded within the smoke detector and surrounding test section. Using the CFD software package Fluent ${ }^{\mathbb{1 1}}$, it is shown that a CFD model geometrically accurate to that of the real UL Smoke Box test section will provide comparable numerical results that could possibly reduce the design iteration efforts currently employed to achieve an acceptable smoke detector design configuration. The model consists of the SA302 smoke detector, the MIC, and the test section itself. To aid in the geometric design, a familiar third party CAD package entitled Solidworks ${ }^{\circledR 20}$ has been utilized in a majority of the model's development. This program along with Fluent's ${ }^{\circledR 18}$ own geometric modeler called $\mathrm{Gambit}^{\circledR 18}$, has been used in tandem to help produce the current geometry and grid model.

Topics such as the nature of CFD, complex geometry reduction, meshing schemes, and types of flow models, will all be discussed in detail. The reader is once again reminded that given a verified and validated computational model from experimental flow characterization, it is foreseeable that the virtual test bed will potentially be able to predict smoke concentrations entering the sensing chambers given an influx of smoke particles.

\subsection{THE NATURE OF COMPUTATIONAL FLUID DYNAMICS}

Computational fluid dynamics, just like experimental and analytical studies, relies on classical fluid mechanic principles that are mathematically represented by a governing system of equations known as the Navier-Stokes (N-S) equations. In short, these equations manifest the conservation of mass, momentum, and energy for fluid flow, which in turn can predict how a fluid behaves under a prescribed set of conditions called boundary conditions. Because the N-S equations are made up of complex, second order, non-linear, partial differential equations (PDEs), a closed-form analytical solution does not exist for most real three-dimensional flow fields. By resolving the continuous temporal and spatial analytical domains into finite points, one can rewrite these complex governing equations in a discrete, algebraic form using a variety of numerical method techniques. These algebraic equations can then be solved at each point, or "node" in the discrete numerical domain. The 
arrangement of these points along the flow field is called the computational grid or mesh, and can be developed in a variety of ways.

The accuracy of these computational equations in obtaining realistic solutions depends on a multitude of factors. In the case of solving analytical equations, one can obtain theoretical accuracy by fully solving the partial differential equation in its entire form under well-posed, approximated circumstances. The accuracy of the discretized partial differential equation used in CFD is different, relying on limitations built into not only the model, but the computer limitations as well. Major factors affecting accuracy include the fineness of the computational mesh, the computational truncation errors produced from the numerical techniques being employed, and the computational truncation error as well as the round-off error imposed by the computer itself. By ensuring a well-posed mesh with proper boundary conditions, however, these errors can be greatly minimized.

The commercial software Fluent ${ }^{\circledR 18}$ employed by this study is versatile in not only the type of flow field it can solve, but also in the various flow characteristics it can report. Particle concentrations, multiphase interaction, and flows involving moving parts are but a few examples of these. Fluent ${ }^{\circledR 18}$ uses the finite volume technique to solve the flow field equations, meaning it treats the domain as an array of conjoined infinitesimal fluid elements fixed in space with the actual fluid moving through its boundaries. Analytically, because the fluid element is fixed in space, this model is considered to be in conservative form. Figure 5.2.1 below depicts the concept.

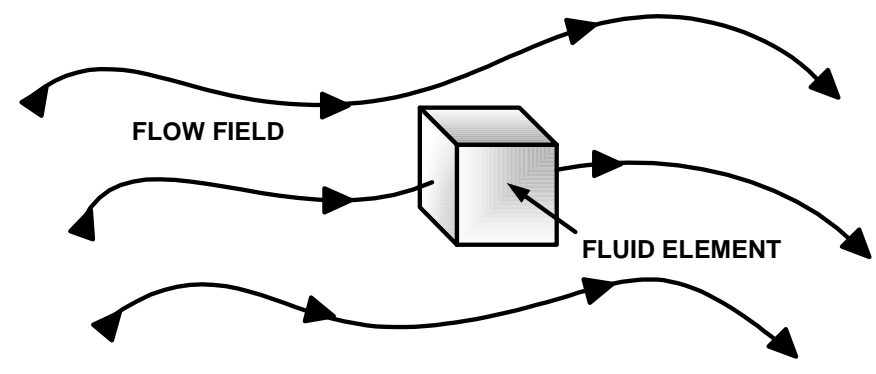

Figure 5.2.1: The general concept of the finite control volume method.

It would be prudent here to emphasize the advantages as well as disadvantages CFD has over experimental testing. For one, CFD can query any point in the flow field for data in which it has calculated, unlike experimental data, where data are often much more coarse dependent on where a measurement was taken. Also, once a CFD model has been verified and validated, with confidence, one can then make minor changes to the model to see how a 
system would behave differently. An example would be creating a validated UL Smoke Box model with the SA302 detector, and then swapping the detector out with a new design and examining how particle concentration changes with respect to a new geometry.

CFD has downsides as well. As stated earlier, a variety of errors can become abundant by creating an ill-posed model, and computer execution time can become very lengthy. However, by imposing realistic boundary conditions on a numerical model with a well-posed mesh - accurate solutions are very plausible making the effort ultimately worthwhile. It cannot be over-emphasized, however, that validation of CFD generated solutions through an independent means such as experimental results is extremely important. No matter what the level of sophisticated CFD code, the computations sometimes converge to non-physical solutions even with a well-posed mesh and realistic boundary conditions. Other concepts related to CFD needing in-depth discussion such as grid generation and solution techniques will be presented as the need arises.

\subsection{GEOMETRIC REDUCTION}

It is well known in the art that the type of computational mesh developed for any CFD problem can either make or break the numerical simulation. In obtaining a quality mesh, the first few steps involve deriving a suitable geometry to which the mesh must be adapted. In examining the geometry files available for the SA302 smoke detector, it was immediately determined that the geometry was too complex for Gambit ${ }^{\circledR 18}$ (computational grid generation software) to create the mesh autonomously without aid from the user. Hence, measures had to be taken to reduce the geometry complexity enough for the objects to be meshed effectively and accurately, but not lose enough detail to the point where the flow field would be significantly altered. One common geometric simplification of a model is to reduce the number of minute faces created by CAD drawing software packages.

The choices in file formats to edit for the SA302 were between IGES and Solidworks ${ }^{\circledR 20}$ assembly part files as provided by BRK Brands, Inc. IGES stands for Initial Graphics Exchange Specification format, and is used primarily for sharing basic geometric data in ASCII format between various CAD programs. These files do not retain program specific instructions on how objects are formulated, but rather just translate the shape "as is." Solidworks ${ }^{20}$ files, on the other hand, maintain a specific parent-child hierarchy of every event or "child" feature defined about a base "parent" object. Because this system is bi- 
directional, meaning it has the ability to conversely de-feature complex parts, a simpler geometry can be readily fashioned from complex architecture. This is very helpful because it not only saves computer power and space from lengthy geometry entries, but it also cuts modeling time nearly in half. The risk of human error in modeling a new smoke detector (a second time) from scratch is eliminated by maintaining the objects original base dimensions - in the end only disposing of minor features such as fillets, chamfers, and slightly oblique edges. These concepts will become more relevant when the computational grid generation methodology is discussed later on. The following discussion extends to each component of the total computational model as each piece was condensed into a more mesh-friendly design. While some parts were easy to work with, others had to be made from scratch.

As shown in Figure 3.1.2, the SA302 smoke detector is comprised mainly of the photoelectric chamber, ion chamber, the upper dome housing, the circuit boards, the deflector plate, along with the base plate and deflector fins enclosing the interior. Because the primary concern lies in developing an accurate representation of the flow field within the interior, utmost care is taken in preserving the interior geometry exposed to the smoke flow. Various figures follow summarizing the reduction process in taking the original Solidworks ${ }^{\circledR 20}$ files, and then de-featuring them into a mesh-friendly geometry. Small gaps became a major issue in attempting to reassemble the entire detector because minute spaces would end up residing between parts - leaving "hidden" spaces not needing to be meshed. Thus, besides reducing the individual parts themselves, some parts were actually merged together to preserve volumetric unity. Figures 5.3.1 and 5.3.2 provide typical examples of how de-featuring works on both the photoelectric and ion chamber sensors compared to the original Solidworks ${ }^{\circledR 20}$ files. Modeling nuisances such as unwanted fillets and chamfers were removed from the original objects, as well as minute curves which should have little impact on the flow field. In all, the number of faces on the entire SA302 original model dropped by over three fourths the total. The same methodology shown in Figures 5.3.1 and 5.3.2 was carried out for the rest of the assembly by successfully merging the base plate, fins, and upper dome together as one piece in an attempt to reduce small gaps and provide a seamless transition for meshing. 


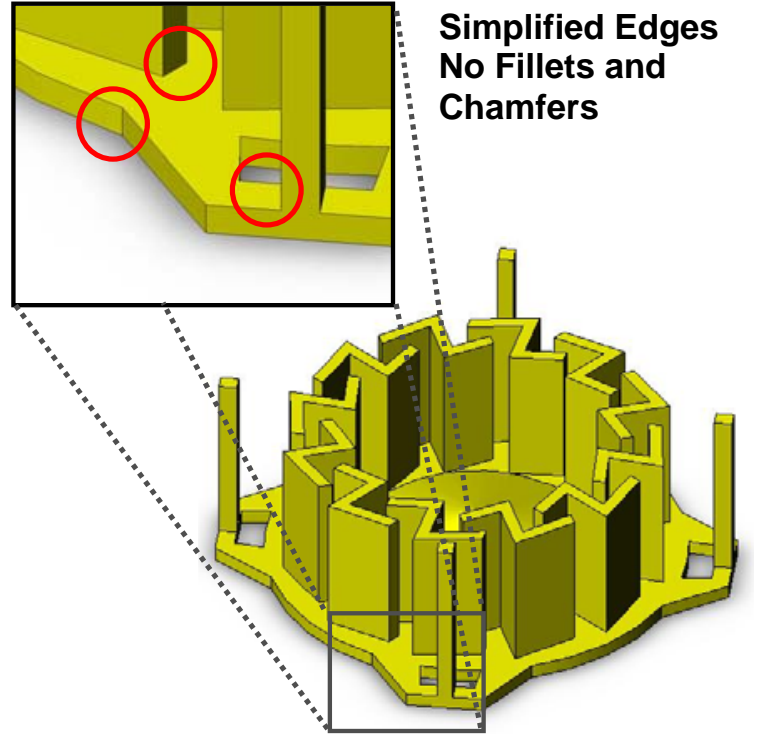

(a.) De-featured Photoelectric Chamber

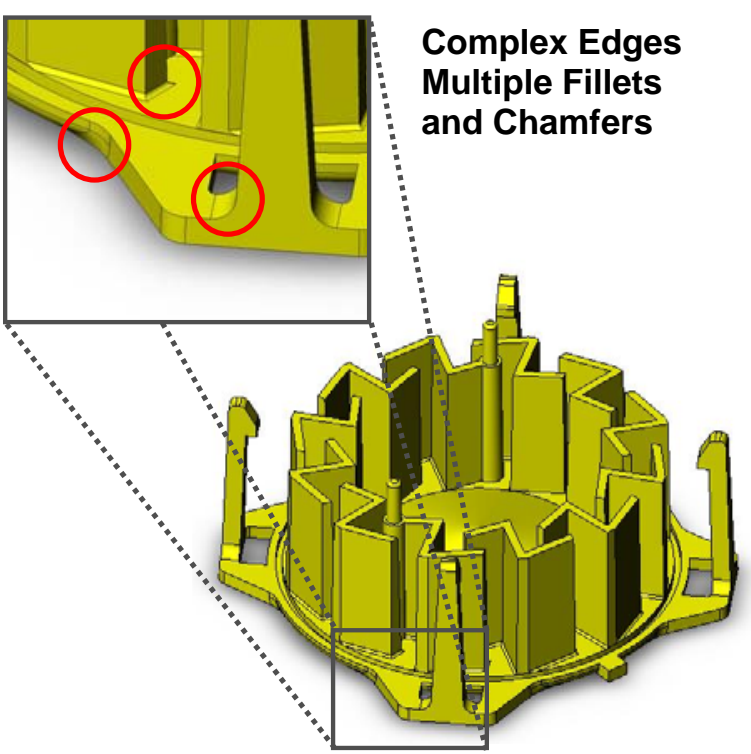

(b.) Original Photoelectric Chamber

Figure 5.3.1: The original photoelectric sensor part compared to the de-featured part.

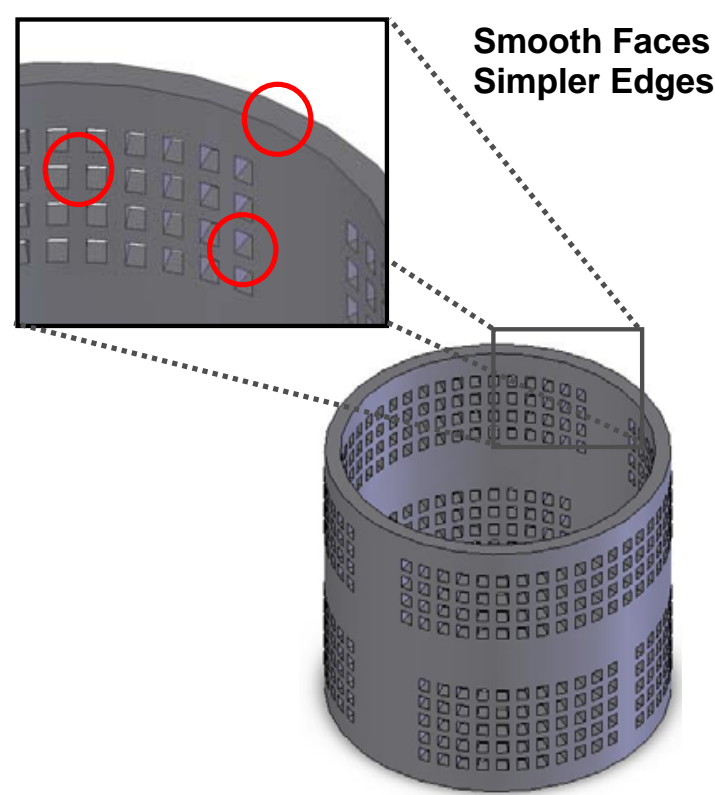

(a.) De-Featured Ion Chamber

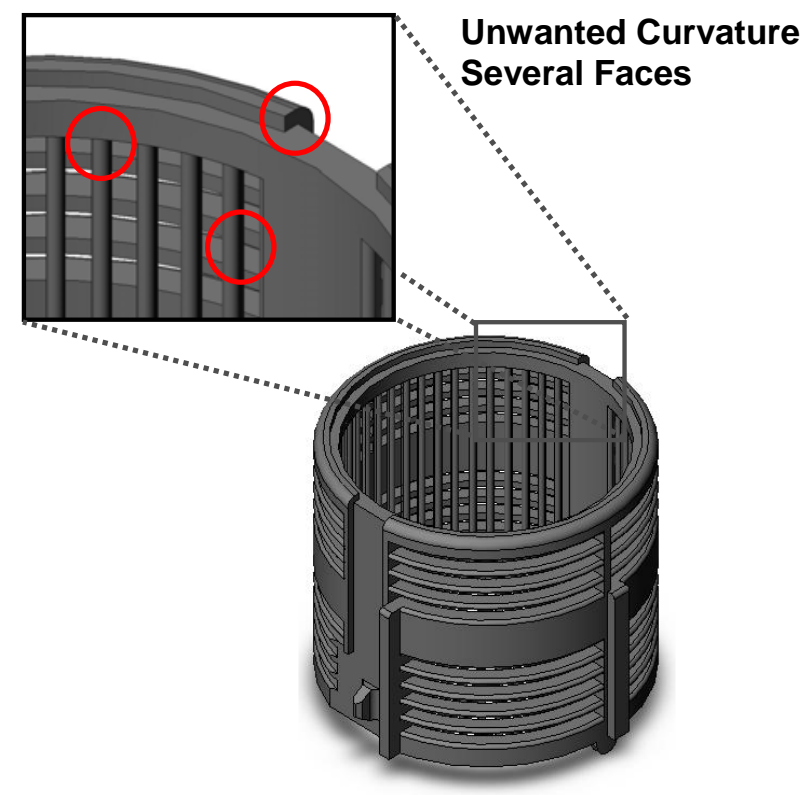

(b.) Original Ion Chamber

Figure 5.3.2: The original ion chamber sensor part compared to the de-featured part.

The ion sensor is the most complex piece in the SA302 that was modeled. It contains the smallest length scale in less than 0.04 in - the width of one of the holes into the settling chamber. In trying to keep the same length scales as close as possible to those in the original geometry, it was necessary to condense the three-dimensional placement of the vertical and 
horizontal ribs into a flat surface to provide a more orthogonal mesh. Curved edges were reduced to seamless entities. Figure 5.3.3 shows where the embossed area underneath the main flow deflector was removed because the experimental SA302 did not contain this added feature. The floor of the experimental version in which all of the base plate components rested on was flat. The actual SA302, however, has this feature because an anti-theft device resides underneath the base. In general, it does not significantly affect the flow patterns for the author's research purposes.

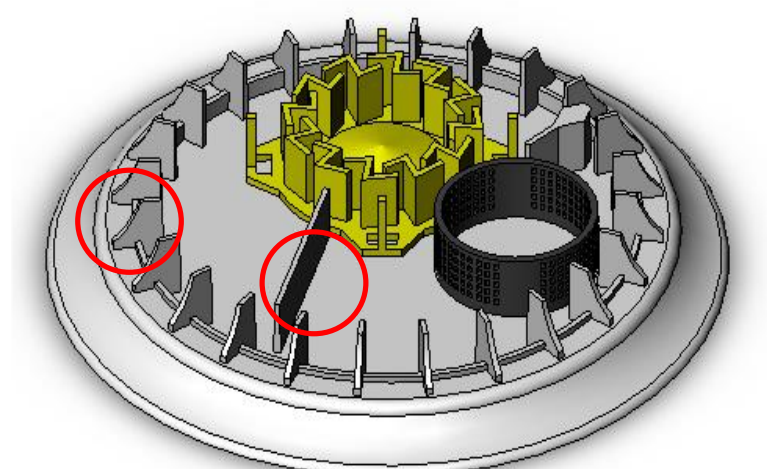

\section{Insect Screen}

Flat Base Plate

Fully Attached

Detached Parts

Embossed Base Plate

(a.) De-featured Base Plate

(b.) Original Base Plate

Figure 5.3.3: The original detector base plate compared to the de-featured assembly.

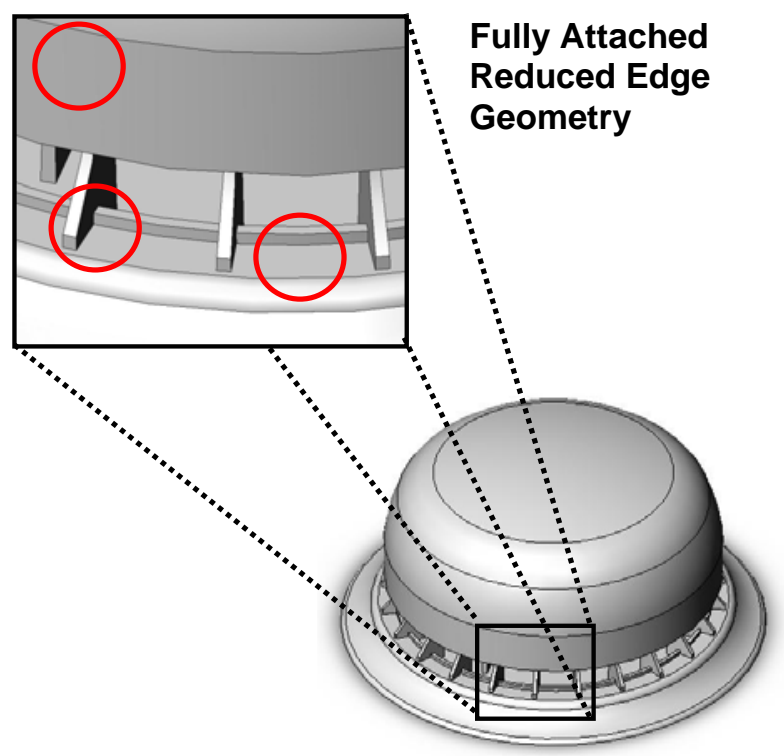

(a.) De-featured SA302 Smoke Detector

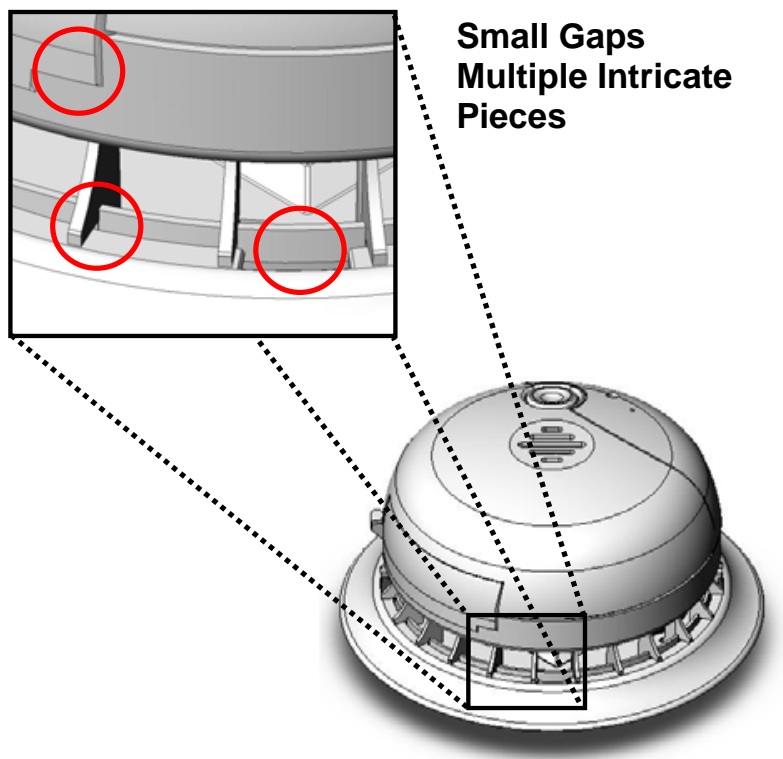

(b.) Original SA302 Smoke Detector

Figure 5.3.4: The original SA302 smoke detector as compared to the de-featured assembly. 
In the end, it was easiest to collapse the numerous individual detector parts (i.e. battery door, led, electrical boards, etc.) into only six actual parts, or volumes. These six parts are: the two sensors, the interior base plate, the detector base plate, the deflector fins, and the upper dome. It was assumed that there was no flow in the interior of the hollow dome containing the electronic circuit boards. Because the interior flow is not significantly affected by the opening surrounding the sensors leading into the dead zone of the hollow dome, the dome was modeled as a solid object. Figure 5.3.5 below illustrates this modeling feature.

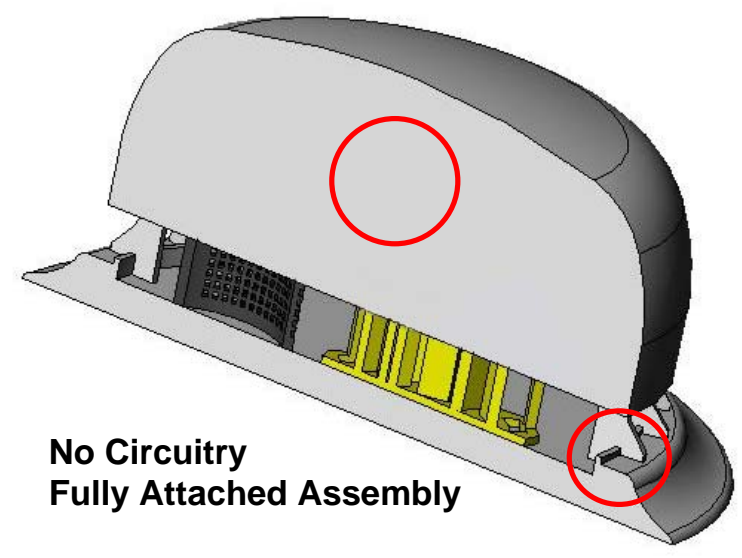

(a.) De-featured Smoke Detector

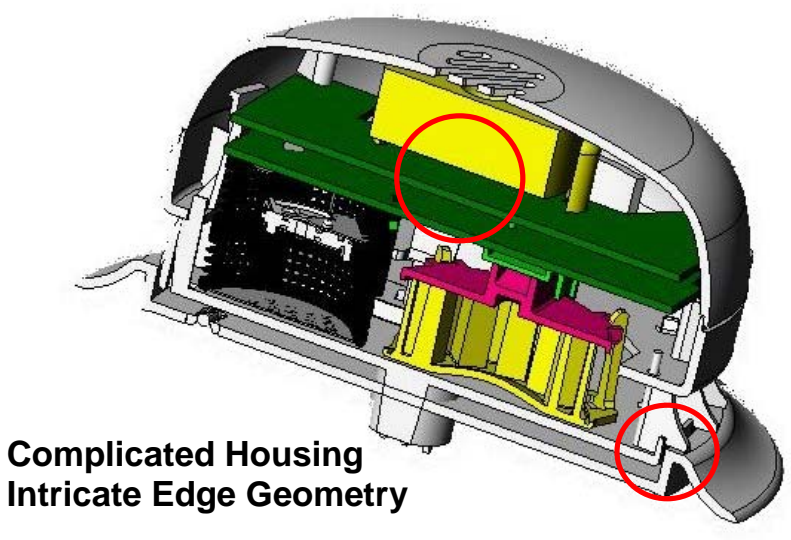

(b.) Original Smoke Detector

Figure 5.3.5: The original detector compared to the de-featured detector sliced along a central cutting plane.

Similar to the detector, the measuring ionization chamber (MIC) has gone through a geometric evolution in its own right. Because there were no original Solidworks ${ }^{\circledR 20}$ files available to edit for the MIC, the MIC geometry was generated from scratch. The MIC design consists of the realized exterior geometry measured with a micrometer, and a simplified internal geometry positioned to govern the main flow patterns. Because sufficient correlation was made between experimental LDA and computational data, the MIC's porous screens were modeled as open inlets into the chamber. Internally, the concentric wall just beyond the outer shell was modeled due to the major role it plays in dictating flow direction upon immediately entering the MIC. The outer shell has been modeled as an infinitely thin wall surface. Modeling this outer shell as an infinitely thin plane as opposed to giving it a real thickness minimizes meshing issues encountered later. Figure 5.3.6 below compares the 
originally modeled MIC with all basic features, with that of the final, de-featured model implemented inside of Fluent ${ }^{\circledR 18}$.

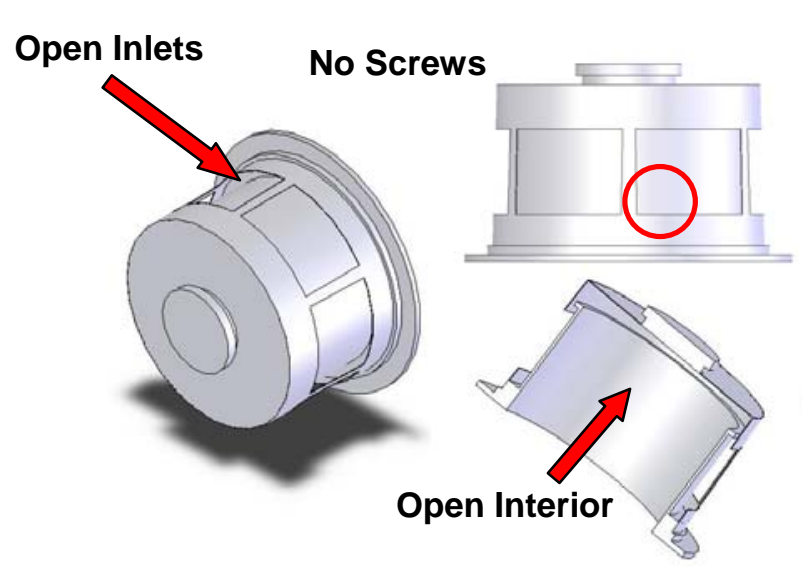

(a.) De-Featured MIC

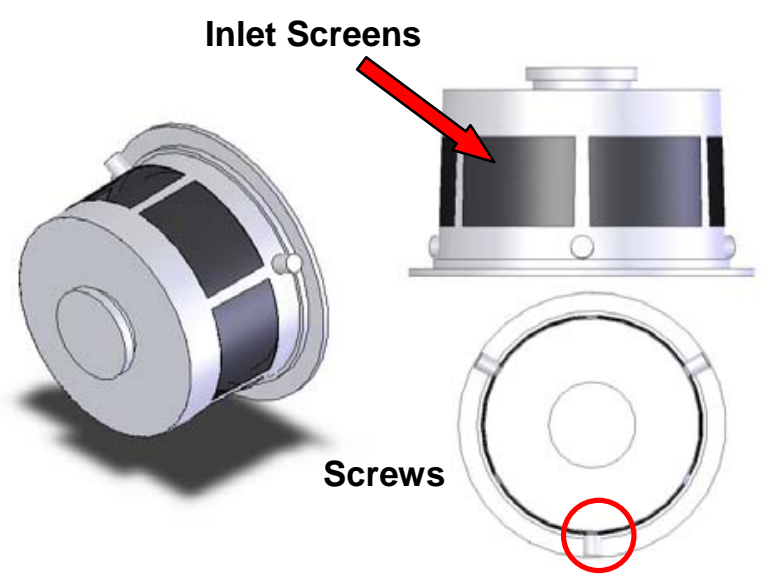

(b.) Original MIC

Figure 5.3.6: A comparison of the originally modeled MIC to that of the de-featured, CFD model.

Generally speaking, the MIC evolved from just a cylinder disrupting the flow field, to a quantified perturbation in the flow delivering $30 \mathrm{l} / \mathrm{min}$ of suction out of the environment. Because the research is focused primarily on flow characterization inside and surrounding the near field of the detector, there is no real need to further evolve the internal geometry of the MIC. Detailed flow and particle tracking within the MIC could become the topic of a completely new study.

\subsection{COMPLETED MODEL}

After each main component was geometrically reduced, an assembly was made in Solidworks ${ }^{\circledR 20}$ connecting all of the various entities. The main components consisted of the SA302 smoke detector, the MIC, the compass plate measuring detector orientation, and the test section itself. This entire test section model was then easily imported into Gambit ${ }^{\circledR 18}$ using ACIS objects. Figure 8 shows a perspective view of the complete Solidworks ${ }^{\circledR 20}$ assembly along with applied boundary conditions. Currently, the specified boundary conditions consist of the main velocity inlet, the velocity outlet derived from MIC suction, the main outflow, and a hole located directly above the detector where internal flow speeds can be measured. These zones will be discussed in more detail later on. 


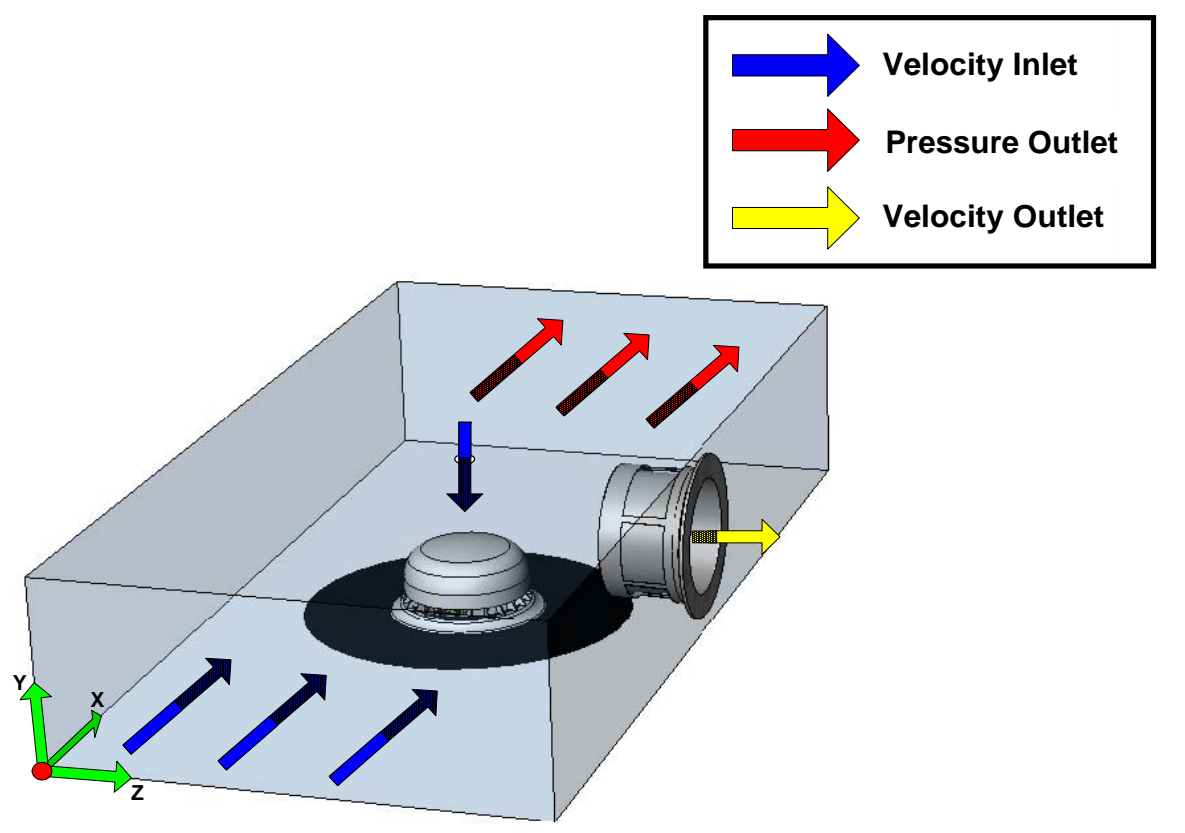

Figure 5.4.1: The total model with inlet and outlet boundary zones as developed in Solidworks ${ }^{\circledR 20}$.

The original idea was to place the computational inlet exactly where laser Doppler anemometry (LDA) data acquisition began, approximately 12 inches downstream of the origin in the $x$ (axial) flow direction. This led to a few minor meshing problems because this plane was coincident with the leading edge of the compass plate on which the detector rests. Placing the inlet zone here created facial intersections leading to highly acute geometric entities. It was finally decided that the computational inlet would be placed in roughly the same plane as the inlet in the real test section for simplicity.

As stated previously, the CFD origin has been defined at the same point where the experimental origin was located - in the corner opposite the MIC against the inlet. In keeping with original Solidworks ${ }^{\circledR 20}$ dimensions, inch units have been retained as the standard unit of length. After measuring smoke box dimensions, the center of the compass plate was placed at coordinate $(18,0,9)$. The detector centered within this plate, has the same centralized coordinates. The MIC attached to the wall opposing the origin, was measured with the center of its base resting at (18.3125,3.5,18 in) - slightly off center with respect to the detector. In all, the computational model is 35.5 in long, 18 in wide, and 7 in tall, as shown in Figure 3.2.4. Although the test section is 36 in long in the axial flow direction, 
prescribed inlet conditions were taken 0.5 in downstream of the actual origin - making the simulated flow field 0.5 in shorter at $35.5 \mathrm{in}$.

In summary, original SA302 IGES files, because they were directly translated from the complete Solidworks ${ }^{\circledR 20}$ assembly, were abandoned for use because Gambit ${ }^{\circledR 18}$ has difficulties importing the IGES format into a solid geometry that could be readily meshed. Problems included (but were not limited to) Gambit ${ }^{\circledR 18}$ only reporting surfaces without attached volumes, along with leaving unexplainable gaps which would ultimately play havoc on the model. Ultimately, it was found that the ACIS file format was both easily exportable from Solidworks ${ }^{\circledR 20}$ and importable into Gambit ${ }^{\circledR 18}$. This is most likely due to the Gambit ${ }^{\circledR 18}$ software being driven by the ACIS kernel. Solid objects were imported as whole volumes, leading to a very well-defined geometry that was easily modifiable in Gambit ${ }^{\circledR 18}$. Due to this reduction, very little effort beyond this is needed in Gambit ${ }^{\circledR 18}$. Because Solidworks ${ }^{\circledR 20}$ is a

much more sophisticated CAD package than Gambit ${ }^{\circledR 18}$, it was worthwhile to spend more modeling time in the Solidworks ${ }^{\circledR 20}$ environment.

\subsection{GRID GENERATION}

When formally introduced to the Gambit ${ }^{\circledR 18}$ software modeling environment, the author executed various test cases with very simplified geometries to get a feel for what to expect from the results. At first, simple cylinders roughly the same size as the detector and MIC were placed within a box the size of the test section. Simple boundary zones were defined that would represent boundary conditions for the N-S equations, and basic solutions were executed and analyzed. As confidence grew from reasonably reported results, the detector along with the MIC originally forged as cylinders evolved to the complete geometric model finally seen in Figure 5.4.1. It is the goal of this section to introduce one of the most important user-related aspects of CFD - computational grid generation. Applying a mesh to the geometric model that can accurately represent flow behavior experienced within the smoke box test section is paramount. The next few sections describe meshing basics, how the spatial grid relates to the computational grid, and flow boundary conditions that serve as the major user defined input to the model.

Meshing any three-dimensional solid object is quite straightforward. What one must first understand, before delving into rugged definitions and their relationships, is that CFD models represent the meshed flow field area - not the actual objects within the flow field. 
Although there are more complicated ways of creating meshes other than what will be stated here, the basics are stated here for brevity.

The most fundamental component of a mesh is a node. A node is an infinitely small point in space that resides in either a two or three-dimensional space. A collection of nodes along a line denote a meshed line, and a collection of meshed lines attached at each endpoint can create a mesh plane - or meshed face. If two separate meshed lines are attached at an endpoint, that intersection is defined by just one node shared by each entity. Just as meshed lines make up meshed faces, a collection of faces can make up a meshed volume. Figure 5.5.1 visualizes these ideas.

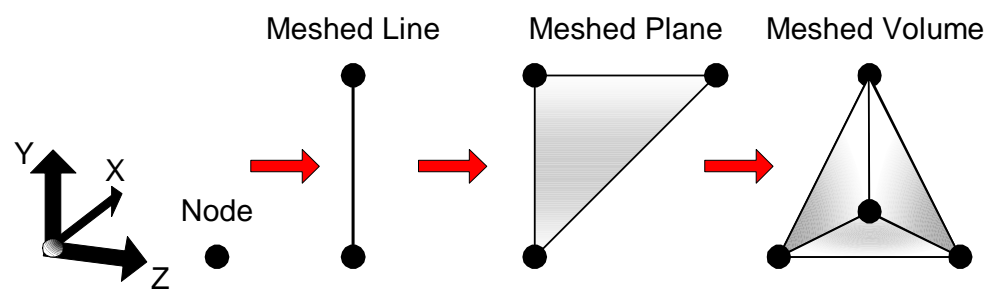

Figure 5.5.1: Mesh hierarchy starting with a node, and ending at a meshed volume.

Because Fluent ${ }^{\circledR 18}$ uses the finite volume method in describing how a fluid behaves, the meshed volume as seen in Figure 5.5.1, represents the same element shown in Figure 5.2.1 - now just with nodes at each facial intersection. It is on these meshed volumetric elements where all of the CFD calculations take place. Meshing, in short, is the art of approximating a real geometry by outlining it from these elements. The more orthogonal the faces of each element are to one another (and in the case of a pyramid or tetrahedron, the more equilateral the faces are), the less trigonometric approximations are needed in calculating wall skewness, obtuseness, etc. This, in turn, is directly proportionate to how well-conditioned the resultant mesh is. An ideal mesh of a simple rectangular duct, for example, would merely be comprised of elements shaped like cubes, all with orthogonal components. Due to various meshing schemes and a multitude of geometry types, different element types exist to serve different purposes. In Gambit ${ }^{\circledR 18}$, two-dimensional geometries can be created using quadrilateral or triangular face elements - all which can be made more refined by adding nodes to midpoints within the elements themselves. Similarly, threedimensional geometries can be built with brick and tetrahedral elements able to be refined in a comparable fashion. The TGrid function will explain this in more detail. 
For the purpose of this research, it was found that tetrahedral, three-dimensional elements using the Gambit ${ }^{\circledR}$ TGrid $^{\circledR 18}$ meshing procedure is the optimal way to mesh the UL Smoke Box three-dimensional flow field. The geometry is too complex to choose otherwise. Tetrahedral meshing, using the same basic principles stated above, discretizes the flow into tetrahedrons - volumetric elements with three faces. Figure 5.5.2 shows its basic shape with nodes attached at each vertex.

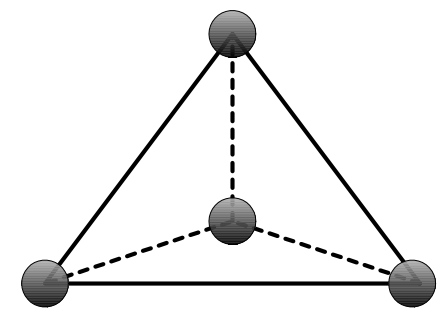

Figure 5.5.2: The 4-node tetrahedral element employed in the computational model.

There are several constraints one can apply to an object before it is meshed to guide the grid generation in a certain direction. With CFD, there is always a compromise. On one hand, the grid needs to be refined enough to approximate the geometry as best it can. On the other hand, the more nodes one adds to the system, the more numbers the computer must crunch equaling more spent time and effort. Also, enough nodes to capture high velocity gradient regions is needed as well. An equilibrium condition can be reached when a mesh or grid is said to have achieved grid independency. When a computational mesh becomes grid independent, it means that any further successive refinement of the grid will not change the value of the dependent variables according to the convergence criteria. In general, it should always be decreased to see if it is an independent solution. The convergence criteria are merely the acceptable errors in the dependent variables (denoted $\epsilon$ ) allowed between iterations, set before iterations begin. The standard, normalized convergence criteria for dependent variables in Fluent ${ }^{\circledR 18}$ is set at 0.001 - which can be expressed as follows:

$$
\frac{\text { Var }_{\text {old }}-\text { Var }_{\text {new }}}{\operatorname{Var}_{\text {new }}}=\in=0.001
$$

This means that if none of the values of the dependent variables change by 0.001 or less after a preceding iteration, the solution is said to have converged. Although the majority of the models ran in Fluent ${ }^{\circledR 18}$ reached converged as high as $1 \times 10^{-6}$, more difficult orientations $\left(0^{\circ}\right)$ had maximum convergence at 0.001. Although convergences varied somewhat between 
orientations, little if any changes in the calculated flow field were noticed. Although this form of convergence is the generally accepted standard for most simple problems, issues can arise by initializing the flow field variables extremely close to the converged, realistic solution because calculated values do not deviate enough to meet such refined convergence criteria. These issues were experienced when initializing the flow field to $30 \mathrm{ft} / \mathrm{min}$ as opposed to $0 \mathrm{ft} / \mathrm{min}$. Although both solutions converged to the same steady-state values presented in the results, the $30 \mathrm{ft} / \mathrm{min}$ initialization "flat-lined" the residues much sooner than those initialized to $0 \mathrm{ft} / \mathrm{min}$.

Because the model is so complex, it would be very laborious to go through and mesh each edge line and then each face. Knowing this, there are several options, or constraints, one can apply to any object before a mesh attempt is made. Three basic constraints easily applicable for edges, faces, and volumes are the interval count, interval size, and shortest edge percentage method. The interval count and size process are similar. Giving an edge an interval count of two, for example, would break a line up into two segments, placing one node in the center and the other two at each endpoint. Interval size works by inputting the size one wants for each interval. To get the same mesh as the one made using interval count, an interval size of $50 \%$ would be used. Using the shortest edge percentage method to mesh an object is different because its gridding behavior is object independent, relative to only one edge in the entire model. The shortest edge percentage scheme takes the shortest edge within the entire model and makes the interval size for whatever is to be meshed a percentage length of that respective shortest edge. In short, it is a simple way to reference the entire grid to one actual size.

Although a variety of meshing methods have been tested, the most dependable meshing scheme has been to first mesh the outer edges of the test section. Afterwards, the edges and then the faces of the compass plate are meshed up to the edge of the detector. Once this is complete, a shortest edge percentage of $100 \%$ is used to volume mesh the entire flow field. One important aspect to understand about the meshing process is that edges must be meshed before faces, and faces meshed before volumes. What this means is that if one were to attempt to volume mesh the entire flow field before doing any primitive meshing, Gambit ${ }^{\circledR 18}$ will first mesh all lines first, then all faces second, and then finally mesh the entire volume last given the specific size function. In doing this, it will use whatever constraints 
were placed before commencing the meshing procedure. If a volume mesh command is given to an entity which already includes a meshed edge, for example, then the volume mesh must conform to the conditions of that meshed edge that have already been established. Unless a completely new mesh is needed without underlying topological factors, a toggle can simply be turned off that would force the removal of old meshes.

Meshing constraints, especially for three-dimensional volumes, help limit the number of nodes in a model. For areas of high interest such as the interior of the smoke detector, a very fine mesh is desired as to characterize the flow as accurately as possible. In other areas such as several diameters beyond the detector, although general flow behavior is sought, a more coarse mesh can be exercised as to not flood the model with millions of unnecessary computational nodes. Also, in areas where there is a lot of activity, such as steep velocity gradients where the flow speed is rapidly changing magnitude and direction, one would want to increase the node fidelity to capture the detail of a rapidly changing flow pattern - such as flow vorticity or wall interaction.

The next few figures depict the completed three-dimensional test section grid. Although there are a few different versions of this model due to detector orientation, graphically they are all nearly identical. In all, there are five complete models; namely, the $0^{\circ}, 315^{\circ}, 270^{\circ}, 180^{\circ}$, and $90^{\circ}$ detector orientations. These models implement the MIC with an infinitely thin planar shell. Because it is difficult to show a three-dimensional tetrahedral mesh, planar slices and walls will be taken from descriptive planes. Figures 5.5 .3 and 5.5.4 show a close-up of the detector and sensors on the inside, respectively. Most figures will be zoomed into this level to capture the fineness of the grid. It is easily identifiable in Figure 5.5.4 to understand the grid fineness about the ion sensor, which is roughly the size of one of its settling chamber holes. Figure 5.5.5 shows the discretized MIC with accompanying velocity outlet boundary condition at its base shaded in blue. One can see from the oblique view how the six screens surrounding the measuring chamber have been modeled as open inlets, as because the screen influence is negligible. Figure 5.5.6 shows the test section walls in their entirety. 


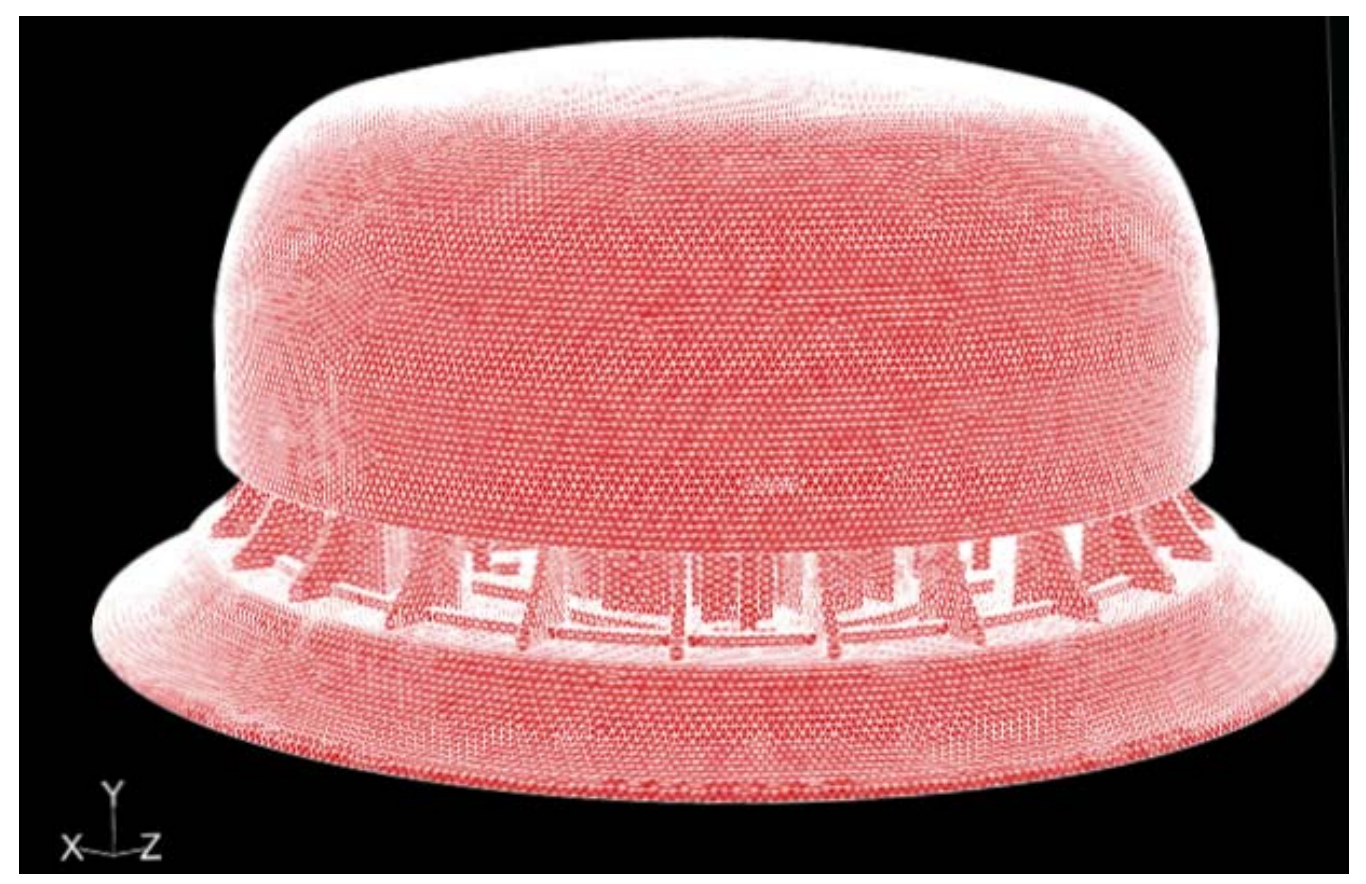

Figure 5.5.3: The surface mesh of the SA302 smoke detector.

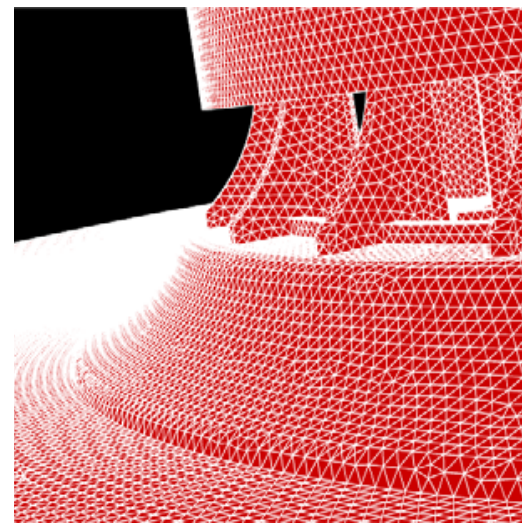

(a.)

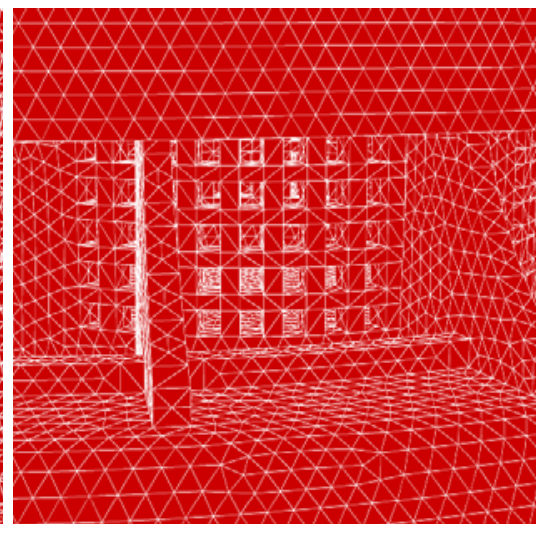

(b.)

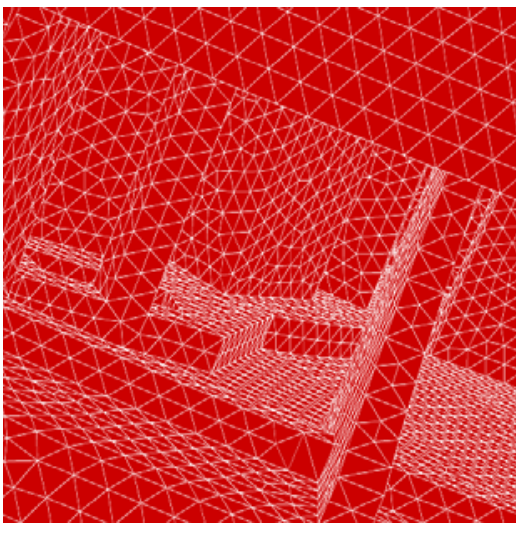

(c.)

Figure 5.5.4: The surface mesh of the detector's respective parts. Images of the (a.) base plate, (b.) ion chamber, and (c.) photoelectric sensor are portrayed. 


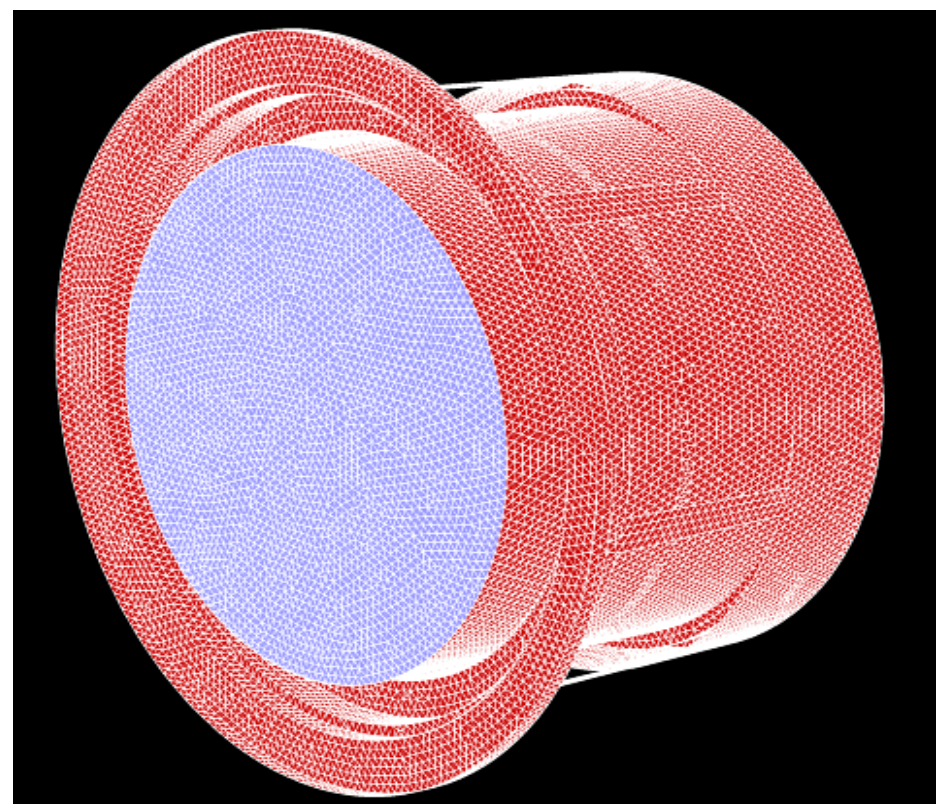

Figure 5.5.5: The surface mesh of the MIC.

It is of interest to note here about Figure 5.5.6 the fineness of the grid present about the hot-wire anemometer access hole on the ceiling and about the MIC in comparison to the rest of the wall. The pressure outlet boundary zone is shaded in blue. By meshing the edges that make up the MIC and measuring hole finer than the test section edges, more flow detail can be captured surrounding these areas where steeper velocity gradients occur. Because the speeds are so slow through the main areas of interest, there is not much emphasis placed on developing a boundary layer grid in these locations.

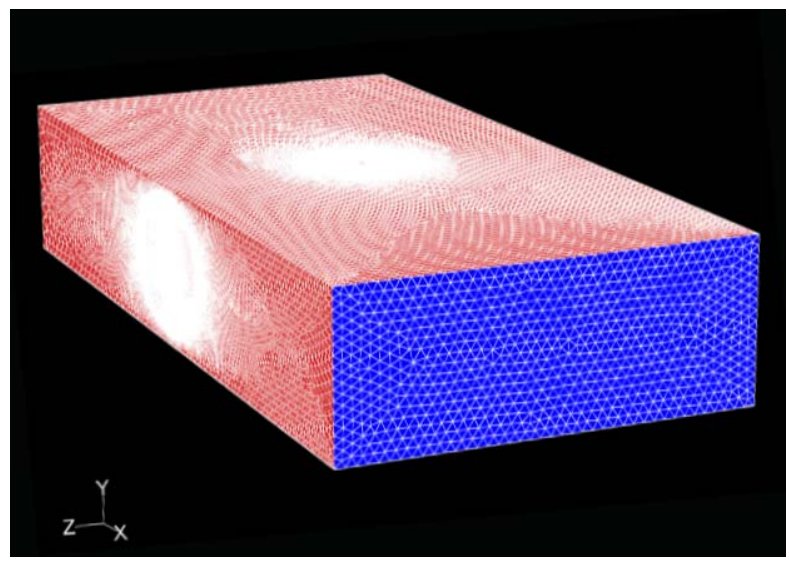

(a.)

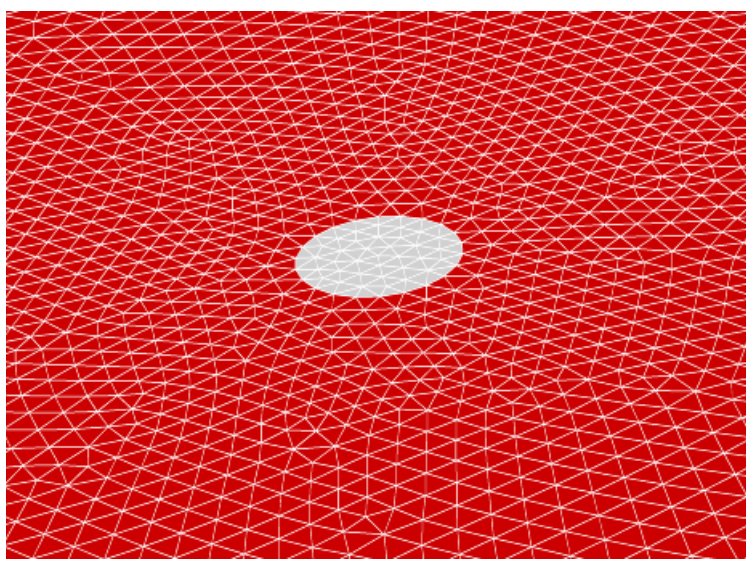

(b.)

Figure 5.5.6: The (a.) test section walls with accompanying pressure outlet zone and (b.) hotwire anemometer access hole. 
The next few figures will depict various interior flow field cross-sections in the test section of the unstructured grid. All walls like the detector and MIC will be solid white, with mesh lines presented in black. The shape of each element is quite deceiving in planar view because the tetrahedron is physically sliced along that plane. Some elements sliced across the center will look like well-posed elements. Others where the slice happened very close to a node will seem very skewed and ill-posed. The purpose of these plots is to only show the mild grid refinement as it approaches the MIC, detector, and hot wire anemometer access hole. Pure white zones denote voids in the flow field where objects are present such as parts of the MIC and the entire detector. Because the shell of the MIC has been modeled as a twodimensional surface, there are no white void space lines for this zone. However, the internal wall which does carry thickness can be observed protruding outward from the MIC base plate. Figure 5.5.7 depicts a planar slice at $x=18$ in right down the middle of the detector and off center of the MIC. Figures 5.5.8 and 5.5.9 yield a closer inspection of the detector and MIC, respectively, in showing tetrahedral sizing constraints from the walls to the outlying flow regimes.

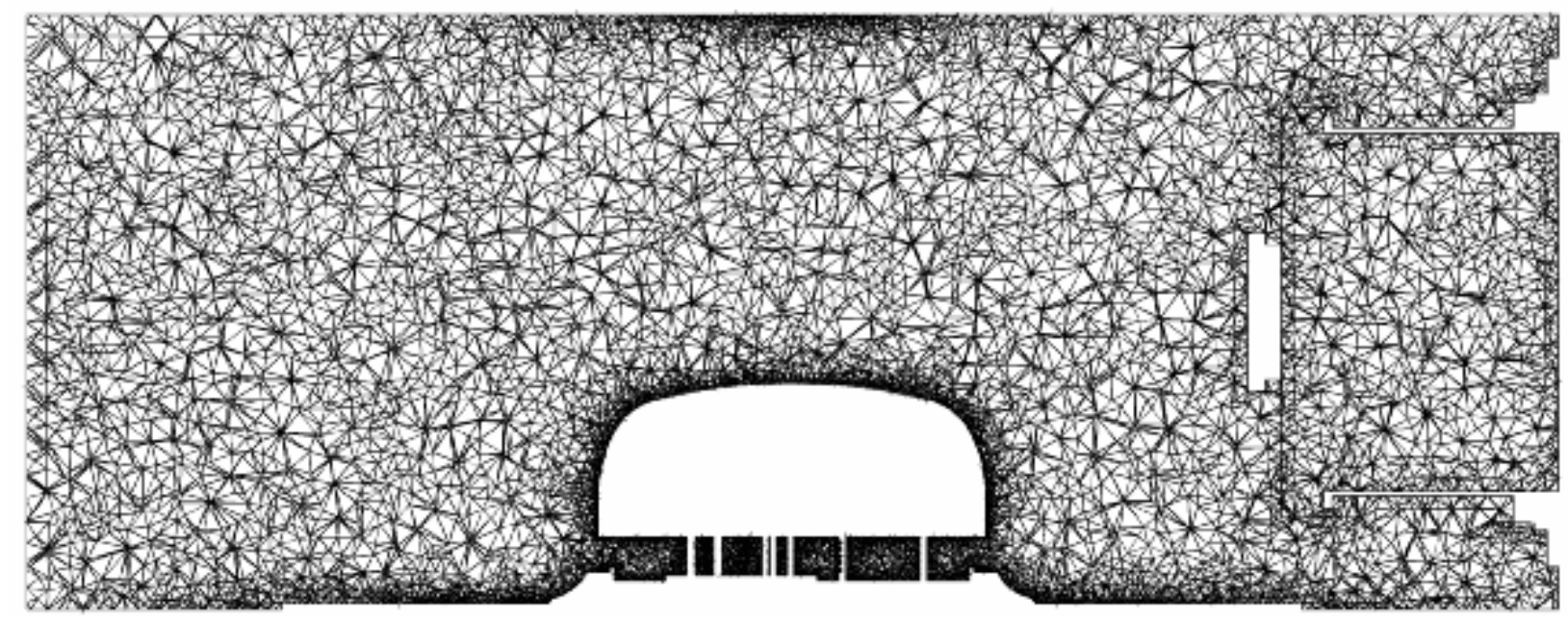

Figure 5.5.7: The interior flow field at the $x=18$ in plane depicting the centerline cut of the SA302 smoke detector and an off-center slice of the MIC on the right. 


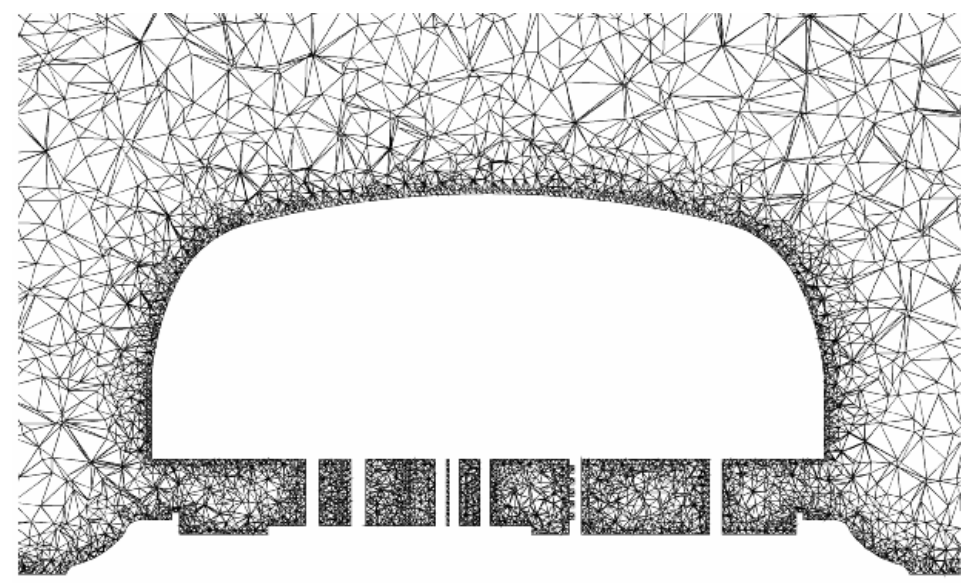

Figure 5.5.8: The same cut as in Figure 5.6.10, but zoomed in on the SA302 smoke detector.

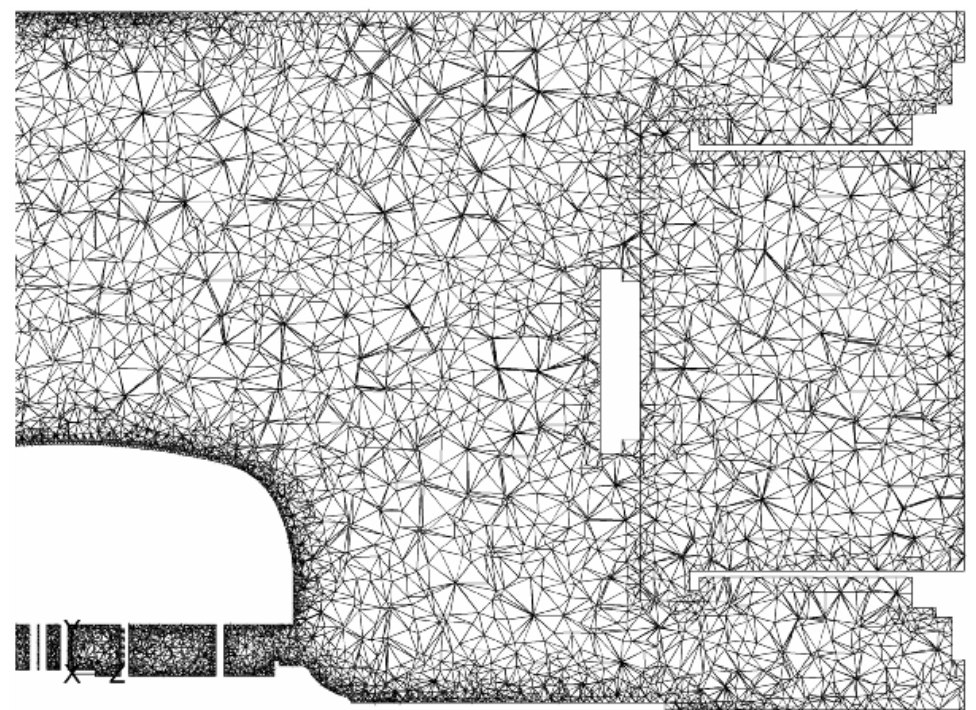

Figure 5.5.9: This figure shows the element size gradients established between the probe access hole, model SA302, and MIC.

Figures 5.5.10 through 5.6.12 depict the mesh at the $y=0.50$ in plane. This plane is an approximate centerline cut of the SA302's opening - which describes a lot of detail where there is high interest. Figure 5.6.10 shows the entire area of the detector easily identifying the fins circumnavigating the sensors. The flow deflector is also easily discernible. 


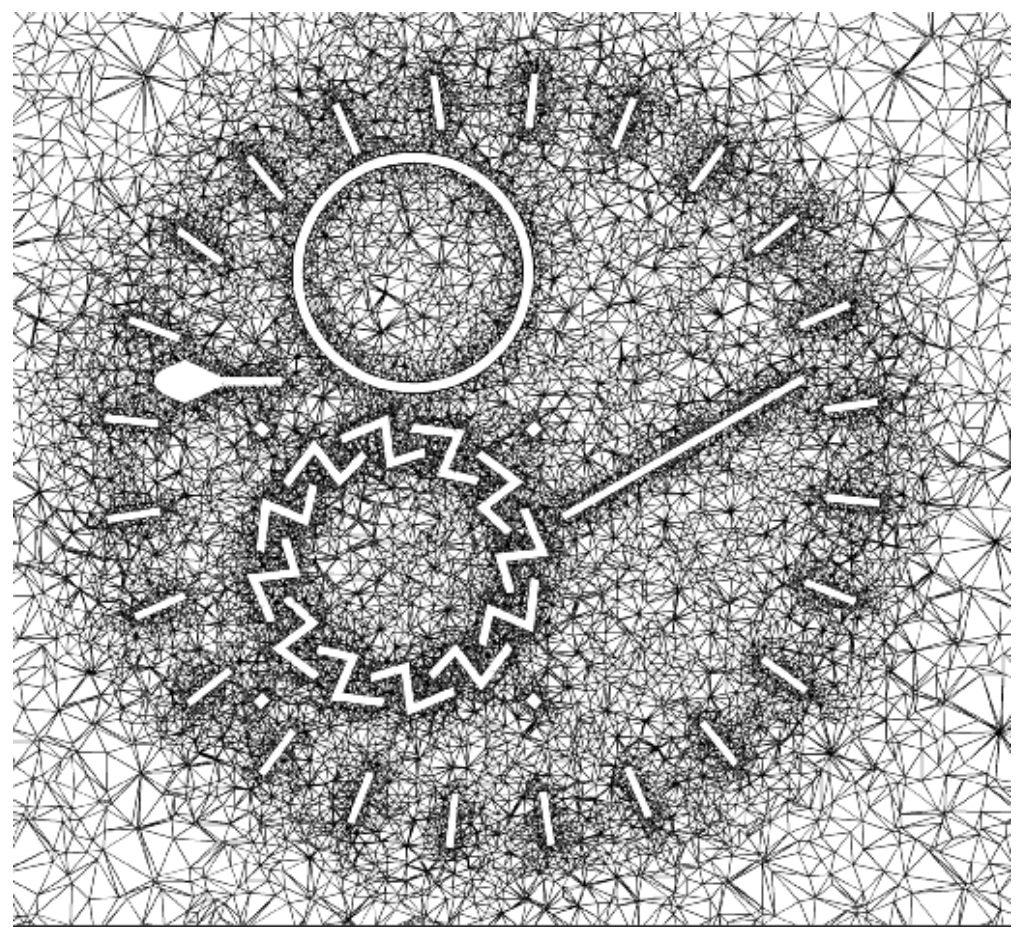

Figure 5.5.10: A detector mesh slice at $y=0.50 \mathrm{in}$.

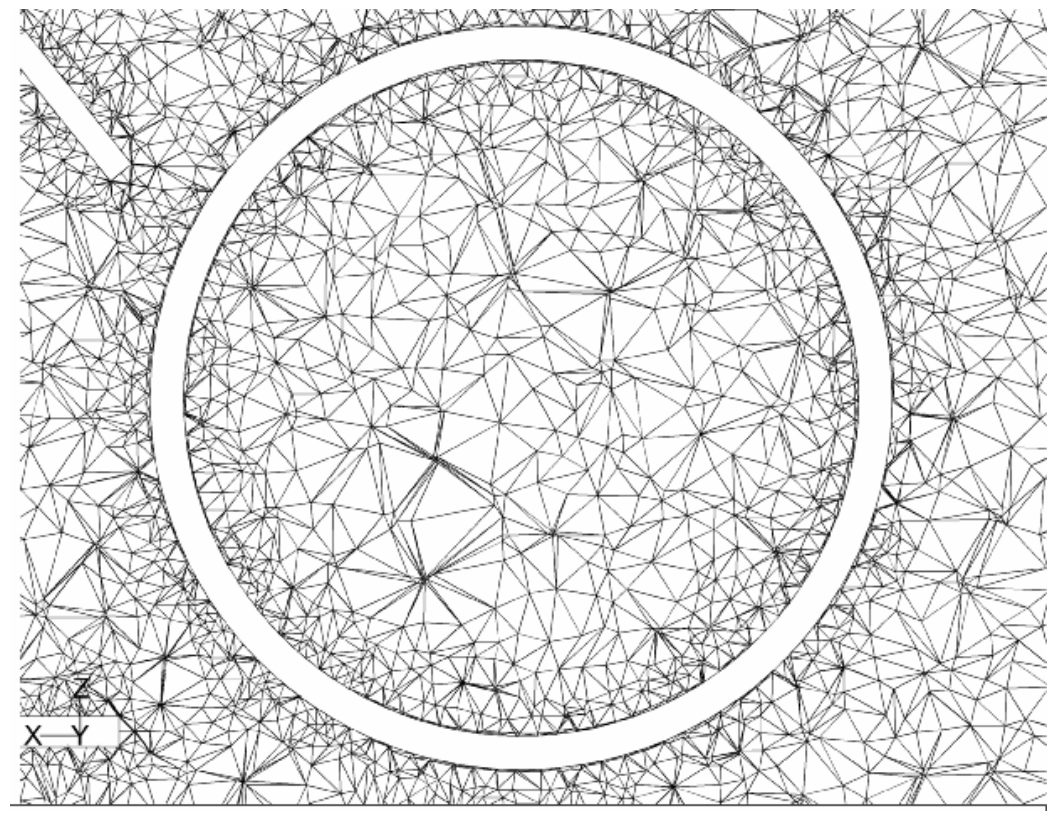

Figure 5.5.11: The ion sensor in the $y=0.50$ in plane. 


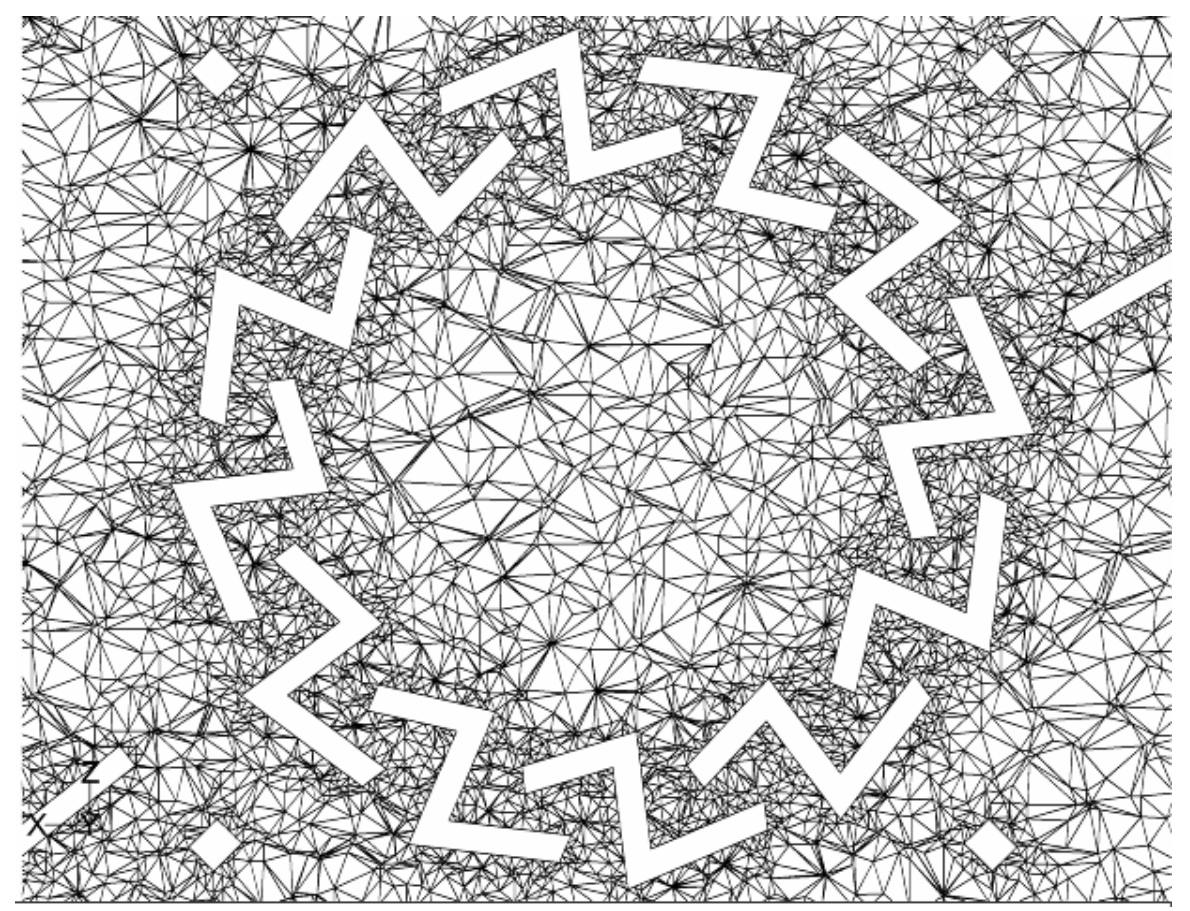

Figure 5.5.12: The photoelectric sensor in the $y=0.50$ in plane.

The average CFD model of the 5 orientation models in this study is comprised of about 2.6 million unstructured tetrahedral elements defined by approximately 560,000 nodes. On a 2.66 Ghz Dual Intel ${ }^{\circledR}$ Xeon $^{\mathrm{TM}}$ Processor with 2 gigabytes of RAM, exportation time of the computational grid alone from Gambit ${ }^{\circledR 18}$ to Fluent ${ }^{\circledR 18}$ takes roughly 40 minutes. Recall, Gambit $^{\circledR 18}$ is the grid generation software and Fluent ${ }^{\circledR 18}$ is the computational code.

Before exporting the mesh to Fluent ${ }^{\circledR 18}$, it is necessary to do a basic grid check to make sure the grid is robust and physically accurate. One major item to check is the element skewness of the entire model. Previously mentioned, skewness is a measure of how obtuse an element is relative to orthogonality. If Fluent ${ }^{\circledR 18}$ receives a mesh that has any element over 0.98 skewness, it will not solve the system and return an error. In Gambit ${ }^{\circledR 18}$, this can be quickly analyzed by selecting the Check Grid command and explore the skewness of the grid. In previous test section models, there were meshing issues that directly related to this problem. The final models, however, show little skewness with the highest skewed element being about 0.85. These elements tend to form between the outer boundary layer of the detector and the approaching larger elements from the test section walls. Whenever there is a major change in the sizing function, often times a few semi-skewed elements will be reported. These few elements, however, often make up less than $1 \%$ of all of the elements, and often do not pose any significant threat to the model's integrity. 
Figures 5.5.13 and 5.5.14 show the shaded faces of the meshed walls. Figure 5.5.13 depict the three main components: the MIC, SA302 smoke detector, and compass plate positioned beneath the detector. The compass plate is used to denote the orientation the detector is in during testing. Fluid mechanically, this plate is fairly important to model because boundary layer effects may come into play even though the flow velocity is so slow.

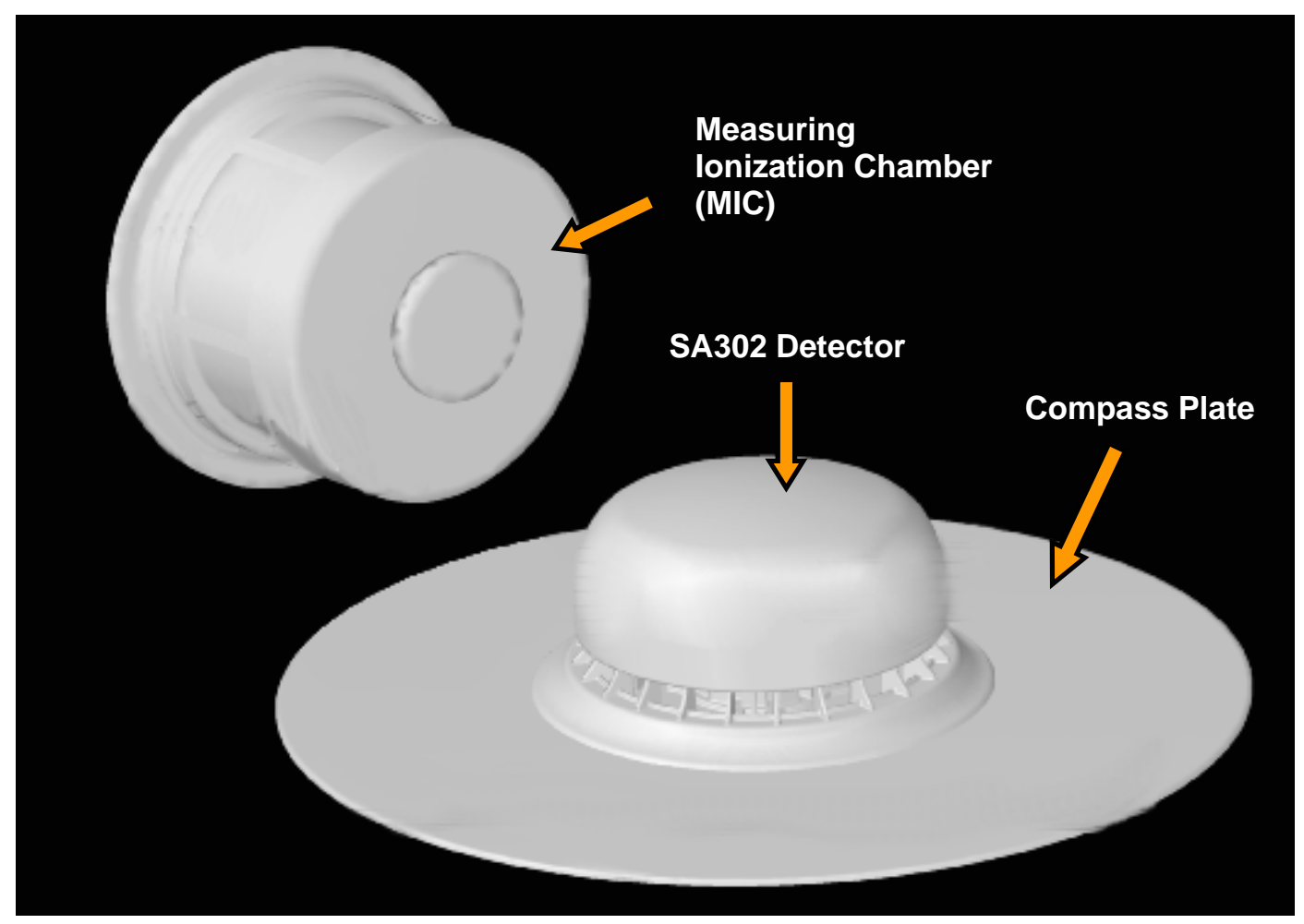

Figure 5.5.13: The lighted wall mesh of the major components in the test section.

For displaying the results of the interior, the detector was defined in two pieces: the base plate and sensors, along with the upper dome. Figure 5.5.14 below shows the detail reached inside the CFD model showing the overall flow area of concern, with a close-up of the two sensing chambers. These elements will be used as back drops when reporting the flow results inside the CFD model for easy flow visualization. Although the sensors in Figure 5.5.14b and c look as if they have no tops, they actually do and are just not activated as to show their interior. 


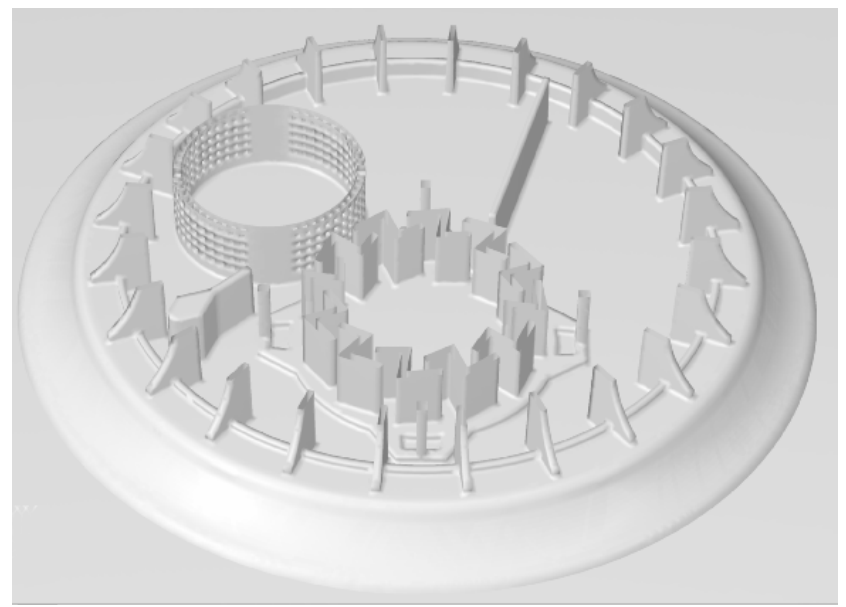

(a.)

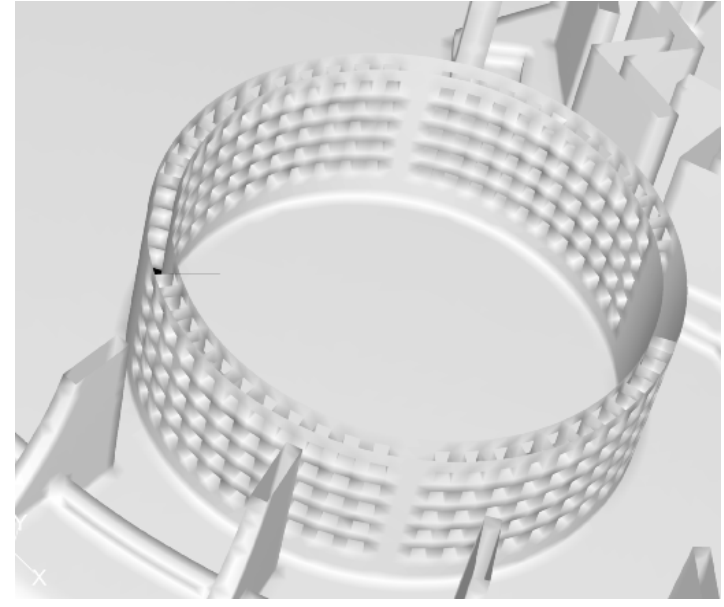

(b.)

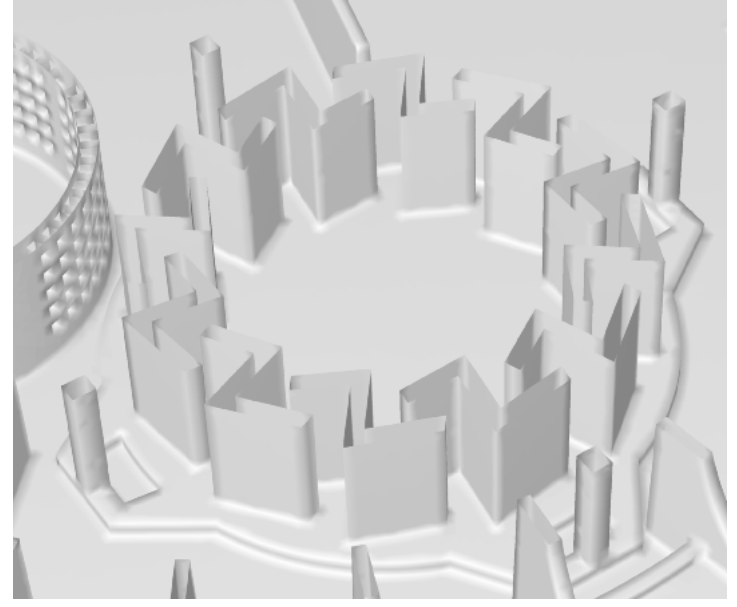

(c.)

Figure 5.5.14: The lighted wall mesh of the SA302, intricately defining the parts of (a.) the entire base plate, (b.) the ion chamber, along with the (c.) photoelectric chamber.

\subsection{BOUNDARY CONDITIONS}

Boundary zones are the next key element in developing a well-posed model. The boundary zones, in short, are zones that impose boundary conditions which place restrictions on the edges and surfaces of a system. These boundary zones are fully analogous to the boundary conditions set for finding solutions to basic differential equations. For twodimensional models, boundary zones are defined along actual edges. For three-dimensional models, boundary zones normally denote face planes. Currently, the boundary zones for the test section are fairly straight-forward.

As mentioned previously, the model begins 0.5 in downstream of the entrance flow straighteners. Here, a velocity inlet boundary condition is imposed along this face of the test section. In preliminary studies, giving this entire face a velocity inlet of $32 \mathrm{ft} / \mathrm{min}$ provided 
very reasonable results when compared to experimental data. Because LDA data were never taken so far upstream of the detector, mainly due to configuration and blockage issues with the laser beam, this inlet condition was quantified using other means. Using a portable hotwire anemometer, 144 equally spaced points at $x=0.5$ in downstream of the flow straightener were measured for their axial, $x$-component velocity. A surface plot depicts this profile in Figure 6.5.1 in the chapter reporting results.

Because the flow field is so close to the flow straightener, it was assumed that there are little three-dimensional effects in this planar region. Thusly, vertical and transverse velocities have been assumed to be negligible. Figure 5.6.1 visualizes the concept behind a flow straightener. Because Fluent ${ }^{\circledR 18}$ does not interpolate between inlet or outlet profile points, it was initially assumed that the 144 experimental data points would need to be smoothed and populated with interpolated points as to create a smoother, more continuous inlet profile. However, after importing the data into the program TableCurve $3 \mathrm{D}^{\circledR}$ to produce a fitted profile for Fluent ${ }^{\circledR 18}$, it was found that differences in the raw and fitted profiles were negligible with respect to downstream results concerning the detector inside of Fluent ${ }^{\circledR 18}$.

\section{Flow Straightener}

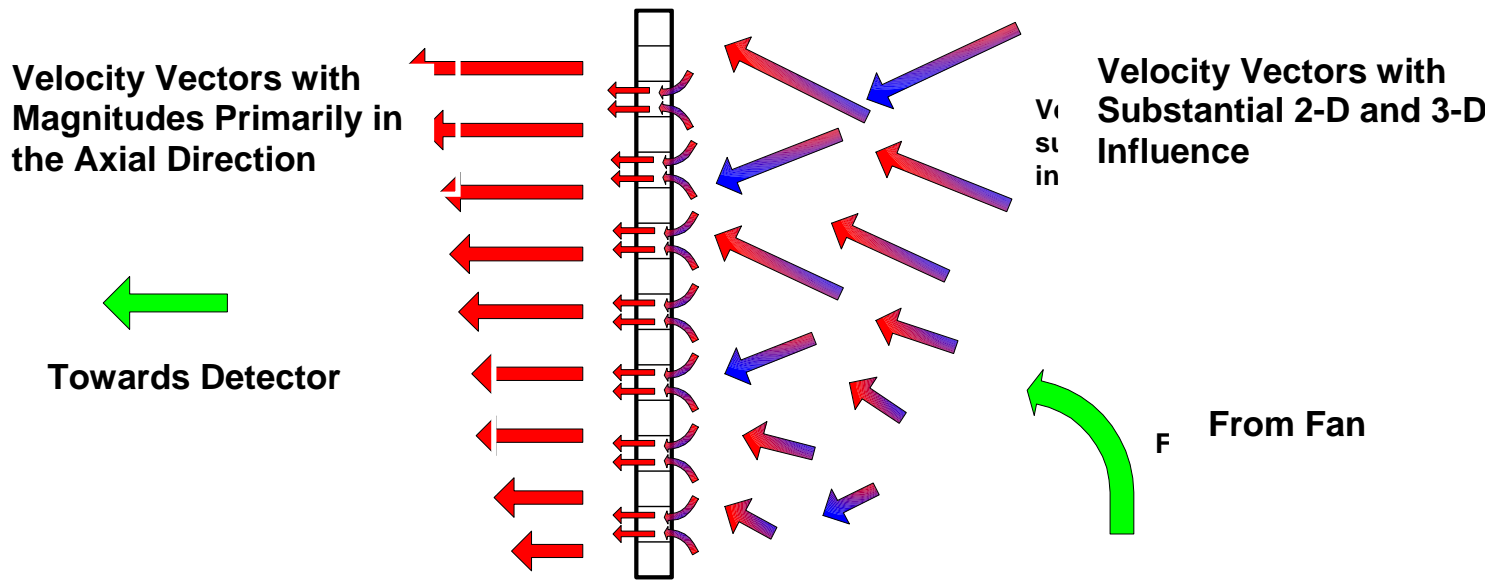

Figure 5.6.1: A simple diagram depicting the functionality of a flow straightener.

On the opposite end of the test section, the two options for an outlet condition consisted of a basic outflow condition, or a pressure outlet condition. The outflow boundary condition is for subsonic cases only, which is quite suitable for this particular low-speed study. Outflow boundary conditions assume pressure and velocity conditions are not known in the region, normally accompanying a slower rate of convergence. The pressure outlet 
condition can be imposed in all flow regimes, and has extra parameters able to be set such as a working gauge pressure and the ability to define backflow parameters if necessary. Due to initial convergence problems, the pressure outlet condition has been imposed on the model using atmospheric outlet conditions (zero gage pressure).

Due to the suction of air at $30 \mathrm{l} / \mathrm{min}$ by the MIC coupled with its intricate design, convergence time grew significantly. The base of the MIC is defined as a velocity outlet condition. Although there is no actual way to define a "velocity outlet" condition in Fluent ${ }^{\circledR 18}$, by choosing the flow direction with respect to the origin being used in the model, one can impose a velocity along with a direction normal to a boundary which can actually transport flow out of the system. The volume flow rate of air the MIC is removing can then easily be converted into a flow velocity, knowing the area of the outlet vent and the fact that volume flow rate is derived from multiplying a flow velocity with a cross-sectional area. The author feels this methodology is best because this leaves the question of how much volumetric flow is entering each inlet face up to the CFD code. As one can see from the diagram in Figure 5.6.2, it is theorized that MIC inlet screens facing the incoming velocity profile will ingest more flow compared to the inlet screens on the opposite side because they are normal to the oncoming flow. Next, oblique screens closest to the oncoming flow receive less flow, with this pattern continuing to the opposite side of the MIC.

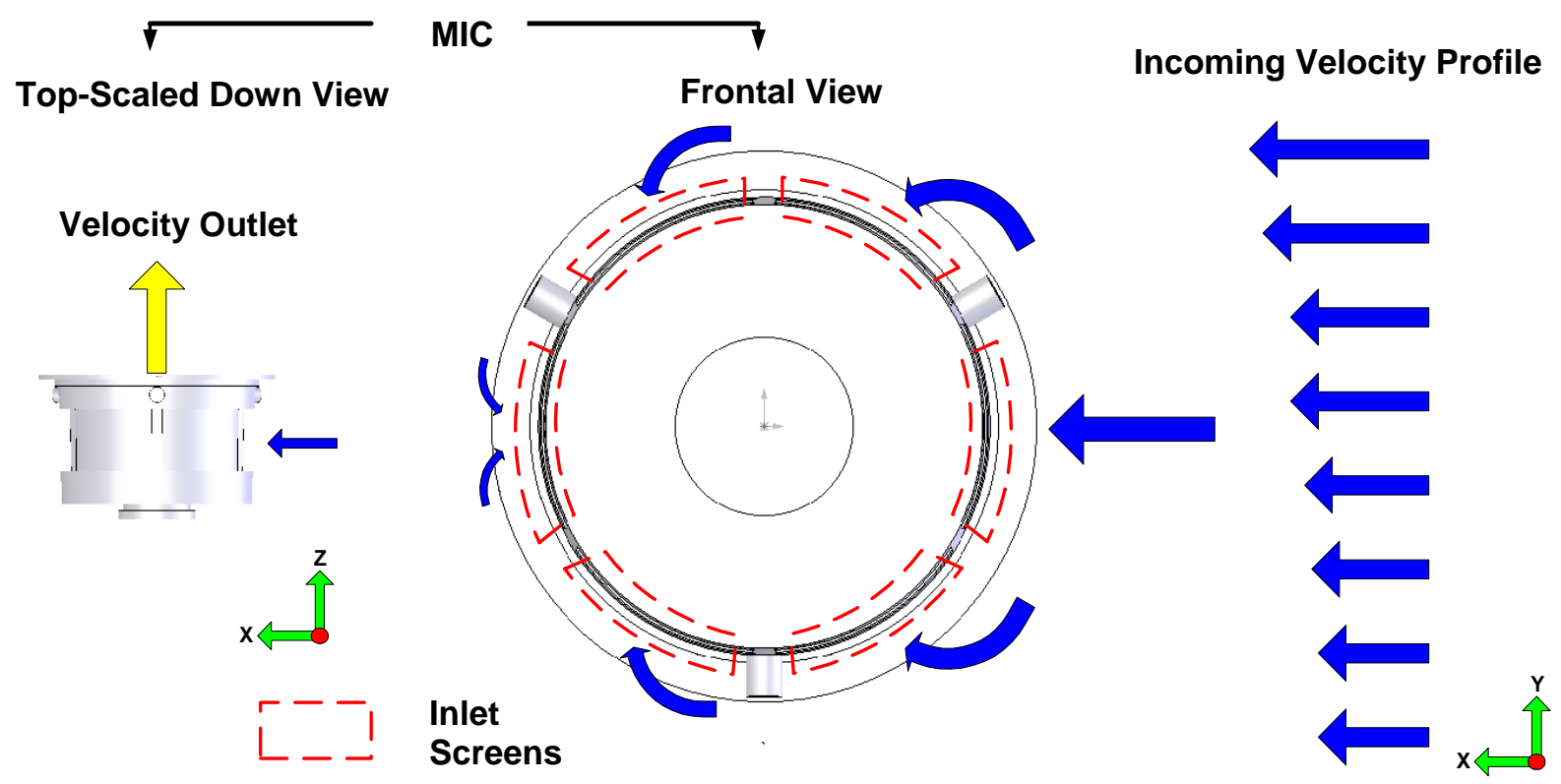

Figure 5.6.2: A simplified diagram describing the probable inlet effects on the MIC screens with respect to flow direction. 
Two boundary zones that were of particular, initial interest were the porous jumps across the insect screen and the MIC inlet screens as shown in Figure 5.6.3. As mentioned earlier, preliminary comparisons between experimental and CFD data did not warrant the need for applying a porous jump condition here because (1), complex porous issues increase the convergence time for the entire model, and (2) this area was a secondary zone in question not significantly affecting the flow characterization of the detector. Hence, the model presented in Figure 5.3.6b was sufficient.

The second porous zone in question is defined by the insect screen around the photochamber sensor used to keep insects and debris from entering. Here, it was found that after a simple pressure loss experiment, there is negligible pressure loss across the screen for Fluent ${ }^{\circledR 18}$ to resolve computationally. The reader is referred to Appendix B for further detail on the results of this study.
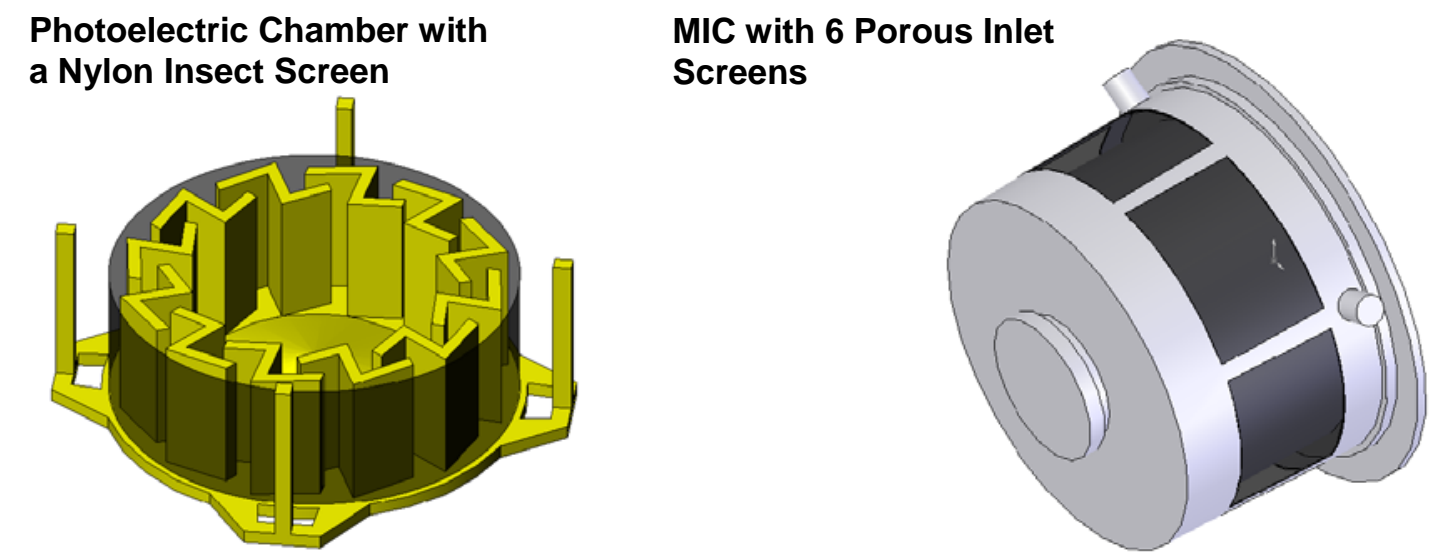

Figure 5.6.3: The photoelectric sensor and MIC with potential porous boundary conditions.

Another boundary condition imposed is a small probe inlet hole in the acrylic sheet located directly above the detector in the smoke box test section. This inlet hole is used to drop a hot-wire anemometer down into the test section while the UL Smoke Box fans are running to ensure a $32 \mathrm{ft} / \mathrm{min}$ flow rate $1 \mathrm{in}$ above the test section floor. Although it only has a $17 / 64^{\text {th }}$ in diameter, the velocities measured across this inlet measure are on the order of almost $400 \mathrm{ft} / \mathrm{min}(\approx 6.5 \mathrm{ft} / \mathrm{sec}$ ). With the smoke box a relatively closed system with added weather stripping to prevent the uncontrolled loss of smoke, this inlet created by the hot wire anemometer access hole is a major source of "make-up" air to replace what is being sucked 
out by the MIC. Because the cross-sectional area is much smaller than the faces of the MIC, however, the speeds are much greater to maintain a constant flow rate of similar magnitude.

\subsection{FLOW MODELS}

Generally speaking, there are two different types of flow, described as laminar and turbulent. Most CFD models are based upon whether or not a flow is laminar or turbulent. The exception to this rule is direct numerical simulation (DNS), which computes the entire three-dimensional flow field in both space and time without resorting to any true turbulent modeling parameters. DNS is often much more costly computationally due to the large grid densities needed to capture turbulent flow fields, and for the resources available for this particular study, not a real option. For complex flow fields such as the UL Smoke Box test section, it is not always easy to determine what type of flow is present, and in most cases, flows exhibit both laminar as well as turbulent zones along with transitional areas. The smoke box experiences a combination of both types of flow patterns. In some instances, such as fully-developed flow through a pipe similar to the one discussed in the pressure drop experiment in Appendix B, the flow is laminar because several diameters downstream, the viscous forces acting on the fluid through the pipe are much greater in magnitude than the inertial forces. This ratio of inertial forces over viscous forces has been defined as the Reynolds number, $R e$. The $R e$ is a function of the freestream velocity, the viscosity and density of the fluid, and a characteristic length. For laminar flows, the N-S equations are solved numerically using governing differential equations - often with grid densities much less than those needed for DNS. ${ }^{18}$

Turbulent flows, on the other hand, are much more common in nature, and unfortunately, much more difficult to model. In general, turbulent flows are characterized by fluctuating velocity fields, that in turn, fluctuate the transport parameters resident in the N-S equations. Because these fluctuations can be of relatively small scale, high frequency and random in nature, they are impossible to solve in closed form. Most turbulence models take these small fluctuations and time average them with respect to the N-S equations, resulting in an adapted set of equations with added variables helping to describe a particular aspect of turbulence. ${ }^{18}$ Because turbulence is a very complex topic in and of itself, discussions hereon about turbulence modeling will be generalized according to just the major advantages and 
disadvantages between the various models. Due to the nature of the smoke box flow, both laminar and turbulence modeling will be examined.

Fluent ${ }^{\circledR 18}$ provides several different types of turbulence models. The options are the Spalart-Allmaras (S-A), the $\kappa-\varepsilon$, the $\kappa-\omega$, and the Reynolds Stress Model (RSM), along with their off-shoots. Historically, the realizable $\kappa-\varepsilon$ and RSM are recommend because of their generalized success in predicting a broad range of turbulent flows. The employed mesh has not had any practical convergence success with the RSM, thus this particular model has been omitted in the results included in this report. The two models that will be broadly compared in this study will be the standard laminar and realizable $\kappa-\varepsilon$ turbulence model.

The basic laminar flow model can be easily modeled using the principles enforced by the conservation of mass and momentum, which are essentially the N-S equations. The conservation of mass states that whatever mass enters a closed system, must also exit that system. It can be expressed mathematically in the following manner: ${ }^{18}$

$$
\frac{\partial \rho}{\partial t}+\nabla \cdot(\rho \vec{v})=0
$$

where $\partial \rho / \partial t$ is the change of fluid density with respect to time, and $\nabla$ (pronounced “del”) is a mathematical operator which represents,

$$
\nabla=\frac{\partial()}{\partial x} \hat{x}+\frac{\partial()}{\partial y} \hat{y}+\frac{\partial()}{\partial z} \hat{z}
$$

$\vec{v}$ is the velocity vector defined as,

$$
\vec{v}=v_{x}+v_{y}+v_{z}
$$

where the subscripts $x, y$, and $z$ here denote velocity vector orientations. Equation 5.7.1 is the general form for conservation of mass, and can be used for both compressible and incompressible flows. The conservation of momentum also stated in three-dimensional form is defined as follows,

$$
\frac{\partial}{\partial t}(\rho \vec{v})+\nabla \cdot(\rho \vec{v} \vec{v})=-\nabla p+\nabla \cdot(\overline{\bar{\sigma}})+\rho \vec{G}+\vec{F}
$$

where $p$ represents pressure, $\overline{\bar{\sigma}}$ is a stress tensor, $\vec{G}$ is a gravitational force, and $\vec{F}$ is a body force. The computational model being implemented in this study, due to the air flow and small smoke particles involved, have negligible gravitational as well as body force effects. The energy equation, in general, is not employed due to the isothermal conditions present in 
the UL Smoke Box. The smoldering cotton wick offers negligible temperature effects in the test section. The reader is referred to the Fluent ${ }^{\circledR 18}$ documentation $^{18}$ for more detail. Fluent ${ }^{\circledR 18}$ discretizes Equations 5.7.1 and 5.7.4 in order to solve the laminar flow model.

The $\kappa-\varepsilon$ turbulence model is a semi-empirical model based upon the model transport equations involving the turbulent kinetic energy, $\kappa$, and its dissipative rate $\varepsilon$. Although $\varepsilon$ is obtained using physical reasoning and bares little likeness to its mathematically exact counterpart, $\kappa$ is derived from an exact equation. As it turned out, the majority of the turbulence models ran in the simulation proved quite unfruitful. Some initial comparative results will be shown in Section 6.6. In general, it will be shown that the laminar model predicts the bulk flow field with much better agreement with the experimental results than the available turbulent models. Hence, a thorough turbulent investigation was ultimately not needed. 


\section{CHAPTER 6: RESULTS}

\subsection{FLOW VISUALIZATION INSIDE THE SA302}

The following few photographs found in Figures 6.1.1 through 6.1.8 reveal captured still images from MPEG movies used to record, in real time, the flow visualization inside the SA302. Each figure is for a different angular orientation of the detector as the flow moves from right to left. From these figures, it is seemingly obvious that the flow tends to maneuver around the ion and photoelectric chamber as they represent obstacles in the path of the flow. The porous settling chamber of the ion sensor and the screen mesh fabric in tandem with the flow impeding path into the photoelectric chamber produces sufficient flow resistance to cause the air flow to approach these bodies as nearly solid objects. While there is a significant concentration of fog smoke inside the detector in Figures 6.1.1 through 6.1.8, there is much less concentration of fog smoke inside the sensing chambers.

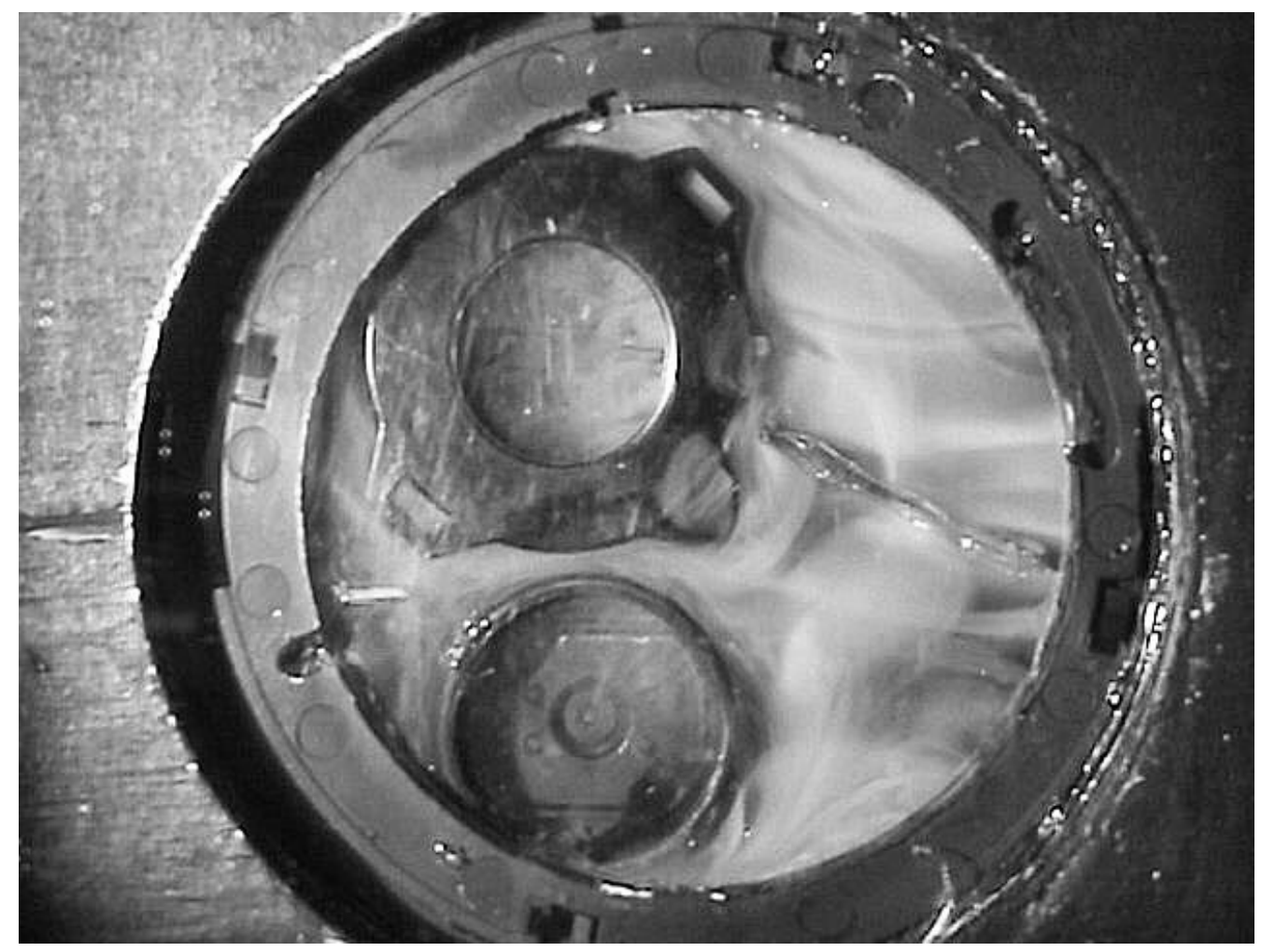

Figure 6.1.1: Flow visualization of the SA302 smoke detector in the UL Smoke Box at $32 \mathrm{ft} / \mathrm{min}$ and orientation angle $0^{\circ}$ (bottom view). 


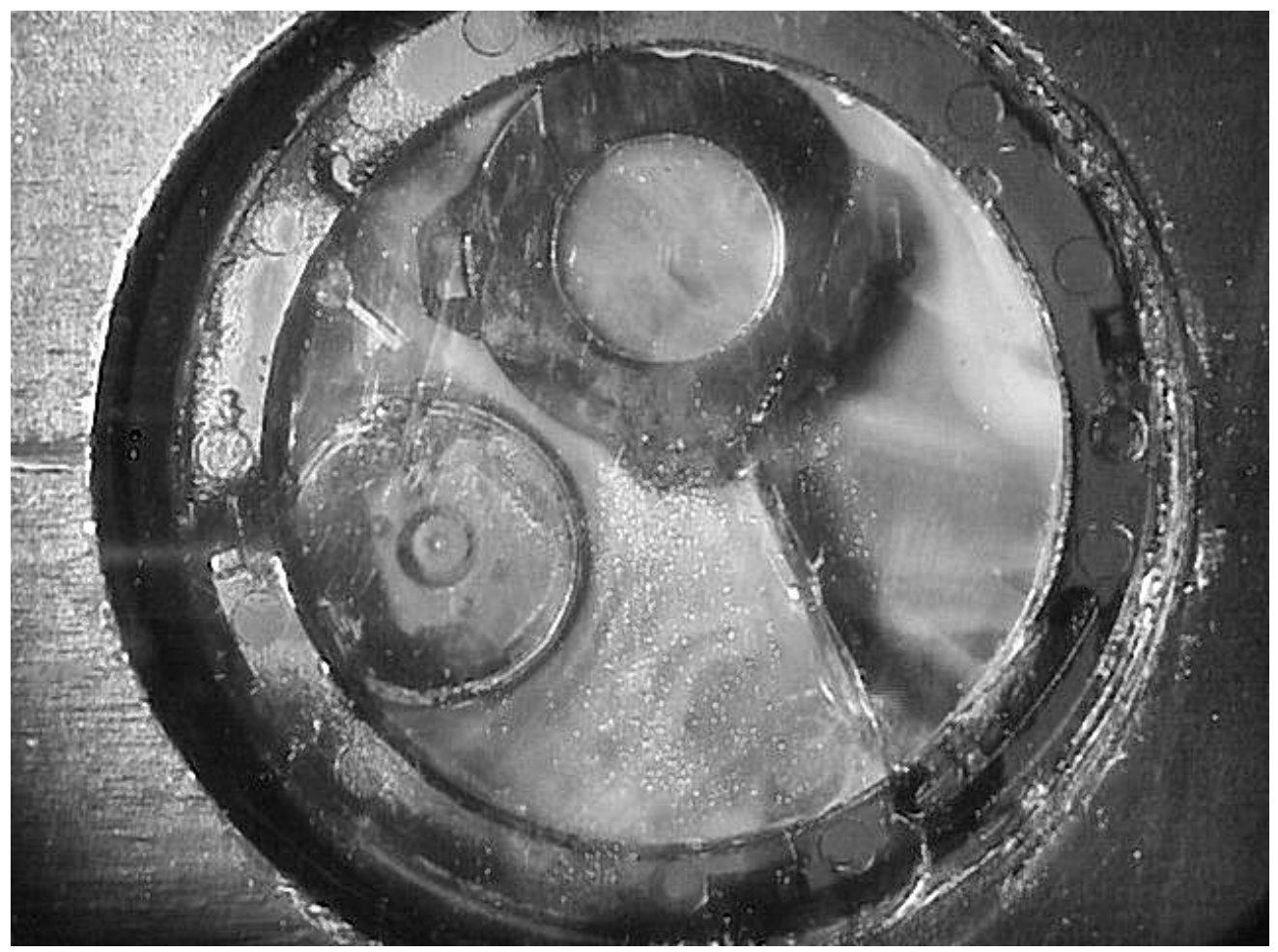

Figure 6.1.2: Flow visualization of the SA302 smoke detector in the UL Smoke Box at $32 \mathrm{ft} / \mathrm{min}$ and orientation angle $45^{\circ}$ (bottom view).

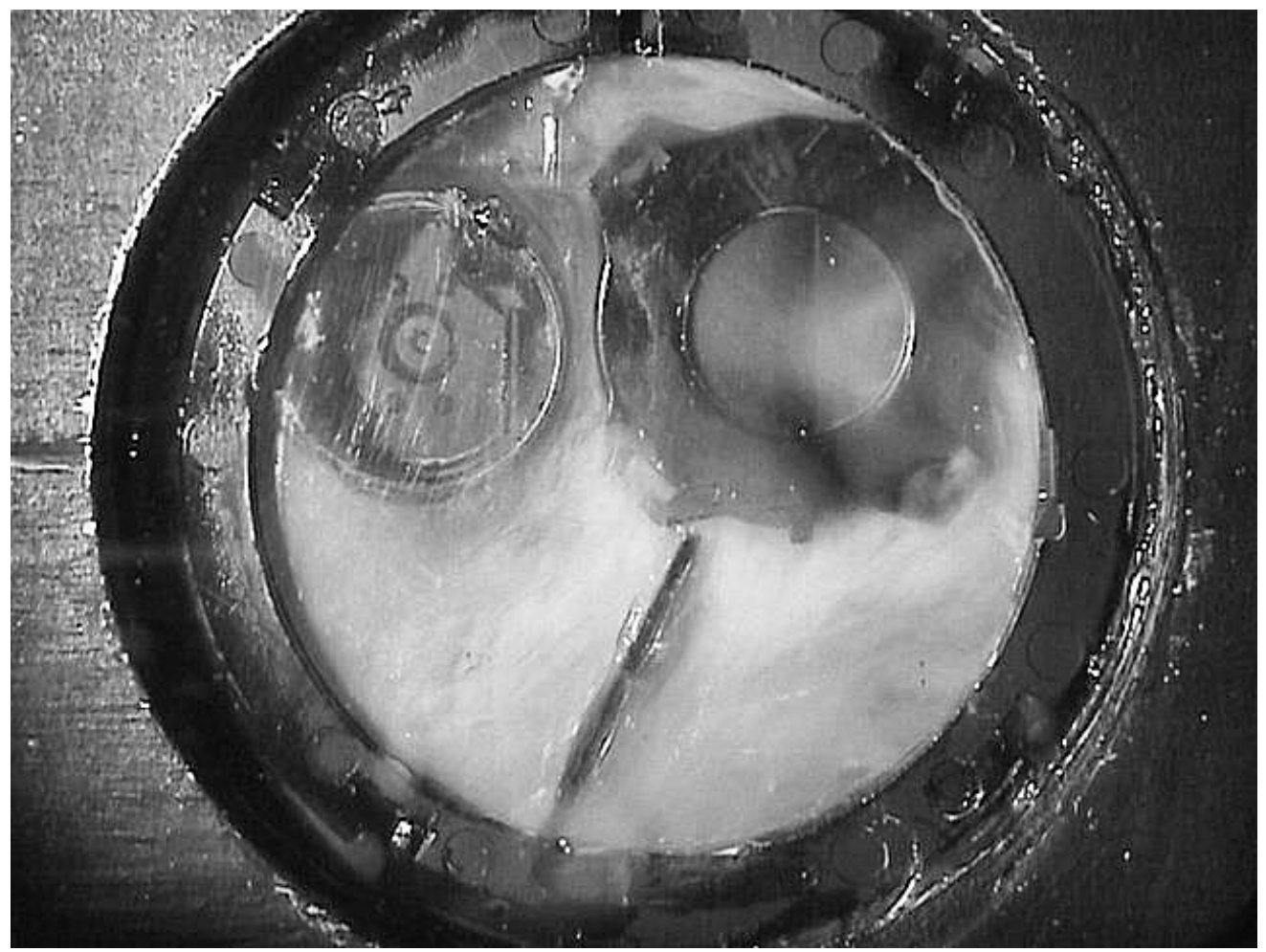

Figure 6.1.3: Flow visualization of the SA302 smoke detector in the UL Smoke Box at $32 \mathrm{ft} / \mathrm{min}$ and orientation angle $90^{\circ}$ (bottom view). 


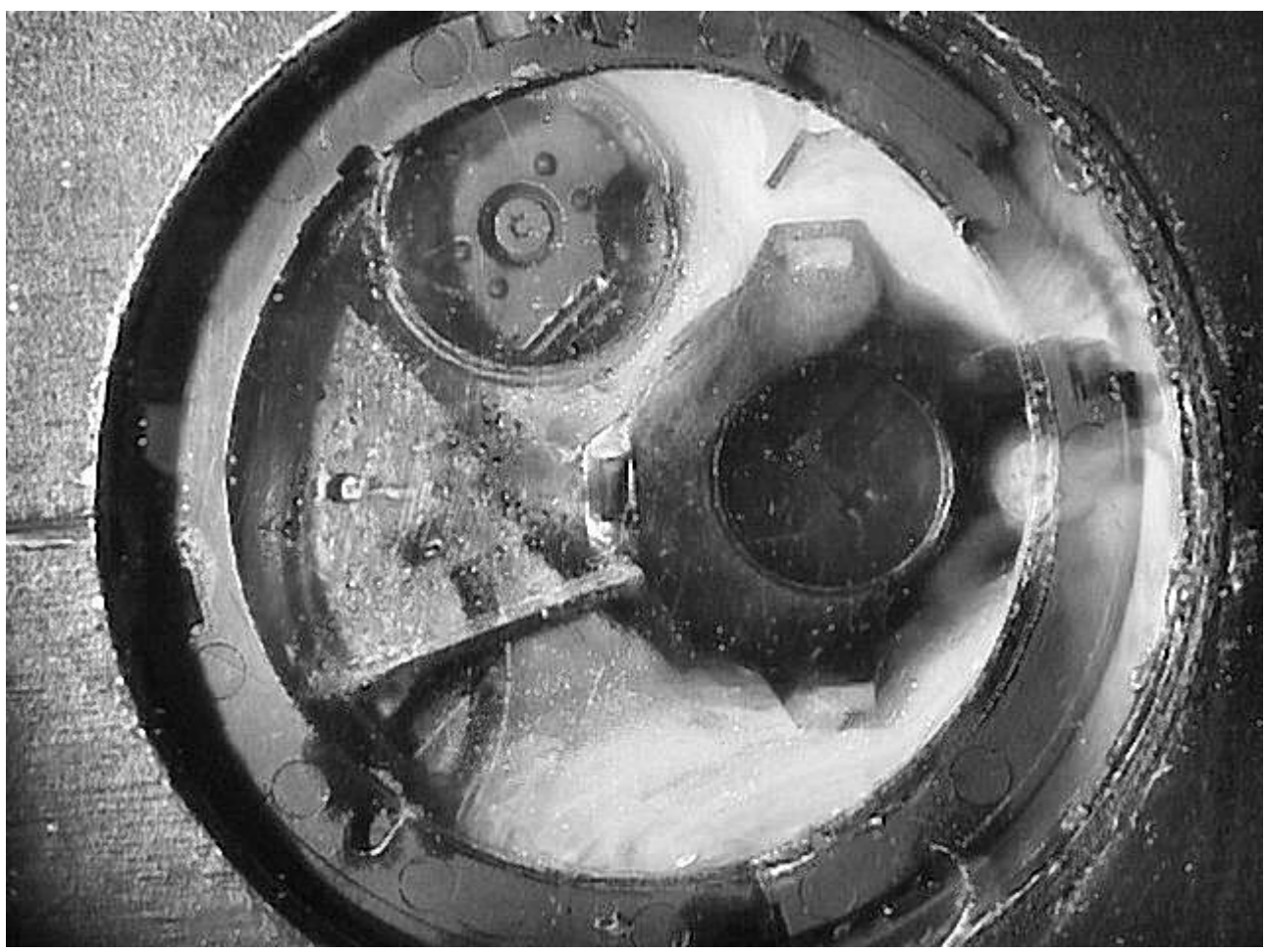

Figure 6.1.4: Flow visualization of the SA302 smoke detector in the UL Smoke Box at $32 \mathrm{ft} / \mathrm{min}$ and orientation angle $135^{\circ}$ (bottom view).

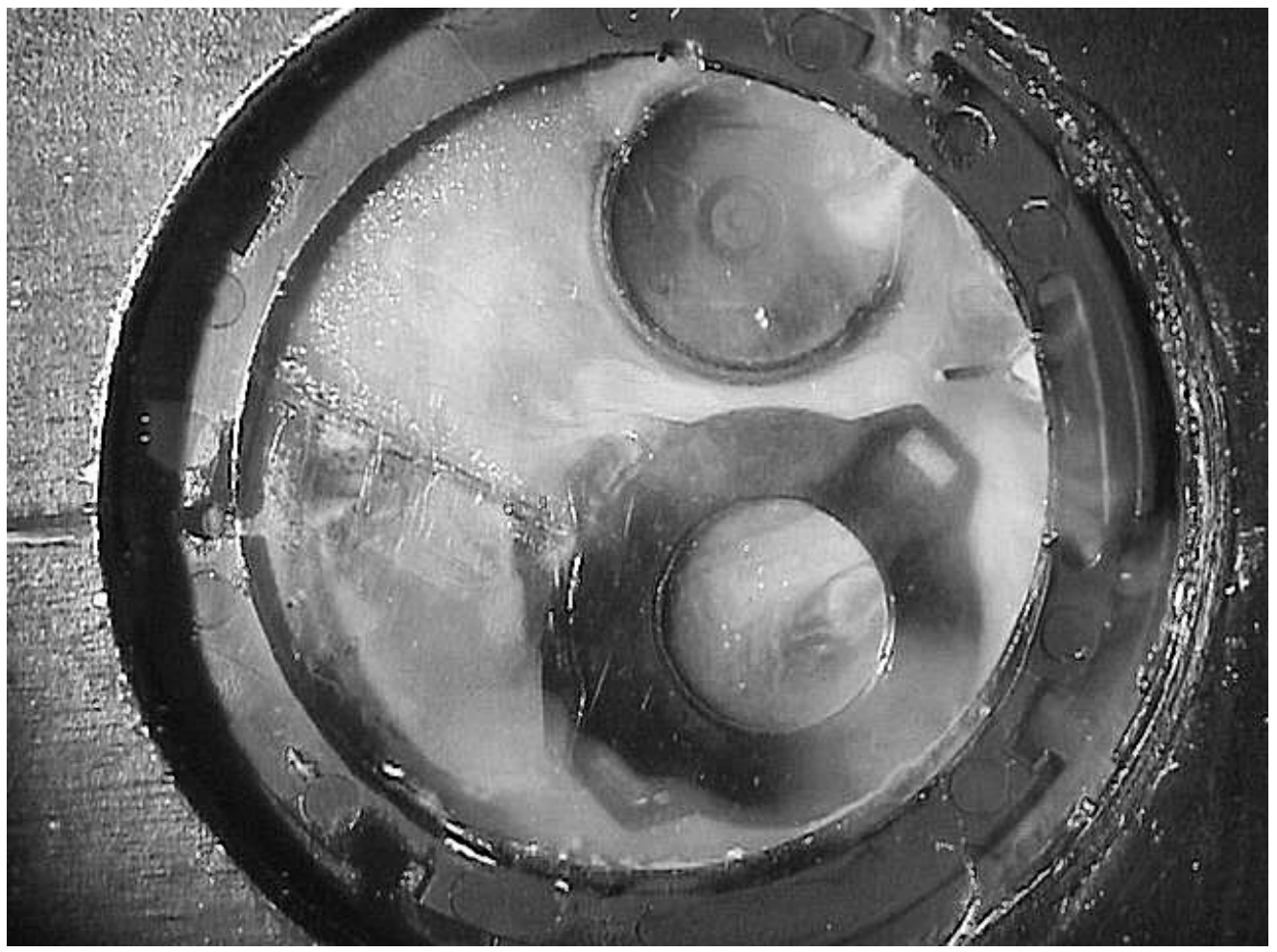

Figure 6.1.5: Flow visualization of the SA302 smoke detector in the UL Smoke Box at $32 \mathrm{ft} / \mathrm{min}$ and orientation angle $180^{\circ}$ (bottom view). 


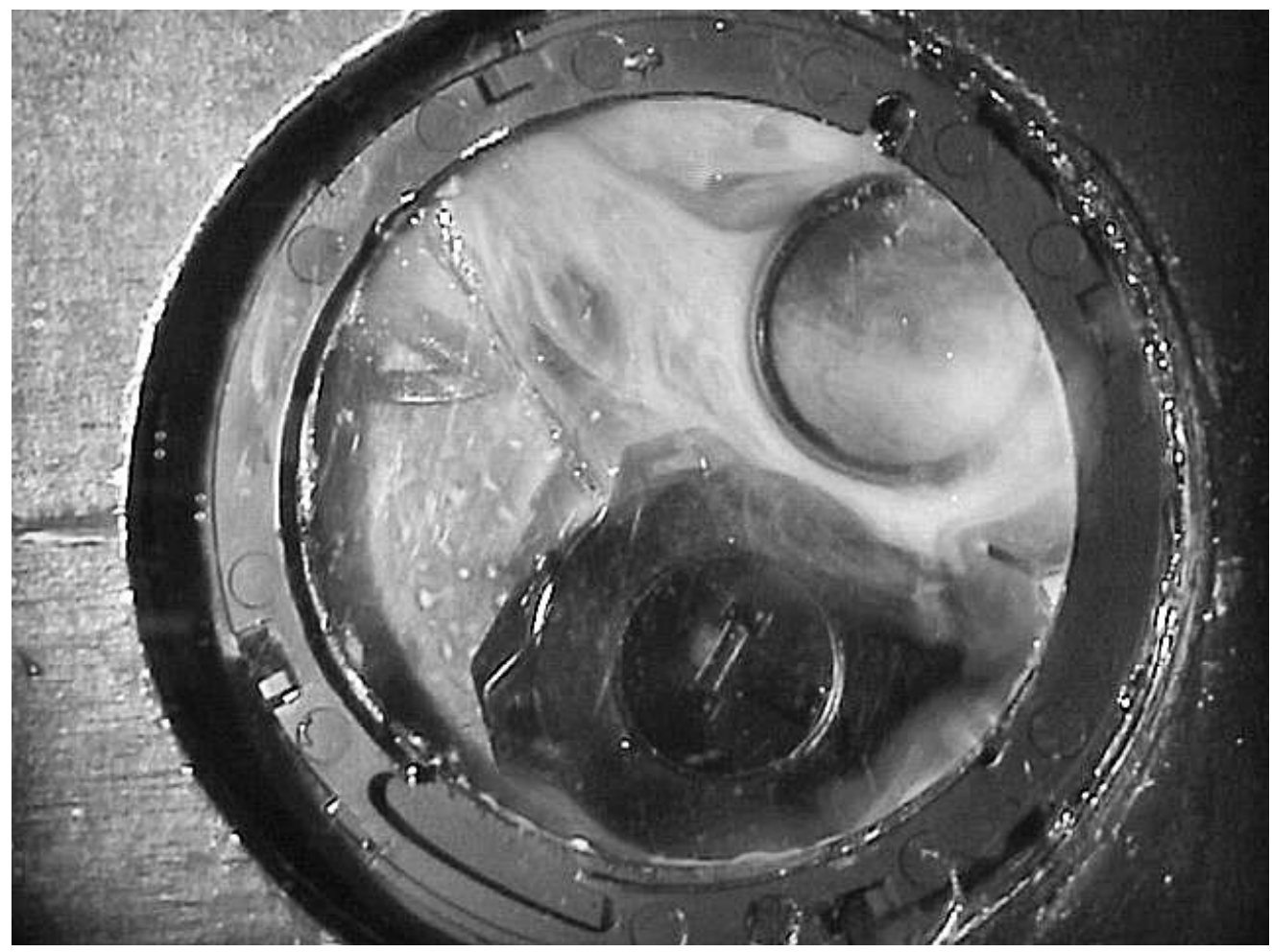

Figure 6.1.6: Flow visualization of the SA302 smoke detector in the UL Smoke Box at $32 \mathrm{ft} / \mathrm{min}$ and orientation angle $225^{\circ}$ (bottom view).

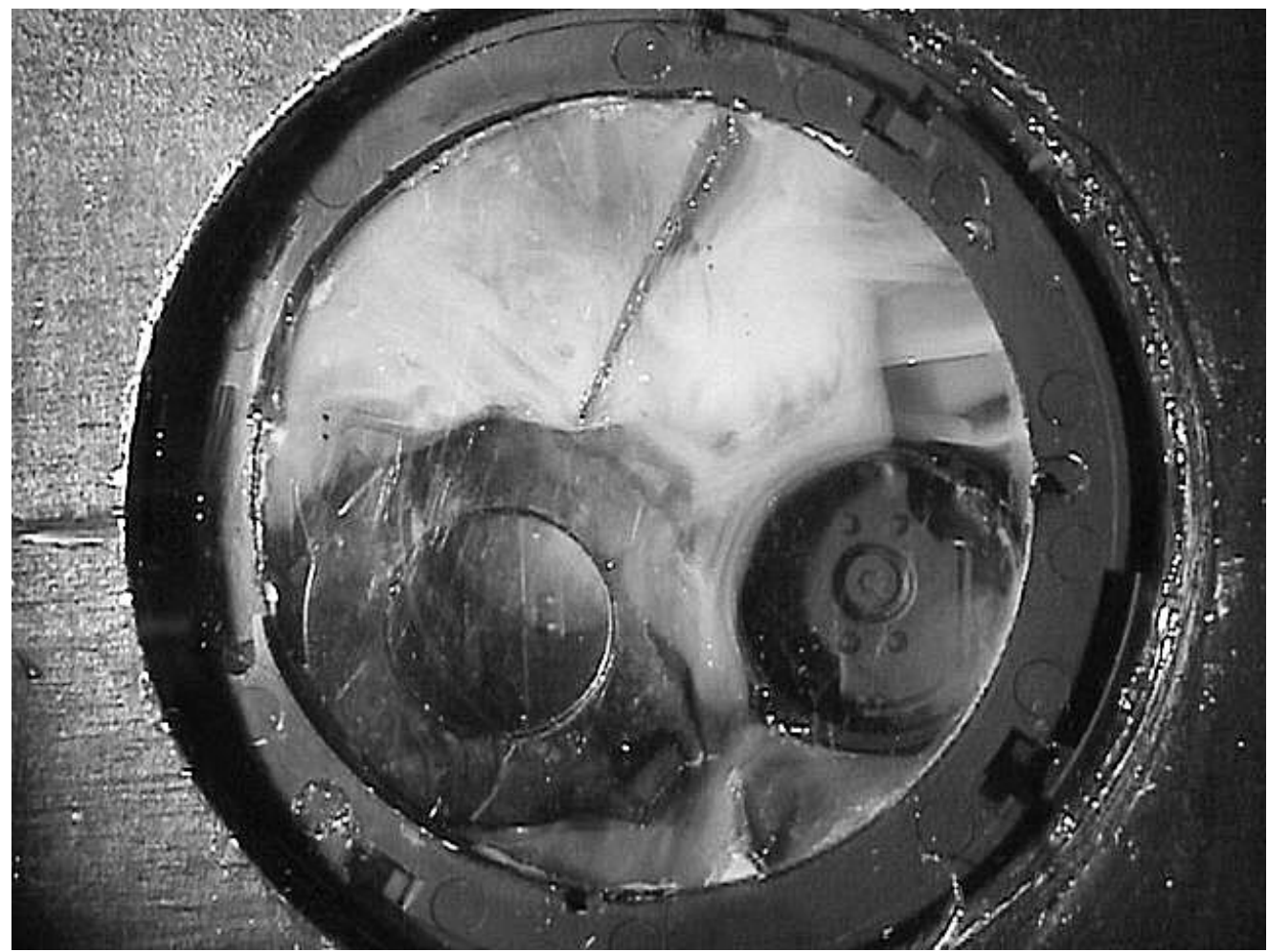

Figure 6.1.7: Flow visualization of the SA302 smoke detector in the UL Smoke Box at $32 \mathrm{ft} / \mathrm{min}$ and orientation angle $270^{\circ}$ (bottom view). 


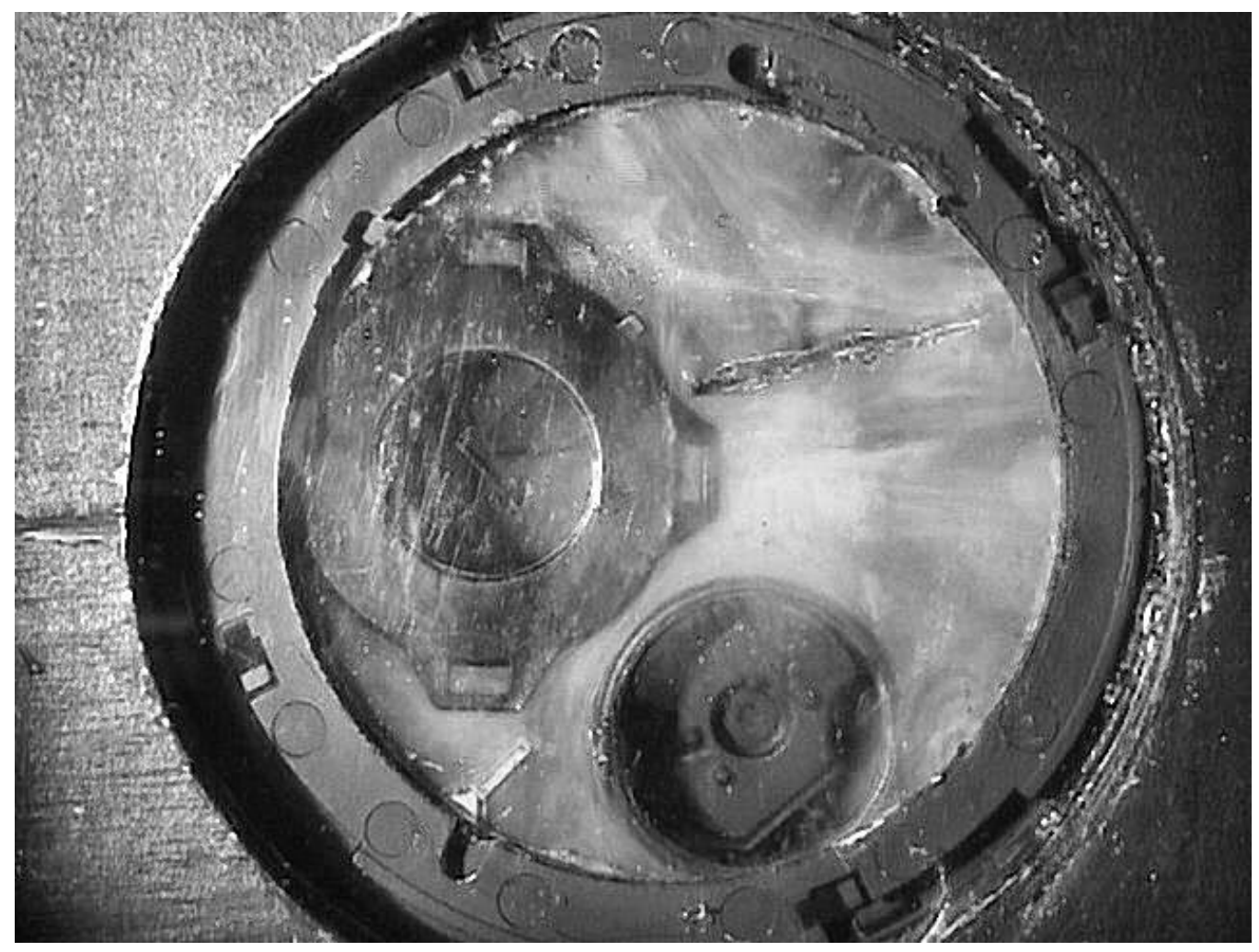

Figure 6.1.8: Flow visualization of the SA302 smoke detector in the UL Smoke Box at $32 \mathrm{ft} / \mathrm{min}$ and orientation angle $315^{\circ}$ (bottom view).

\subsection{FLOW VISUALIZATION INSIDE THE UL SMOKE BOX}

Figures 6.2.1 through 6.2.10 are still photographs captured from a digital video taken of flow in the UL Smoke Box near the SA302 smoke detector. A laser light sheet was used to illuminate the selected planes of the flow while theatrical fog smoke was used to make the field visible. Figures 6.2.1 through 6.2.3 show the wake region aft of the smoke detector by illuminating a vertical $(y-z)$ plane in the test section of $x=21$ in, $x=22$ in, $x=23$ in, respectively. The perspective taken in each of these figures is from the upstream viewpoint through the acrylic window on the top of the UL Smoke Box. In each of these figures, the complex wake region is revealed with significant secondary flow patterns in the $y$-z plane. The influence of the MIC causes the wake downstream of the smoke detector to be nonsymmetric, and appear more pronounced on the right side in each case. The coarse resolution of these images are the result from the low light levels required in the UL Smoke Box to ensure a sharp contrast of the illuminated flow field with respect to the background test chamber. 


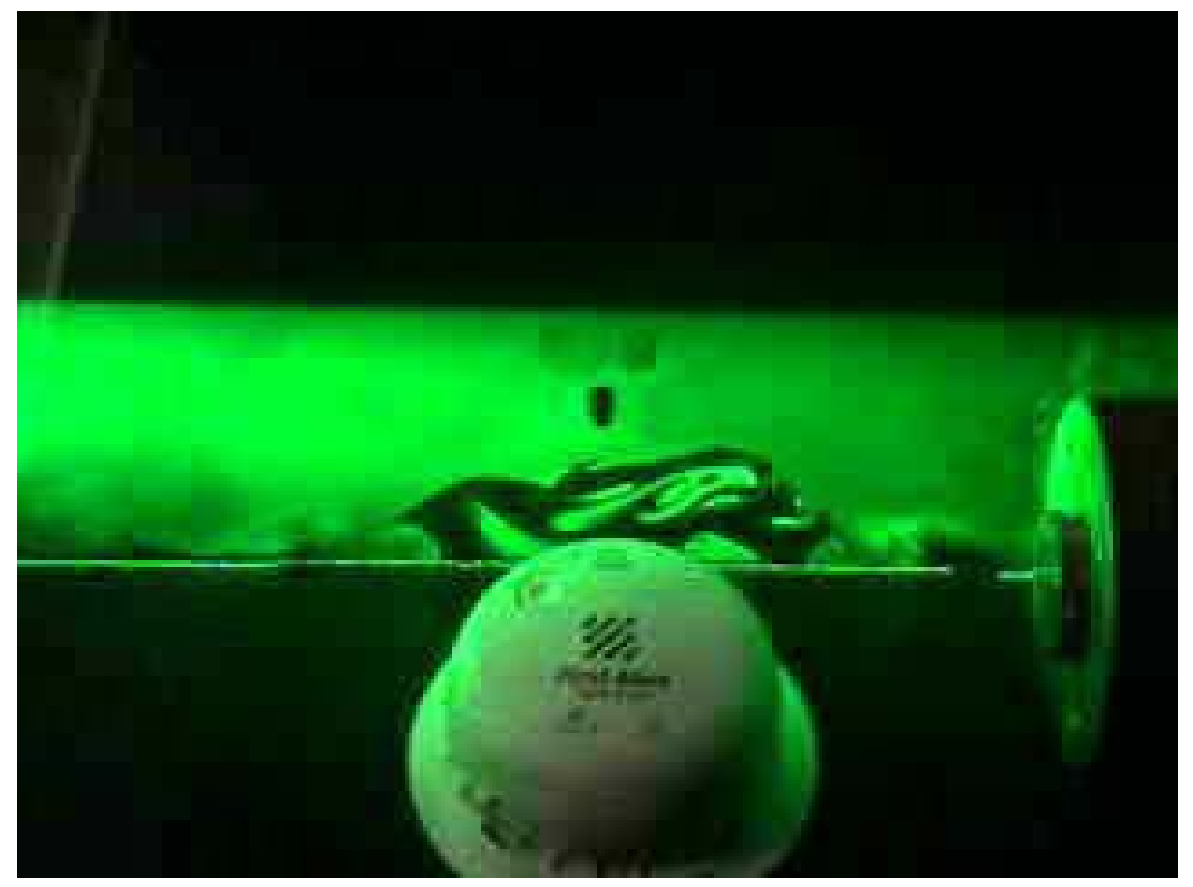

Figure 6.2.1: Flow visualization in the $y-z$ plane of the UL Smoke Box at $x=21$ in.

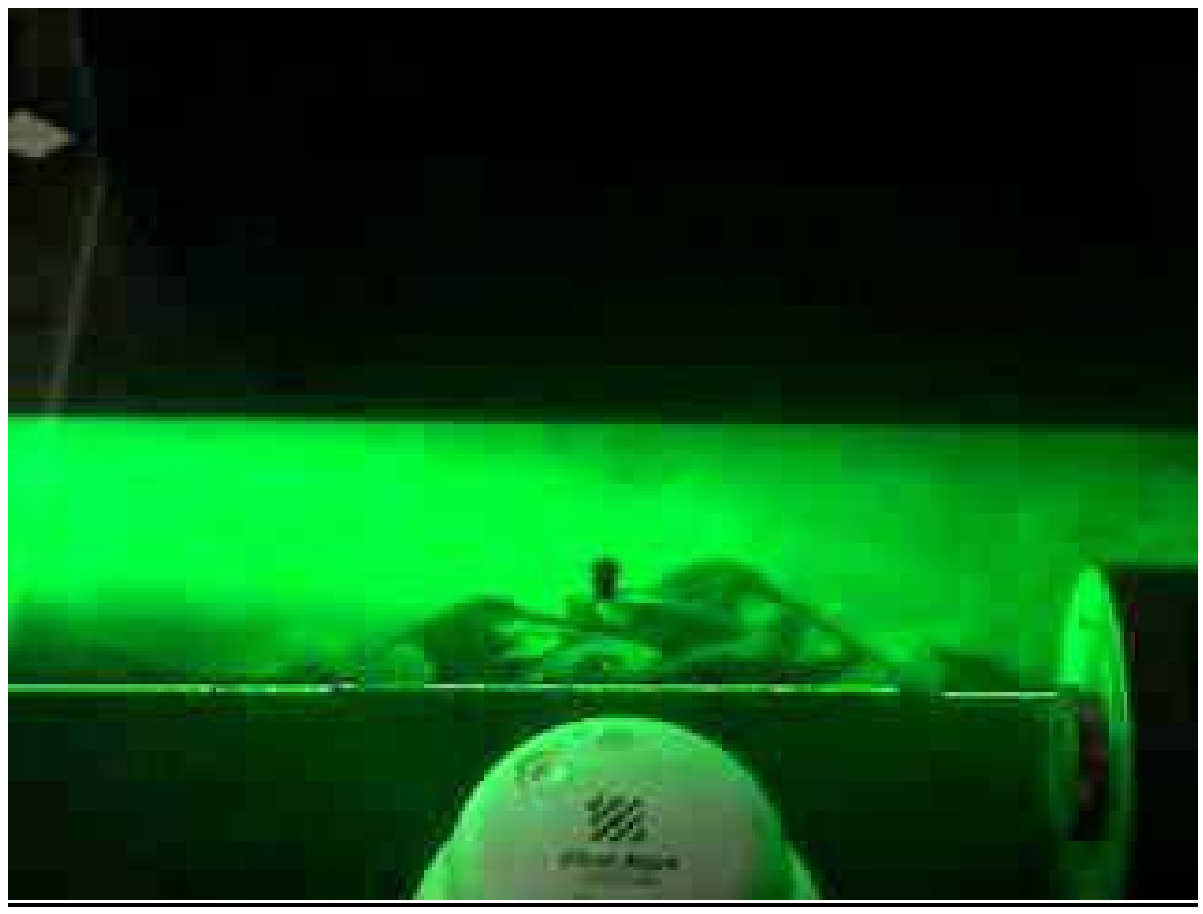

Figure 6.2.2: Flow visualization in the $y-z$ plane of the UL Smoke Box at $x=22$ in. 


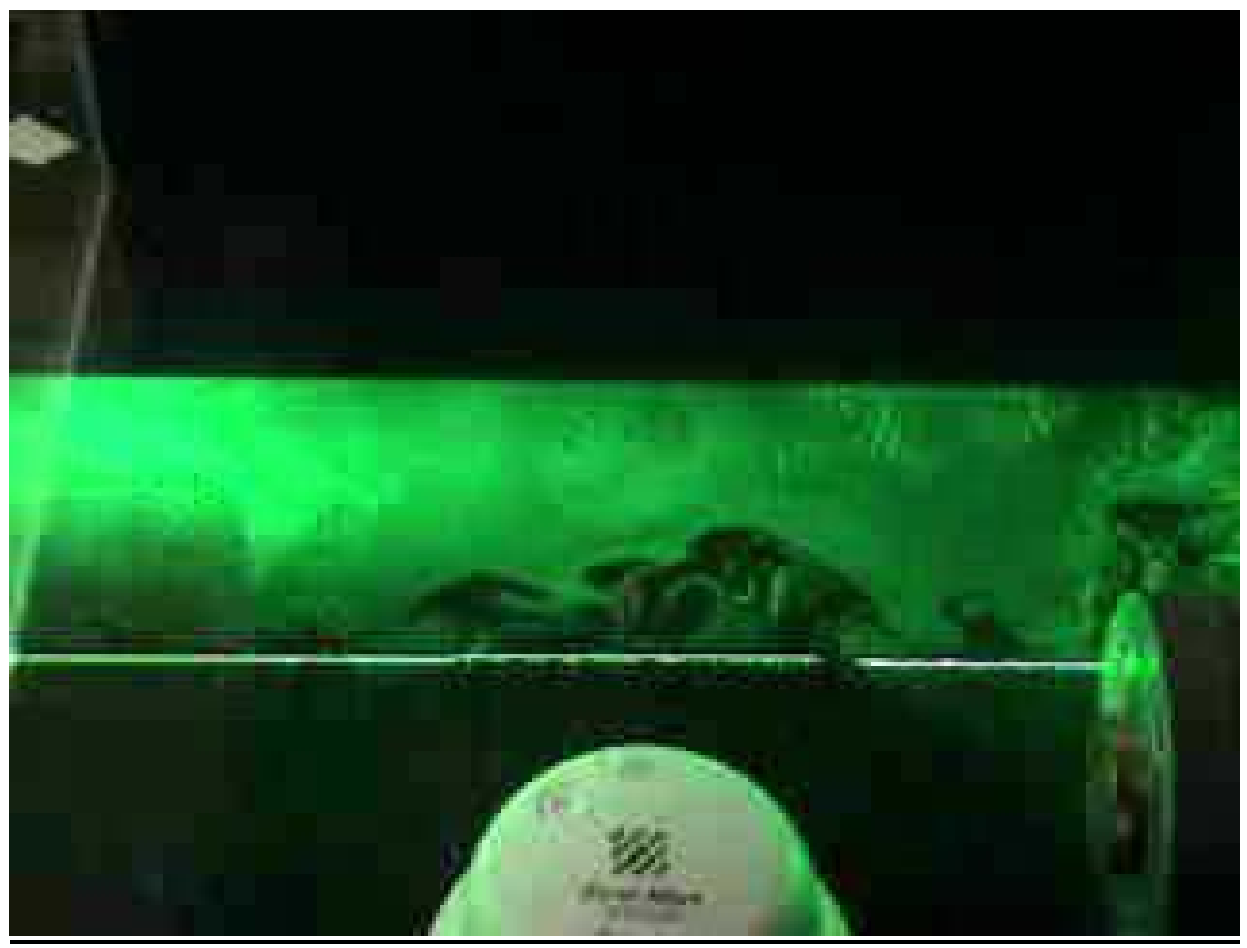

Figure 6.2.3: Flow visualization in the $y-z$ plane of the UL Smoke Box at $x=23$ in.

Figures 6.2.4 through 6.2.7 show the flow patterns in the test section approaching the smoke detector at various horizontal $(x-z)$ planes above the test section floor. Figures 6.2.4 and 6.2.5 illustrate that near the floor at $y=0.25$ in and $0.50 \mathrm{in}$, respectively, the flow turns significantly to the left as it approaches the smoke detector. This behavior is verified further by the LDA velocity measurements to be presented in Section 6.4 and is attributed to the presence of the MIC on the right hand side of the test section, forcing the gross fluid motion toward an unobstructed side of the test section. This effect is less pronounced at horizontal $(x-z)$ planes located at $y=1.0$ in and at $y=2.0$ in above the floor. The flow has higher velocity and, thus, higher momentum further above the floor of the test section and is, therefore, more resistant to the flow blockage of the presence of the MIC compared to flow near the floor. 


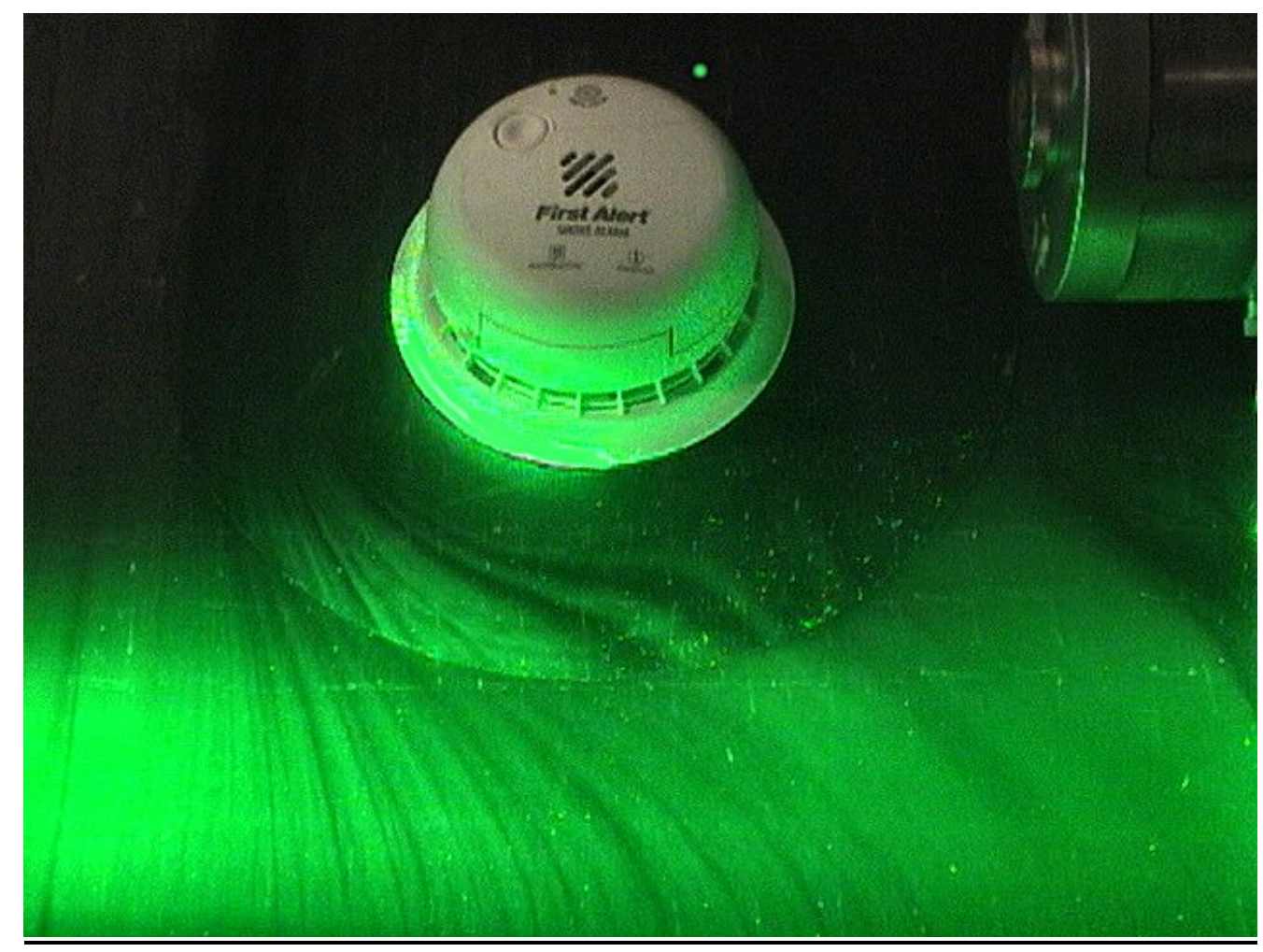

Figure 6.2.4: Flow visualization in the $x-z$ plane in the UL Smoke Box at $y=0.25$ in.

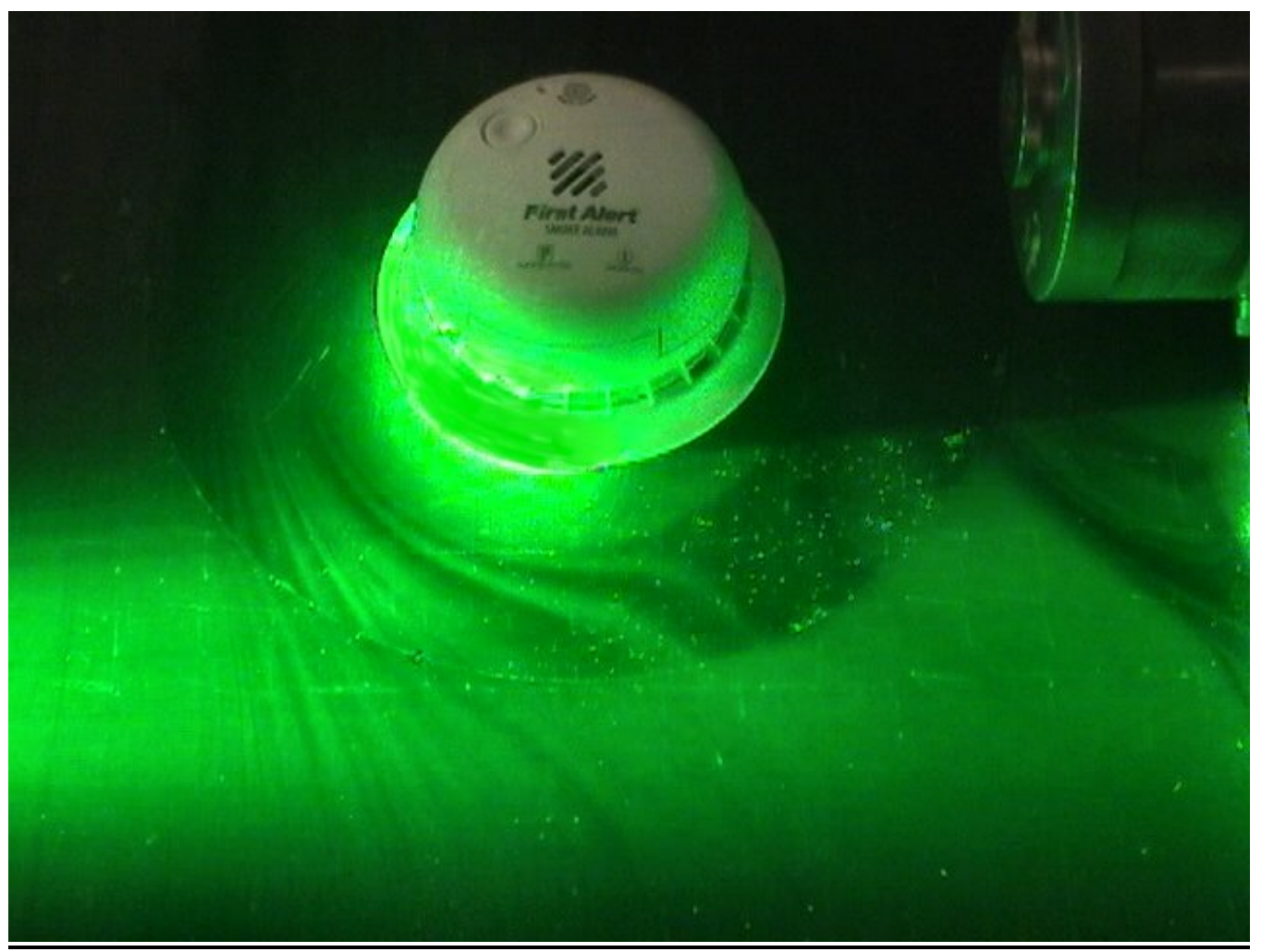

Figure 6.2.5: Flow visualization in the $x-z$ plane in the UL Smoke Box at $y=0.50$ in. 


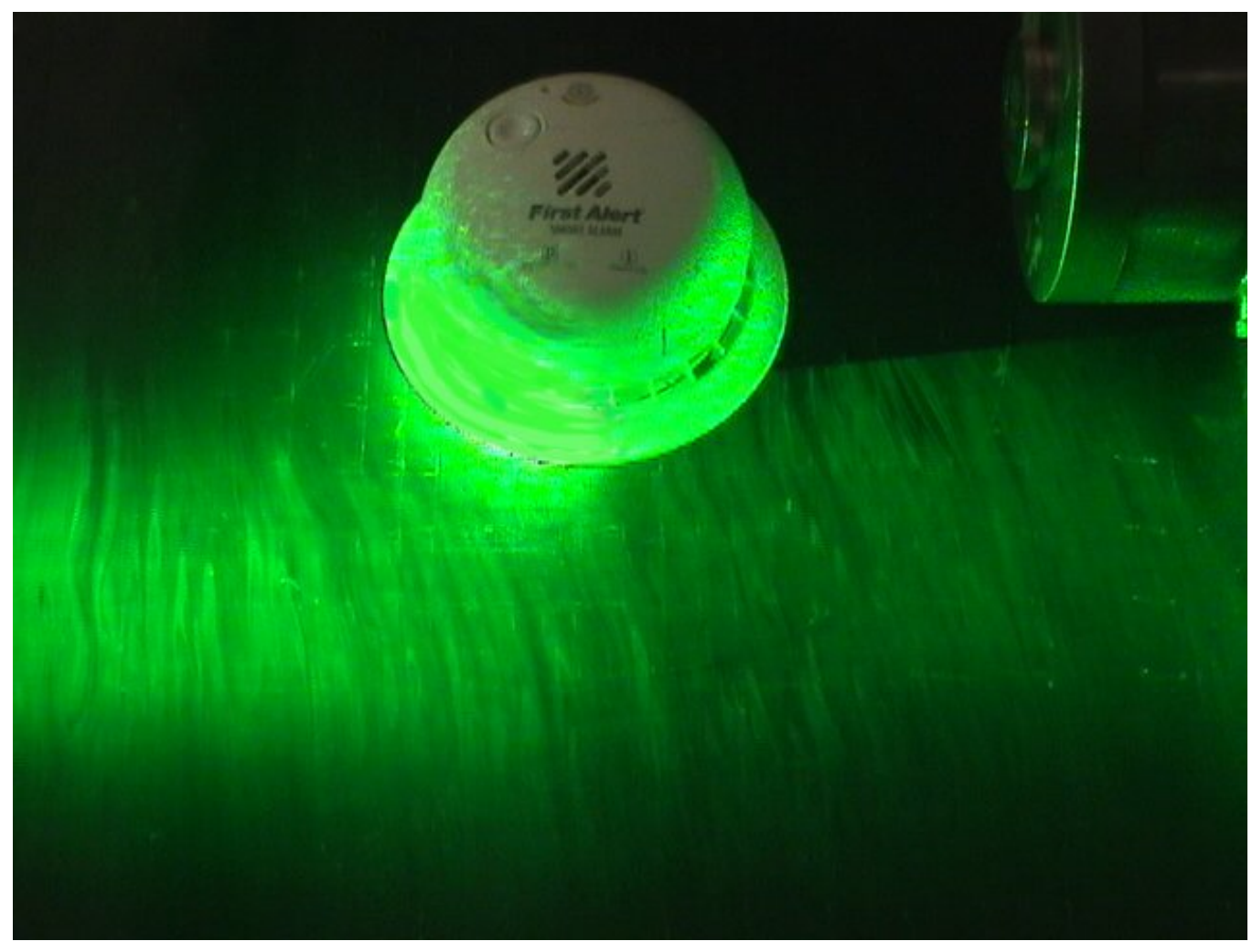

Figure 6.2.6: Flow visualization in the $x-z$ plane in the UL Smoke Box at $y=1.0$ in.

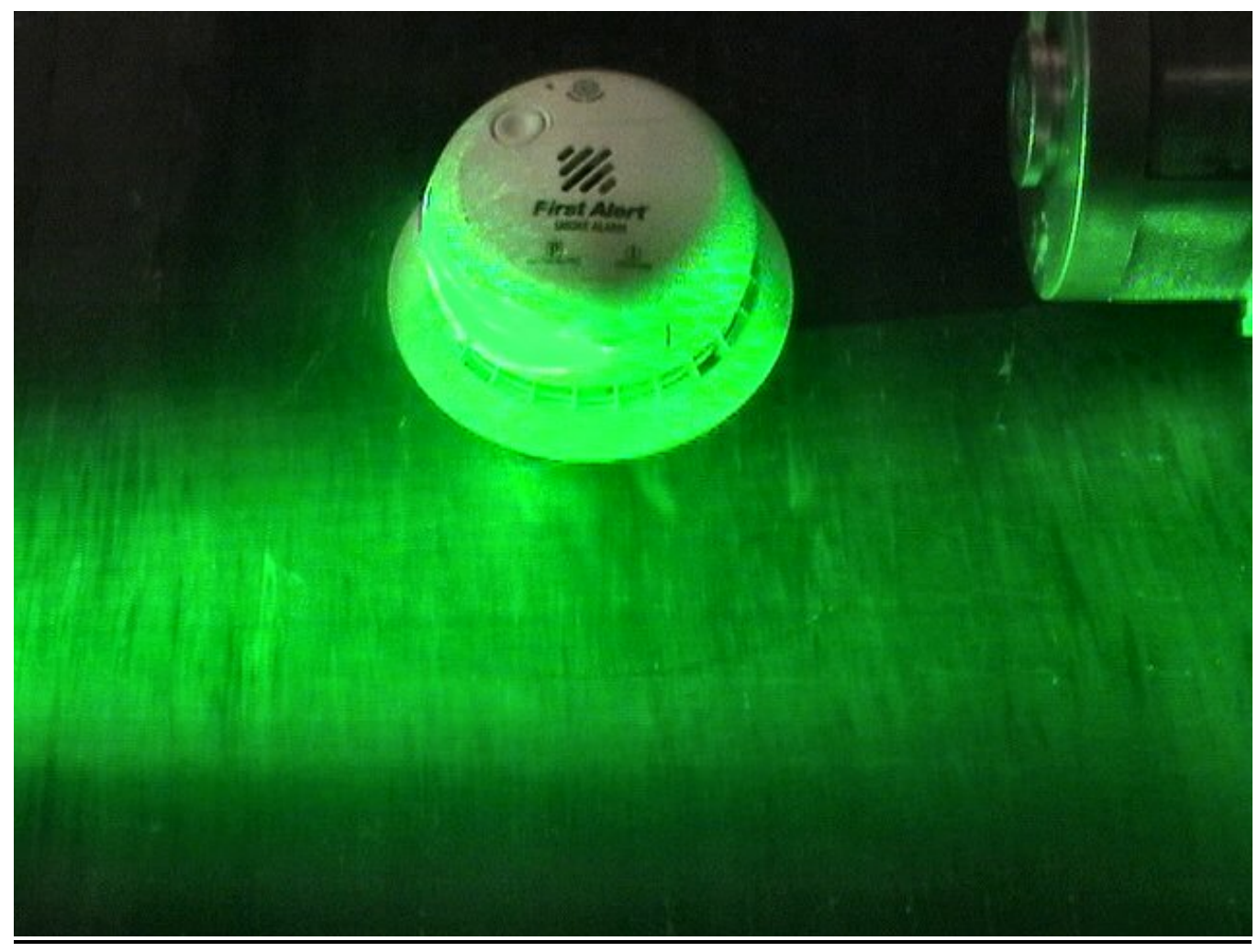

Figure 6.2.7: Flow visualization in the $x-z$ plane in the UL Smoke Box at $y=2.0$ in. 
Figures 6.2.8 through 6.2.10 depict the flow patterns in the wake of the smoke detector in horizontal $(x-z)$ planes above the test section floor at $y=0.25$ in, $y=0.50$ in, $y=$ $1.0 \mathrm{in}$, and $y=2.0 \mathrm{in}$, respectively. These figures illustrate the classical, separated flow present in the wake of a cylindrical cross-section body at low Reynolds numbers $(R e)$ less than a few 100,000. The $R e$ for this flow using the smoke detector body diameter as the characteristic length is on the order of 1400 . The $R e$ is a non-dimensional parameter in fluid mechanics to characterize the relative dominance of inertial forces versus viscous forces in a flow field. The higher the Re, the more inertial forces dominate the viscous forces and the more likely the flow is turbulent. The classic “Von Karman's Vortex Street” is presented as alternating flow vortices are shed on either side of the detector body in the wake. There are locations directly behind the detector body where the flow is stagnant or is moving slightly backwards toward the deflector due to the separation of the flow. This is much like the aerodynamic stall of an aircraft wing flying at too steep an angle of attack where the flow separates from the surface of a wing. The LDA velocity measurements show that no resolvable amount of wake flows backwards into the detector and ultimately, into the sensors.

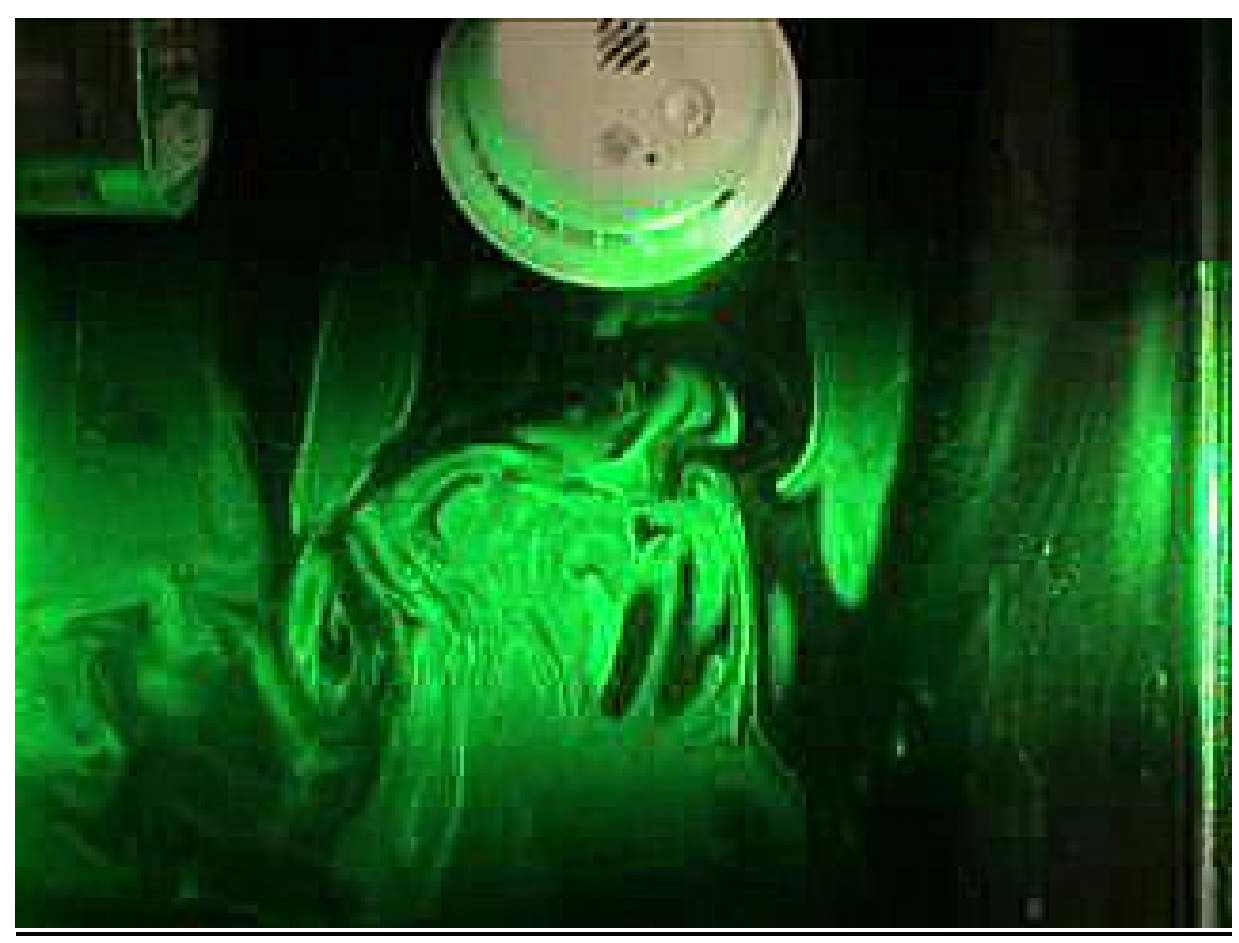

Figure 6.2.8: Flow visualization in the wake of the SA302 Smoke Detector at $y=0.25$ in. 


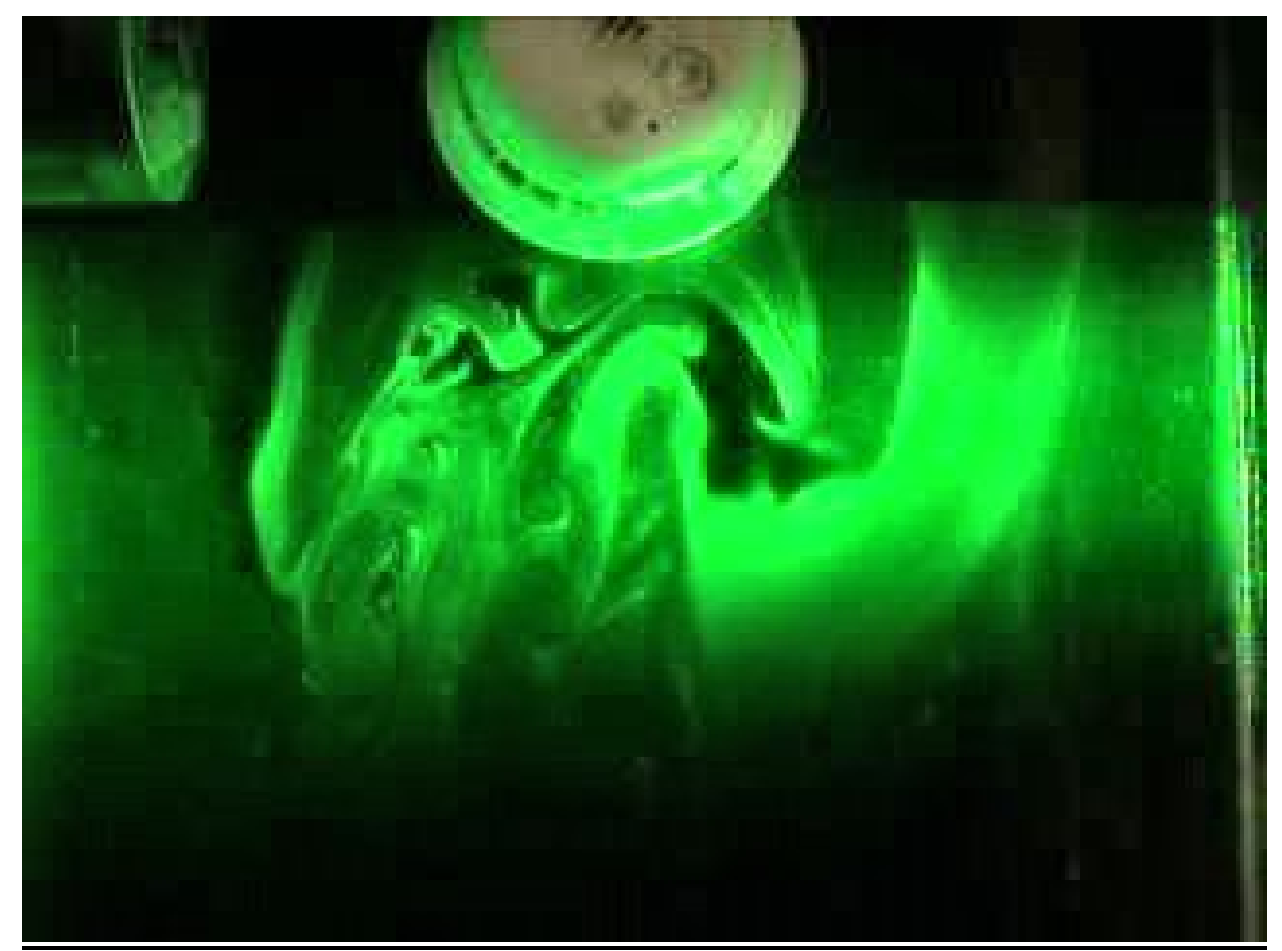

Figure 6.2.8: Flow visualization in the wake of the SA302 Smoke Detector at $y=0.50$ in.

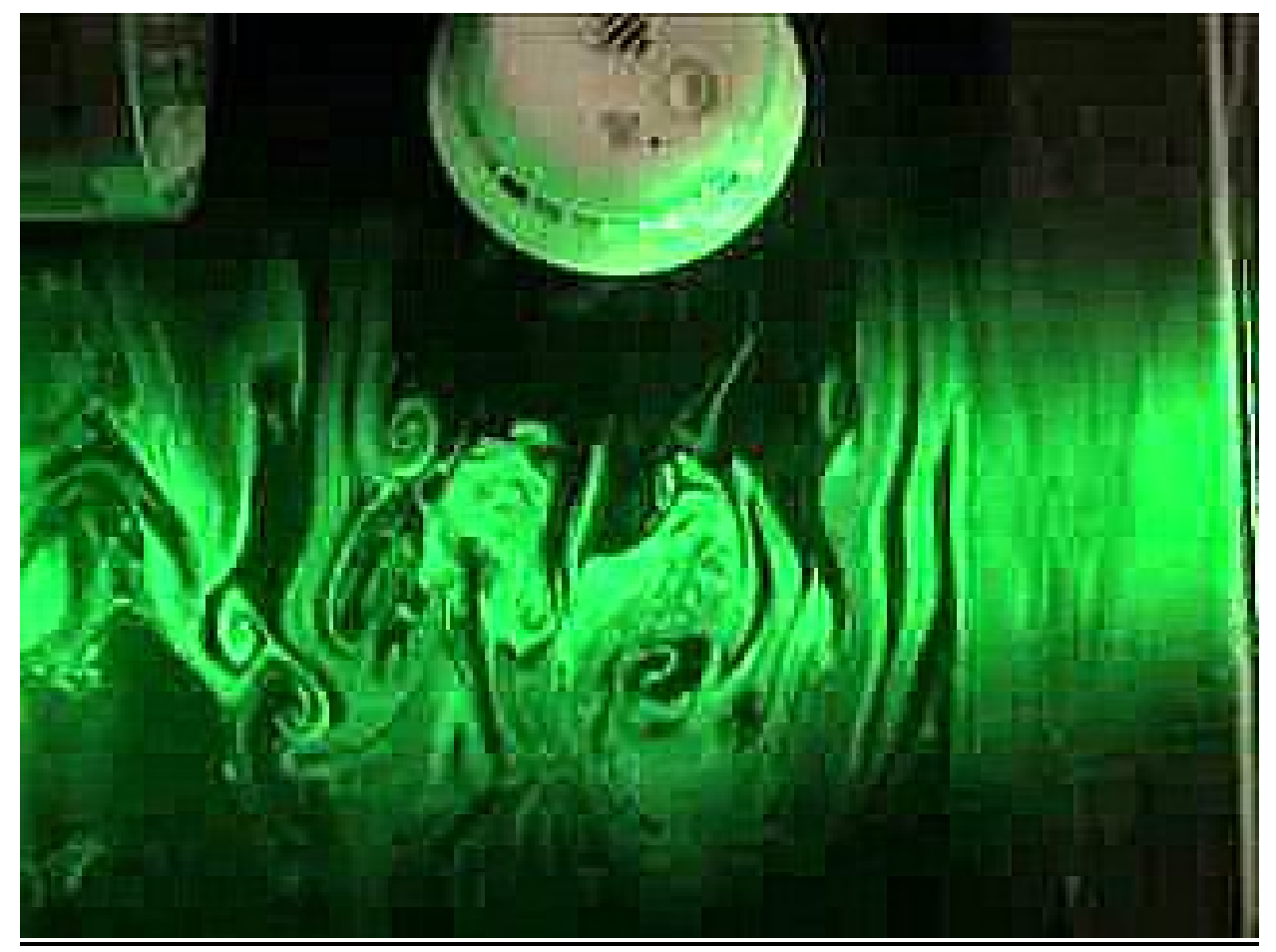

Figure 6.2.9: Flow visualization in the wake of the SA302 Smoke Detector at $y=1.0$ in. 


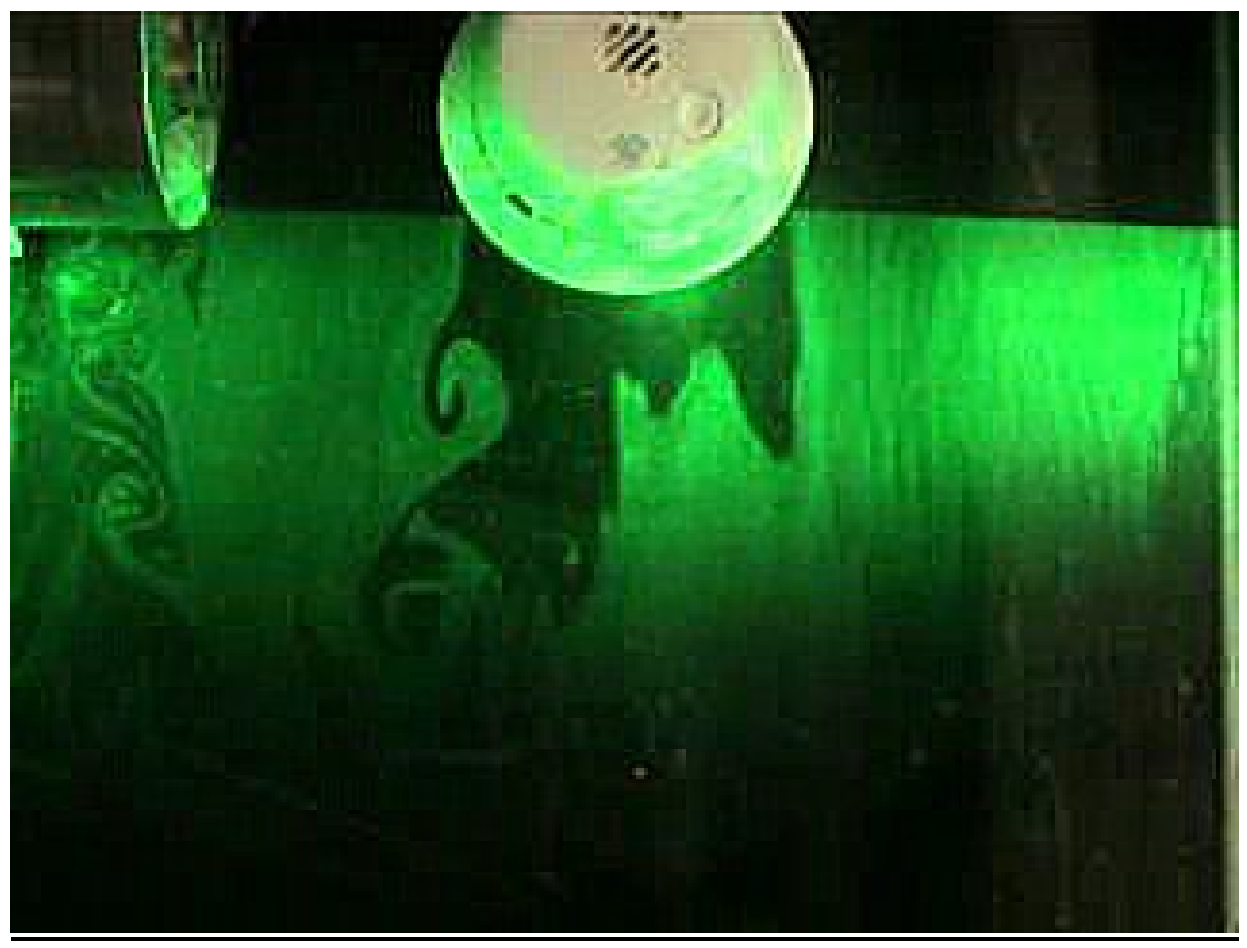

Figure 6.2.10: Flow visualization in the wake of the SA302 Smoke Detector at $y=2.0 \mathrm{in}$.

\subsection{SA302 VELOCITY MEASUREMENTS USING LDA}

Figures 6.2.8 through 6.2.12 show vector representations of velocities measured inside the smoke detector at the mid-plane of the detector smoke entrance (horizontal plane). These vectors are in the $u-w$ velocity plane, and the length of each vector represents its magnitude in relation to the reference vector shown in each figure. The vector fields show that the air (and smoke) tends to behave as if the sensing chambers are nearly solid bodies as is evidenced by most of the flow moving around rather than through these bodies. The velocity magnitudes measured inside the sensing chambers were on the order of a few percent of the freestream smoke box axial velocity of $32 \mathrm{ft} / \mathrm{min}$. As expected, the sensor positioned most upstream has the highest velocity of the two.

The effects of the flow dividers are very clearly evident in these figures as is the effect of one sensor positioned in front on the other. As expected, the sensor positioned most upstream has the highest velocity of the two inside. Plots were created with a multiple of packages, using TECPLOT v7.2 ${ }^{\circledR 21}$ to create velocity magnitudes. 


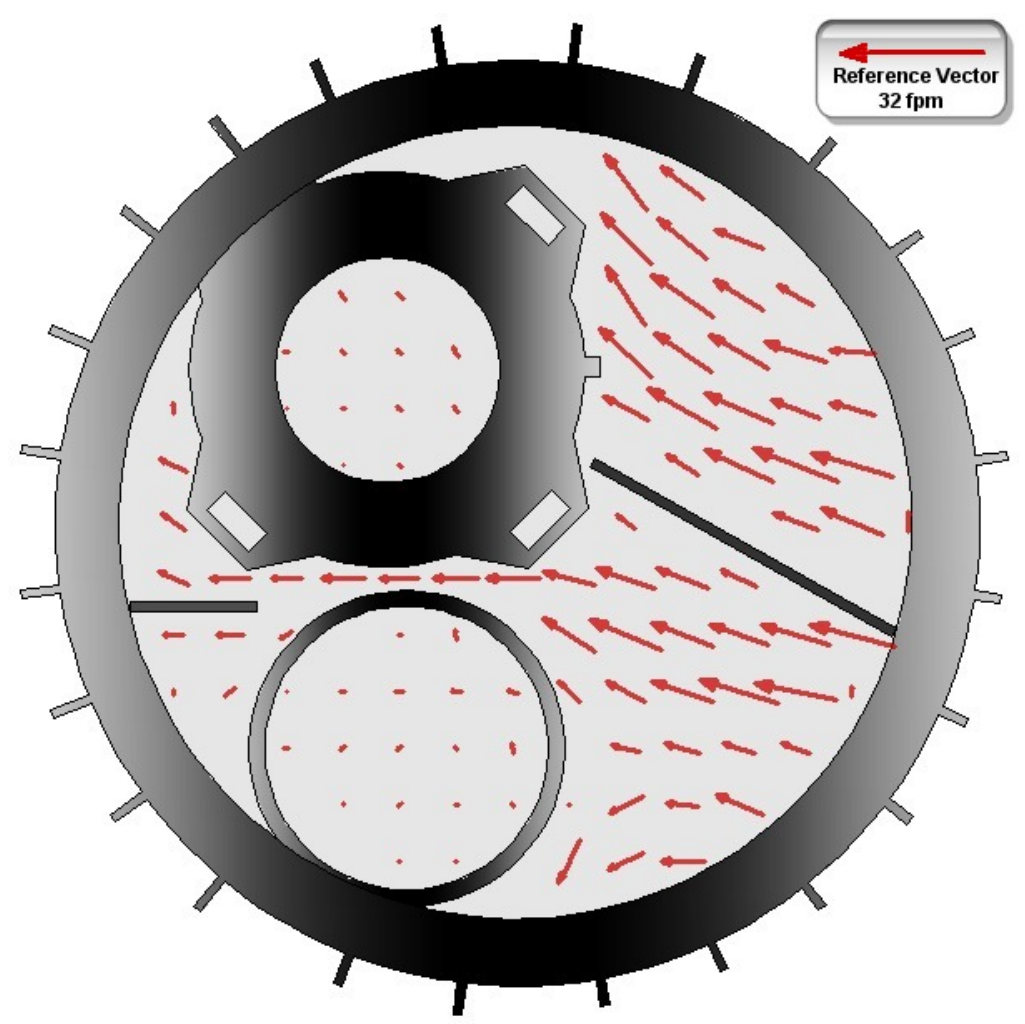

Figure 6.3.1: Flow velocity vectors inside the SA302 Smoke Detector as measured by laser Doppler anemometry at an orientation of $0^{\circ}$ (bottom view).

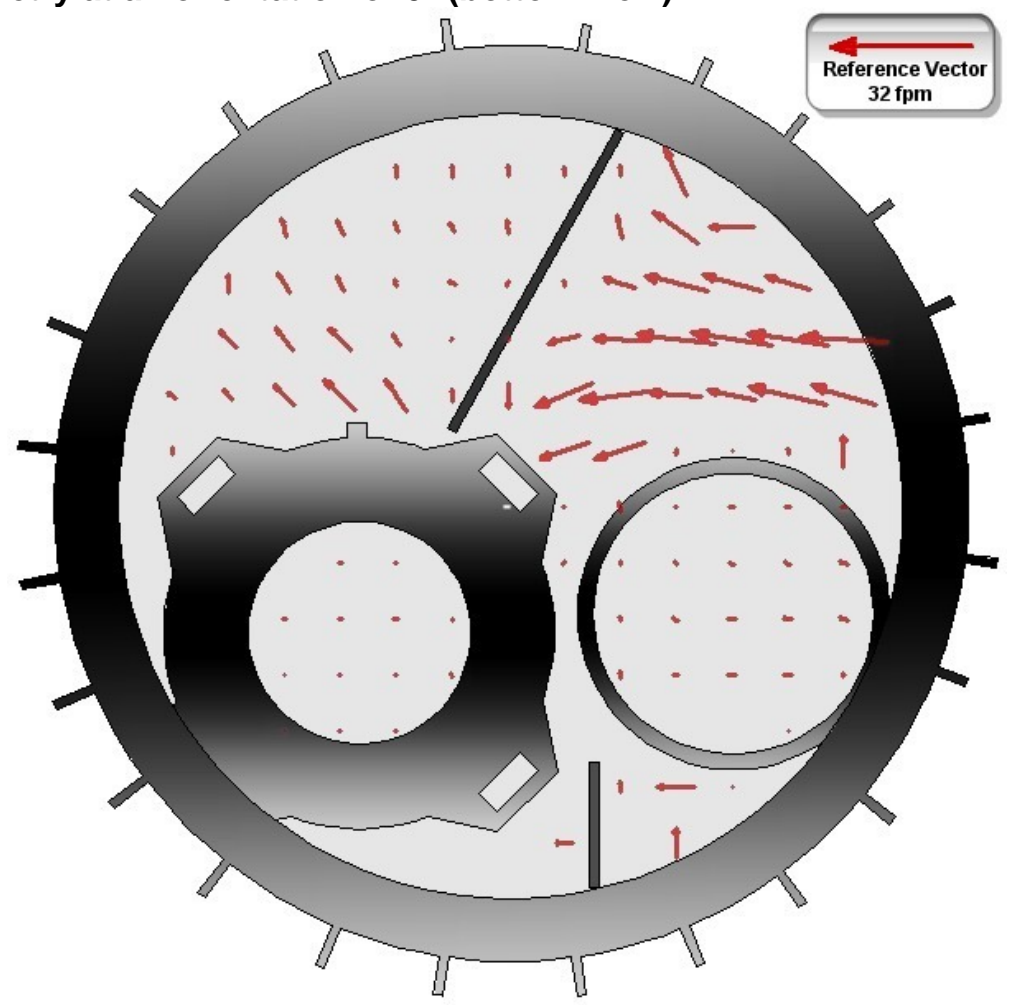

Figure 6.3.2: Flow velocity vectors inside the SA302 Smoke Detector as measured by laser Doppler anemometry at an orientation of $90^{\circ}$ (bottom view). 


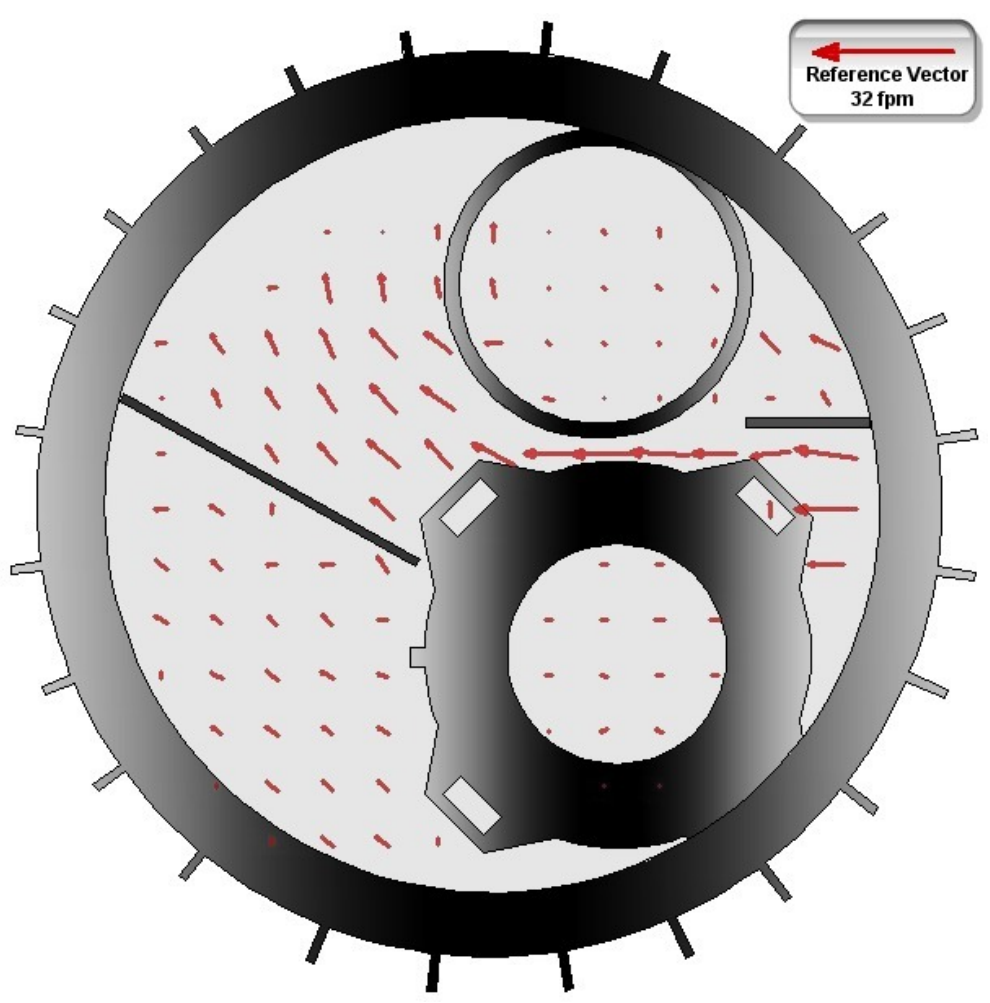

Figure 6.3.3: Flow velocity vectors inside the SA302 Smoke Detector as measured by laser Doppler anemometry at an orientation of $180^{\circ}$ (bottom view).

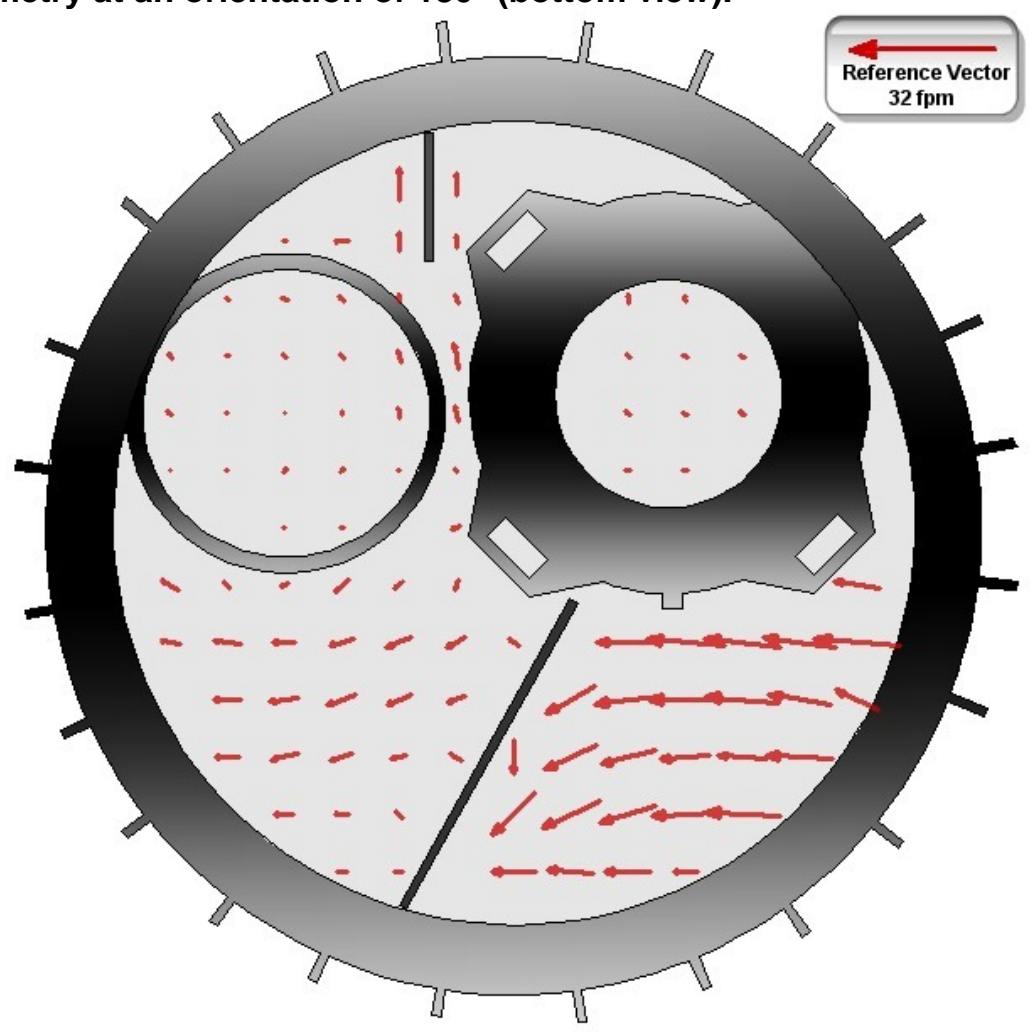

Figure 6.3.4: Flow velocity vectors inside the SA302 Smoke Detector as measured by laser Doppler anemometry at an orientation of $270^{\circ}$ (bottom view). 


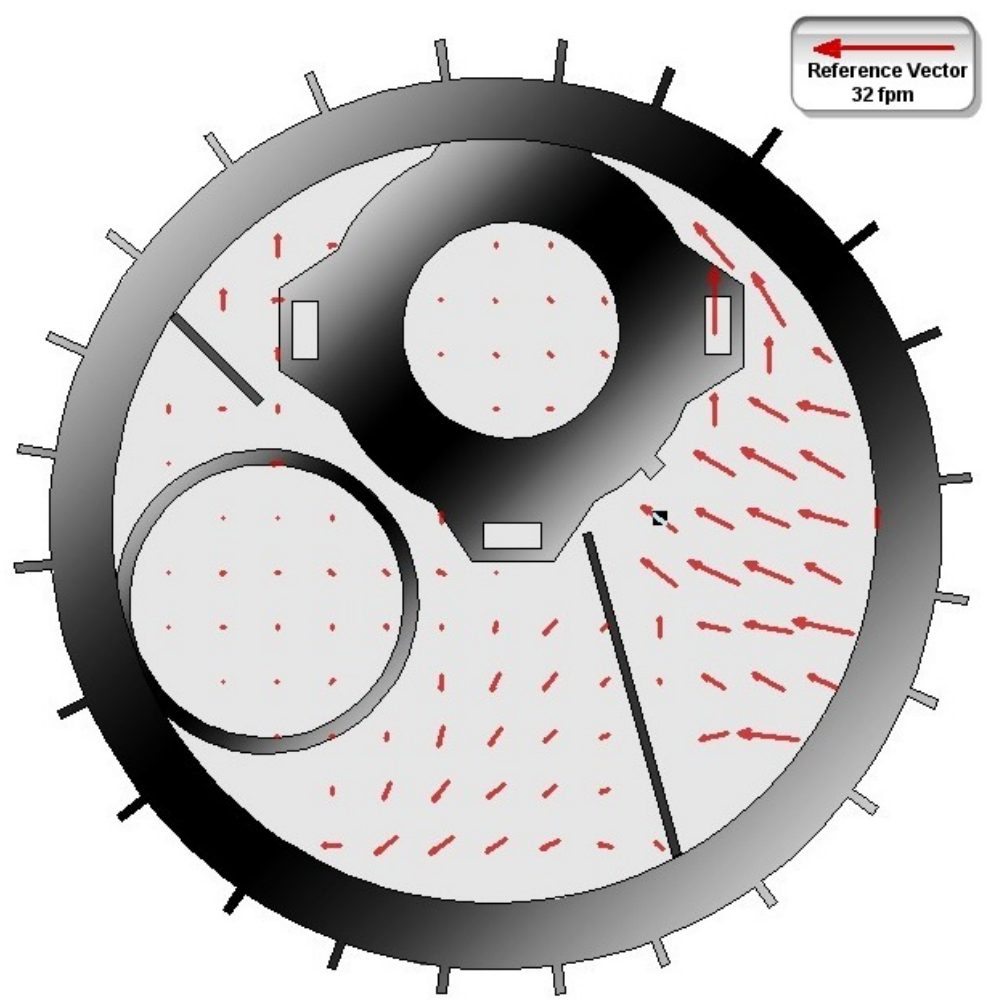

Figure 6.3.5: Flow velocity vectors inside the SA302 Smoke Detector as measured by laser Doppler anemometry at an orientation of $315^{\circ}$ (bottom view).

Figures 6.3.6 through 6.3.10 show the results of the flow visualization inside the SA302 smoke detector overlaid with the velocity vectors as measured by the laser Doppler anemometer. The view is from the bottom of the detector and the velocity measurements from which the vectors were constructed were acquired at the horizontal mid-plane of the detector body. UL Smoke Box test section flow is moving from right to left for each figure. The flow visualization still photographs in these figures represent just one frame of the MPEG video footage captured for each orientation. Notice that the flow field behaviors captured by the flow visualization technique and LDA velocity vectors are quite similar. It is also obvious that the flow velocities inside the ion and photoelectric chamber are very small in magnitude compared to the smoke box free stream velocity. 


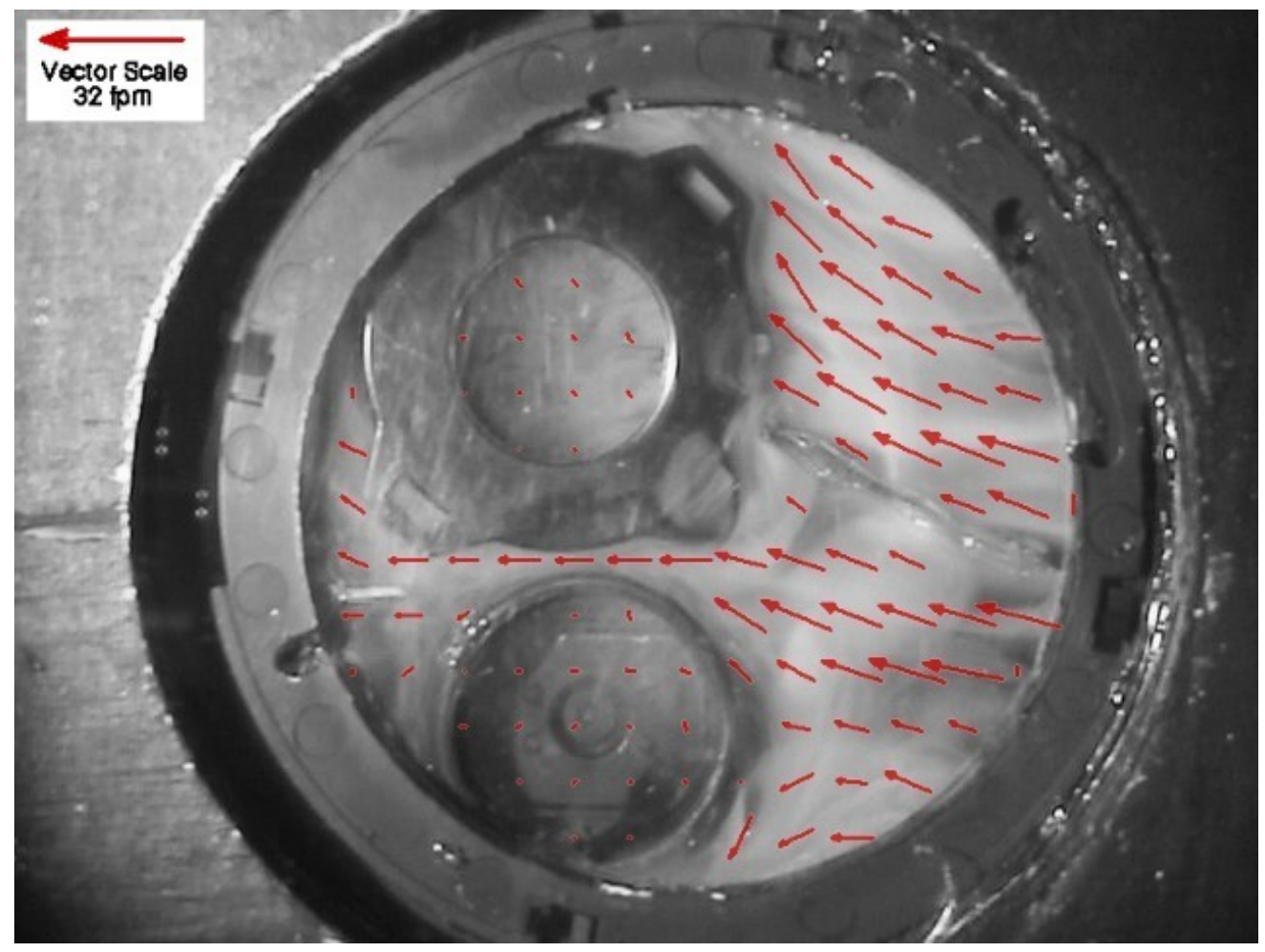

Figure 6.3.6: Air flow velocity vectors inside the SA302 smoke detector mid-plane as measured by LDA superposed on a flow visualization image at $0^{\circ}$ (bottom view).

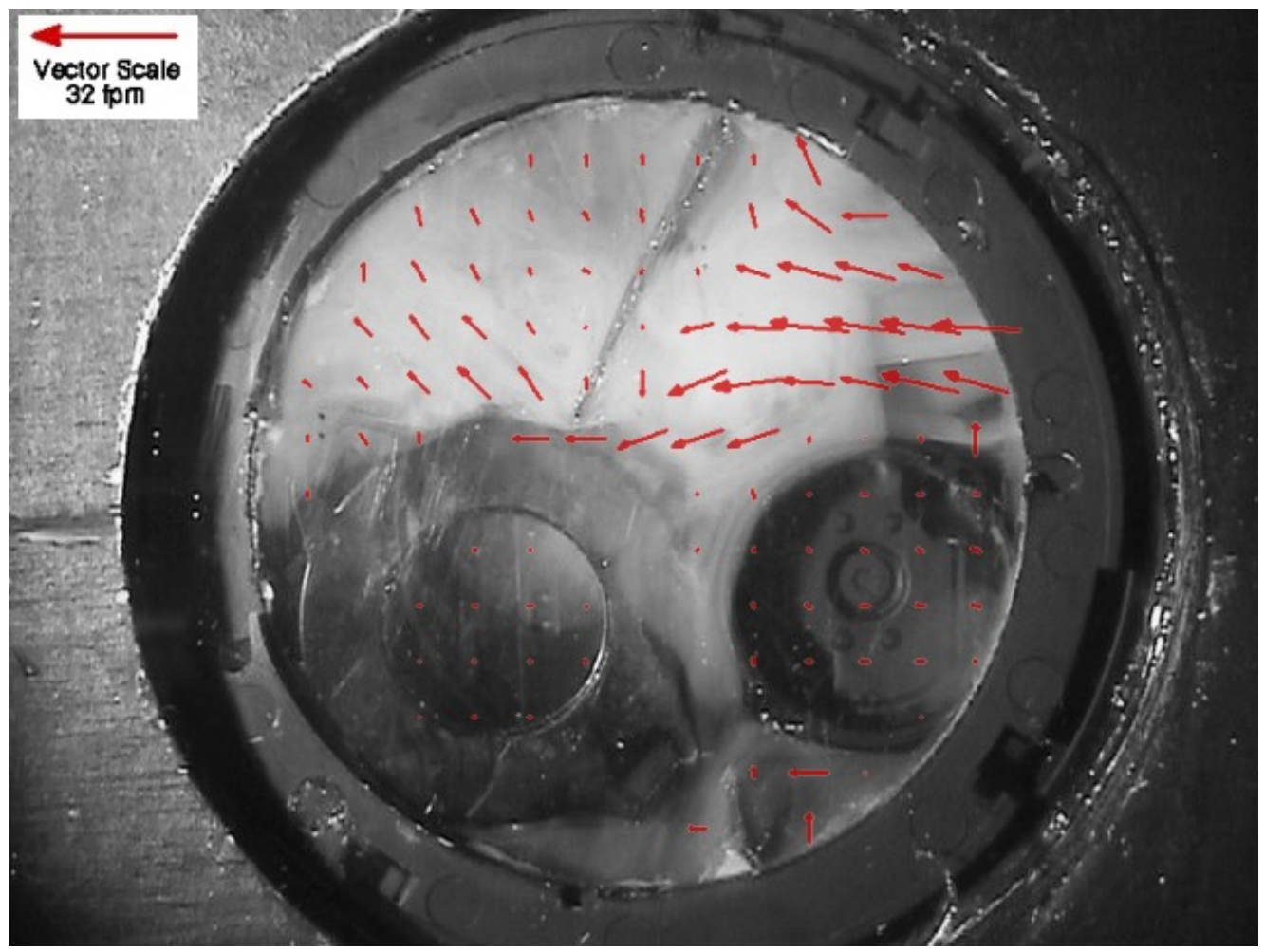

Figure 6.3.7: Air flow velocity vectors inside the SA302 smoke detector mid-plane as measured by LDA superposed on a flow visualization image at $90^{\circ}$ (bottom view). 


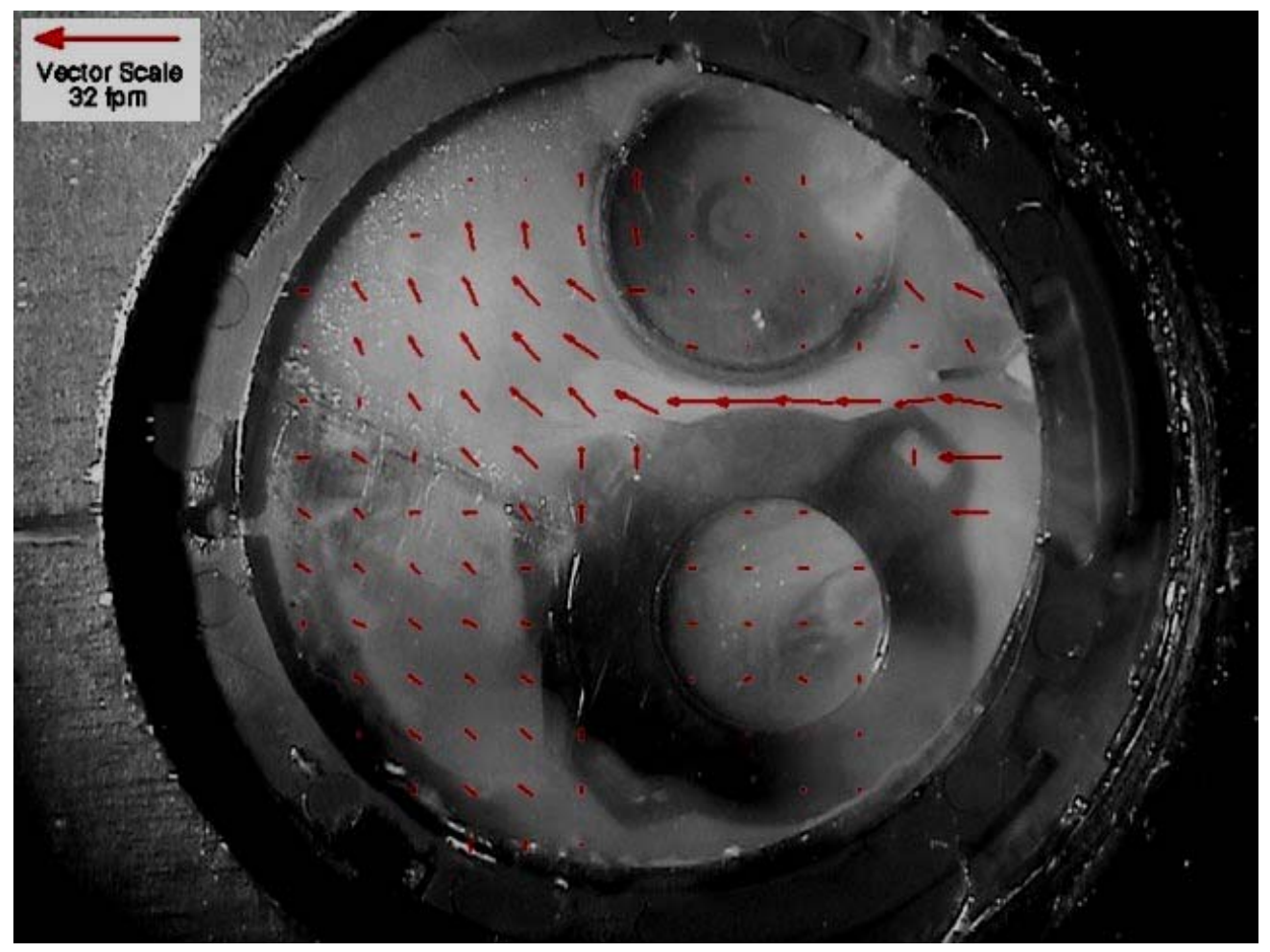

Figure 6.3.8: Air flow velocity vectors inside the SA302 smoke detector mid-plane as measured by LDA superposed on a flow visualization image at $180^{\circ}$ (bottom view).

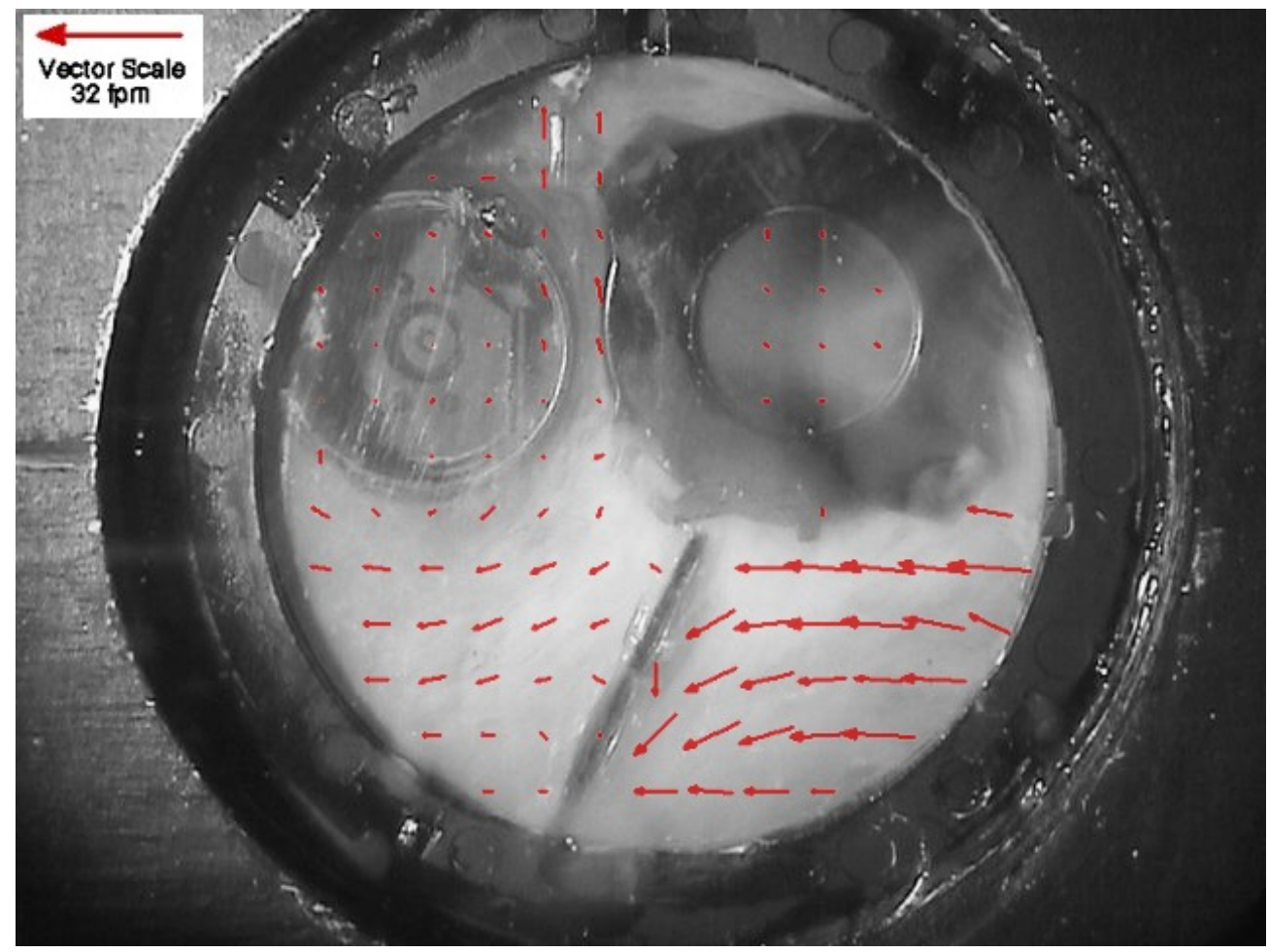

Figure 6.3.9: Air flow velocity vectors inside the SA302 smoke detector mid-plane as measured by LDA superposed on a flow visualization image at $270^{\circ}$ (bottom view). 


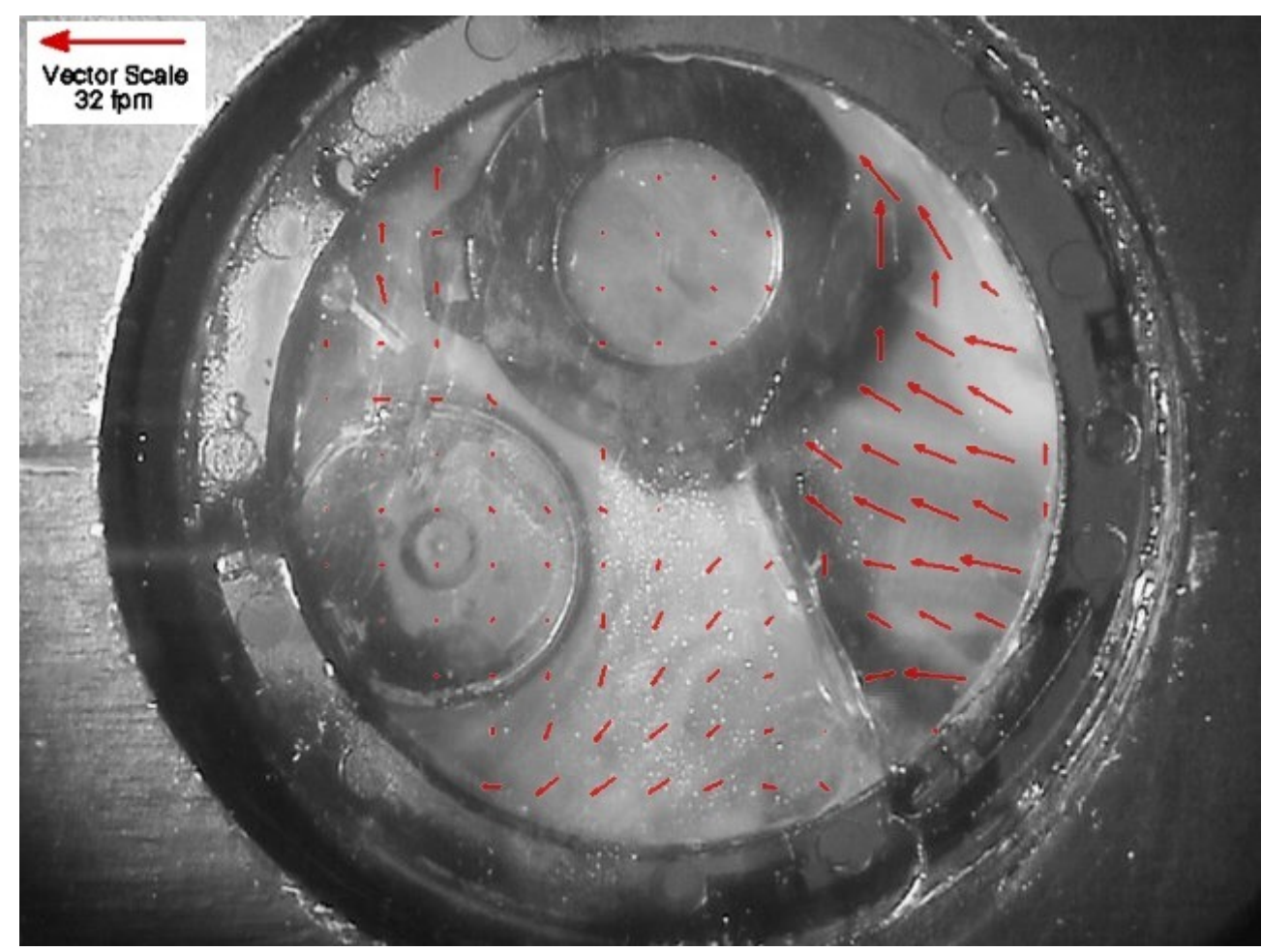

Figure 6.3.10: Air flow velocity vectors inside the SA302 smoke detector mid-plane as measured by LDA superposed on a flow visualization image at $315^{\circ}$ (bottom view).

Figures 6.3.11 through 6.3.15 show the flow velocity vectors and streamlines inside the SA302 smoke detector and inside the ion chamber and photoelectric chamber determined from the LDA data as the orientation angle of the smoke detector is varied. Recall the grid to define the physical locations of these LDA velocity measurements is shown in Figure 4.4.2. The Tecplot ${ }^{\circledR 21}$ software used to plot the flow streamlines numerically interpolates between the velocity measurements locations to compute velocities in the complete field. The streamlines here only show direction of the flow path and do not convey any velocity magnitude information. The velocity vectors, however, do show both velocity magnitude and direction of the flow in the $x$-z plane. The velocity magnitude can be compared to the reference vector in each of the figures. Each figure shows the overall flow field inside the detector and a detailed view of the corresponding flow field inside the sensors shown in the overall field. In some of these figures, it is noted that the computer interpolated streamlines appear to pass through the flow divider plates in the detector - when that is not physically possible. The interpolation software used to generate these streamline plots from the experimentally obtained velocity data did not recognize the presence of a solid object. It is 
also noted, however, that the flow divider plates did not entirely span the space between the detector base and the electronics compartment base, permitting a small amount of flow to pass over the flow dividers.

Figure 6.3.11 shows the flow behavior inside the detector oriented at $0^{\circ}$ in the smoke box. The long flow divider tends to move the flow in a diagonal direction towards the photochamber and results in the flow moving through the photo-chamber in a diagonal direction as well. The detailed flow inside the ion chamber reveals the presence of a vortex flow, although quite low speed. The flow velocity inside the detector is on the order of 10 to 15 $\mathrm{ft} / \mathrm{min}$ while the flow velocity inside the ion chamber and the photo-chamber is on the order of 1 to $2 \mathrm{ft} / \mathrm{min}$. The flow speed and direction inside the detector and its sensing chambers differs greatly from the flow outside the detector in the smoke box.

Figure 6.3.12 shows the flow behavior inside the detector oriented at $315^{\circ}$ in the smoke box. Here, the long flow divider tends to block direct flow to the ion chamber and causes the flow to divert downward due to flow separation downstream of the divider. The result is a lower average flow velocity inside the ion chamber compared to the $0^{\circ}$ detector orientation shown in Figure 6.3.11. The flow direction inside the photo-chamber is quite similar to that shown in Figure 6.3.11 but with a slightly reduced magnitude.

Figure 6.1.13 shows the flow behavior inside the detector oriented at $270^{\circ}$ in the smoke box. In this orientation, direct flow to the ion chamber is prevented since the ion chamber is in the direct wake of the photoelectric chamber. While the flow inside the photoelectric chamber ranges from parallel to the smoke box flow direction to somewhat diagonal in direction, the flow inside the ion chamber is very significantly effected by the flow in the low speed wake of the photo-chamber and the flow separation at the tip of the long flow divider. The flow inside the ion chamber has the features of a pair of low speed counter-rotating vortices and the associated very curved streamlines patterns.

Figure 6.3.14 shows the flow behavior inside the detector oriented at $180^{\circ}$ in the smoke box. As expected, in this favorable orientation, the flow enters both sensing chambers with the minimum flow resistance relative to the other detector orientations tested. The flow velocity magnitudes inside the ion chamber range from 2 to $5 \mathrm{ft} / \mathrm{min}$ while the flow velocity magnitudes inside the photoelectric chamber range up to $2 \mathrm{ft} / \mathrm{min}$. 
Figure 6.3.15 shows the flow behavior inside the detector oriented at $90^{\circ}$ in the smoke box. In this orientation, flow into the ion chamber is not obstructed by the photo-chamber or flow dividers, and therefore, experiences flow velocity magnitudes up to $3 \mathrm{ft} / \mathrm{min}$. The shorter flow divider directs flow into the ion chamber to cause the flow direction to shift upward near the downstream edge. The flow velocity magnitudes in the photo-chamber are on the order of 0.5 to $1.0 \mathrm{ft} / \mathrm{min}$, much lower values than experienced inside the ion chamber since the photoelectric chamber is located in the wake of the ion chamber in this detector orientation.

It is evident due to the highest velocities measured that the $180^{\circ}$ orientation provided the slowest ionization sensor velocities, and the $270^{\circ}$ orientation provided the fastest photoelectric sensor velocities. 


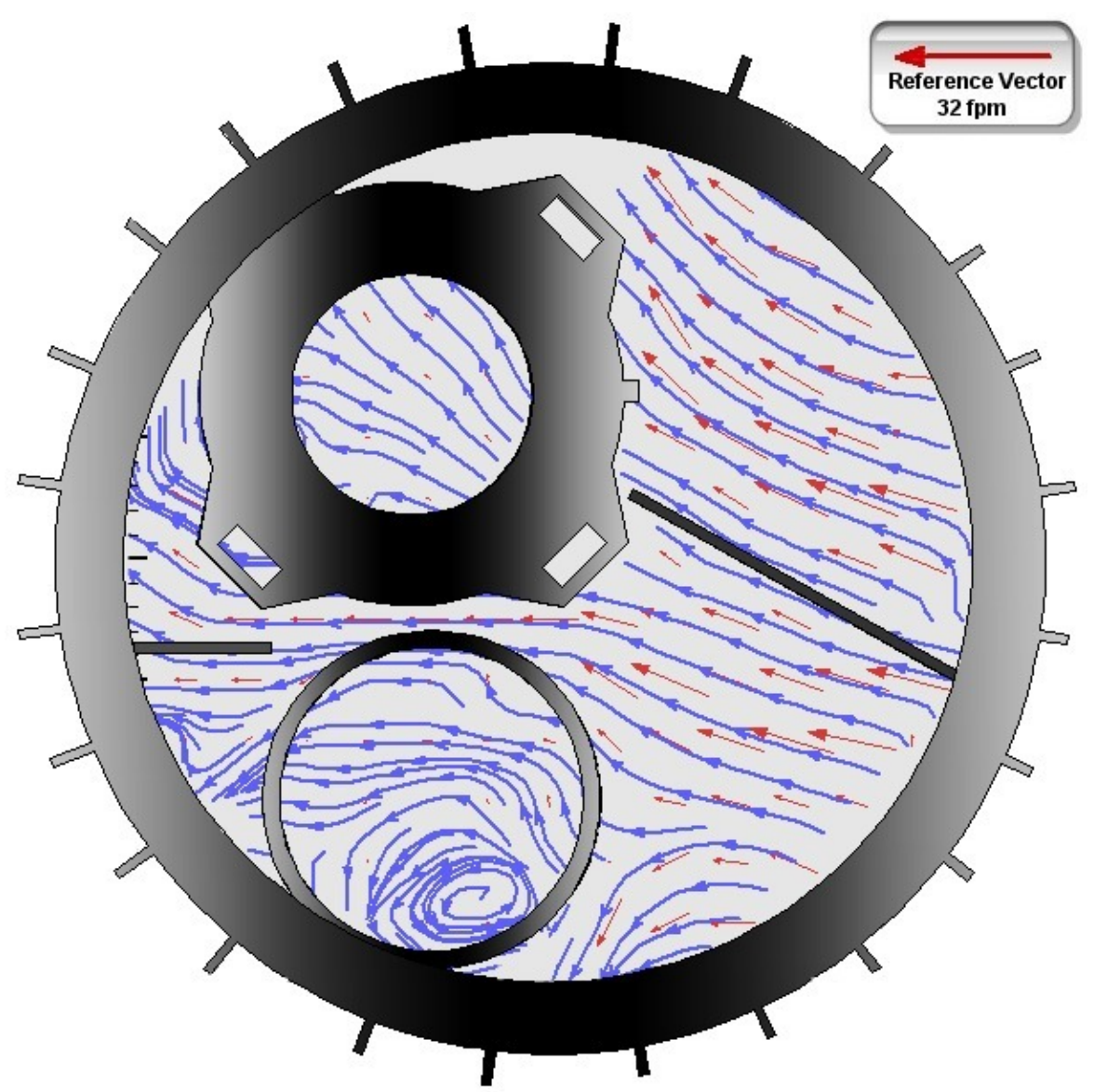

(a.)

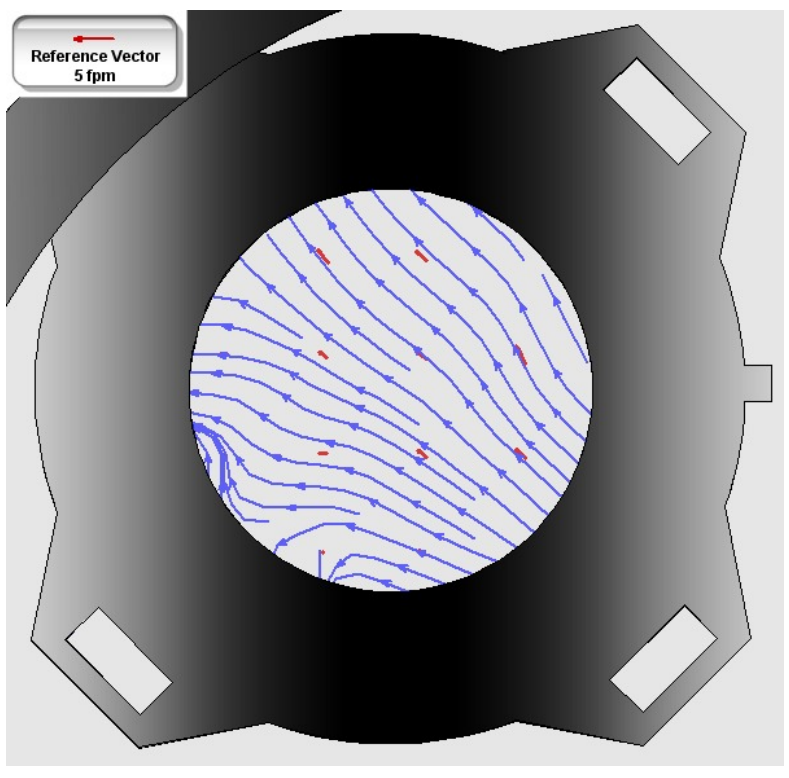

(b.)

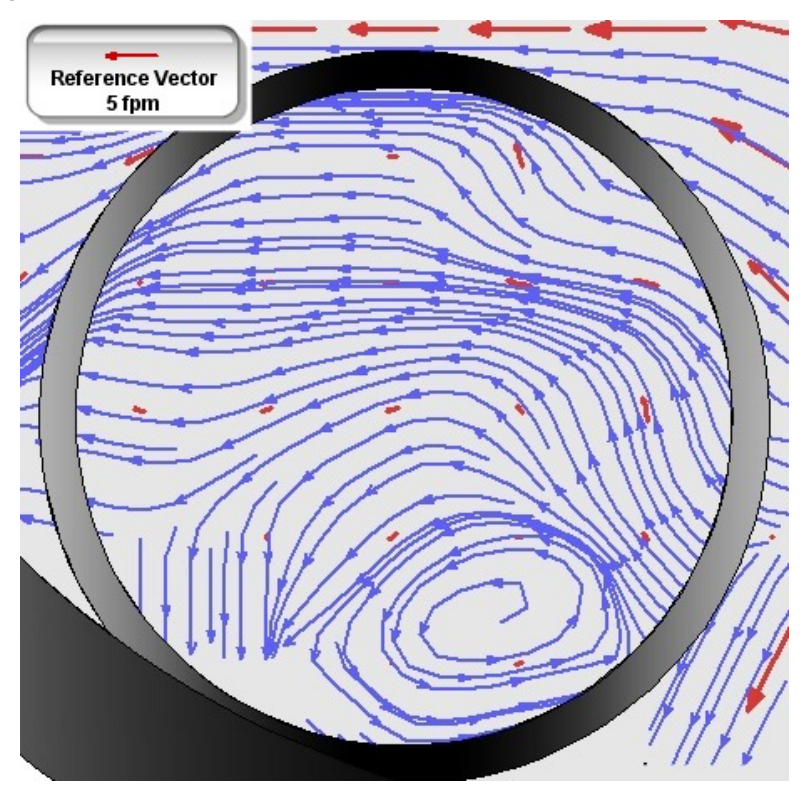

(c.)

Figure 6.3.11: The $u-v$ velocity vectors and streamlines in the SA302 smoke detector at the mid-plane of the detector flow channel at $0^{\circ}$ (bottom view) (a, b, and c denote the detector, photo, and ion chamber respectively.) 


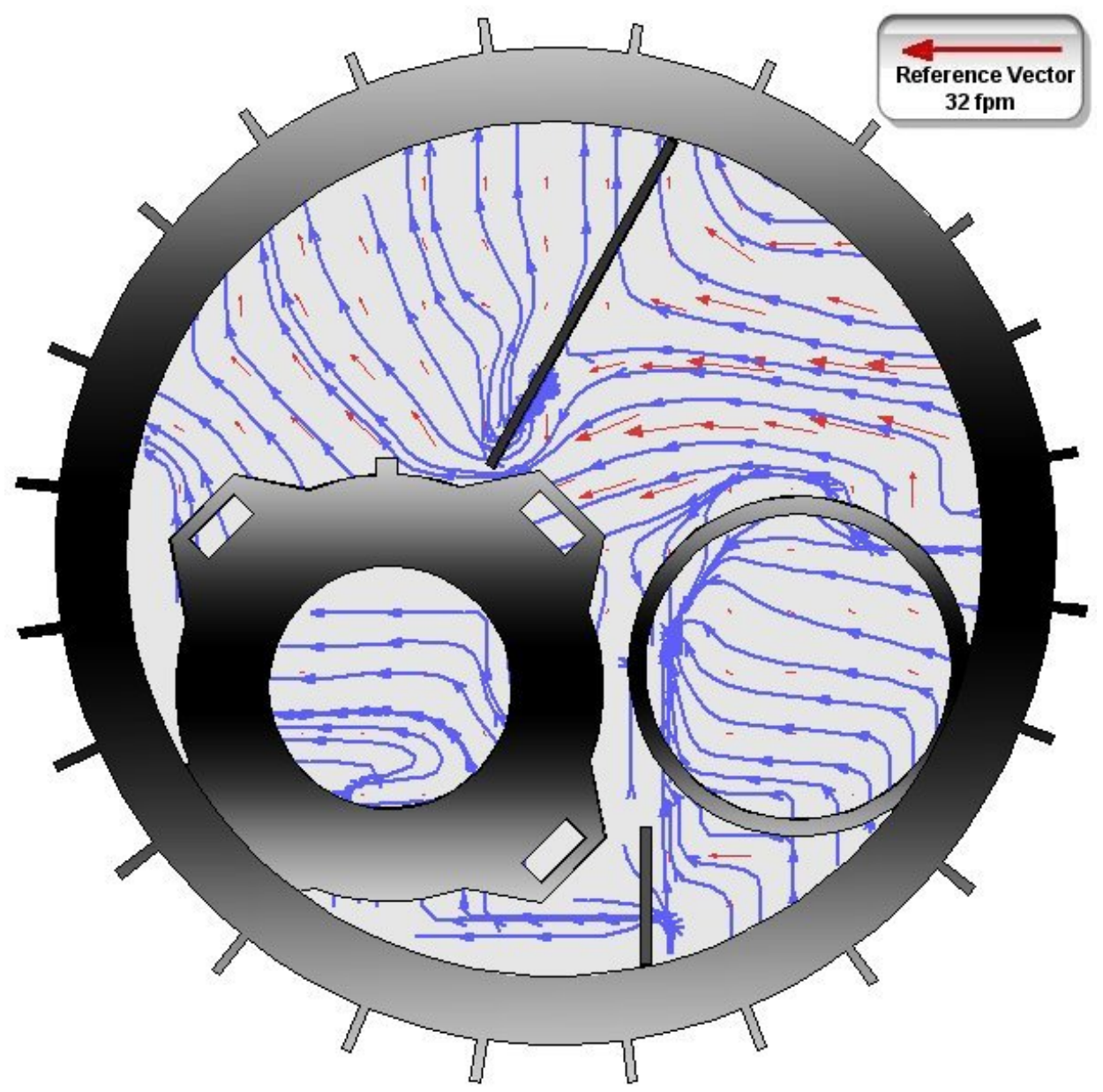

(a.)

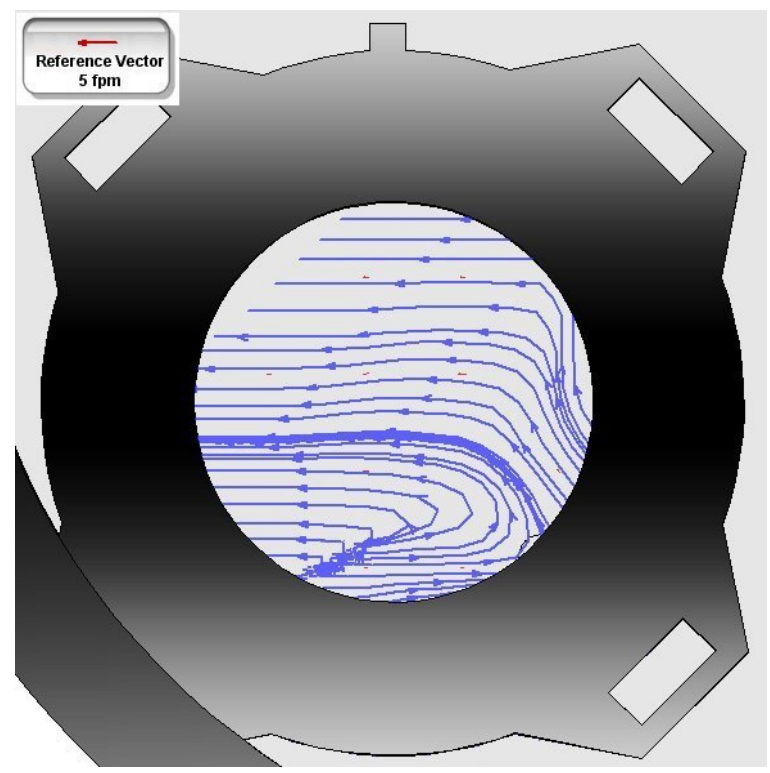

(b.)

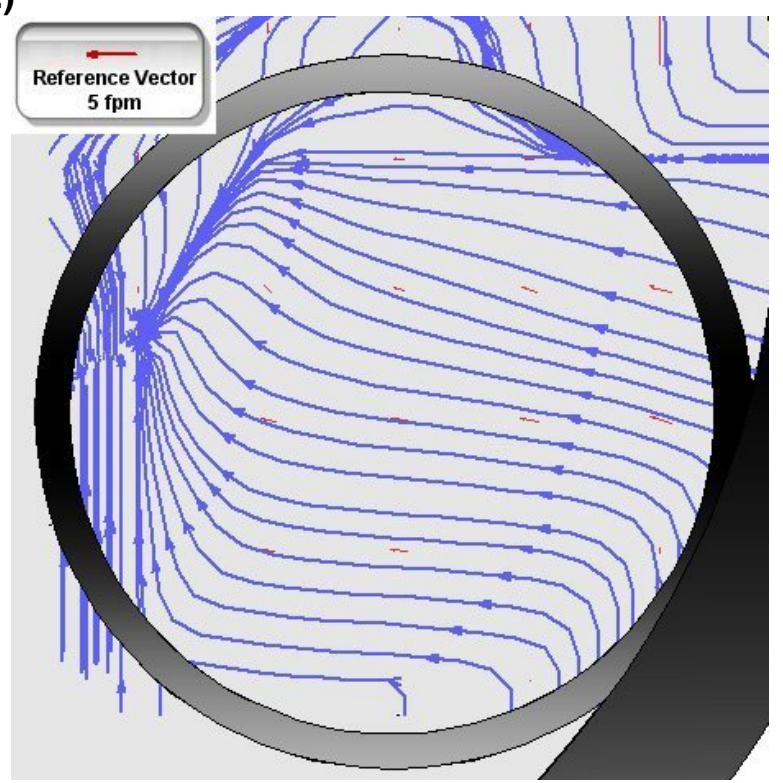

(c.)

Figure 6.3.12: The $u-v$ velocity vectors and streamlines in the SA302 smoke detector at the mid-plane of the detector flow channel at $90^{\circ}$ (bottom view) $(a, b$, and c denote the detector, photo, and ion chamber respectively.) 


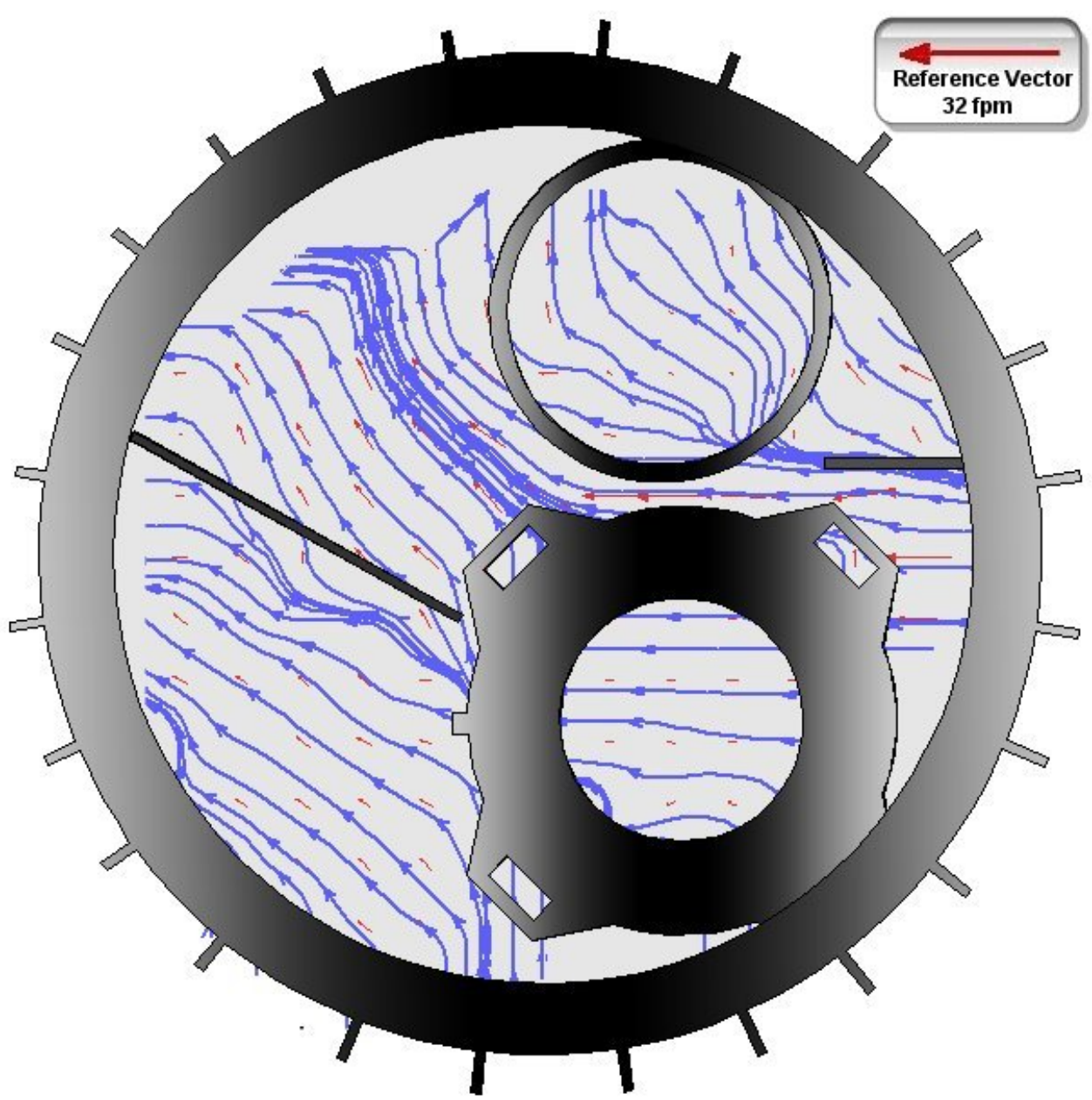

(a.)

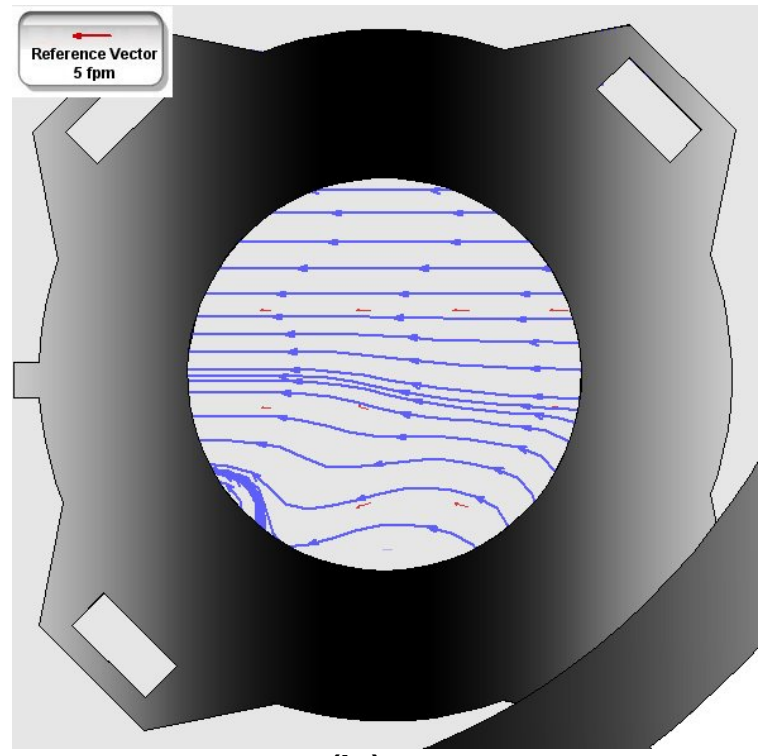

(b.)

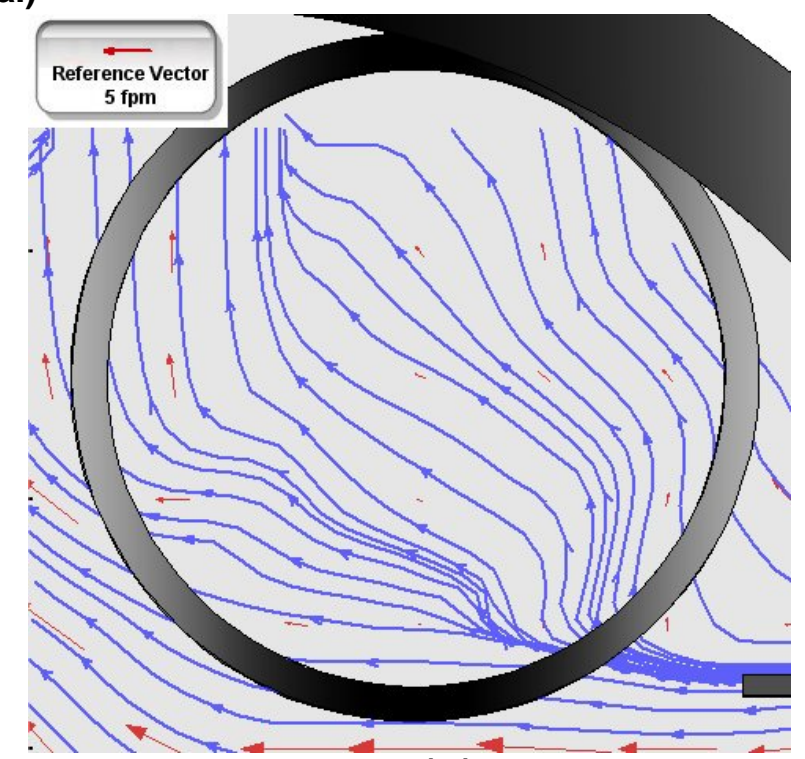

(c.)

Figure 6.3.13: The $u-v$ velocity vectors and streamlines in the SA302 smoke detector at the mid-plane of the detector flow channel at $180^{\circ}$ (bottom view) (a, b, and c denote the detector, photo, and ion chamber respectively.) 


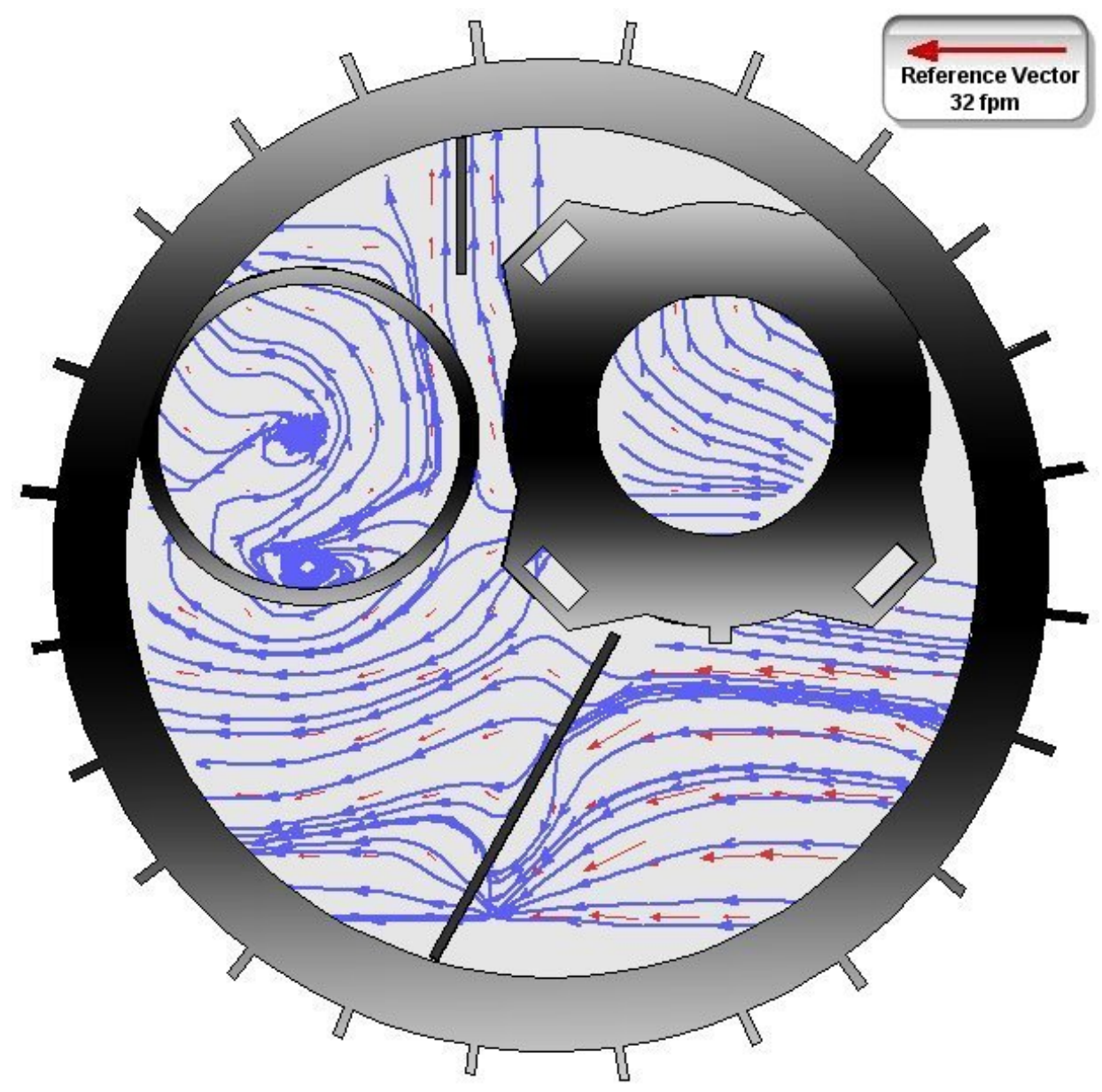

(a.)

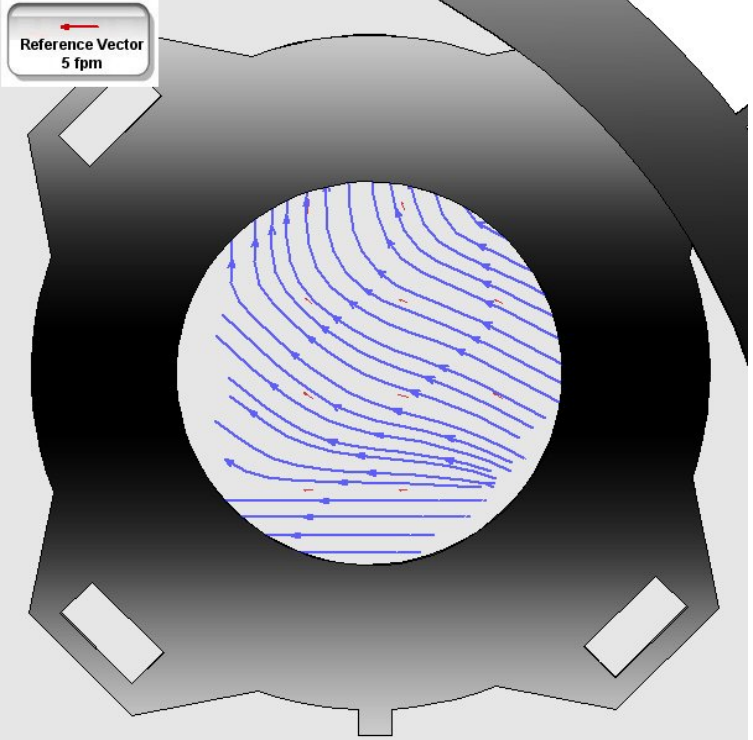

(b.)

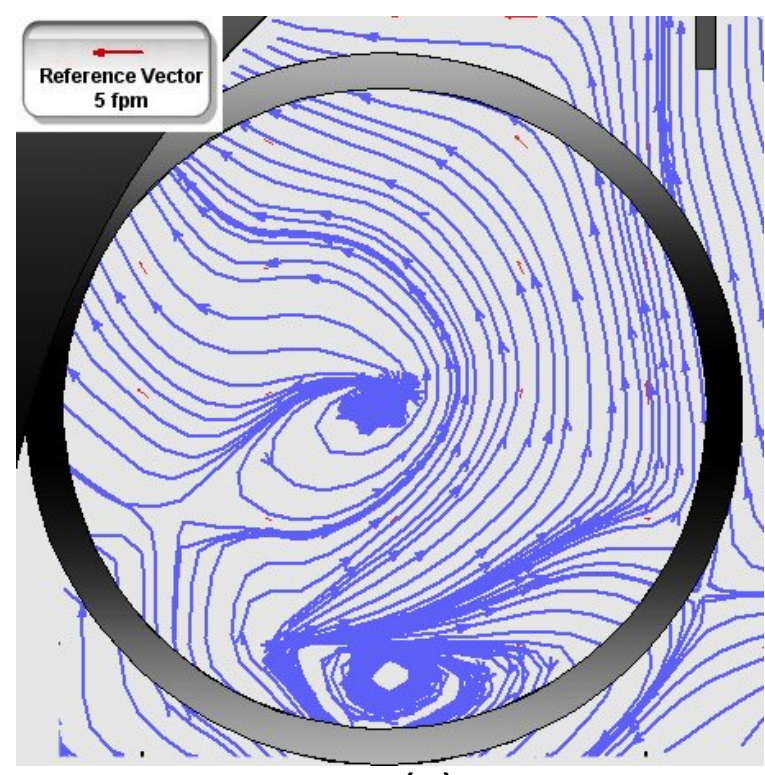

(c.)

Figure 6.3.14: The $u-v$ velocity vectors and streamlines in the SA302 smoke detector at the mid-plane of the detector flow channel at $270^{\circ}$ (bottom view) ( $a, b$, and c denote the detector, photo, and ion chamber respectively.) 


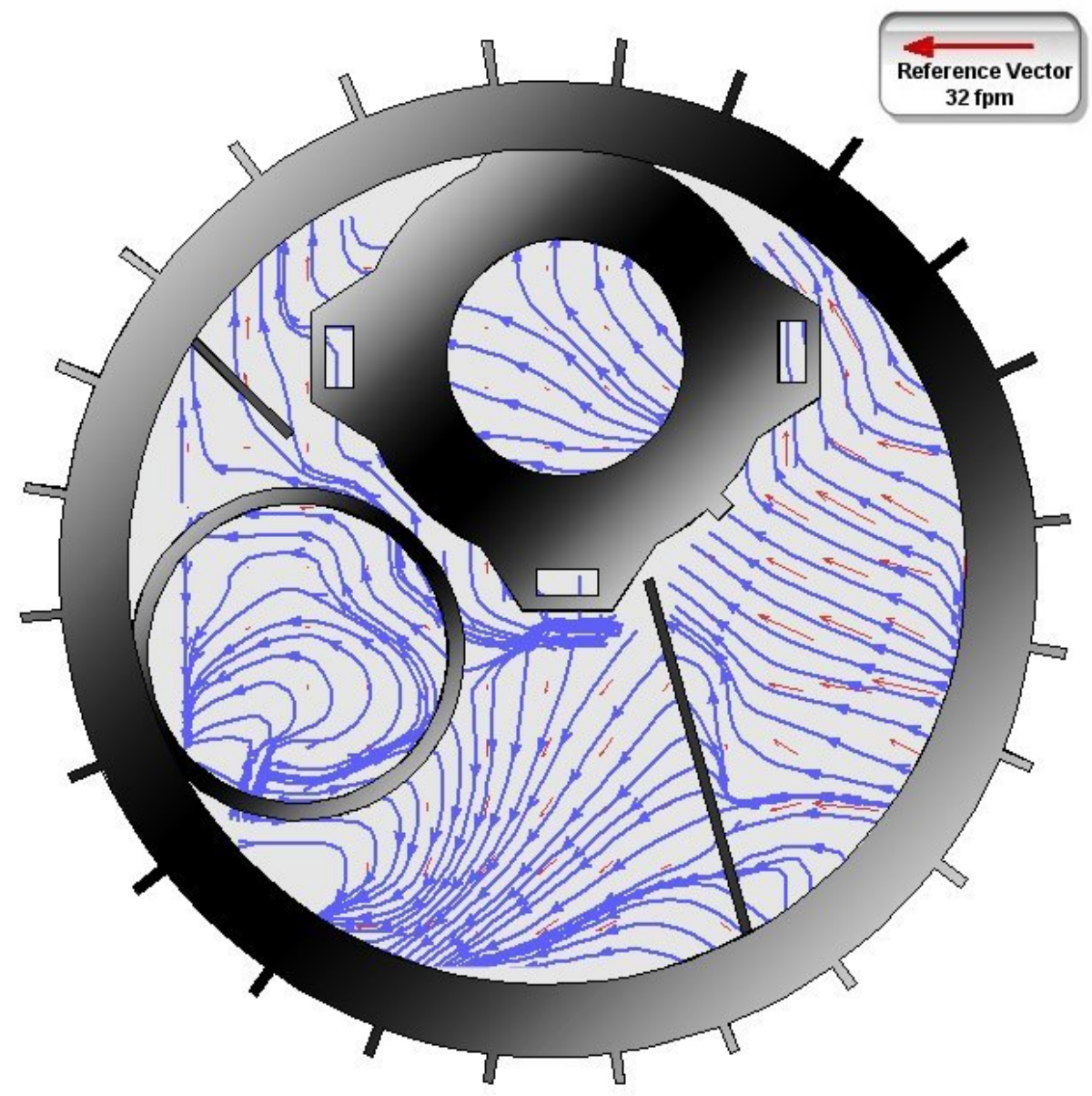

(a.)

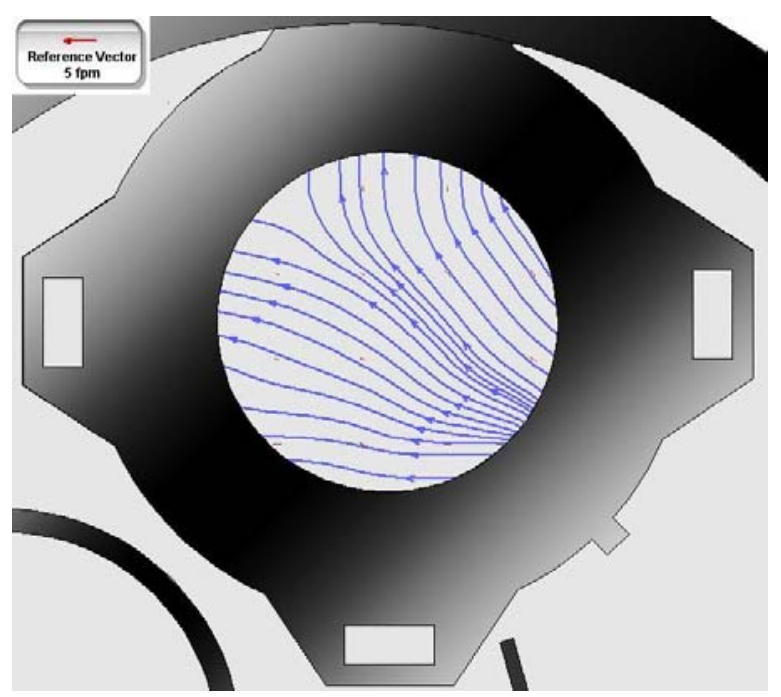

(b.)

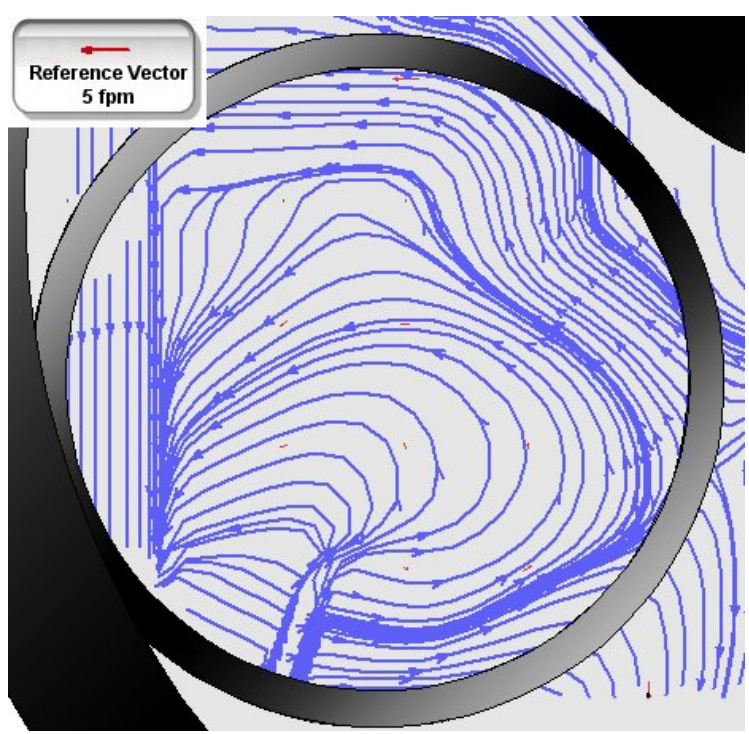

(c.)

Figure 6.3.15: The $u-v$ velocity vectors and streamlines in the SA302 smoke detector at the mid-plane of the detector flow channel at $315^{\circ}$ (bottom view) (a, b, and c denote the detector, photo, and ion chamber respectively.) 


\subsection{UL SMOKE BOX VELOCITY MEASUREMENTS USING LDA}

Figures 6.4.1 through 6.4.20 show the $u-w$ velocity vectors and streamlines at various vertical, $y$-coordinate levels in the smoke box with an SA302 smoke detector in the test section. In each figure, the top image illustrates the vertical level of the cross-section of the smoke box test section where the velocity vectors and the flow streamlines are shown in the following image on the same page. The green arrows on the legend plot indicate the viewing direction.

In Figures 6.4.1 and 6.4.2 for $y=0.25$ in, it is clear that the flow approaching the smoke detector is moving away from the MIC in the transverse direction. This behavior was also illustrated by the laser flow visualization in Figure 6.2.4. The presence of the MIC very significantly affects the flow near the floor of the test section in the boundary layer. In the wake of the detector, there are regions of separated and re-circulating flows patterns where the flow actually moves backwards toward the detector.

Figure 6.4.4 illustrates the velocity vectors and flow streamlines near the detector for $y=0.5 \mathrm{in}$. The flow behavior is similar to the $y=0.25$ in plane, but the transverse flow component is weaker as was observed in Figure 6.2.4 as well. A significant wake region exists aft of the detector. Figures 6.4.5 through 6.4.16 show the velocity vectors and the streamline patterns for the smoke box for the horizontal planes $y=1$ in through $y=6$ in. The flow patterns exhibited by these figures are quite intuitive. There is a wake present downstream of the detector until the measurement plane level increases to $y=3$ in (Figure 6.4.9 and 6.4.10) where the plane is above the highest most point of the detector. The influence of the presence of the MIC cannot be ignored in any of the test cases since it tends to push the streamlines away from its flat surface toward the center of the test section, basically acting as a solid body in the flow field. In the wake of the detector, vortices are traced by the streamlines following much of the flow pattern observed in the flow visualization plots found in Section 6.2. 


\section{Legend}
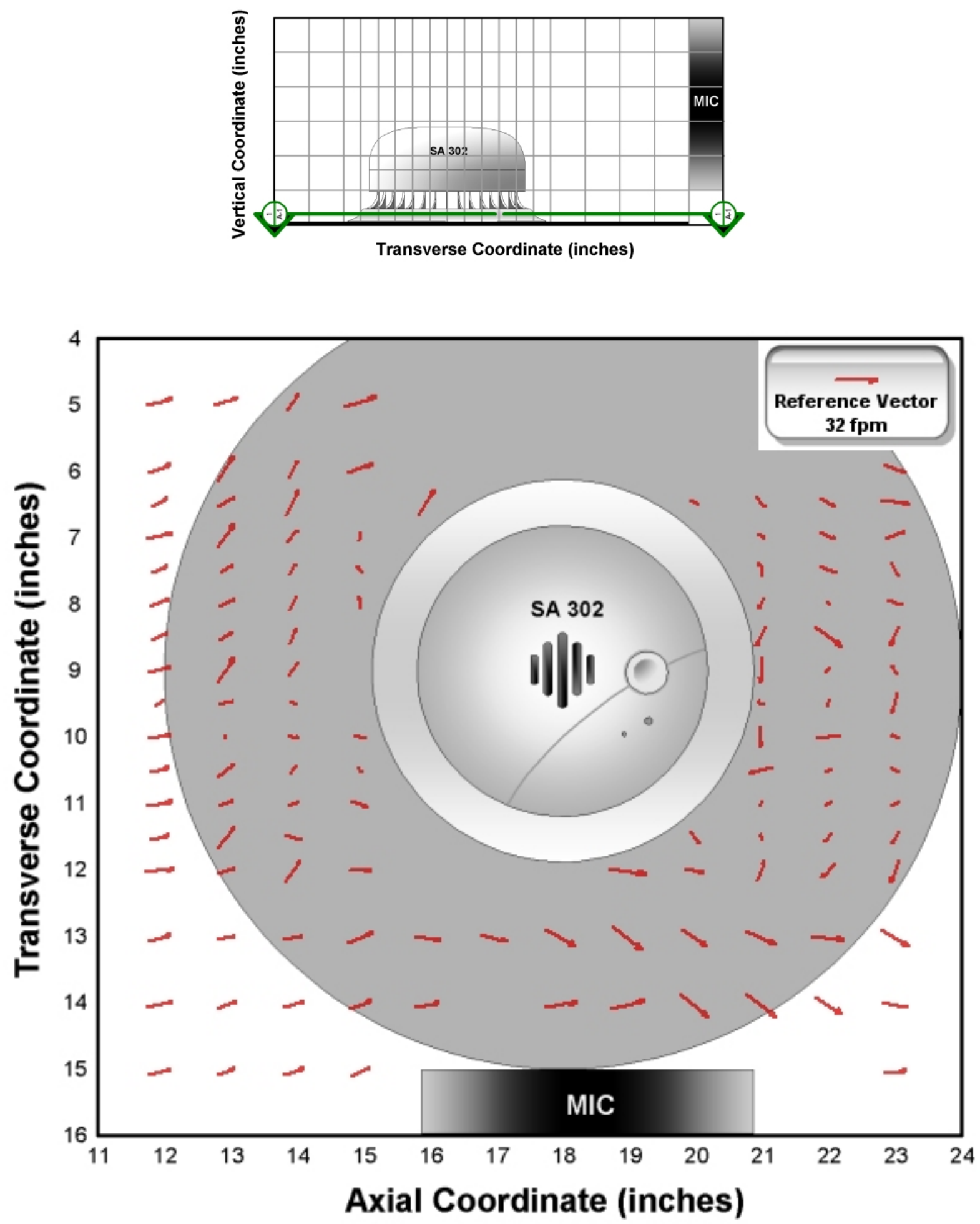

Figure 6.4.1: The $u-w$ velocity vectors in the UL Smoke Box test section at $y=0.25$ in. 


\section{Legend}
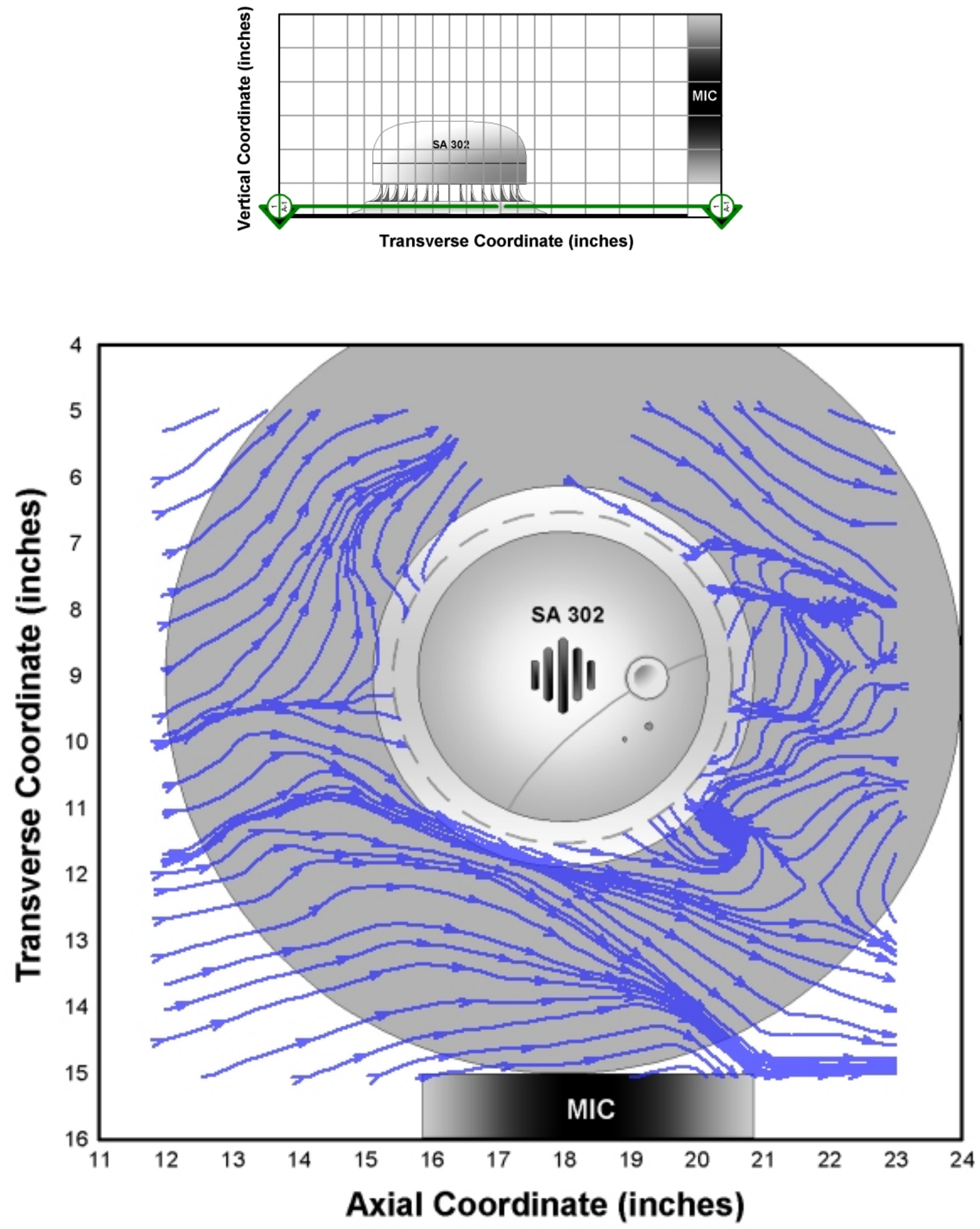

Figure 6.4.2: The $u$-w streamlines in the UL Smoke Box test section at $y=0.25$ in. 


\section{Legend}
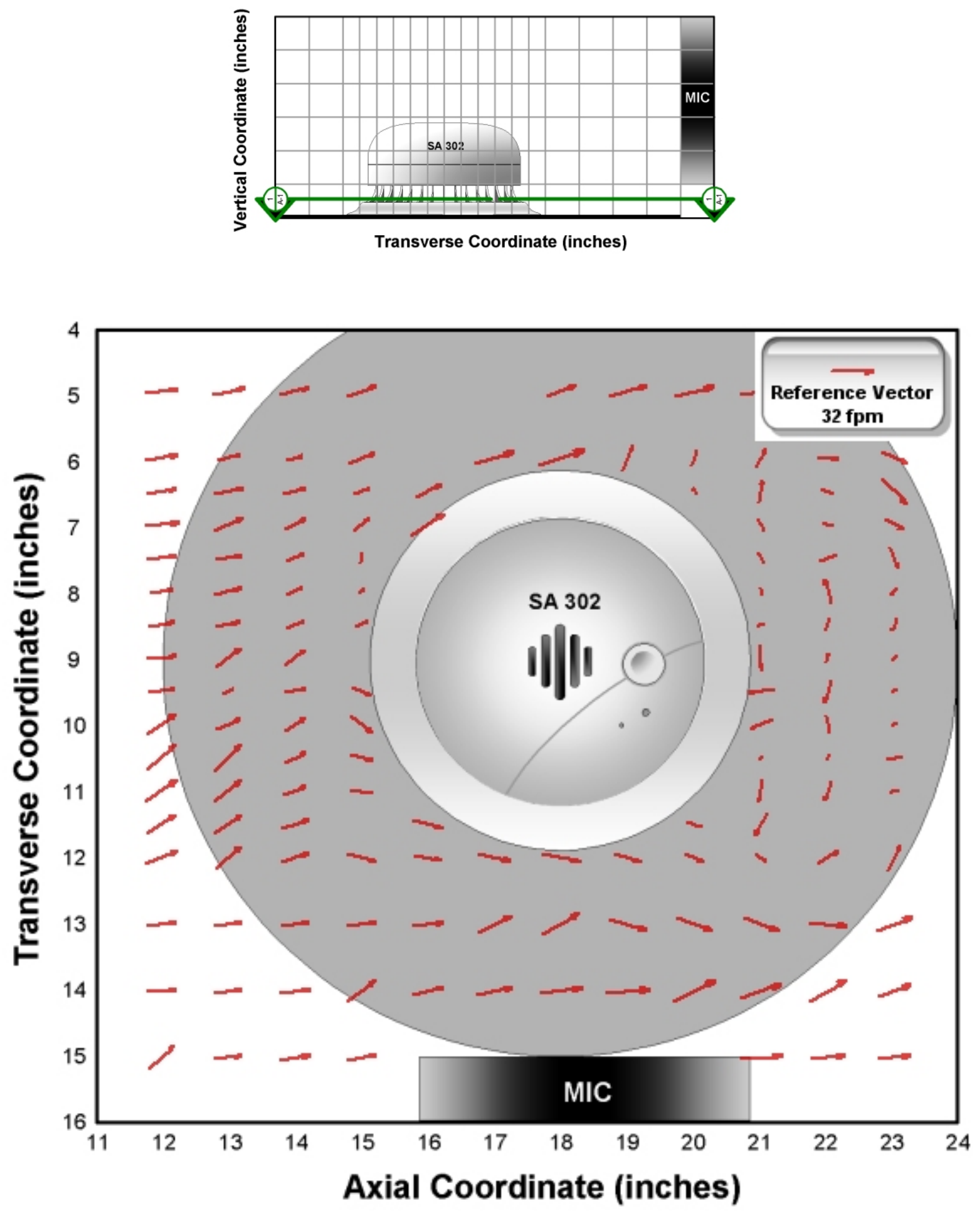

Figure 6.4.3: The $u-w$ velocity vectors in the UL Smoke Box test section at $y=0.50$ in. 


\section{Legend}
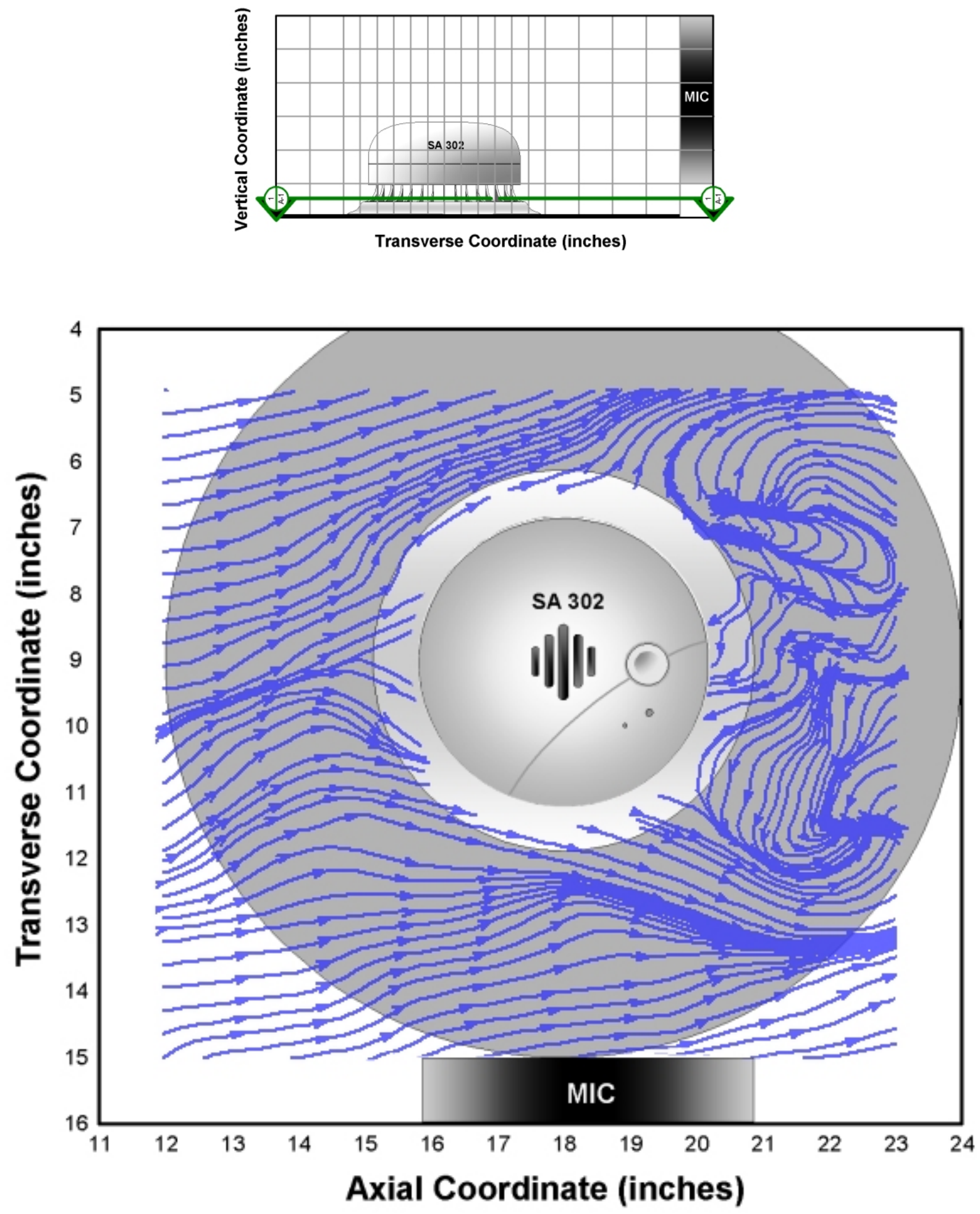

Figure 6.4.4: The $u$-w streamlines in the UL Smoke Box test section at $y=0.50 \mathrm{in}$. 


\section{Legend}
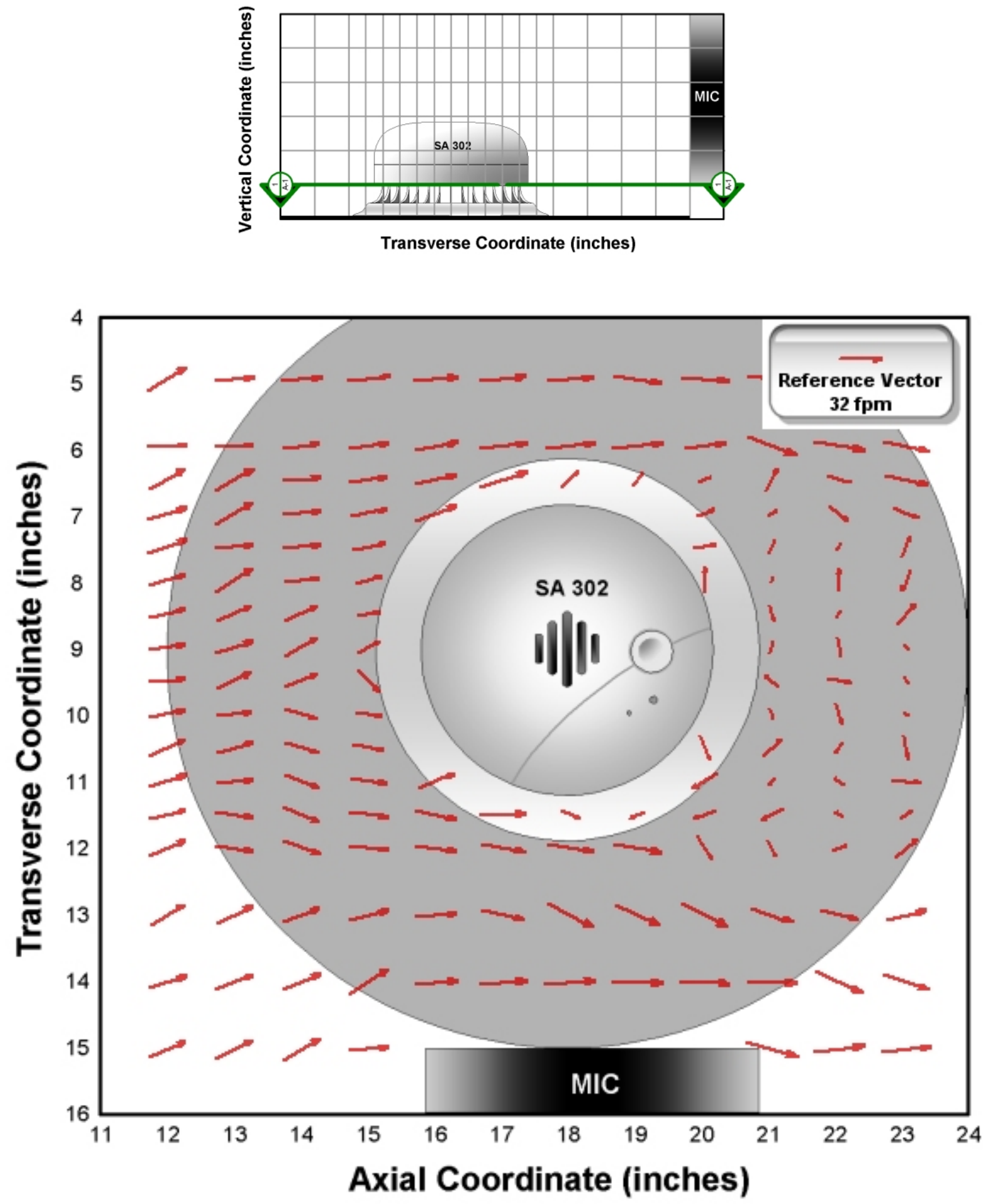

Figure 6.4.5: The $u-w$ velocity vectors in the UL Smoke Box test section at $y=1.0$ in. 


\section{Legend}
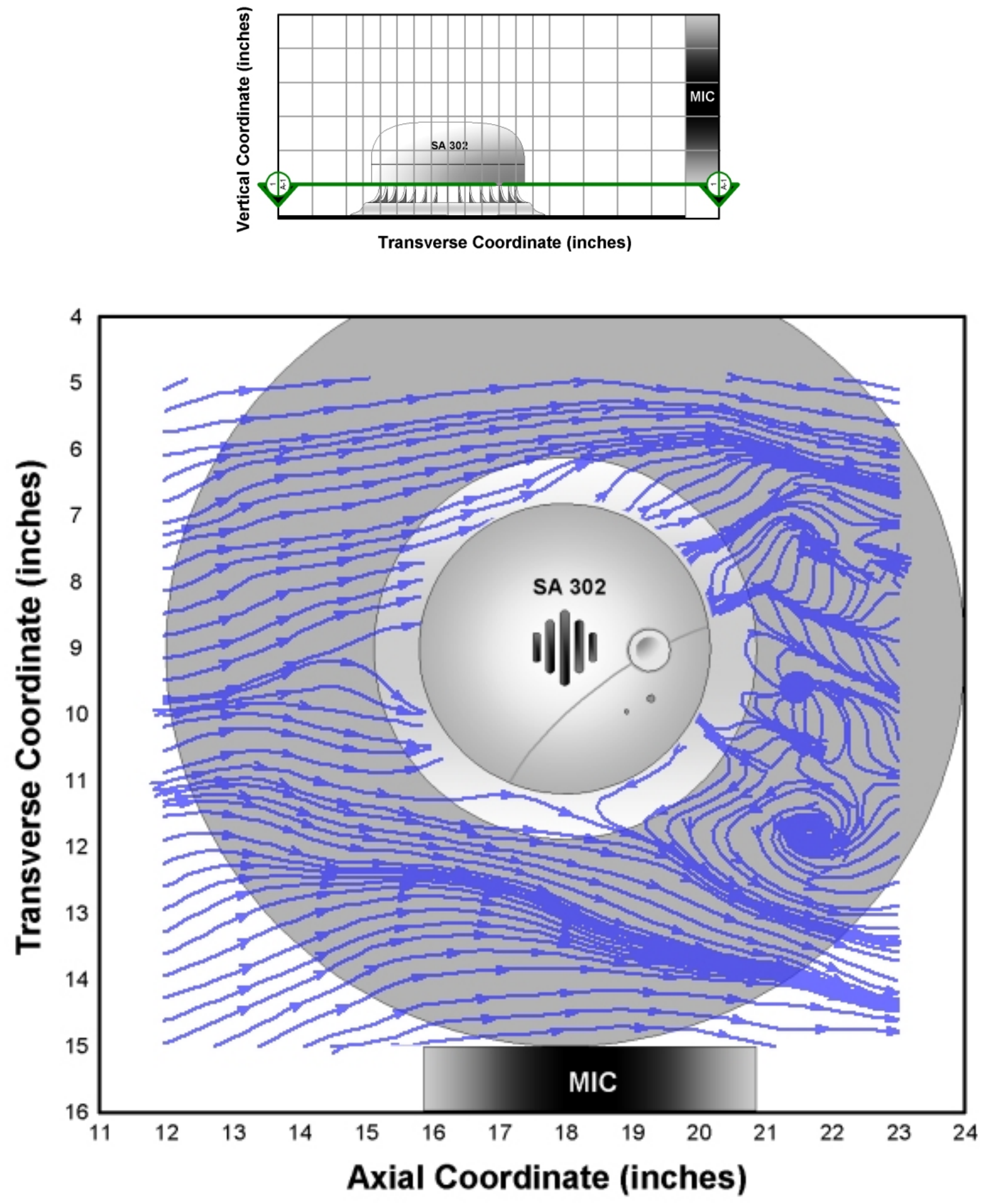

Figure 6.4.6: The $u-w$ streamlines in the UL Smoke Box test section at $y=1.0$ in. 


\section{Legend}
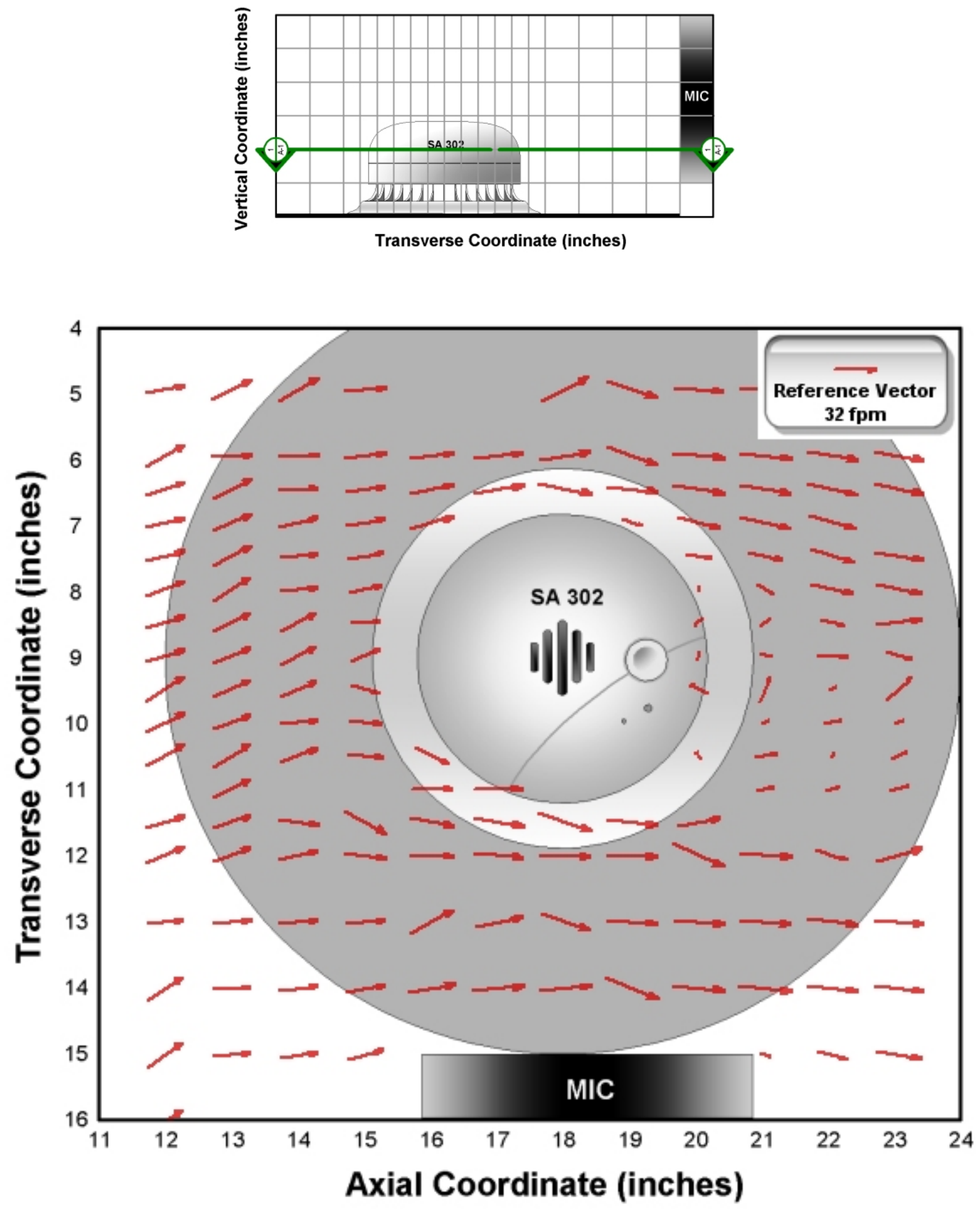

Figure 6.4.7: The $u-w$ velocity vectors in the UL Smoke Box test section at $y=2.0$ in. 


\section{Legend}
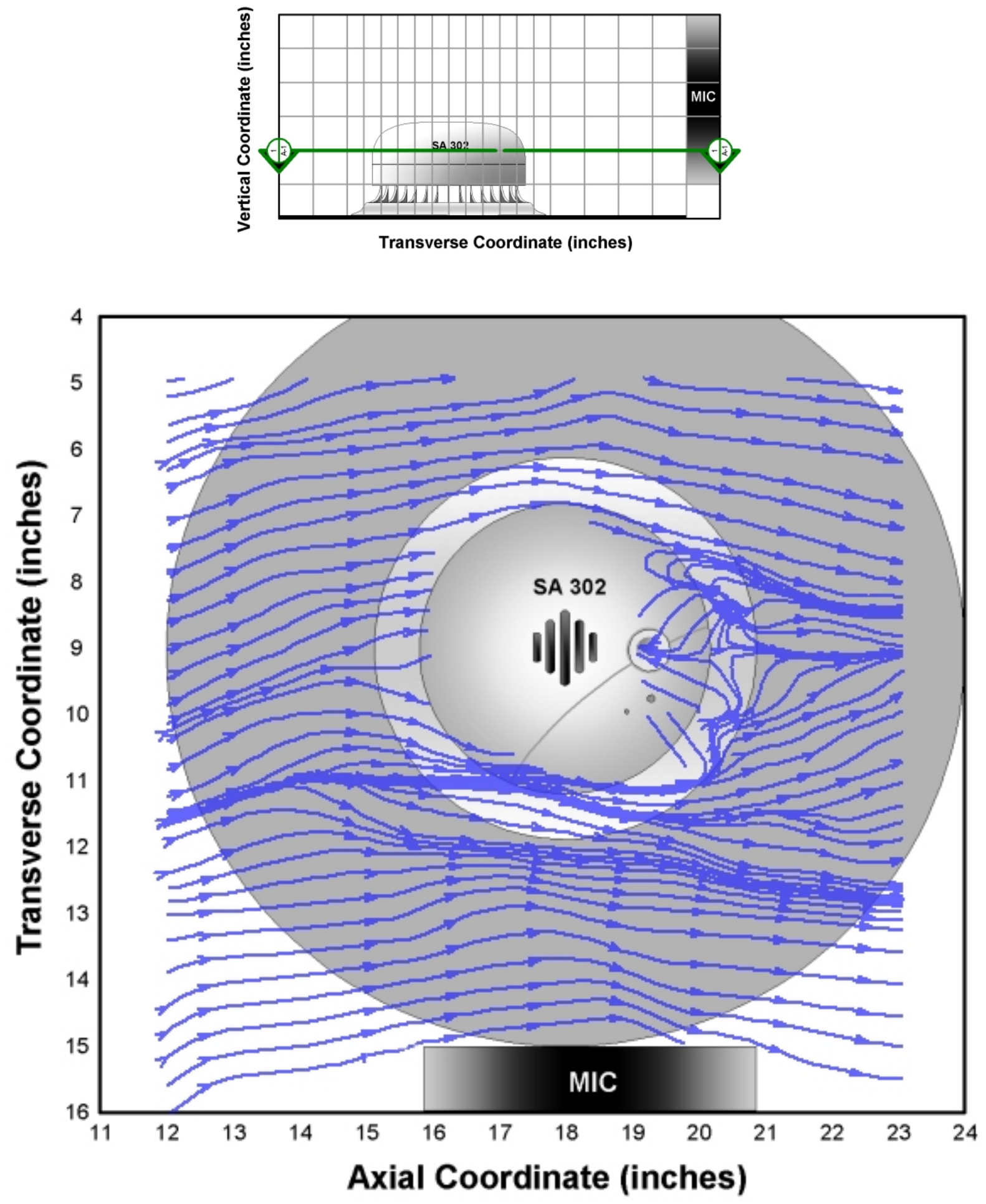

Figure 6.4.8: The $u$-w streamlines in the UL Smoke Box test section at $y=2.0$ in. 


\section{Legend}
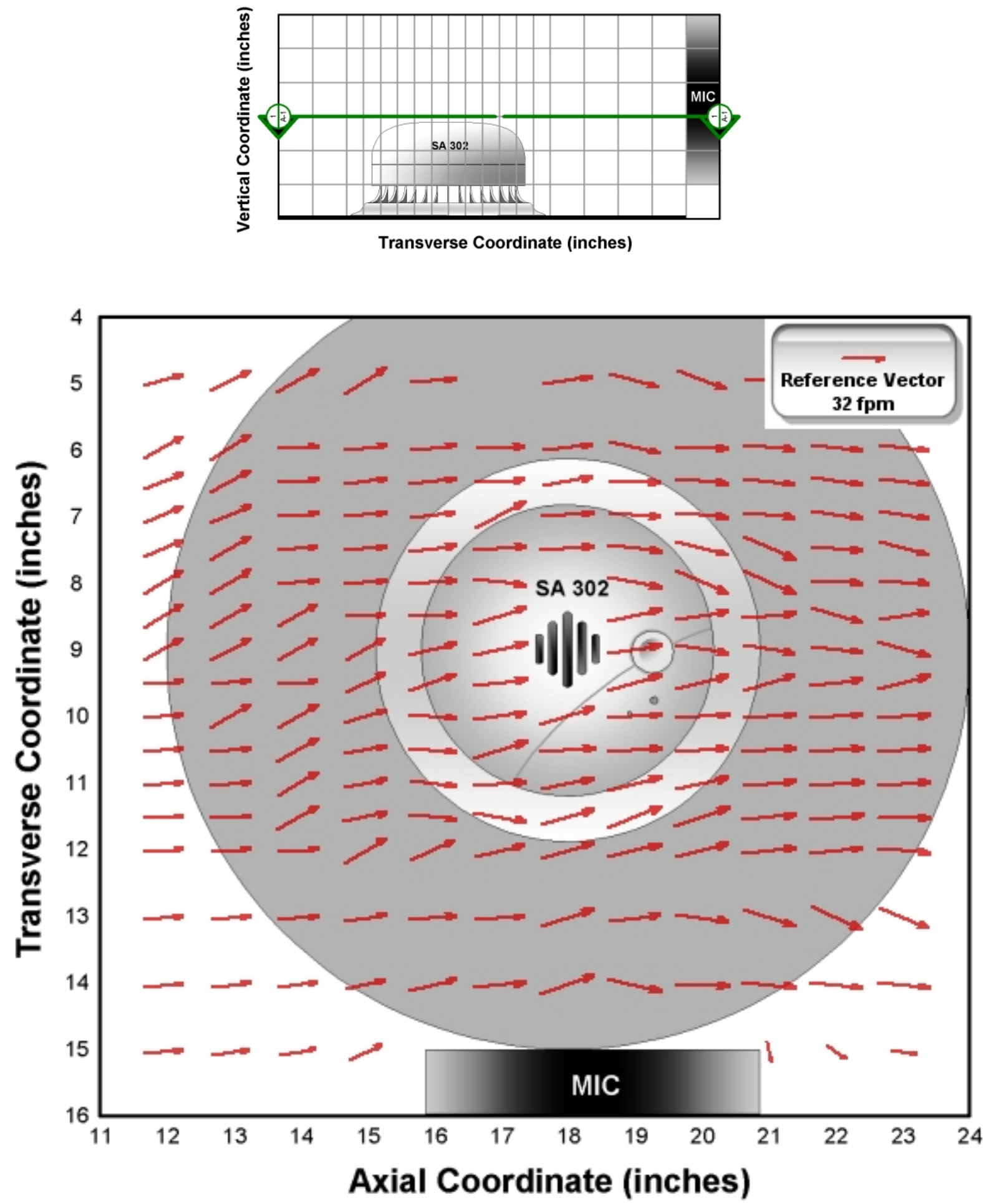

Figure 6.4.9: The $u$ - $w$ velocity vectors in the UL Smoke Box test section at $y=3.0$ in. 


\section{Legend}
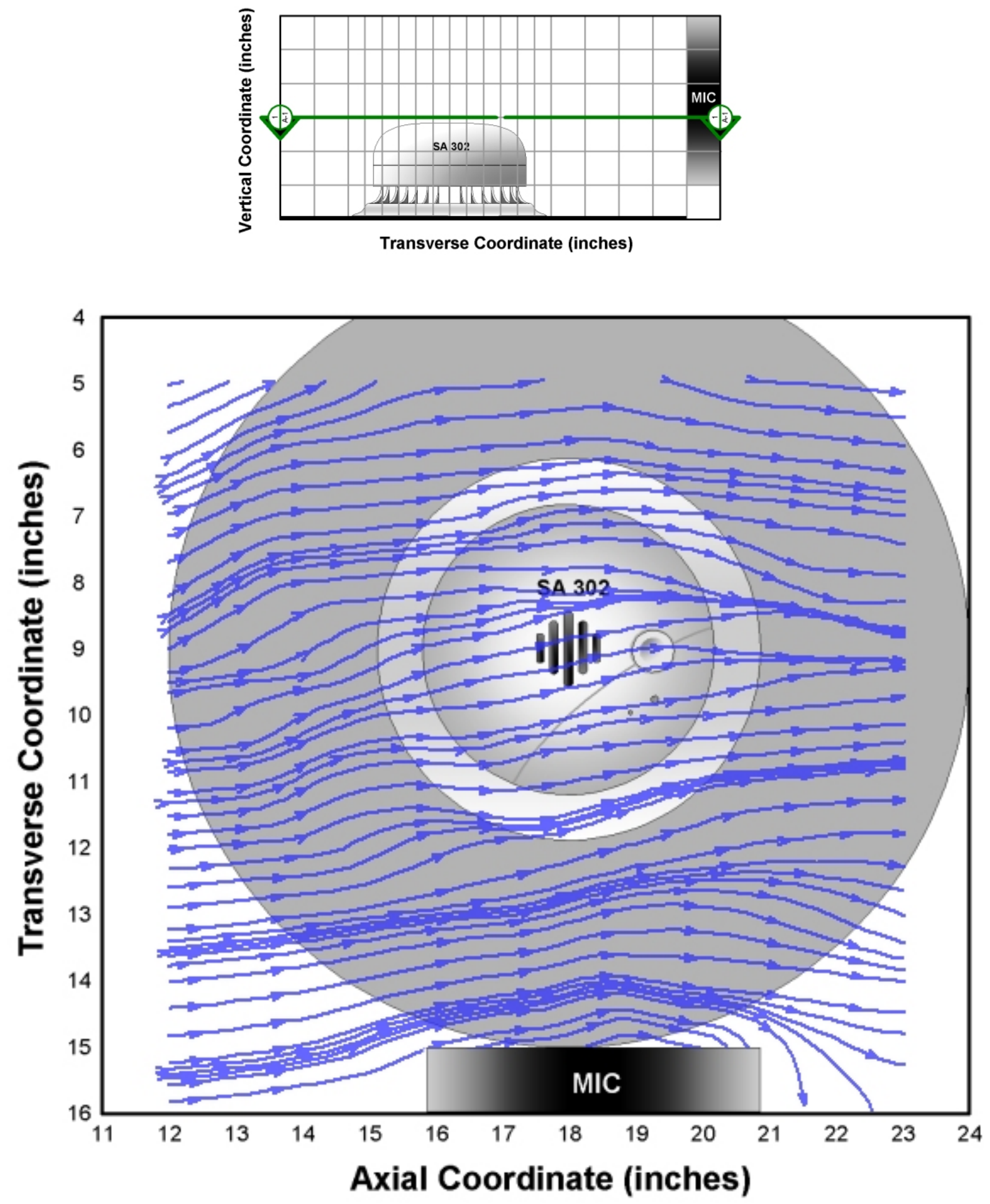

Figure 6.4.10: The $u-w$ streamlines in the UL Smoke Box test section at $y=3.0$ in. 


\section{Legend}
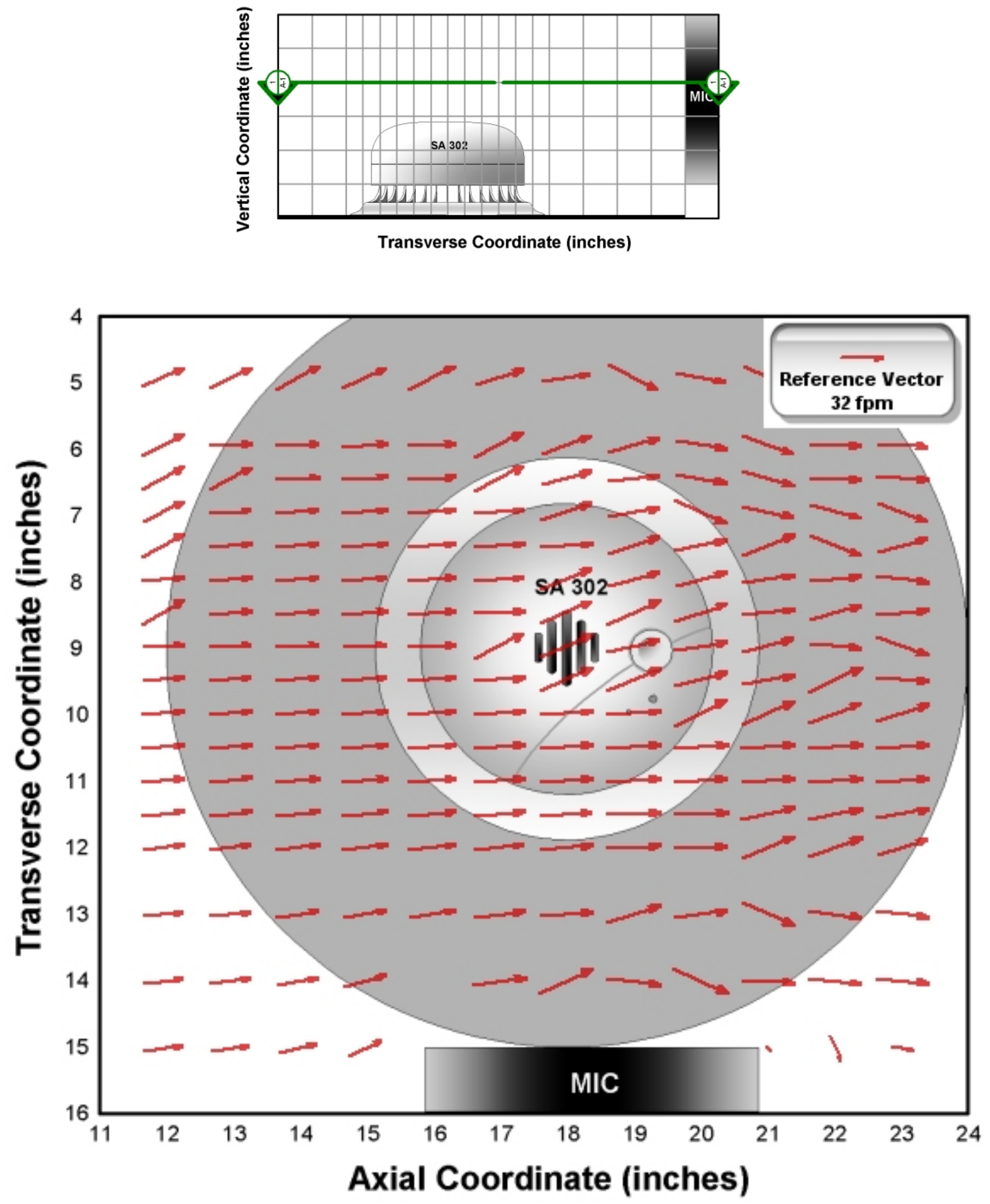

Figure 6.4.11: The $u$ - $w$ velocity vectors in the UL Smoke Box test section at $y=4.0$ in. 


\section{Legend}
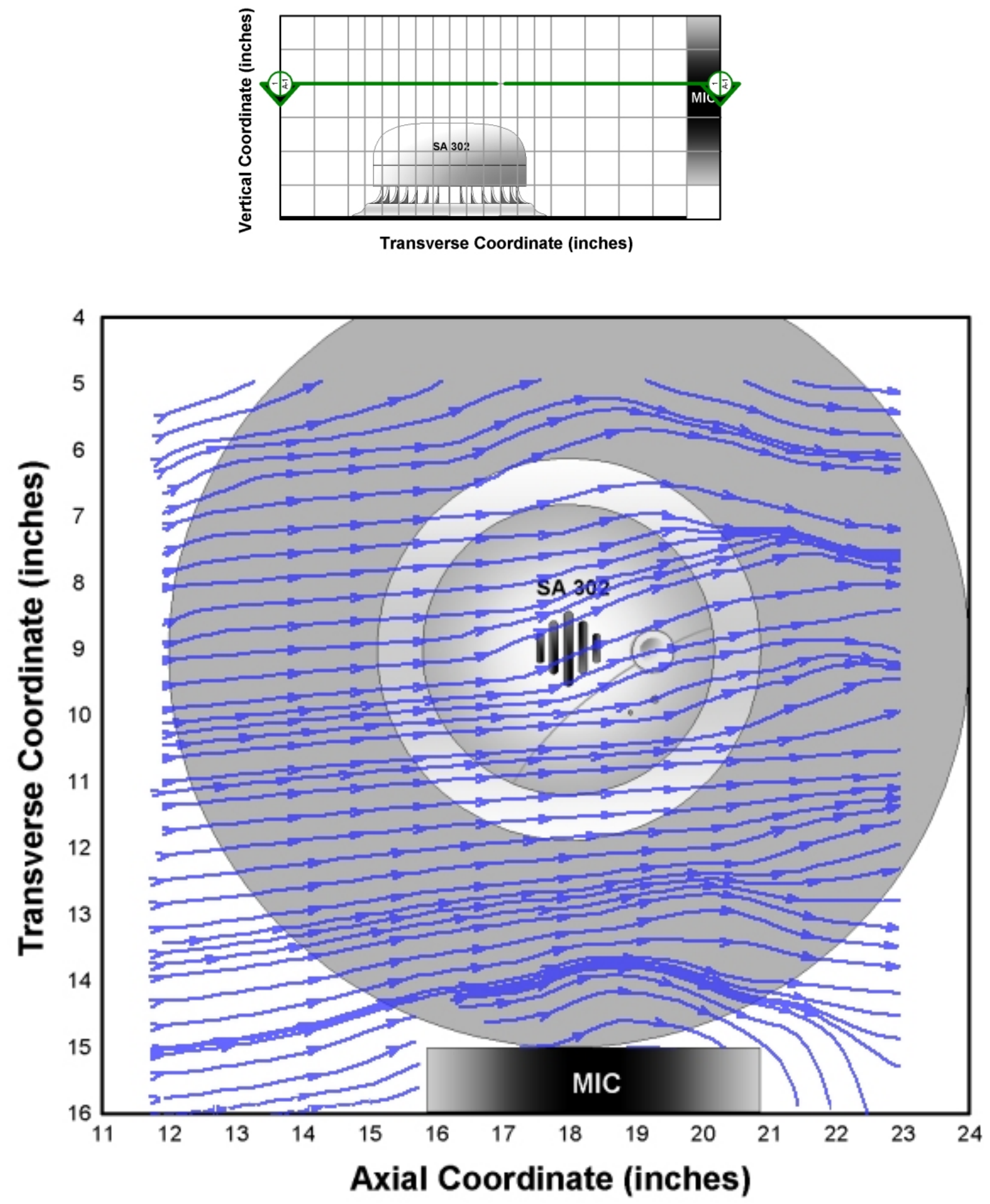

Figure 6.4.12: The $u-w$ streamlines in the UL Smoke Box test section at $y=4.0$ in. 


\section{Legend}
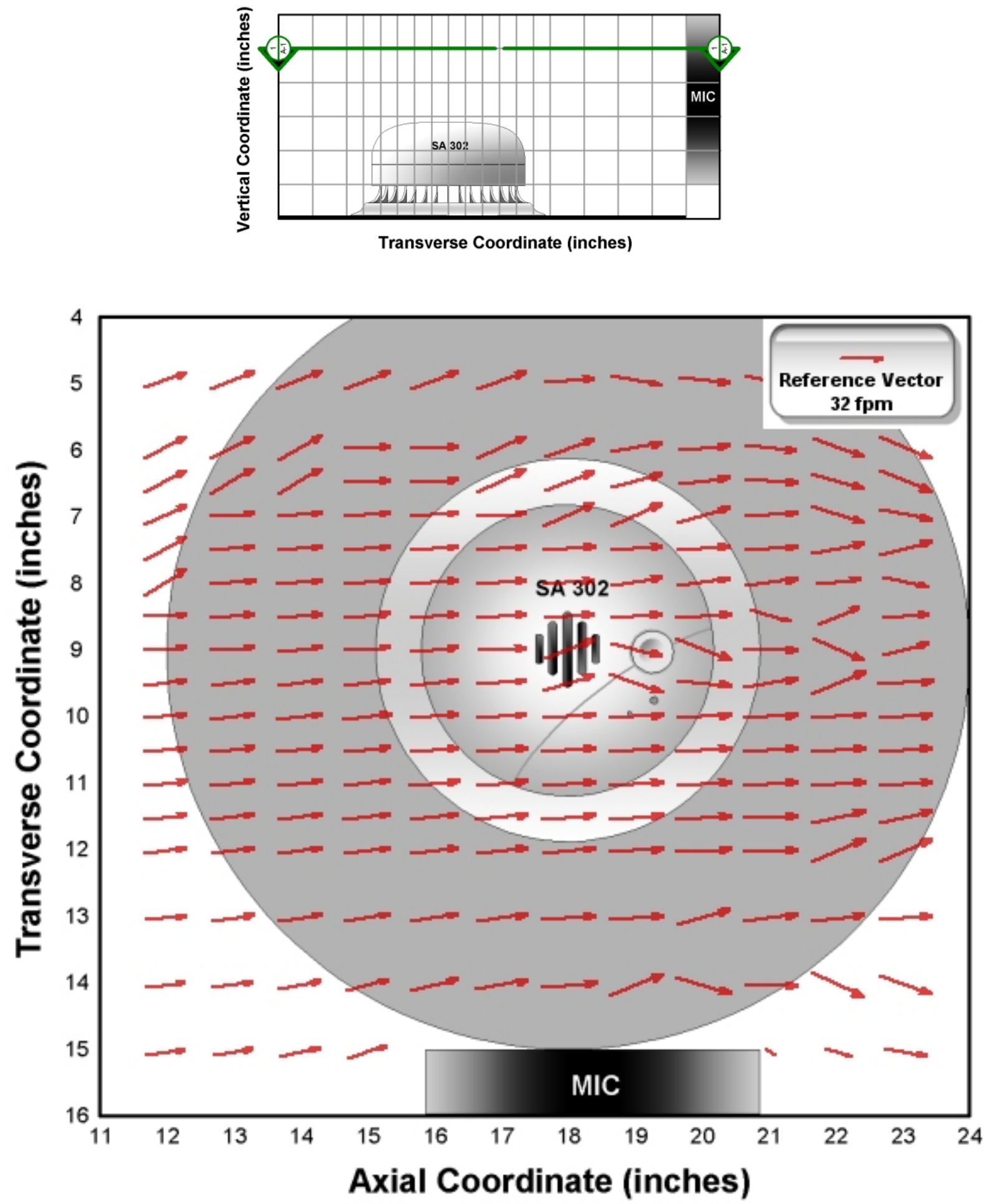

Figure 6.4.13: The $u$-w velocity vectors in the UL Smoke Box test section at $y=5.0$ in. 


\section{Legend}
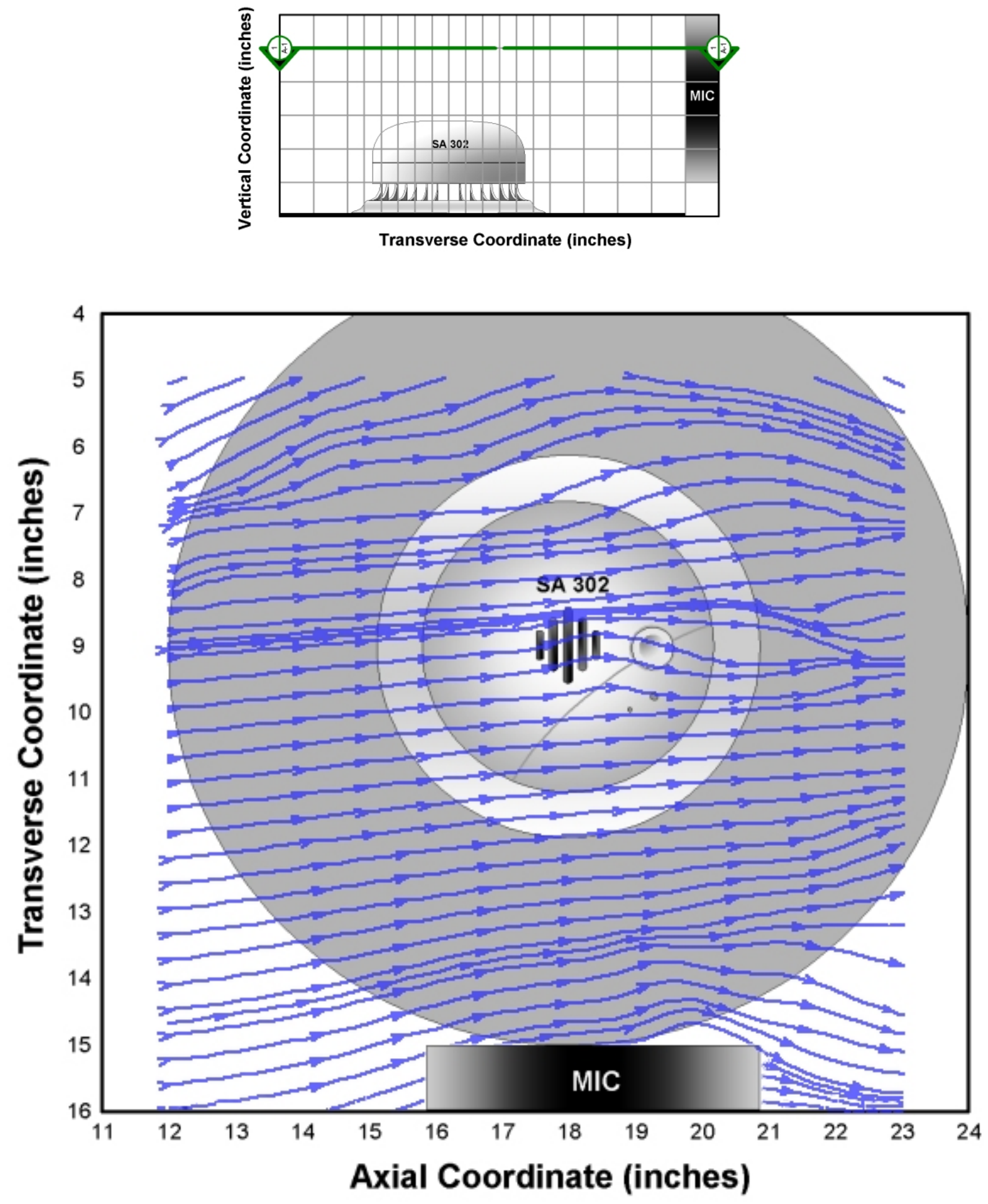

Figure 6.4.14: The $u$-w streamlines in the UL Smoke Box test section at $y=5.0$ in. 


\section{Legend}
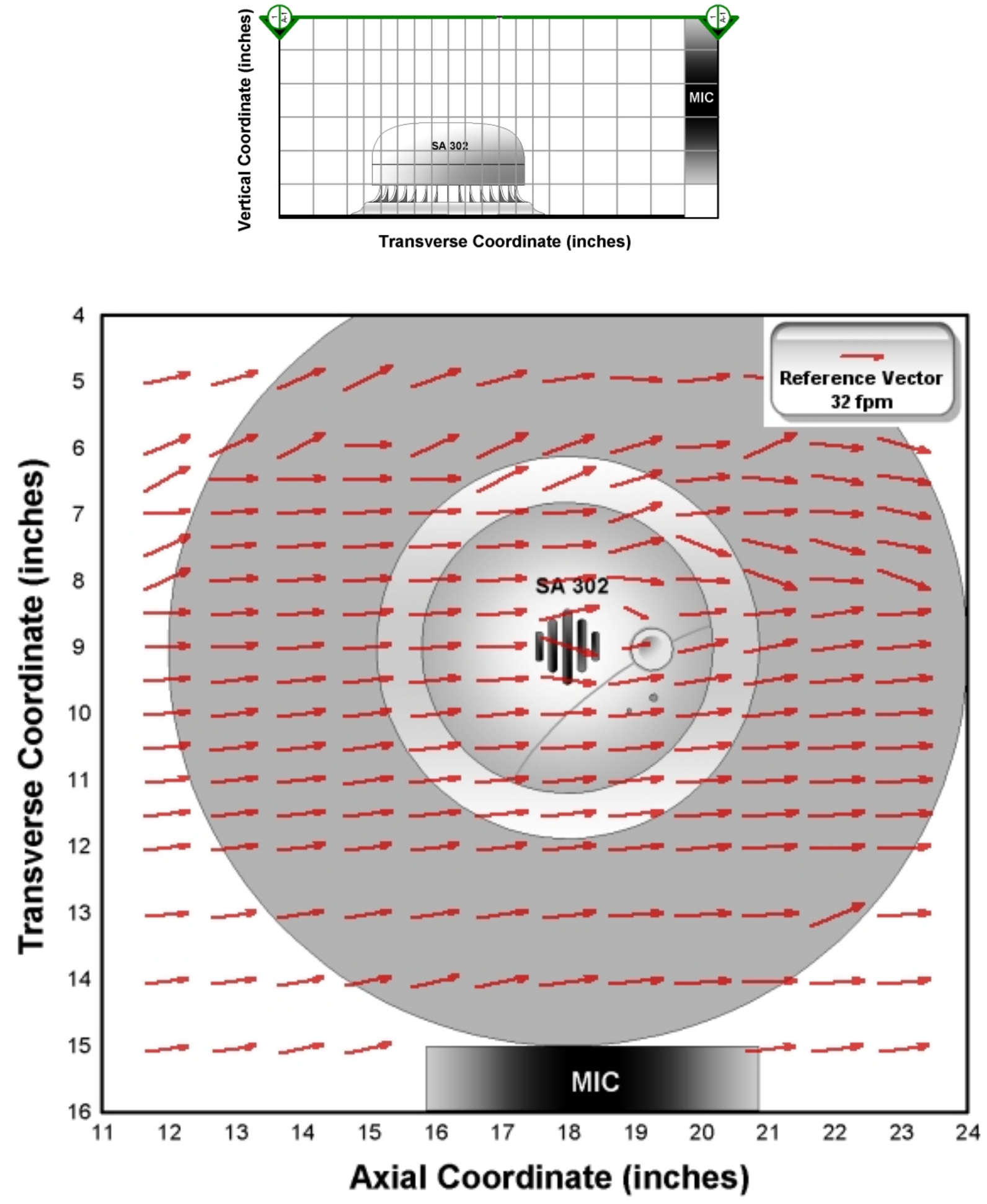

Figure 6.4.15: The $u$ - $w$ velocity vectors in the UL Smoke Box test section at $y=6.0$ in. 


\section{Legend}
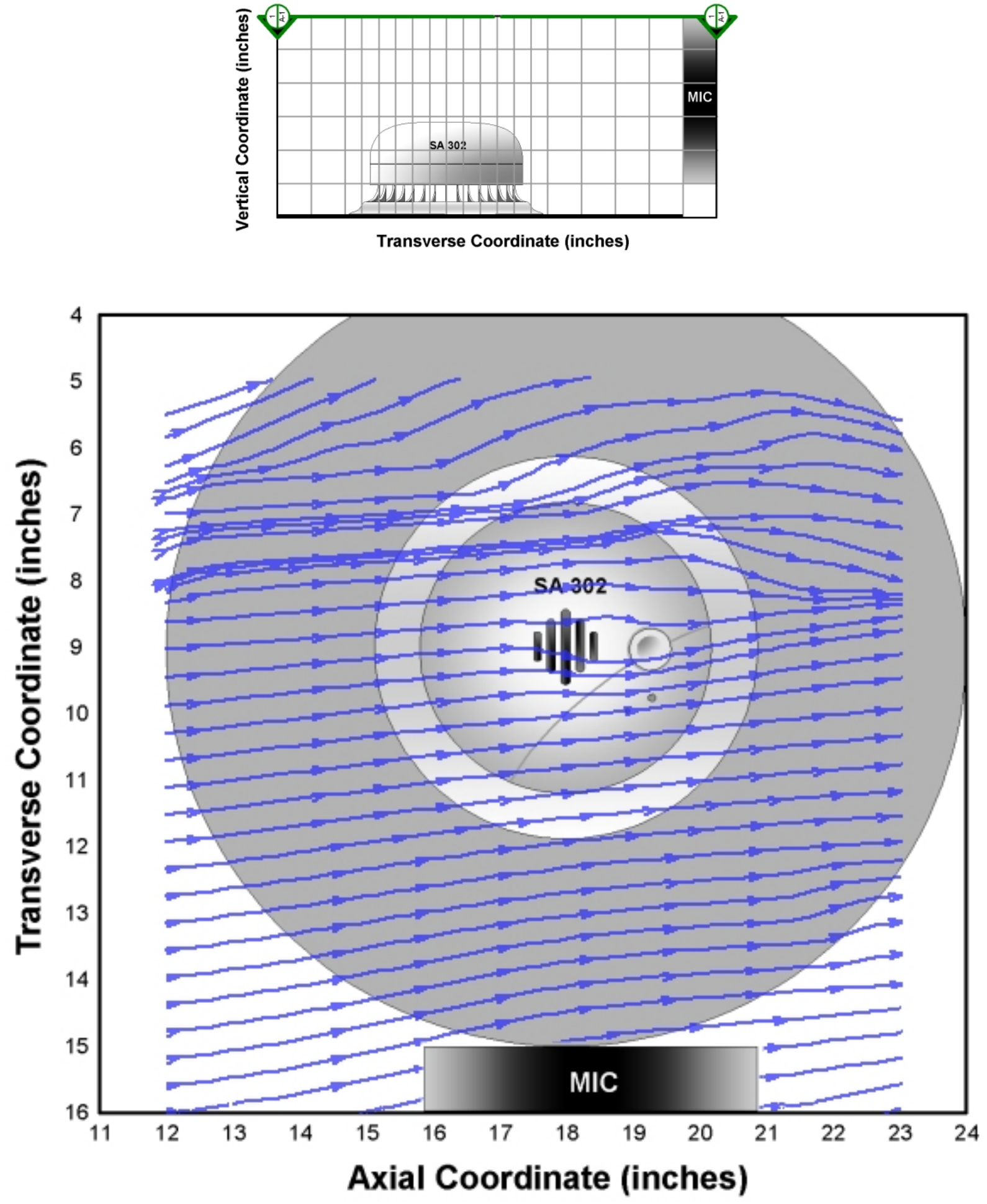

Figure 6.4.16: The $u$-w streamlines in the UL Smoke Box test section at $y=6.0$ in. 
Figures 6.4.17 through 6.4.29 show the $u-v$ velocity vectors and streamlines at various transverse, z-coordinate levels in the smoke box with an SA302 smoke detector in the test section. In each figure, the top image illustrates the transverse level of cross-section of the smoke box test section where the velocity vectors, and the flow streamlines are shown in the two following images on the same page. The green arrows on the legend plot indicate the viewing angle. The transverse coordinate, $z$, is zero at the wall of the smoke box test section opposite the MIC and increases positively to the MIC wall at $z=18 \mathrm{in}$. The range of the transverse, $z$-coordinate in the plots is from $z=3$ in to $z=15 \mathrm{in}$. Note at the $z=9 \mathrm{in}$, the $u-v$ plane is located at the mid-transverse section of the detector. For values of $z<9$ in, the velocity vector plots and the streamlines plots are viewed from the MIC side of the test section prospective, or the reverse angle view for the plots for $z<9 \mathrm{in}$. This helps to show more detail near the detector perimeter.

All of the $x-y$ planes near the detector show the effect of the detector on the smoke box flow, mainly that the flow must move up and over the detector, and must accelerate to due so. The flow separates and forms a wake behind the detector. It is important to note that at transverse, $z$ locations ranging from 8 to 10 in (Figures 6.2.22 through 6.2.24), some flow streamlines can be observed moving toward the opening of the detector sensing area. At $z=$ 15 (Figure 6.4.29), the measurement plane is at the vertical face of the MIC which is why data are absent in the center portion of the plot. 


\section{Legend}

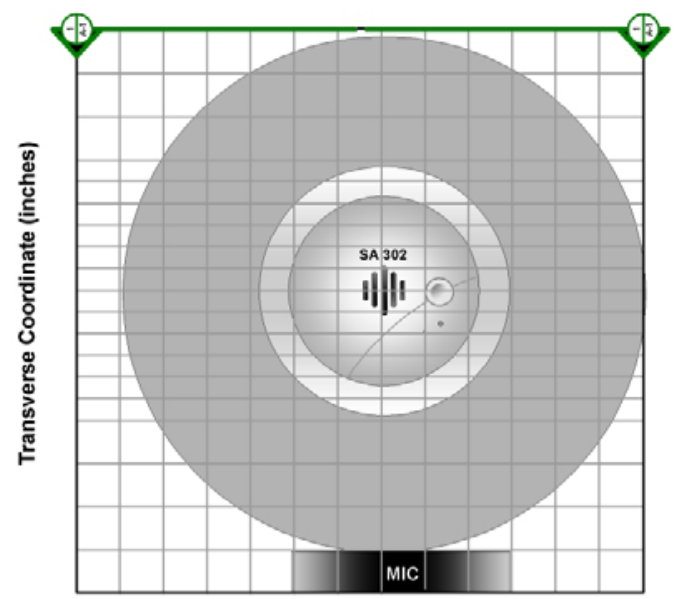

Axial Coordinate (inches)
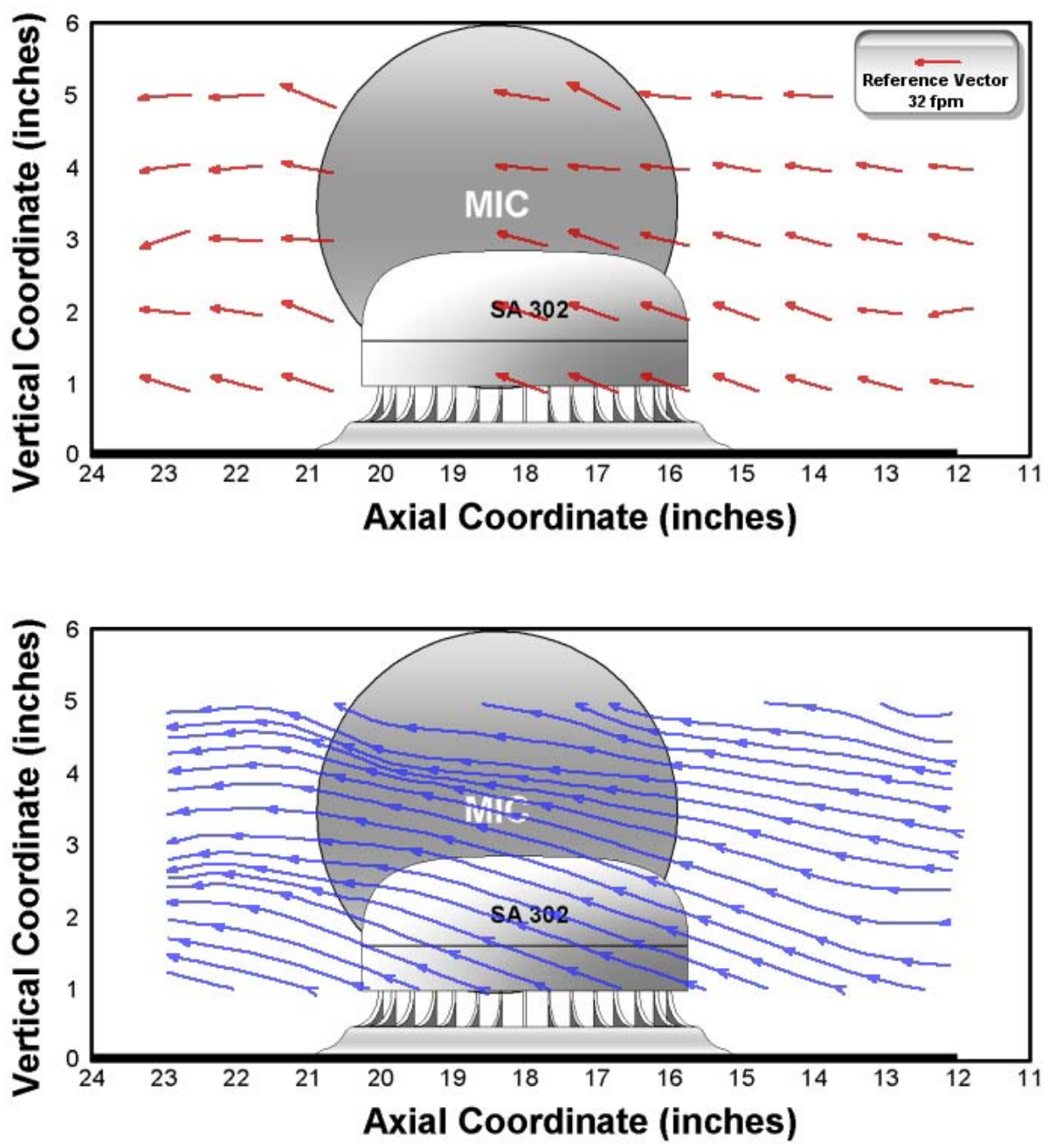

Figure 6.4.17: The $u-v$ velocity vectors and streamlines in the UL Smoke Box test section at $z$ $=3.0$ in. 


\section{Legend}

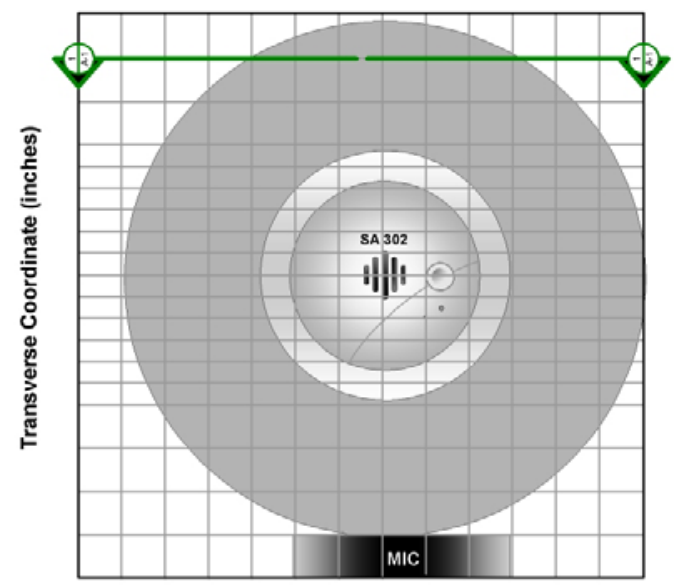

Axial Coordinate (inches)
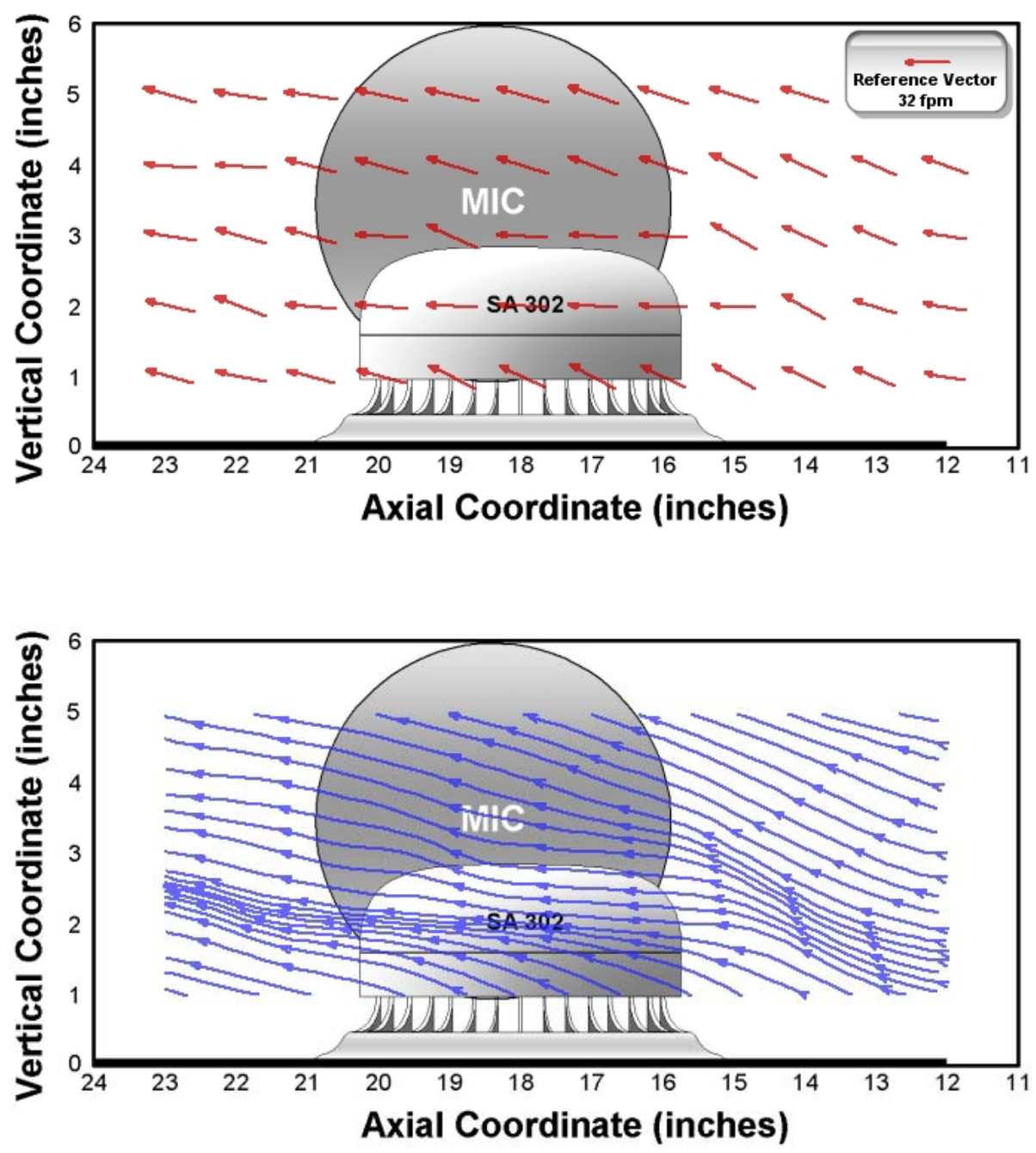

Figure 6.4.18: The $u-v$ velocity vectors and streamlines in the UL Smoke Box test section at $z$ $=4.0 \mathrm{in}$. 


\section{Legend}

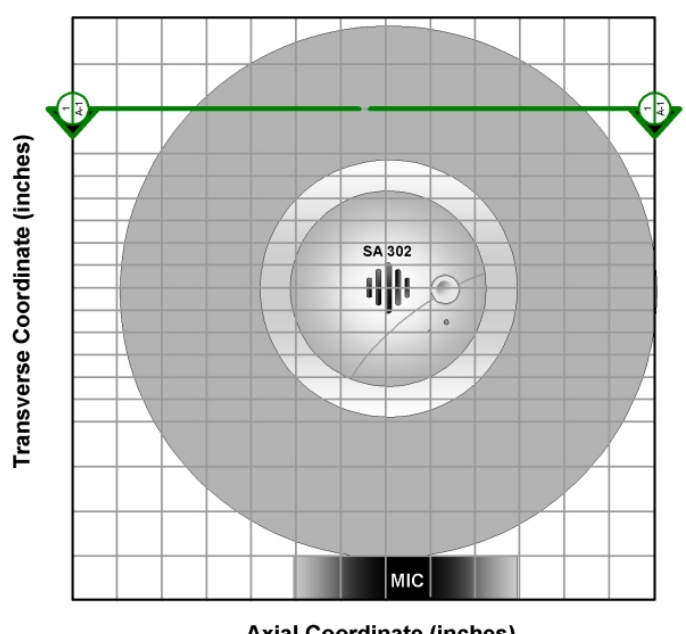

Axial Coordinate (inches)
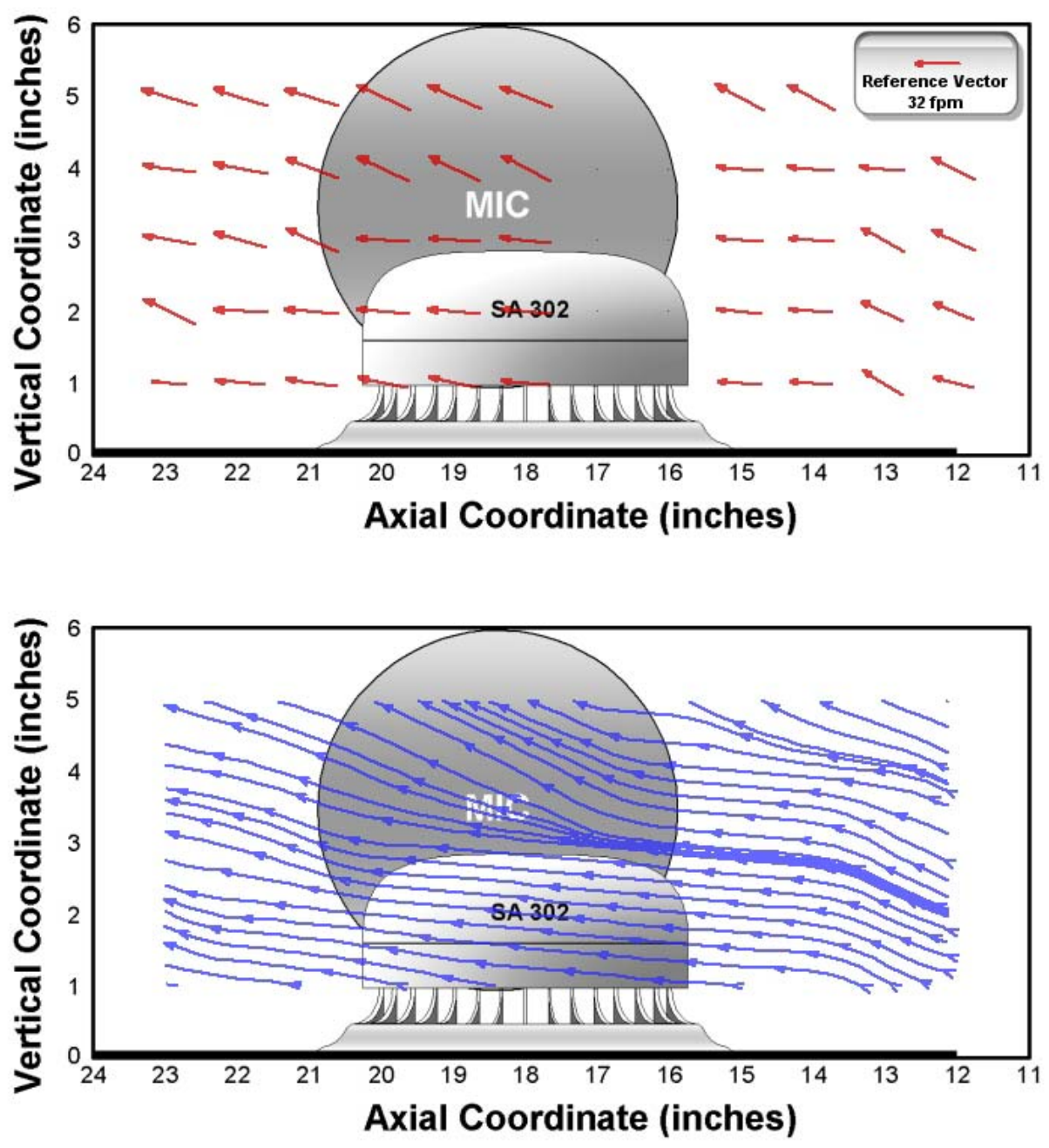

Figure 6.4.19: The $u-v$ velocity vectors and streamlines in the UL Smoke Box test section at $z$ $=5.0$ in. 


\section{Legend}

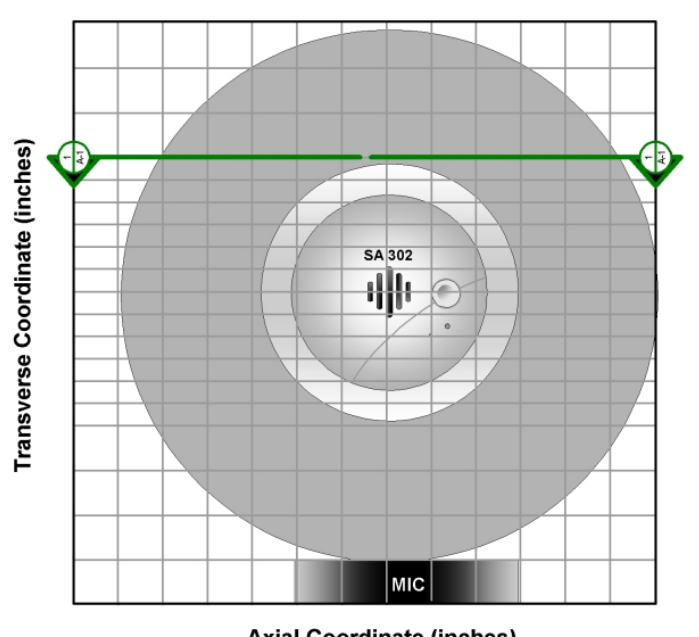

Axial Coordinate (inches)
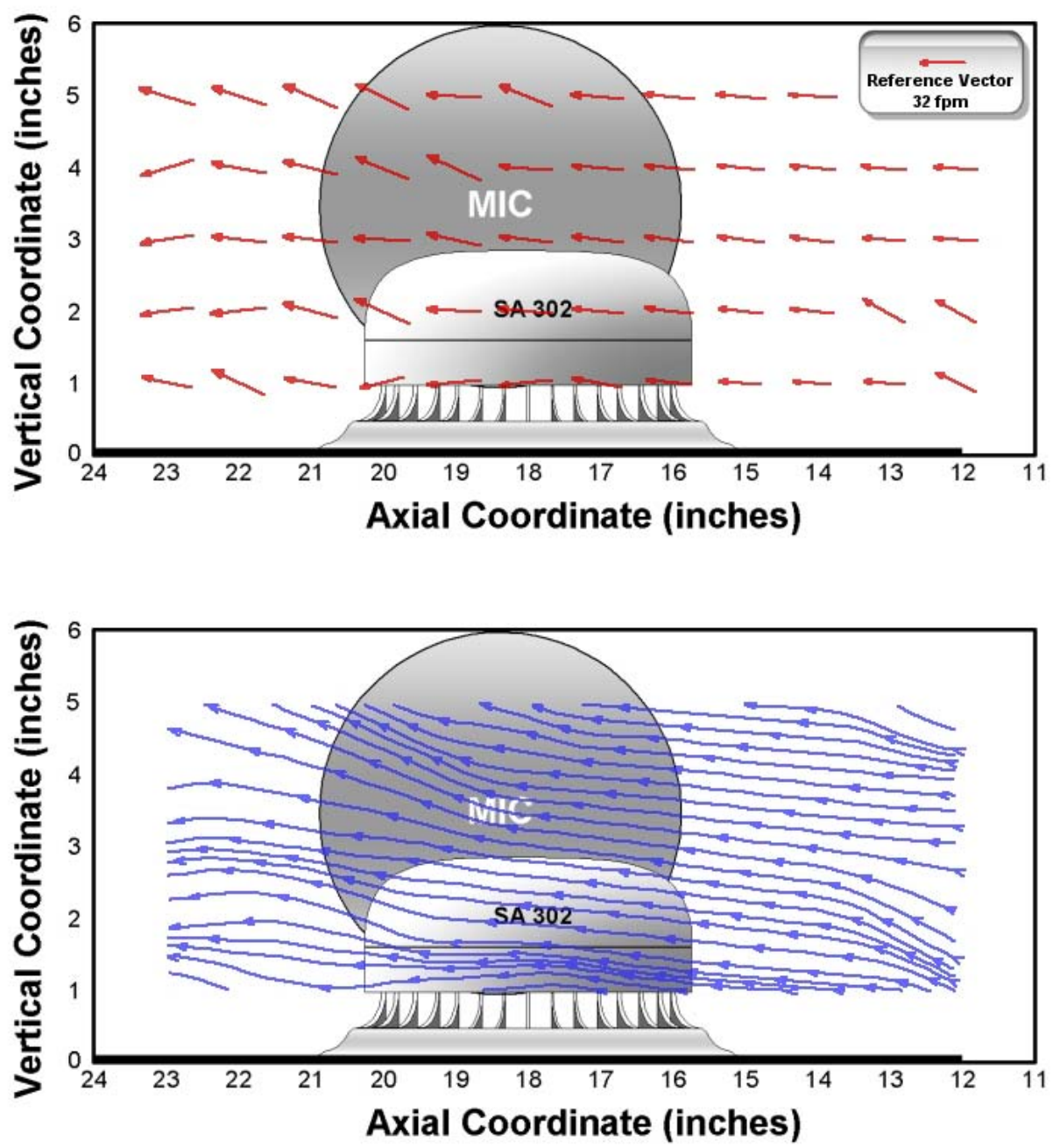

Figure 6.4.20: The $u-v$ velocity vectors and streamlines in the UL Smoke Box test section at $z$ $=6.0 \mathrm{in}$. 


\section{Legend}
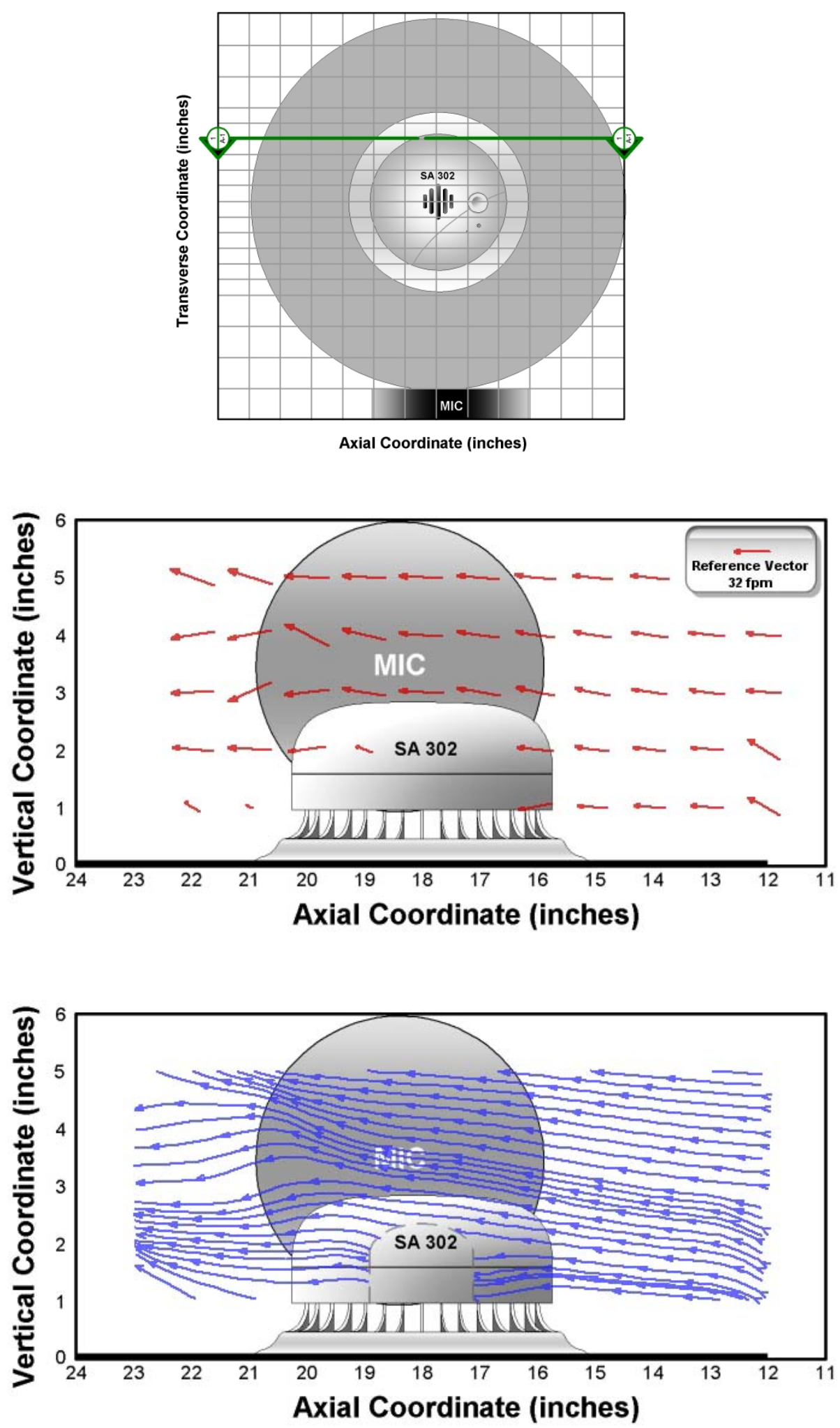

Figure 6.4.21: The $u-v$ velocity vectors and streamlines in the UL Smoke Box test section at $z$ $=7.0 \mathrm{in}$. 


\section{Legend}
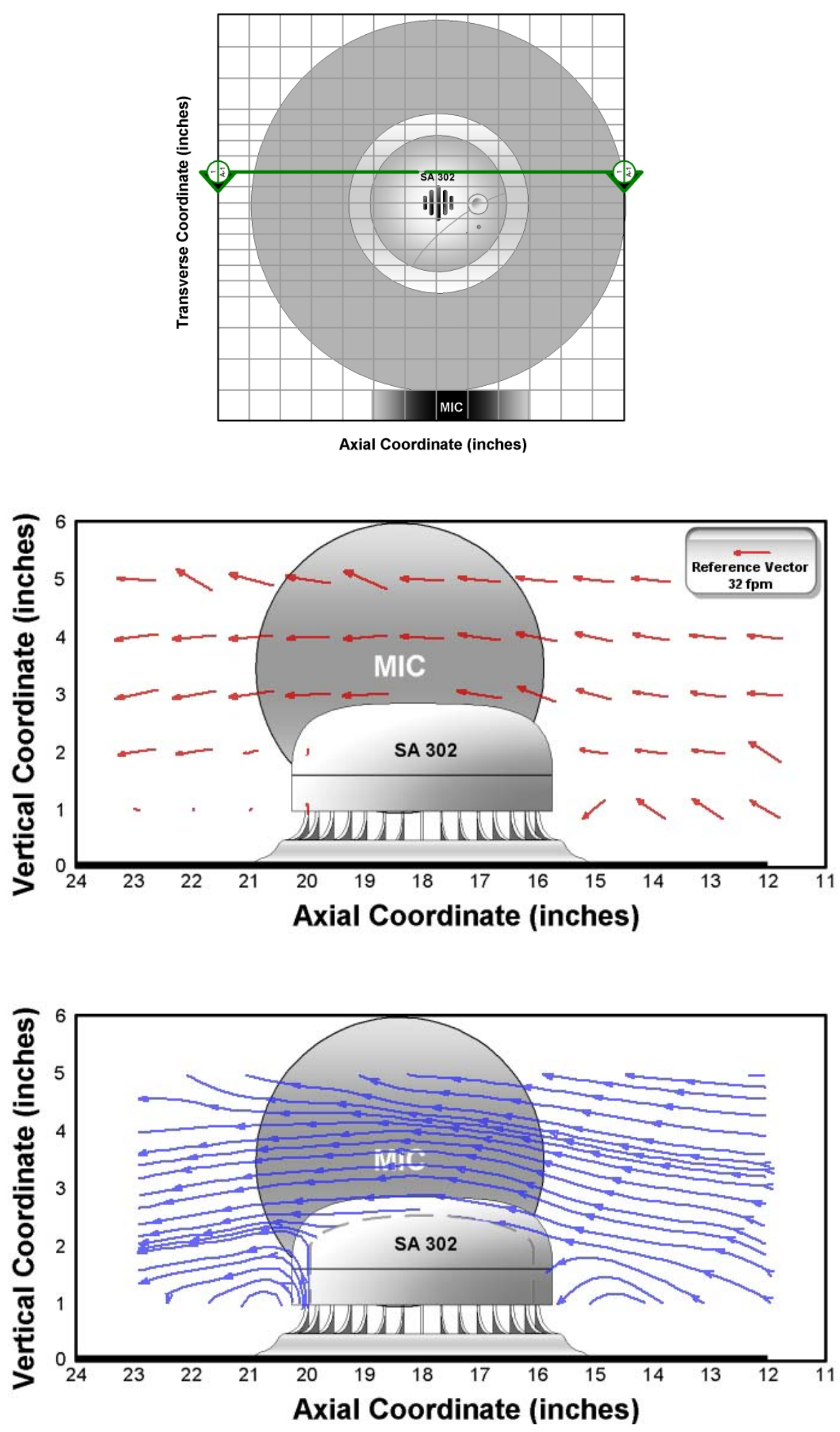

Figure 6.4.22: The $u-v$ velocity vectors and streamlines in the UL Smoke Box test section at $z$ $=8.0 \mathrm{in}$. 


\section{Legend}

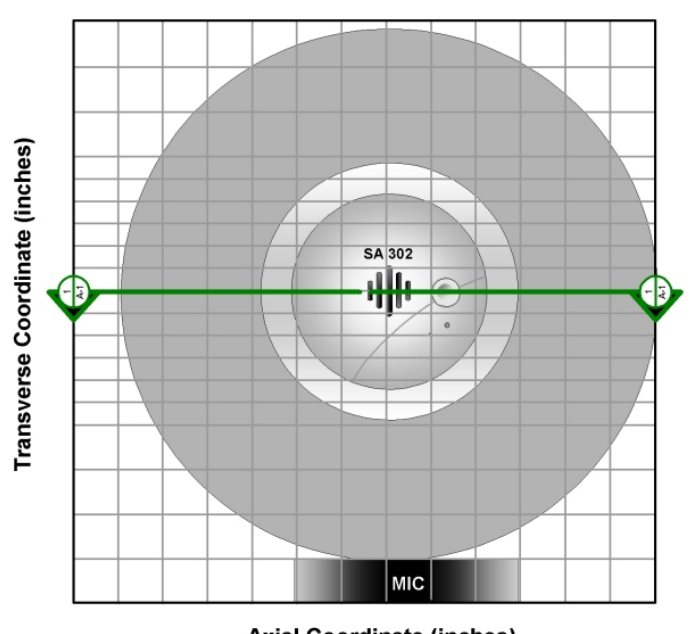

Axial Coordinate (inches)
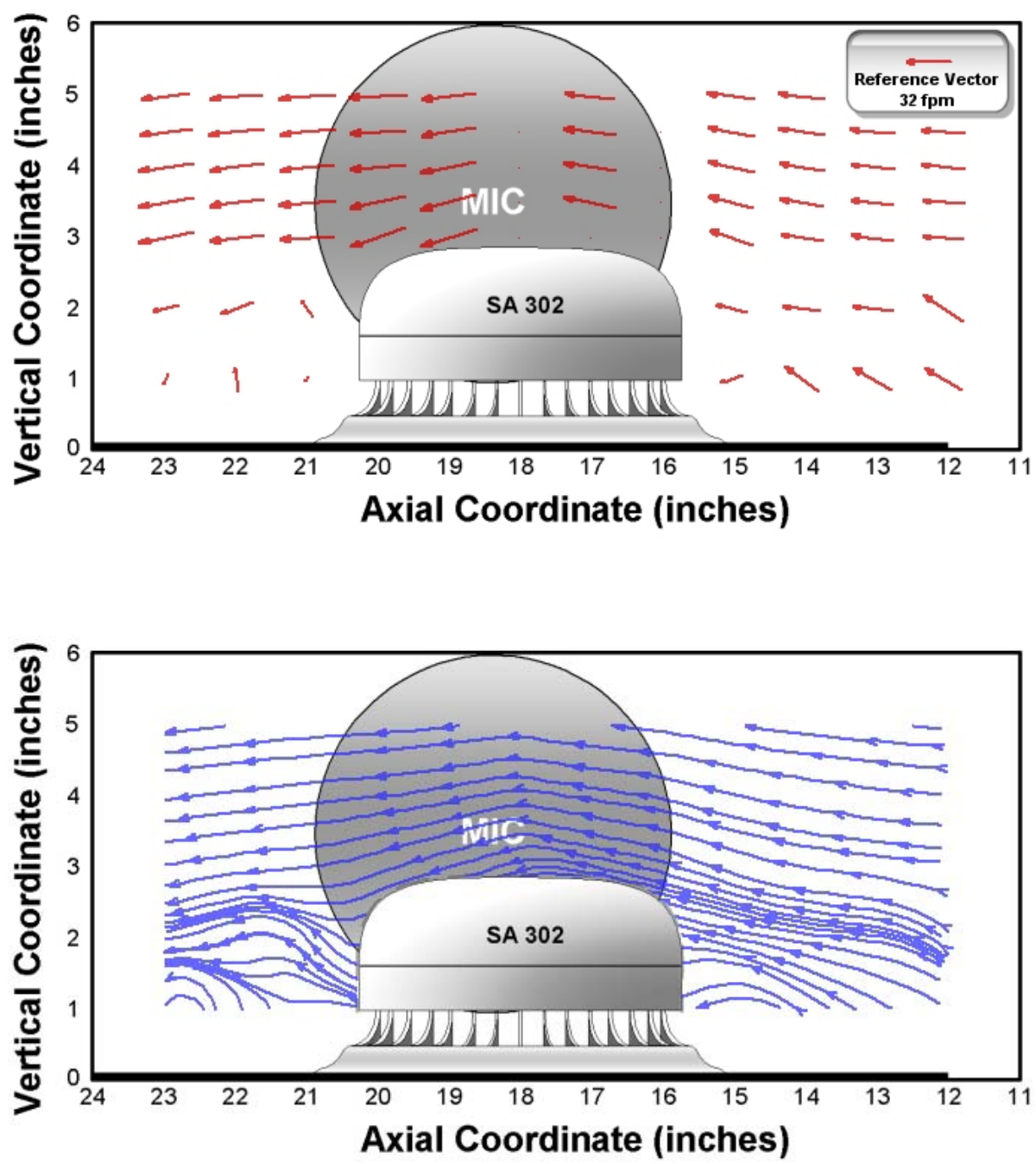

Figure 6.4.23: The $u-v$ velocity vectors and streamlines in the UL Smoke Box test section at $z$ $=9.0$ in. 


\section{Legend}

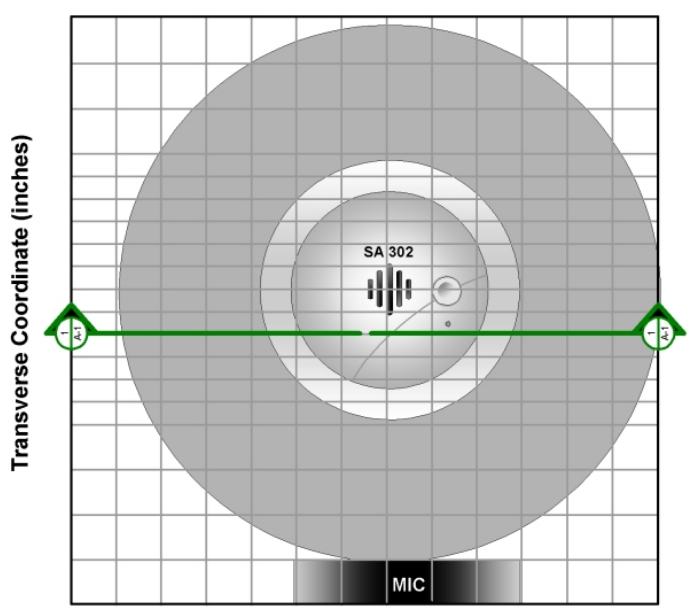

Axial Coordinate (inches)
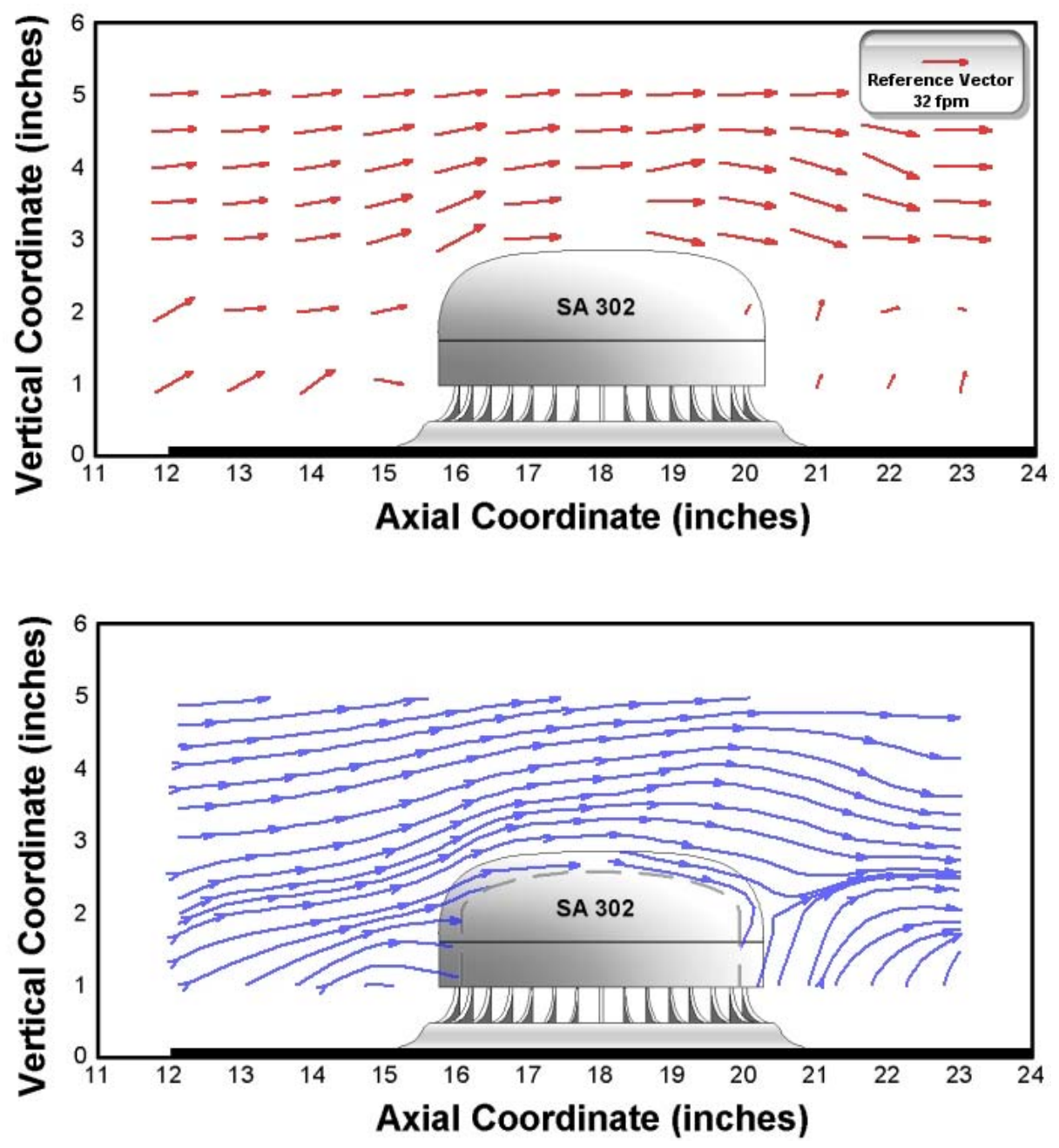

Figure 6.4.24: The $u-v$ velocity vectors and streamlines in the UL Smoke Box test section at $z$ $=10.0$ in (reverse angle view). 


\section{Legend}
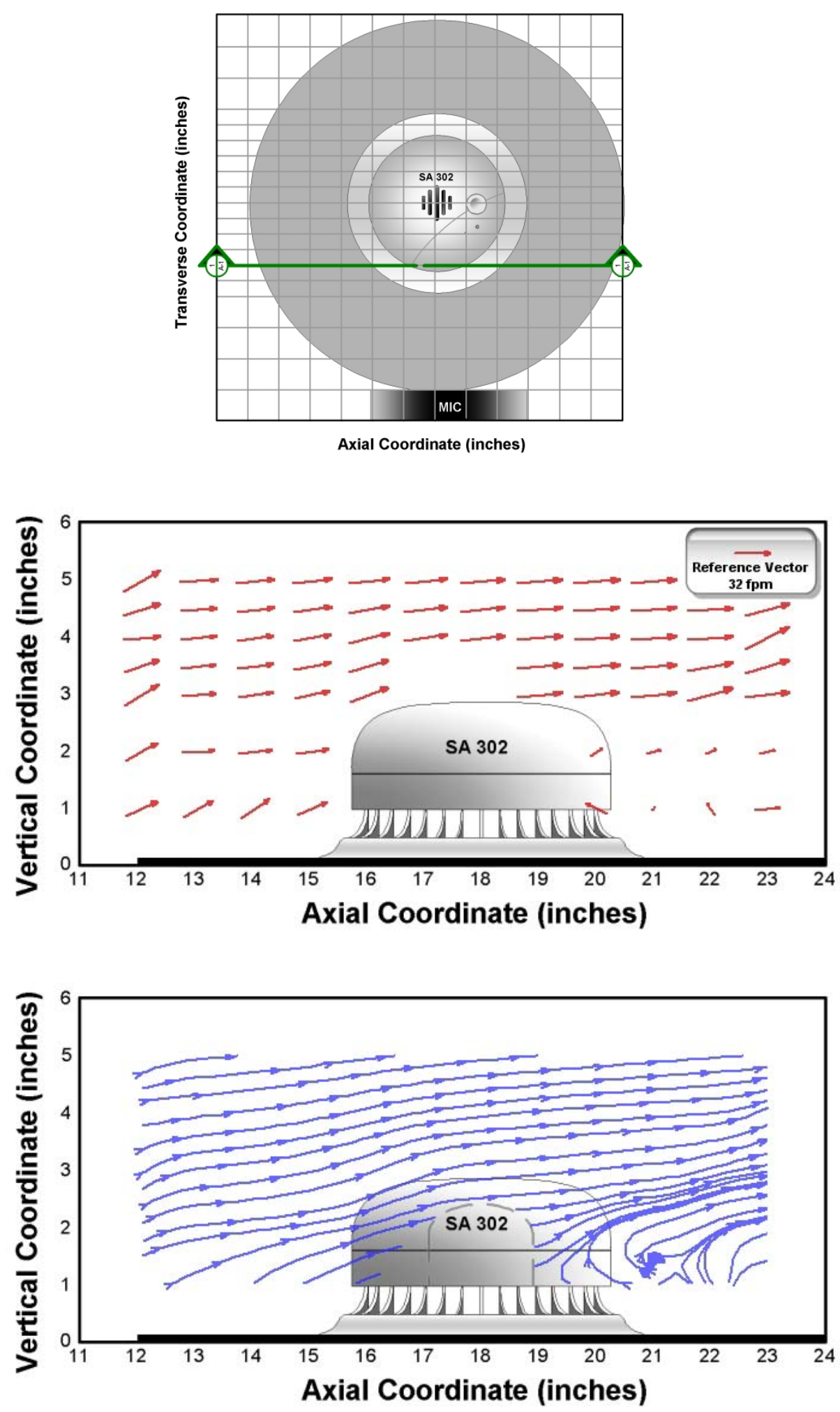

Figure 6.4.25: The $u-v$ velocity vectors and streamlines in the UL Smoke Box test section at $z$ $=11.0$ in (reverse angle view). 


\section{Legend}

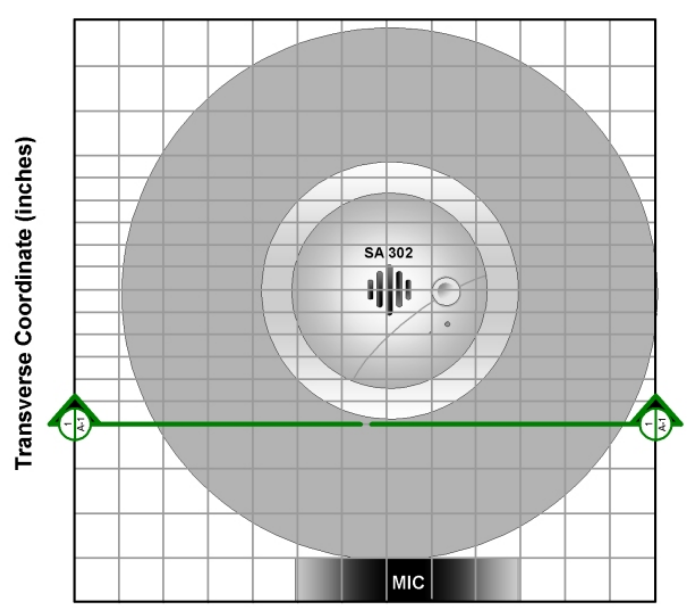

Axial Coordinate (inches)
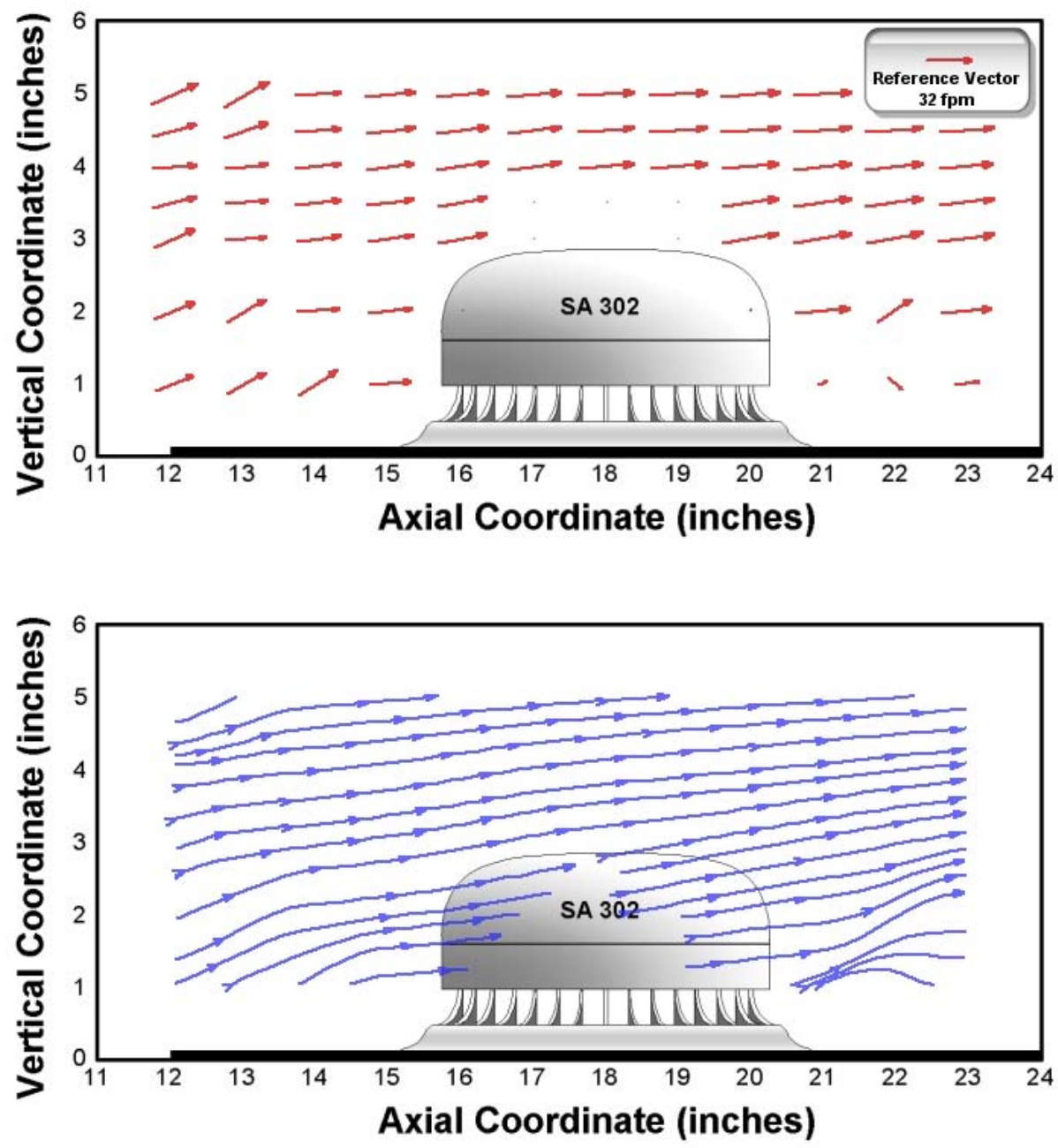

Figure 6.4.26: The $u-v$ velocity vectors and streamlines in the UL Smoke Box test section at $z=$ 12.0 in (reverse angle view). 


\section{Legend}

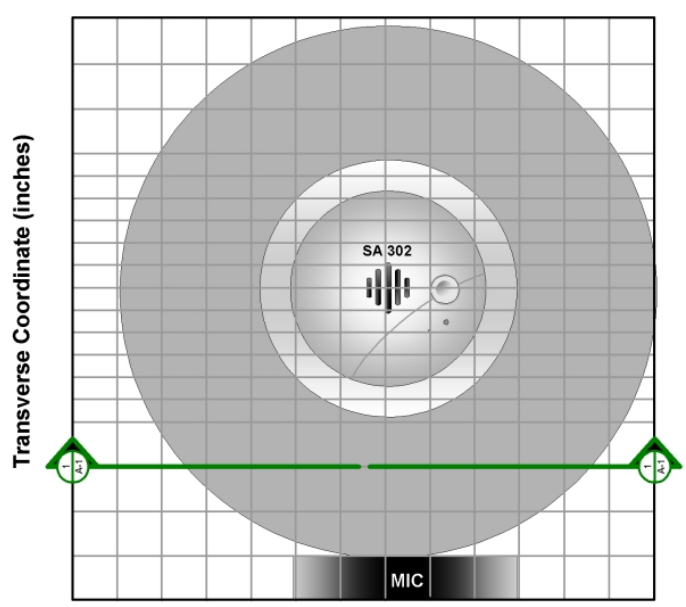

Axial Coordinate (inches)
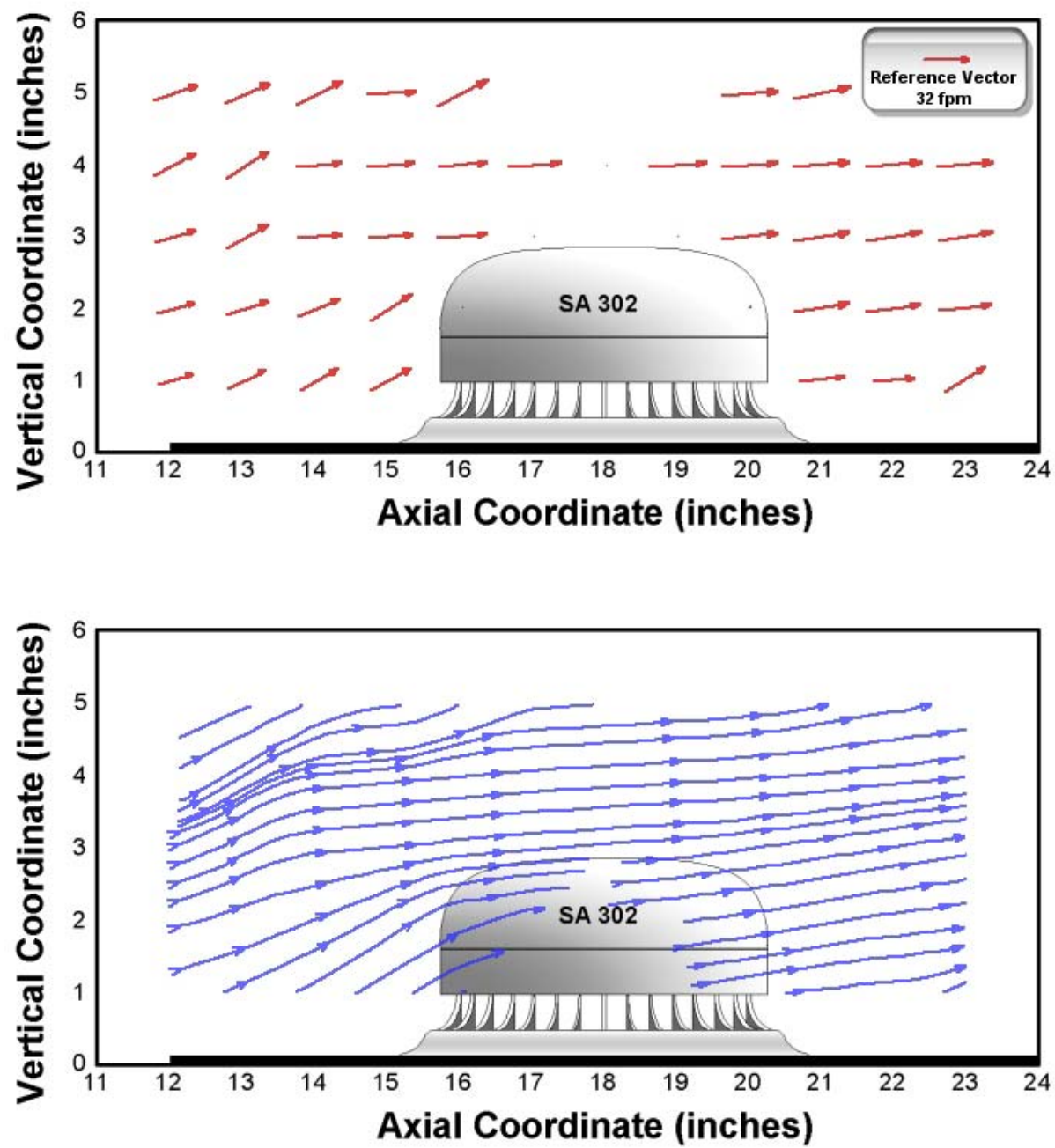

Figure 6.4.27: The $u-v$ velocity vectors and streamlines in the UL Smoke Box test section at $z=$ 13.0 in (reverse angle view). 


\section{Legend}

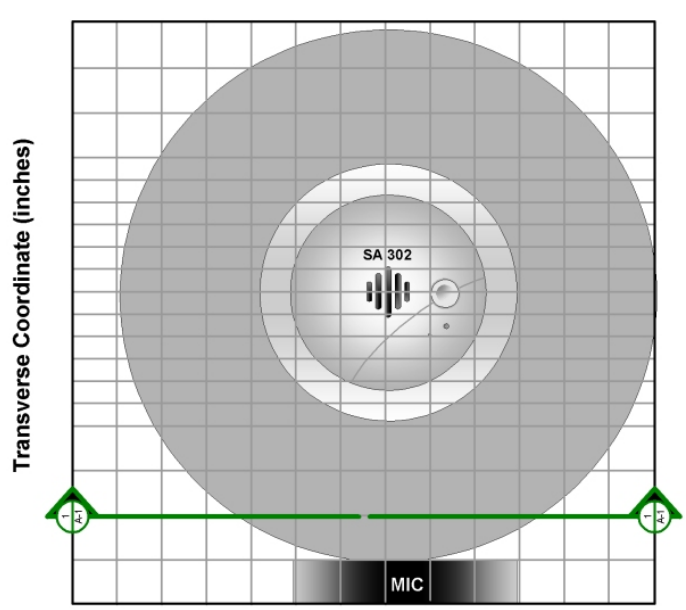

Axial Coordinate (inches)
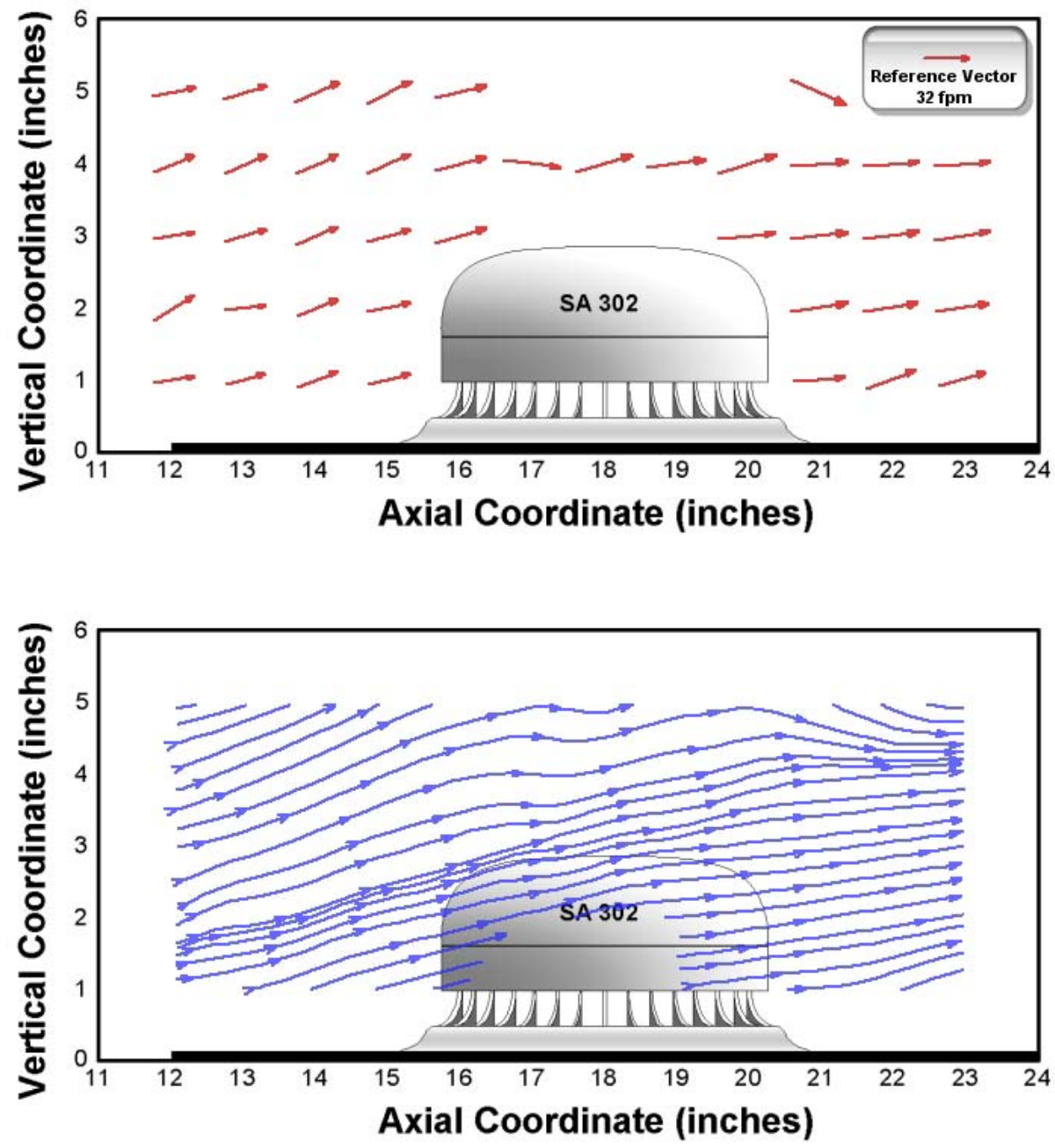

Figure 6.4.28: The $u-v$ velocity vectors and streamlines in the UL Smoke Box test section at $z=$ 14.0 in (reverse angle view). 


\section{Legend}

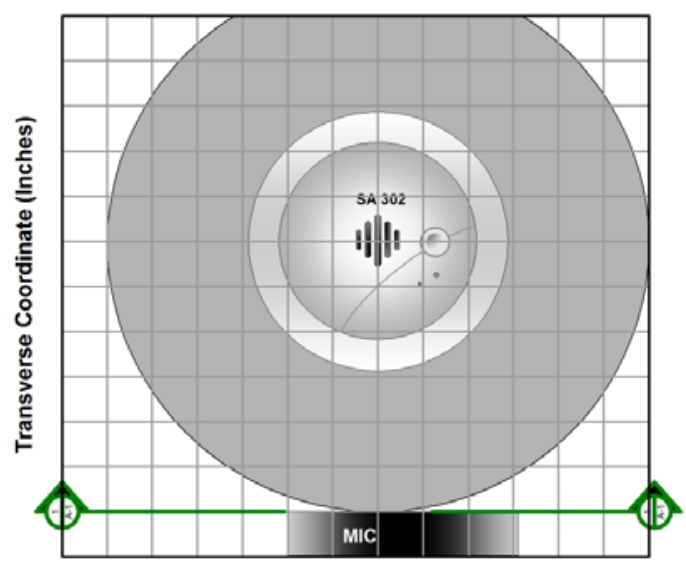

Axial Coordinate (Inches)
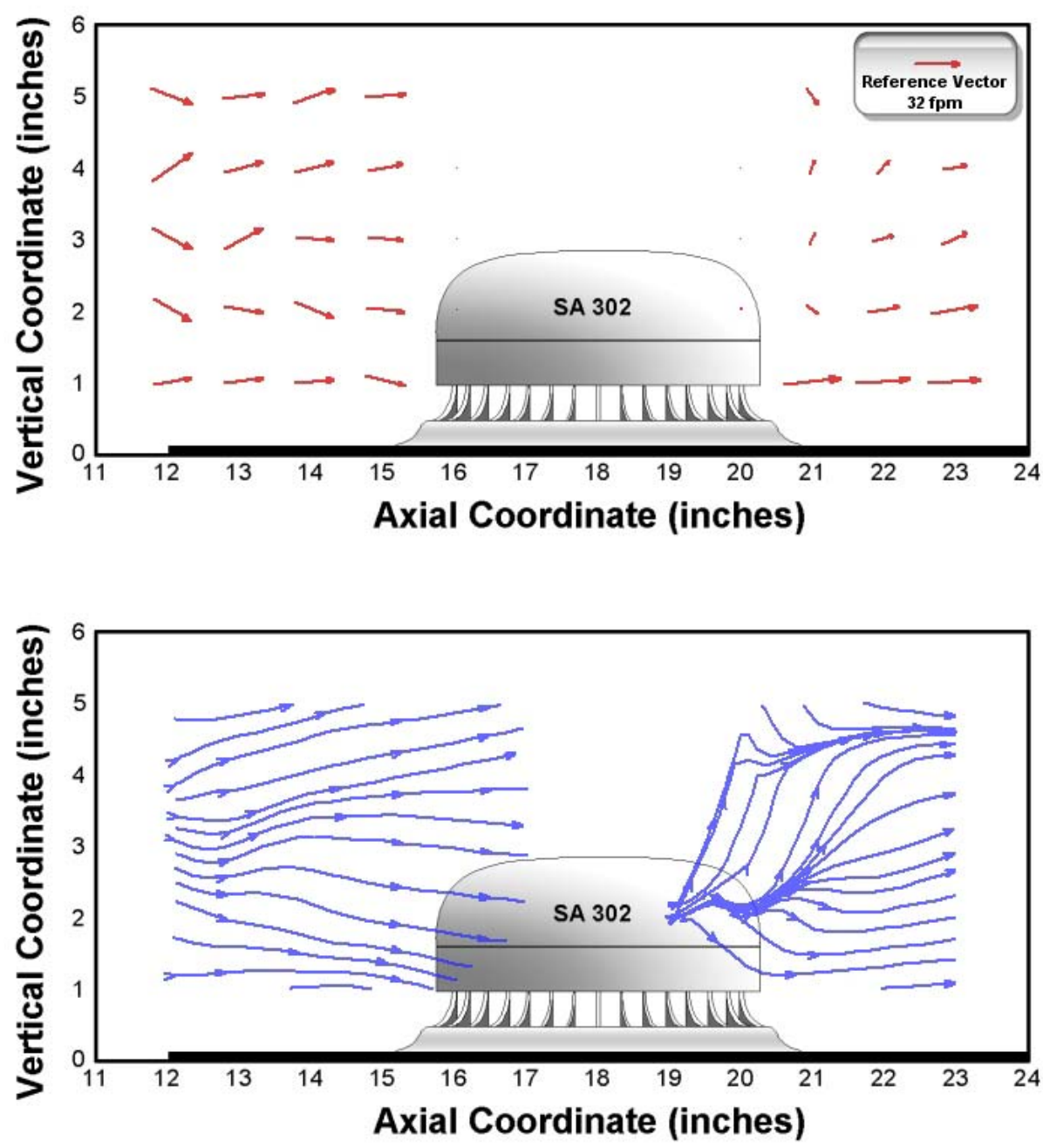

Figure 6.4.29: The $u-v$ velocity vectors and streamlines in the UL Smoke Box test section at $z=$ 15.0 in (reverse angle view). 
Figures 6.4.30 through 6.4.41 show the $v$-w velocity vectors and streamlines at various axial, $x$-coordinate levels in the smoke box with an SA302 smoke detector in the test section. In each of the figures a legend plot is shown to orient the reader for proper perspective for the vector and streamline plots that follow. The arrows on the cross-section indication on the legend plot indicate the direction of the view.

Figures 6.4.30 through 6.4.36 clearly show the effect the MIC has on the flow as it approaches the mid-axial plane of the detector. The flow is forced away from the MIC and towards the detector. As the flow approaches the detector at $x=12,13,14$, and 15 in (Figures 6.4.30 through 6.4.33, the flow moves up and over and around the detector body as is illustrated by the streamlines plots. Due to limitations on the LDA beam configuration, no vertical velocity data were acquired below $y=1 \mathrm{in}$. Also, for transverse $z$ locations greater than 16 in, no vertical velocity data were acquired at axial locations between and including $x$ $=16$ in and $x=20$ in since the detector eclipsed the laser beams needed to measure the vertical velocity in that region. For values of $x>18 \mathrm{in}$, the velocity vector plots and the streamlines plots are viewed from the downstream side of the test section prospective, or the reverse angle view for the plots for $x<18 \mathrm{in}$. This helps to show more detail near the detector perimeter.

Figures 6.4.39 through 6.4.41 show the $v-w$ velocity vectors and streamlines in the wake of the detector. As can be observed from these figures, the wake region is highly chaotic and is characterized by large scale vortices that were also captured in the flow visualization pictures in Figures 6.1.1 through 6.1.3 shown from a similar upstream prospective. 


\section{Legend}
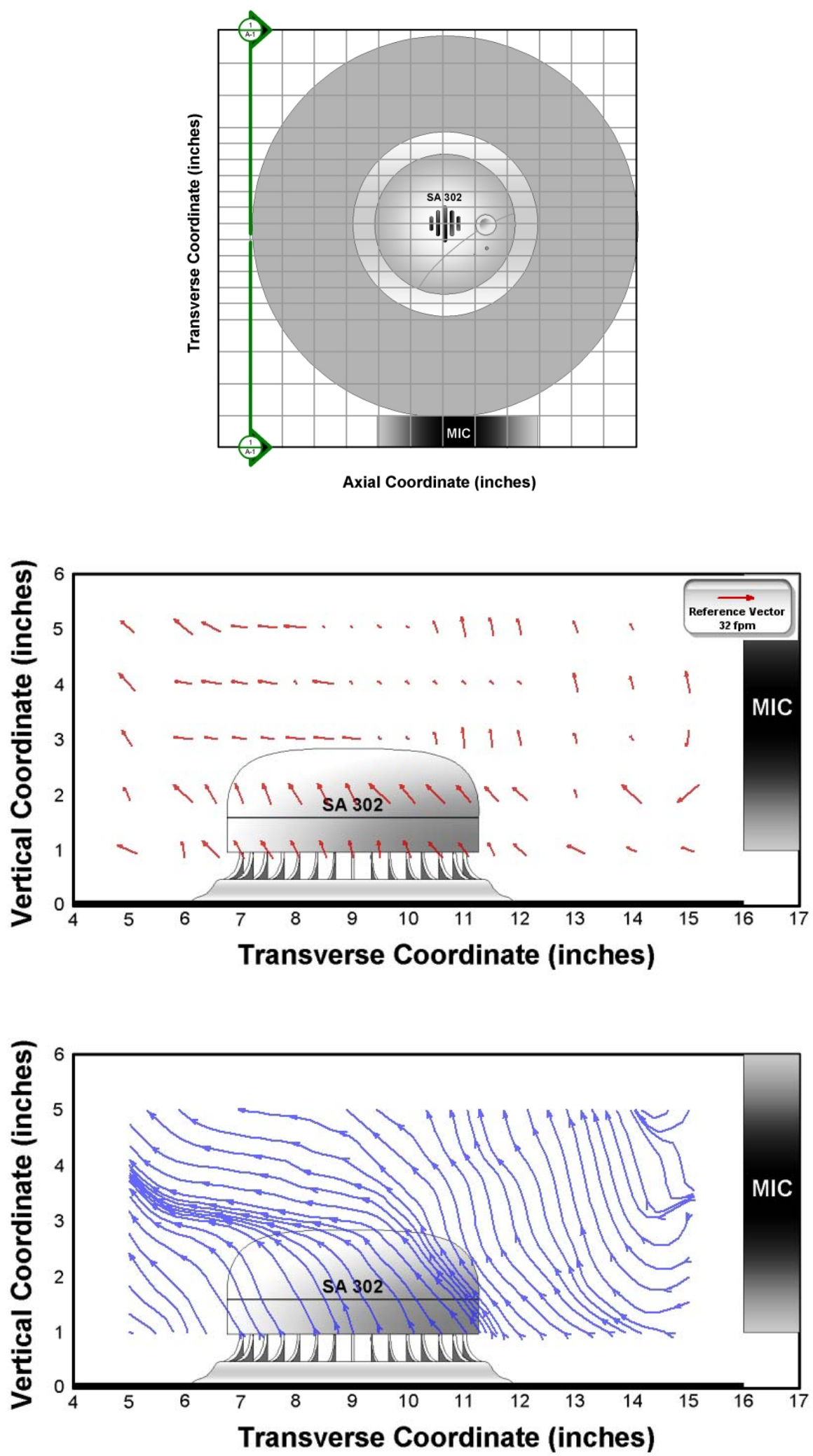

Figure 6.4.30: The $v$ - $w$ velocity vectors and streamlines in the UL Smoke Box test section at $x$ $=12.0 \mathrm{in}$. 

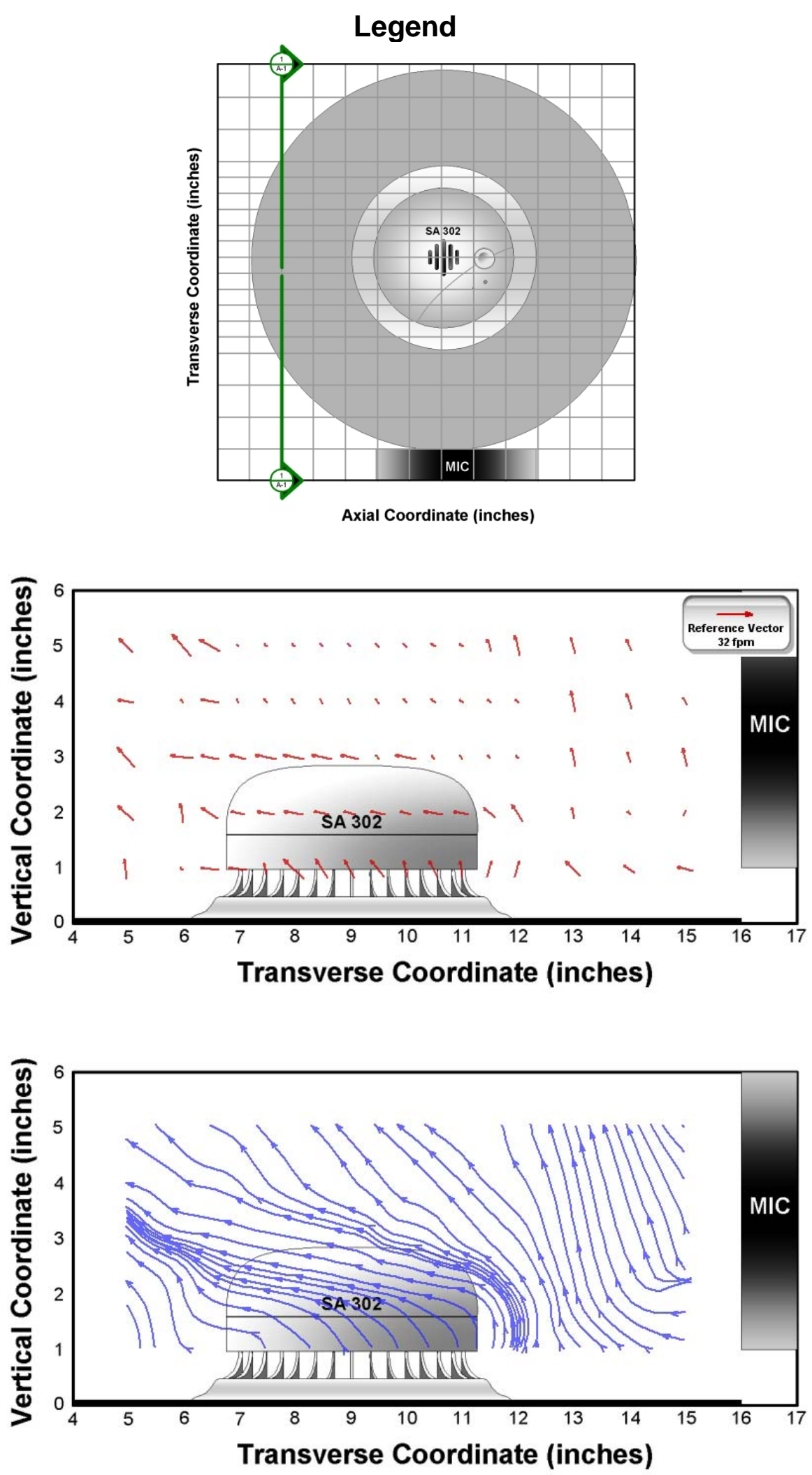

Figure 6.4.31: The $v$ - $w$ velocity vectors and streamlines in the UL Smoke Box test section at $x$ $=13.0$ in. 


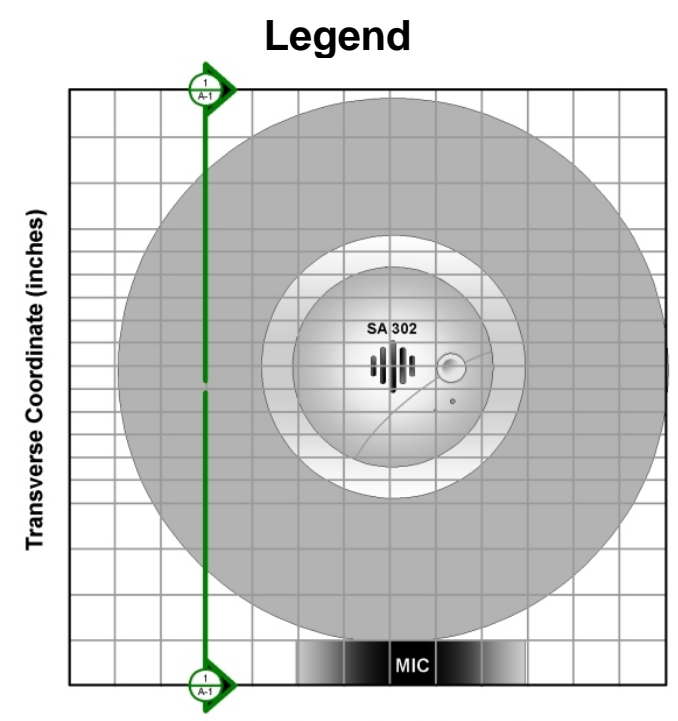

Axial Coordinate (inches)
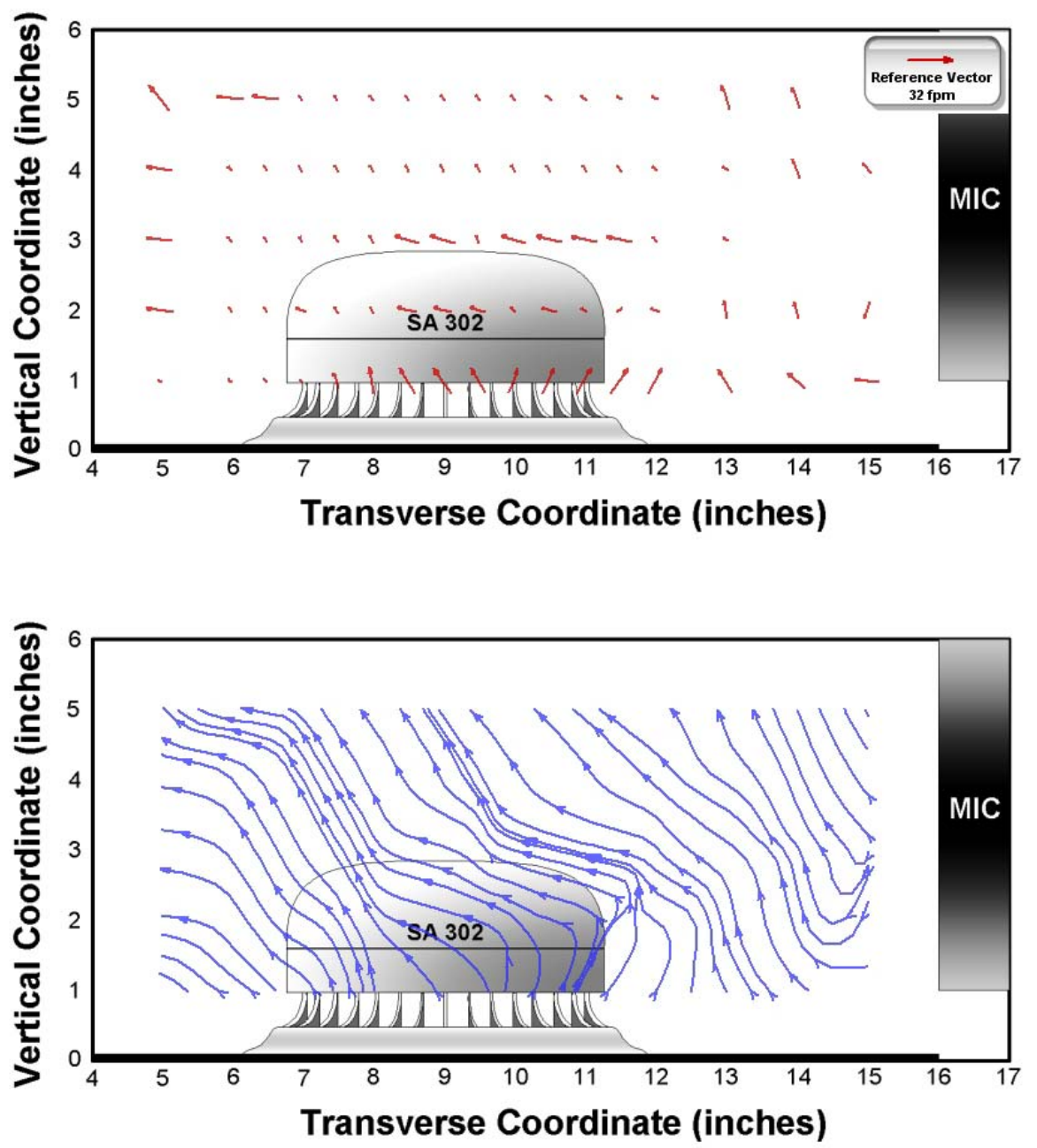

Figure 6.4.32: The $v$ - $w$ velocity vectors and streamlines in the UL Smoke Box test section at $x$ $=14.0$ in. 

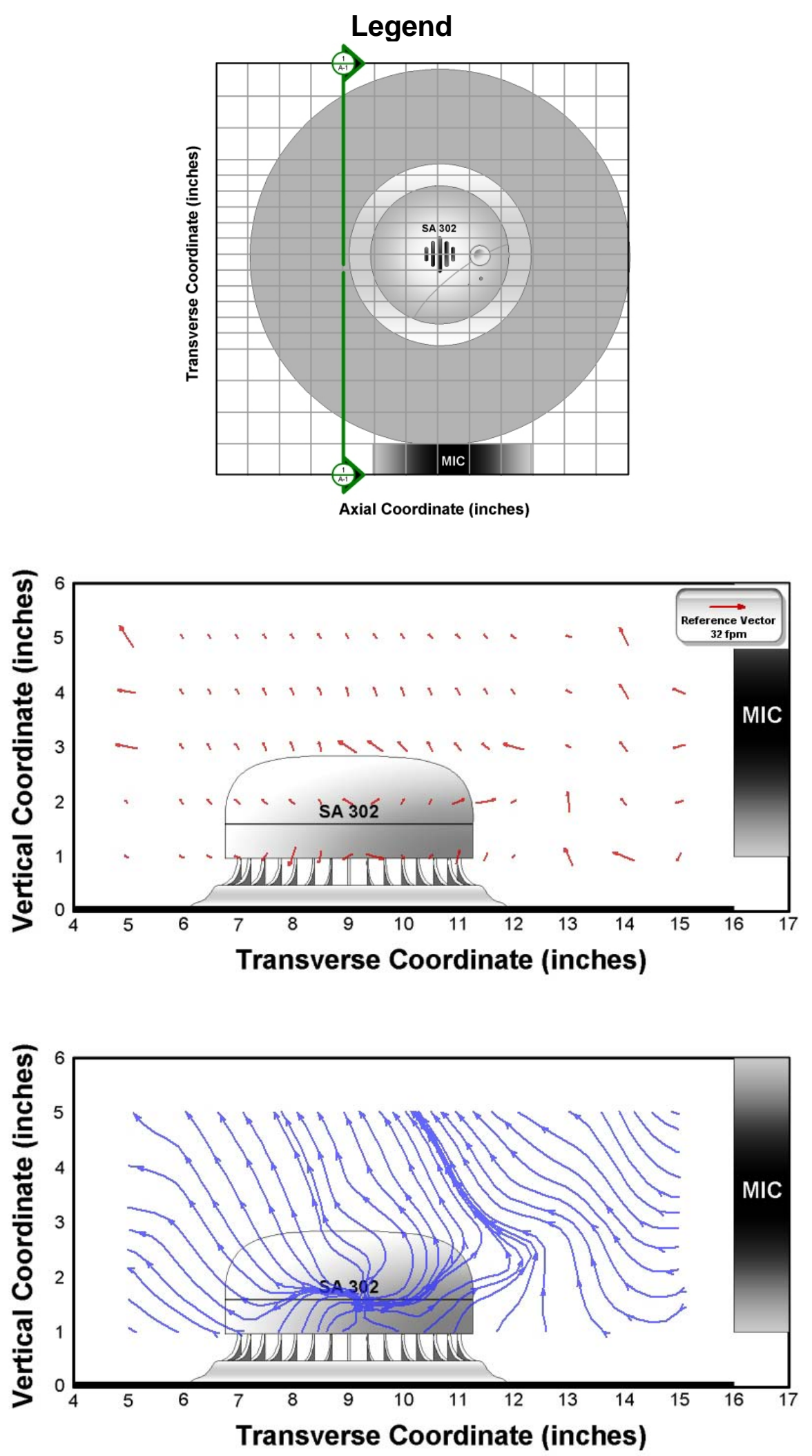

Figure 6.4.33: The $v$ - $w$ velocity vectors and streamlines in the UL Smoke Box test section at $x$ $=15.0$ in. 

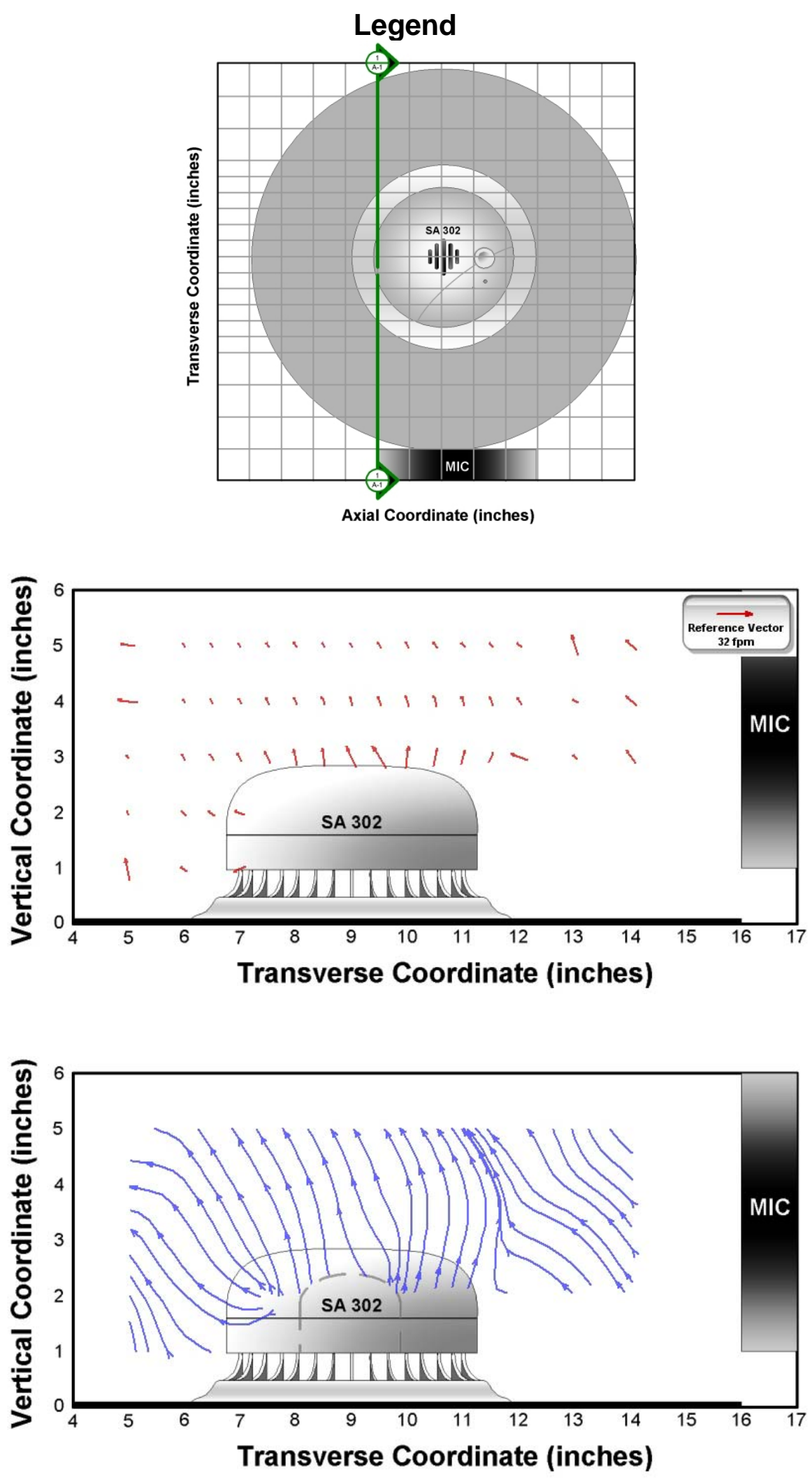

Figure 6.4.34: The $v$ - $w$ velocity vectors and streamlines in the UL Smoke Box test section at $x$ $=16.0$ in. 

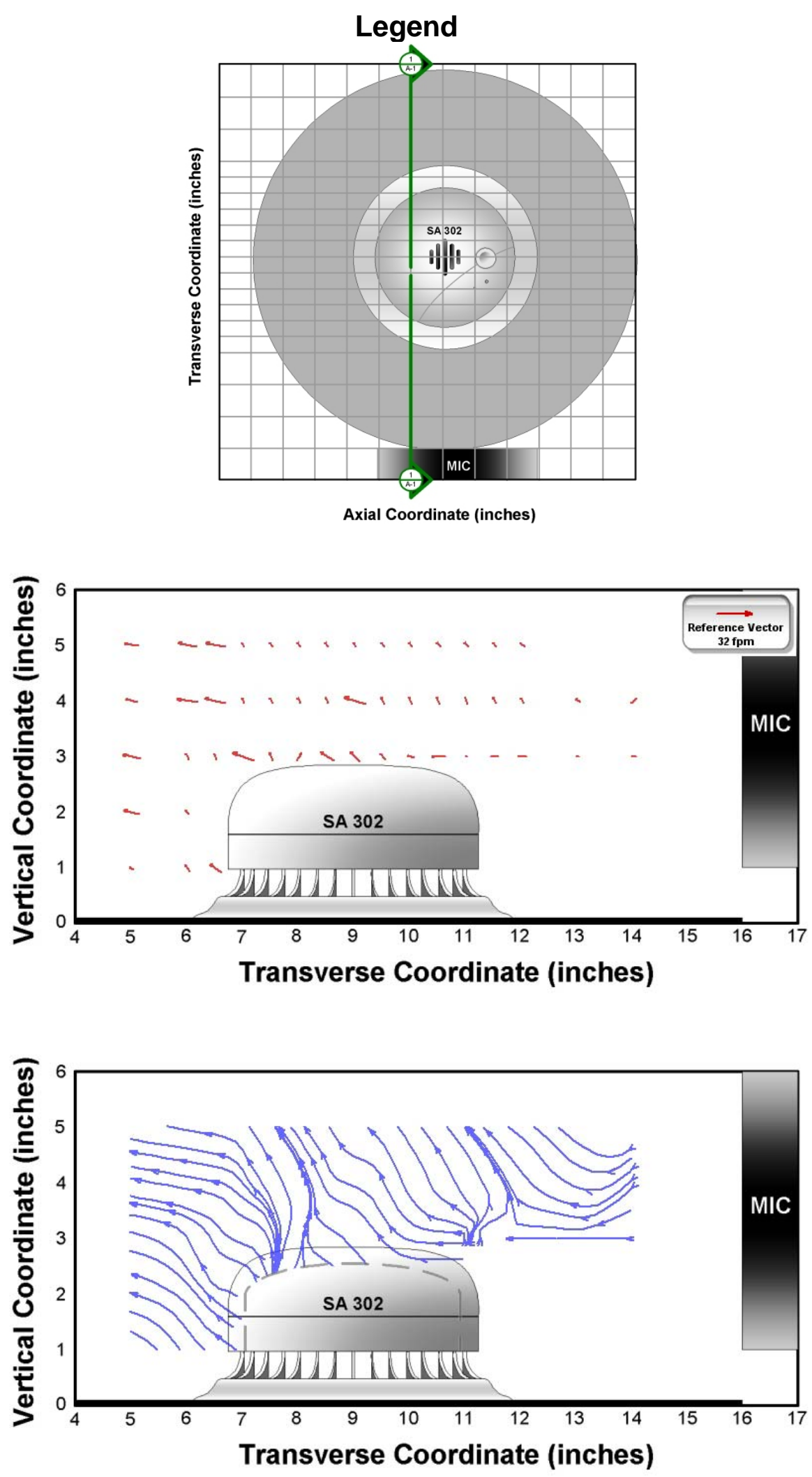

Figure 6.4.35: The $v$ - $w$ velocity vectors and streamlines in the UL Smoke Box test section at $x$ $=17.0 \mathrm{in}$. 

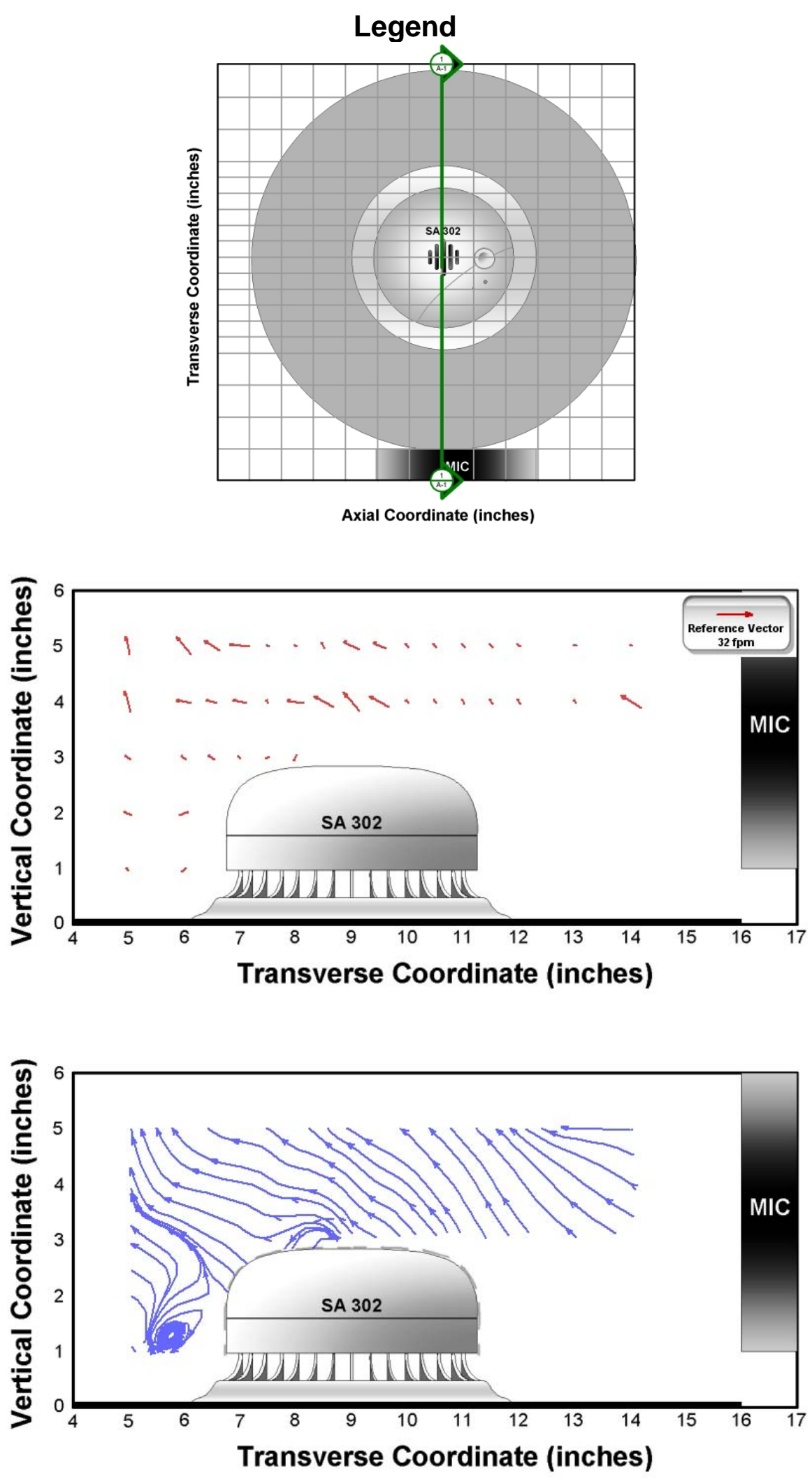

Figure 6.4.36: The $v-w$ velocity vectors and streamlines in the UL Smoke Box test section at $x$ $=18.0$ in. 

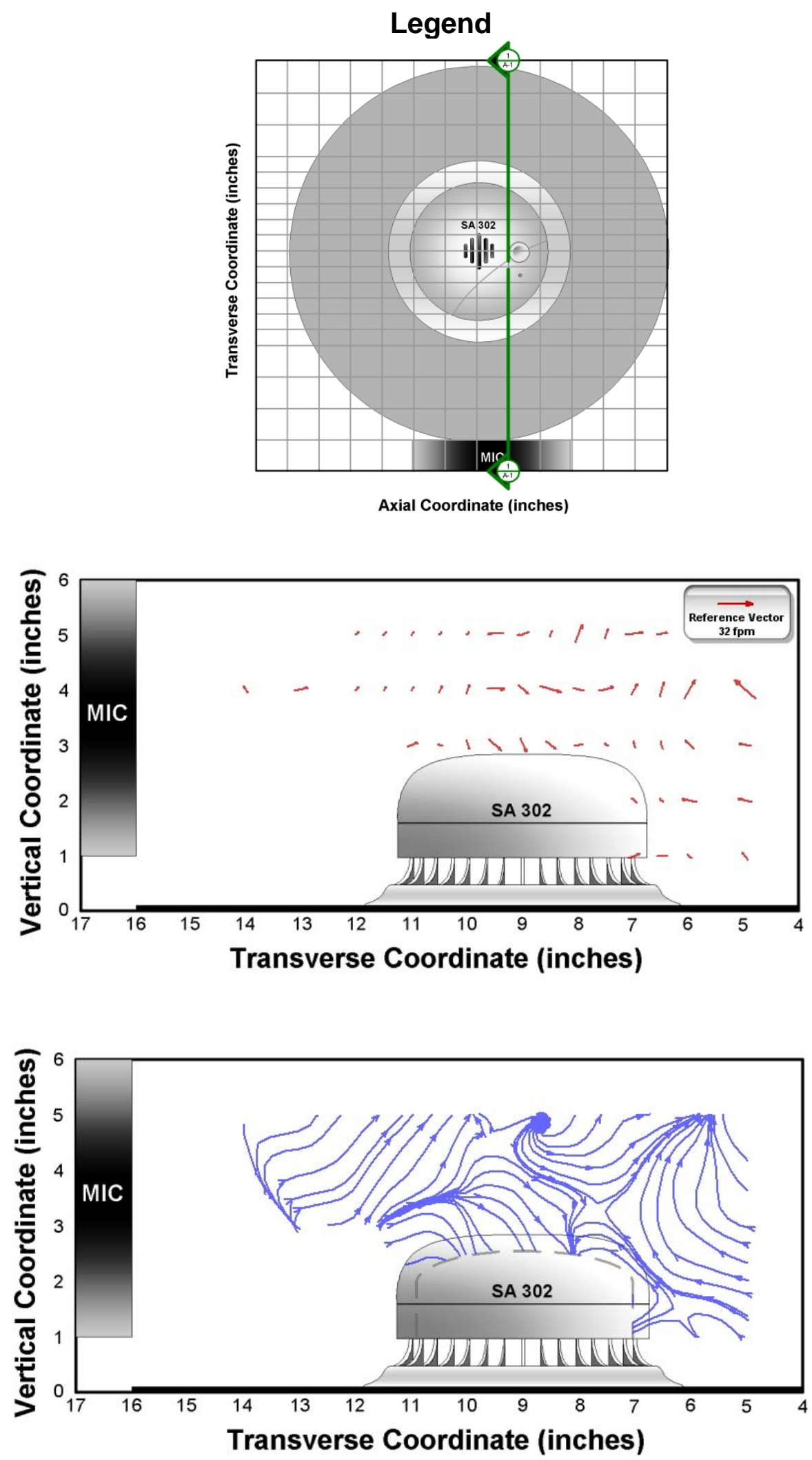

Figure 6.4.37: The $v-w$ velocity vectors and streamlines in the UL Smoke Box test section at $x$ $=19.0$ in (reverse angle view). 

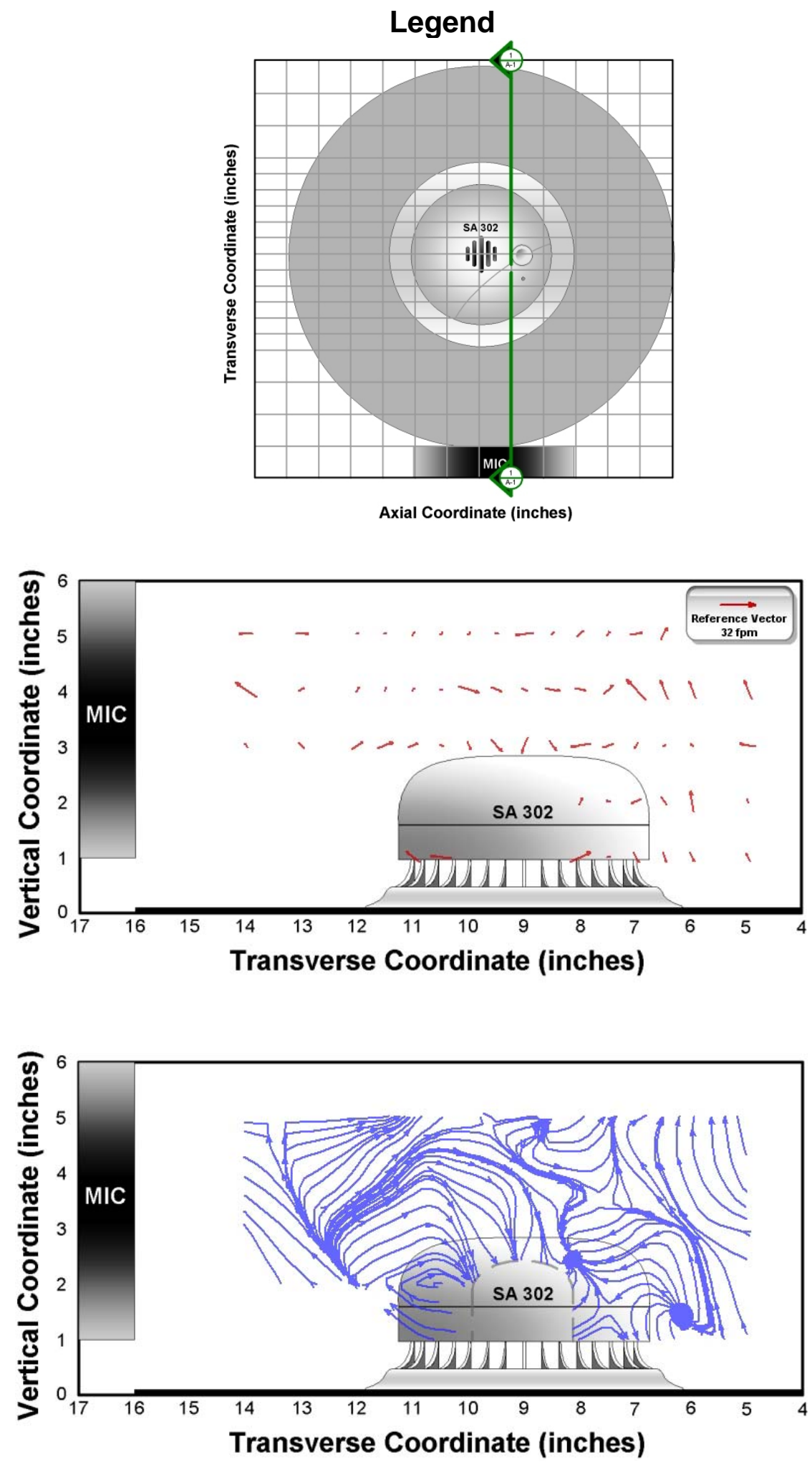

Figure 6.4.38: The $v$ - $w$ velocity vectors and streamlines in the UL Smoke Box test section at $x$ $=20.0$ in (reverse angle view). 

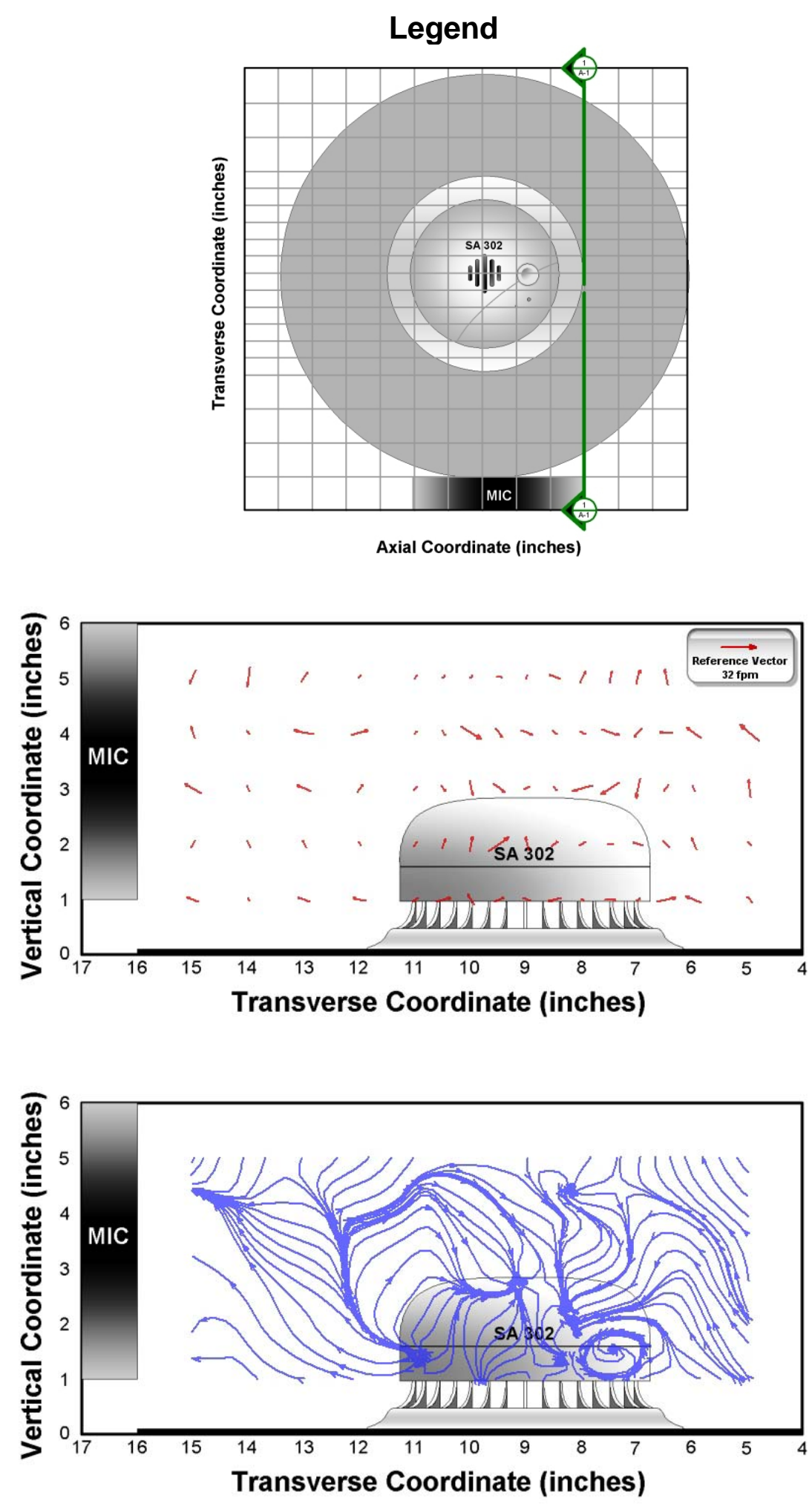

Figure 6.4.39: The $v$-w velocity vectors and streamlines in the UL Smoke Box test section at $x$ $=21.0$ in (reverse angle view). 

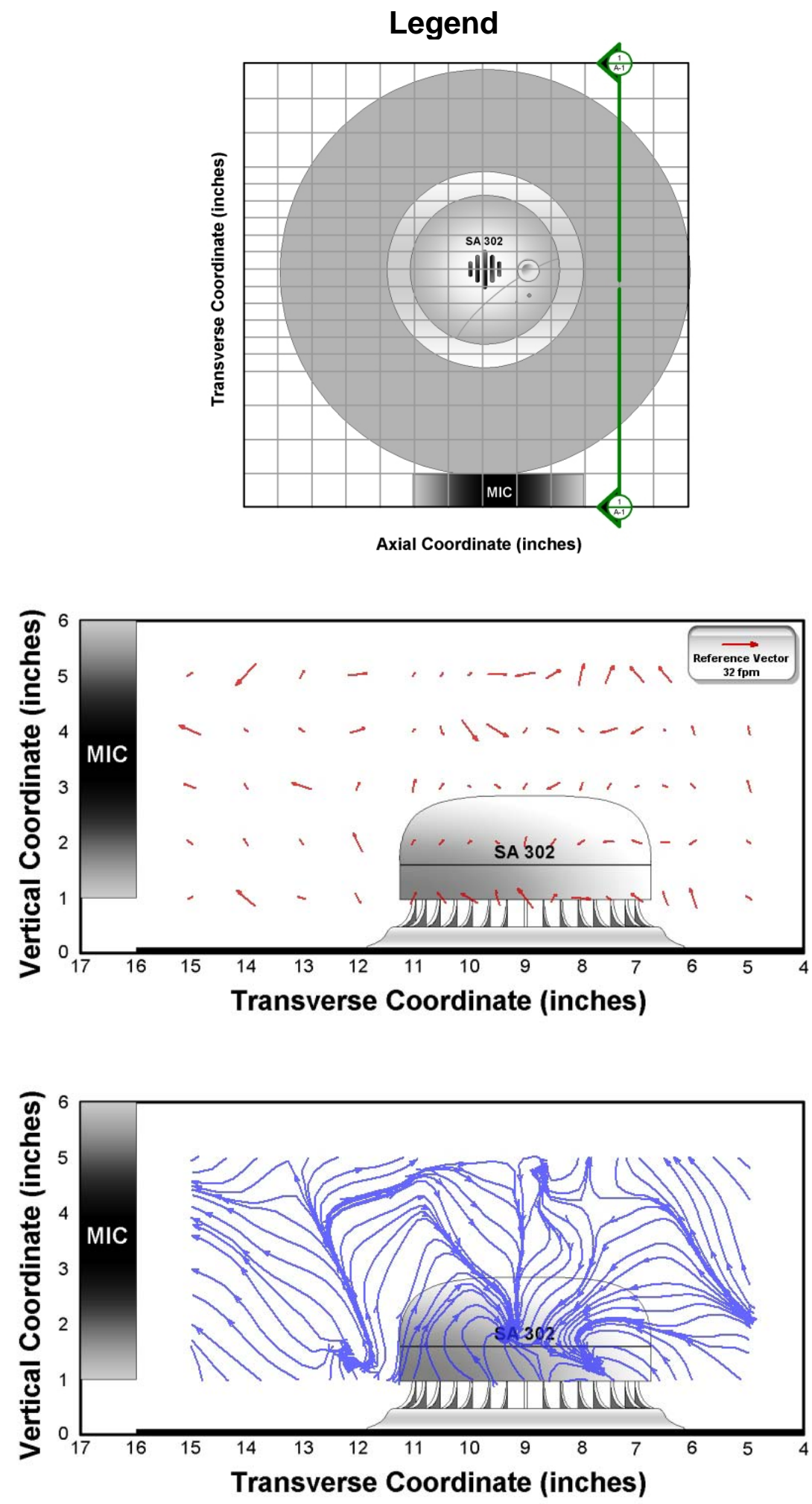

Figure 6.4.40: The $v$ - $w$ velocity vectors and streamlines in the UL Smoke Box test section at $x$ $=22.0$ in (reverse angle view). 

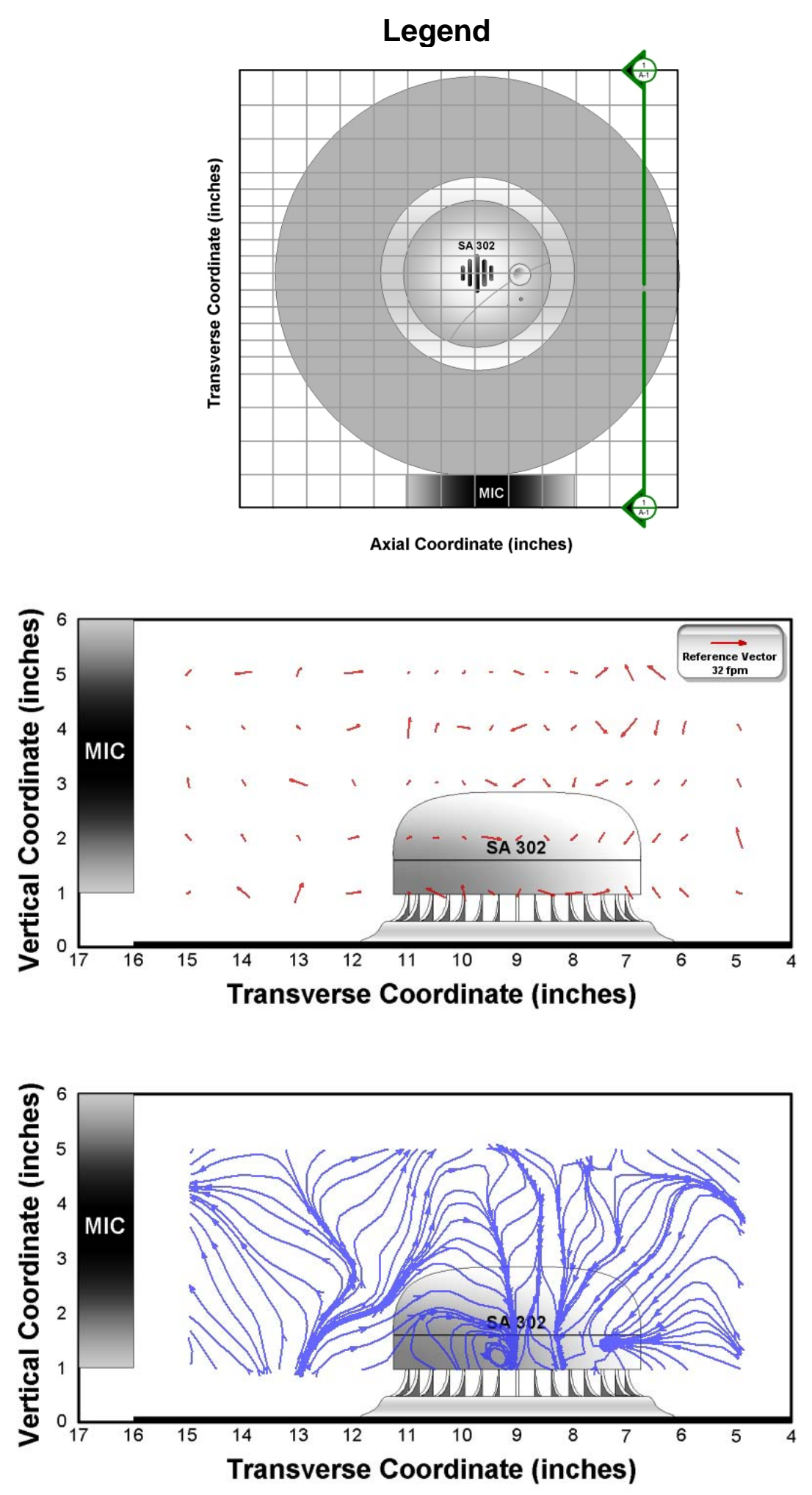

Figure 6.4.41: The $v$-w velocity vectors and streamlines in the UL Smoke Box test section at $x$ $=23.0$ in (reverse angle view). 


\subsection{UL SMOKE BOX INLET PROFILE RESULTS USING HOT-WIRE ANEMOMETRY}

Figure 6.5.1 shows the surface plot of the 144 hot-wire anemometer data points taken approximately $0.5 \mathrm{in}$, in front of the flow straightener entrance to the test section. Taking data too closely to the flow straightener would increase uncertainty. The hot-wire anemometer results were corrected given a calibration graph knowing the atmospheric conditions of the day. Turbulence intensities did not vary higher than 1 to $2 \%$ in measuring the free stream velocity. As one would suspect from the geometry of the UL Smoke Box, the bulk airflow makes a circular bend in navigating from the bottom of the UL Smoke Box to the top in meeting the test section flow straightener. Because of these flow conditions, the outer velocities closest to the test section ceiling exhibit higher velocity magnitudes than those near the floor. As one can see, even though the UL $217^{5}$ standard only requires the flow 1 in above the floor to be $32 \mathrm{ft} / \mathrm{min}$ where the detector sits, variations still exist moving in the vertical coordinate.

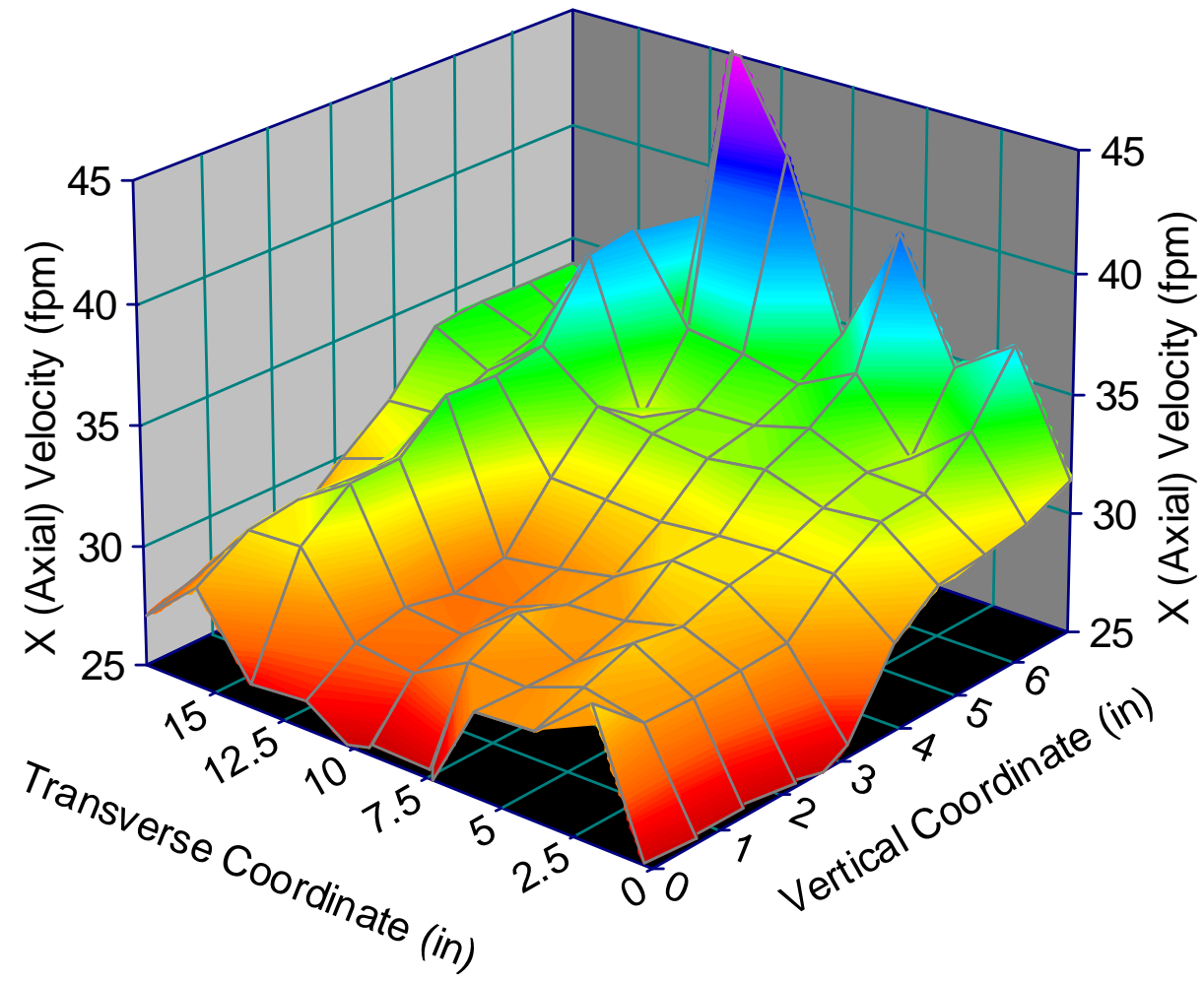

Figure 6.5.1: A surface plot of the inlet profile depicting the $x$, axial velocity as measured by hot-wire anemometry. 


\subsection{UL SMOKE BOX AND MODEL SA302 SMOKE DETECTOR VELOCITY PREDICTIONS USING CFD}

The following sets of figures show quantitative results reported by the CFD model of the UL Smoke Box test section. The data will be broken down in terms of detector orientation; namely, the $0^{\circ}, 90^{\circ}, 180^{\circ}, 270^{\circ}$, and $315^{\circ}$ orientation, in order to compare to experimental LDA data taken in the same manner. Because the smoke detector's external geometry is axisymmetric along the vertical coordinate regardless of orientation, the upstream air flow does not experience any significant change in flow pattern while varying detector orientation. Because of this and the fact that this study is primarily concerned with the immediate area around and inside the smoke detector, computational results concerning the entire test section (external to the detector) will only be reported for one orientation $\left(0^{\circ}\right)$. As the reader will eventually find, the external test section flow for all SA302 smoke detector orientations is largely the same. In general, the test section results are to support the global conclusion that the CFD model does an adequate job in characterizing the significant flow patterns experimentally witnessed inside the real UL Smoke Box, yielding sufficient agreement to warrant the use of CFD for prediction modeling.

One of the first issues to be addressed is grid independence. For this case, there were five levels of grid refinement to show an approach to grid independency. Figure 6.6.1 depicts the analysis done. To depict these results, the author selected a rake of data 1 in above the test section floor at $\mathrm{x}=15 \mathrm{in}$, which is nearly tangent to the front of the SA302's top dome. A visual legend depicts the rake's position in the right hand corner of Figure 6.6.1, with a red block arrow denoting flow direction. This convention will be used throughout the remainder of this report in presenting rake comparisons between LDA and CFD data. Using merely number of cells as the nomenclature used to define grid sizing seemed inadequate for defining the refines of the CFD model because unique meshing sequences had to be taken into account as discussed previously in designing the overall grid. Hence, the models are defined based upon the number of nodal intervals placed along the length, width, and height of the test section; namely, $80 \times 40 \times 16$ ( $\approx 360,000$ nodes), $110 \times 55 \times 23(\approx 390,000$ nodes $), 115 \times 60 \times 24(\approx 490,000$ nodes $)$, and $125 \times 63 \times 25(\approx 560,000$ nodes). Due to the restriction on computer resource available, models with larger magnitudes of grid refinement were not able to be assessed. Thus, although grid 
independence was not truly achieved with respect to the entire model, very comparative results between the grid with the highest nodal density (denoted $125 \times 63 \times 25$ in Figure 6.6.1) and LDA data warrant valid conclusions drawn from this study. With each respective test section size, there corresponded a proportional sizing constraint placed upon the MIC housing and compass plate sitting below the detector.

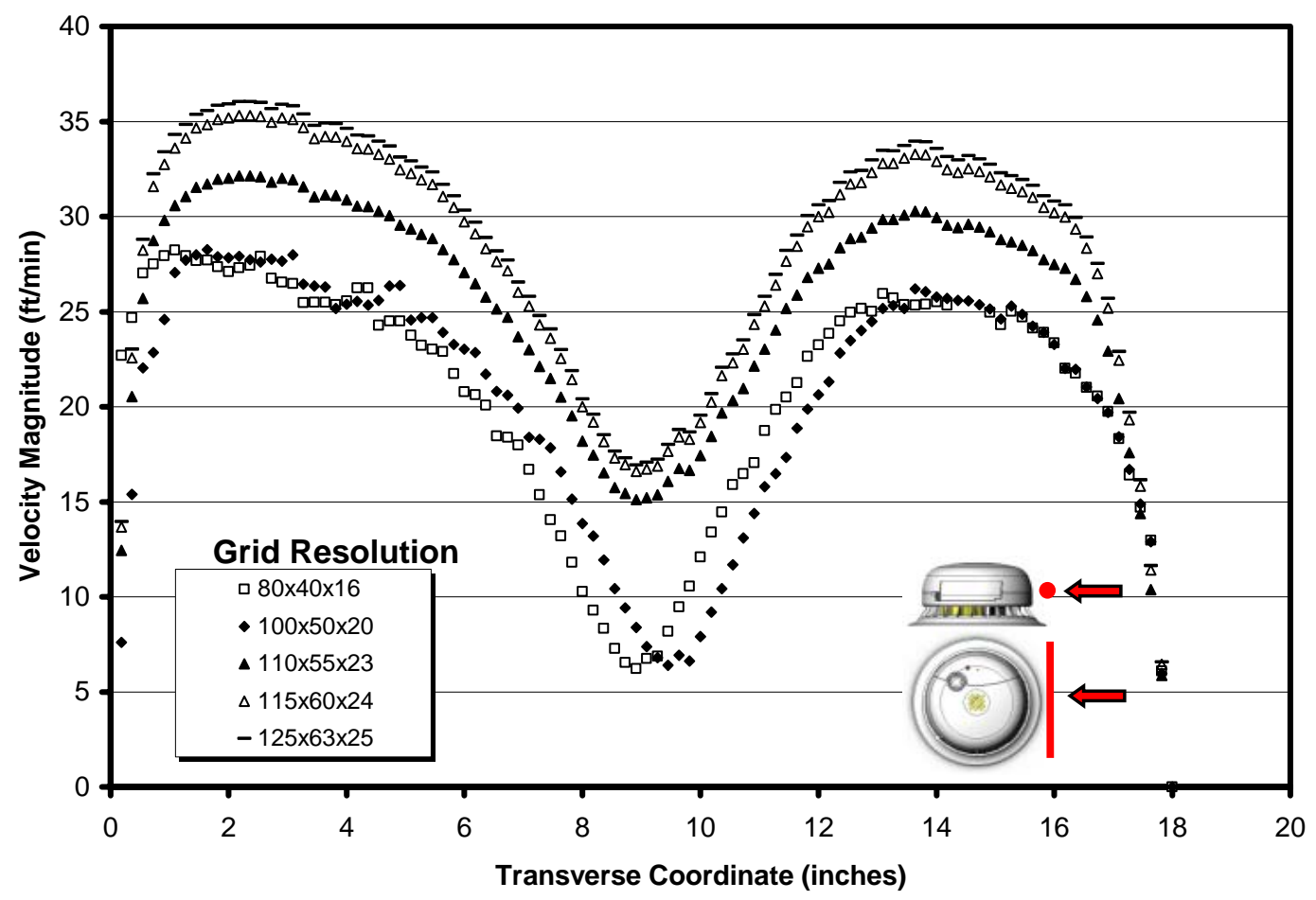

Figure 6.6.1: A plot depicting grid independence for 5 computational models.

For all models, a volumetric constraint of $100 \%$ of the shortest edge $(\approx 0.037$ in) was used to mesh outward from the detector to meet the surrounding walls. Other, more simplistic methods were used, but were not successful. This meshing system was explored on the $0^{\circ}$ orientation, and implemented on the rest of the models due to the excellent mesh repeatability. Total nodal values for each model varied by approximately 10,000 nodes between each model.

The final models reported in these results are $2^{\text {nd }}$ order, implicitly solved unstructured grids using the standard laminar flow model. In short, $2^{\text {nd }}$ order accuracy means two higher order terms are resolved within the Taylor series expansion leading to a truncation error of only $\Delta x^{2}$ (where $\Delta x<<1$ ), whereas $1^{\text {st }}$ order accuracy satisfies a truncation error of only $\Delta x$. 
While $1^{\text {st }}$ order accuracy only predicts the slope of the function being discretized, $2^{\text {nd }}$ order accuracy goes beyond that in predicting the concavity of the function as well.

Initially, there was a study conducted on whether or not to use a laminar or turbulent model to rapidly assess the future movement of the research focus using a $1^{\text {st }}$ order model to estimate test section response. If one calculates the Reynolds number for the test section based upon the diameter of the detector roughly assuming a cylinder in an external flow field, the Re is approximately 1400 . On the other hand, if one assumes the system mimics interior duct flow, taking the hydraulic diameter of the test section as the characteristic length $(\approx 10$ in) spawns a Re of about 2800 - which is on the brink of transitioning from laminar to turbulent flow. Without a smoke detector centered within the test section, flow visualization results show flow patterns staying consistently laminar at least halfway through the box where the flow field is introduced to the MIC, whereby the flow begins to break up in the wake of the MIC. Preliminary models employed various turbulence models, and the one that provided the best turbulent results were those from the realizable $\kappa-\varepsilon$ model discussed earlier. Again, the realizable $\kappa-\varepsilon$ model was chosen due to its widespread history of being robust for a wide variety of turbulent flow fields.

Figures 6.6.2 through 6.6.10 show comparisons made between the realizable $\kappa-\varepsilon$ turbulent model and the laminar flow model for different test section planes. From Figures 6.6.2 and 6.6.3, the discernible stagnation area resident at the forefront of the detector can be seen as the flow towards the middle of the floor begins to slow down increasingly as the vertical $x$-y contour planes approach the detector as well as the MIC. As one can see, the dissipative forces associated with the turbulent model greatly restricted the presence of an unsymmetrical wake (Figure 6.6.4), which the laminar model readily reports. If one recalls, the experimental data found both from the flow visualization (Section 6.2) and experimental test section LDA (Section 6.4) depict an unsymmetrical wake behind the detector as well showing that the laminar model compares largely better to the experimental data than does the turbulent case. The probe inlet shown in Figures 6.6.6 and 6.6.7 also readily present the major differences between the laminar and turbulent models in showing how the realizable $\kappa$ $\varepsilon$ dissipates the large incoming velocity jet from the probe inlet vary significantly as compared to the laminar model. Figure 6.6.8 compares the velocity vector, profile view of the swirling wake in the laminar model to that of the predominantly stagnant results reported 
by the turbulent model. Figure 6.6.9 shows the significant spatial differences between the laminar and turbulent models calculating the size of the boundary layer about the detector. Figure 6.6.10 shows another indication of the unsymmetrical wake meeting the large velocity gradient from the probe inlet in the laminar model, and conversely no significant interaction from the turbulent case. In almost all instances, a much smaller turbulent boundary layer is depicted compared to a fairly more resolved one from the laminar cases. Because of the quickly established similarities between the laminar model and LDA data, turbulence modeling was quickly abandoned.

One important note when studying velocity contour and vector plots is interpreting the range in which the data are being reported. All of the plots shown here have their own distinct range of values. This is to ensure a maximum resolution is maintained for each plot as to discern areas of very slowly changing velocity gradients such as those found inside the detector, and especially the sensing chambers resident inside. 


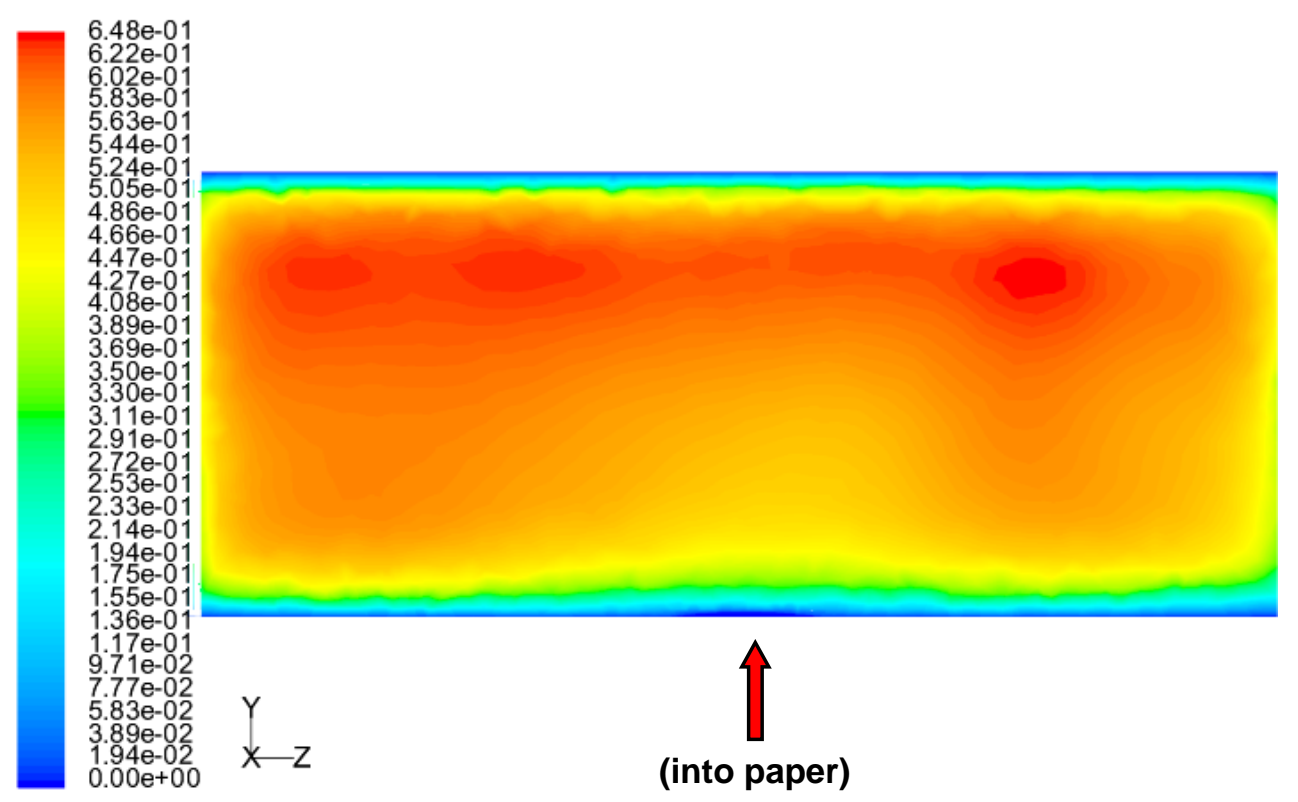

(a.) Laminar Model

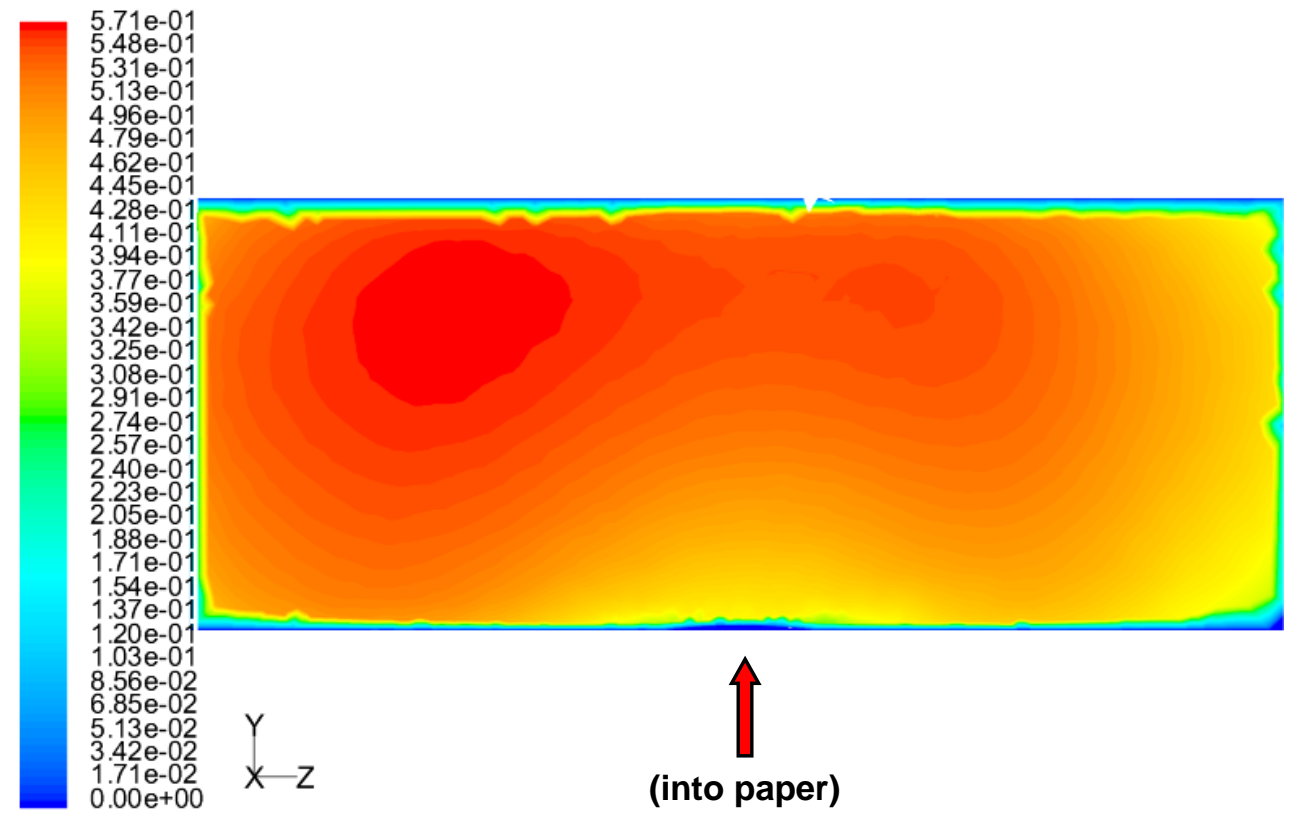

(a.) Realizable $\kappa-\varepsilon$ Turbulence Model

Figure 6.6.2: A velocity contour comparison between the (a.) laminar and (b.) realizable $\kappa-\varepsilon$ turbulence models for the $x=12$ in approach plane. 


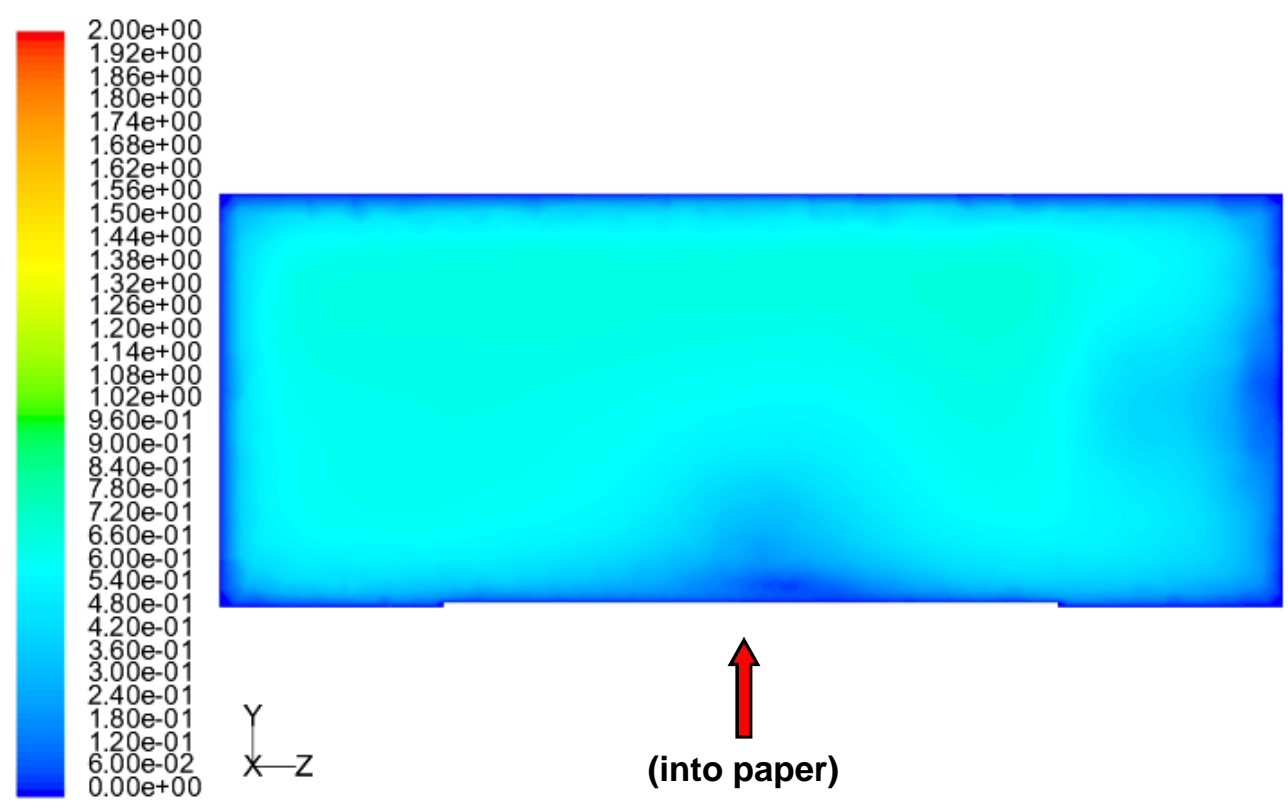

(a.) Laminar Model

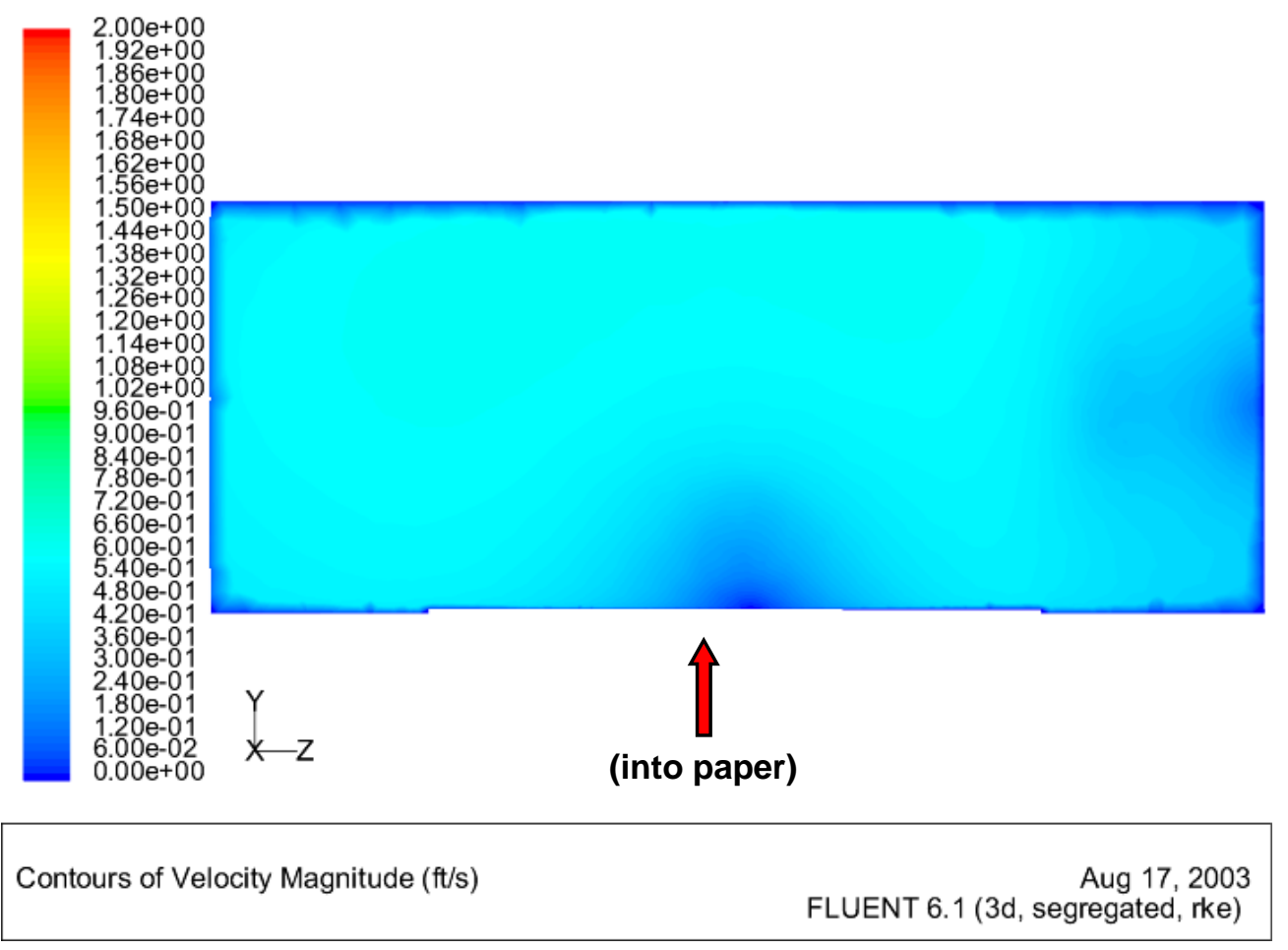

(a.) Realizable $\kappa-\varepsilon$ Turbulence Model

Figure 6.6.3: A velocity contour comparison between the (a.) laminar and (b.) realizable $\kappa-\varepsilon$ turbulence models for the $x=15$ in approach plane. 


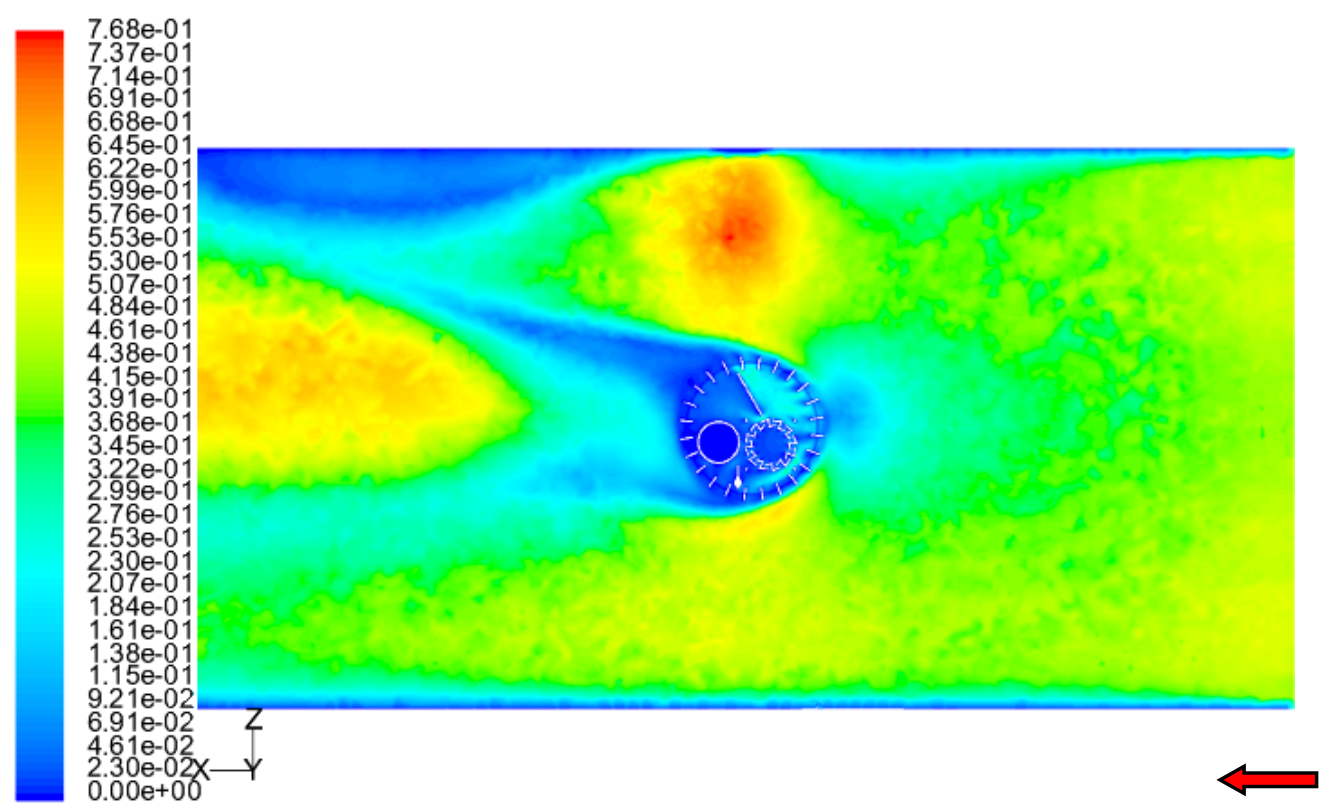

(a.) Laminar Model

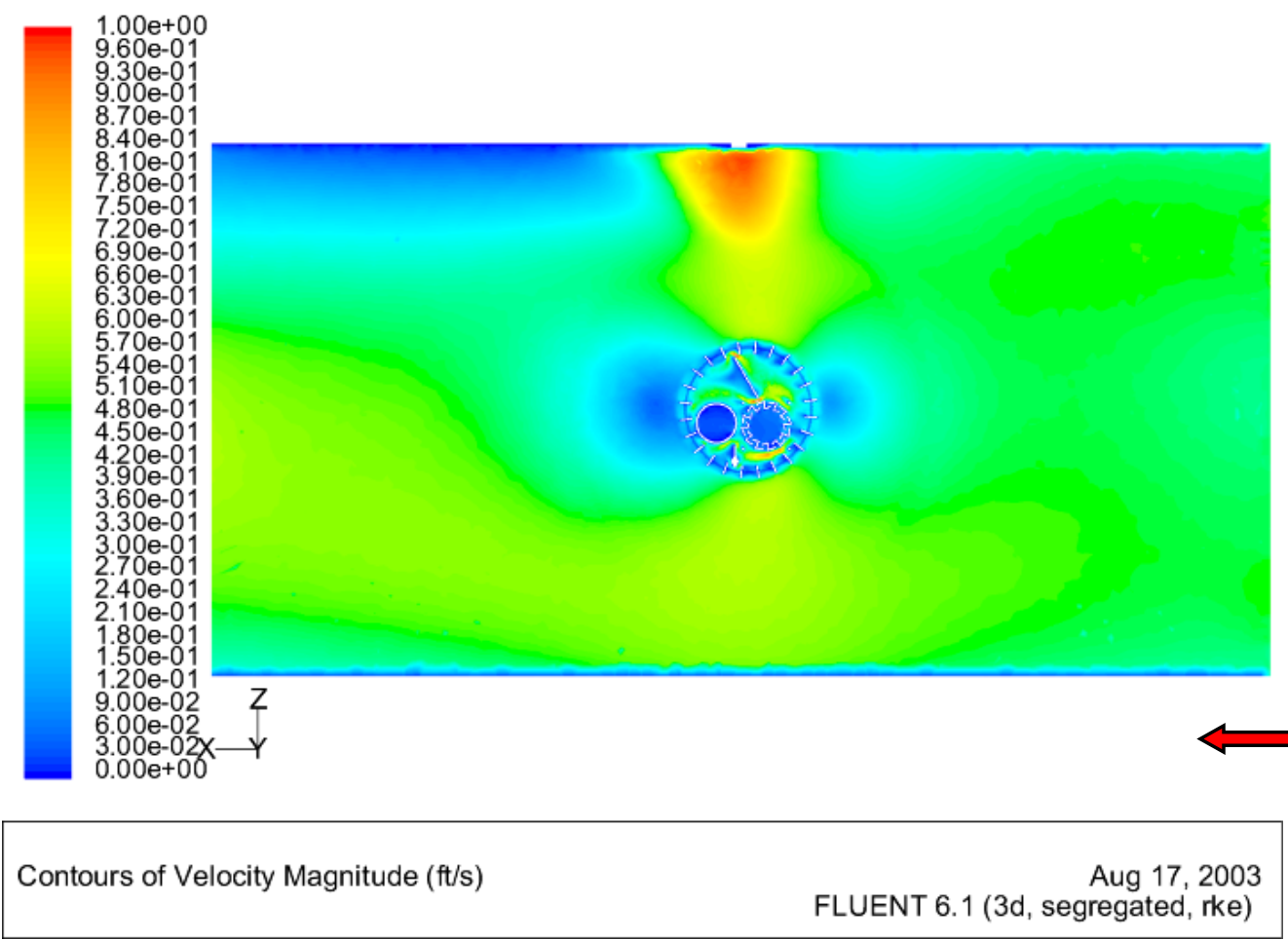

(a.) Realizable $\kappa-\varepsilon$ Turbulence Model

Figure 6.6.4: A velocity contour comparison between the (a.) laminar and (b.) realizable $\kappa-\varepsilon$ turbulence models for the $y=0.50$ in plane (mid-plane of the detector's sensing elements). 


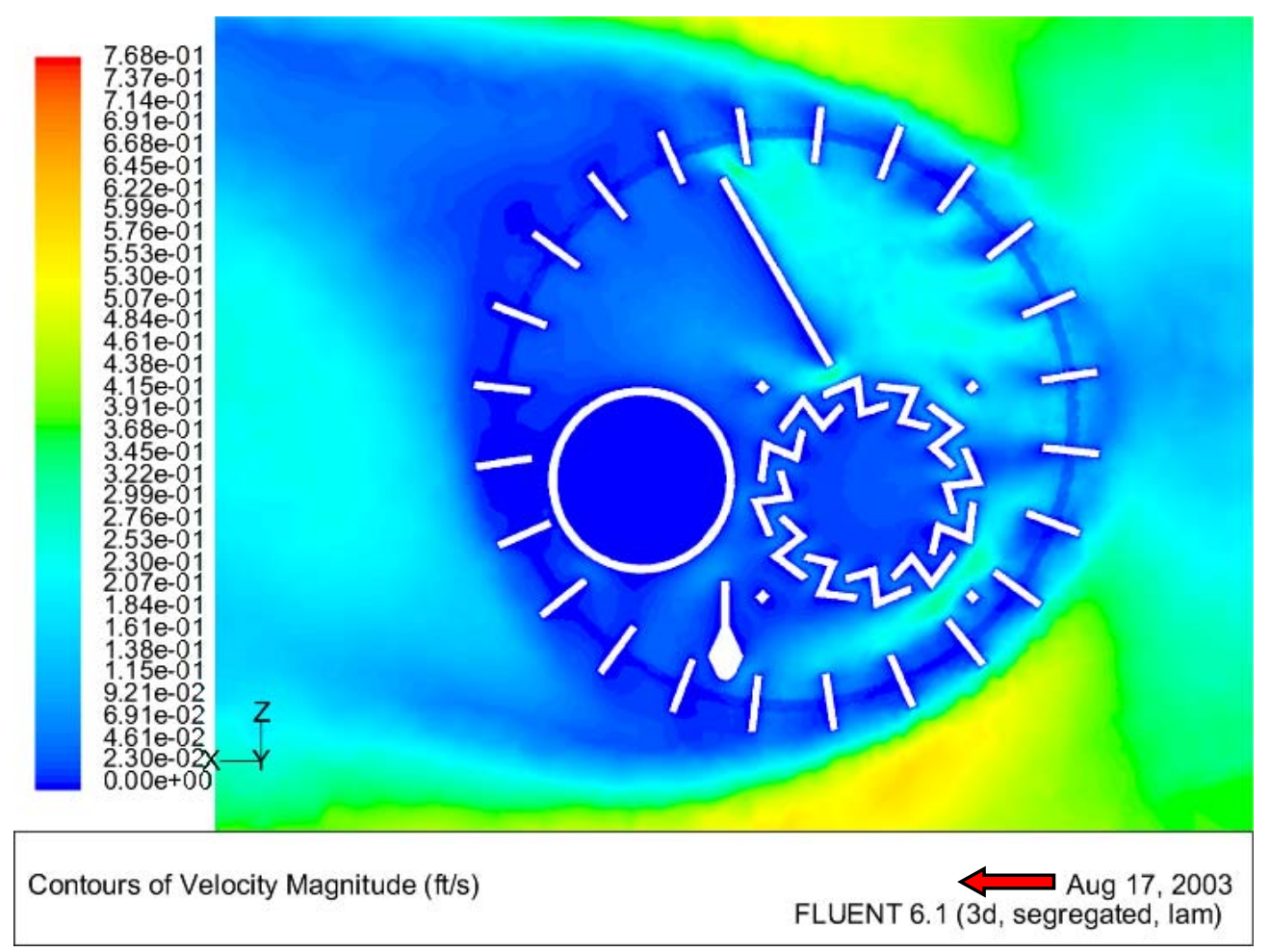

(a.) Laminar Model

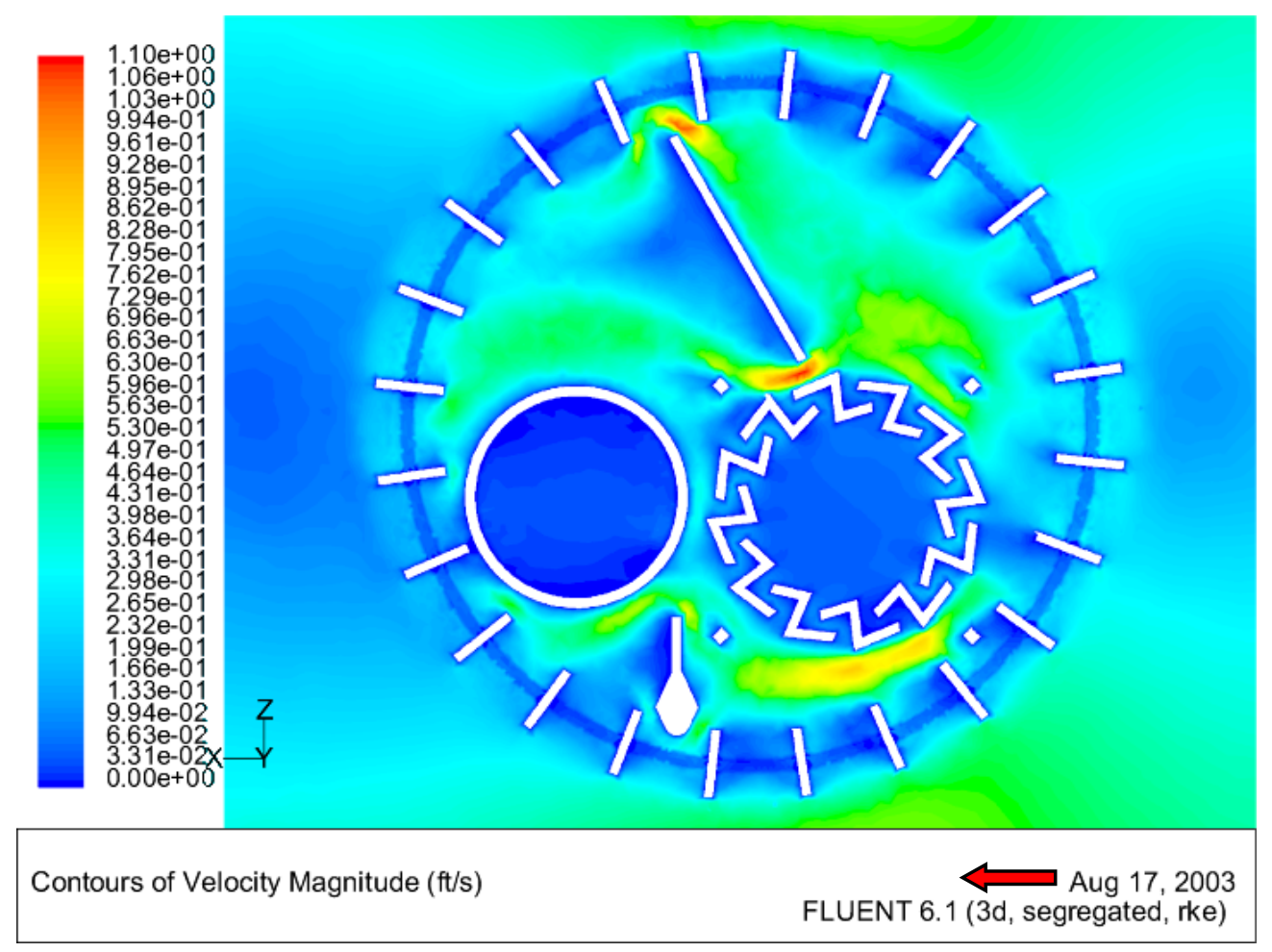

(a.) Realizable $\kappa-\varepsilon$ Turbulence Model

Figure 6.6.5: A velocity contour comparison between the (a.) laminar and (b.) realizable $\kappa-\varepsilon$ turbulence models for the $y=0.50$ in plane (mid-plane of the detector's sensing elements). 


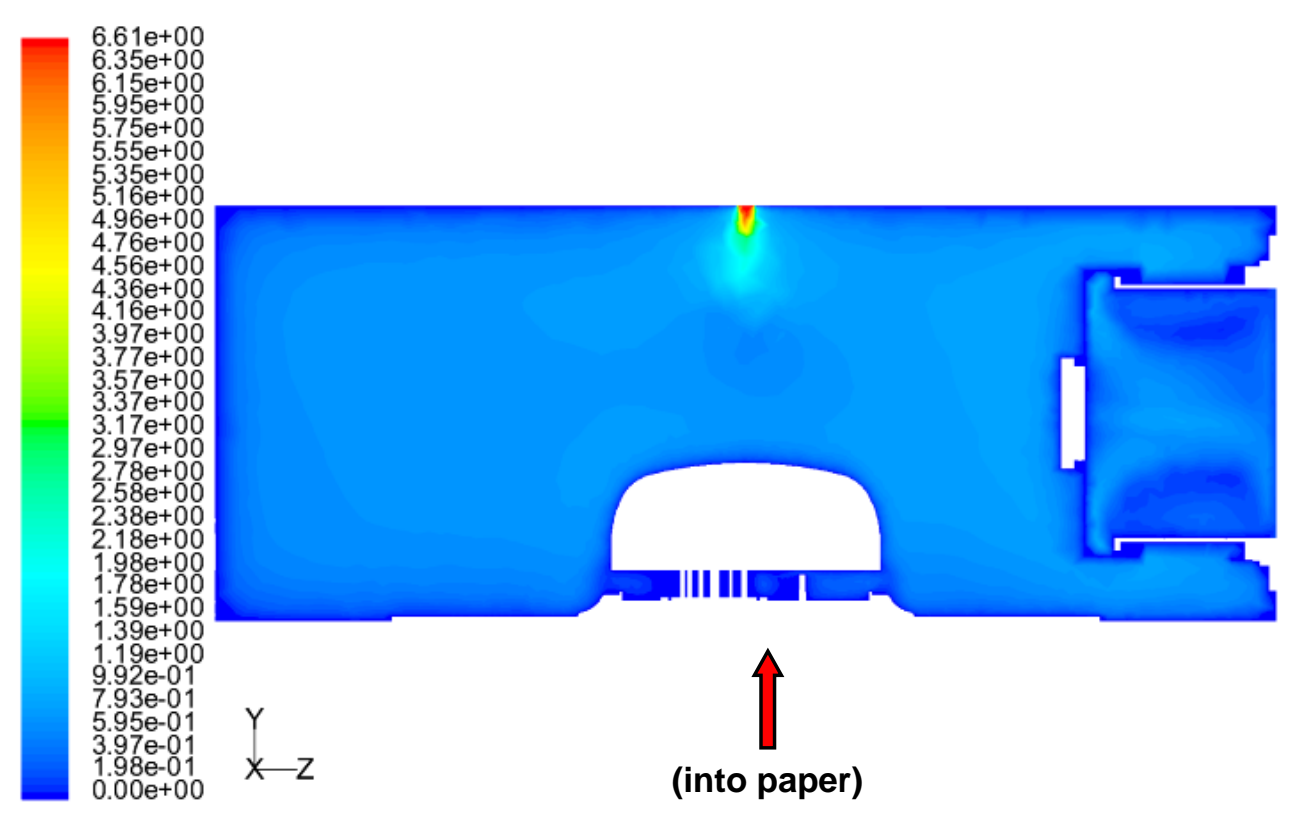

(a.) Laminar Model

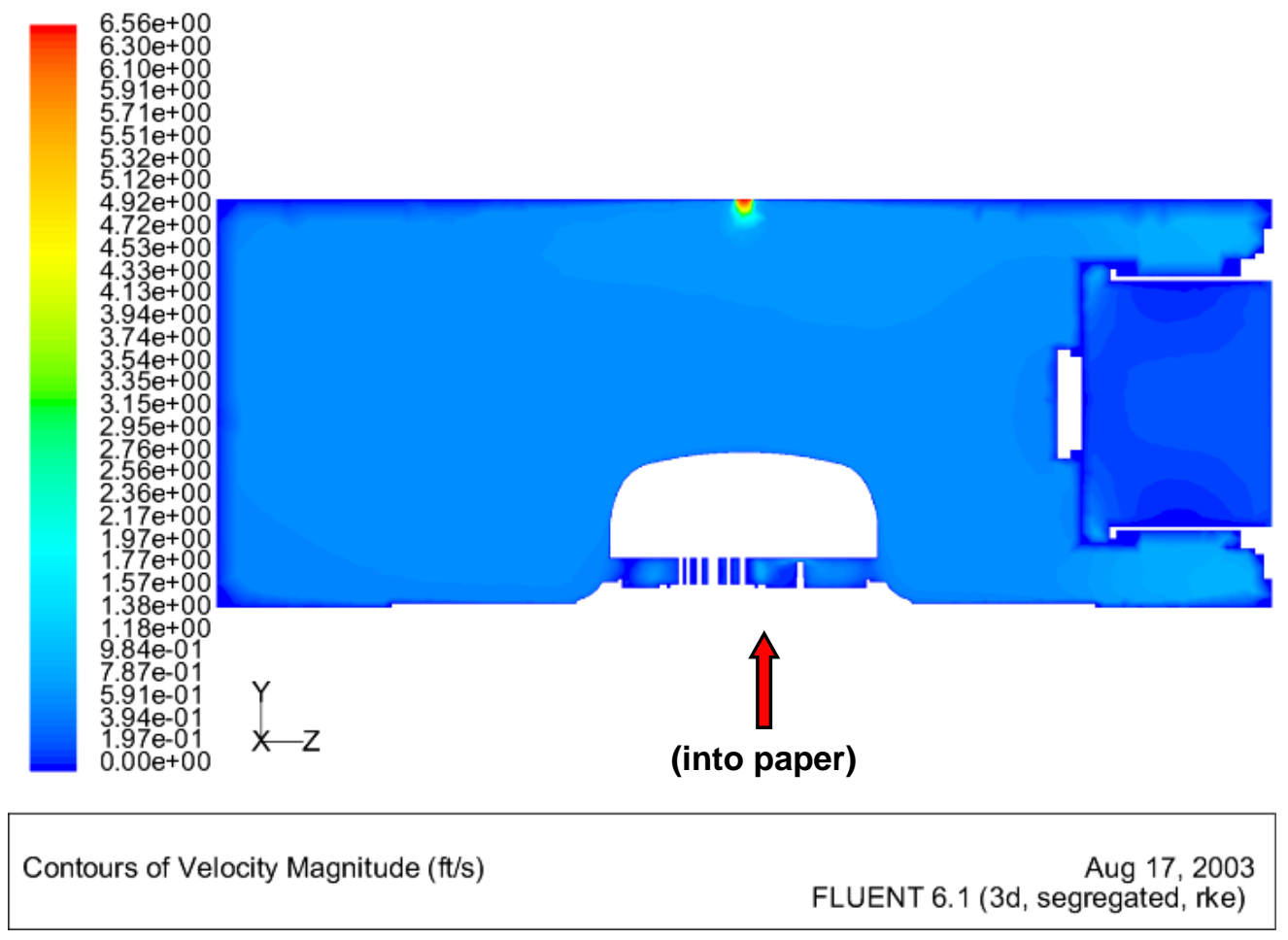

(a.) Realizable $\kappa-\varepsilon$ Turbulence Model

Figure 6.6.6: A velocity contour comparison between the (a.) laminar and (b.) realizable $\kappa-\varepsilon$ turbulence models for the $x=18$ in plane, which is positioned at the center of the probe inlet. 


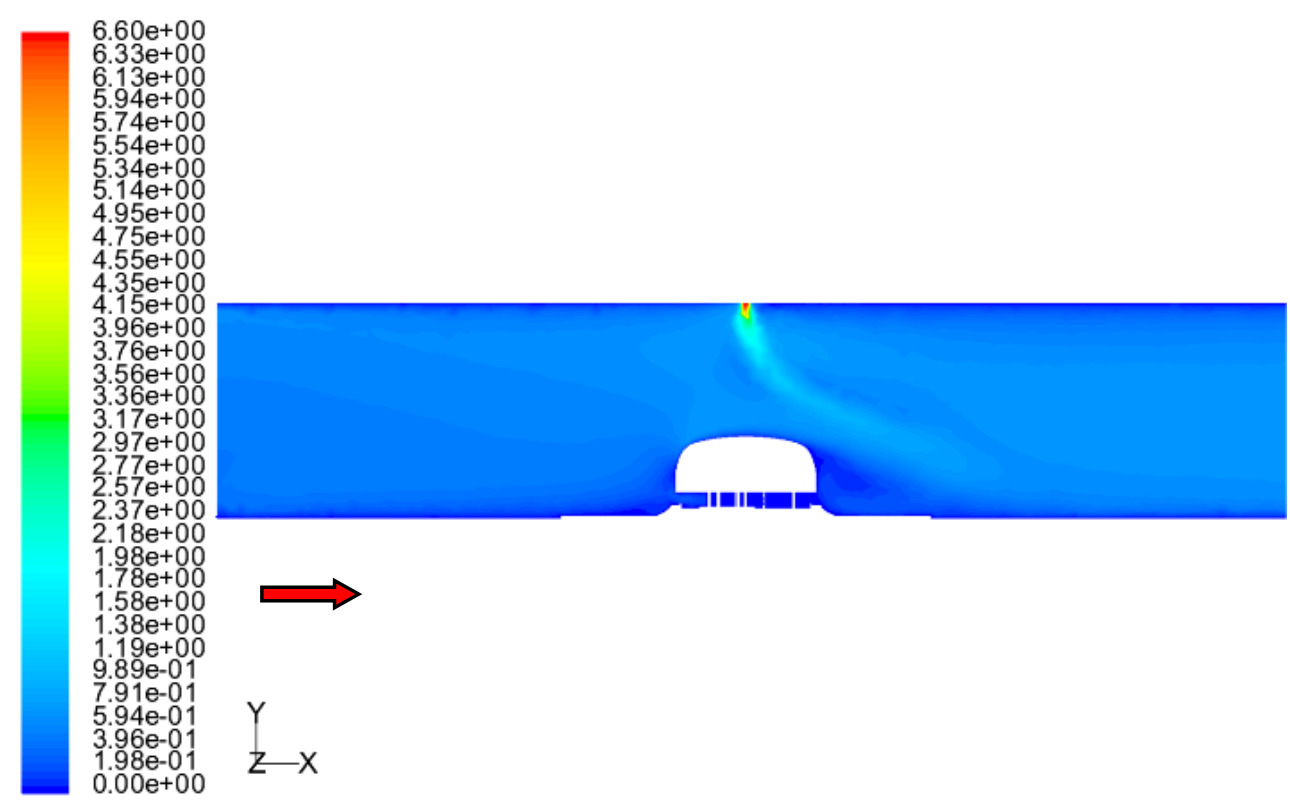

(a.) Laminar Model

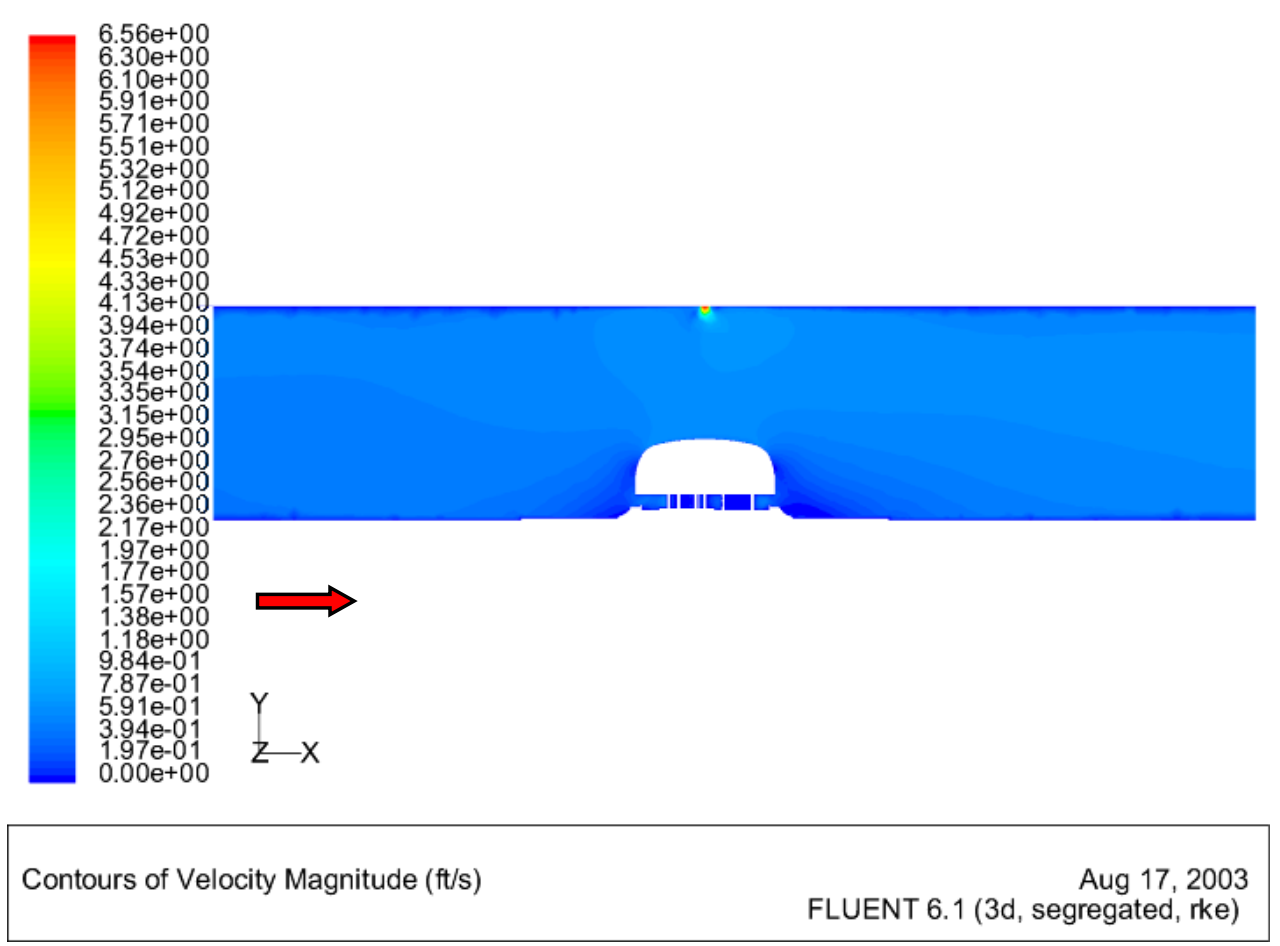

(a.) Realizable $\kappa-\varepsilon$ Turbulence Model

Figure 6.6.7: A velocity contour comparison between the (a.) laminar and (b.) realizable $\kappa-\varepsilon$ turbulence models for the $z=9$ in plane detailing the velocity gradients induced by the probe inlet. 


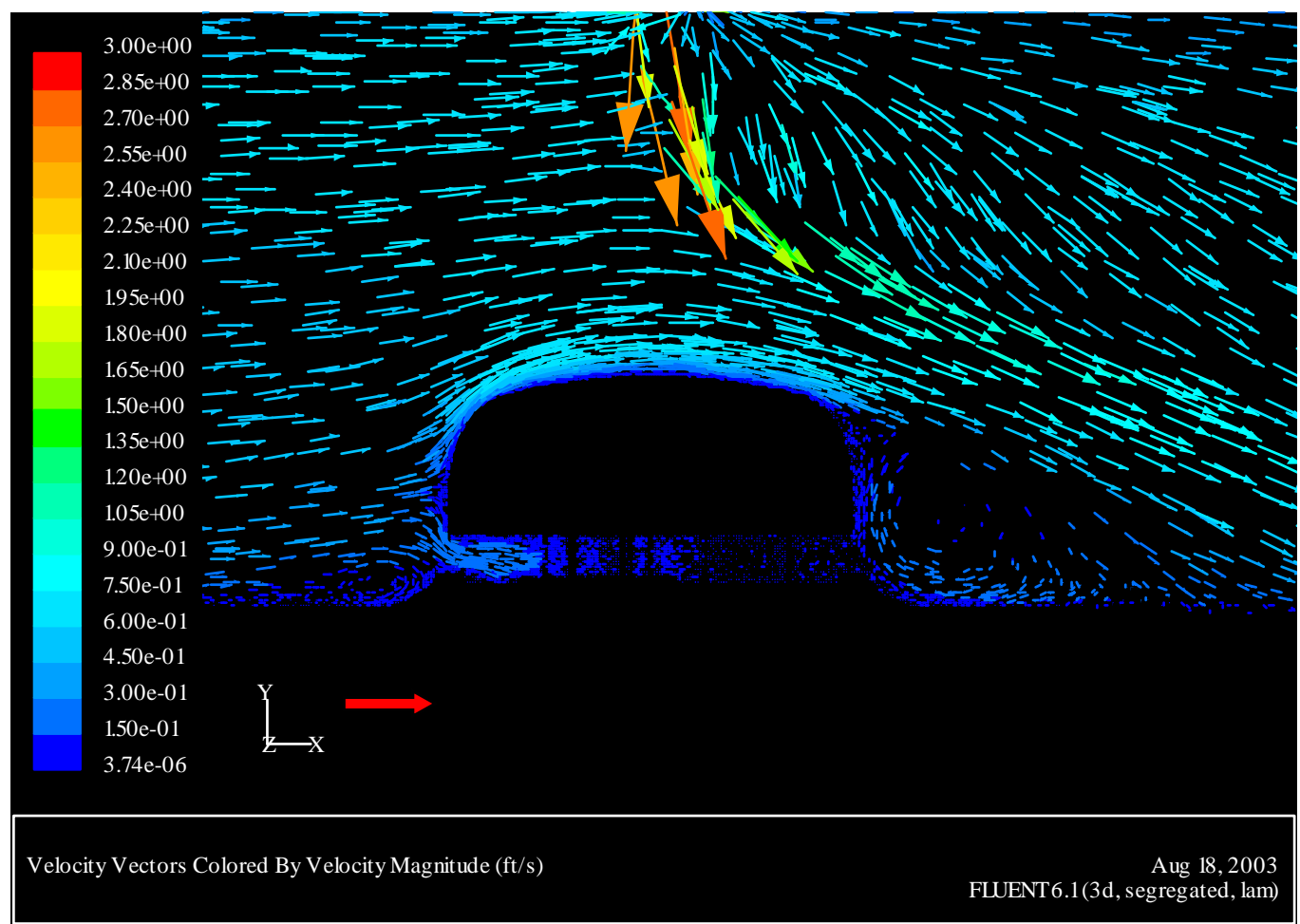

(a.) Laminar Model

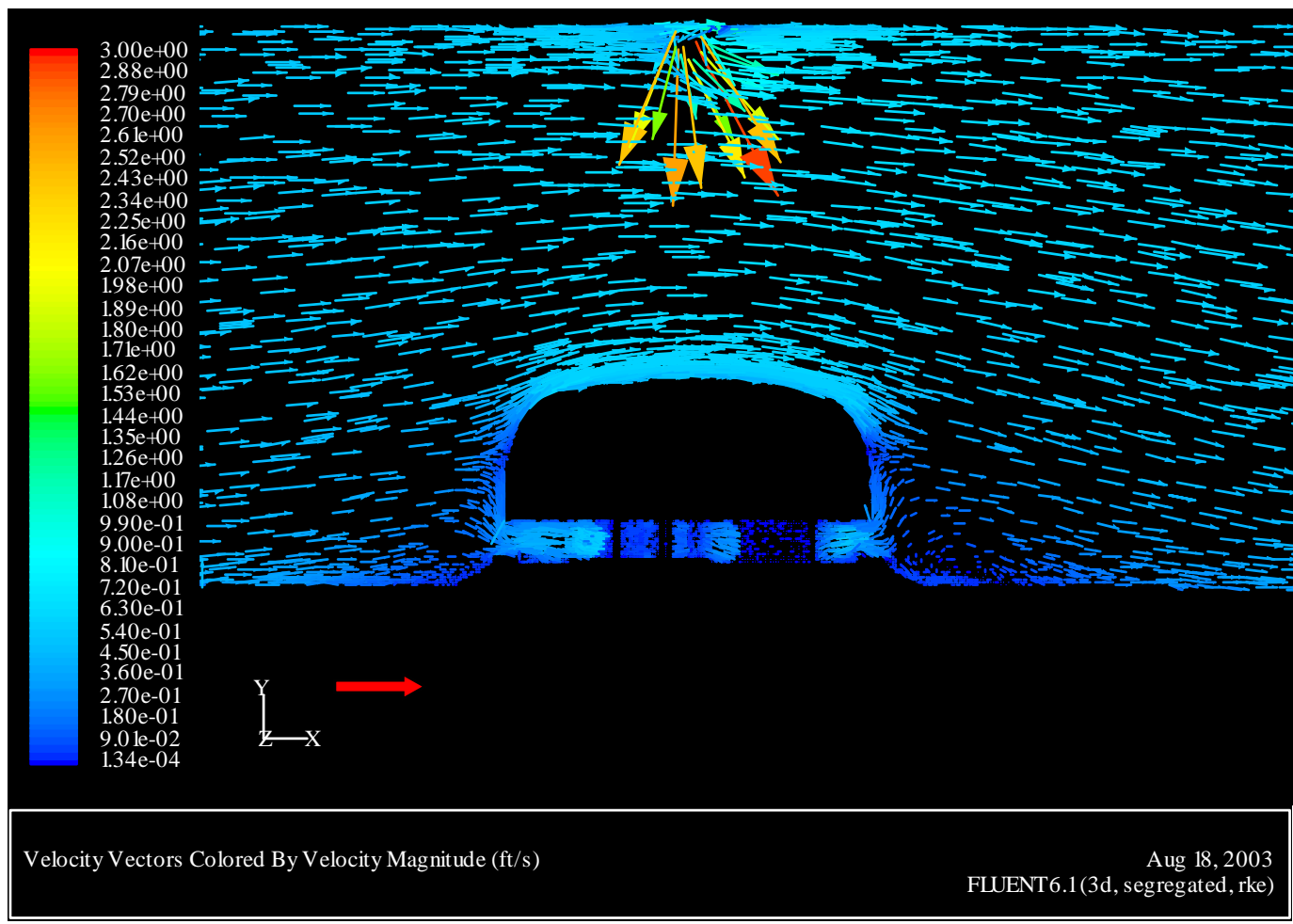

(a.) Realizable $\kappa-\varepsilon$ Turbulence Model

Figure 6.6.8: A velocity vector comparison between the (a.) laminar and (b.) realizable $\kappa-\varepsilon$ turbulence models for the $z=9$ in plane. 


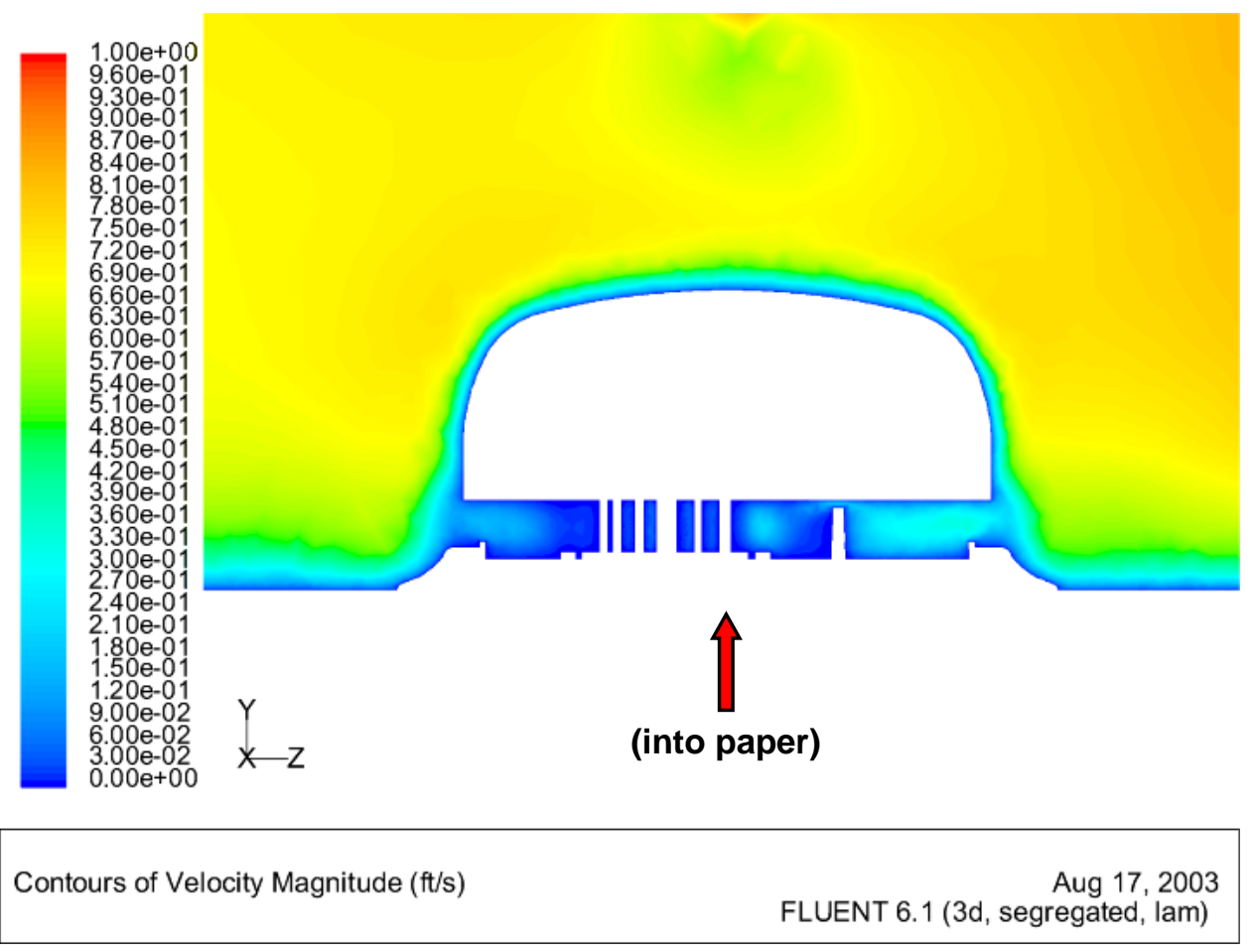

(a.) Laminar Model

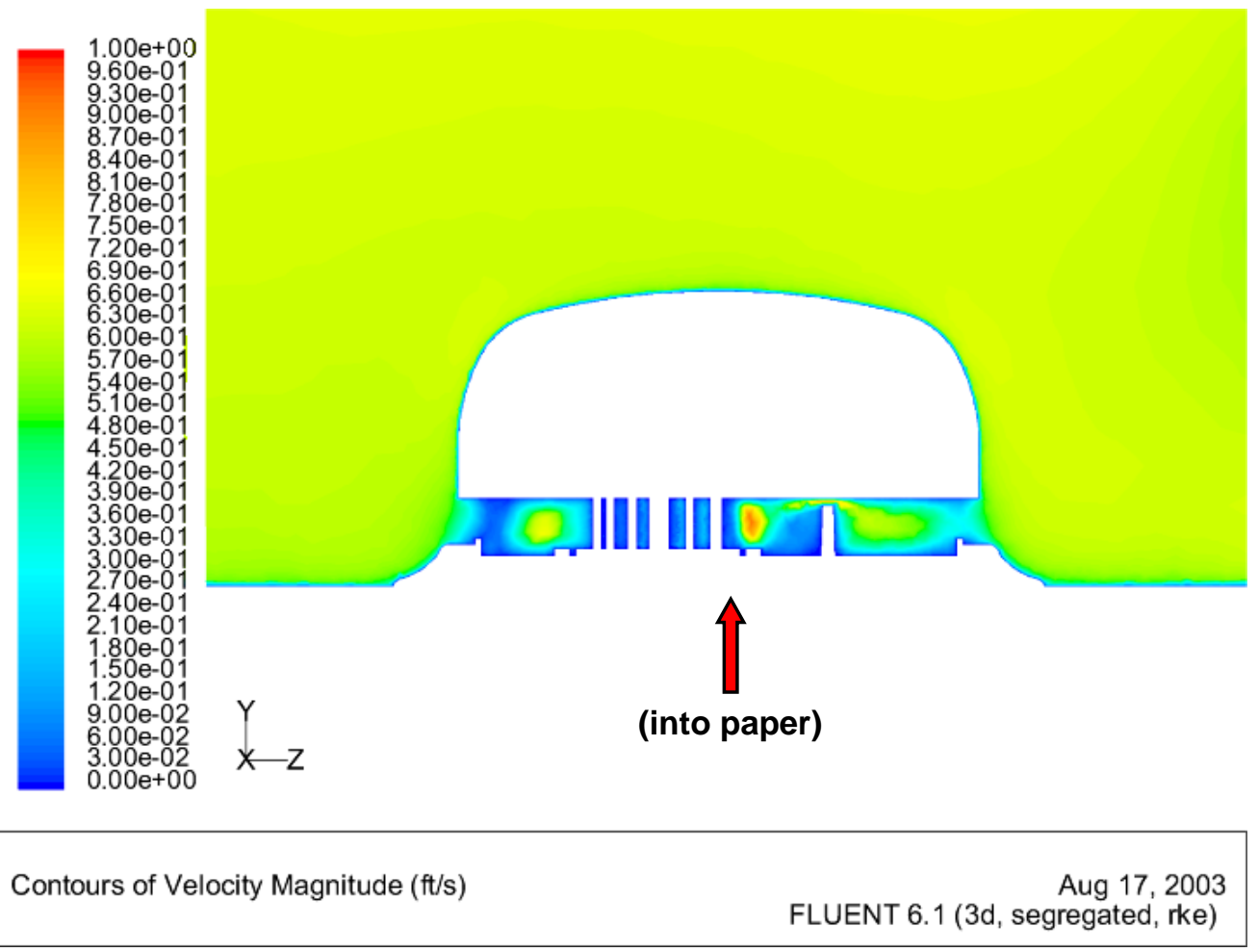

(a.) Realizable $\kappa-\varepsilon$ Turbulence Model

Figure 6.6.9: A comparison of velocity contours between the (a.) laminar and (b.) realizable $\kappa-$ $\varepsilon$ turbulence models for the $x=18$ in plane visualizing the flow about the SA302. 


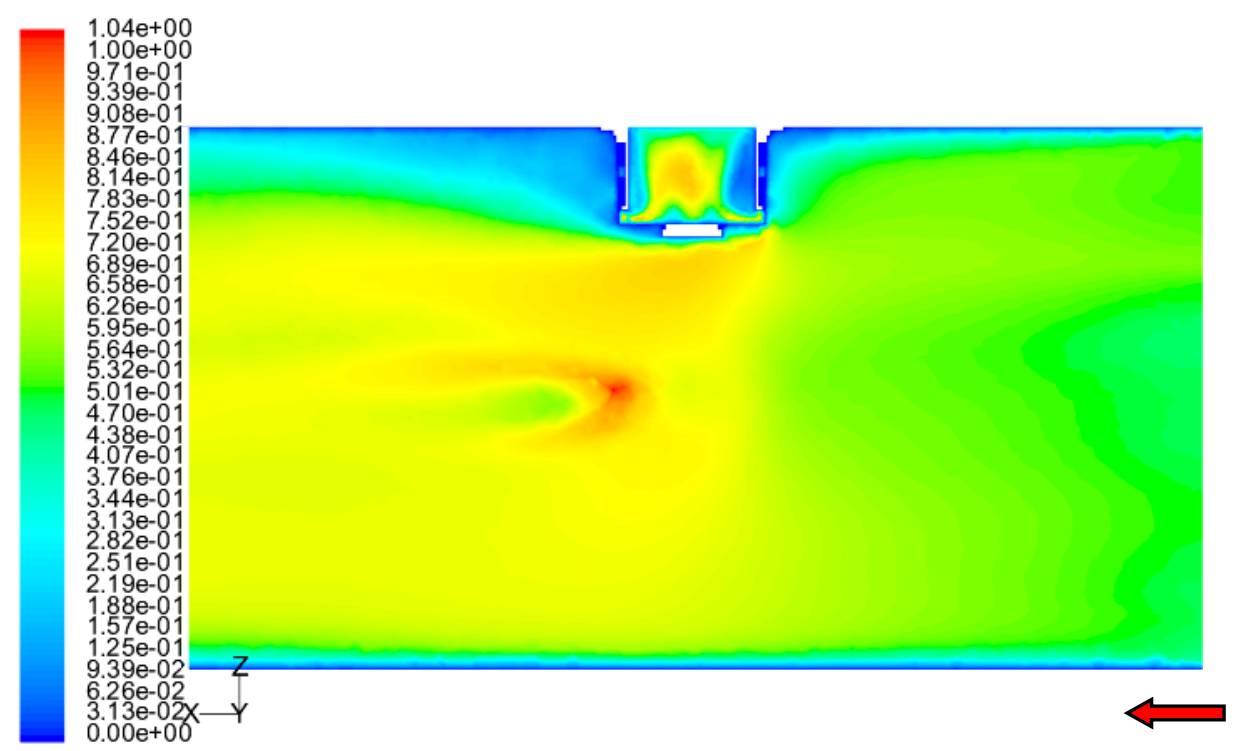

Contours of Velocity Magnitude (ft/s)

Aug 17, 2003 FLUENT 6.1 (3d, segregated, lam)

(a.) Laminar Model

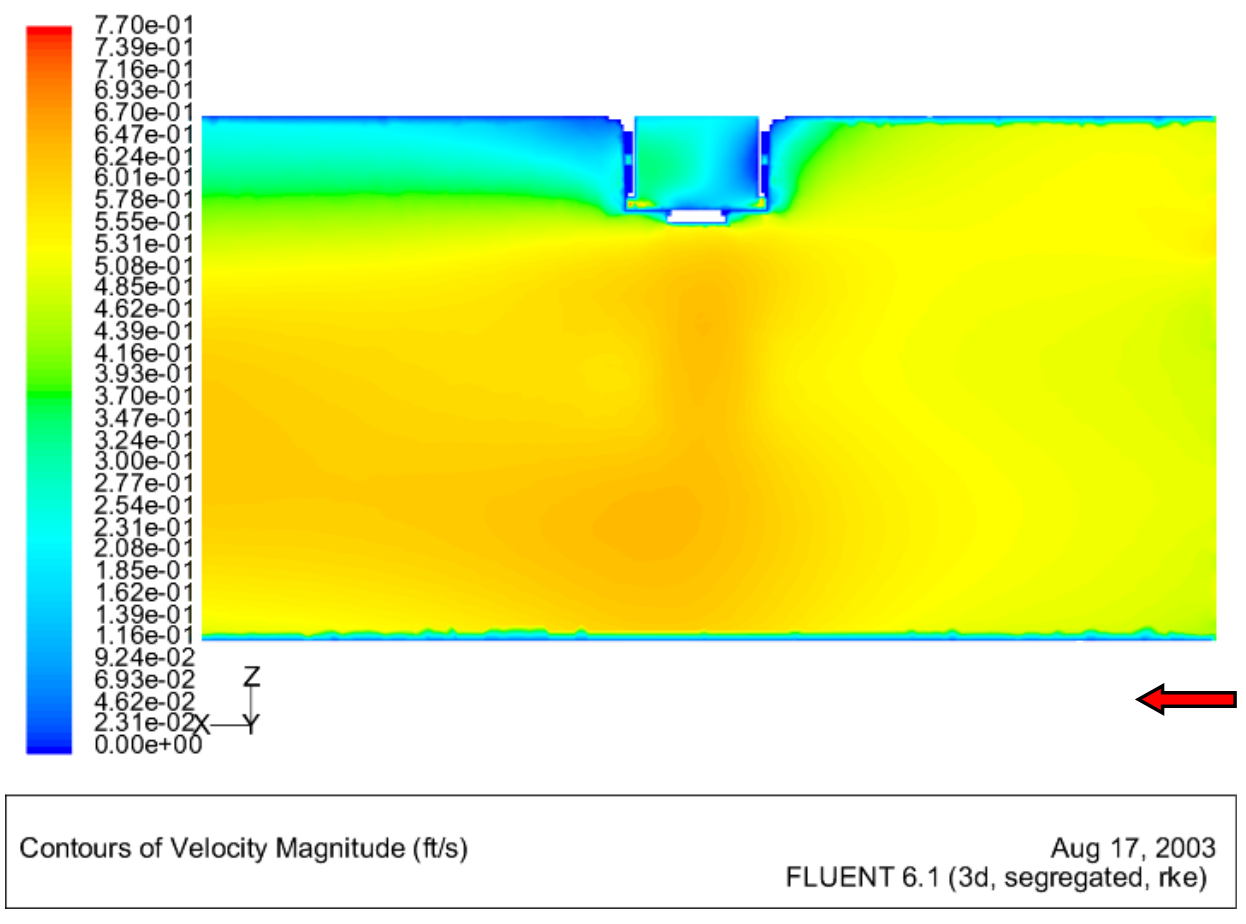

(a.) Realizable $\kappa-\varepsilon$ Turbulence Model

Figure 6.6.10: A comparison between the (a.) laminar and (b.) realizable $\kappa-\varepsilon$ turbulence models for the $y=3.5$ in plane (mid-plane of the test section). 
The following set of Figures 6.6.11 through 6.6.75 depict the final results of the grid independent $125 \times 63 \times 25$ ( $\approx 530,000$ node $) 2^{\text {nd }}$ Order model for all orientations. Within these figures lay velocity vector and contour plots that describe not only the flow field surrounding the smoke detector, but the intricate flow patterns calculated inside the detector housing as well.

Figures 6.6.11 through 6.6.31 show complete gathered results for the $0^{\circ}$ detector orientation. In Figure 6.6.11, the unsymmetrical wake of the detector is once again evident as witnessed in the preliminary first order cases, although this time with more nearly twice as much grid resolution. In Figure 6.6.12, a velocity vector plot visualizes a reverse flow issue encountered with all of the orientation models. In examining the upper left hand corner of the plot, one can see a re-circulating region induced by the presence of the extended region of the upper side of the detector wake interacting with the upstream influences of the MIC body. All models, although eventually converged with nearly zero if any reverse flow at the pressure outlet boundary condition, dealt with this issue. Because LDA data primarily consisted of the areas close to the detector, comparative data were not available to make any straightforward conclusions on whether or not this flow reversal realistically exists. However, because it is significantly downstream of the main area of focus, its presence propagating back an unrealistic affect on the interior of the detector to ultimately distort critical results is assumed to be negligible.

In Figures 6.6.13 and 6.6.14, the wake is more heavily described showing a close inspection of the unsymmetrical wake using both a contour and velocity plot. The unsymmetrical wake, save for the detector orientation, is very repeatable in all orientations.

Figures 6.6.15 and 6.6.16 report the actual flow characterization inside of the SA302 at $0^{\circ}$ orientation. It will eventually be seen that the $0^{\circ}$ orientation permits the highest flow velocities deepest into the detector because the flow is not initially impeded by either of the sensors. The only obstacle is the main flow deflector controlling the flow direction toward each respective source. Here, the deflector seems to play a minor role in delivering a comparable amount of smoke particles to each sensor with respect to the oncoming flow direction because they are side by side one another with minimal forward blockage.

Figures 6.6.17 through 6.6.20 report the velocity contour and vector plots inside the ionization and photoelectric chambers, respectively. Here, as will be a common theme for 
nearly all orientations, shows the ionization chamber with substantially slower velocities ( $\approx 1$ to $2 \mathrm{ft} / \mathrm{min}$ ) as compared to that of the photoelectric chamber ( $\approx 3$ to $5 \mathrm{ft} / \mathrm{min}$ ). This could be due to a number of geometric reasons, but more likely due to the more shielded design the ionization chamber design presents compared to that of the photoelectric chamber, as well as the main flow deflector in this instance channeling flow more normally to the sides of the photoelectric chamber. The ionization chamber, in this orientation, receives smoke particles off a re-circulating region created from the angle of it to the main flow deflector.

Figure 6.6.21 and 6.6.22 depict similar upstream results as those presented earlier, only with more resolution due to the increased number of cells. Figure 6.6.23 through 6.6.25 report the presence of the probe inlet again also with more refinement. Figure 6.6.25 shows the entire $y-z$ centerline plane of the test section depicting the vertical velocity vectors from the probe velocity inlet and how it affects the overall flow stream. This concept will be visualized more so later on.

Figures 6.6.26 through 6.6.29 depict test section visualization denoting each main components influence on the entire model. In Figure 6.6.26, pathlines are released from about one in above the test section floor near the main inlet, allowing them to disperse through the flow field following the path of essentially, what a massless particle would endure in a velocity flow field. From this figure, the unsymmetrical wake clearly evident in three-dimensional space. It could be theorized here that that the unsymmetrical wake could be due in large part to the presence of the MIC. Figure 6.6.27 depicts the chaotic path the pathlines flowing through MIC take upon being released a couple of inches from the test section's main inlet. Figure 6.6.28 shows the interaction between the smoke detector's wake and the large velocity gradient present (starting at $\approx 390 \mathrm{ft} / \mathrm{min}$ ) at the mouth of the probe inlet. As one can see, the effects of the small diameter hole $\left(17 / 64^{\text {th }}\right.$ in) propagates deep into the system. Figure 6.6.29 shows all three major influences in one setting, denoting a possible overall effect on the asymmetry present in the detector's wake.

Figures 6.6.30 and 6.6.31 show pathlines moving through the actual detector sensors. Figure 6.6.30 shows a top down perspective with a basic rake of 100 pathlines generally placed at the mouth of the detector inlet, showing how the flow field would transport smoke particles. Figure 6.6.31 seeds more of a special interest area right in front of the sensors, showing pathlines invading each sensing chamber. 


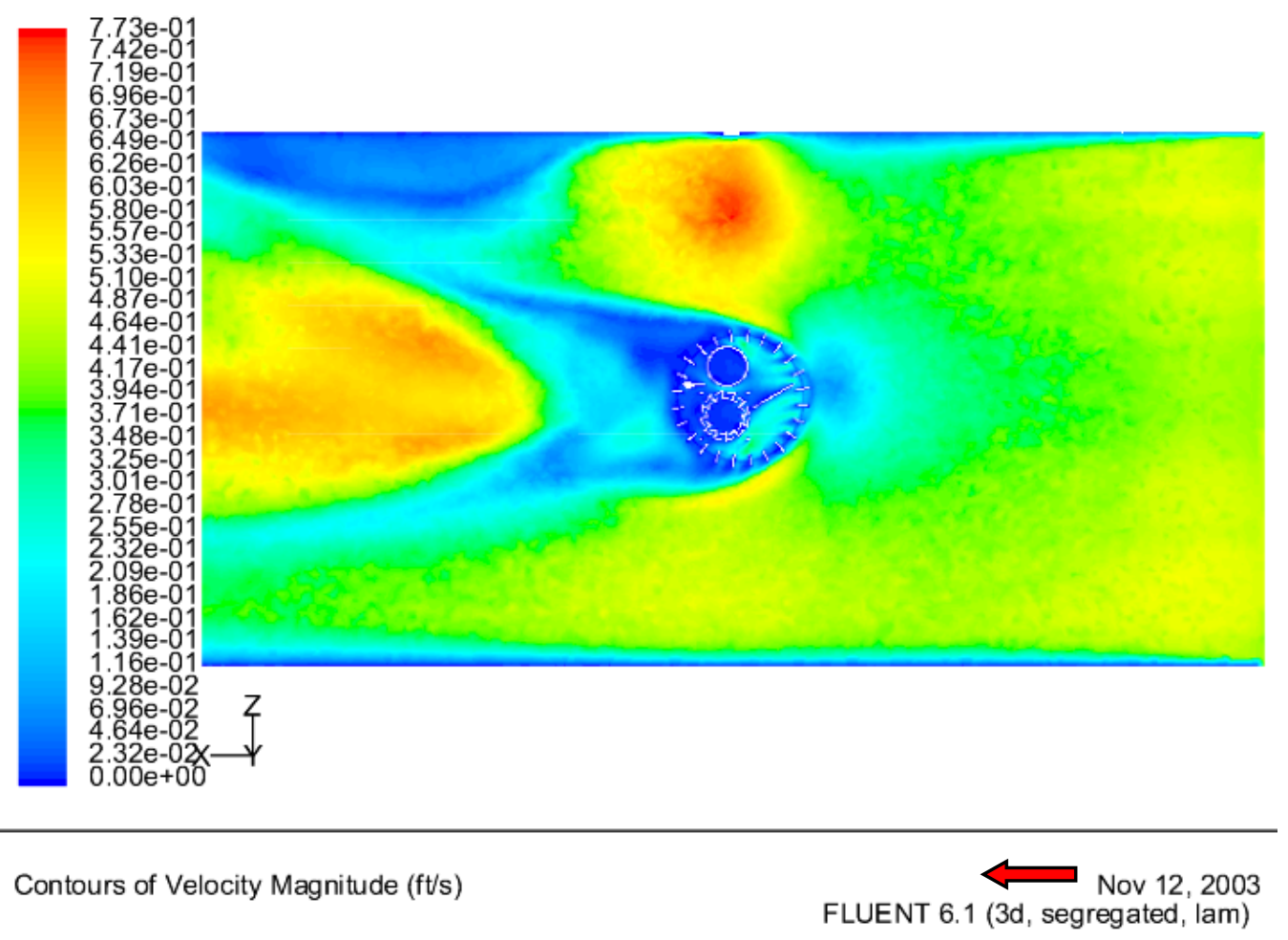

Figure 6.6.11: A velocity contour plot using the laminar model depicting the $y=0.50$ in plane for the $0^{\circ}$ orientation.

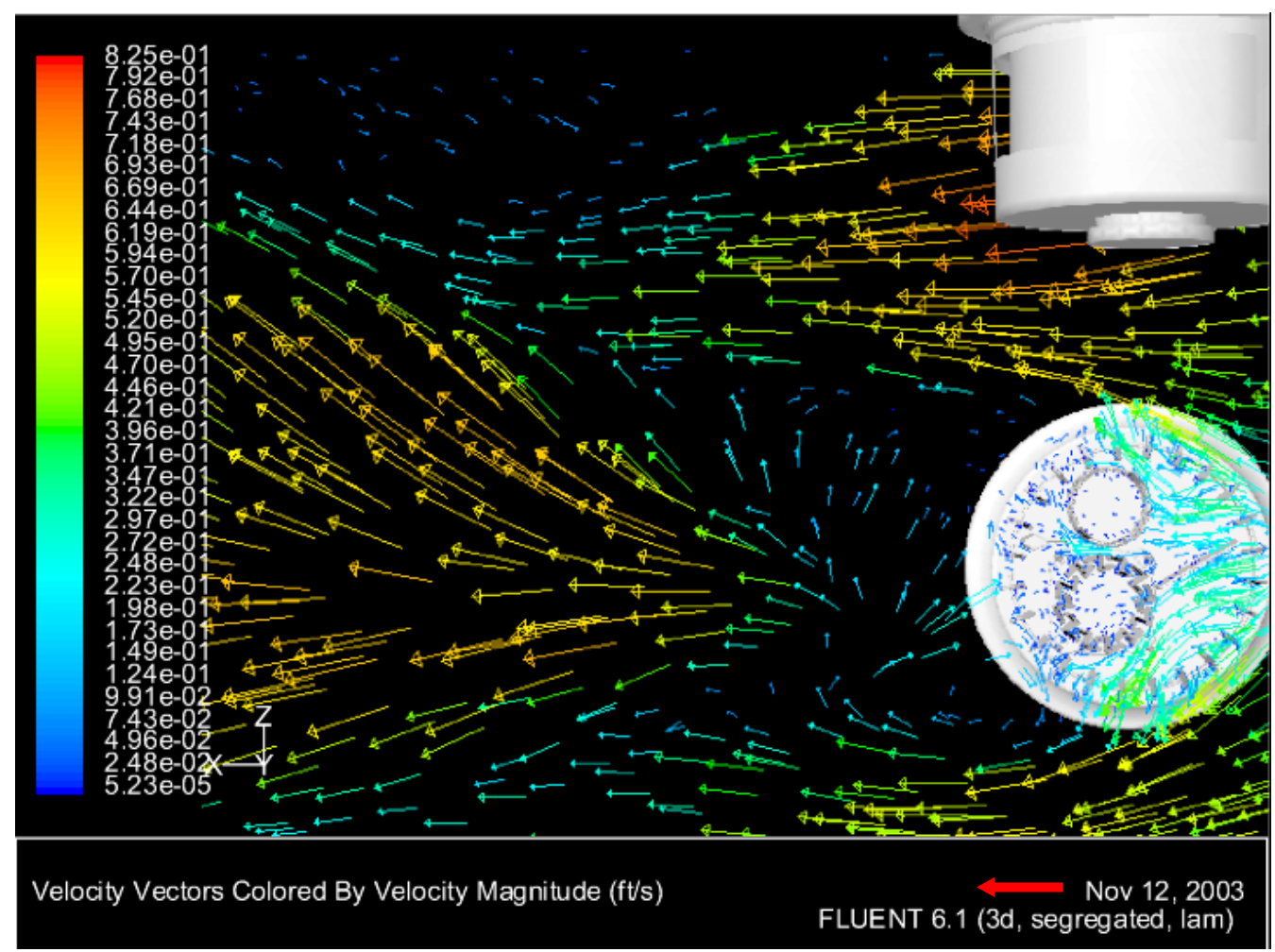

Figure 6.6.12: A velocity vector plot using the laminar model depicting the wake of the detector approaching the pressure outlet boundary in the $y=0.50$ in plane. 


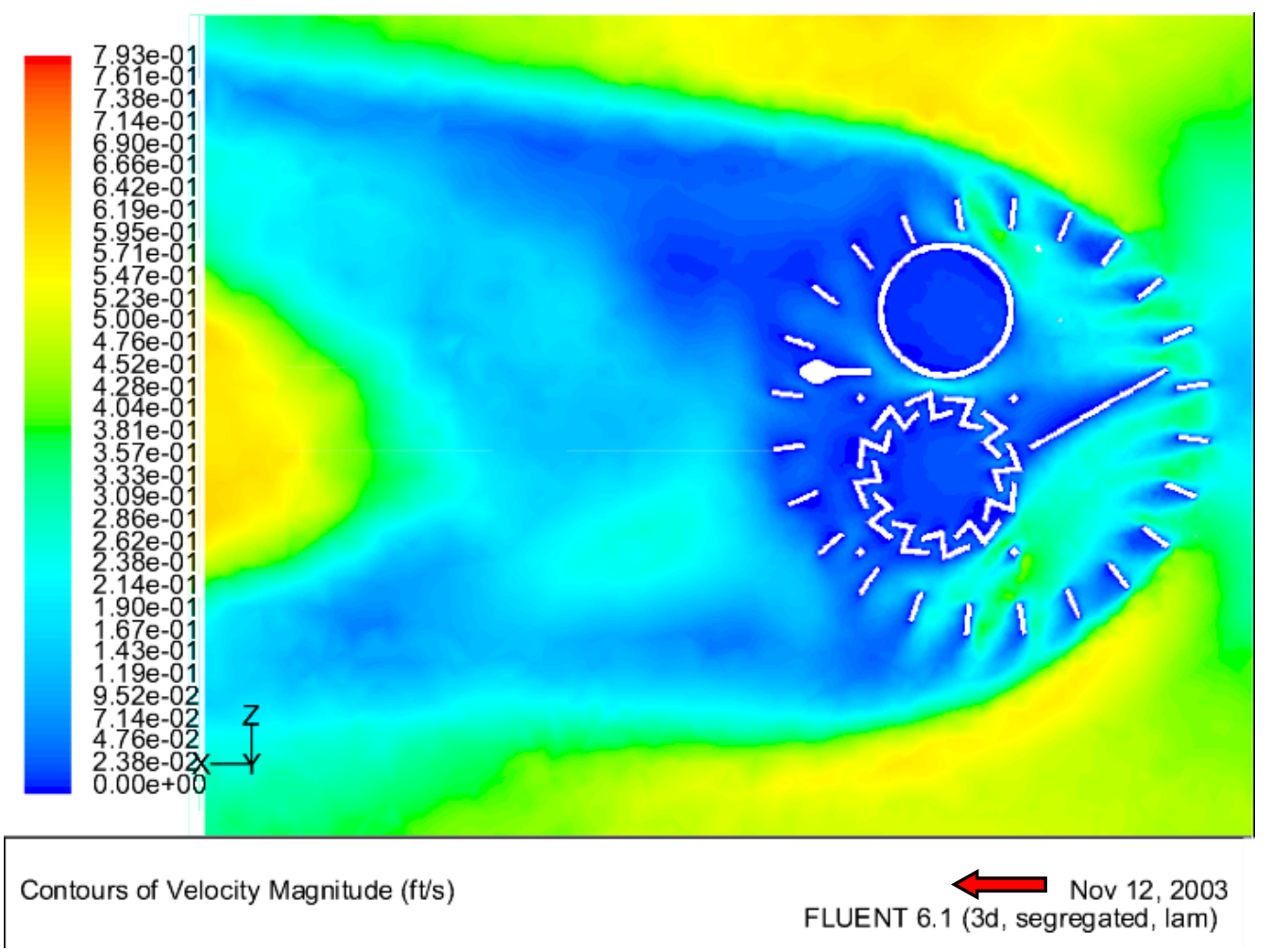

Figure 6.6.13: A velocity contour plot using the laminar model depicting the detector and its wake in the $y=0.50$ in plane for the $0^{\circ}$ orientation.

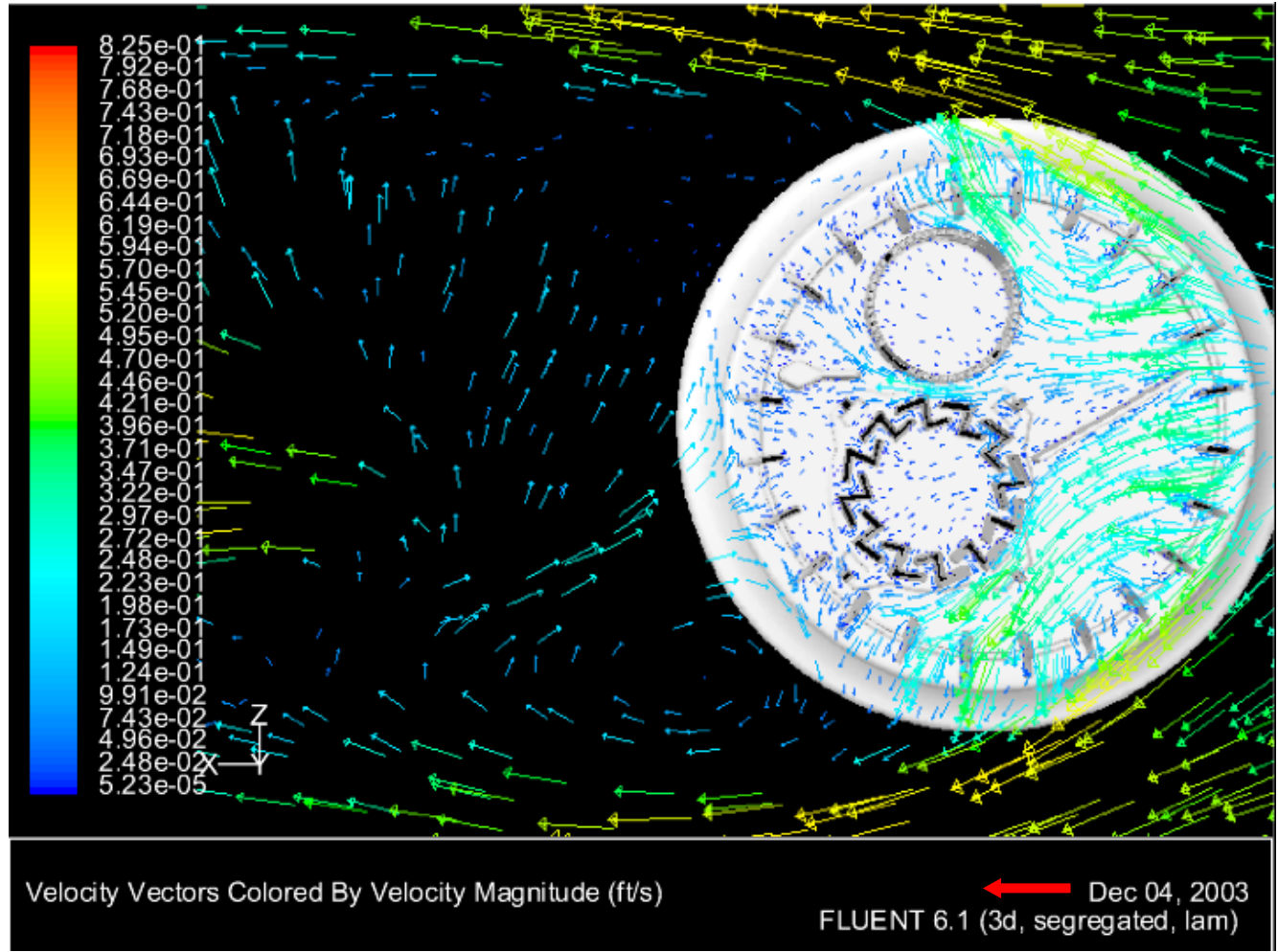

Figure 6.6.14: A velocity vector plot using the laminar model depicting the wake of the detector approaching the pressure outlet boundary in the $y=0.50$ in plane ( $0^{\circ}$ Orientation). 


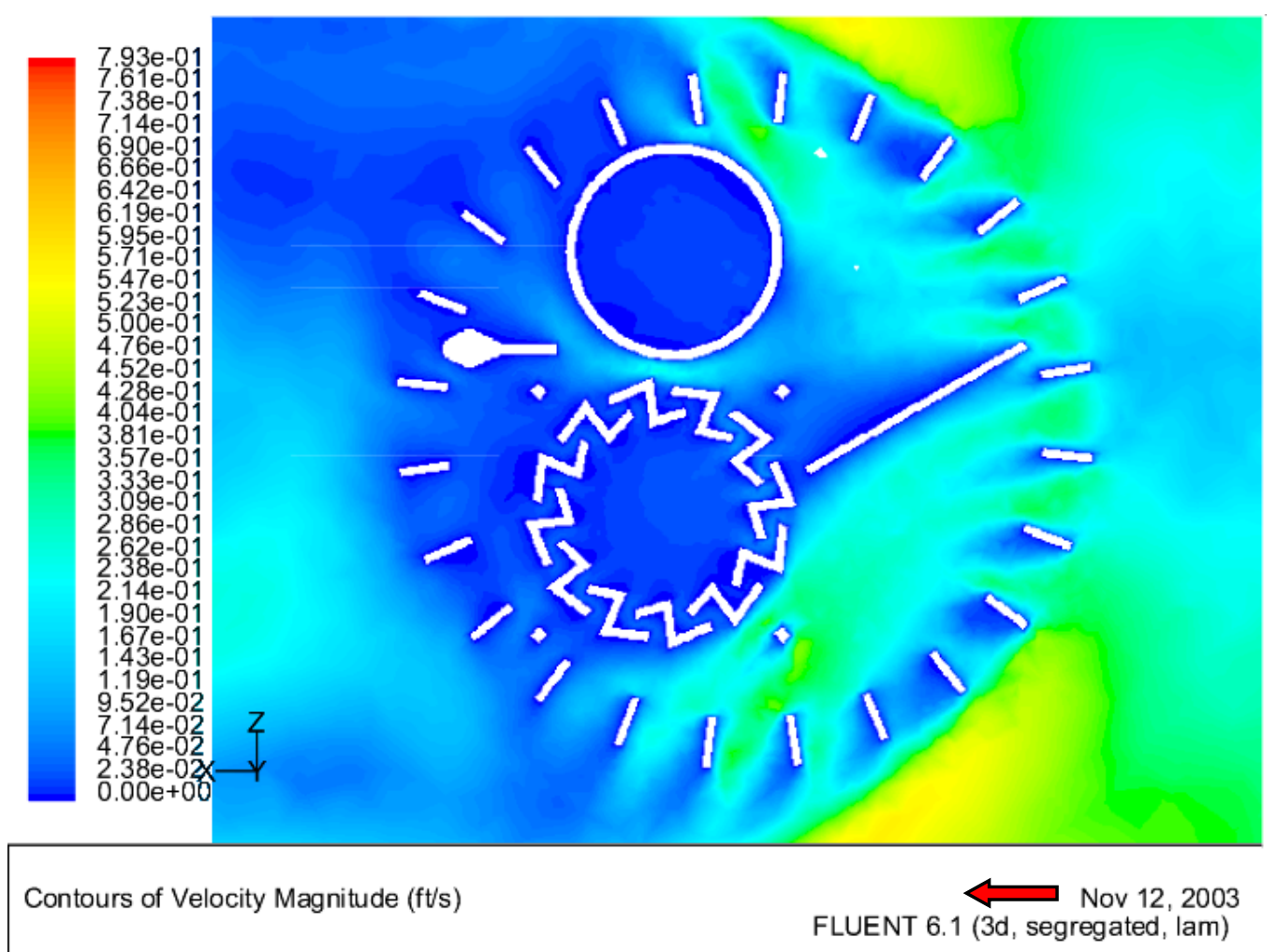

Figure 6.6.15: A velocity contour plot using the laminar model depicting the detector in the $y=0.50$ in plane for the $0^{\circ}$ orientation.

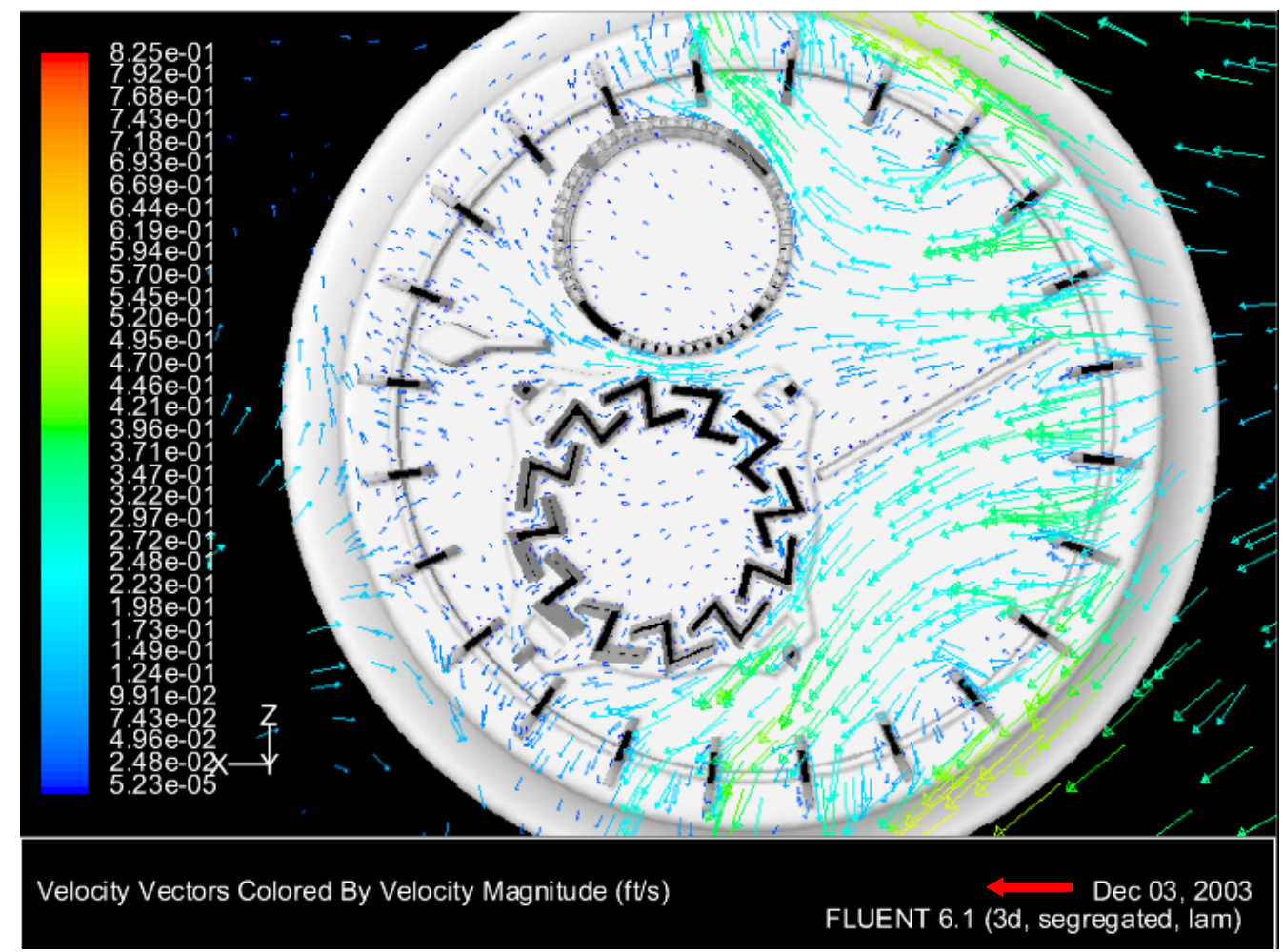

Figure 6.6.16: A velocity vector plot using the laminar model depicting the detector in the $y=$ 0.50 in plane for the $0^{\circ}$ orientation. 

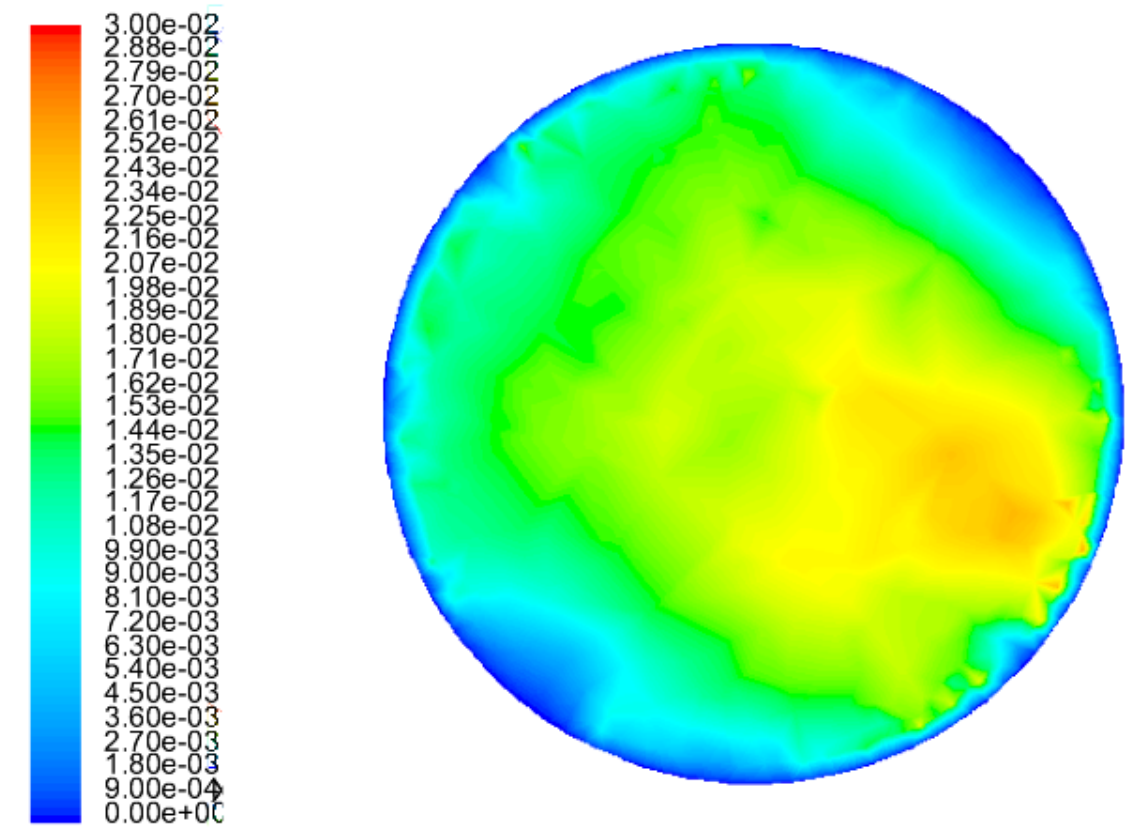

Contours of Velocity Magnitude (ft/s)

FLUENT 6.1 (3d, segregated, lam)

Figure 6.6.17: A velocity contour plot using the laminar model depicting the ionization chamber in the $y=0.50$ in plane for the $0^{\circ}$ orientation.

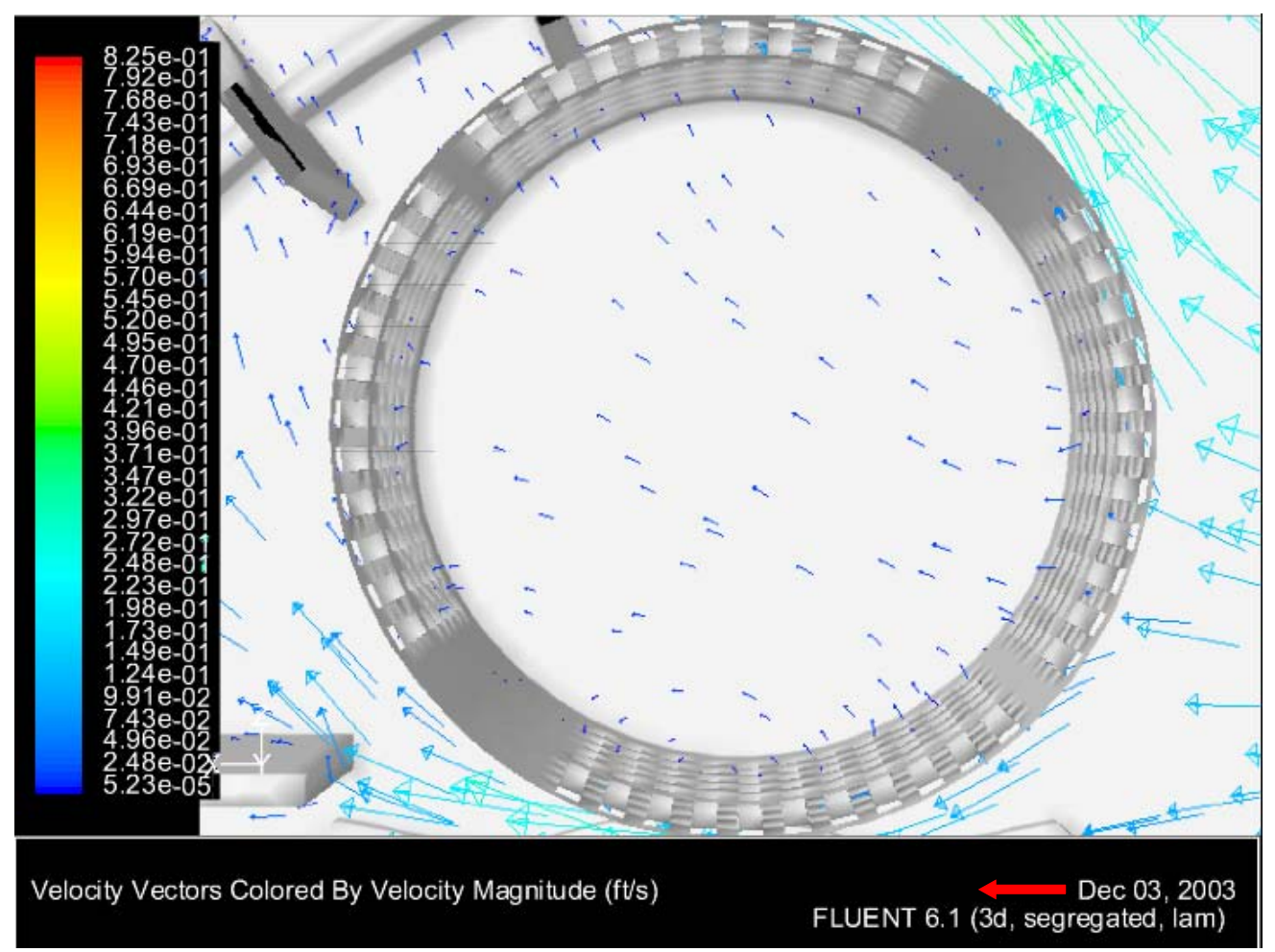

Figure 6.6.18: A velocity vector plot using the laminar model depicting the ionization chamber in the $y=0.50$ in plane for the $0^{\circ}$ orientation. 


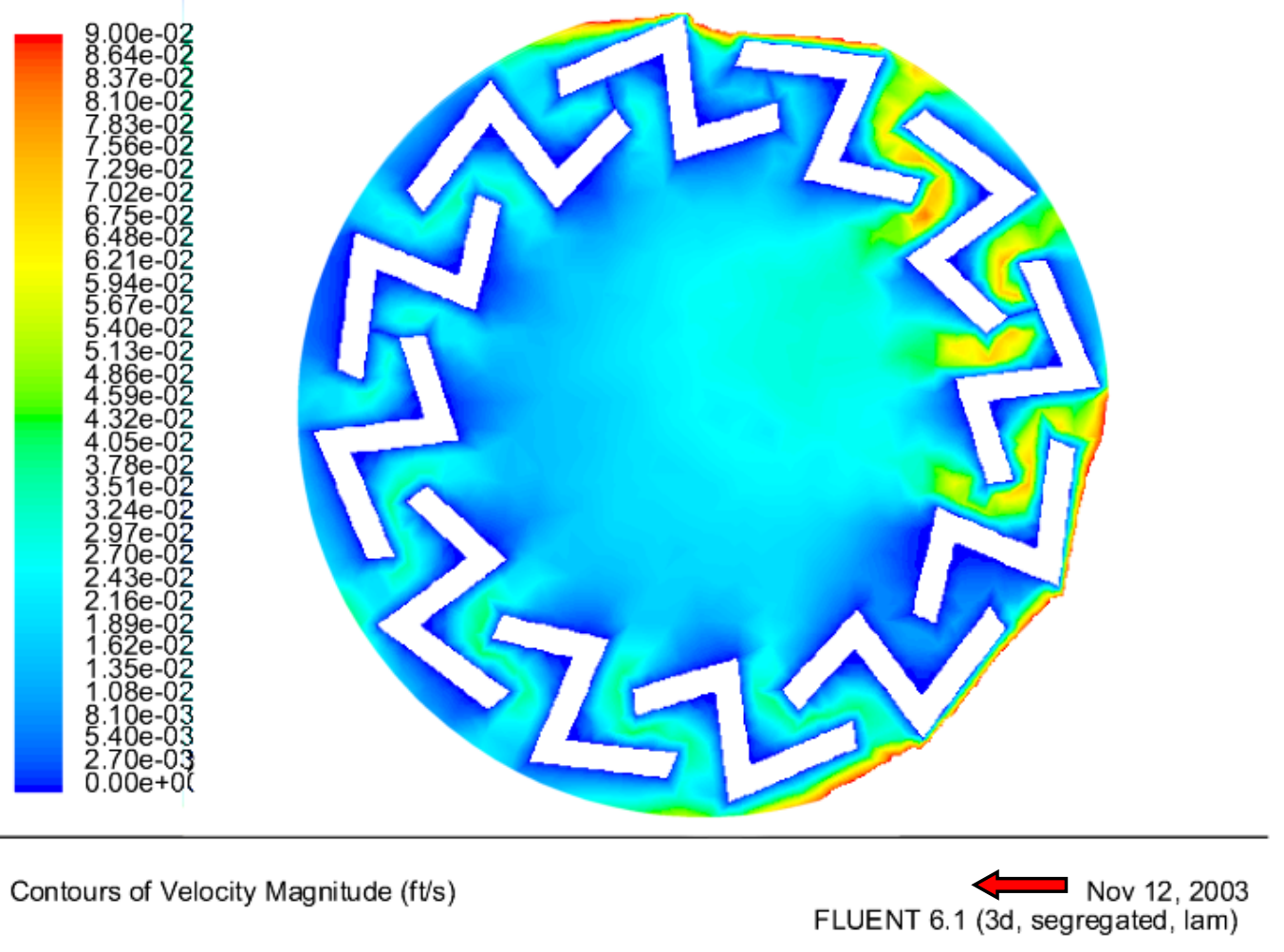

Figure 6.6.19: A velocity contour plot using the laminar model depicting the photoelectric chamber in the $y=0.50$ in plane for the $0^{\circ}$ orientation.

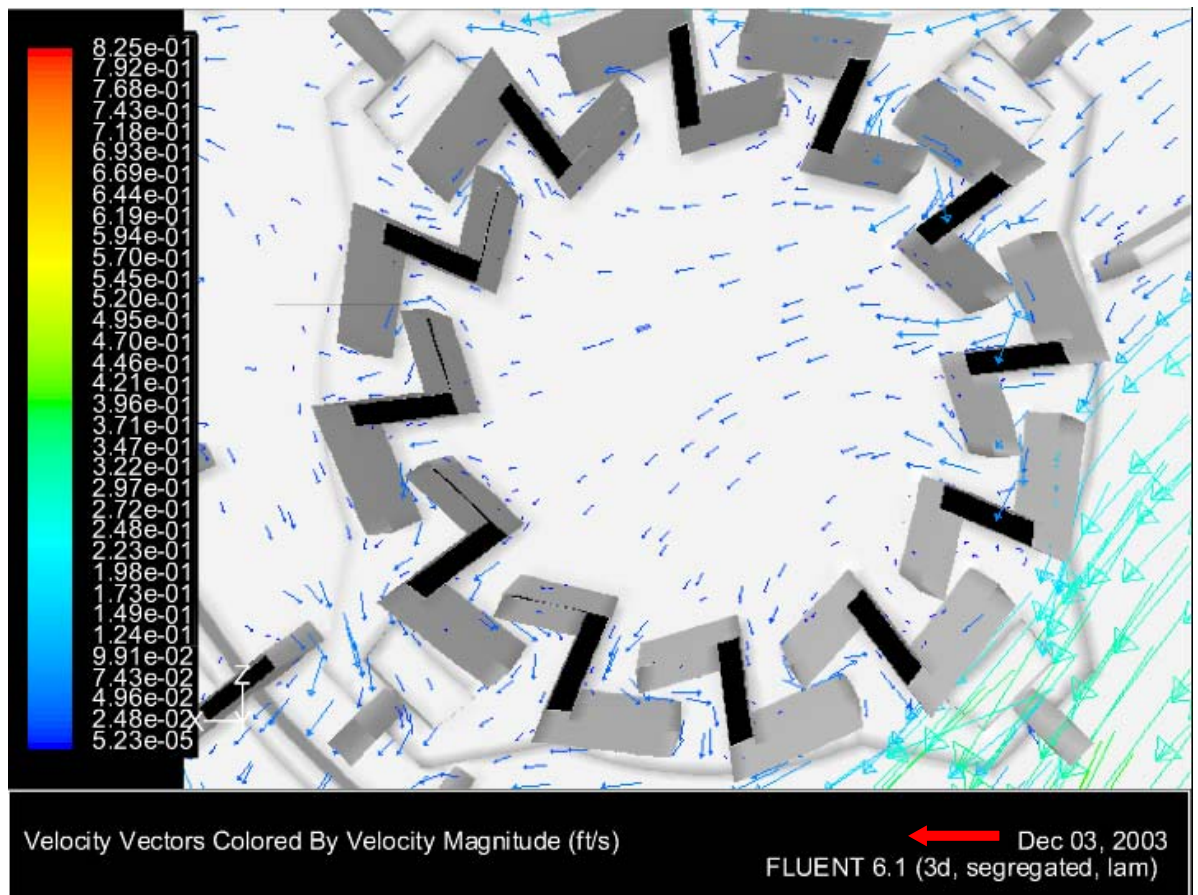

Figure 6.6.20: A velocity vector plot using the laminar model depicting the photoelectric chamber in the $y=0.50$ in plane for the $0^{\circ}$ orientation. 


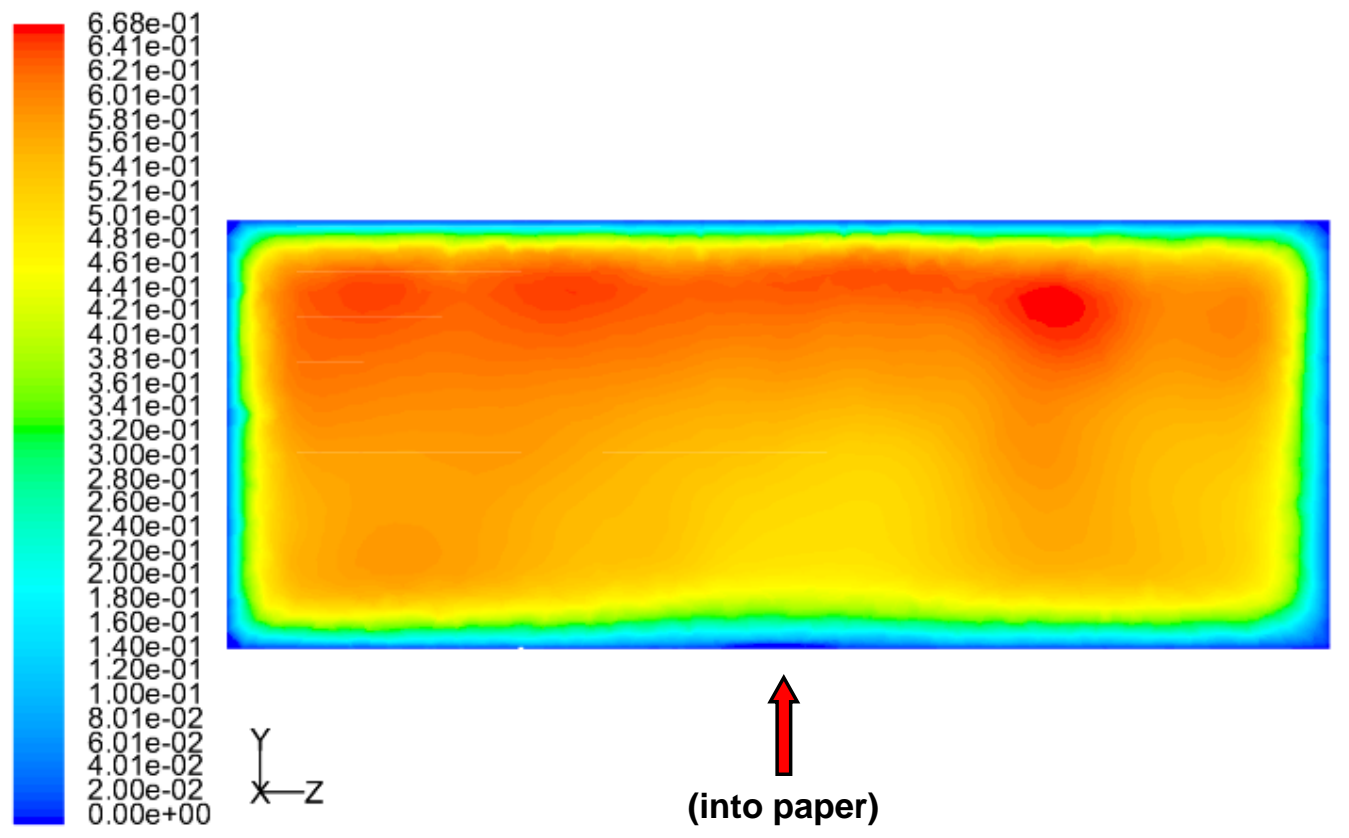

Contours of Velocity Magnitude (ft/s)

Nov 12, 2003

FLUENT 6.1 (3d, segregated, lam)

Figure 6.6.21: A velocity contour plot using the laminar model depicting the $x=12$ in approach plane.
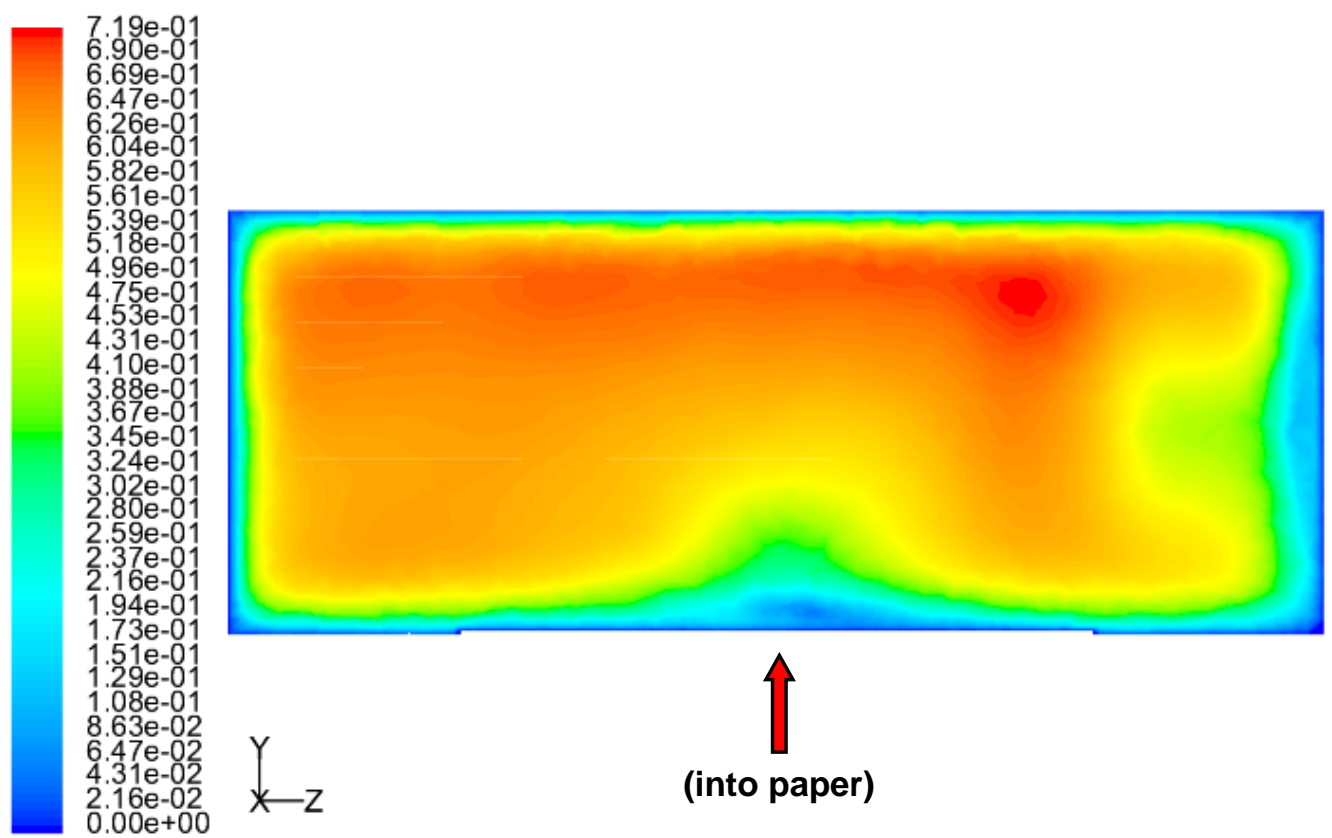

(into paper)

Contours of Velocity Magnitude ( $\mathrm{ft} / \mathrm{s})$

Nov 12, 2003

FLUENT 6.1 (3d, segregated, lam)

Figure 6.6.22: A velocity contour plot using the laminar model depicting the $x=15$ in approach plane. 


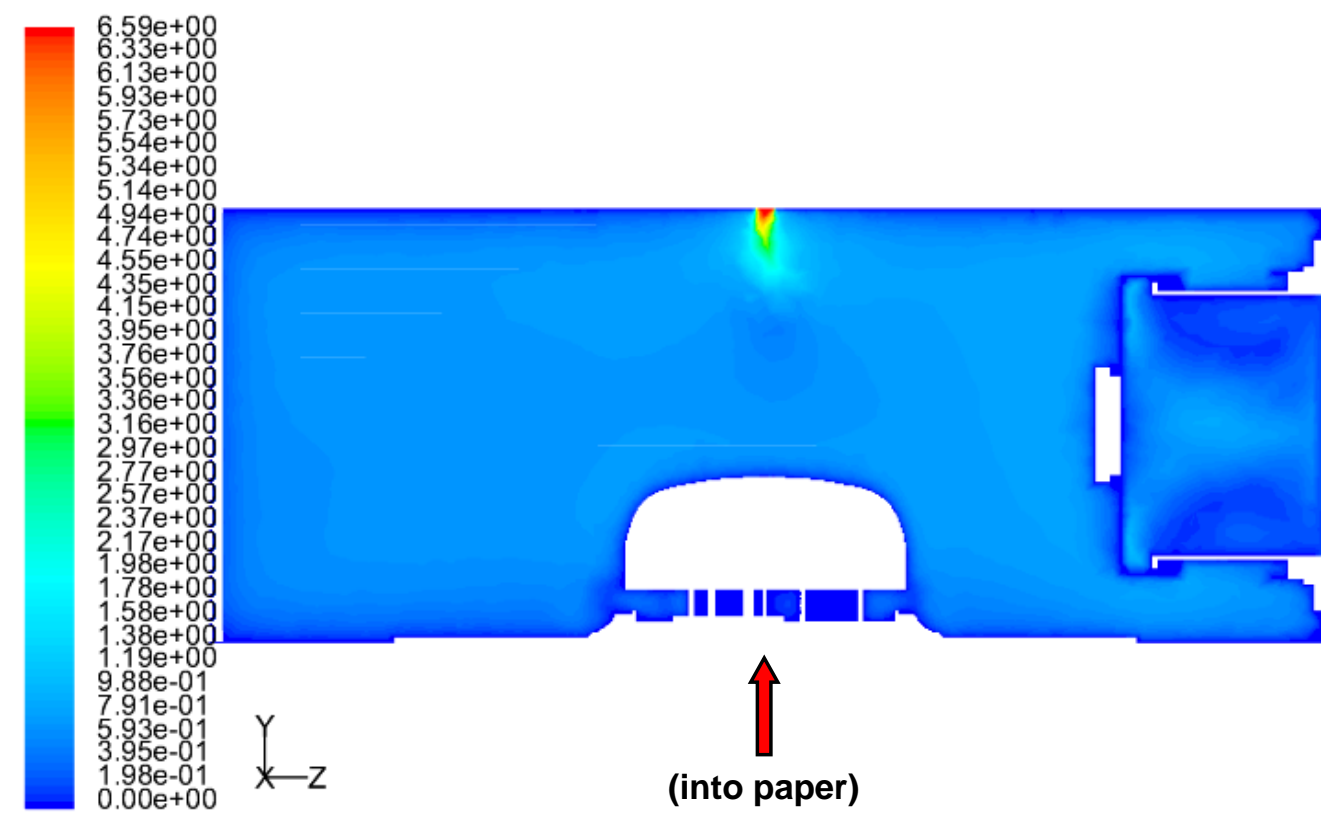

Figure 6.6.23: A velocity contour plot using the laminar model depicting the $x=18$ in plane (center cut of the SA302 detector and slightly off-center that of the MIC).

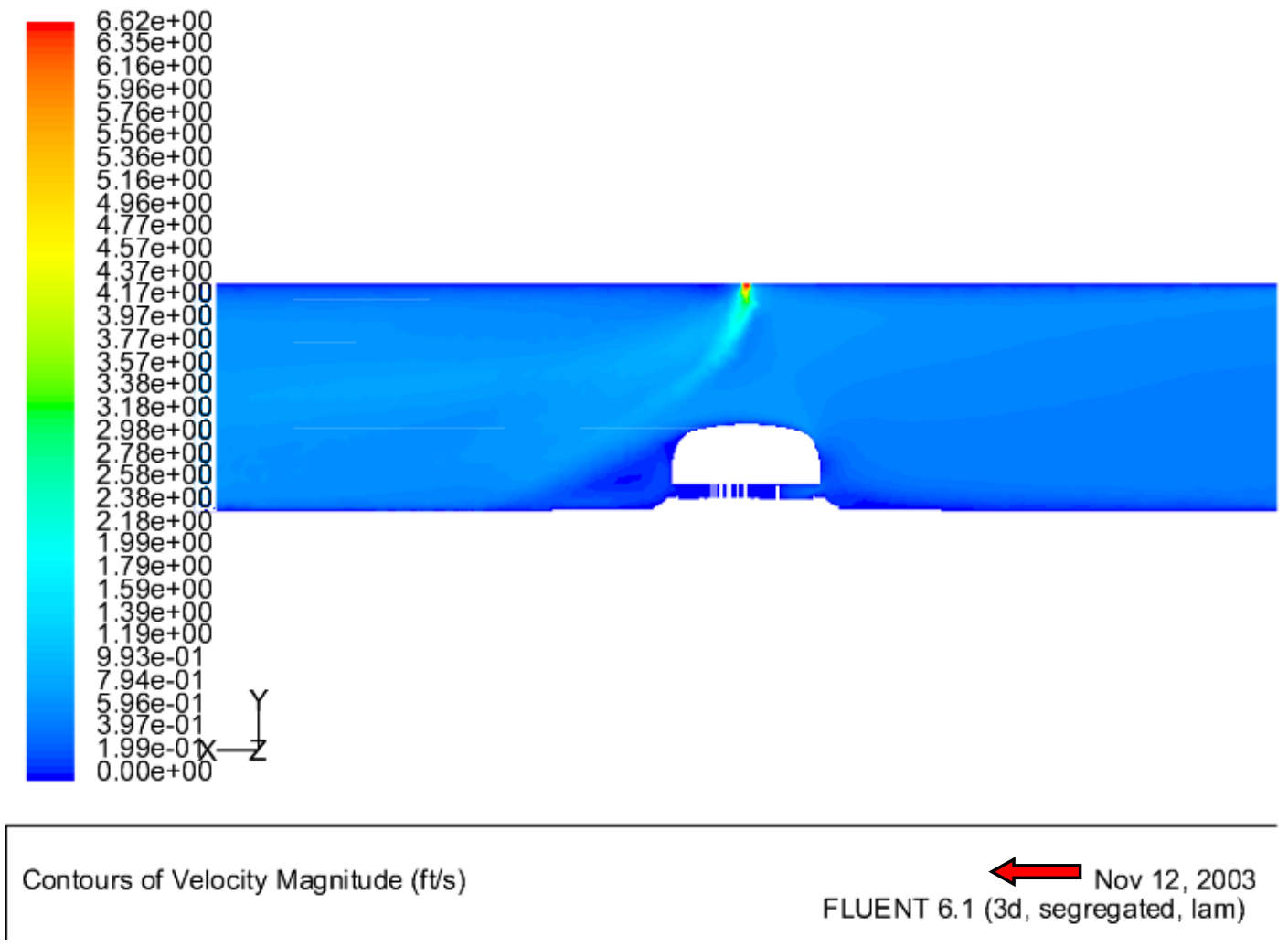

Figure 6.6.24: A velocity contour plot using the laminar model depicting the $z=9$ in plane (center cut of the SA302 detector and probe inlet). 


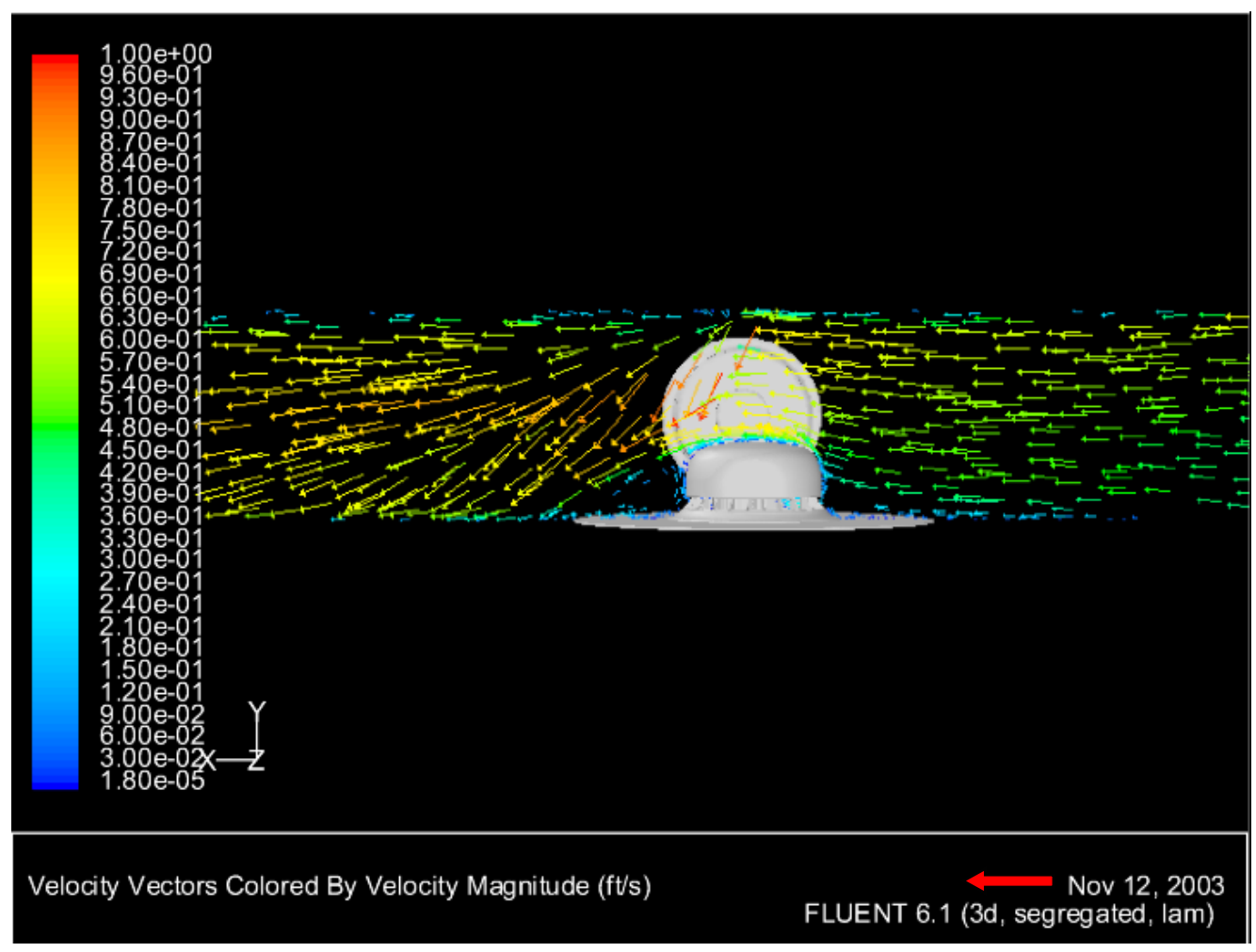

Figure 6.6.25: A velocity vector plot using the laminar model depicting the $z=9$ in plane (center cut of the SA302 detector and probe inlet).

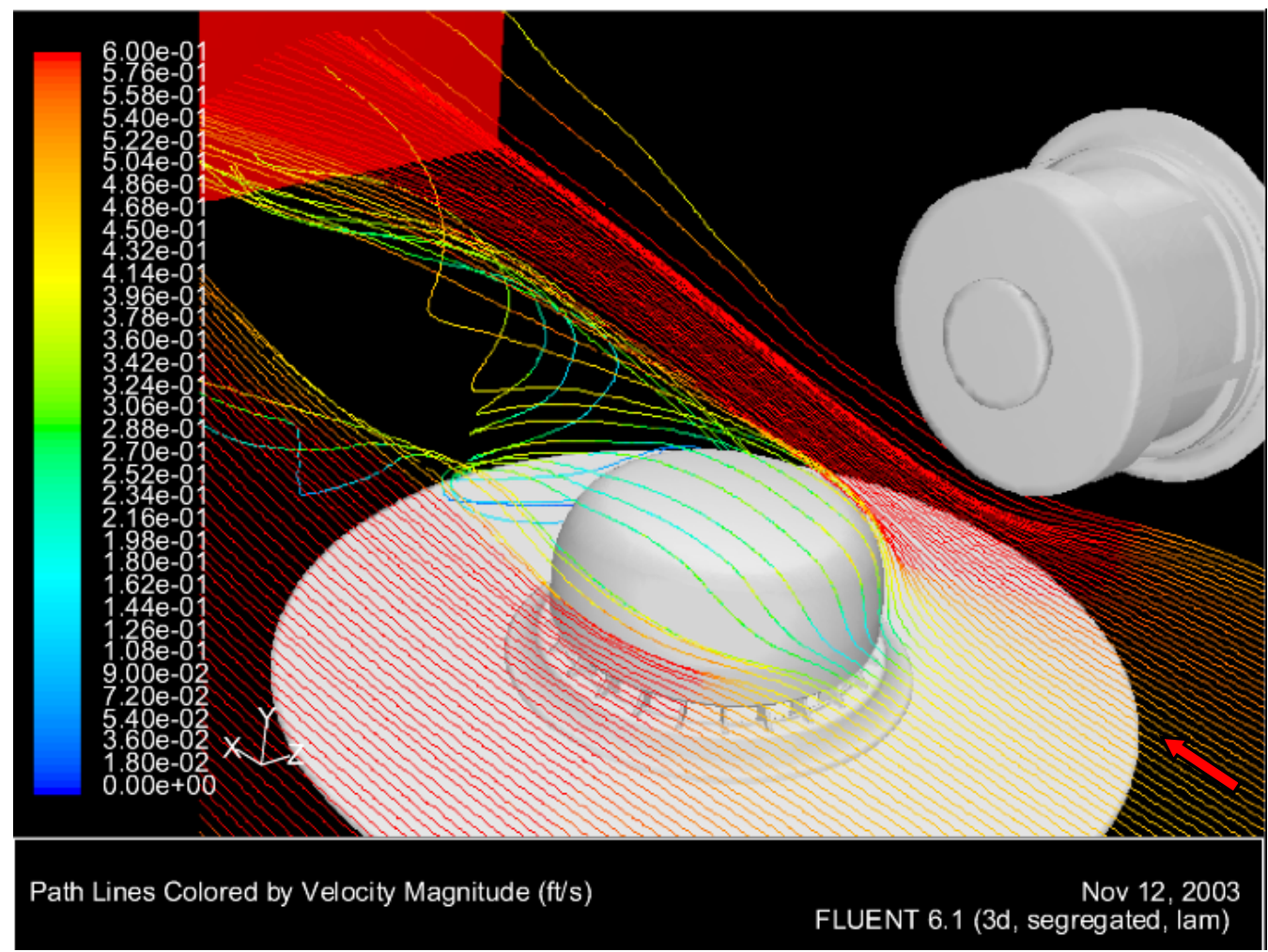

Figure 6.6.26: Test section flow visualization showing a pathline injection 1 in above the floor near the main inlet. 


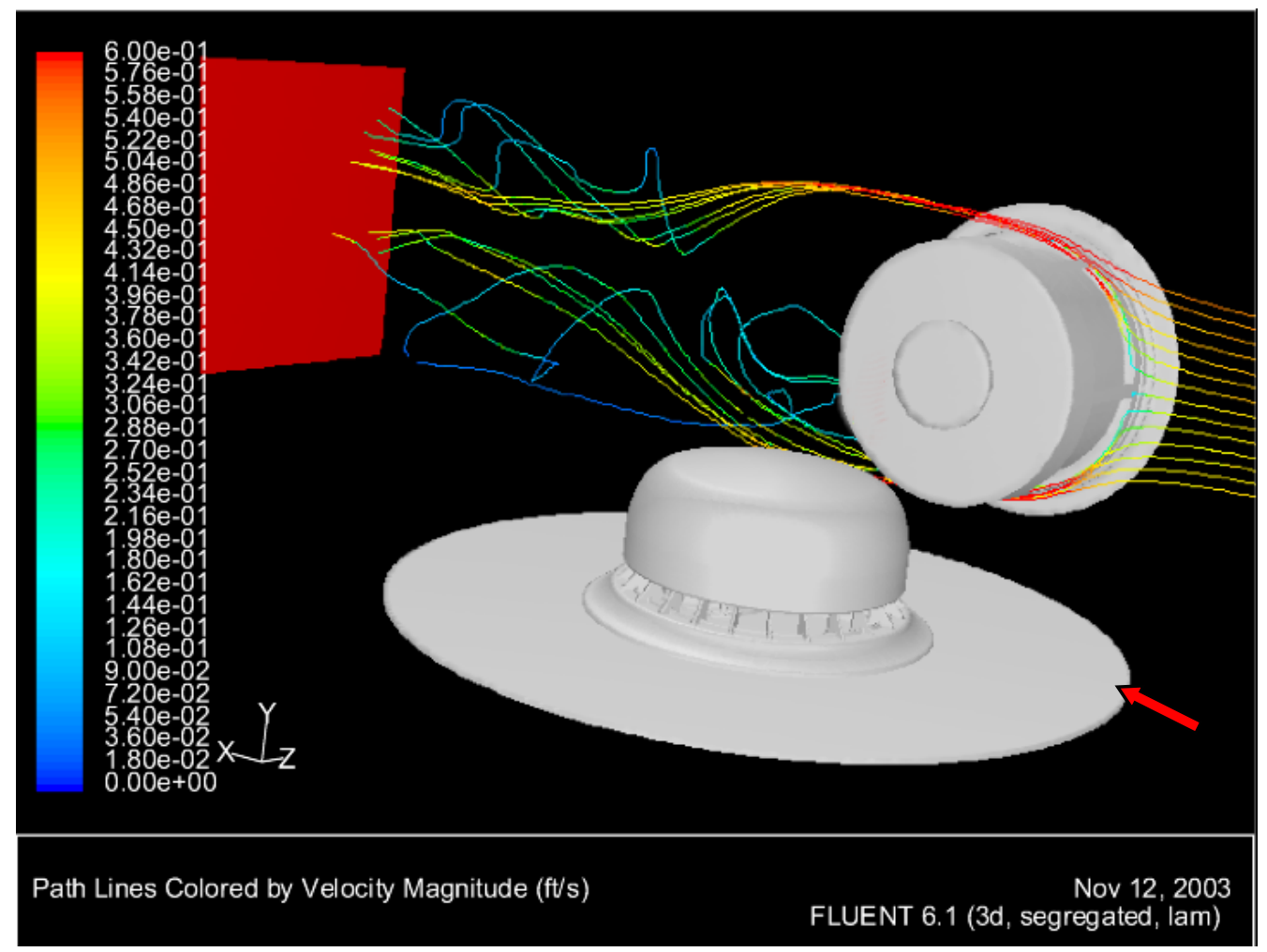

Figure 6.6.27: Test section flow visualization showing a pathline injection near the main inlet depicting the wake of the MIC.

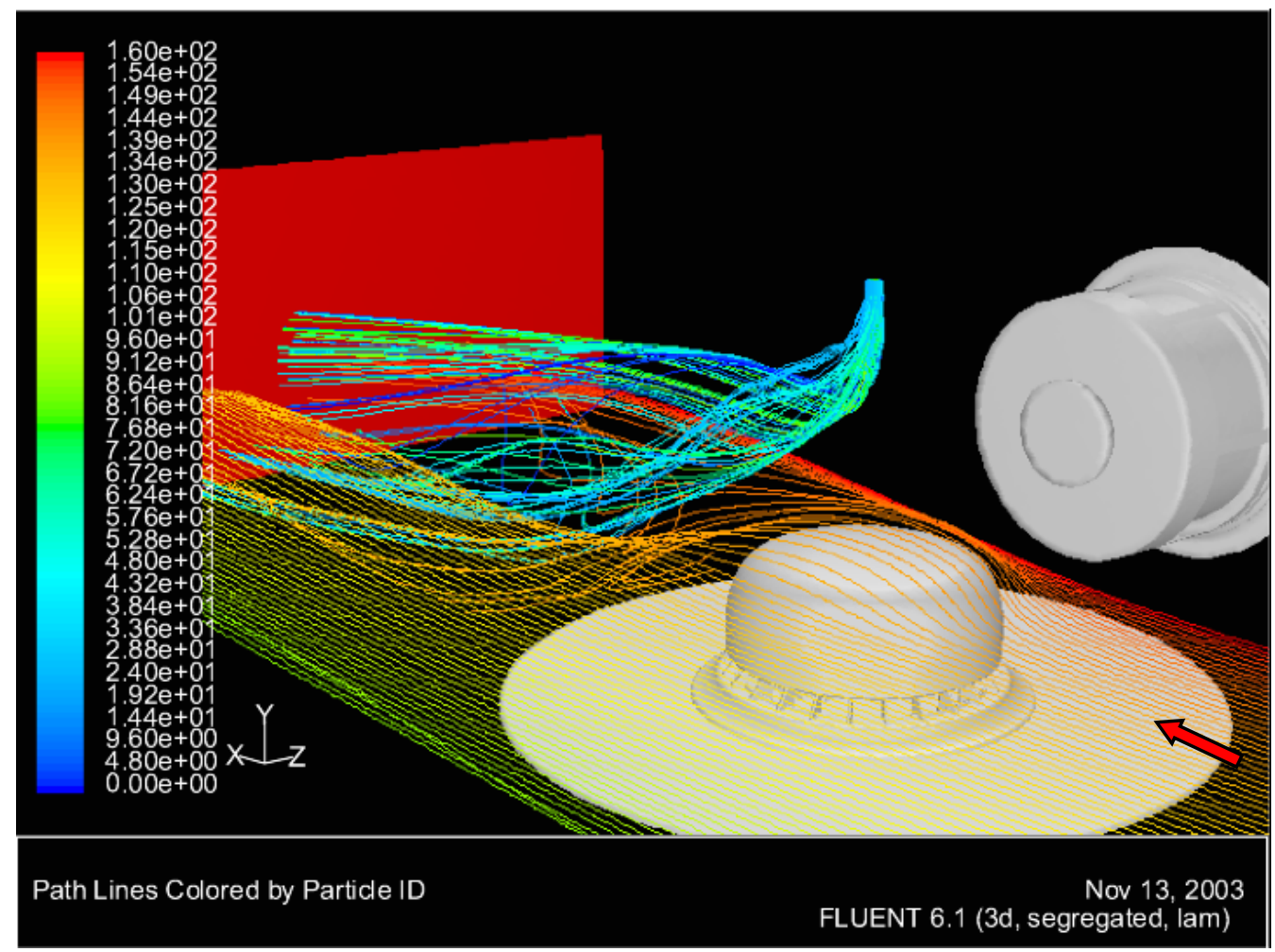

Figure 6.6.28: Test section flow visualization showing the interaction using pathlines between the probe velocity inlet injection and the main inlet flow. 


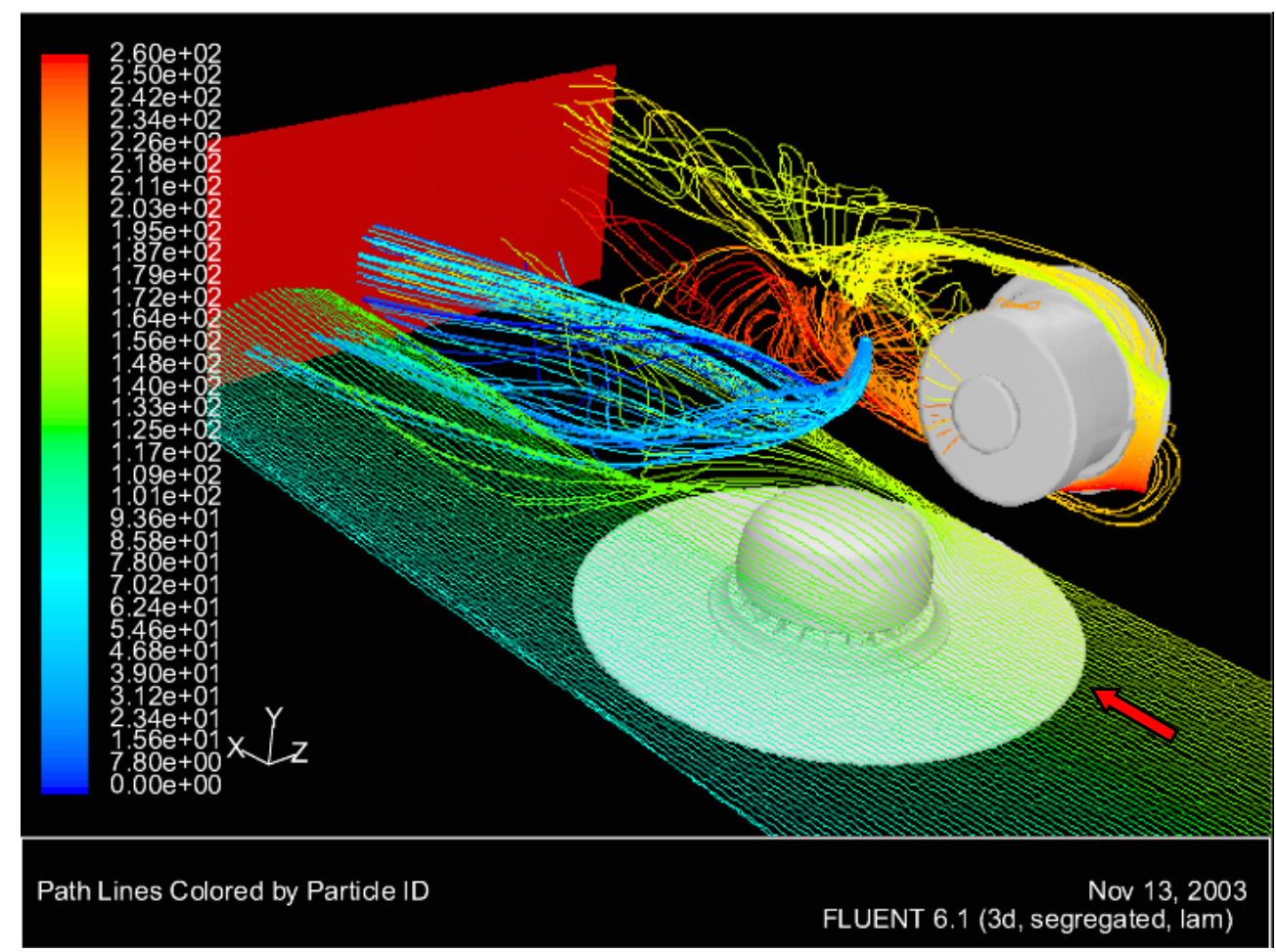

Figure 6.6.29: Test section flow visualization showing the combined interaction, using pathlines, of the presence of the MIC, SA302, and probe inlet.

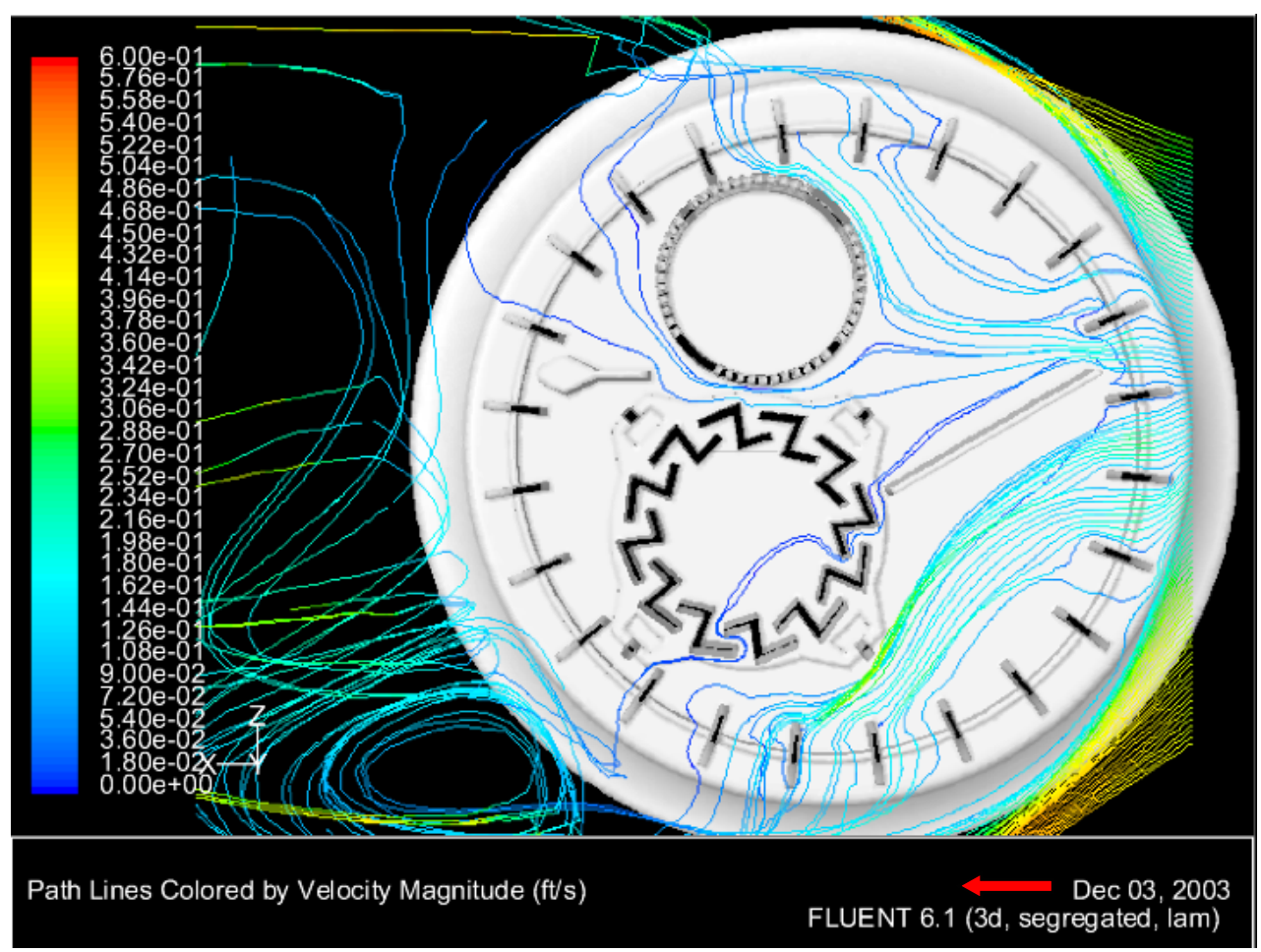

Figure 6.6.30: Detector flow visualization showing the route of 100 pathlines injected 1 in above the floor near the entrance to the SA302 smoke detector ( $0^{\circ}$ orientation). 


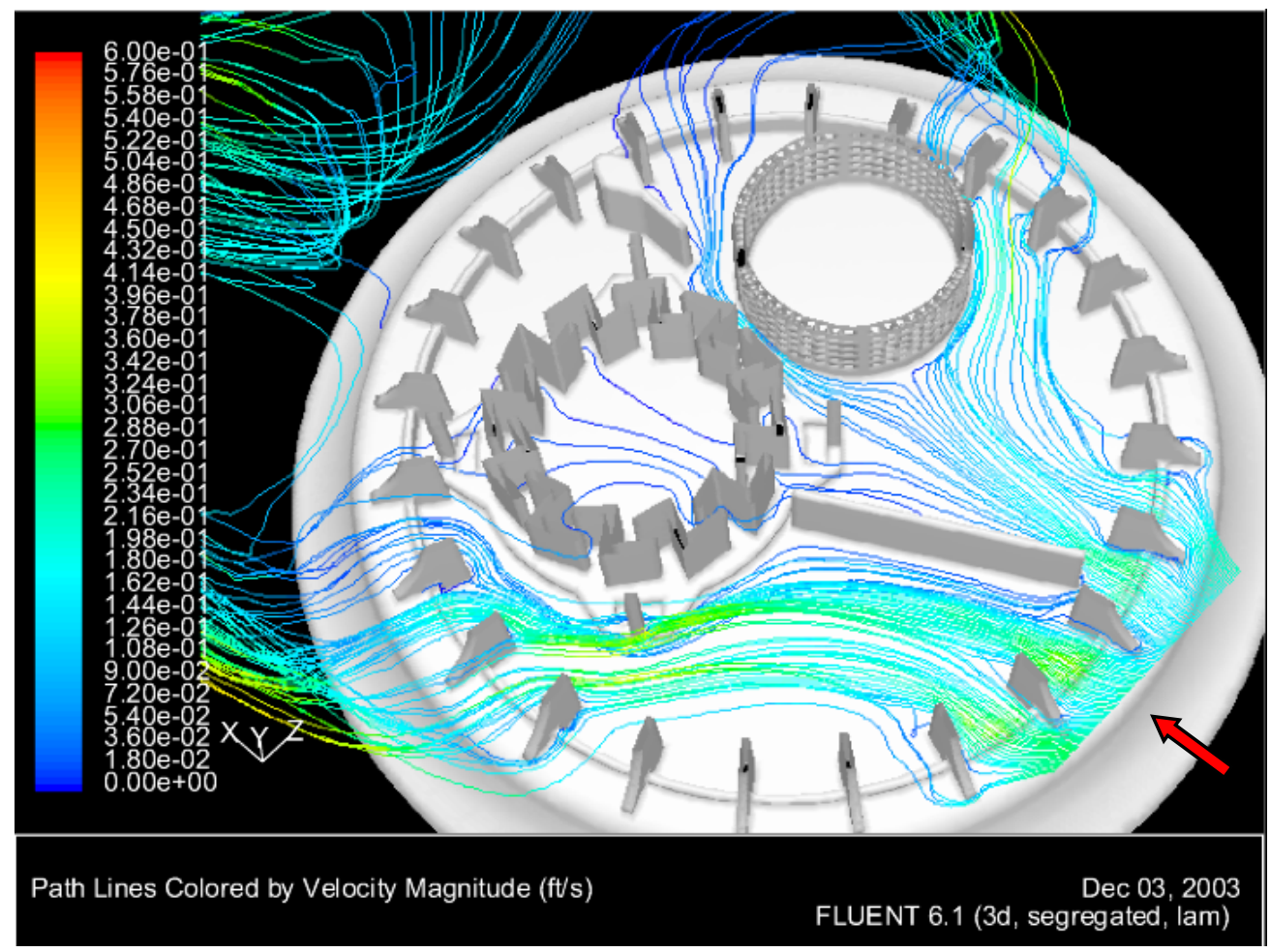

Figure 6.6.31: Detector flow visualization showing possible flow patterns inside the sensing elements of the SA302 ( $0^{\circ}$ orientation).

Figures 6.6.32 through 6.6.42 describe the computational results found for the $90^{\circ}$ orientation. Very similar to the $0^{\circ}$ orientation, the velocity and vector contour plots in Figures 6.6.32 through 6.6.34 outside the detector remain essentially the same. Figures 6.6.35 and 6.6.36 introduce the close-up flow characteristics, showing the photoelectric sensor in the wake of the ionization chamber. Here, although the ionization sensor is the first to meet the oncoming flow, the contour plots in Figures 6.6.37 and 6.6.39 for the ionization and photoelectric sensors, respectively, once again indicate the photoelectric sensor has velocities entering at nearly twice the speed as that of the ion sensor. The only significant role the main flow deflector plays in this orientation is to force flow through the southern side of the photoelectric chamber as seen in Figure 6.3.36. Figures 6.6.41 and 6.6.42 show pathlines injected at the base of the detector showing path flow through the sensing elements. 


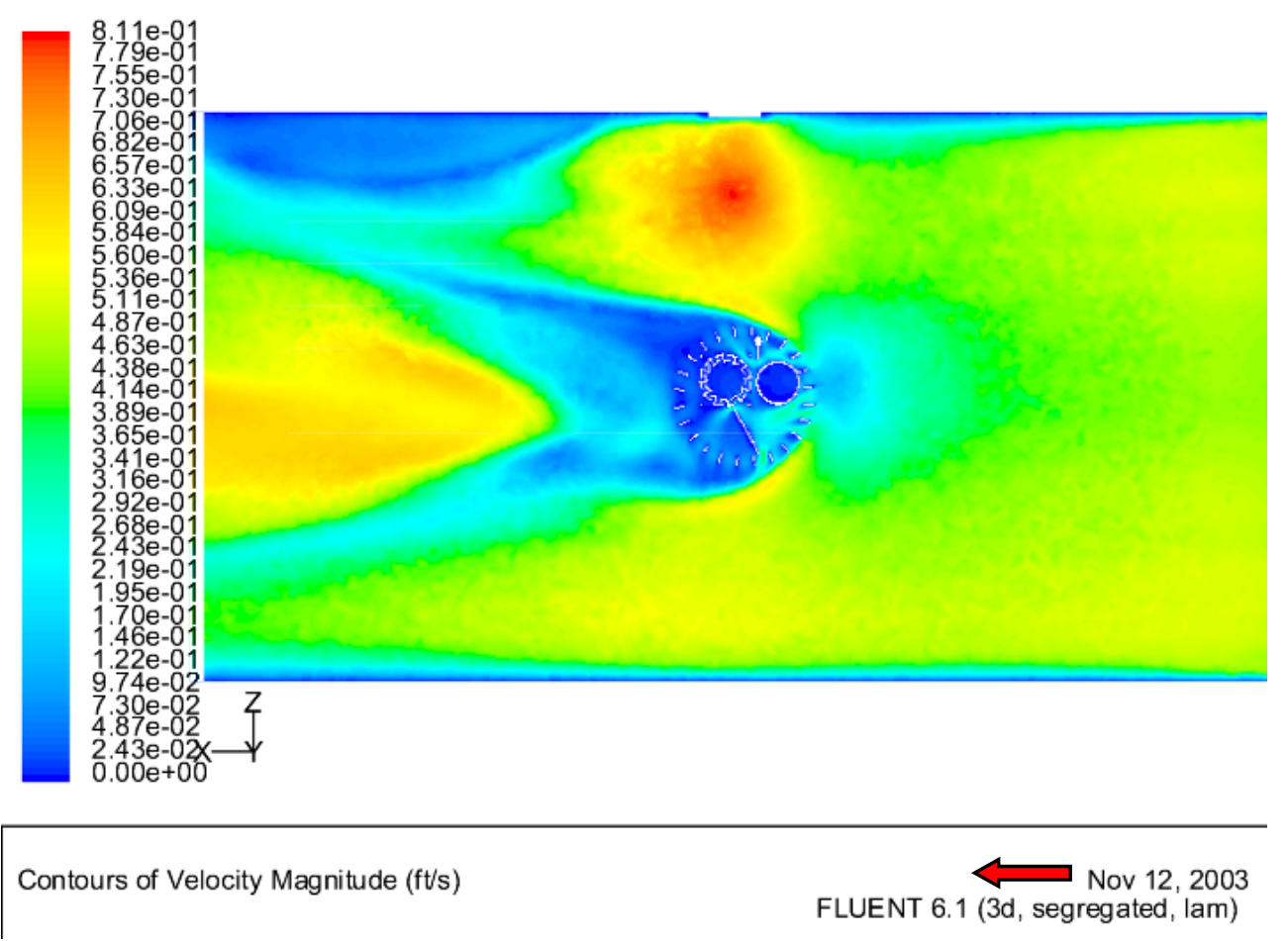

Figure 6.6.32: A velocity contour plot using the laminar model depicting the $y=0.50$ in plane for the $90^{\circ}$ orientation.

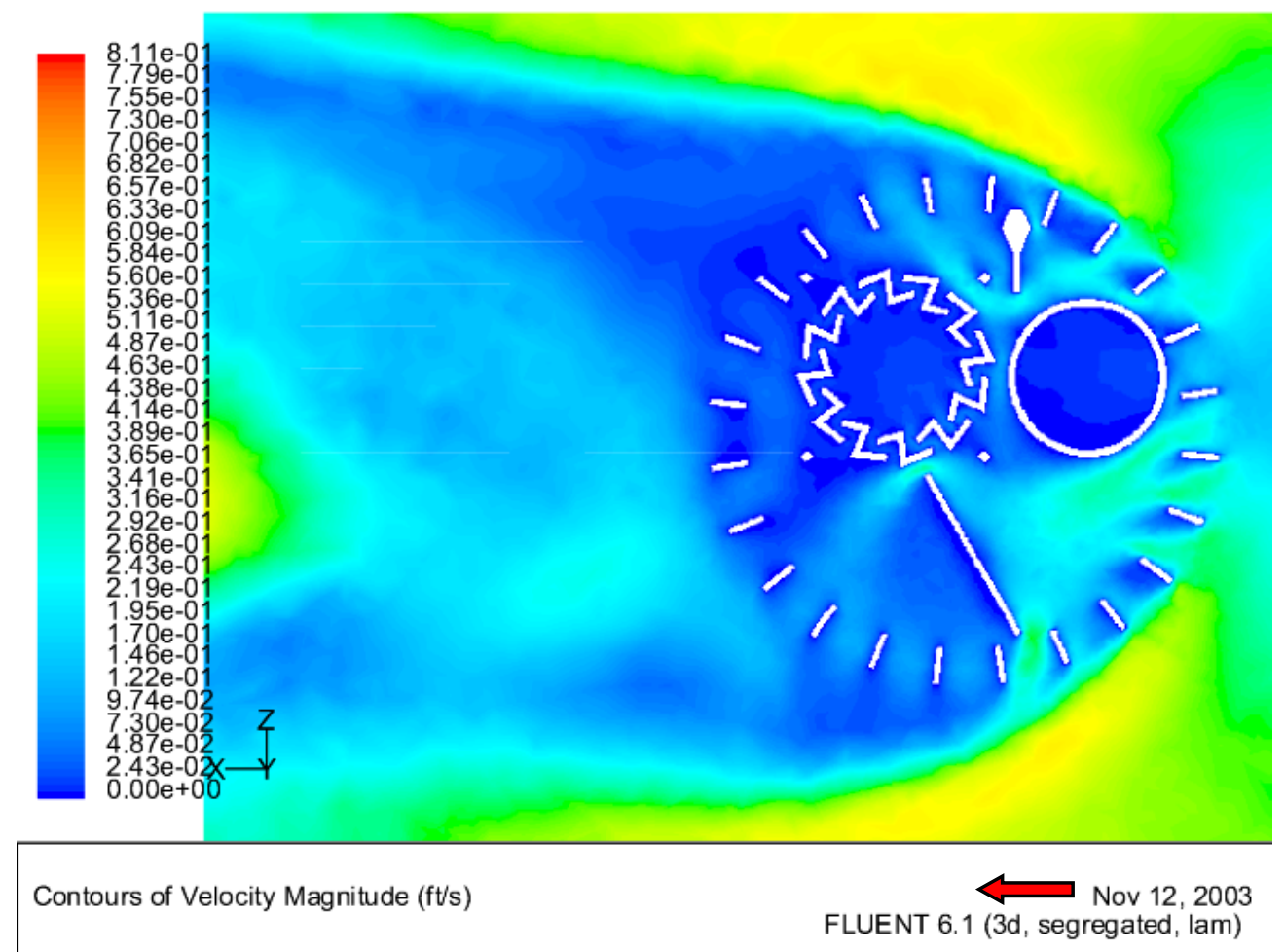

Figure 6.6.33: A velocity contour plot using the laminar model depicting the detector and its wake in the $y=0.50$ in plane for the $90^{\circ}$ orientation. 


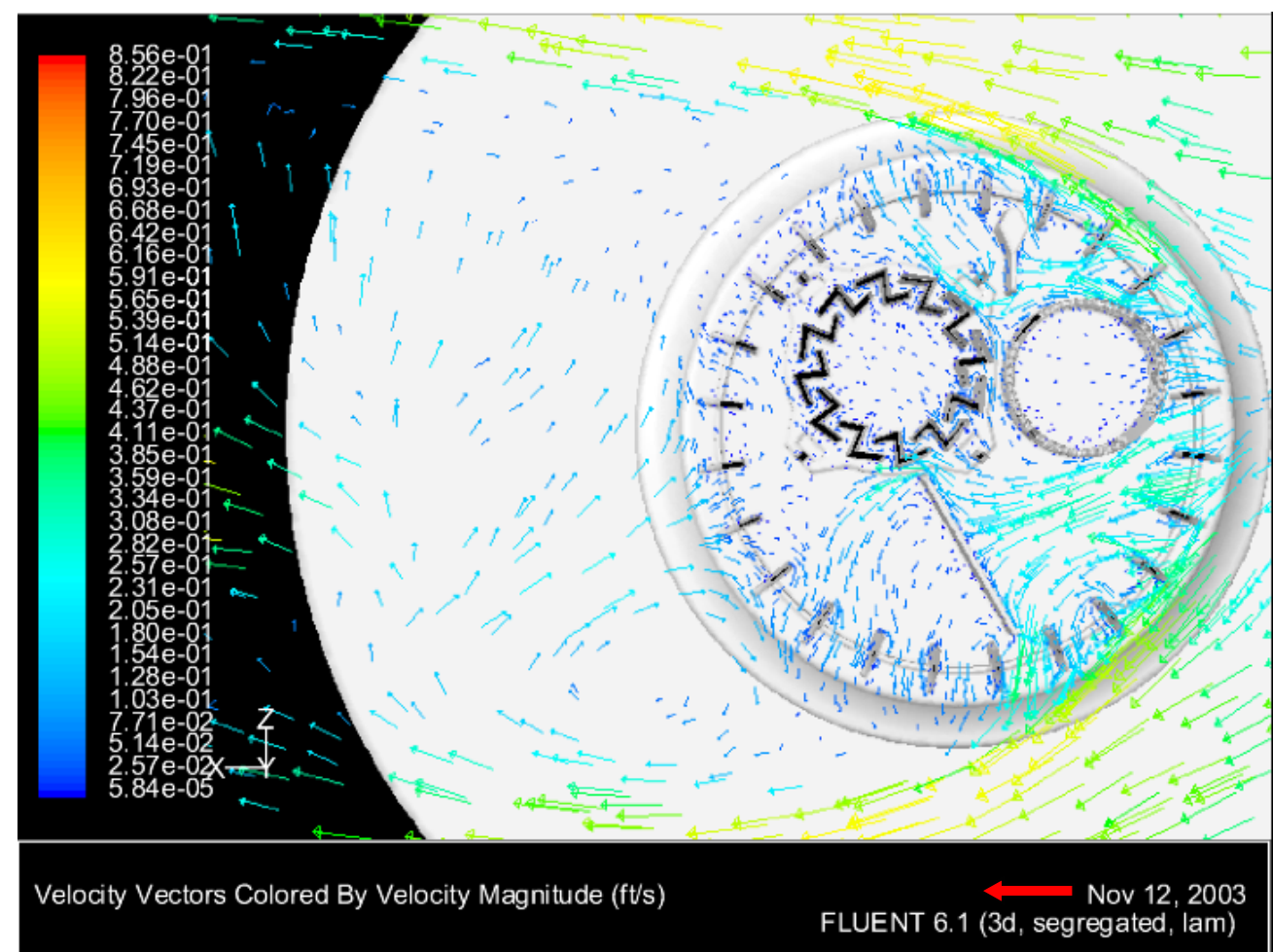

Figure 6.6.34: A velocity vector plot using the laminar model depicting the wake of the detector approaching the pressure outlet boundary in the $y=0.50$ in plane $\left(90^{\circ}\right.$ Orientation).

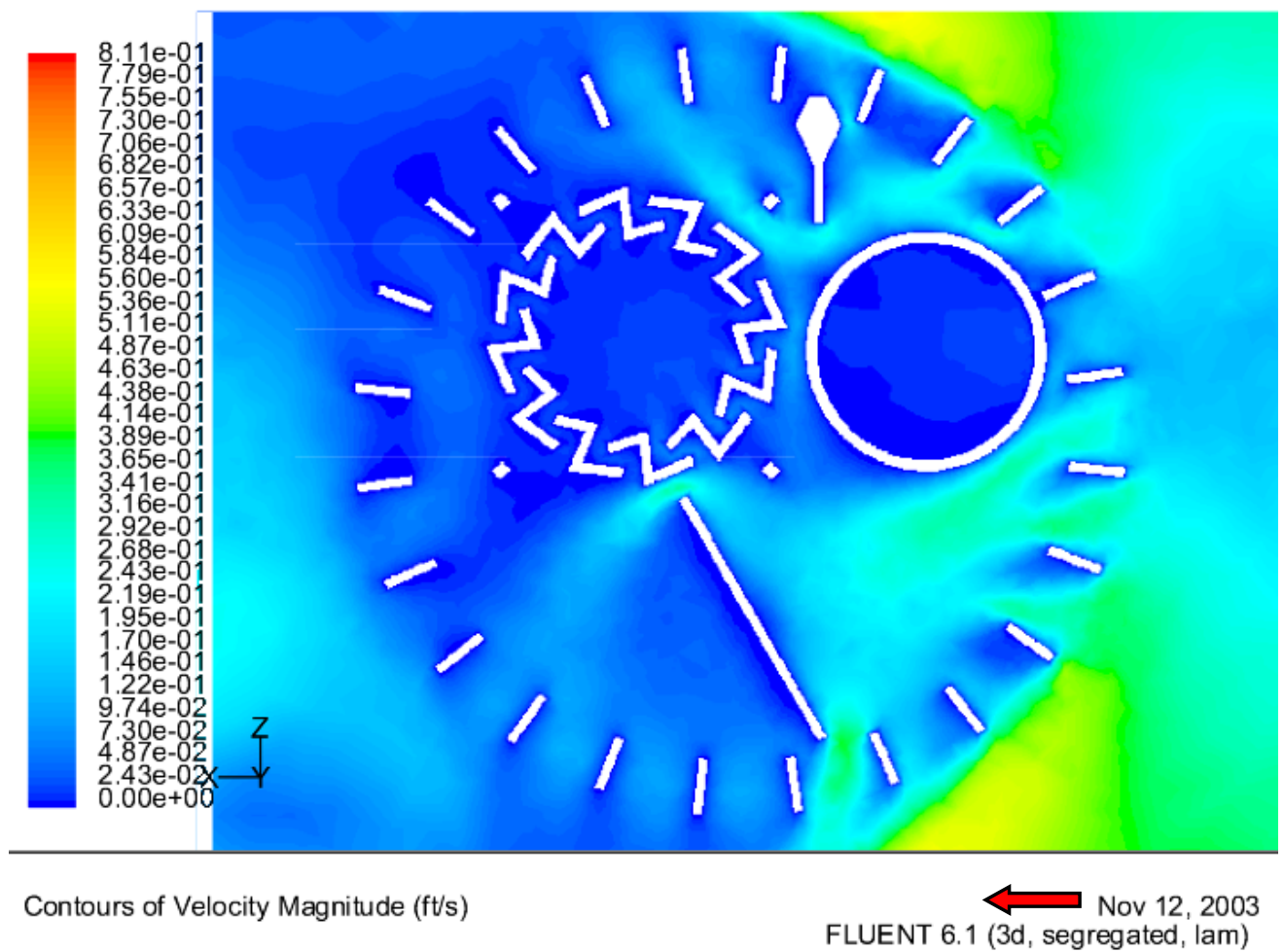

Figure 6.6.35: Detector flow visualization showing possible flow patterns inside the sensing elements of the SA302 $\left(90^{\circ}\right.$ orientation). 


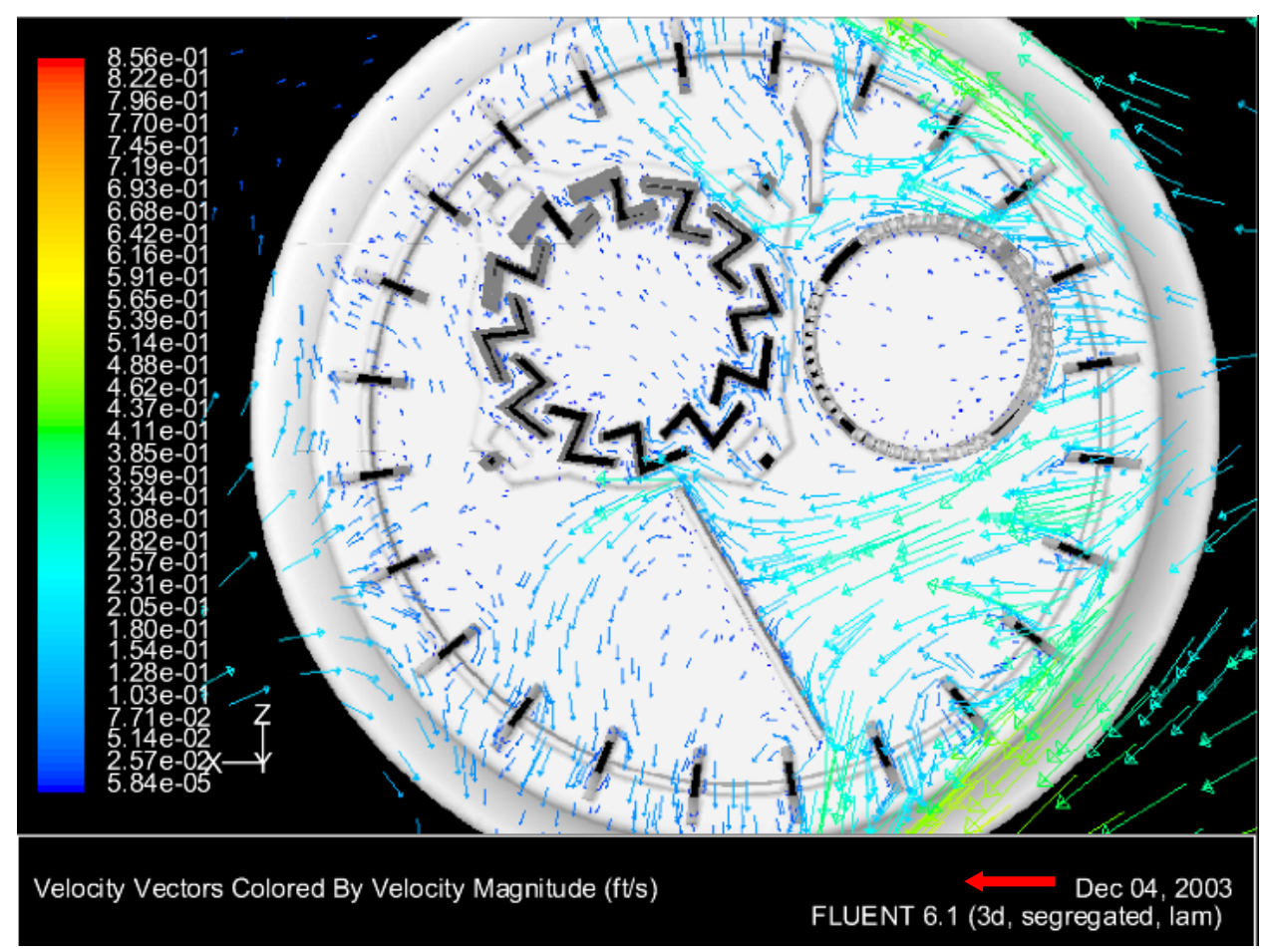

Figure 6.6.36: A velocity vector plot using the laminar model depicting the detector in the $y=$ 0.50 in plane for the $90^{\circ}$ orientation.
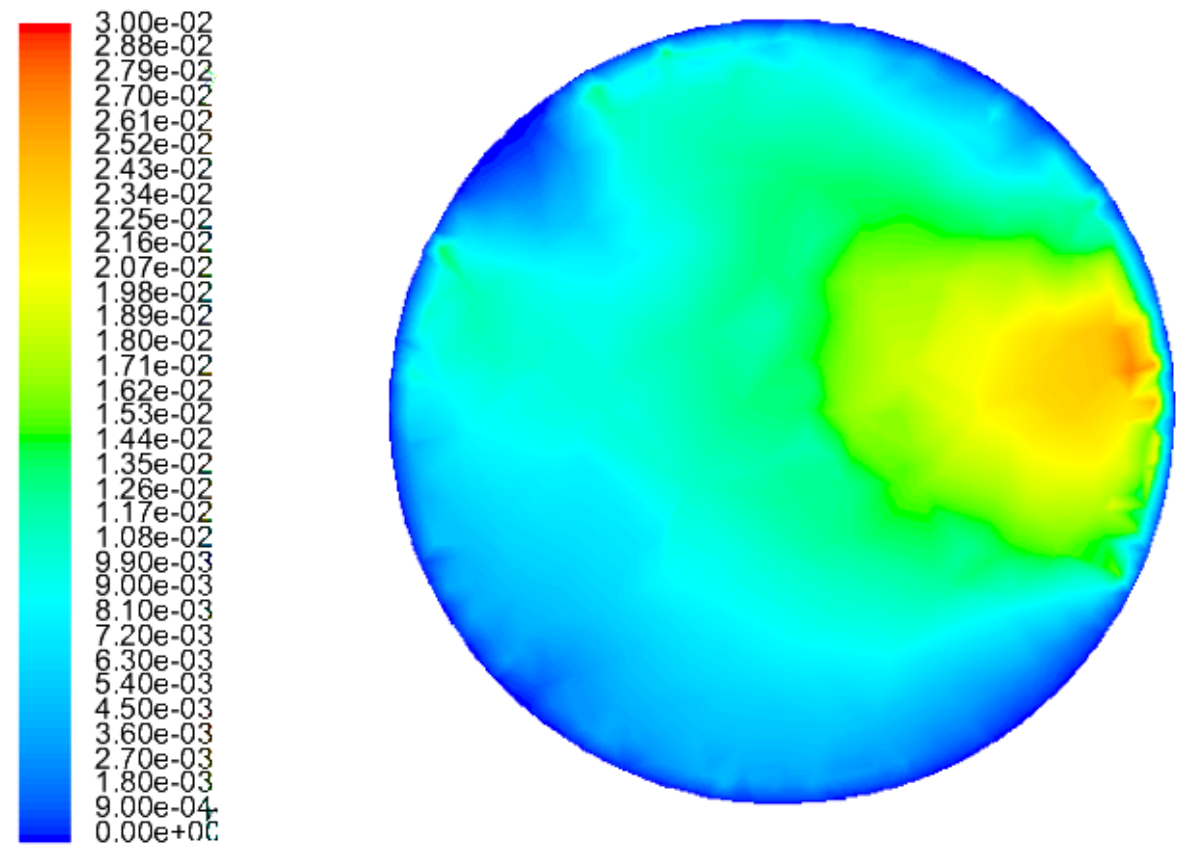

Contours of Velocity Magnitude (ft/s)

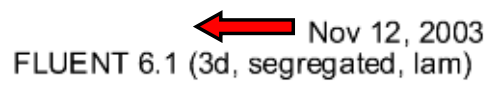

Figure 6.6.37: A velocity contour plot using the laminar model depicting the ionization chamber in the $y=0.50$ in plane for the $90^{\circ}$ orientation. 


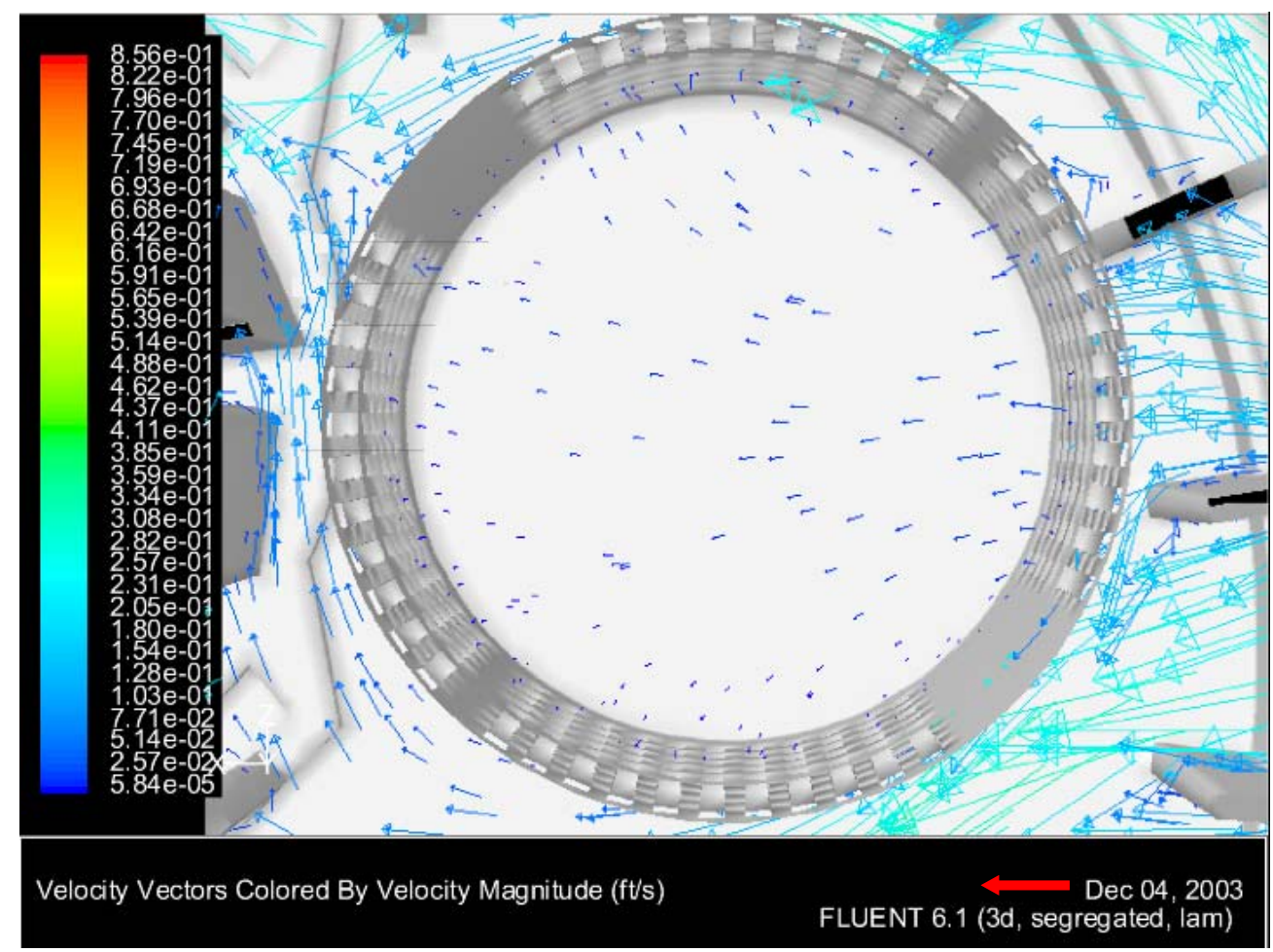

Figure 6.6.38: A velocity vector plot using the laminar model depicting the ionization chamber in the $y=0.50$ in plane for the $90^{\circ}$ orientation.
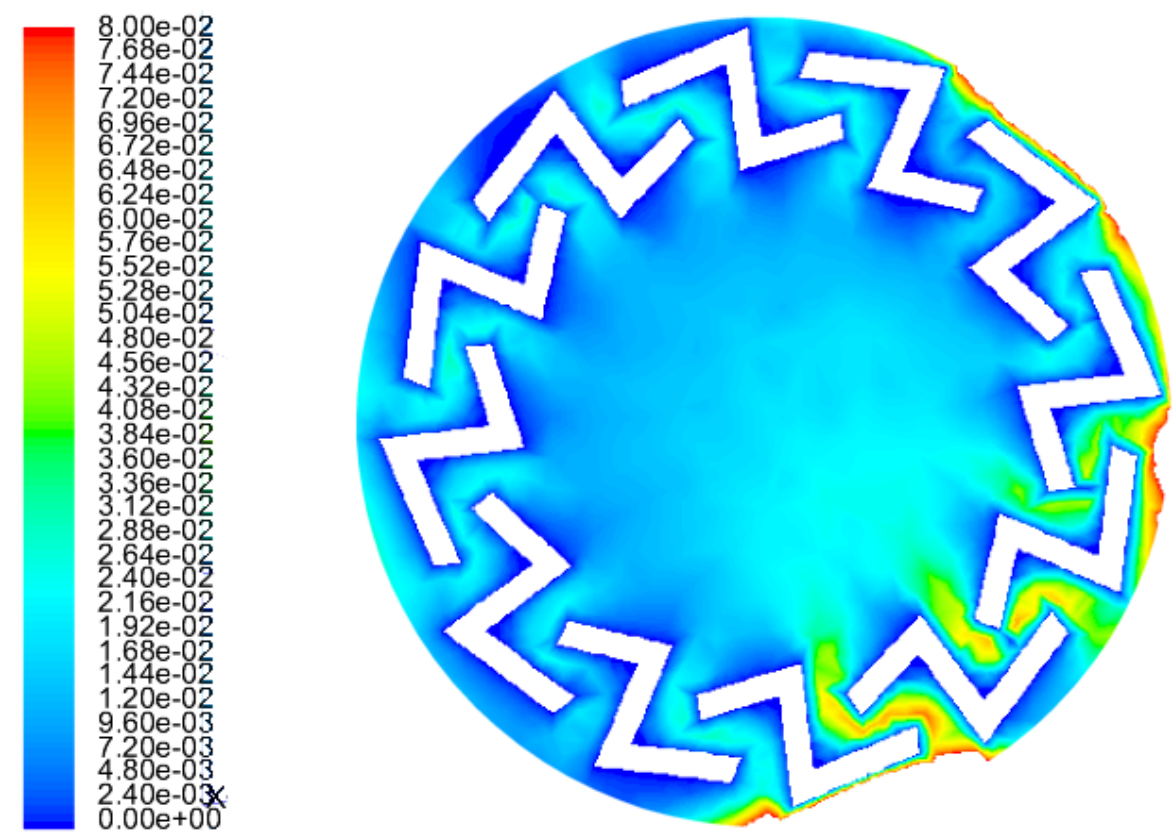

Contours of Velocity Magnitude (ft/s)

Nov 12, 2003

FLUENT 6.1 (3d, segregated, lam)

Figure 6.6.39: A velocity contour plot using the laminar model depicting the photoelectric chamber in the $y=0.50$ in plane for the $90^{\circ}$ orientation. 


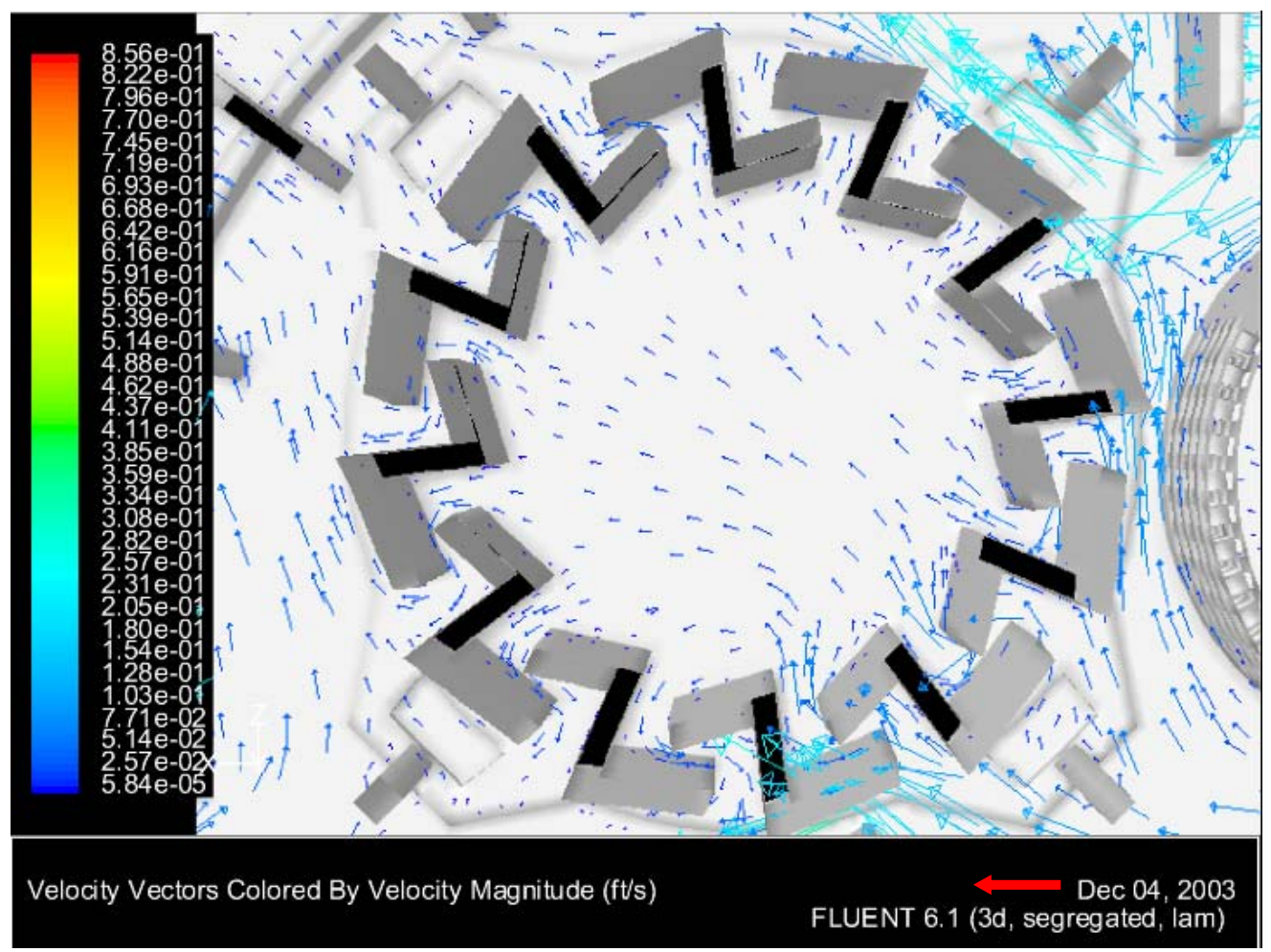

Figure 6.6.40: A velocity vector plot using the laminar model depicting the photoelectric chamber in the $y=0.50$ in plane for the $90^{\circ}$ orientation.

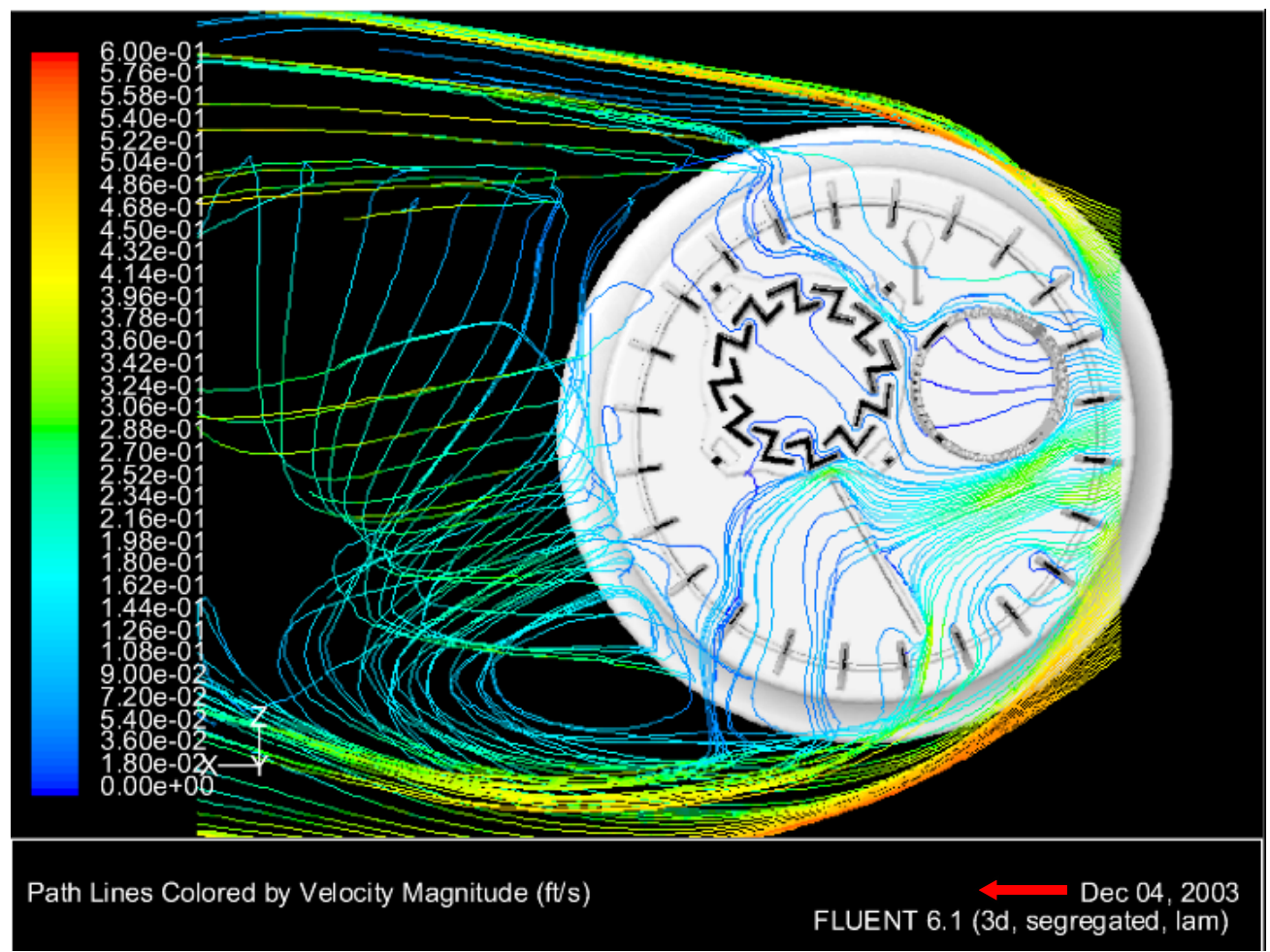

Figure 6.6.41: Detector flow visualization showing the route of 100 pathlines injected 1 in above the floor near the entrance to the SA302 smoke detector $\left(90^{\circ}\right.$ orientation). 


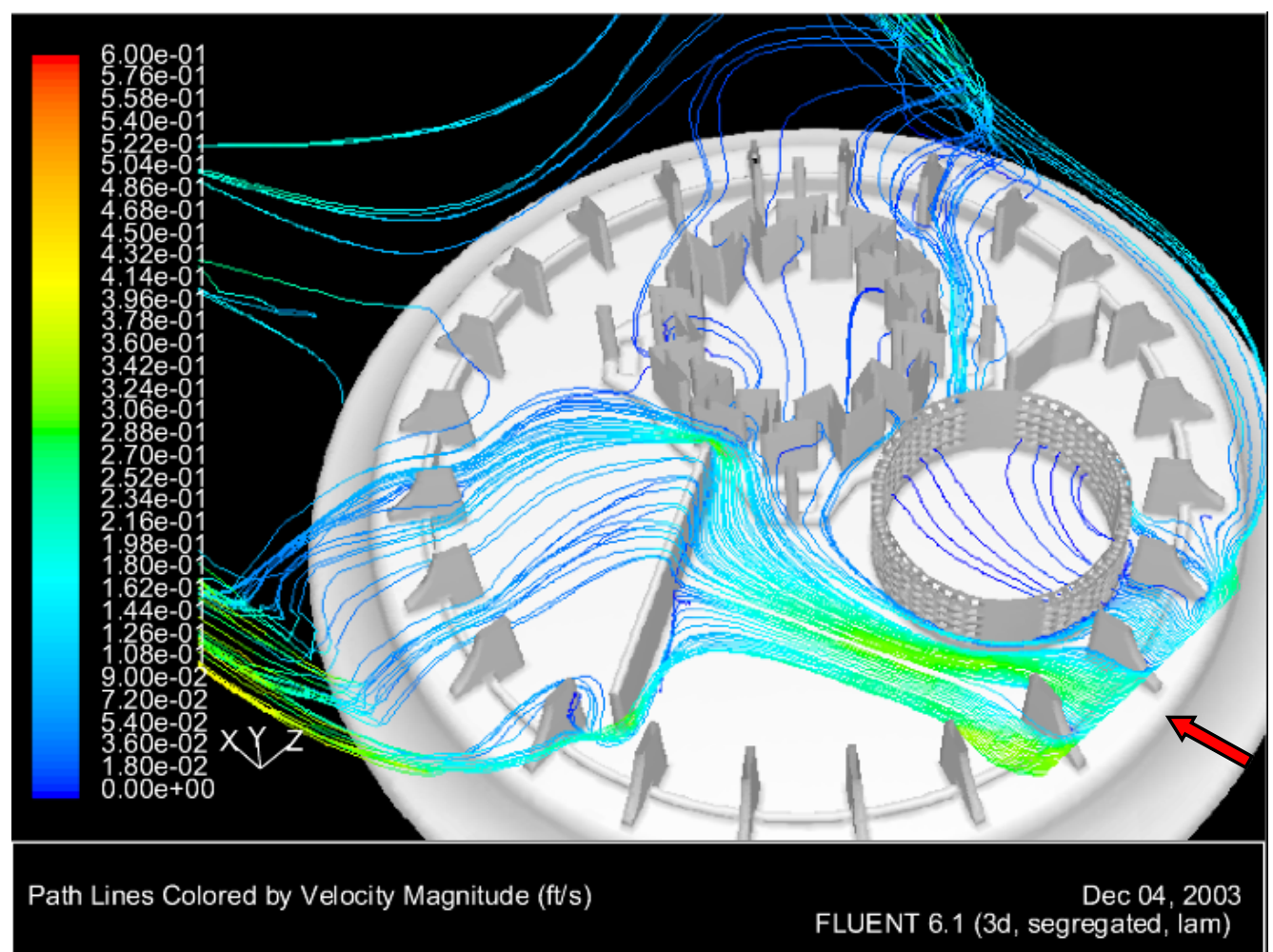

Figure 6.6.42: Detector flow visualization showing possible flow patterns inside the sensing elements of the SA302 $\left(90^{\circ}\right.$ orientation).

Figures 6.4.43 through 6.4.53 depict the results for the $180^{\circ}$ orientation. This orientation is unique in that both of the sensing chambers face the incoming flow head on. Although the flow velocities inside of the ionization chamber still stay relatively between 1 and $2 \mathrm{ft} / \mathrm{min}$ like the previous orientations, velocities inside the photoelectric chamber reach speeds of nearly $8 \mathrm{ft} / \mathrm{min}$. From the contour and velocity vector plots one can determine the incoming flow angle due to the higher range of speeds culminating along the entire right hand side of the photoelectric chamber.

It is of mild interest to also note here the slight change in the wake of the detector due to the rounded sensor bodies in the forefront giving the velocities a smooth curved surface off which to roll. Because of this, their wakes are slightly less chaotic in nature. From Figures 6.6.52 and 6.6.53, one can see this orientation has no trouble in introducing a large influx of air flow into both its sensing elements. 


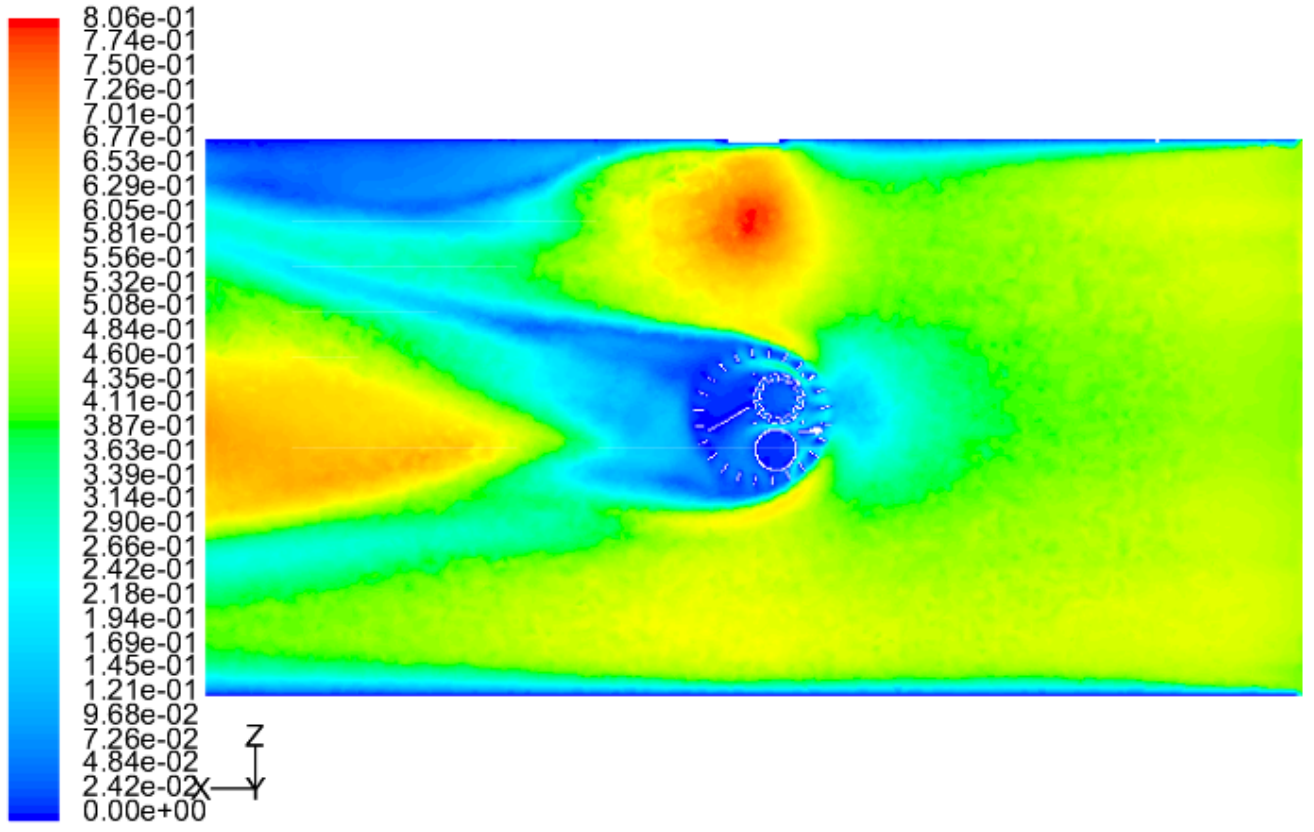

Contours of Velocity Magnitude (ft/s)

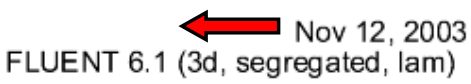

Figure 6.6.43: A velocity contour plot using the laminar model depicting the $y=0.50$ in plane for the $180^{\circ}$ orientation.

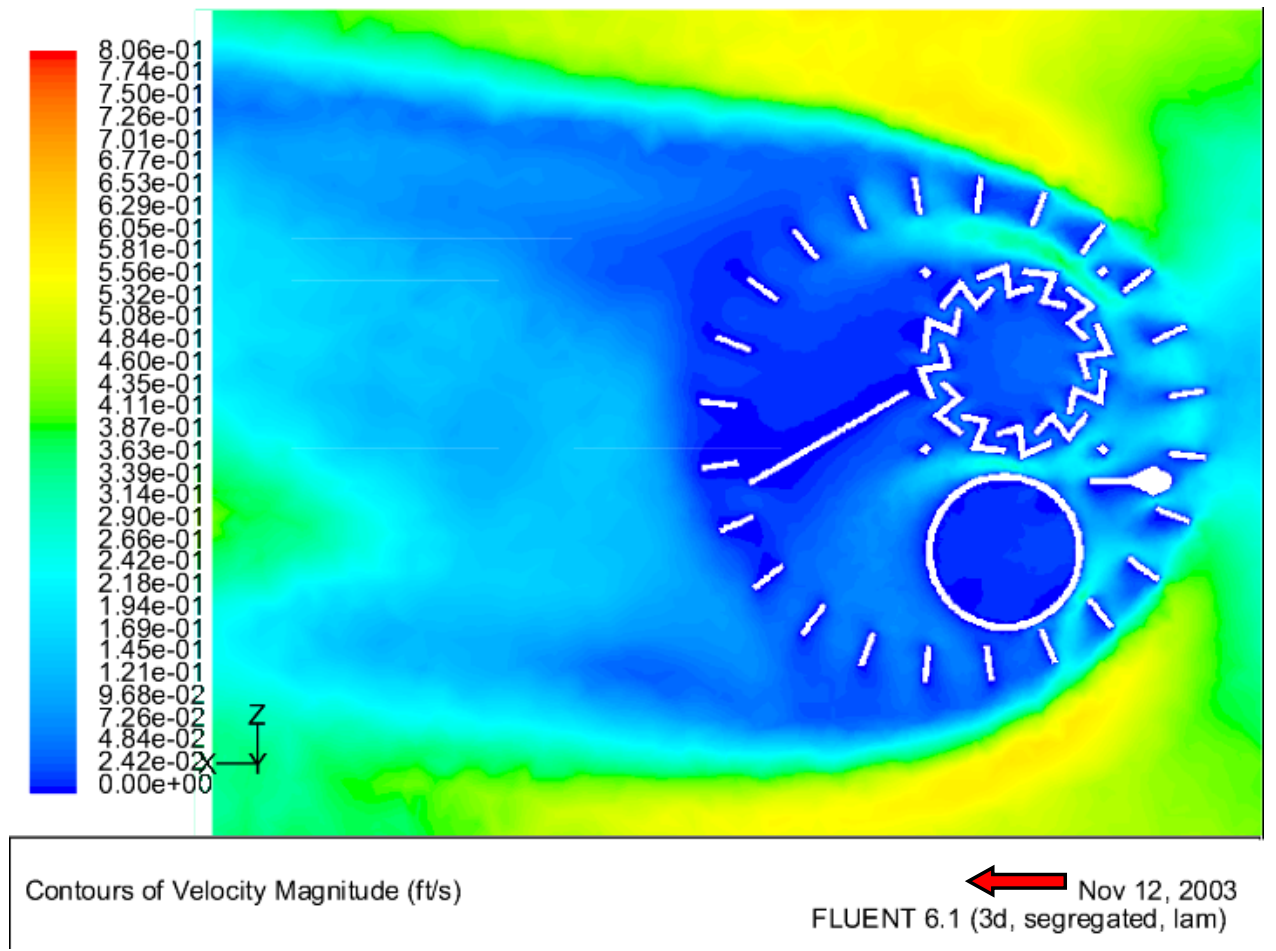

Figure 6.6.44: A velocity contour plot using the laminar model depicting the detector and its wake in the $y=0.50$ in plane for the $180^{\circ}$ orientation. 


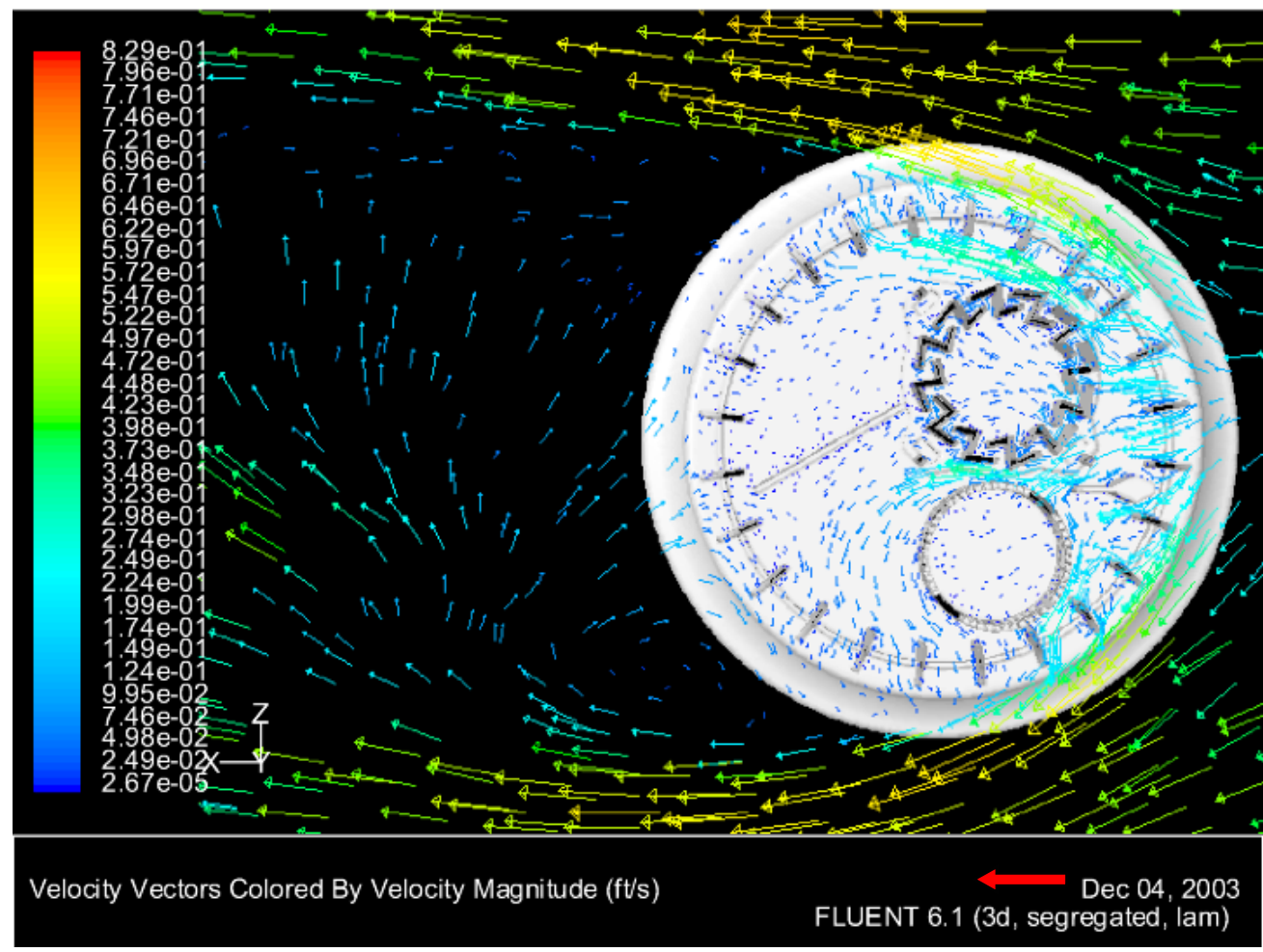

Figure 6.6.45: A velocity vector plot using the laminar model depicting the wake of the detector approaching the pressure outlet boundary in the $y=0.50$ in plane $\left(180^{\circ}\right.$ Orientation $)$.

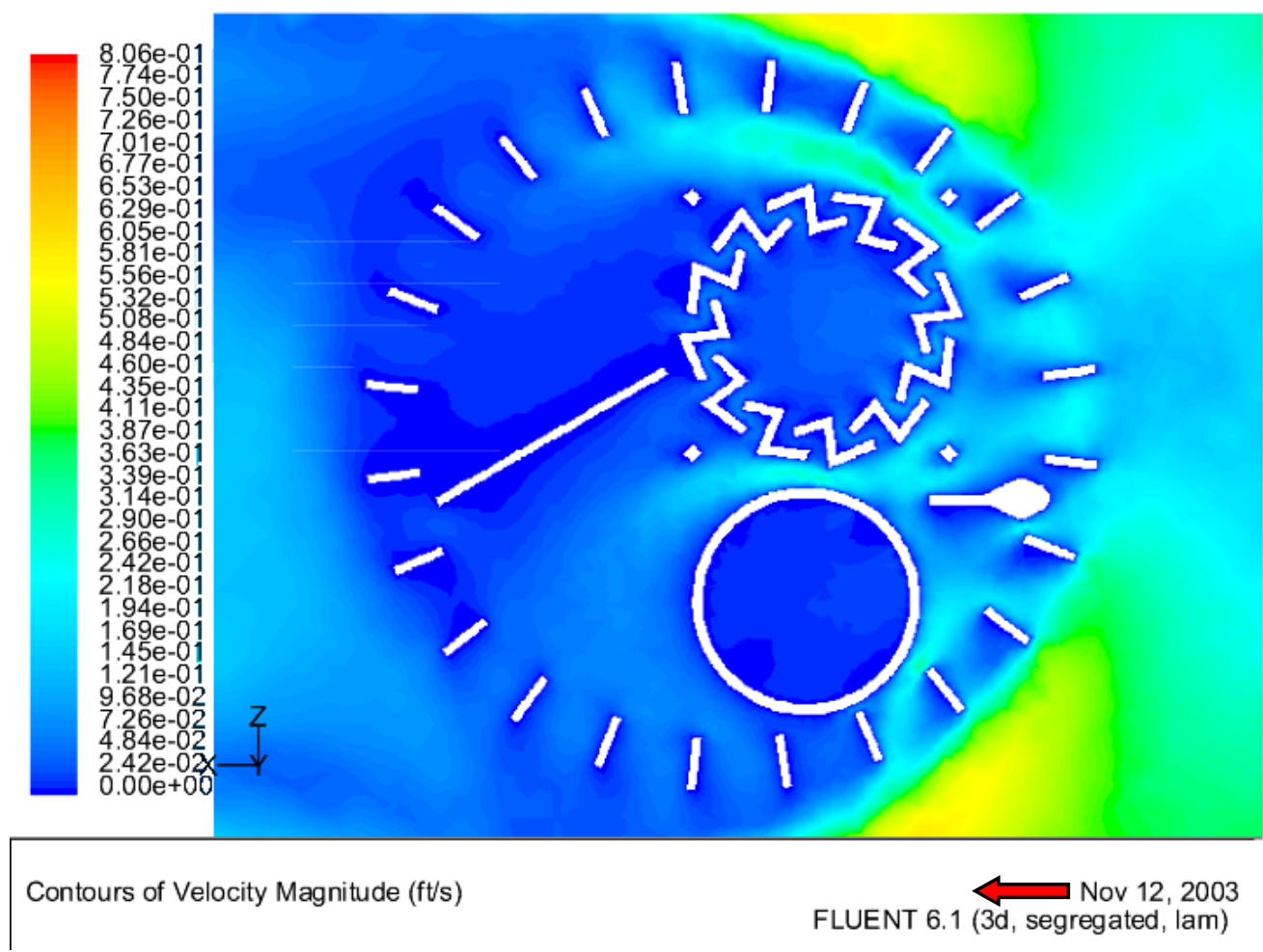

Figure 6.6.46 Detector flow visualization showing possible flow patterns inside the sensing elements of the SA302 $\left(180^{\circ}\right.$ orientation). 


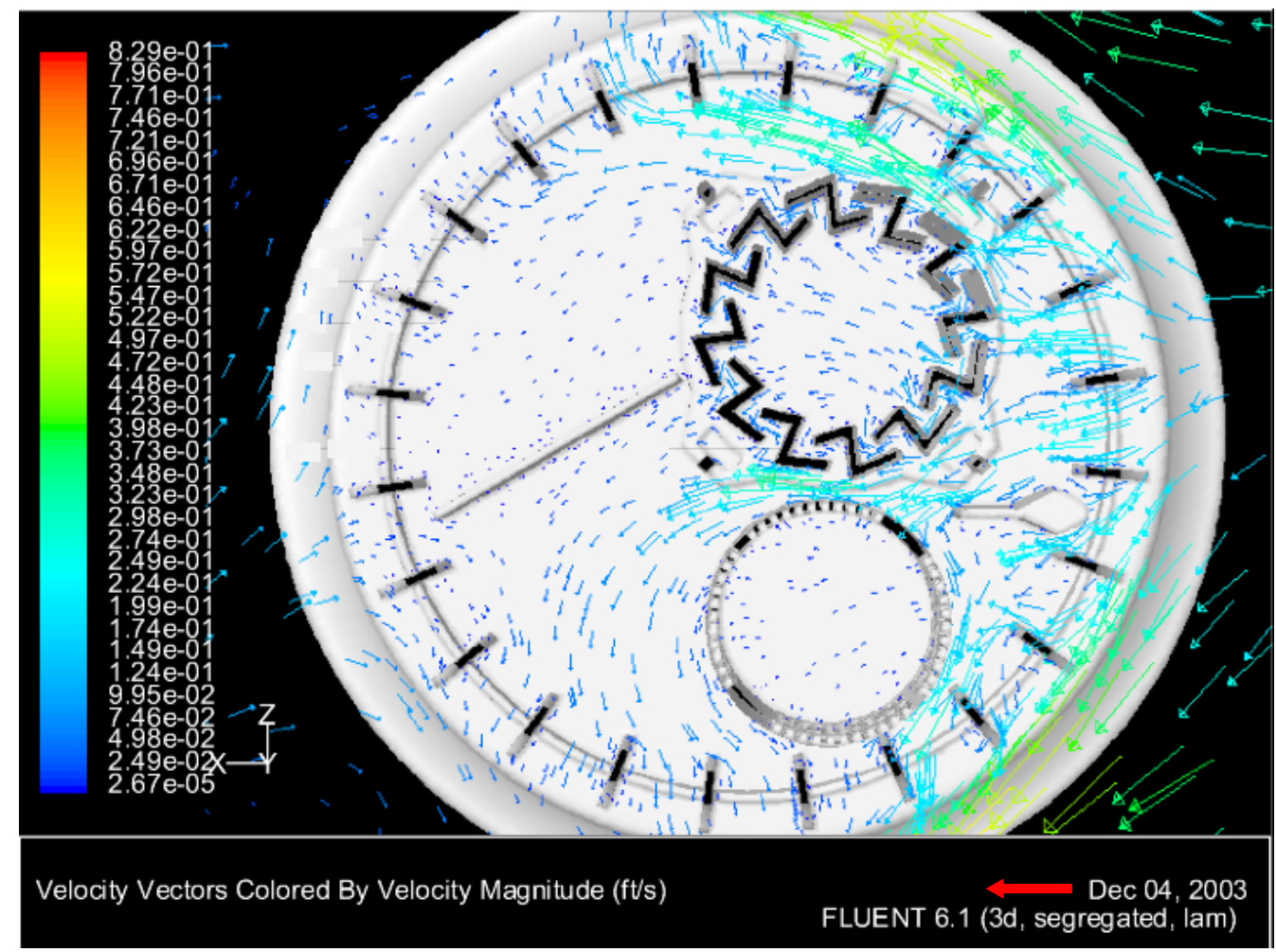

Figure 6.6.47 A velocity vector plot using the laminar model depicting the detector in the $y=$ 0.50 in plane for the $180^{\circ}$ orientation.
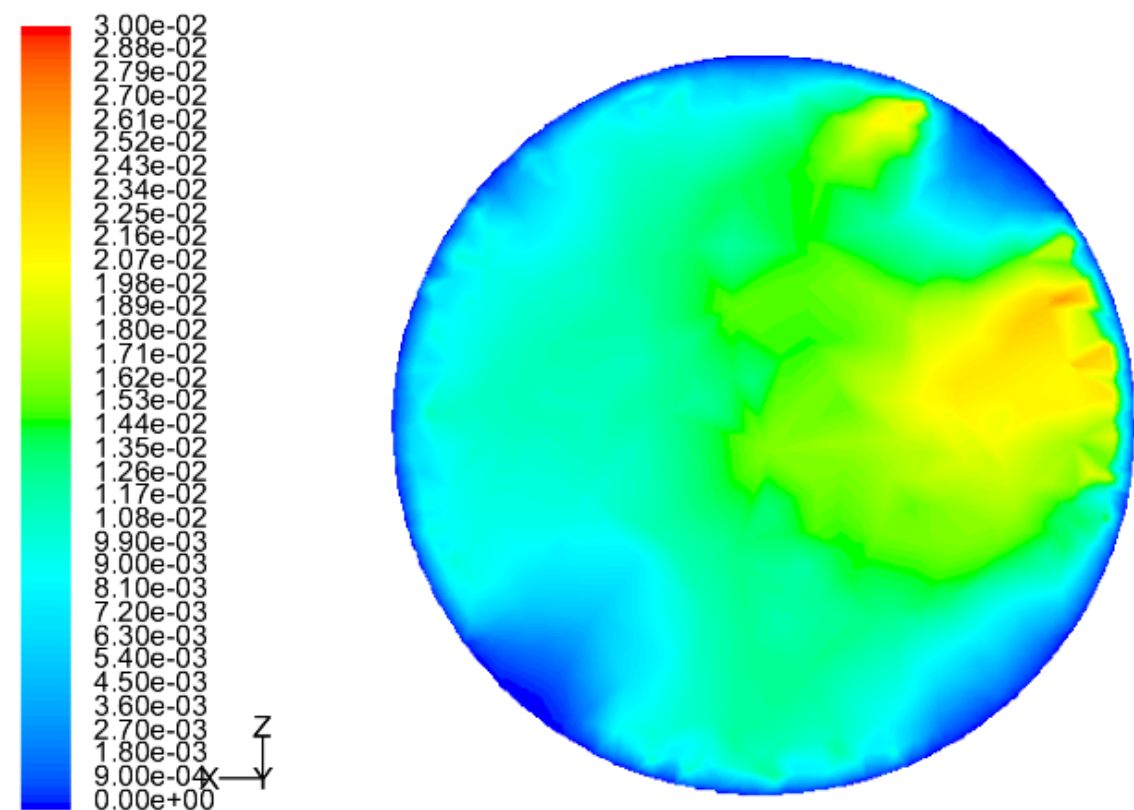

Contours of Velocity Magnitude ( $\mathrm{ft} / \mathrm{s}$ )

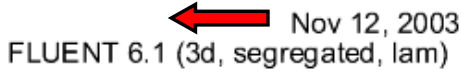

Figure 6.6.48 A velocity contour plot using the laminar model depicting the ionization chamber in the $y=0.50$ in plane for the $180^{\circ}$ orientation. 


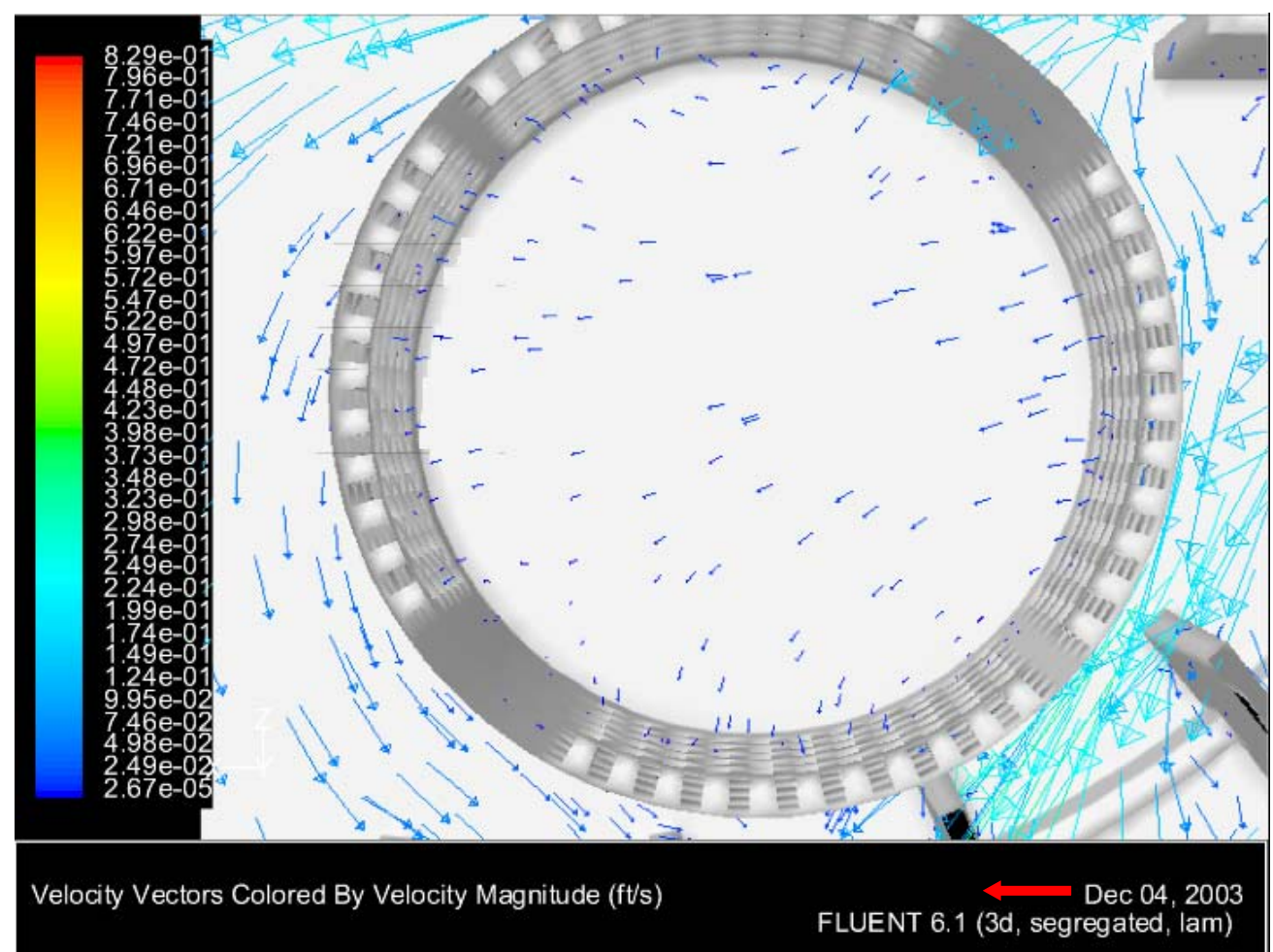

Figure 6.6.49 A velocity vector plot using the laminar model depicting the ionization chamber in the $y=0.50$ in plane for the $180^{\circ}$ orientation.

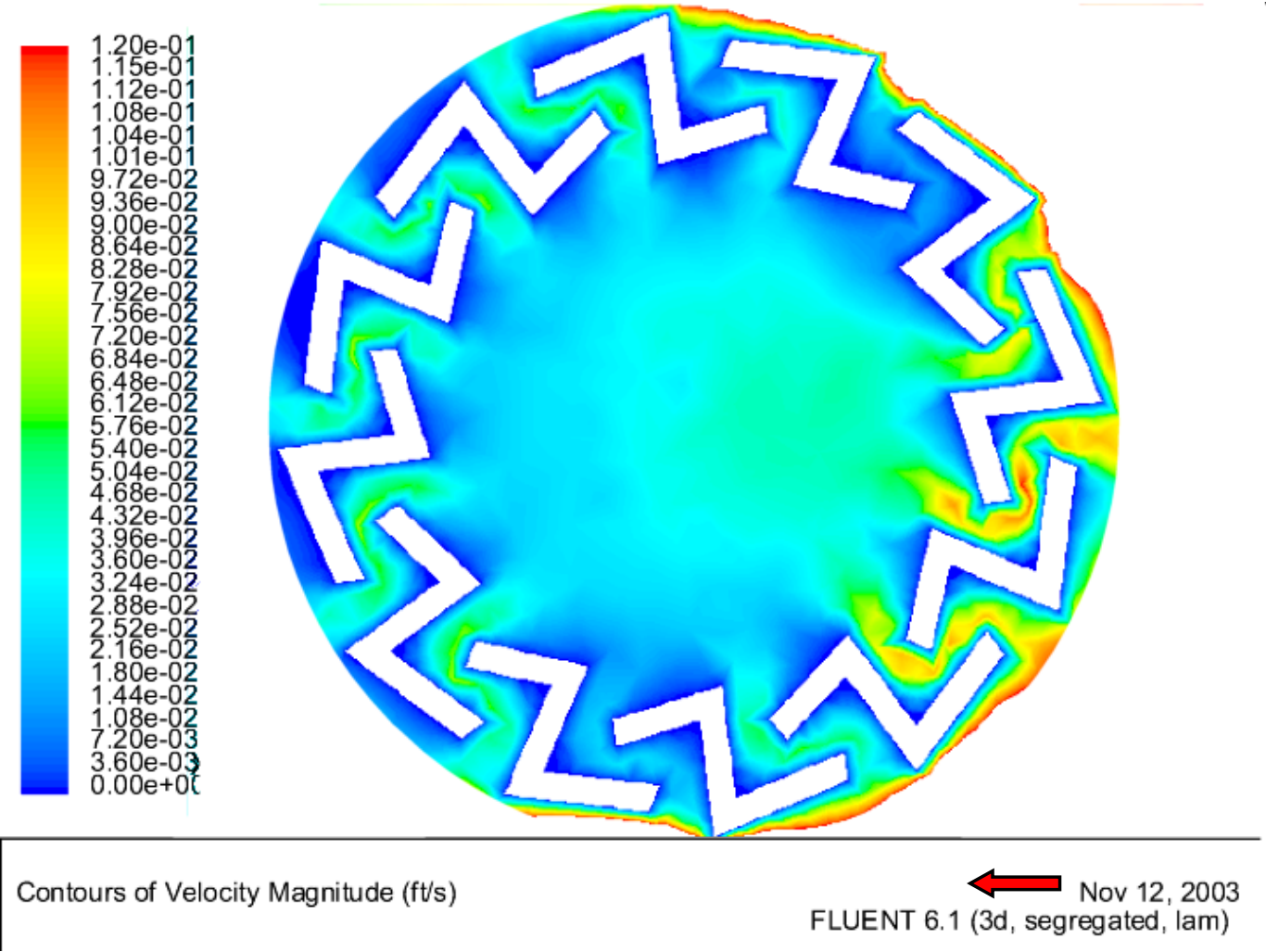

Figure 6.6.50 A velocity contour plot using the laminar model depicting the photoelectric chamber in the $y=0.50$ in plane for the $180^{\circ}$ orientation. 


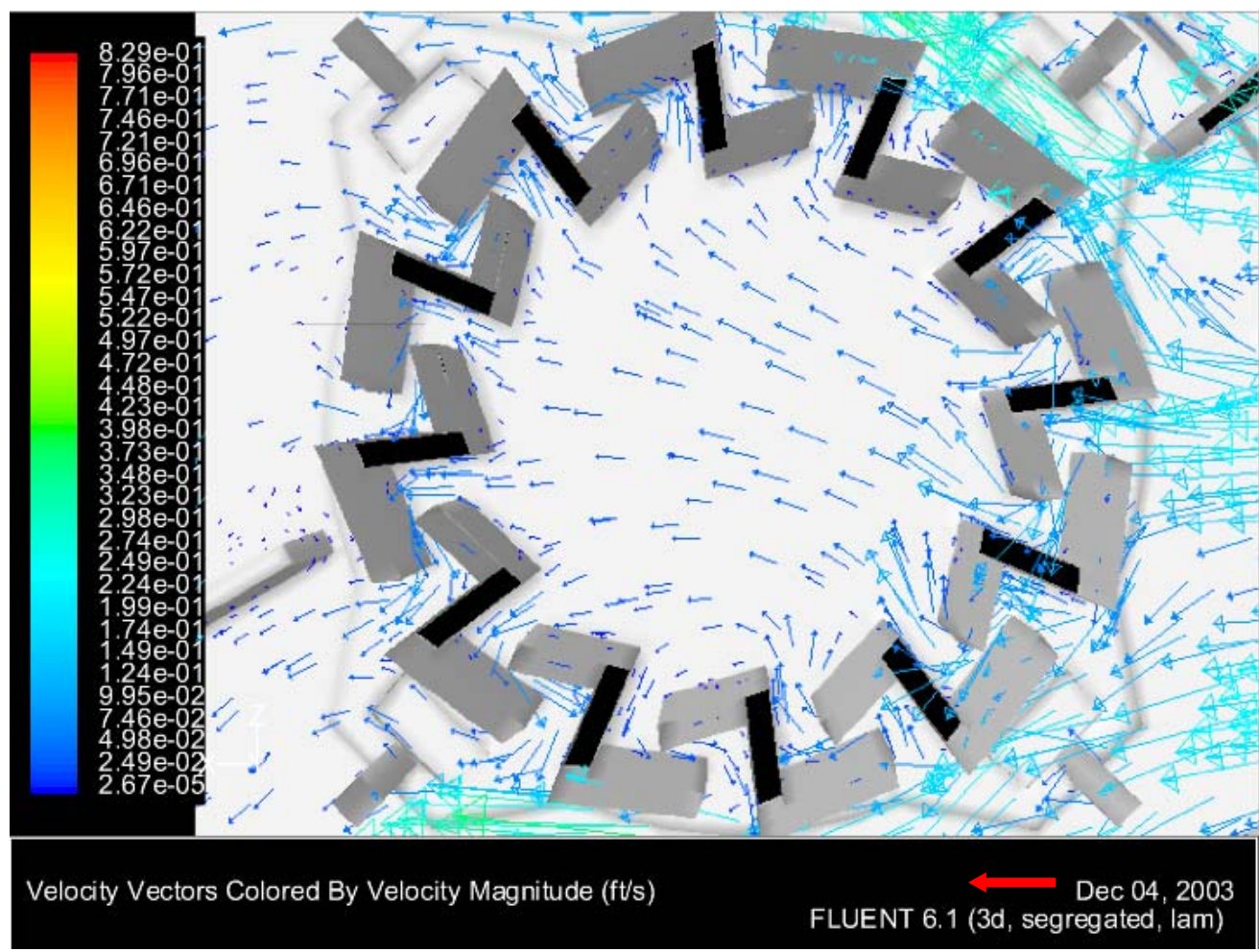

Figure 6.6.51 A velocity vector plot using the laminar model depicting the photoelectric chamber in the $y=0.50$ in plane for the $180^{\circ}$ orientation.

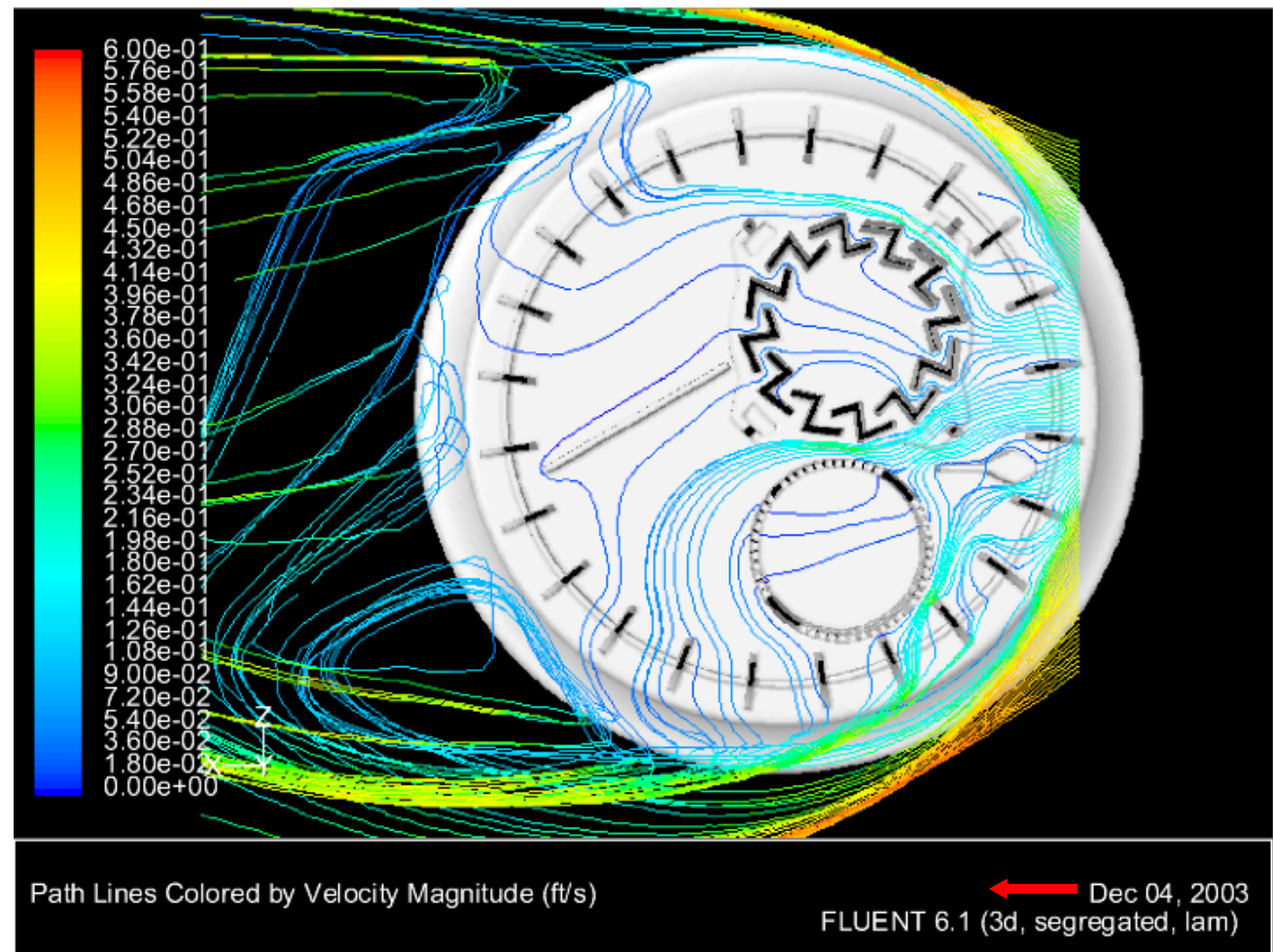

Figure 6.6.52 Detector flow visualization showing the route of 100 pathlines injected 1 in above the floor near the entrance to the SA302 smoke detector $\left(180^{\circ}\right.$ orientation). 


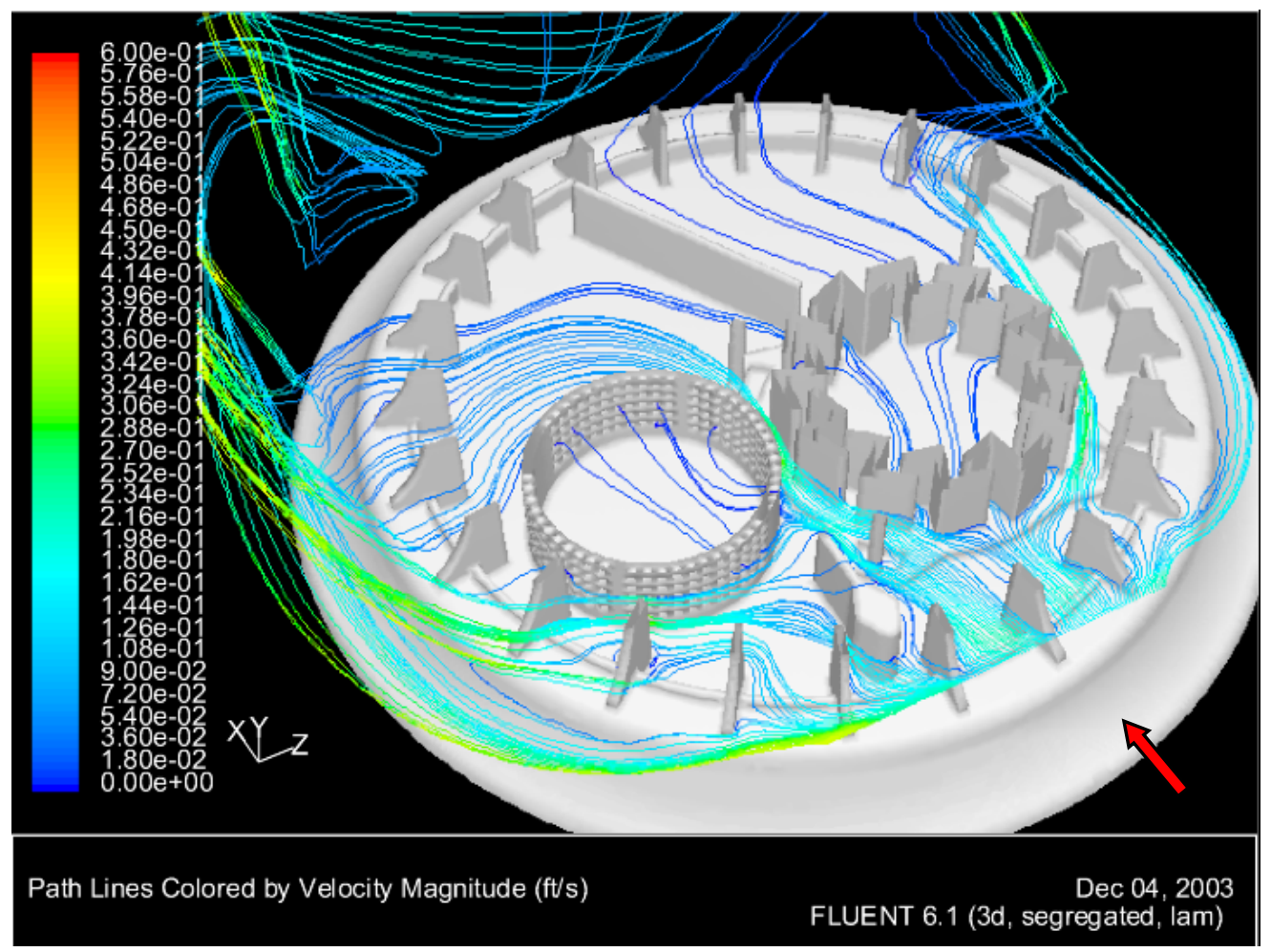

Figure 6.6.53 Detector flow visualization showing possible flow patterns inside the sensing elements of the SA302 $\left(180^{\circ}\right.$ orientation).

Figures 6.6.54 through 6.6.64 report the results for the $270^{\circ}$ orientation. In this orientation, the ionization sensor is in the wake of the photoelectric chamber. The detector's wake much like the earlier figures resembles the same basic flow patterns. In this instance, the photoelectric chamber is fed the majority of its air flow from the right hand side of its baffles normal to the oncoming flow direction, and the ionization sensor, as seen in Figures 6.6.59 and 6.6.60, receives most of its smoke particles from the bottom outside edge. According to the pathline predictions in Figures 6.6.63 and 6.6.64, the ionization sensor receives very little flow from the wake of the photoelectric sensor. In this instance, the main flow deflector does more harm than good because it is deflecting the majority of air back out of the semi-enclosure. Only a minute amount of rapidly moving flow is able to slip between the photoelectric sensor and deflector to deliver particles to the top half of the ionization sensor. Because of this orientation, ionization sensor speeds are on the predicted order of less than $0.5 \mathrm{ft} / \mathrm{min}$ with photoelectric sensor speeds once again ranging on the high end close to $8 \mathrm{ft} / \mathrm{min}$. 


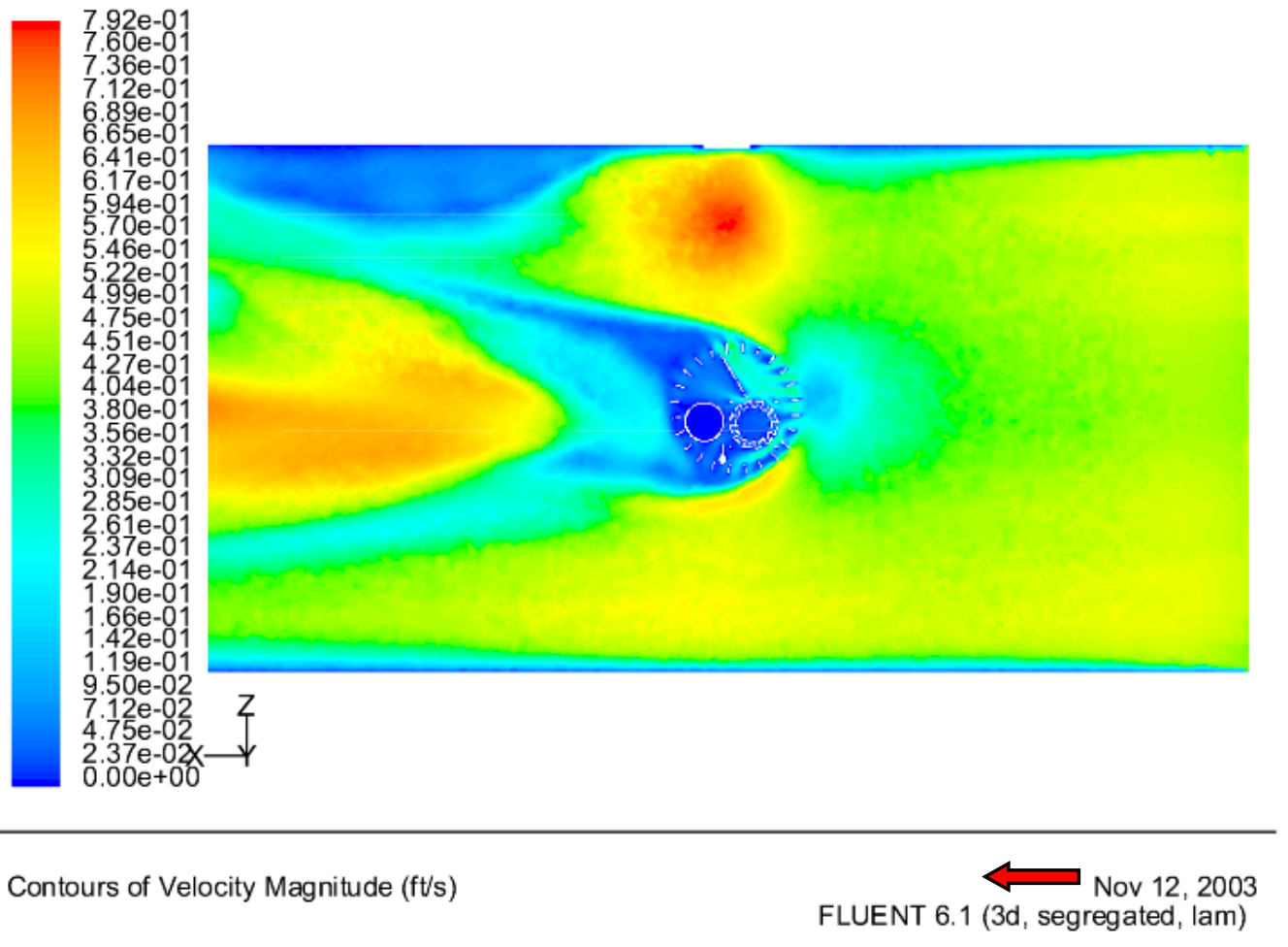

Figure 6.6.54: A velocity contour plot using the laminar model depicting the $y=0.50$ in plane for the $270^{\circ}$ orientation.

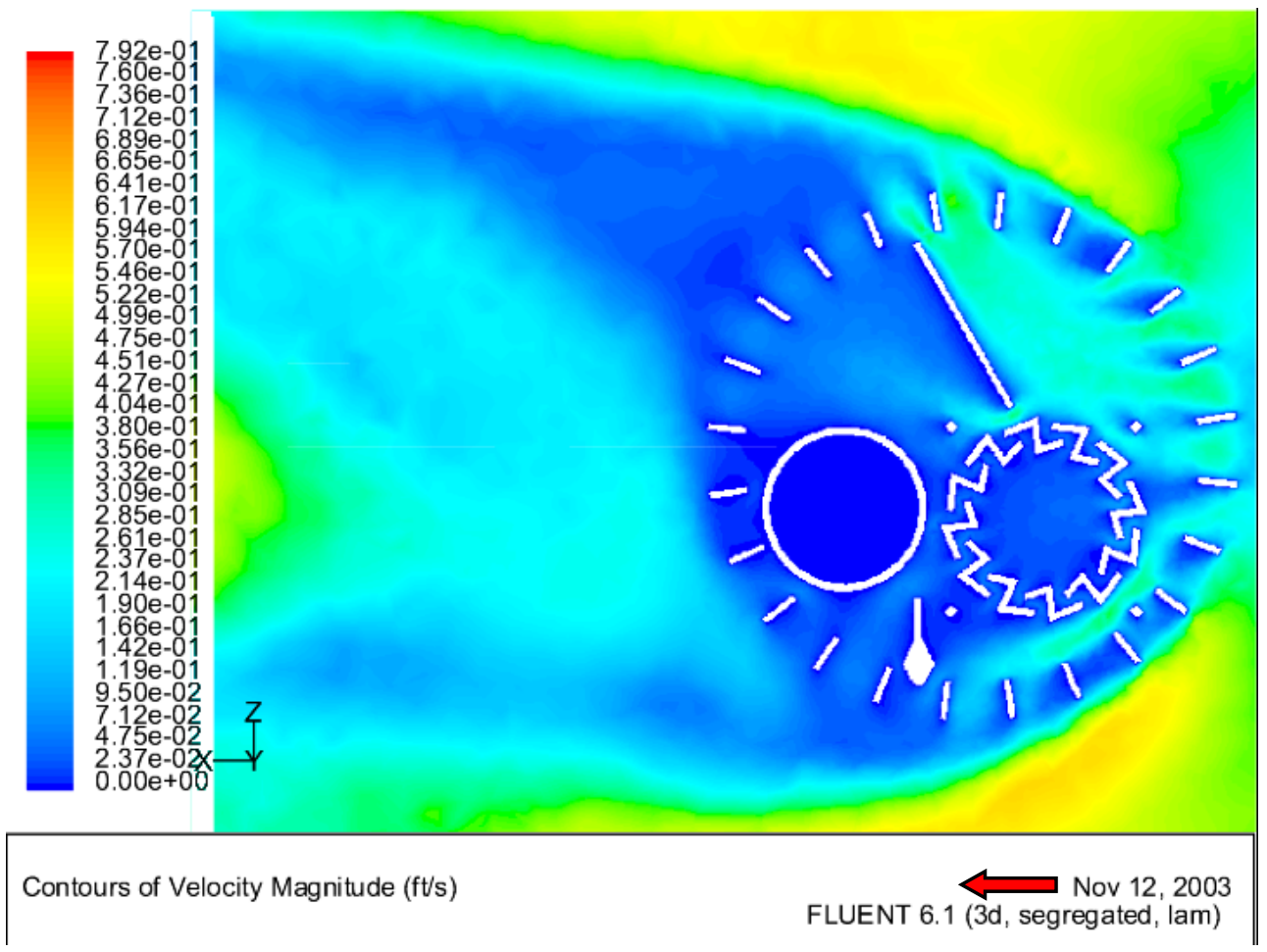

Figure 6.6.55: A velocity contour plot using the laminar model depicting the detector and its wake in the $y=0.50$ in plane for the $270^{\circ}$ orientation. 


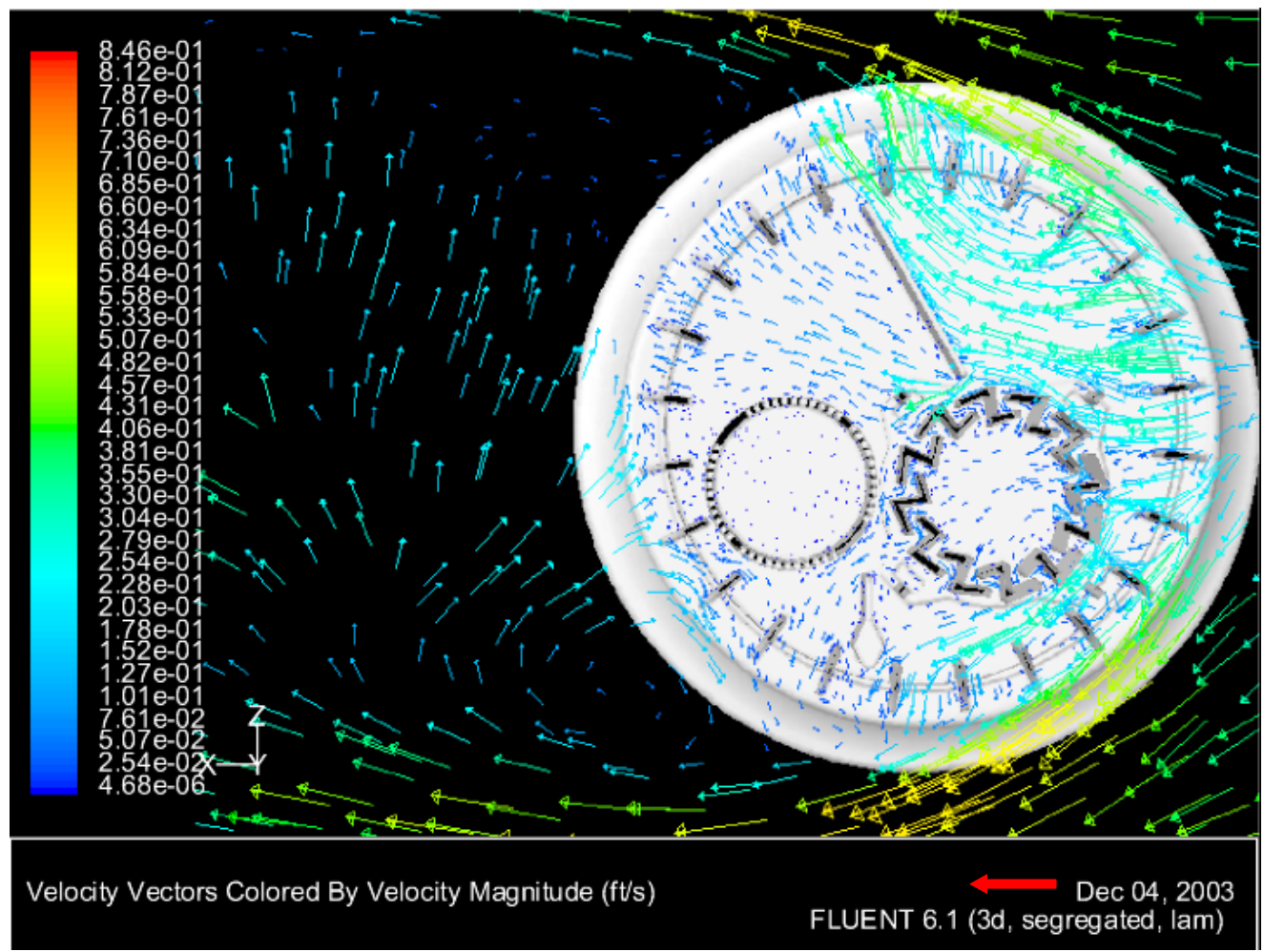

Figure 6.6.56: A velocity vector plot using the laminar model depicting the wake of the detector approaching the pressure outlet boundary in the $y=0.50$ in plane $\left(270^{\circ}\right.$ Orientation).

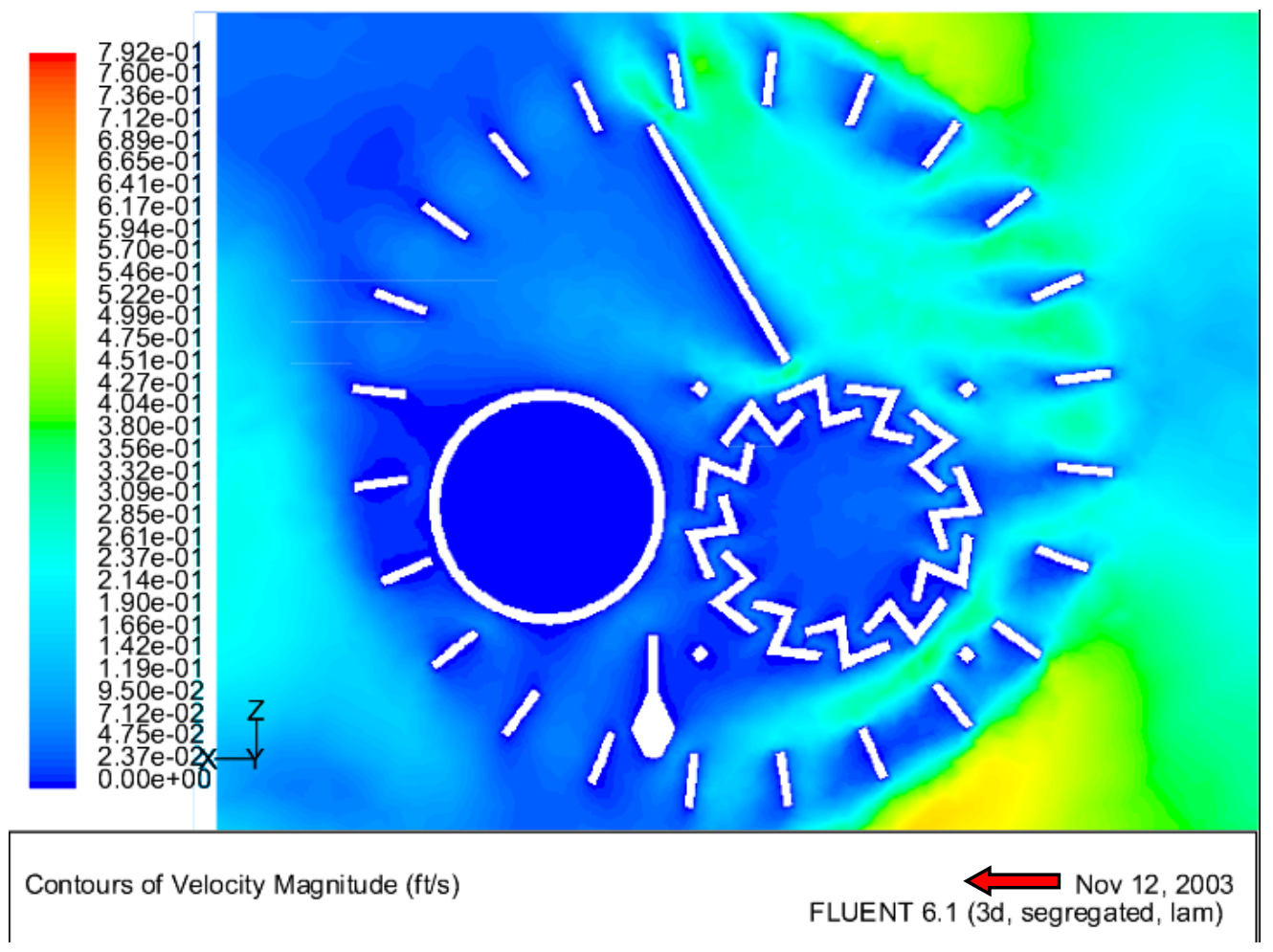

Figure 6.6.57 Detector flow visualization showing possible flow patterns inside the sensing elements of the SA302 $\left(270^{\circ}\right.$ orientation). 


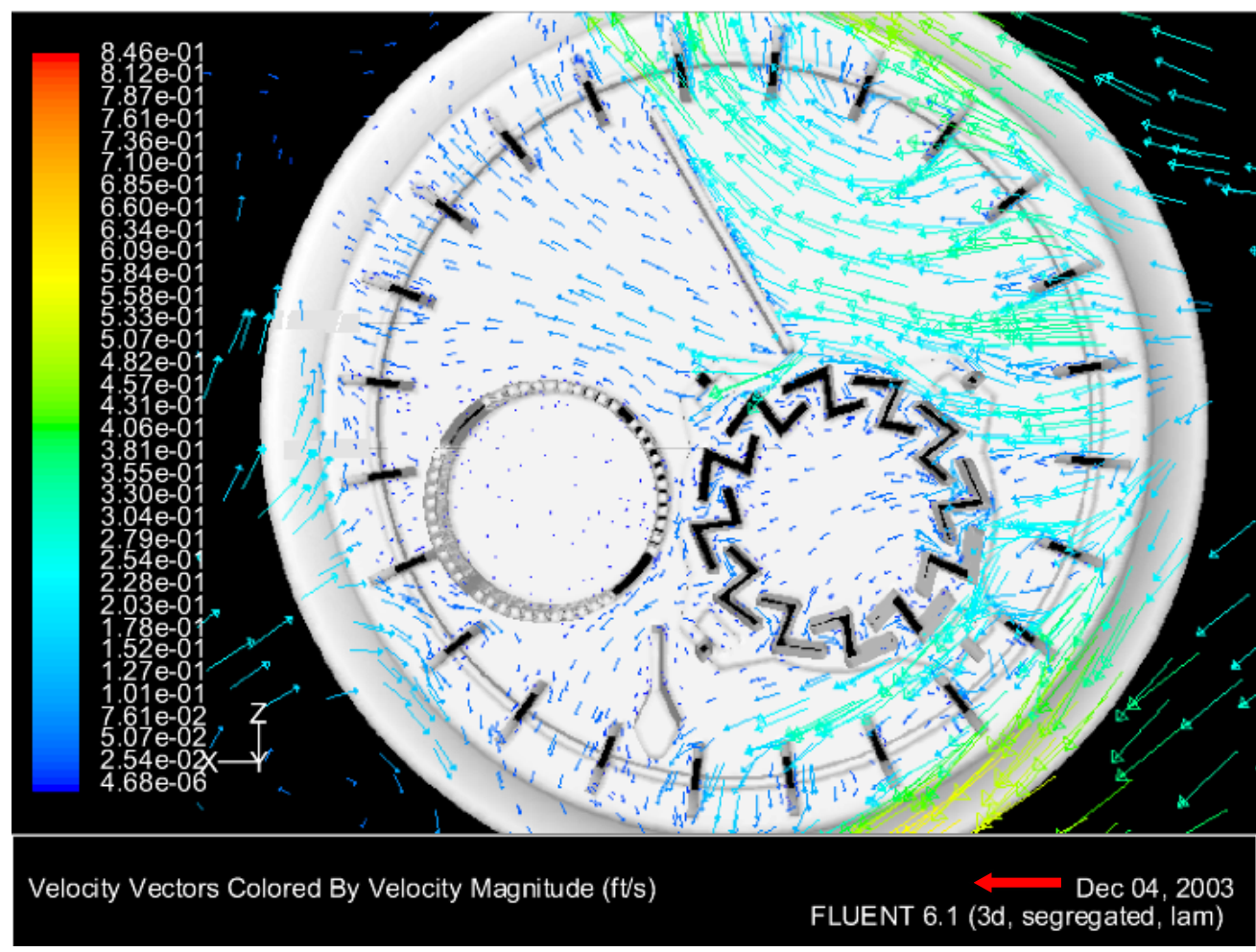

Figure 6.6.58 A velocity vector plot using the laminar model depicting the detector in the $y=$ 0.50 in plane for the $270^{\circ}$ orientation.
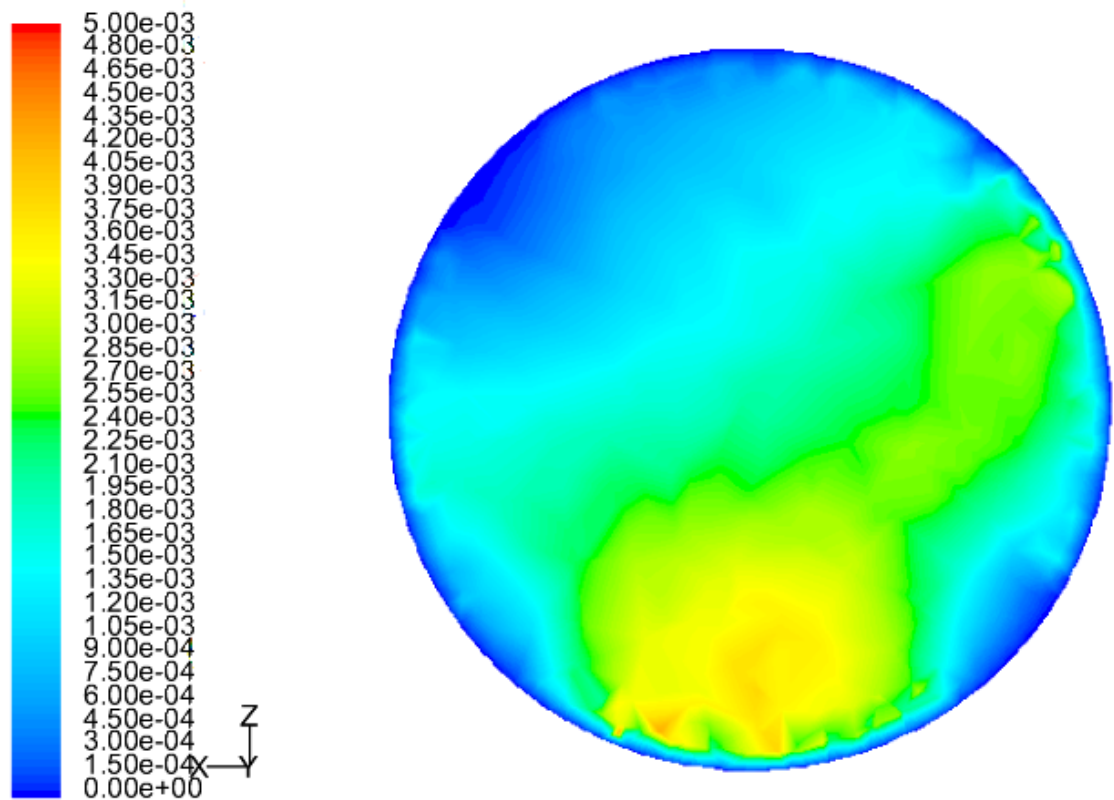

Contours of Velocity Magnitude (ft/s)

FLUENT 6.1 (3d, segregated, lam)

Figure 6.6.59 A velocity contour plot using the laminar model depicting the ionization chamber in the $y=0.50$ in plane for the $270^{\circ}$ orientation. 


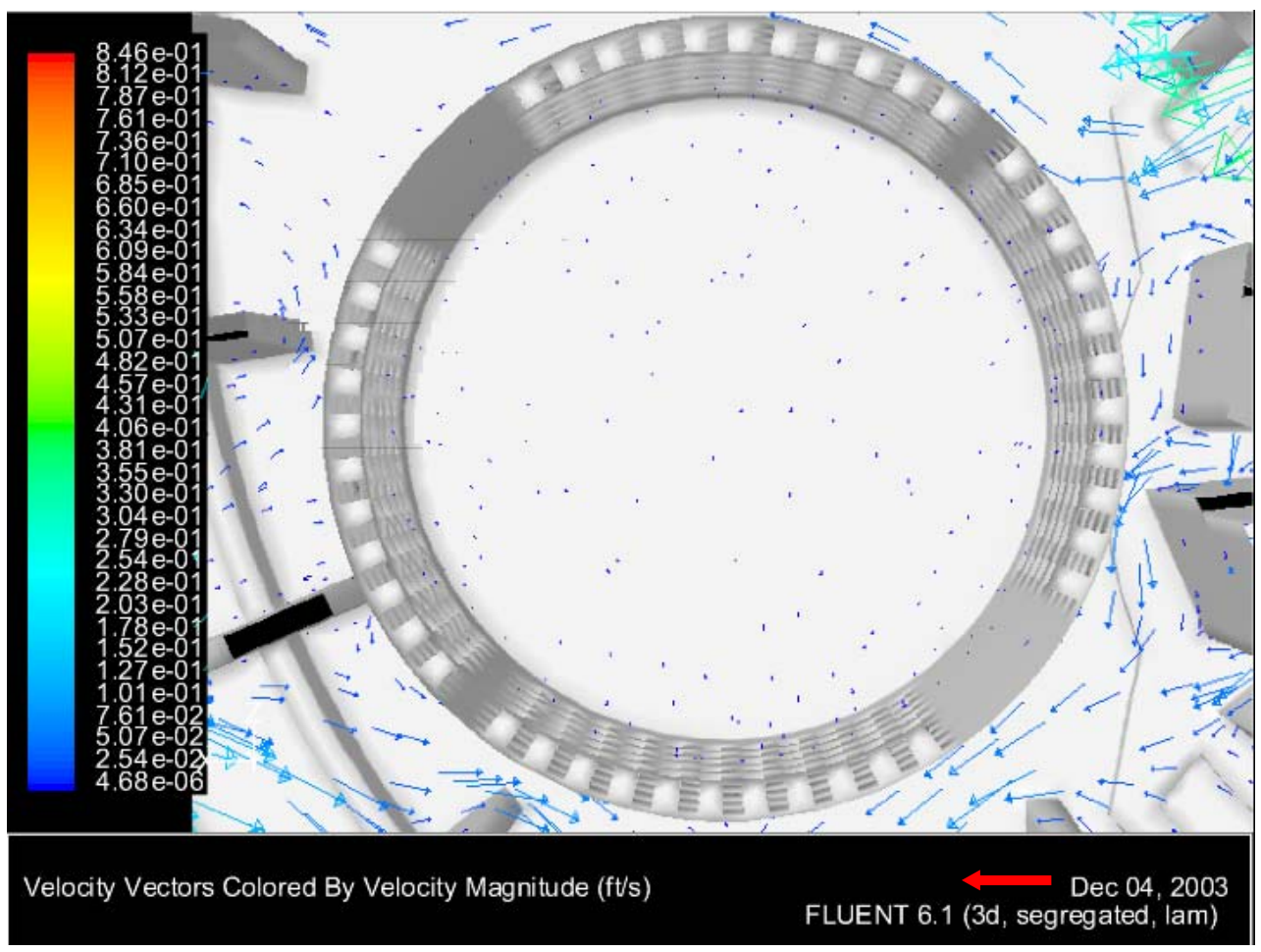

Figure 6.6.60 A velocity vector plot using the laminar model depicting the ionization chamber in the $y=0.50$ in plane for the $270^{\circ}$ orientation.
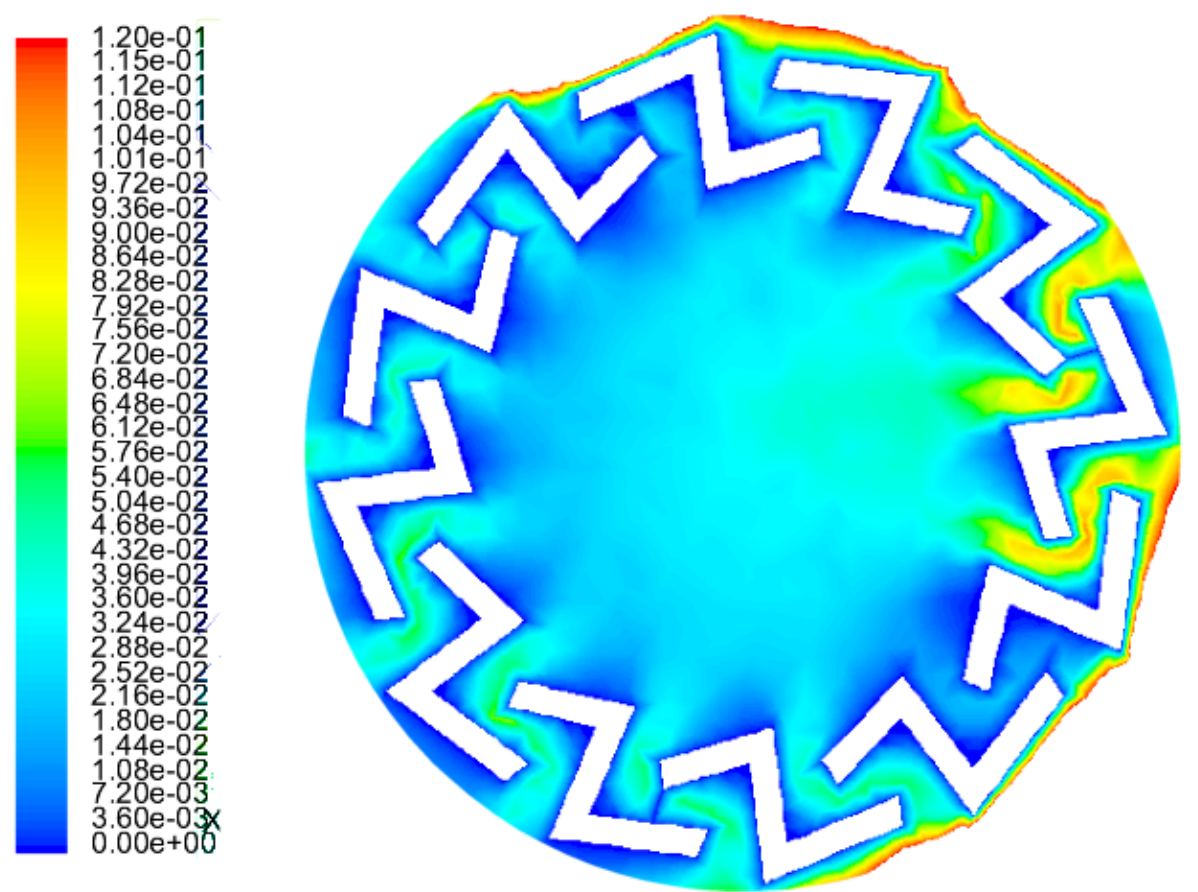

Contours of Velocity Magnitude (ft/s)

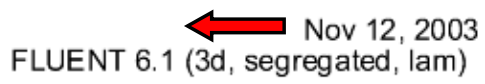

Figure 6.6.61 A velocity contour plot using the laminar model depicting the photoelectric chamber in the $y=0.50$ in plane for the $270^{\circ}$ orientation. 


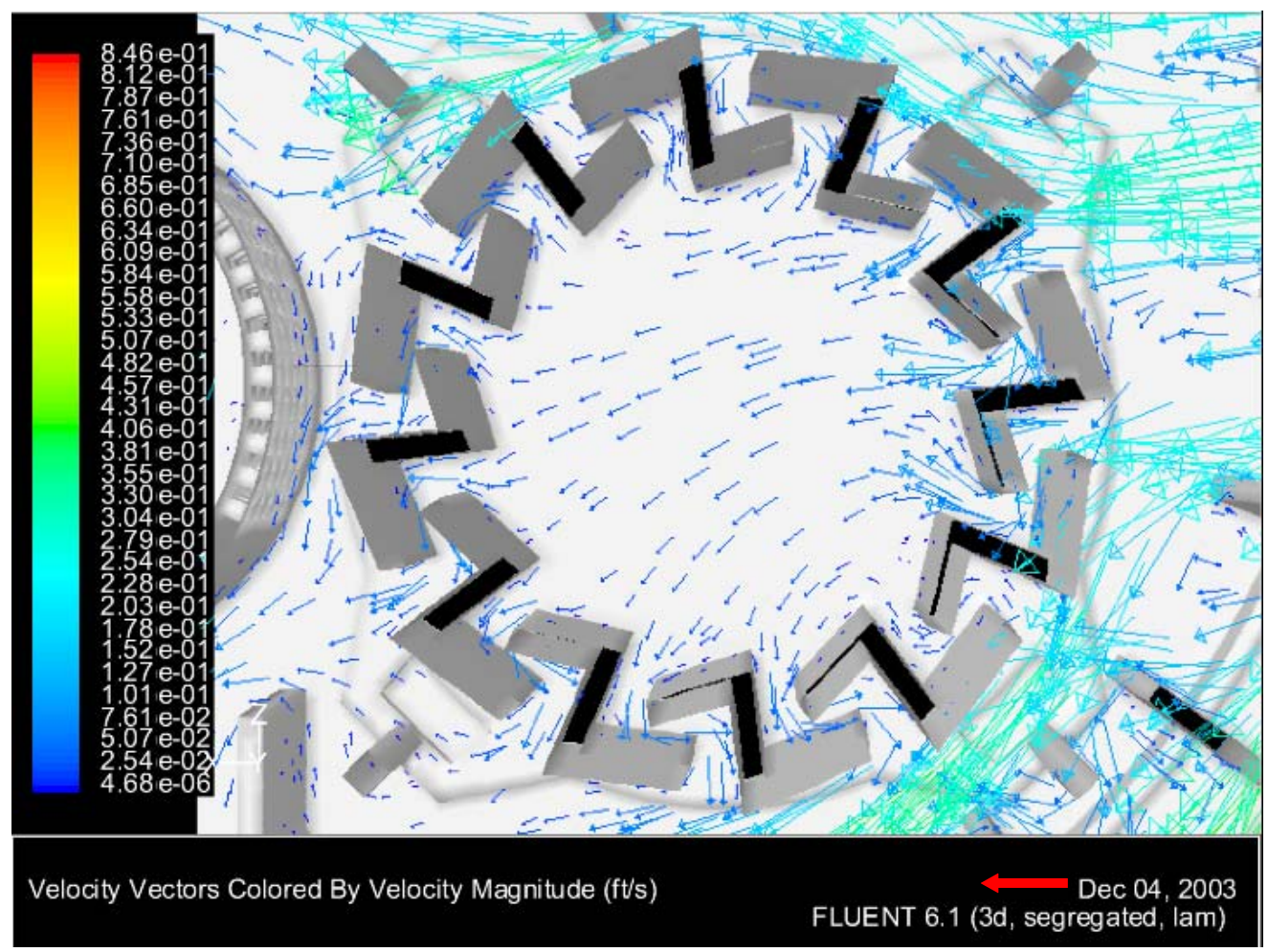

Figure 6.6.62 A velocity vector plot using the laminar model depicting the photoelectric chamber in the $y=0.50$ in plane for the $270^{\circ}$ orientation.

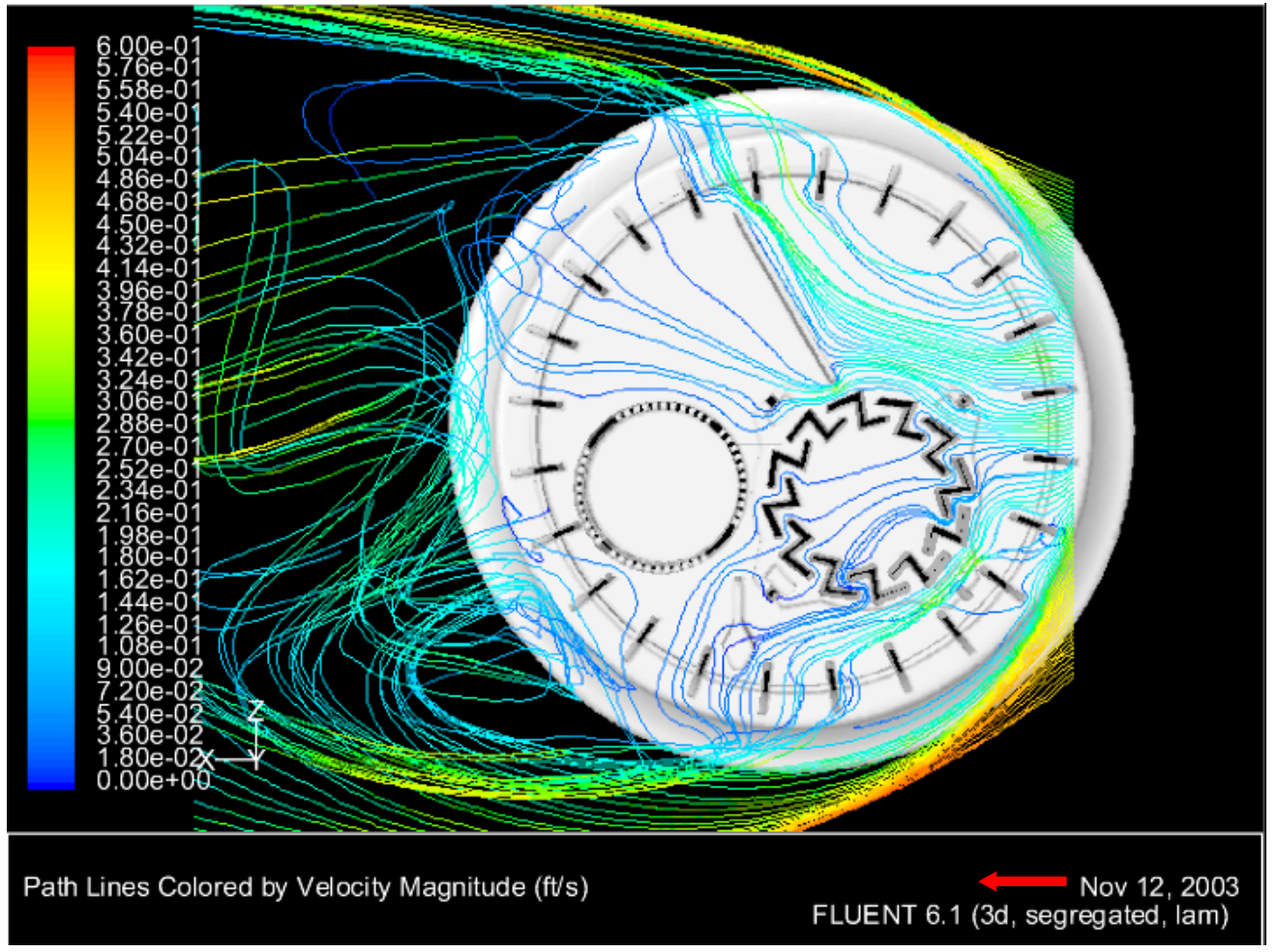

Figure 6.6.63 Detector flow visualization showing possible flow patterns inside the sensing elements of the SA302 $\left(270^{\circ}\right.$ orientation). 


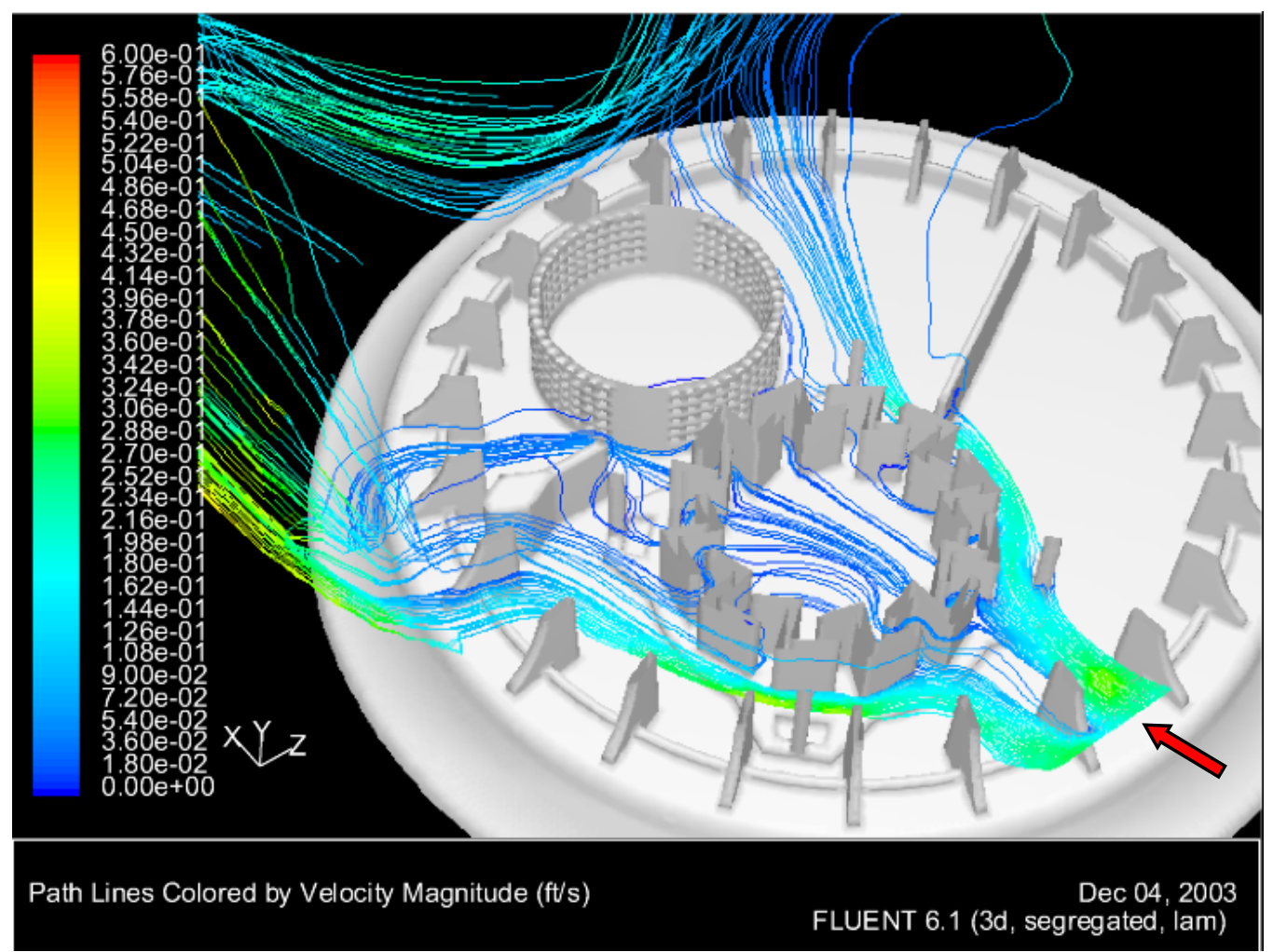

Figure 6.6.64 Detector flow visualization showing the route of 100 pathlines injected 1 in above the floor near the entrance to the SA302 smoke detector $\left(270^{\circ}\right.$ orientation).

Figures 6.6.65 through 6.6.75 depict the results for the $315^{\circ}$ orientation computational model. This orientation was picked specifically to characterize the effects of the ionization sensor being completely in the wake of the detector. As one can see from Figures 6.6.65 and 6.6.66, the entire detector wake is slightly different from those in previous orientations, in large part due to the separation of flow as it passes across the deflector normal to its path. From this, a re-circulating region seems to be more prominent in the top half of the wake as compared to previous models. In comparing velocity magnitudes experienced by both sensing elements, the computational model predicts the photoelectric chamber to reach internal speeds of up to almost $10 \mathrm{ft} / \mathrm{min}$. However, the ionization chamber being in the wake of the main flow deflector experiences speeds on the order of only $1 \mathrm{ft} / \mathrm{min}$. Figures 6.6.74 and 6.6.75 also predict a relatively low likelihood that a significant amount of smoke particles will enter the ionization chamber first in comparison to the photoelectric chamber. 


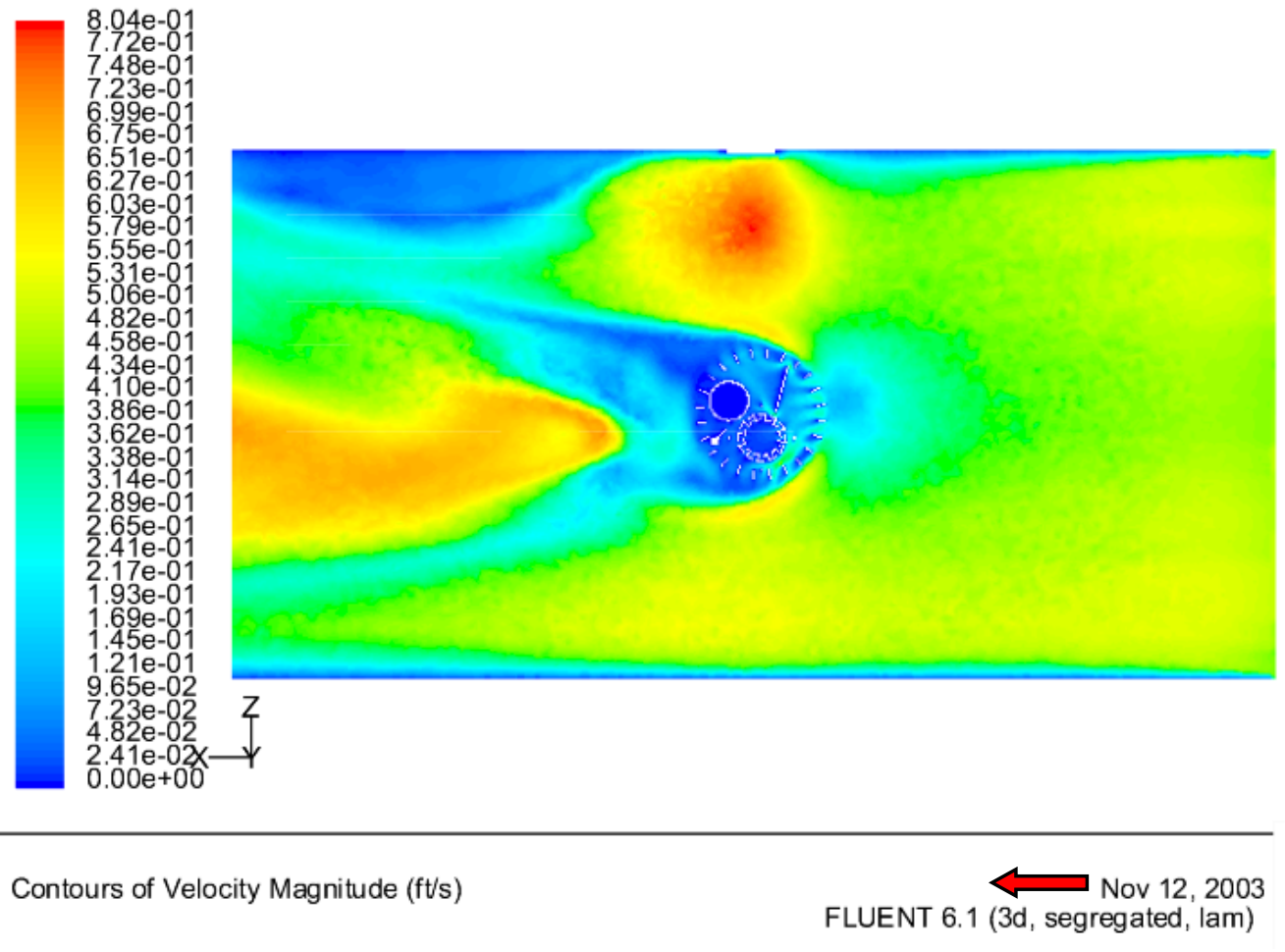

Figure 6.6.65: A velocity contour plot using the laminar model depicting the $y=0.50$ in plane for the $315^{\circ}$ orientation.

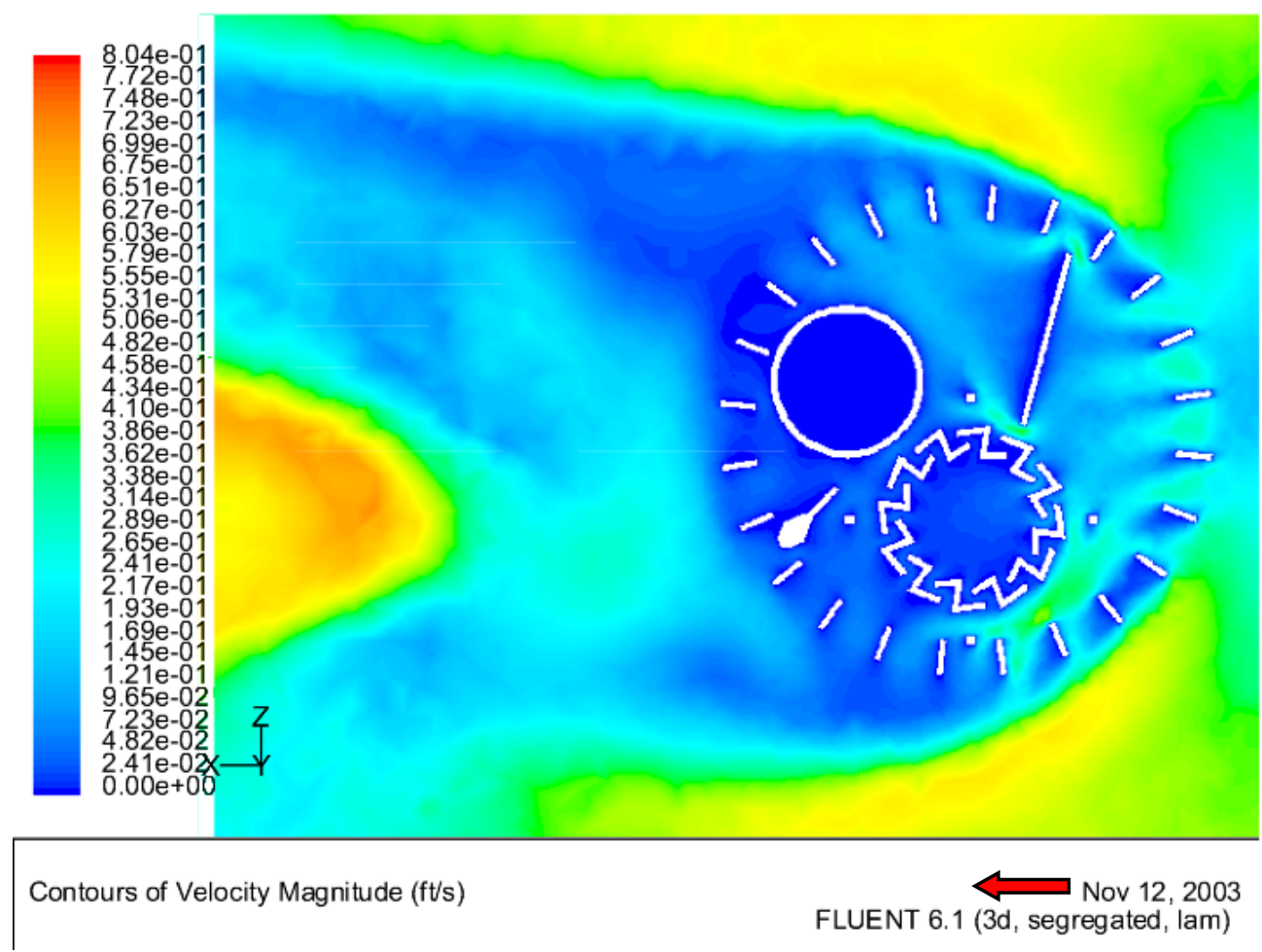

Figure 6.6.66: A velocity contour plot using the laminar model depicting the detector and its wake in the $y=0.50$ in plane for the $315^{\circ}$ orientation. 


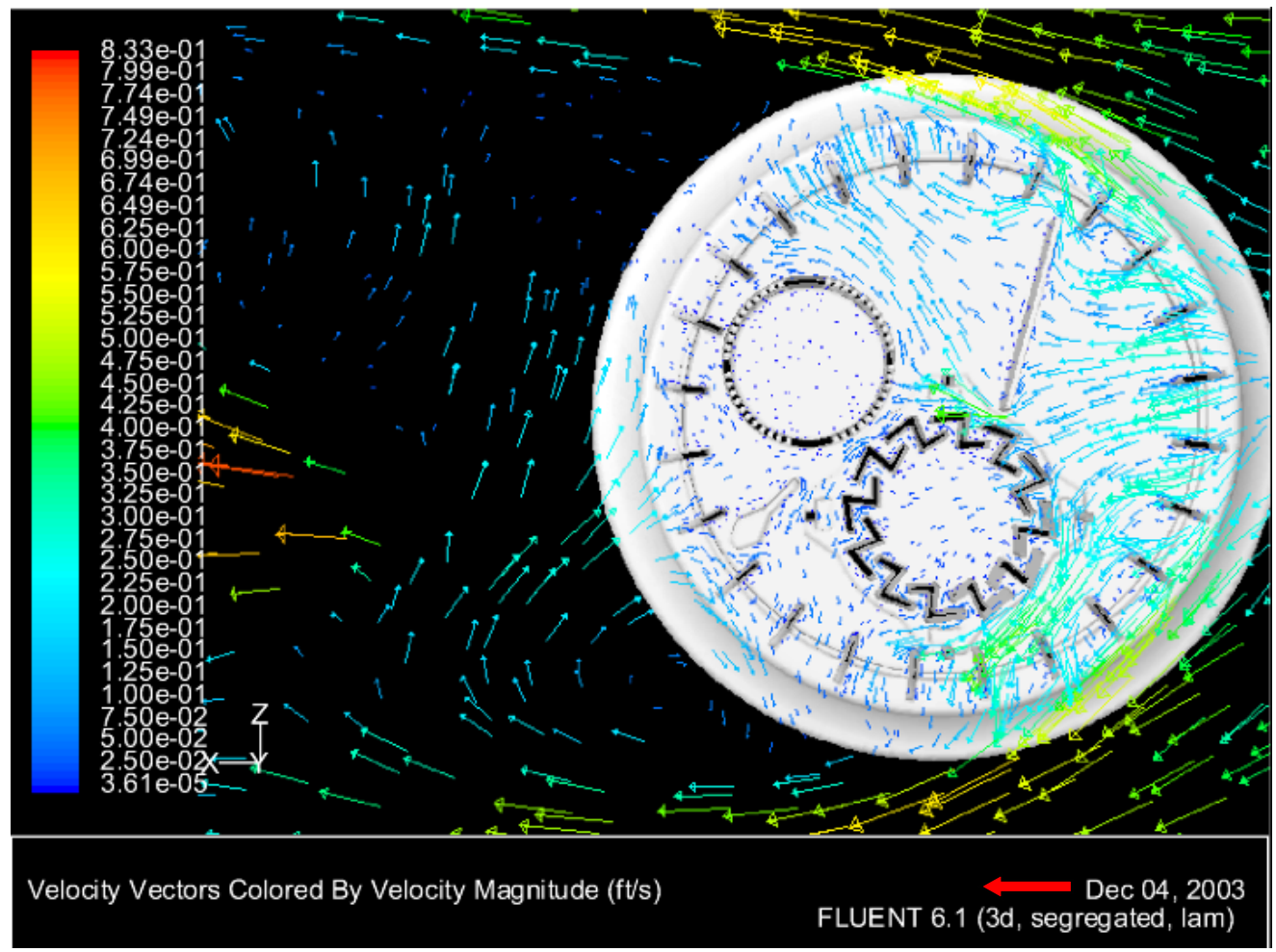

Figure 6.6.67: A velocity vector plot using the laminar model depicting the wake of the detector approaching the pressure outlet boundary in the $y=0.50$ in plane ( $315^{\circ}$ Orientation).

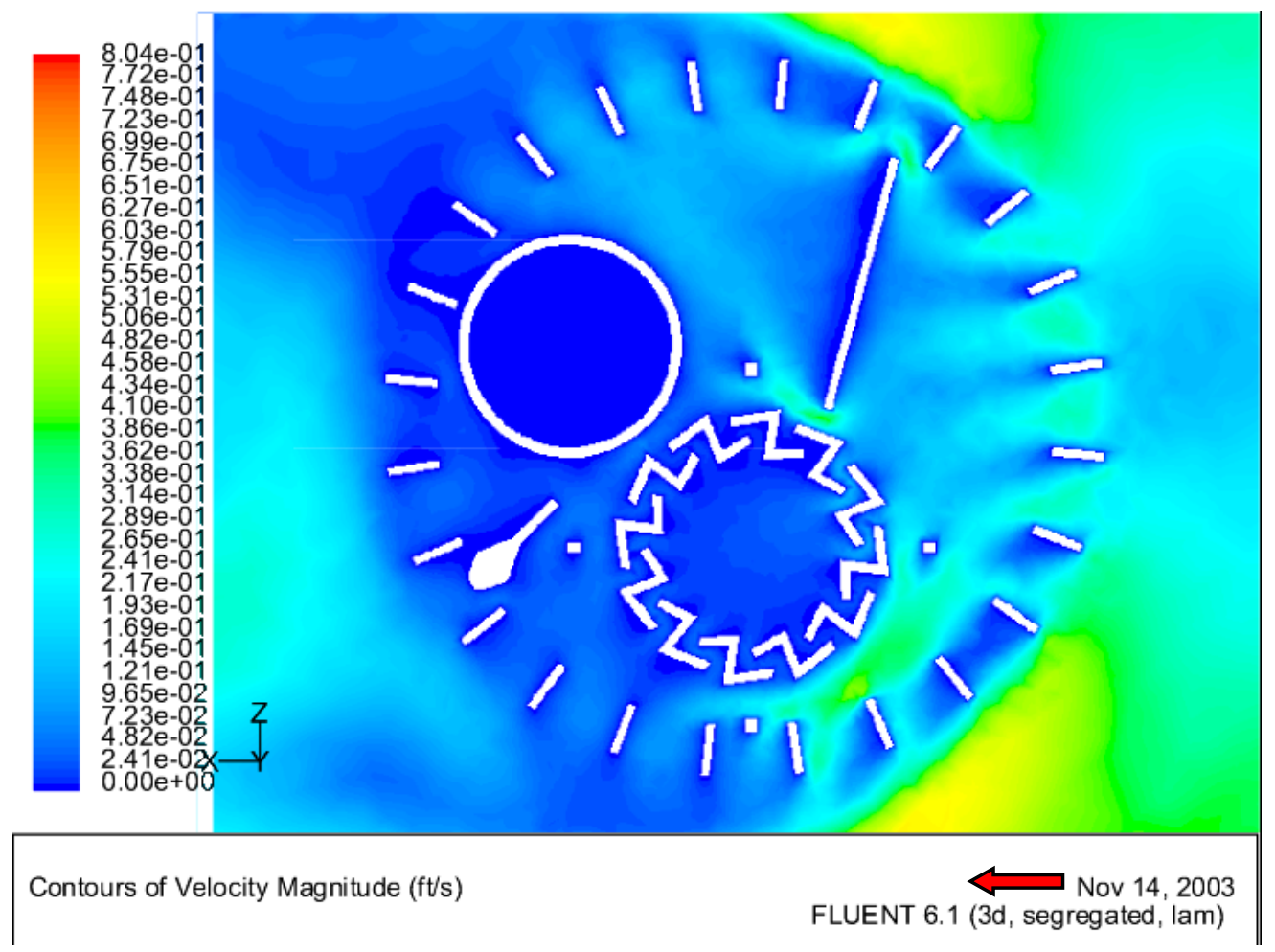

Figure 6.6.68 Detector flow visualization showing possible flow patterns inside the sensing elements of the SA302 (315 ${ }^{\circ}$ orientation). 


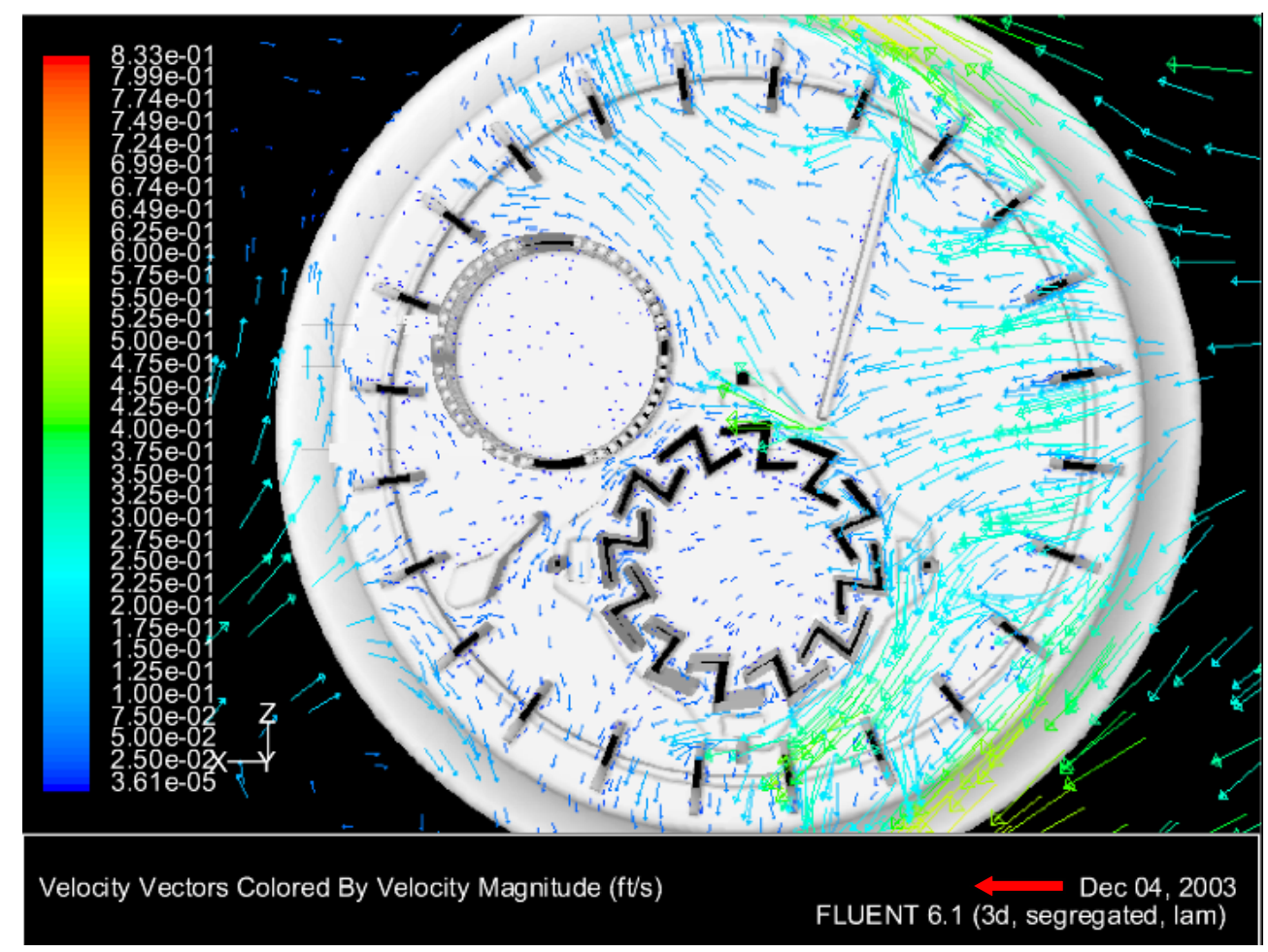

Figure 6.6.69 A velocity vector plot using the laminar model depicting the detector in the $y=$ 0.50 in plane for the $315^{\circ}$ orientation.
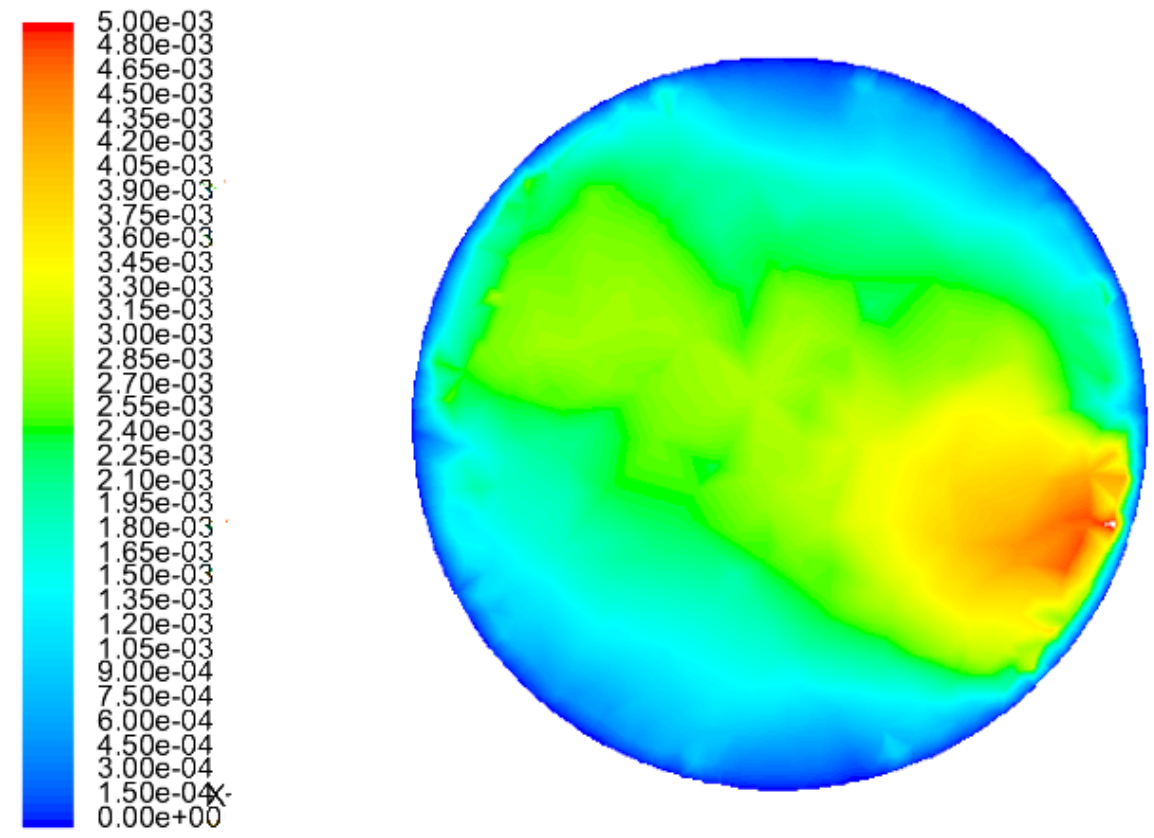

Contours of Velocity Magnitude (ft/s)

FLUENT 6.1 (3d, segregated, lam)

Figure 6.6.70 A velocity contour plot using the laminar model depicting the ionization chamber in the $y=0.50$ in plane for the $315^{\circ}$ orientation. 


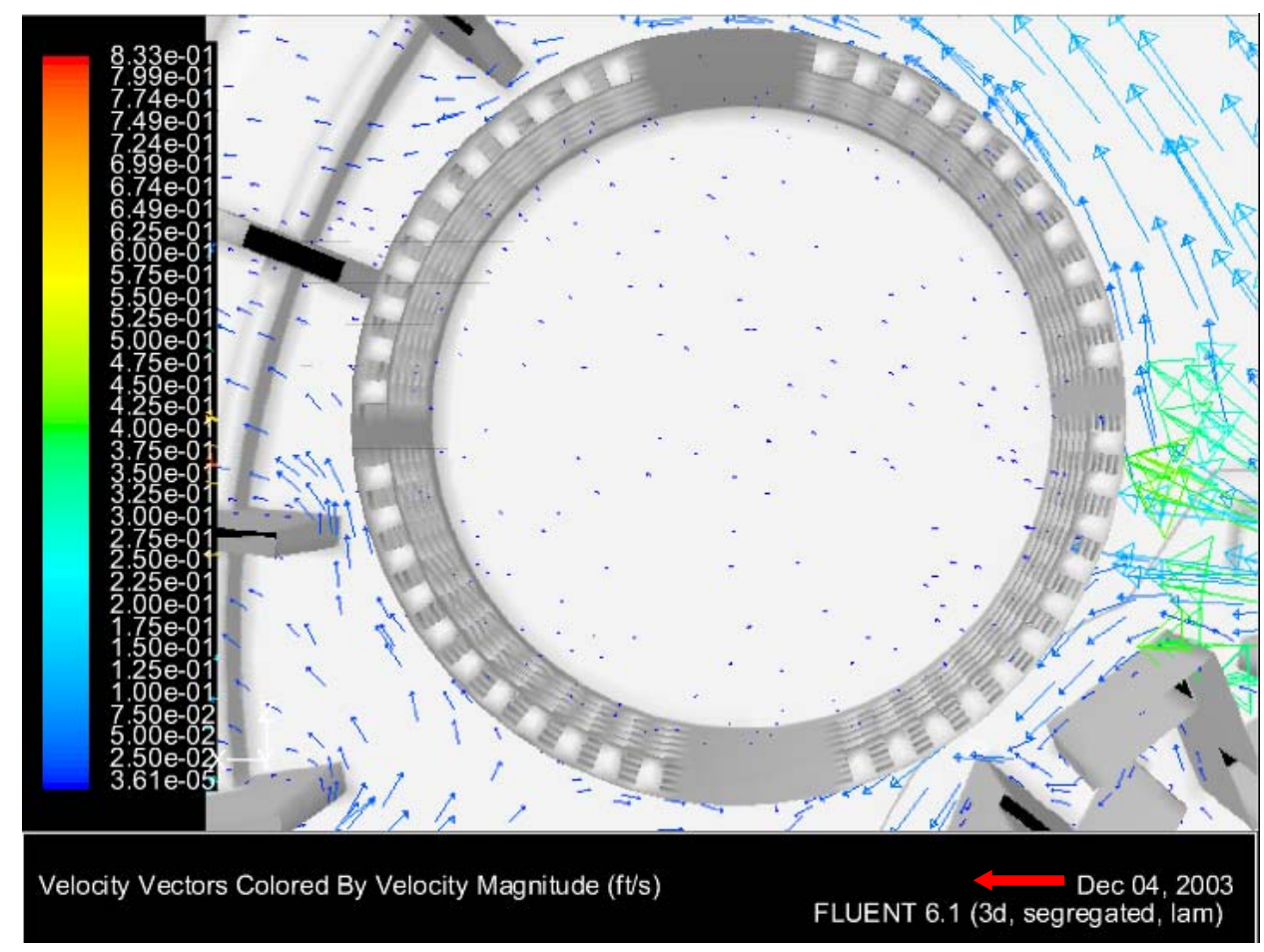

Figure 6.6.71 A velocity vector plot using the laminar model depicting the ionization chamber in the $y=0.50$ in plane for the $315^{\circ}$ orientation.
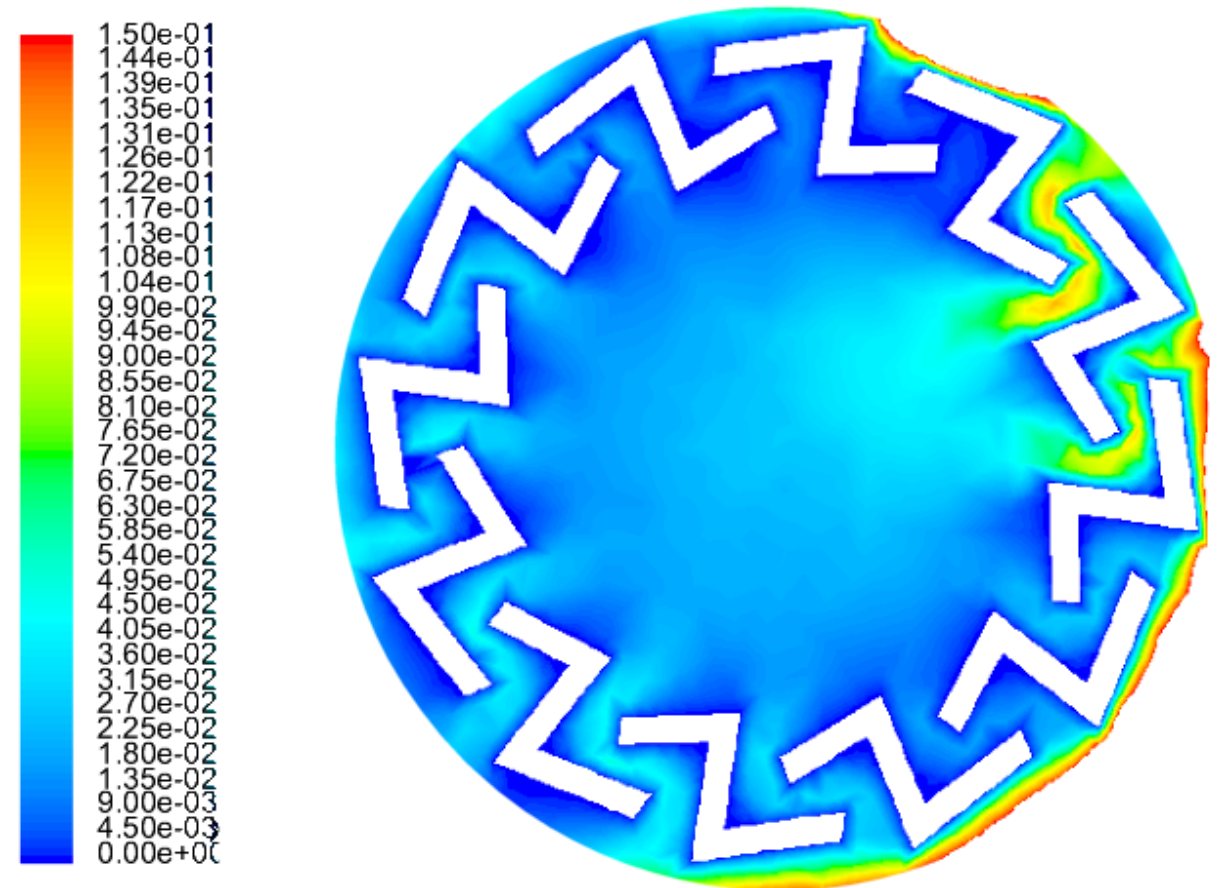

Contours of Velocity Magnitude (ft/s)

Nov 12, 2003

FLUENT 6.1 (3d, segregated, lam)

Figure 6.6.72 A velocity contour plot using the laminar model depicting the photoelectric chamber in the $y=0.50$ in plane for the $315^{\circ}$ orientation. 


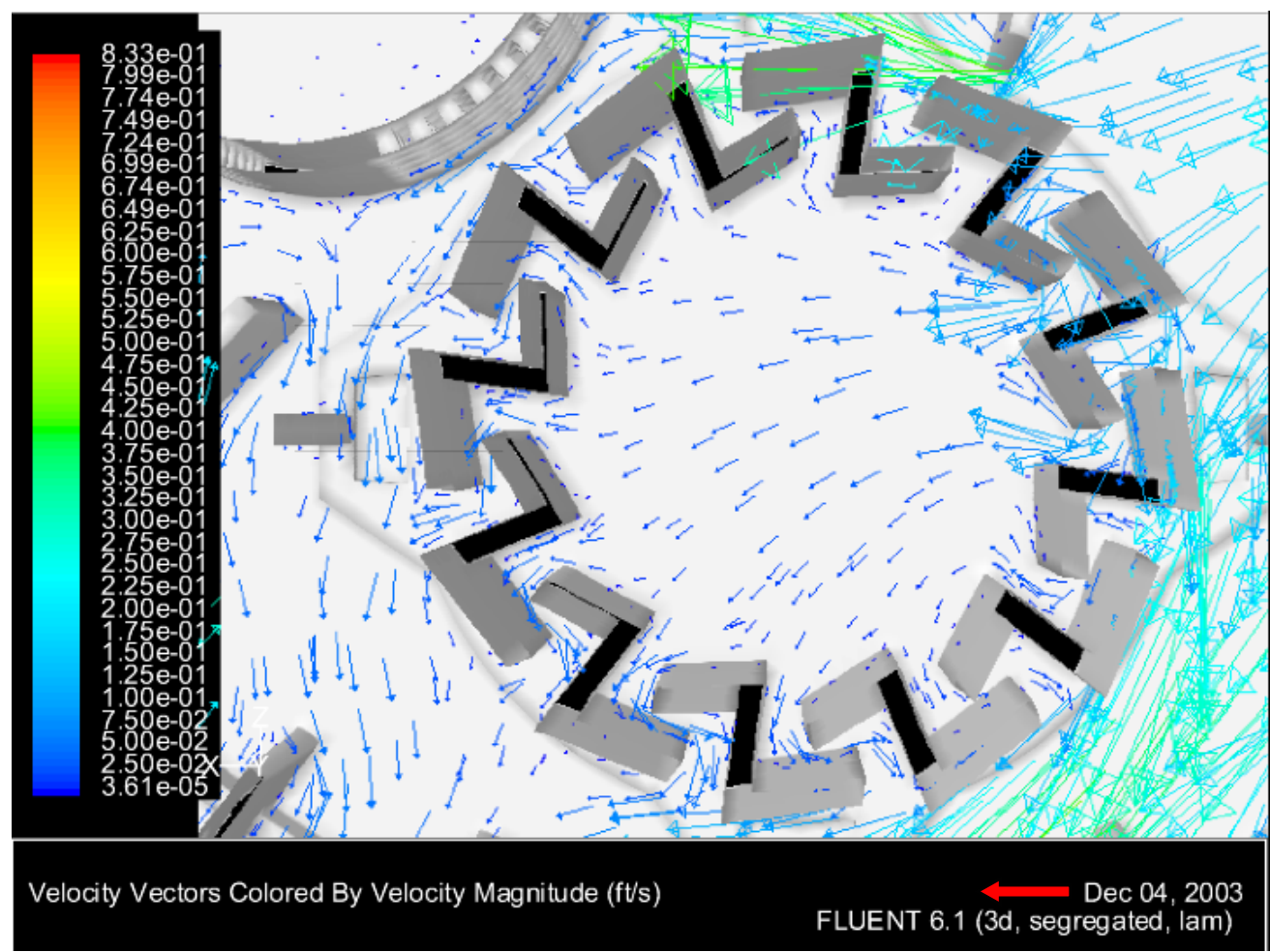

Figure 6.6.73 A velocity vector plot using the laminar model depicting the photoelectric chamber in the $y=0.50$ in plane for the $315^{\circ}$ orientation.

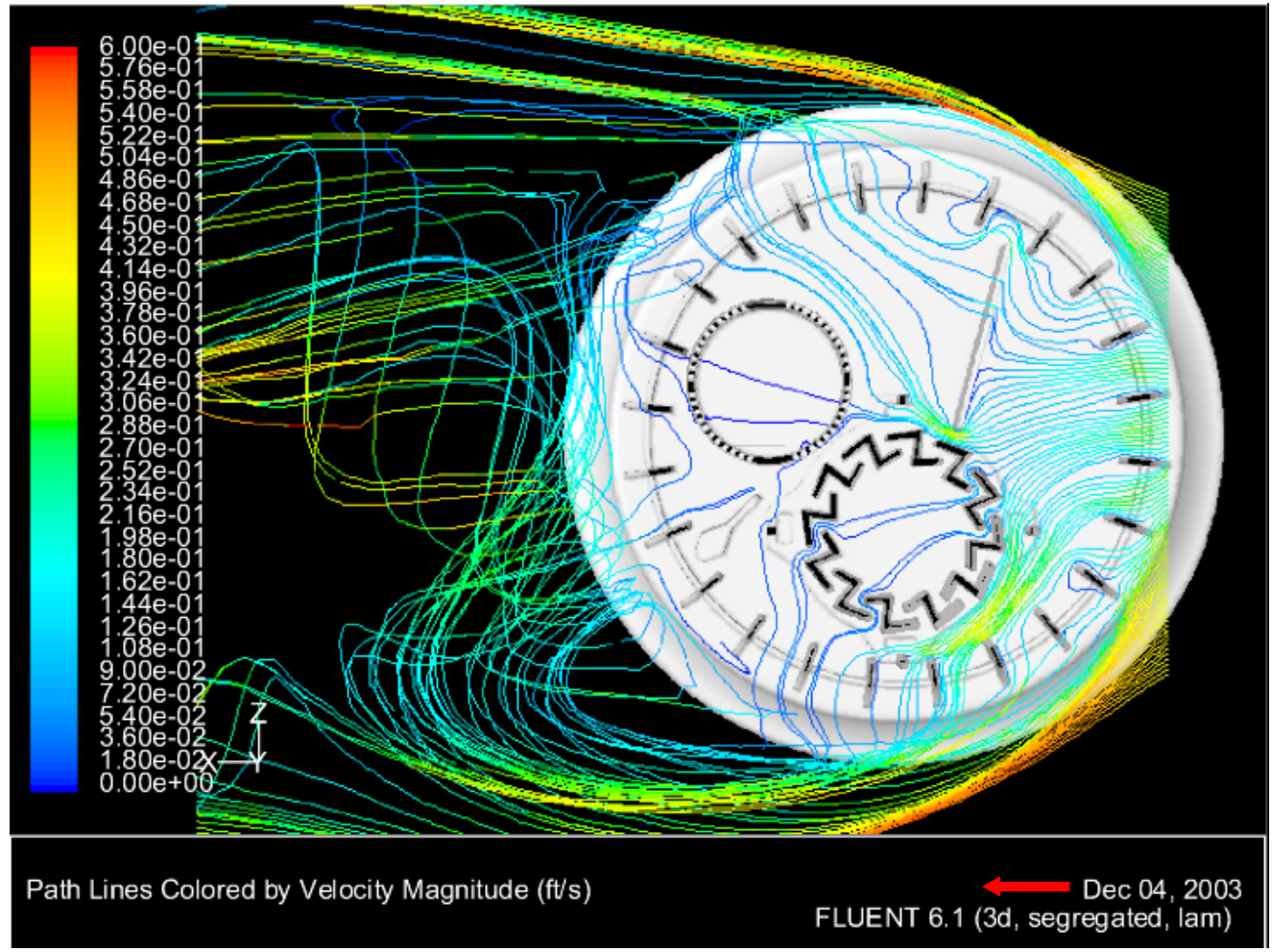

Figure 6.6.74 Detector flow visualization showing the route of 100 pathlines injected 1 in above the floor near the entrance to the SA302 smoke detector ( $315^{\circ}$ orientation). 


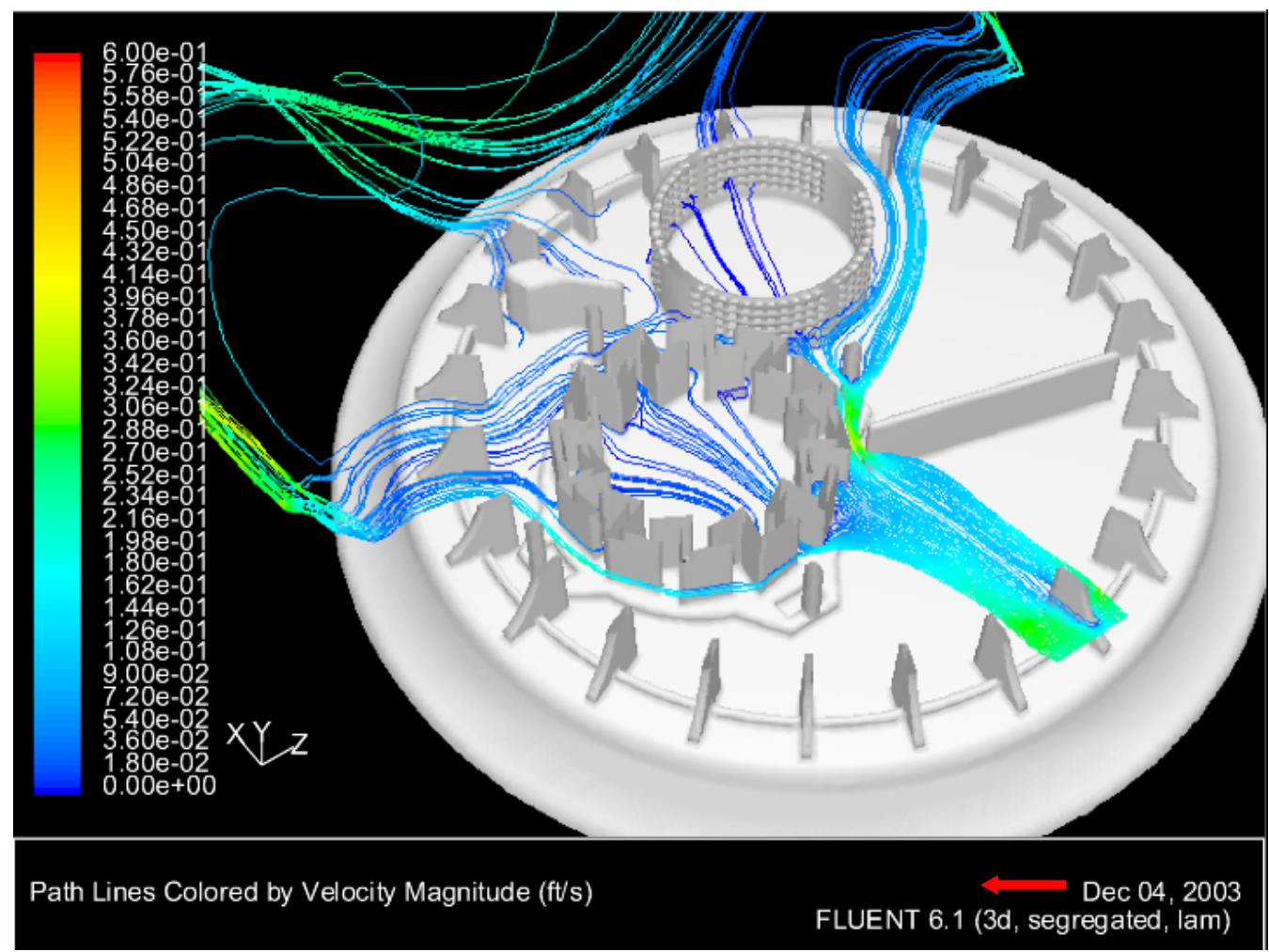

Figure 6.6.75 Detector flow visualization showing possible flow patterns inside the sensing elements of the SA302 (315 ${ }^{\circ}$ orientation).

\subsection{UL SMOKE BOX LDA VS. CFD VELOCITY COMPARISONS}

Figures 6.7.1 through 6.7.11 show $x-y$ plots comparing actual experimental LDA data against results predicted computationally by Fluent ${ }^{\circledR 18}$. The data being compared are $u, v$, and $w$ velocity rakes in the surrounding vicinity of the detector. Transverse rakes are shown for 1 in above the test section floor at $x=12,13,14,15,21,22$, and 23 in, then at $x=18$ in, rakes at $y=3,4$, and $5 \mathrm{in}$. The comparisons are designed such that the approach planes can be compared, as well as locations above the detector between the probe inlet and upper dome, and likewise downstream in the wake of the detector. Visual legends are placed where applicable on the plots to convey the location of the rake being compared highlighted in red, with the rest in the subset shaded black. An accompanying red arrow denotes the main axial flow direction.

In general, results pertaining to the axial, $u$ velocity component were excellent, with comparative error often less than $10 \%$. This should not come as quite a surprise, because much time was spent on developing the inlet condition with regard to the flow straightener 
producing nearly uniform axial flow, which was evident in the flow visualization. However, discrepancies begin to arise when comparing the $v$ and $w$ component data approaching the detector, for reasons not quite easily concluded. In analyzing Figures 6.7.2, 6.7.5, 6.7.8 and 6.7.11, one can see that as the flow approaches the detector, comparative results get meagerly better in both the $v$ and $w$ components due to the presence of the detector forcing the velocity vectors upward and away and away from the MIC. For some rakes of experimental LDA, w component data, points seemed to be a bit more scattered than one would expect. A major issue that arises with the $x=18$ in data is the large velocity gradient directed downward due to the presence of the probe inlet sucking air down rapidly. This part of the computational model is highly reactive in this area of the flow field data showing evidence in Figures 6.7.14, 6.7.17, and 6.7.20. The $v$ and $w$ component LDA data are more sporadically placed in this zone, calculating velocities somewhat more chaotically due to the more turbulent nature of the incoming jet mixing with the main axial fluid motion. This area causes greater turbulence intensities making it harder to get a realistic experimental feel for the speeds in this area. The computational model, on the other hand, intuitively reports the increased $v$ component velocities as the flow curves over the top of the detector, but then drastically decreases negatively once the probe inlet is reached, and then recovers positive on the opposite side near the MIC.

The $w$ component CFD data seems to compare more accurately with that of the LDA data as it approaches the MIC as well. As the flow field motions toward the detector and MIC, $w$ component velocities negatively increase in size up until it reaches the MIC because its presence forces it back towards the detector in the opposite direction. The flow visualization supports these interpretations nicely (See Figures 6.2.5 through 6.2.7).

Strikingly, data in the wake of the detector in Figures 6.7.22 through 6.7.30 compares relatively very well with respect to all three velocity components. The steady, unsymmetrical wake appears to be captured well within 10-15\% comparative error between experimental measurements and CFD prediction with low turbulence intensities, with larger turbulence intensities resident along the centerline behind the detector. The asymmetry is best visualized in the $u$, axial component velocities where velocities on the right side closest to the MIC suction are stronger. Globally, the present CFD laminar model seems to adequately capture the major flow influences witnessed inside the test section. 


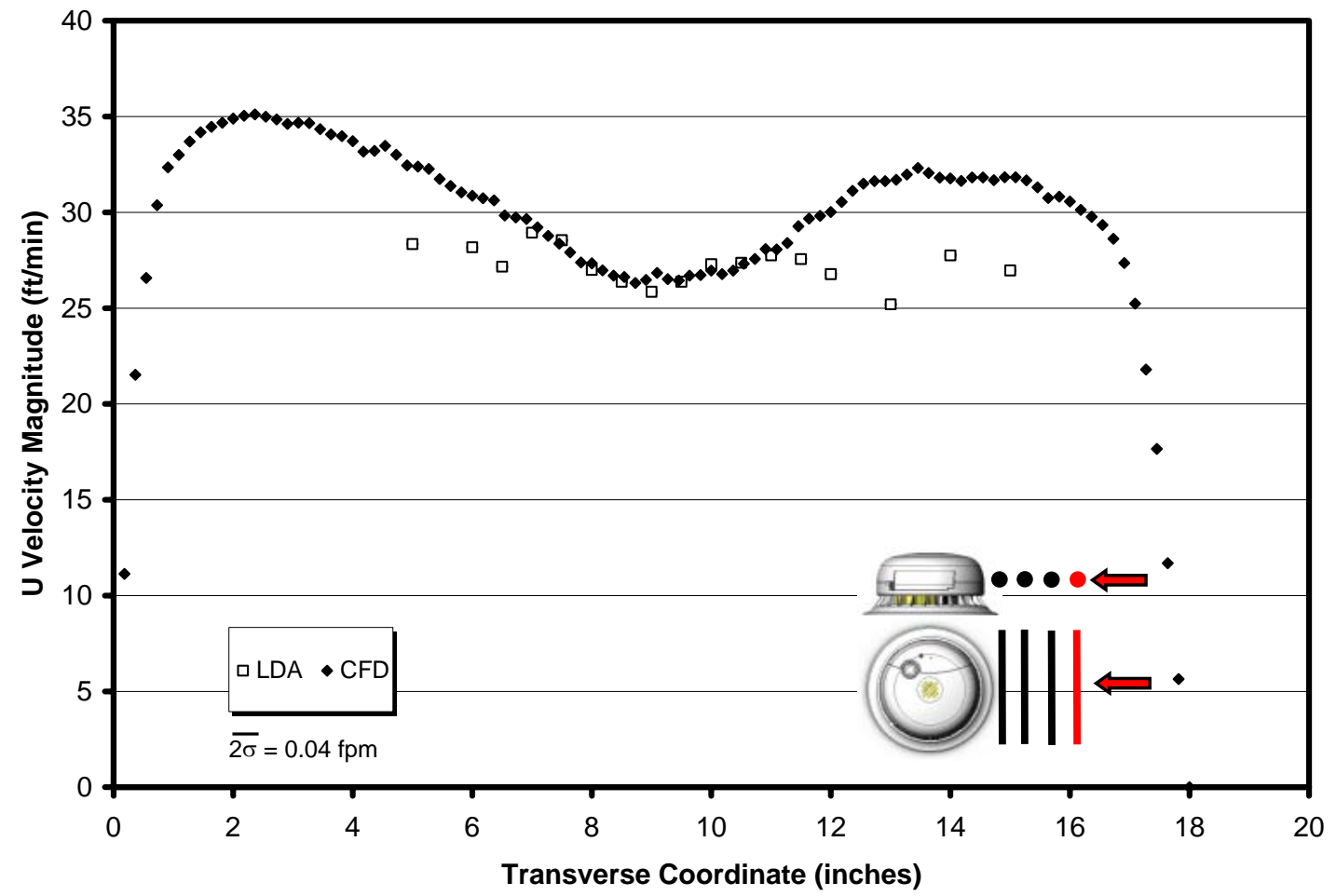

Figure 6.7.1: UL Smoke Box $u$ velocity comparisons between LDA and CFD at the $y=1$ in, $x=$ 12 in position spanning the transverse, $z$ direction.

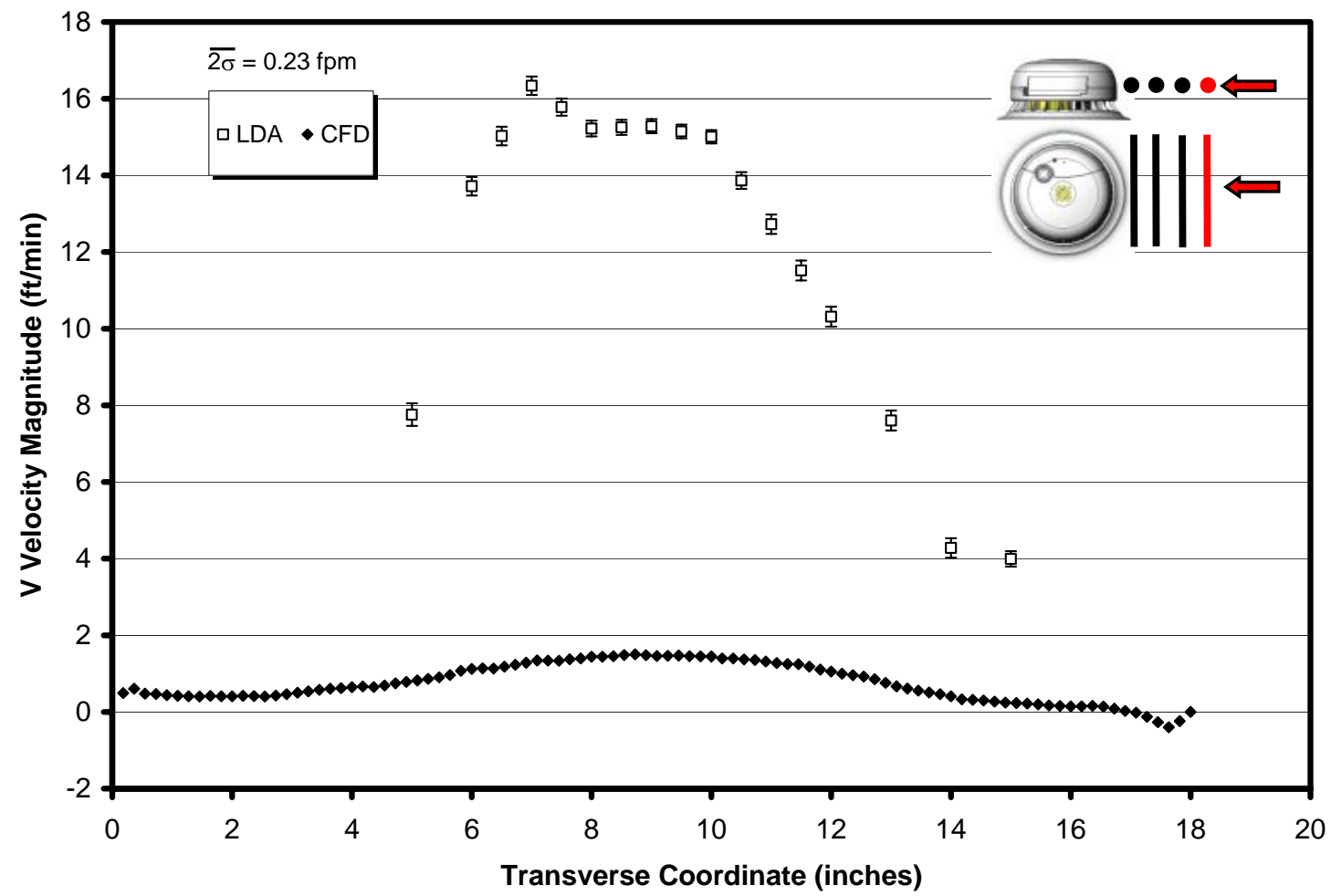

Figure 6.7.2: UL Smoke Box $v$ velocity comparisons between LDA and CFD at the $y=1$ in, $x=$ 12 in position spanning the transverse, $z$ direction. 


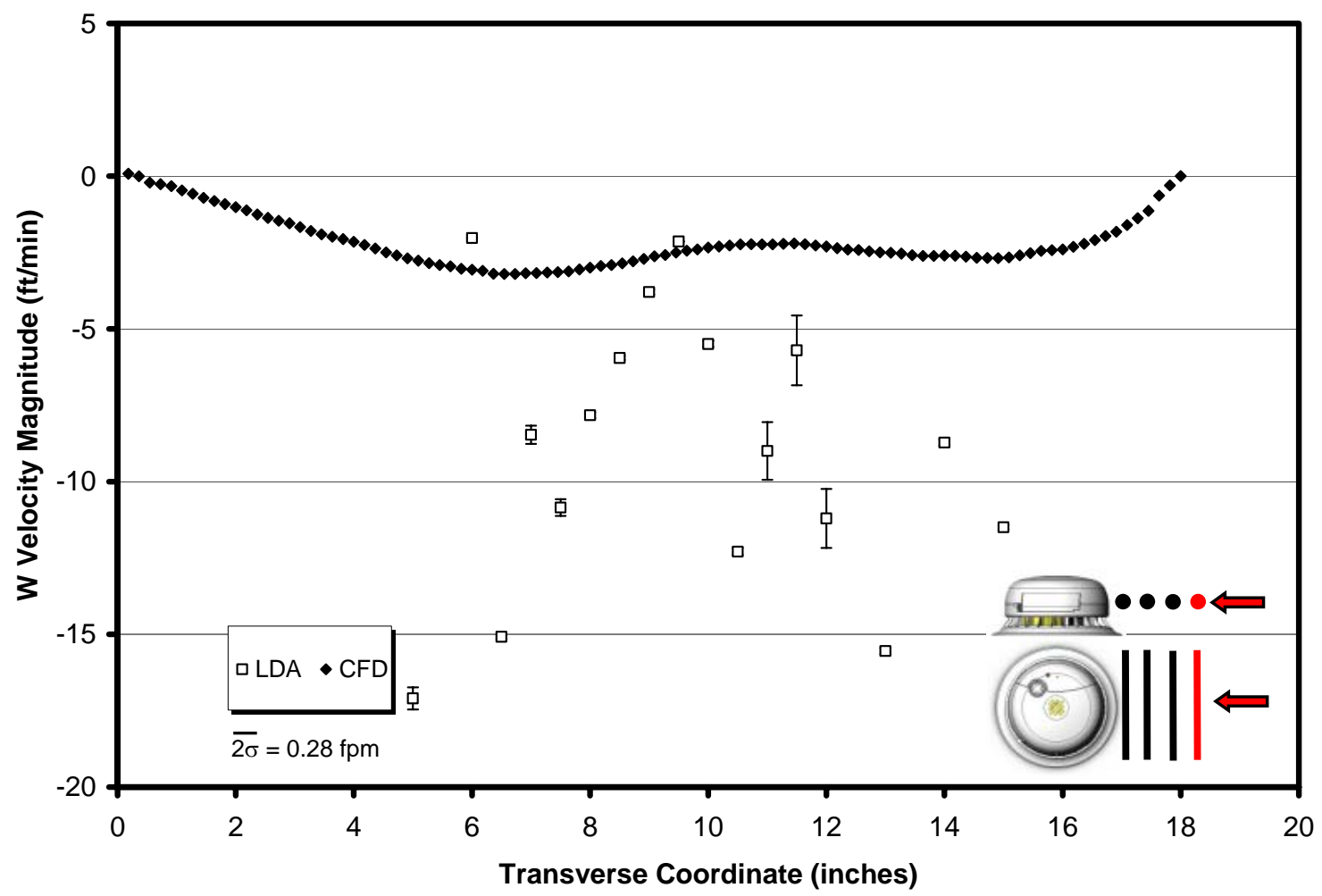

Figure 6.7.3: UL Smoke Box $w$ velocity comparisons between LDA and CFD at the $y=1$ in, $x=$ 12 in position spanning the transverse, $z$ direction.

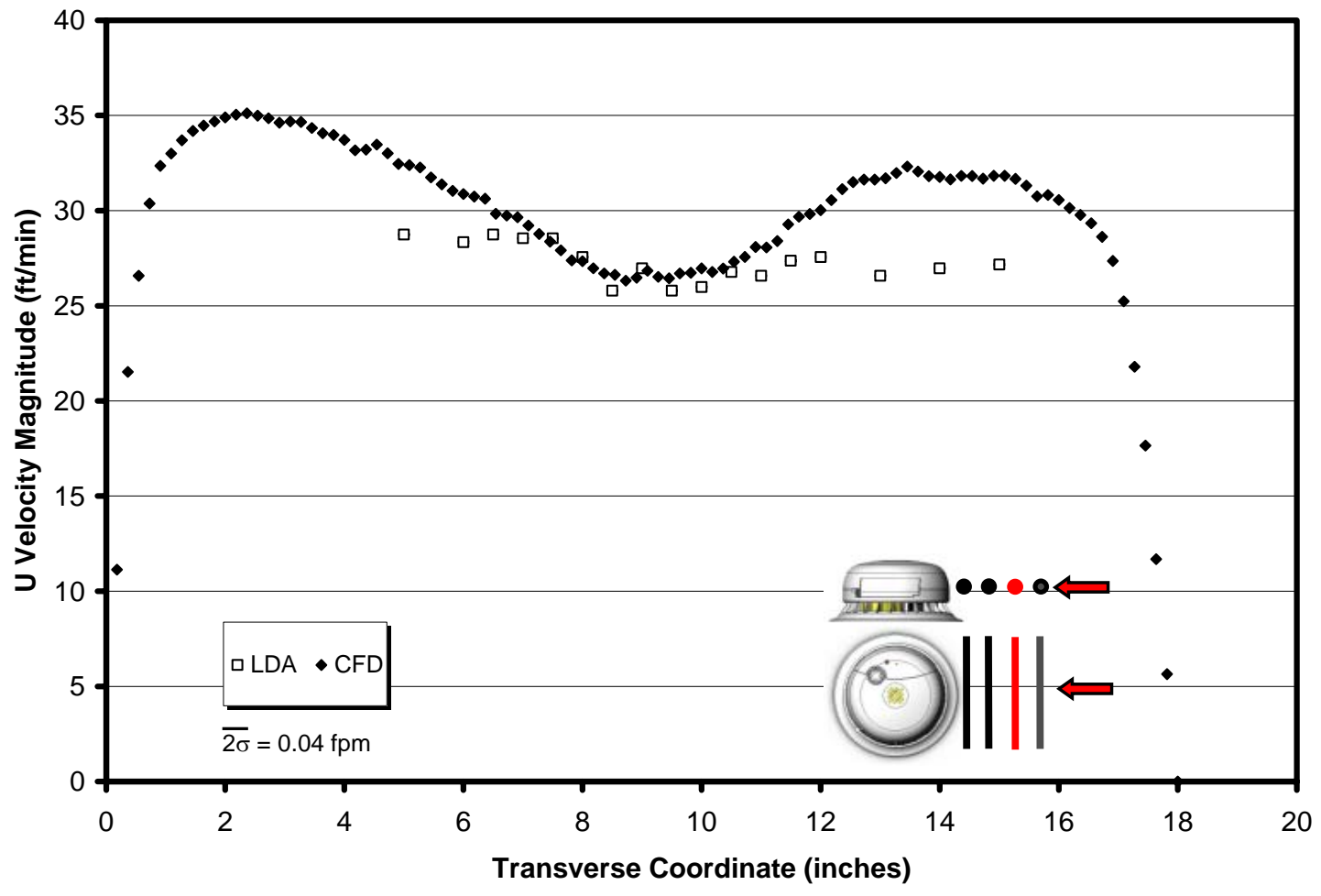

Figure 6.7.4: UL Smoke Box $u$ velocity comparisons between LDA and CFD at the $y=1$ in, $x=$ 13 in position spanning the transverse, $z$ direction. 


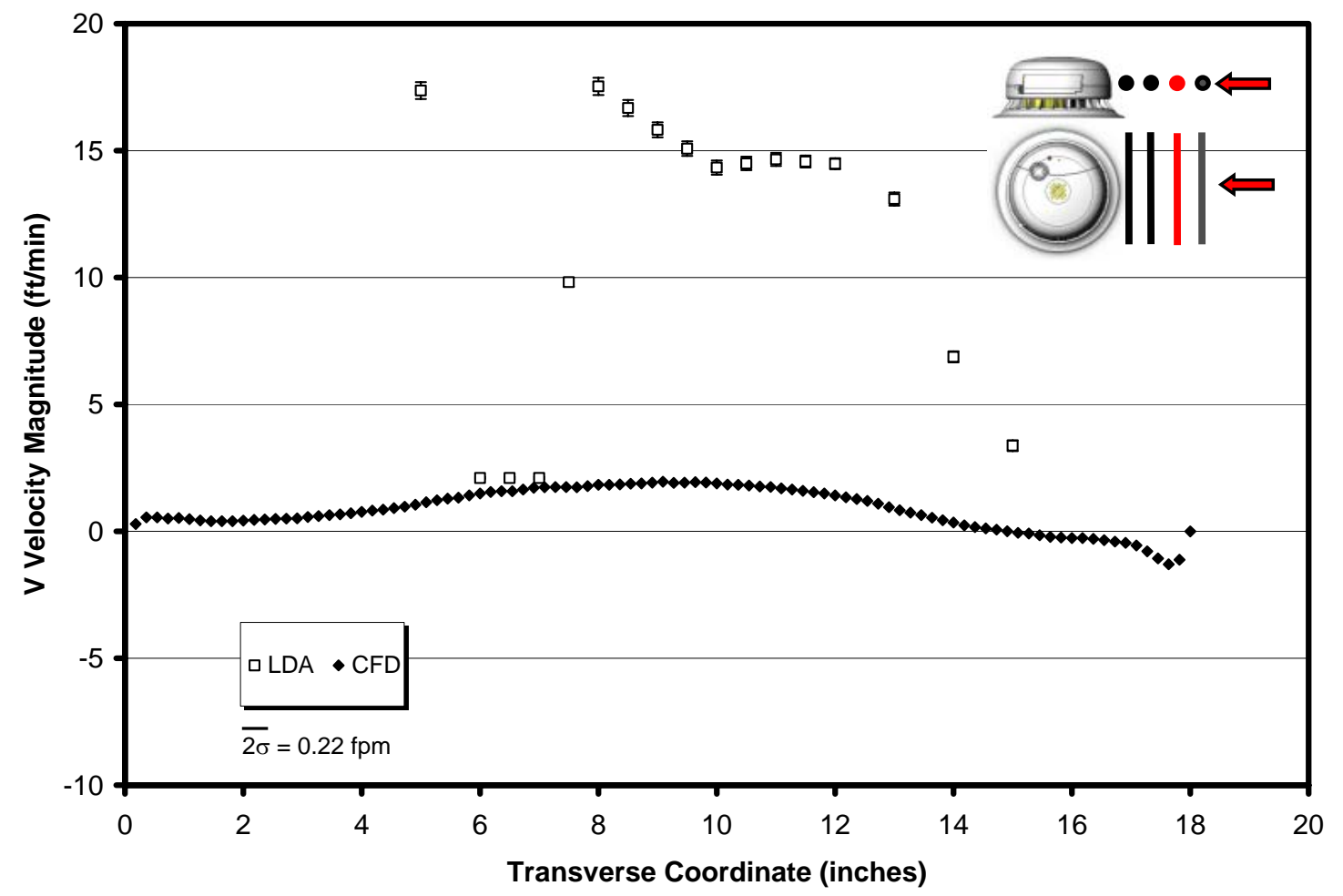

Figure 6.7.5: UL Smoke Box $v$ velocity comparisons between LDA and CFD at the $y=1$ in, $x=$ 13 in position spanning the transverse, $z$ direction.

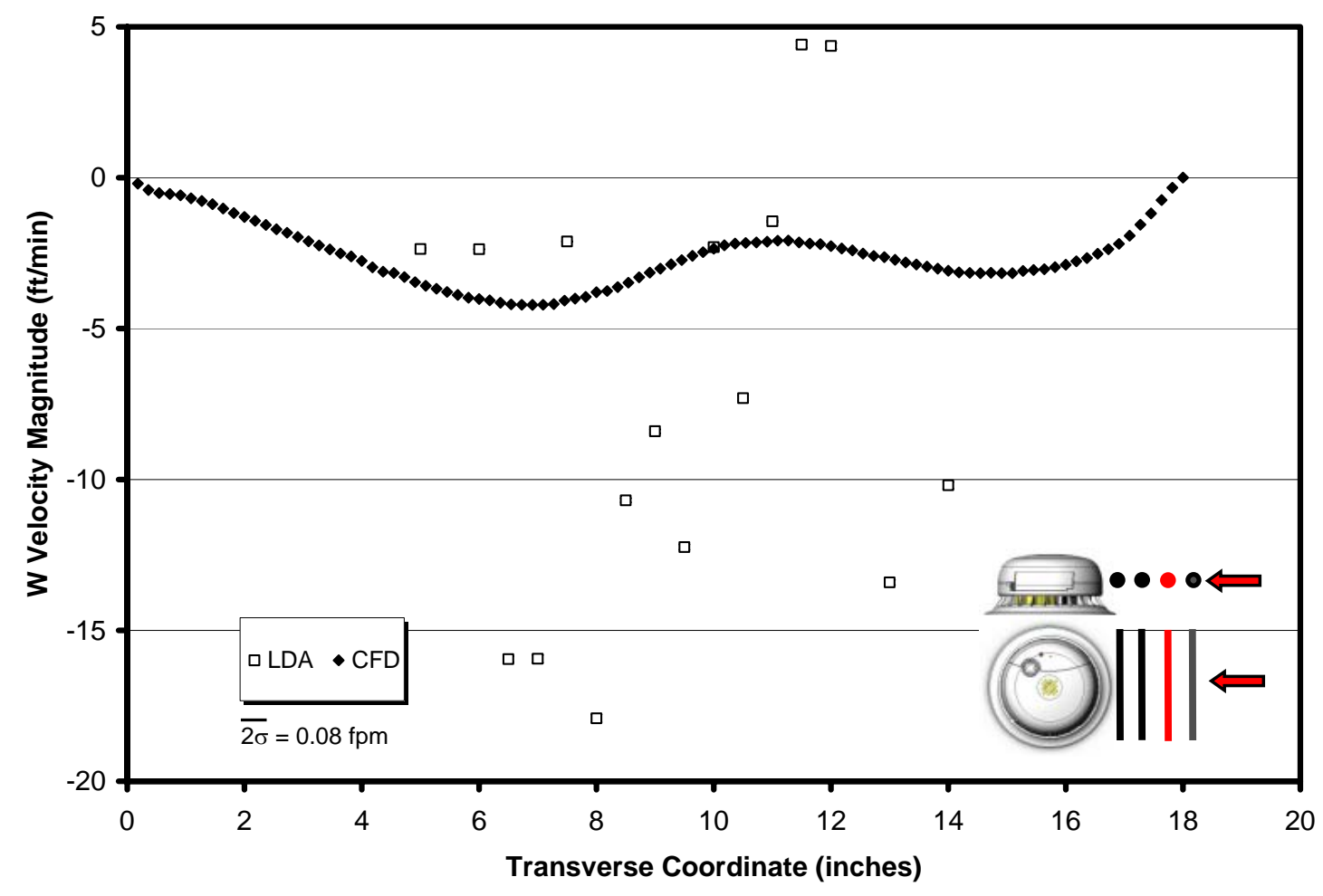

Figure 6.7.6: UL Smoke Box $w$ velocity comparisons between LDA and CFD at the $y=1$ in, $x=$ 13 in position spanning the transverse, $z$ direction. 


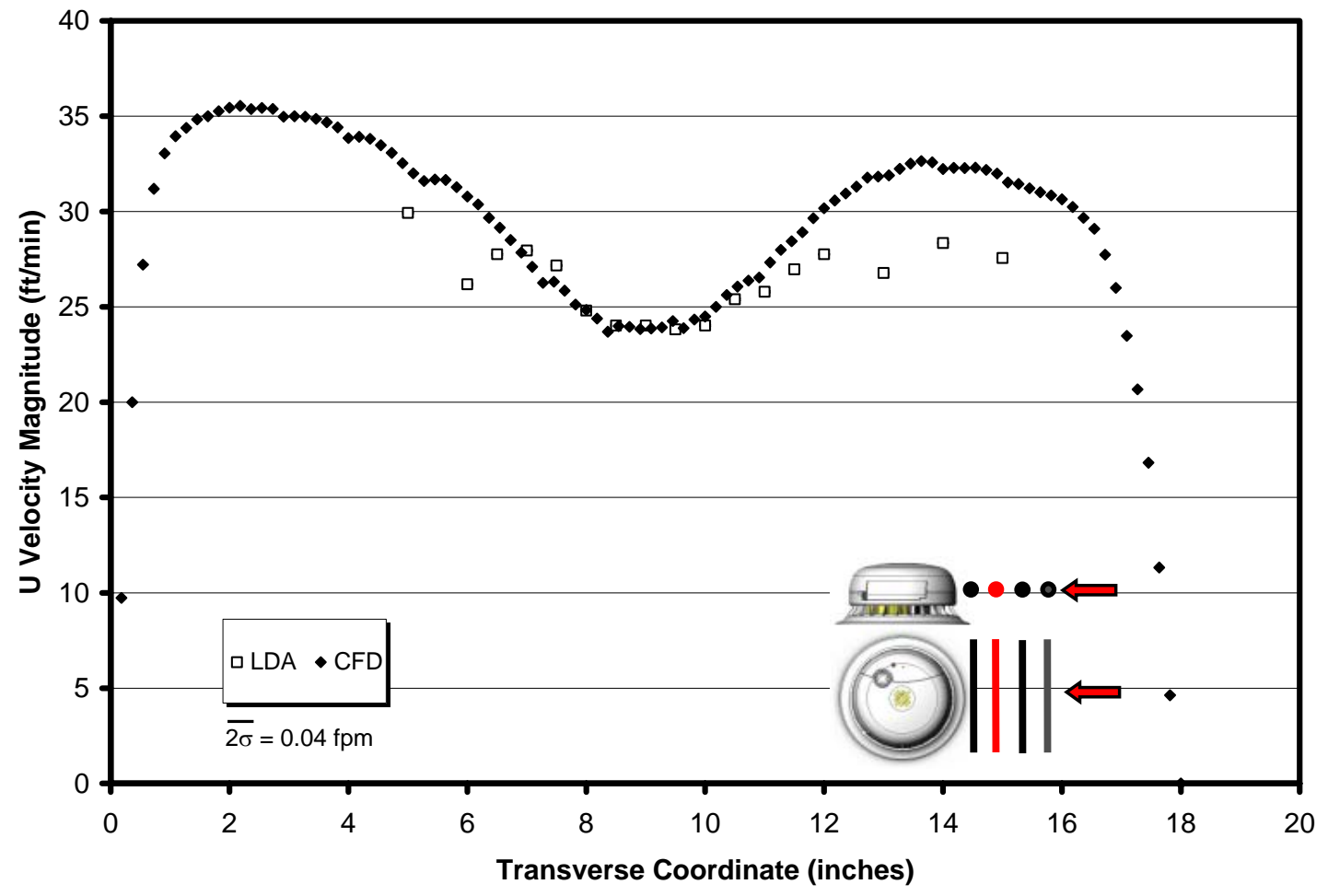

Figure 6.7.7: UL Smoke Box $u$ velocity comparisons between LDA and CFD at the $y=1$ in, $x=$ 14 in position spanning the transverse, $z$ direction.

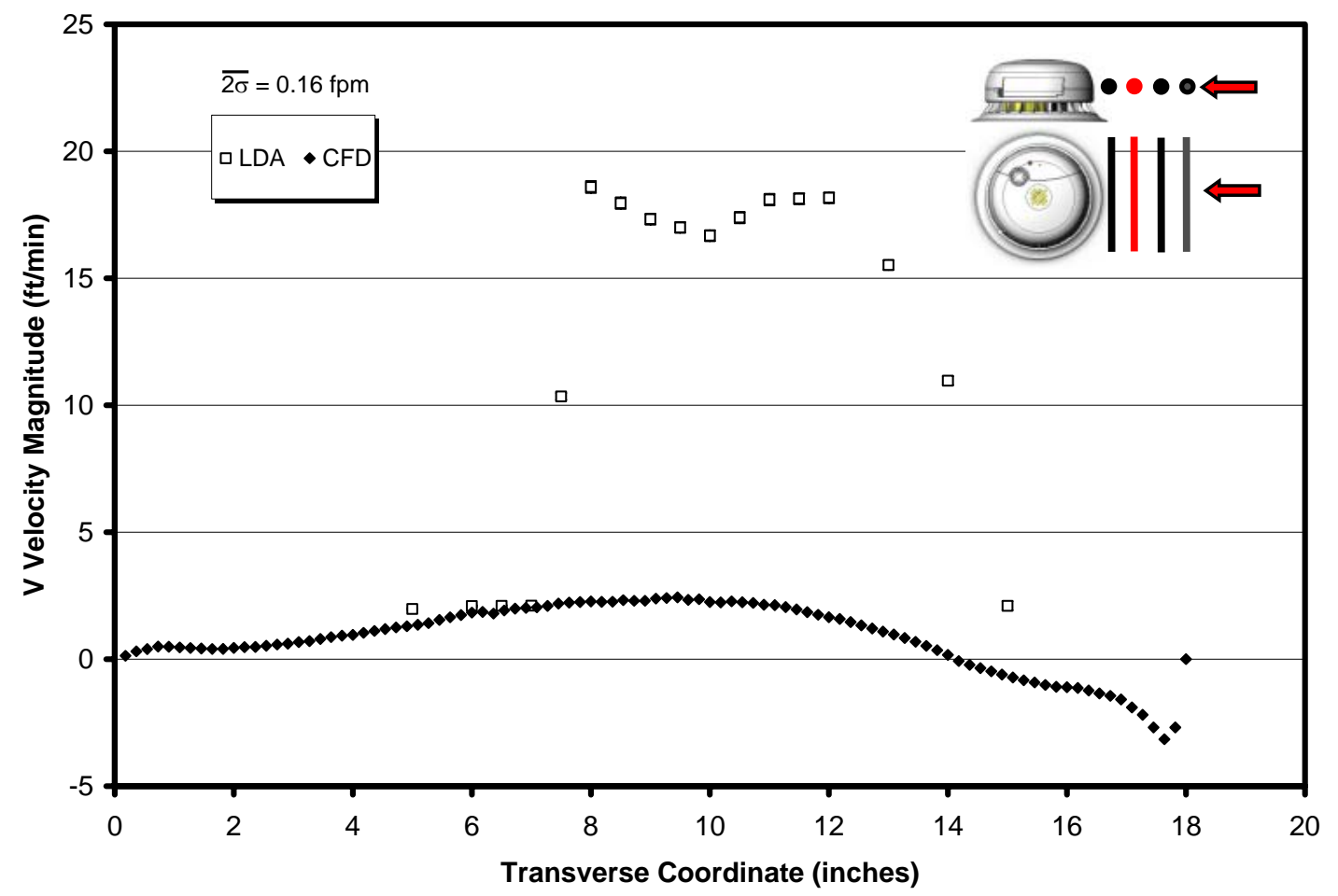

Figure 6.7.8: UL Smoke Box $v$ velocity comparisons between LDA and CFD at the $y=1$ in, $x=$ 14 in position spanning the transverse, $z$ direction. 


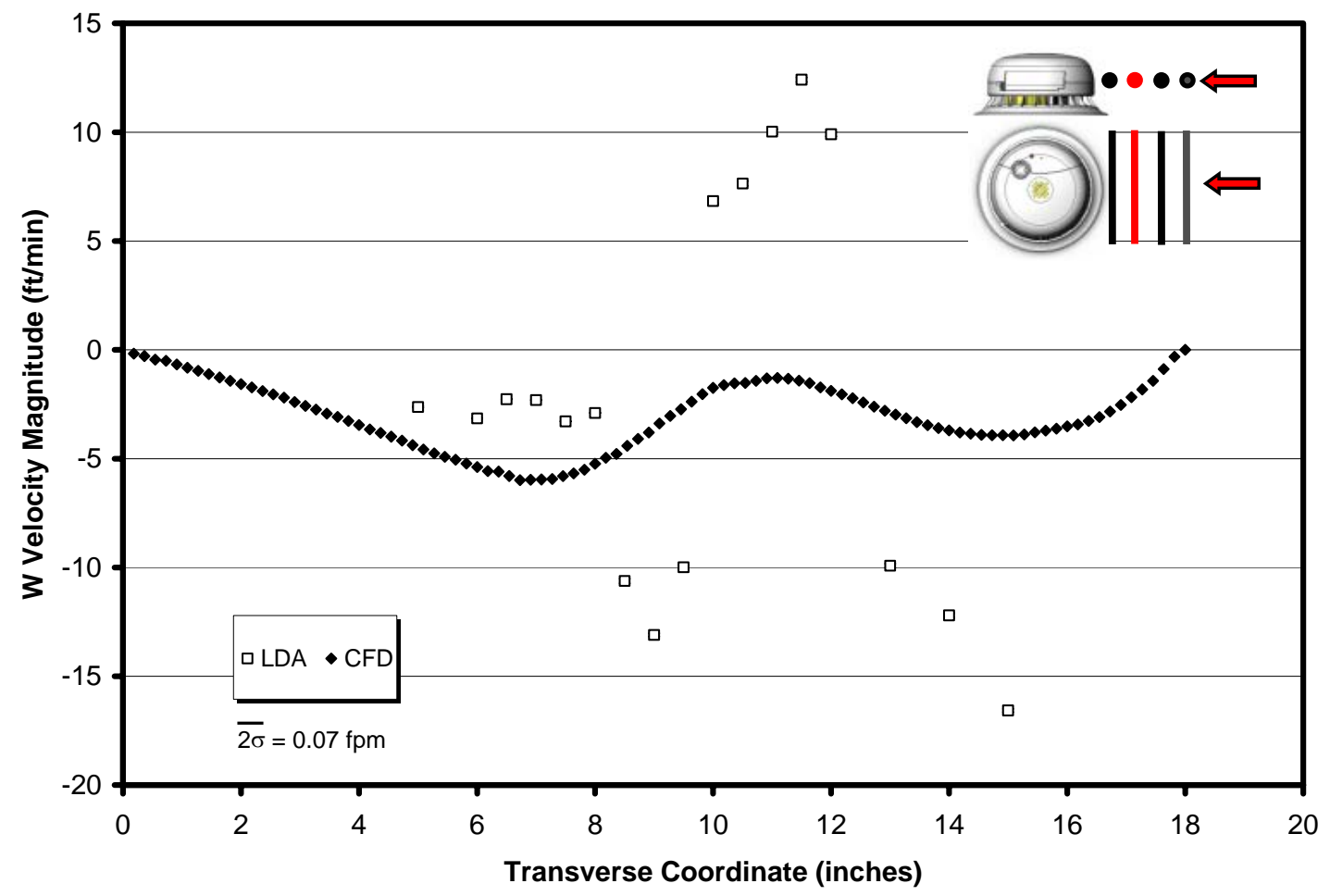

Figure 6.7.9: UL Smoke Box $w$ velocity comparisons between LDA and CFD at the $y=1$ in, $x=$ 14 in position spanning the transverse, $z$ direction.

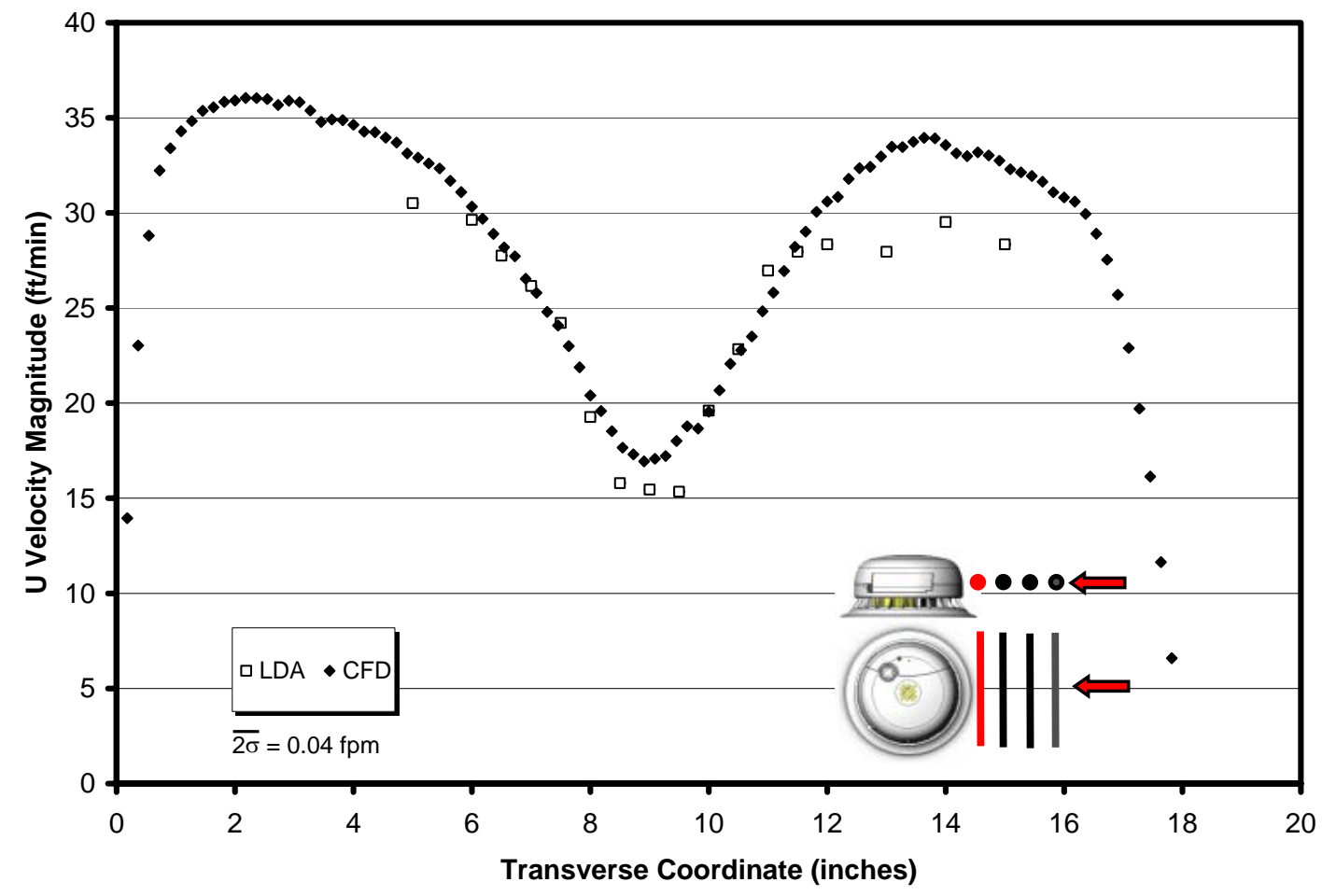

Figure 6.7.10: UL Smoke Box $u$ velocity comparisons between LDA and CFD at the $y=1$ in, $x$ $=15$ in position spanning the transverse, $z$ direction. 


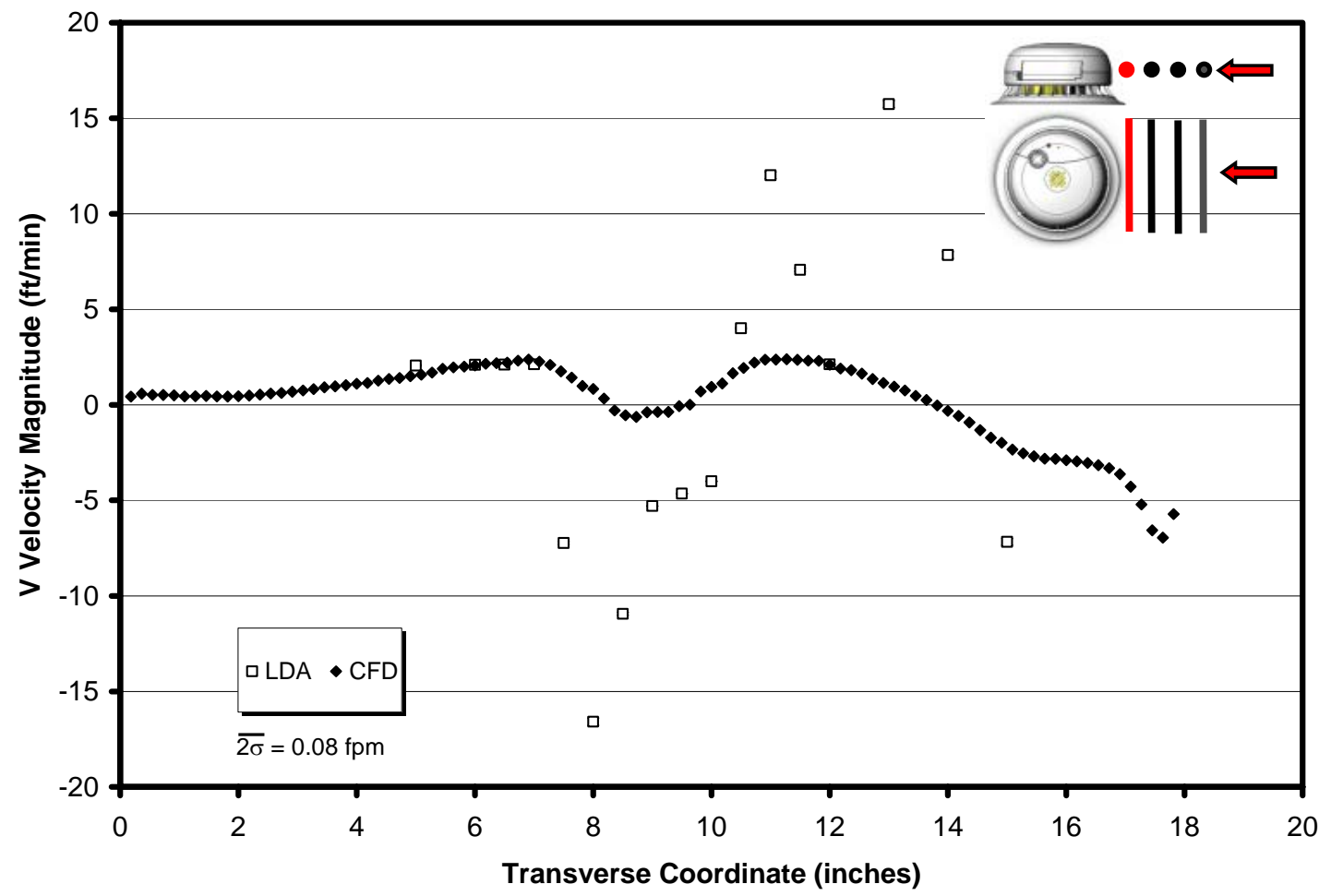

Figure 6.7.11: UL Smoke Box $v$ velocity comparisons between LDA and CFD at the $y=1$ in, $x$ $=15$ in position spanning the transverse, $z$ direction.

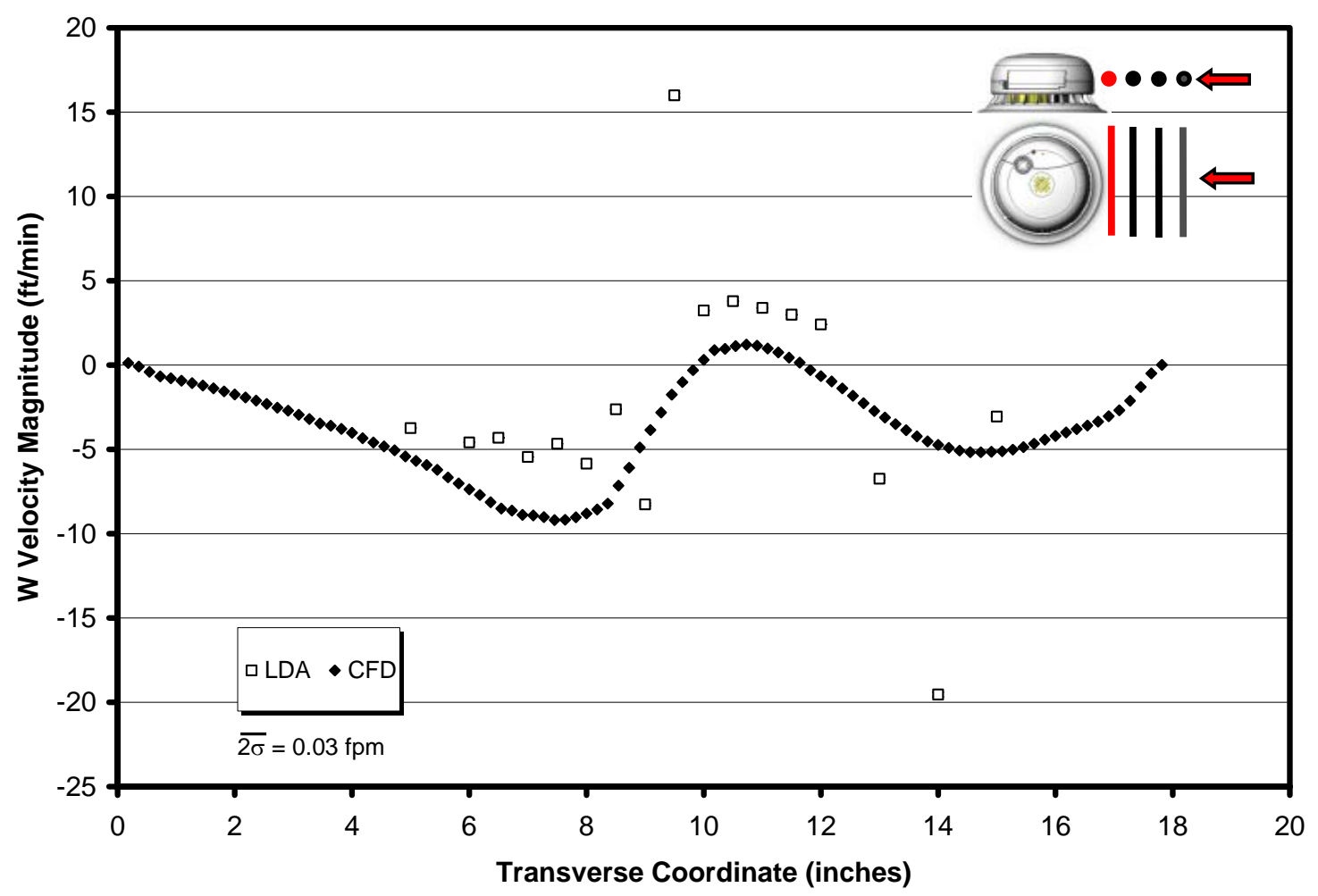

Figure 6.7.12: UL Smoke Box $w$ velocity comparisons between LDA and CFD at the $y=1$ in, $x$ $=15$ in position spanning the transverse, $z$ direction. 


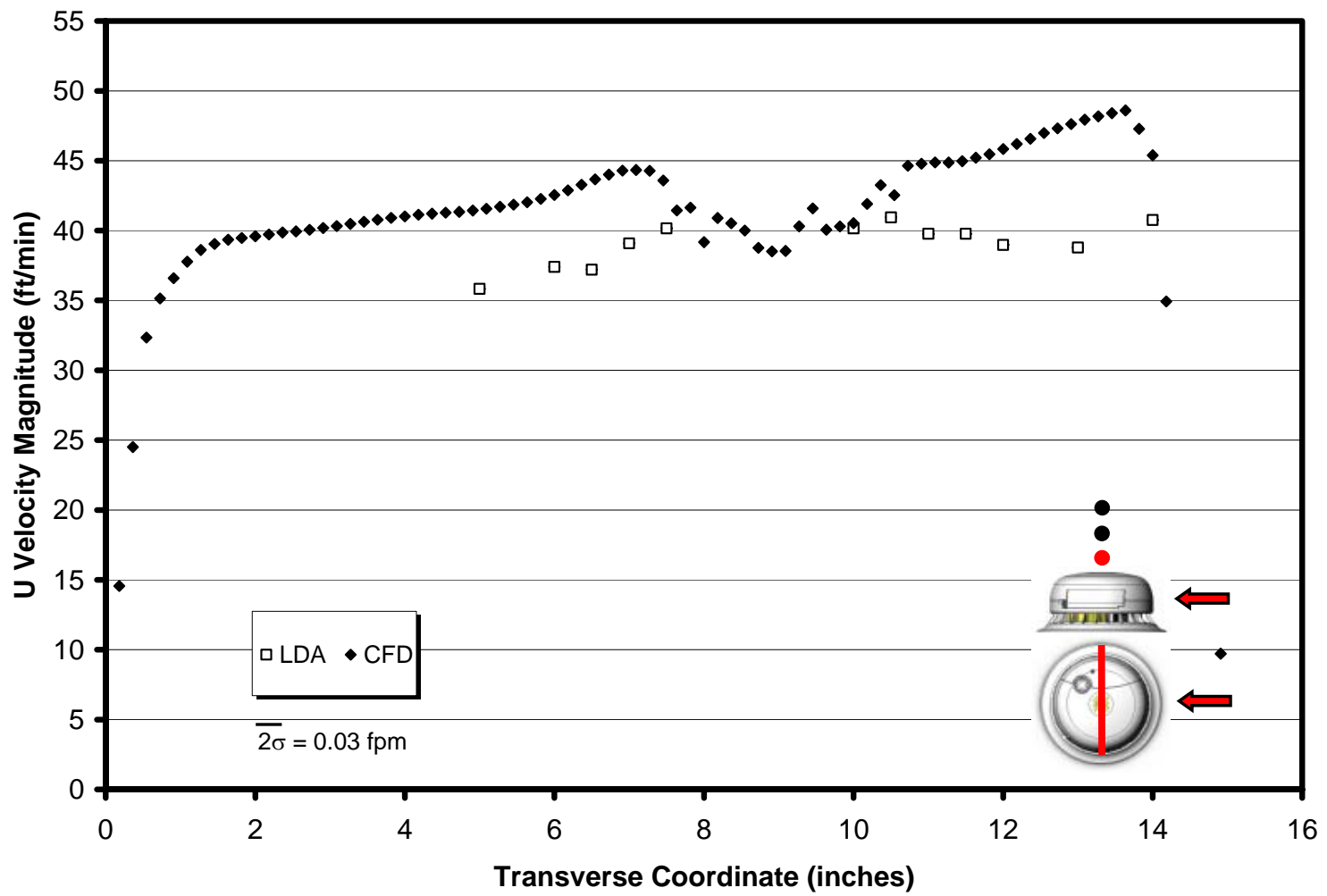

Figure 6.7.13: UL Smoke Box $u$ velocity comparisons between LDA and CFD at the $x=18$ in, $y$ $=3$ in position spanning the transverse, $z$ direction.

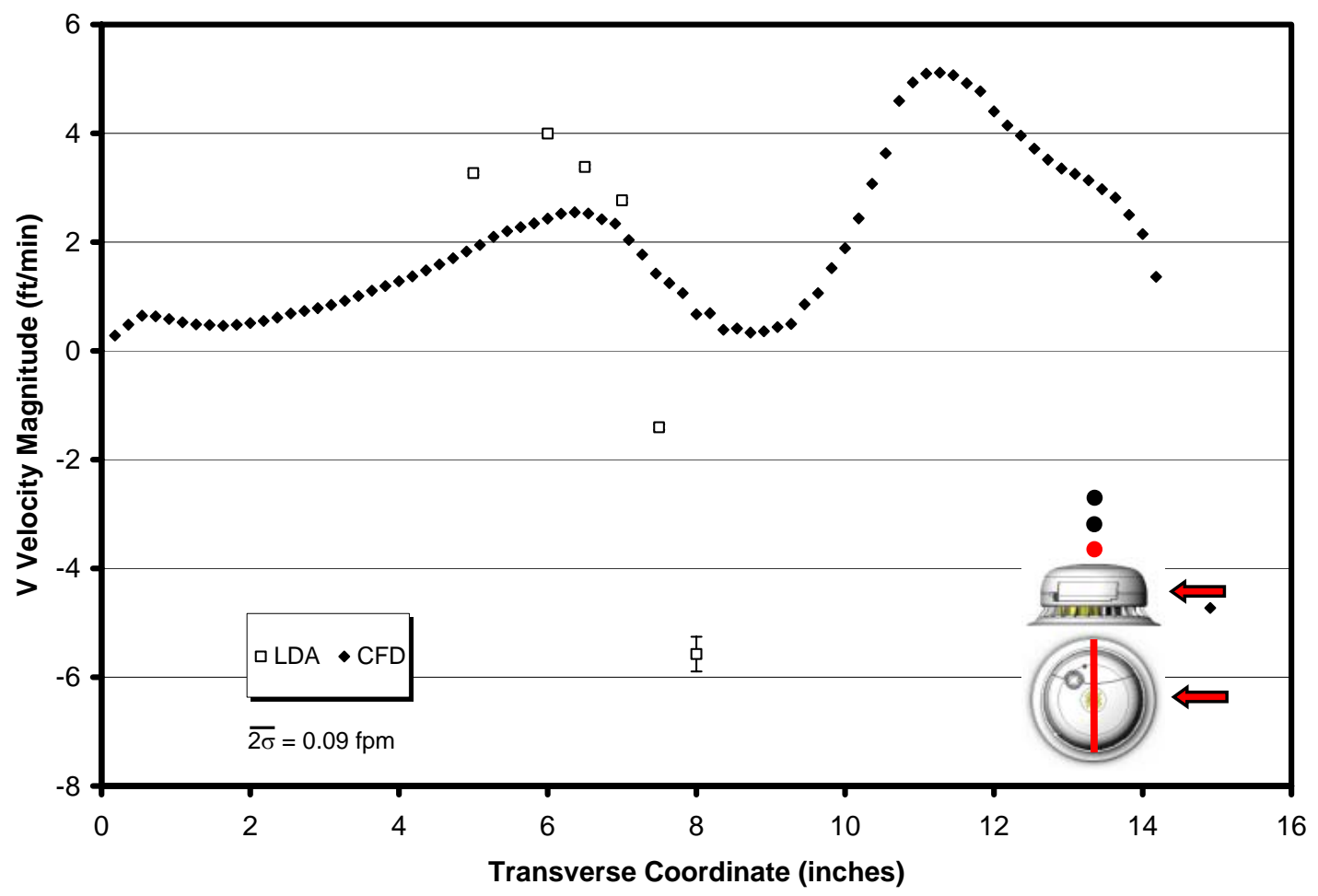

Figure 6.7.14: UL Smoke Box $v$ velocity comparisons between LDA and CFD at the $x=18$ in, $y$ $=3$ in position spanning the transverse, $z$ direction. 


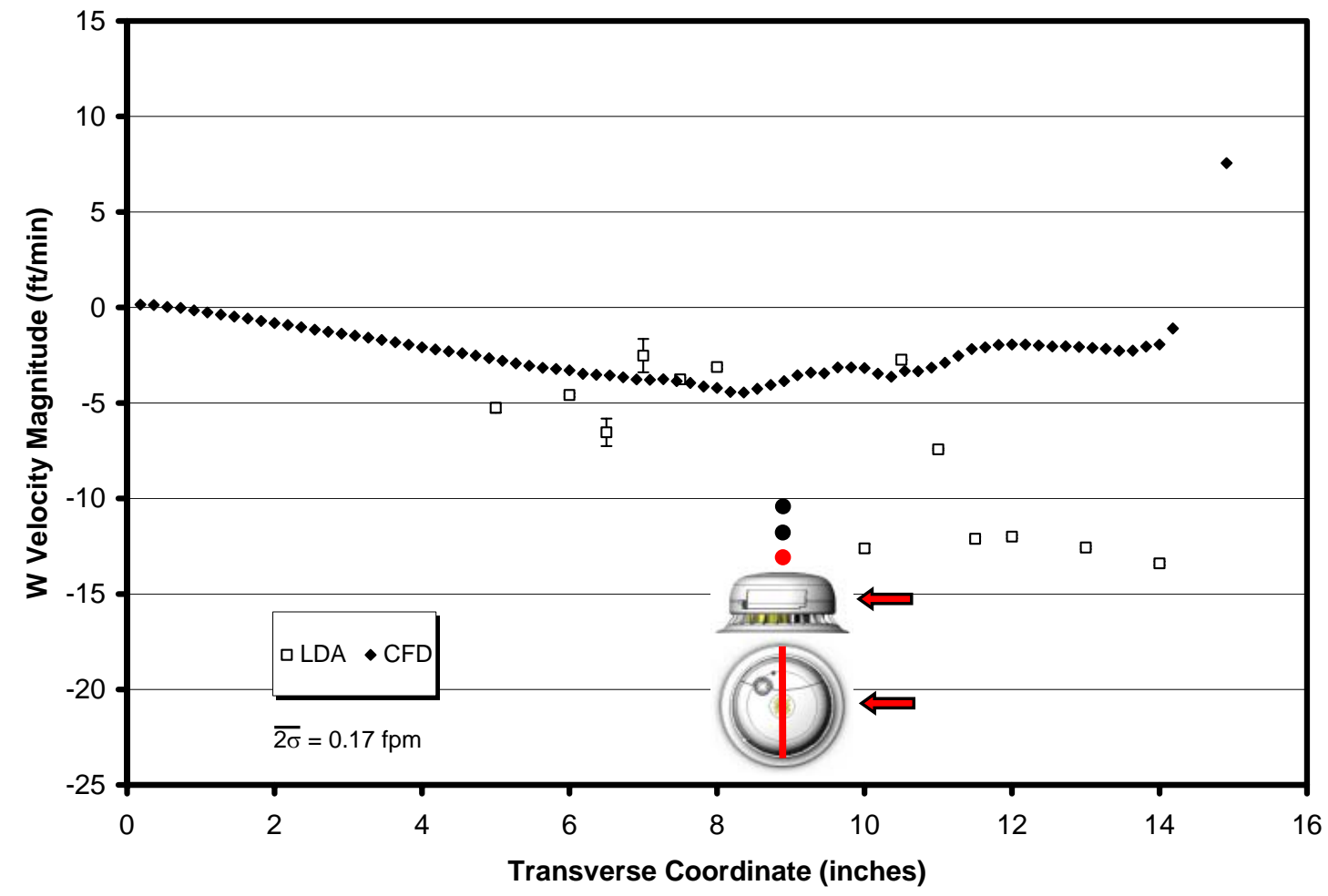

Figure 6.7.15: UL Smoke Box $w$ velocity comparisons between LDA and CFD at the $x=18$ in, $y$ $=3$ in position spanning the transverse, $z$ direction.

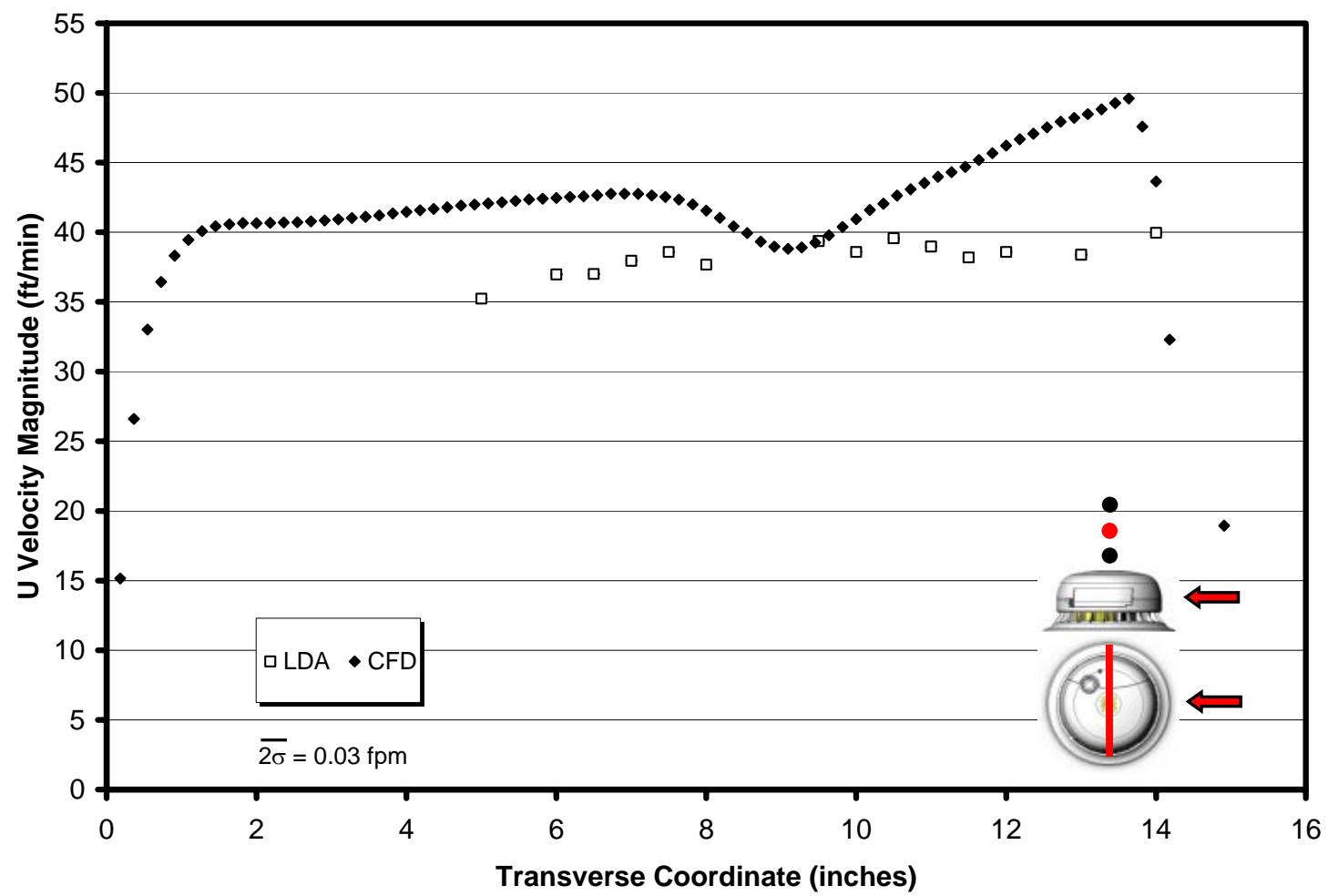

Figure 6.7.16: UL Smoke Box $u$ velocity comparisons between LDA and CFD at the $x=18$ in, $y$ $=4$ in position spanning the transverse, $z$ direction. 


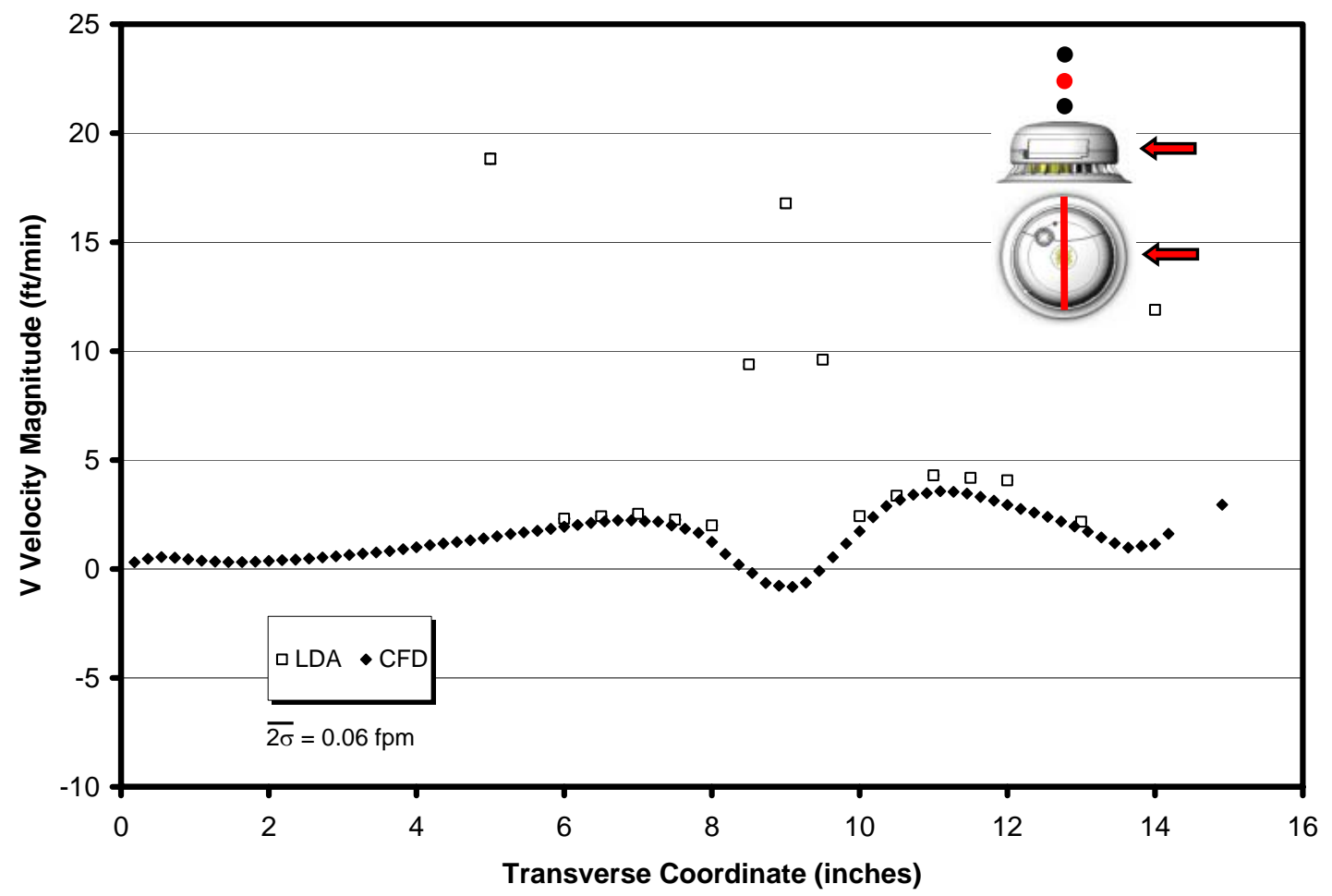

Figure 6.7.17: UL Smoke Box $v$ velocity comparisons between LDA and CFD at the $x=18$ in, $y$ $=4$ in position spanning the transverse, $z$ direction.

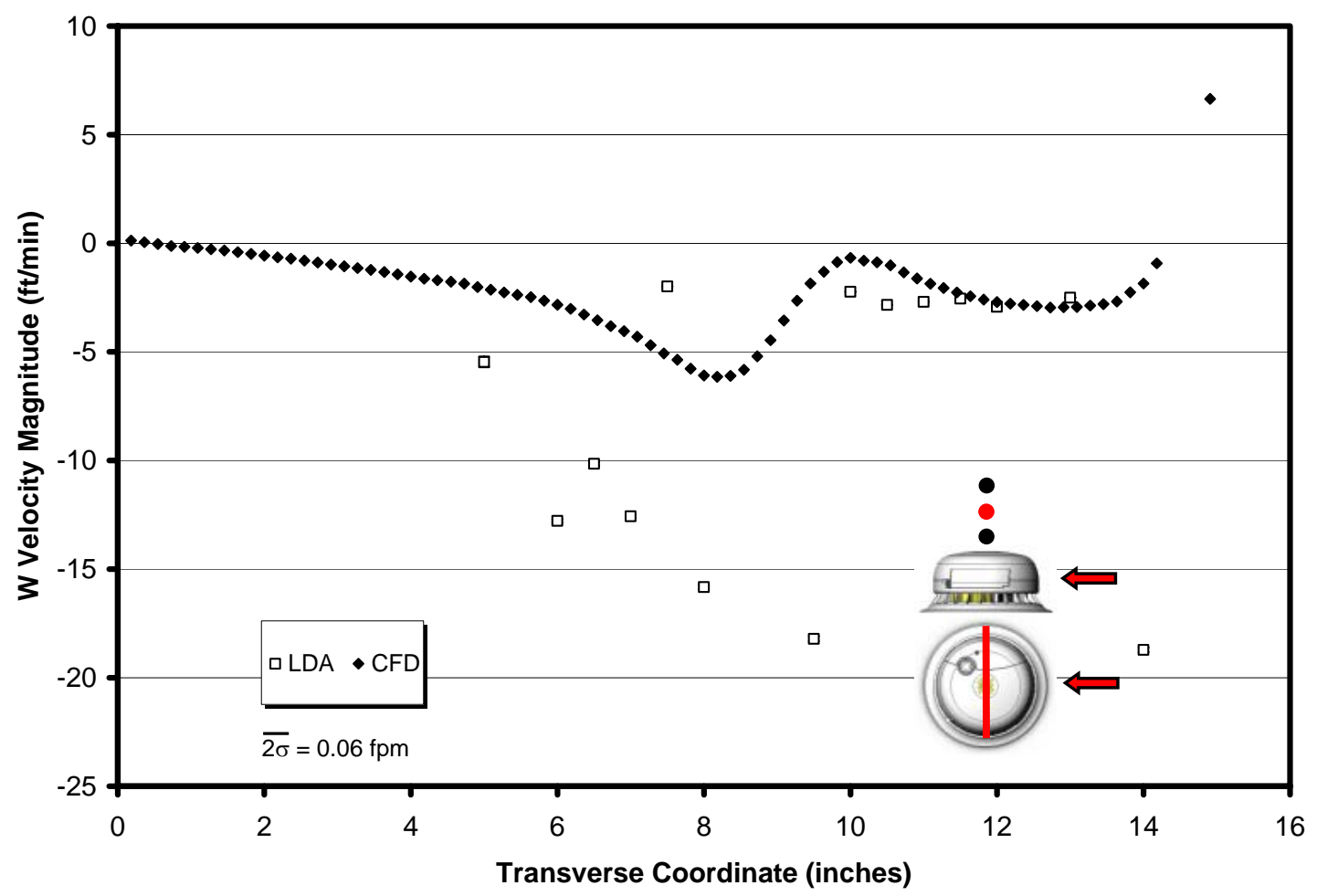

Figure 6.7.18: UL Smoke Box $w$ velocity comparisons between LDA and CFD at the $x=18$ in, $y$ $=4$ in position spanning the transverse, $z$ direction. 


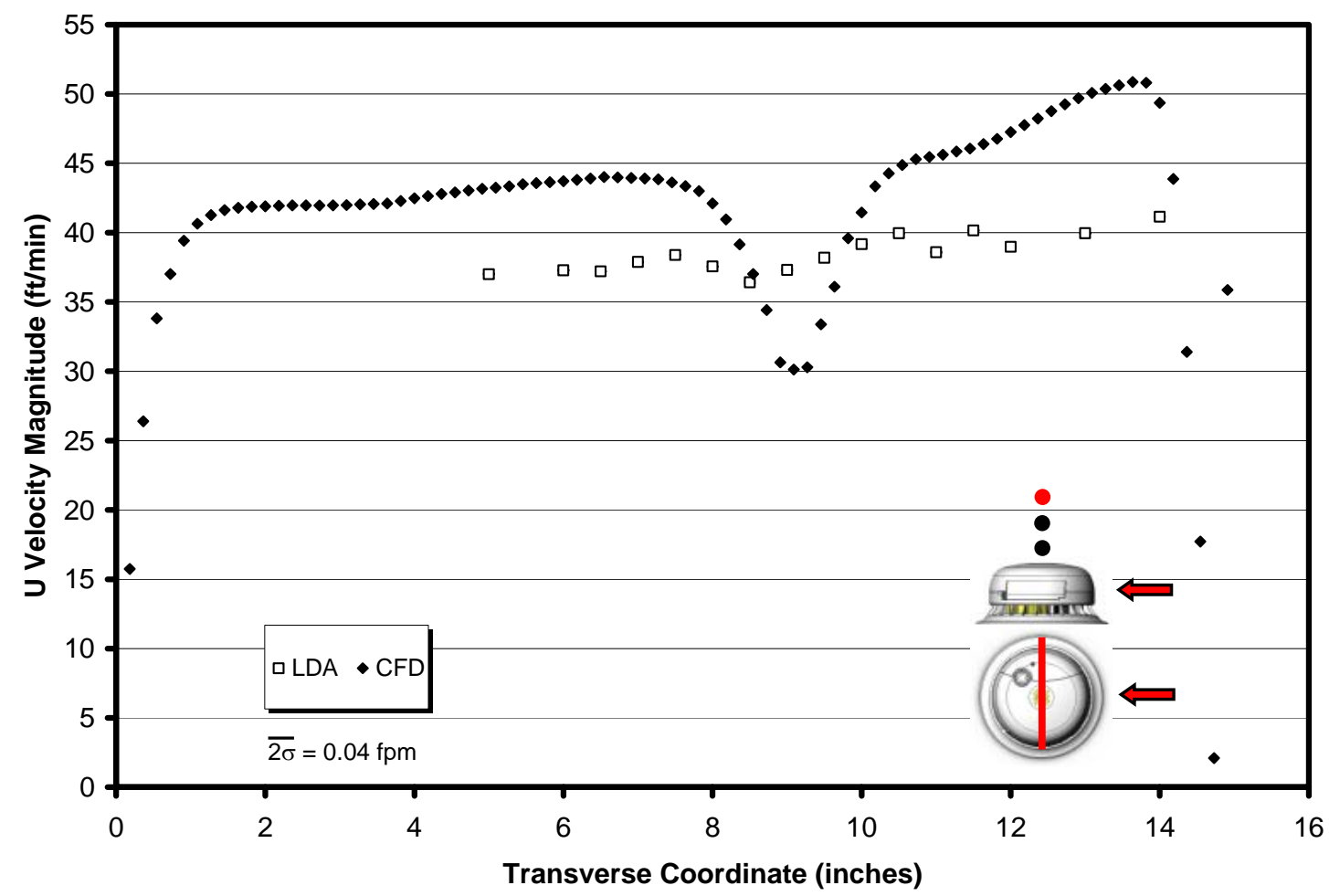

Figure 6.7.19: UL Smoke Box $u$ velocity comparisons between LDA and CFD at the $x=18$ in, $y$ $=5$ in position spanning the transverse, $z$ direction.

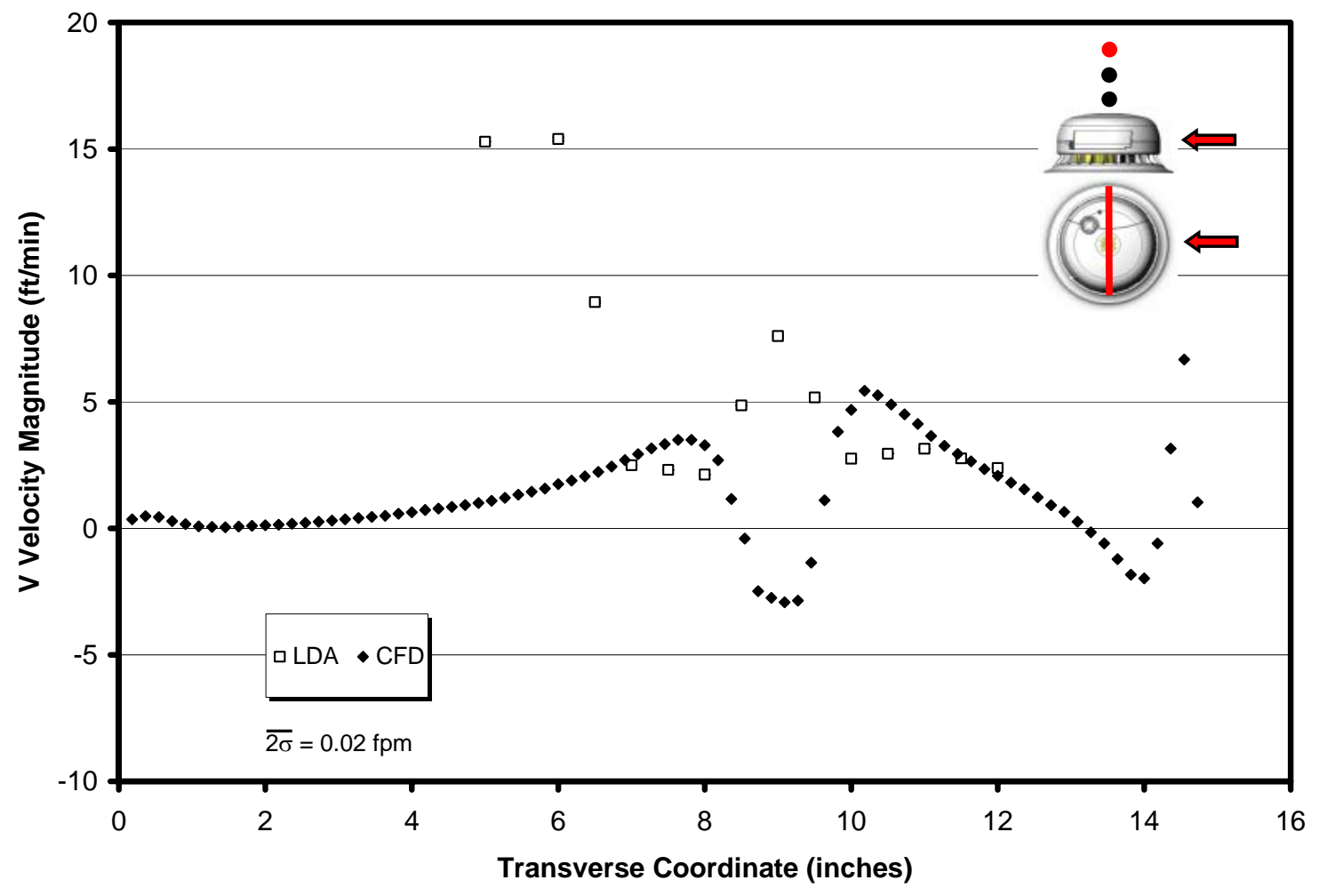

Figure 6.7.20: UL Smoke Box $v$ velocity comparisons between LDA and CFD at the $x=18$ in, $y$ $=5$ in position spanning the transverse, $z$ direction. 


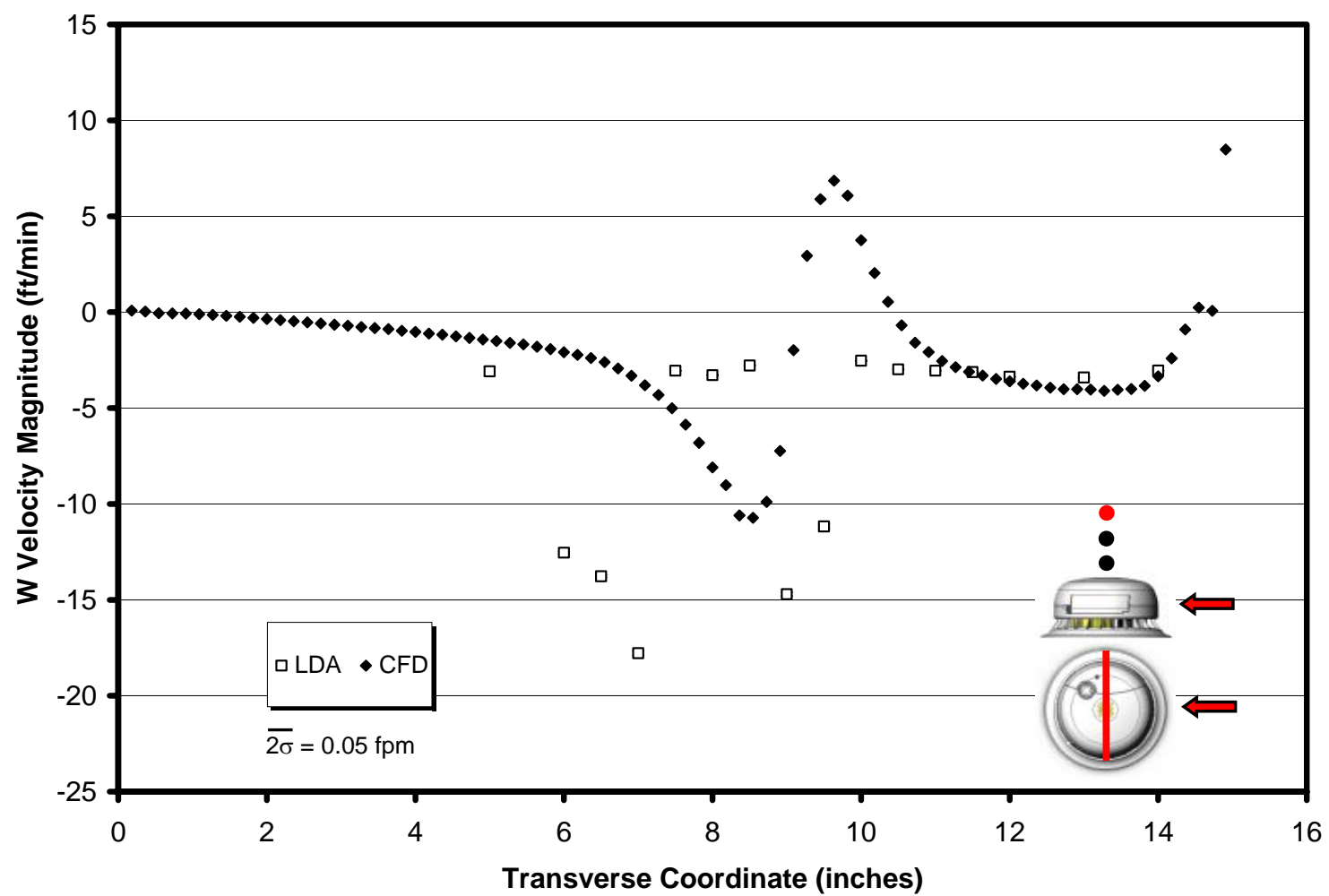

Figure 6.7.21: UL Smoke Box $v$ velocity comparisons between LDA and CFD at the $x=18$ in, $y$ $=5$ in position spanning the transverse, $z$ direction.

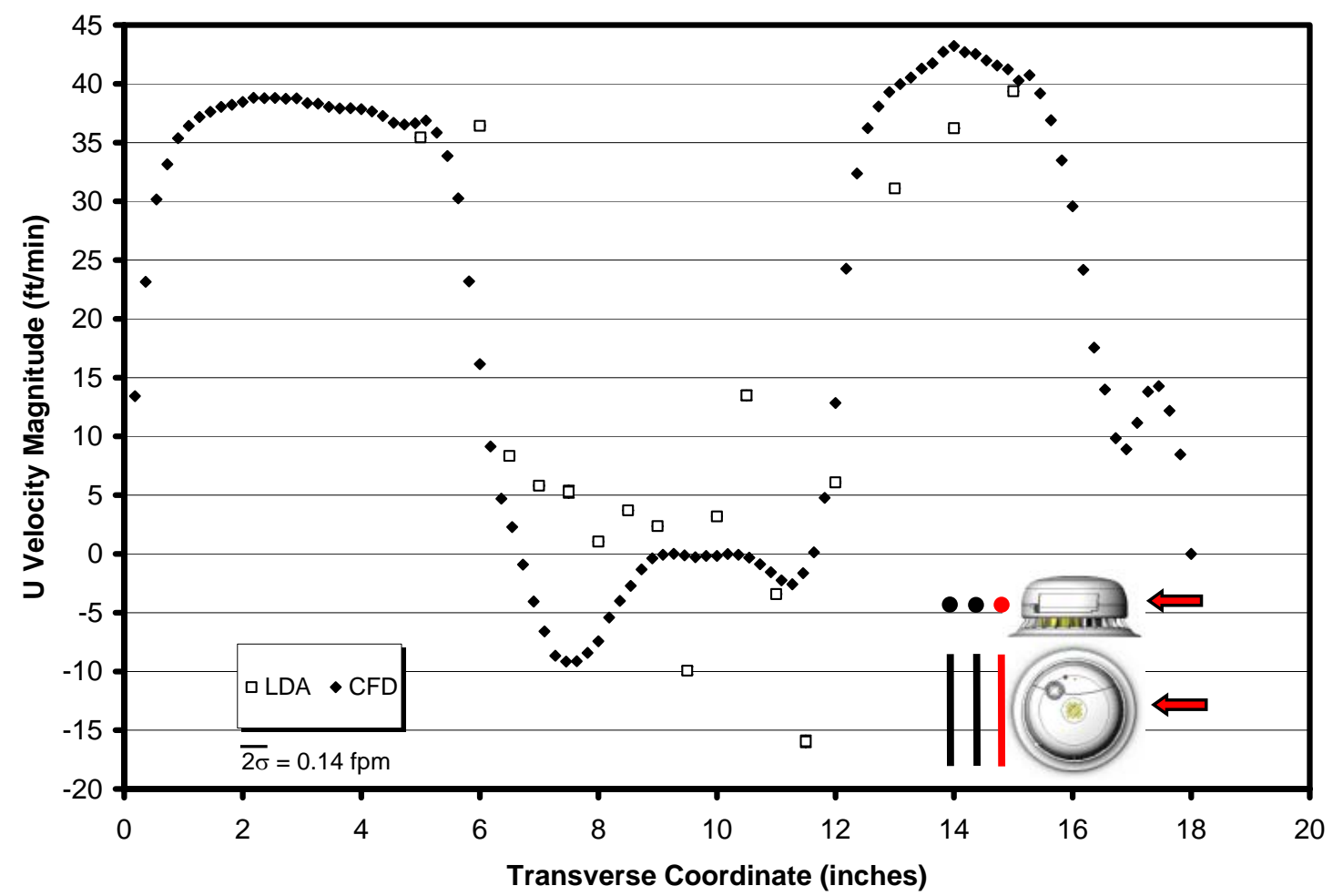

Figure 6.7.22: UL Smoke Box $u$ velocity comparisons between LDA and CFD at the $x=21$ in, $y$ $=1$ in position spanning the transverse, $z$ direction. 


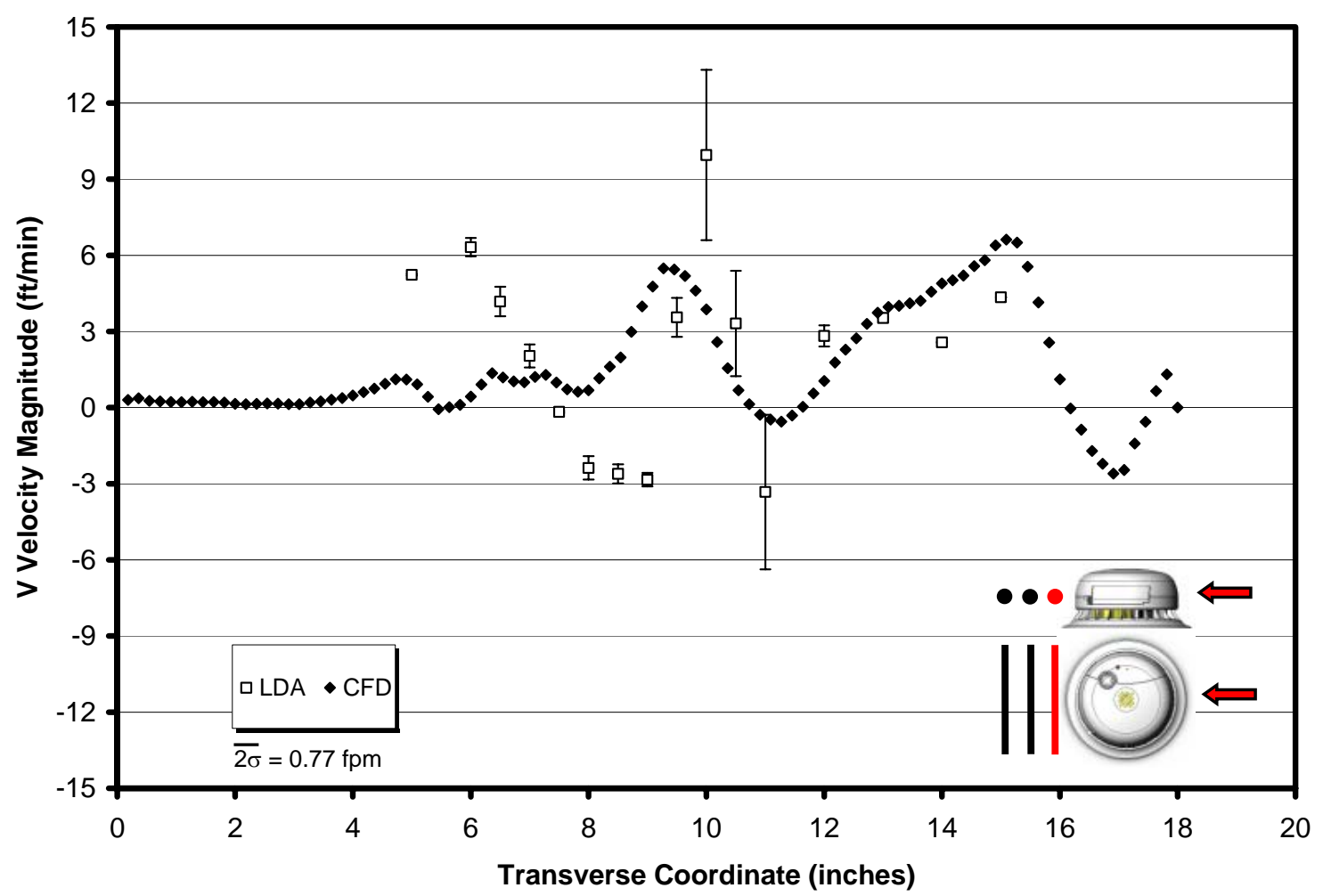

Figure 6.7.23: UL Smoke Box $v$ velocity comparisons between LDA and CFD at the $x=21$ in, $y$ $=1$ in position spanning the transverse, $z$ direction.

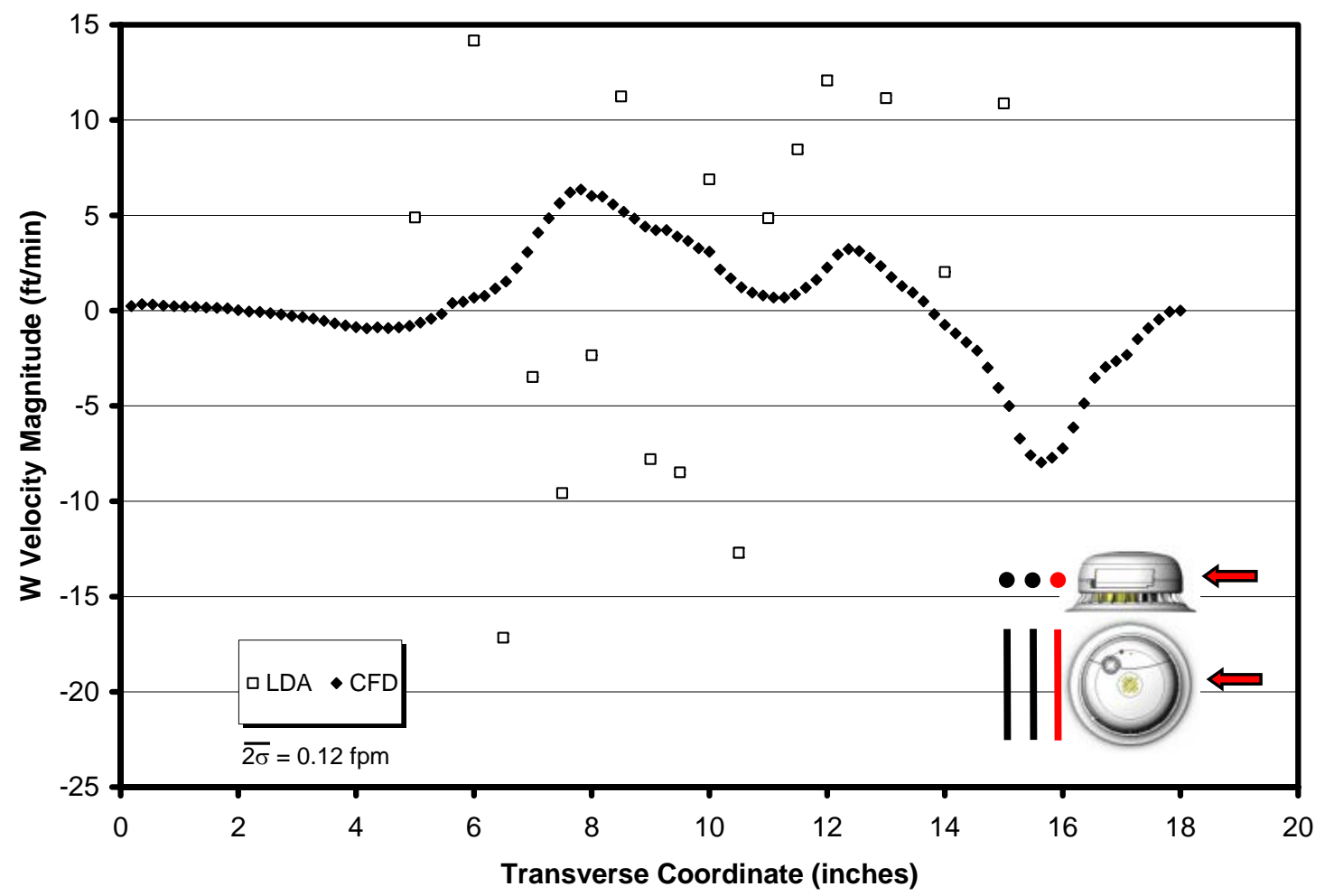

Figure 6.7.24: UL Smoke Box $w$ velocity comparisons between LDA and CFD at the $x=21$ in, $y$ $=1$ in position spanning the transverse, $z$ direction. 


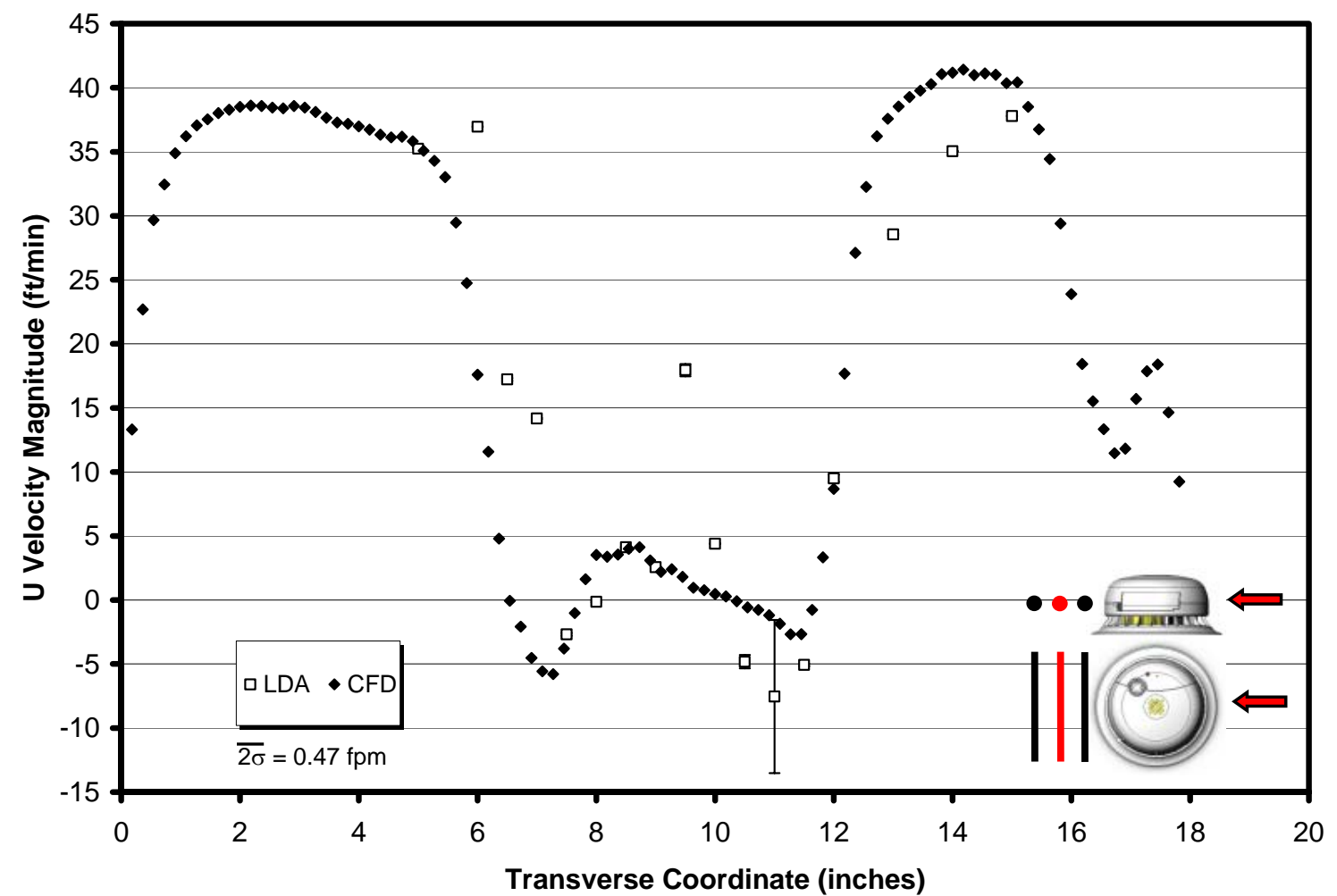

Figure 6.7.25: UL Smoke Box $u$ velocity comparisons between LDA and CFD at the $x=22$ in, $y$ $=1$ in position spanning the transverse, $z$ direction.

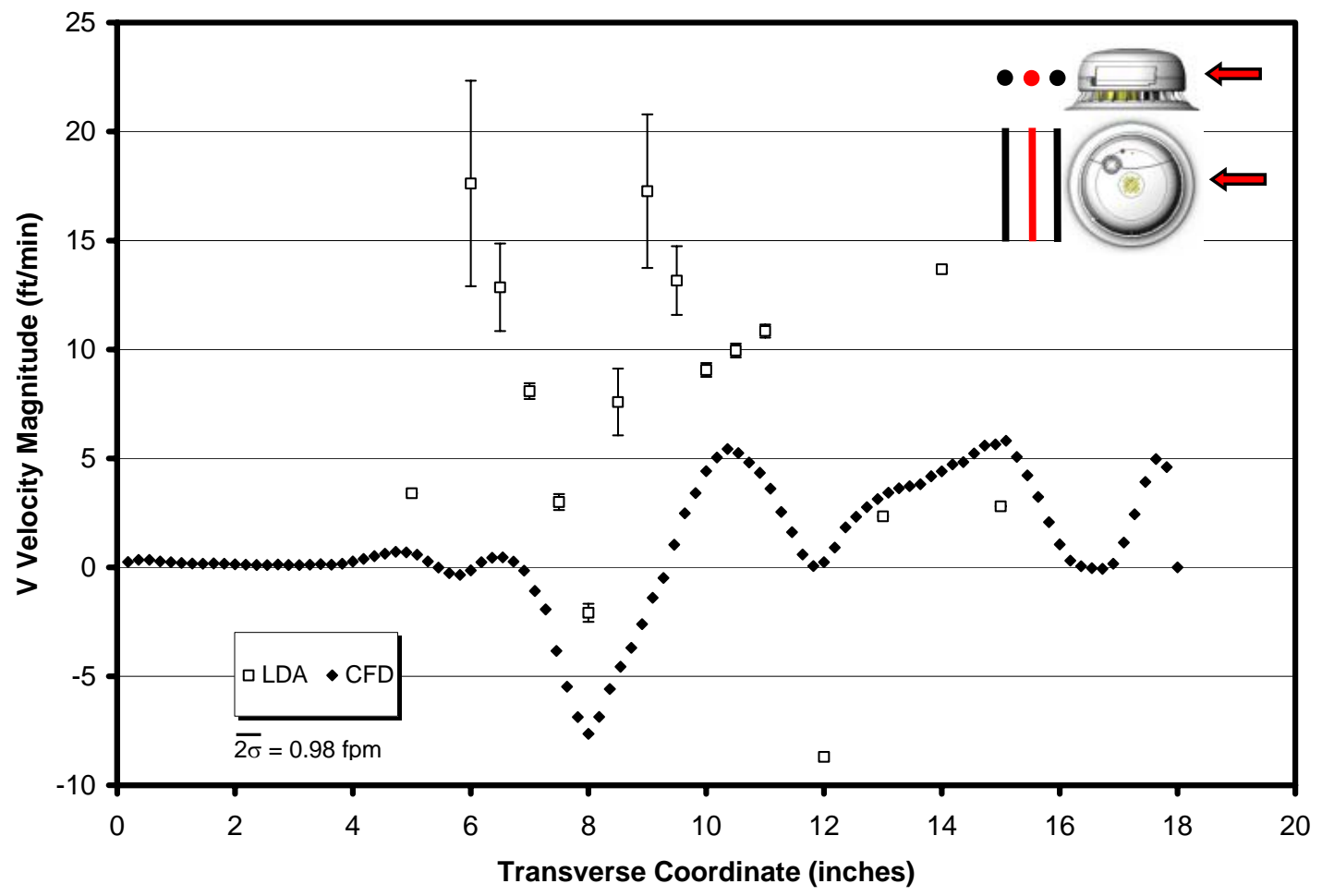

Figure 6.7.26: UL Smoke Box $v$ velocity comparisons between LDA and CFD at the $x=22$ in, $y$ $=1$ in position spanning the transverse, $z$ direction. 


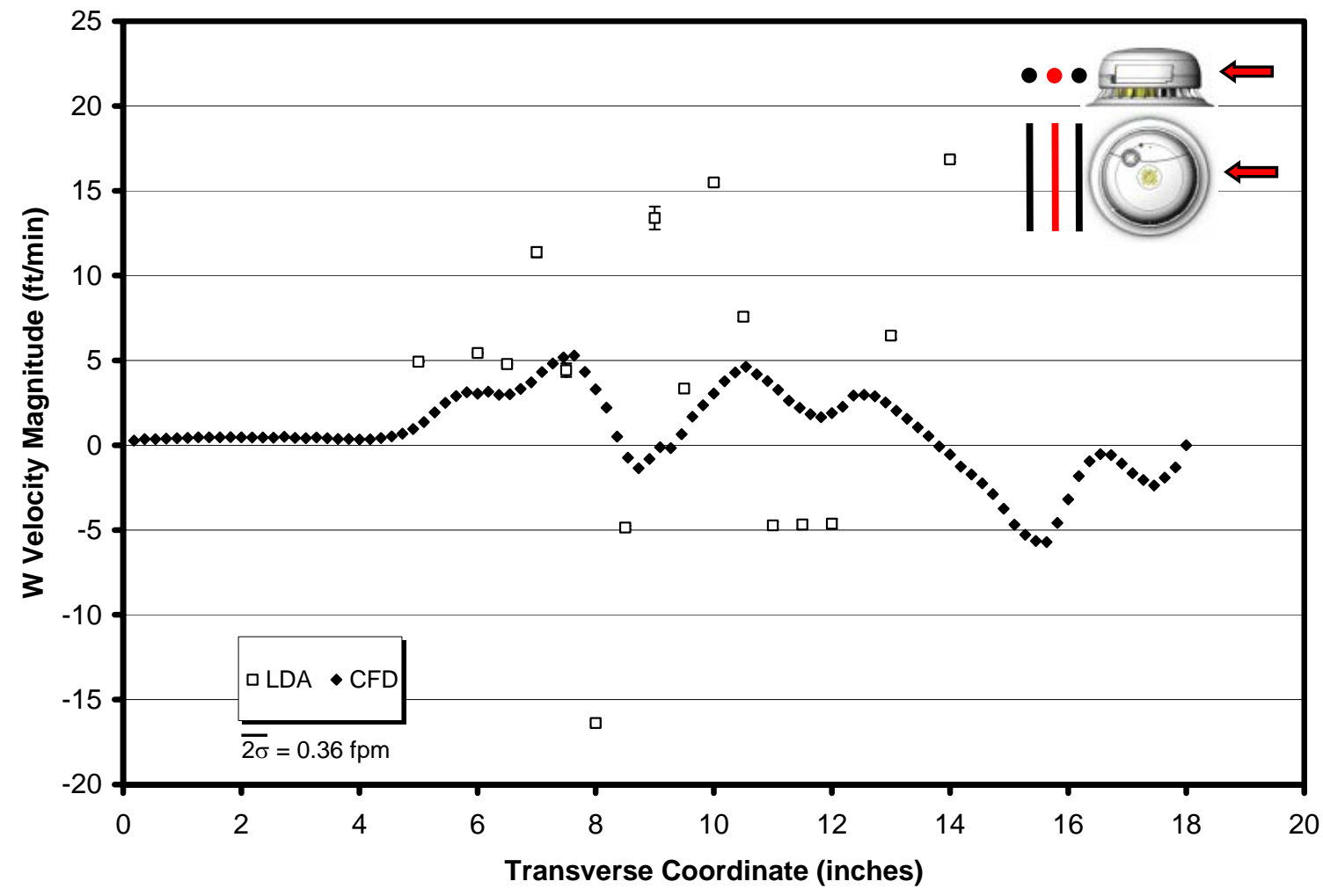

Figure 6.7.27: UL Smoke Box $w$ velocity comparisons between LDA and CFD at the $x=22$ in, $y$ $=1$ in position spanning the transverse, $z$ direction.

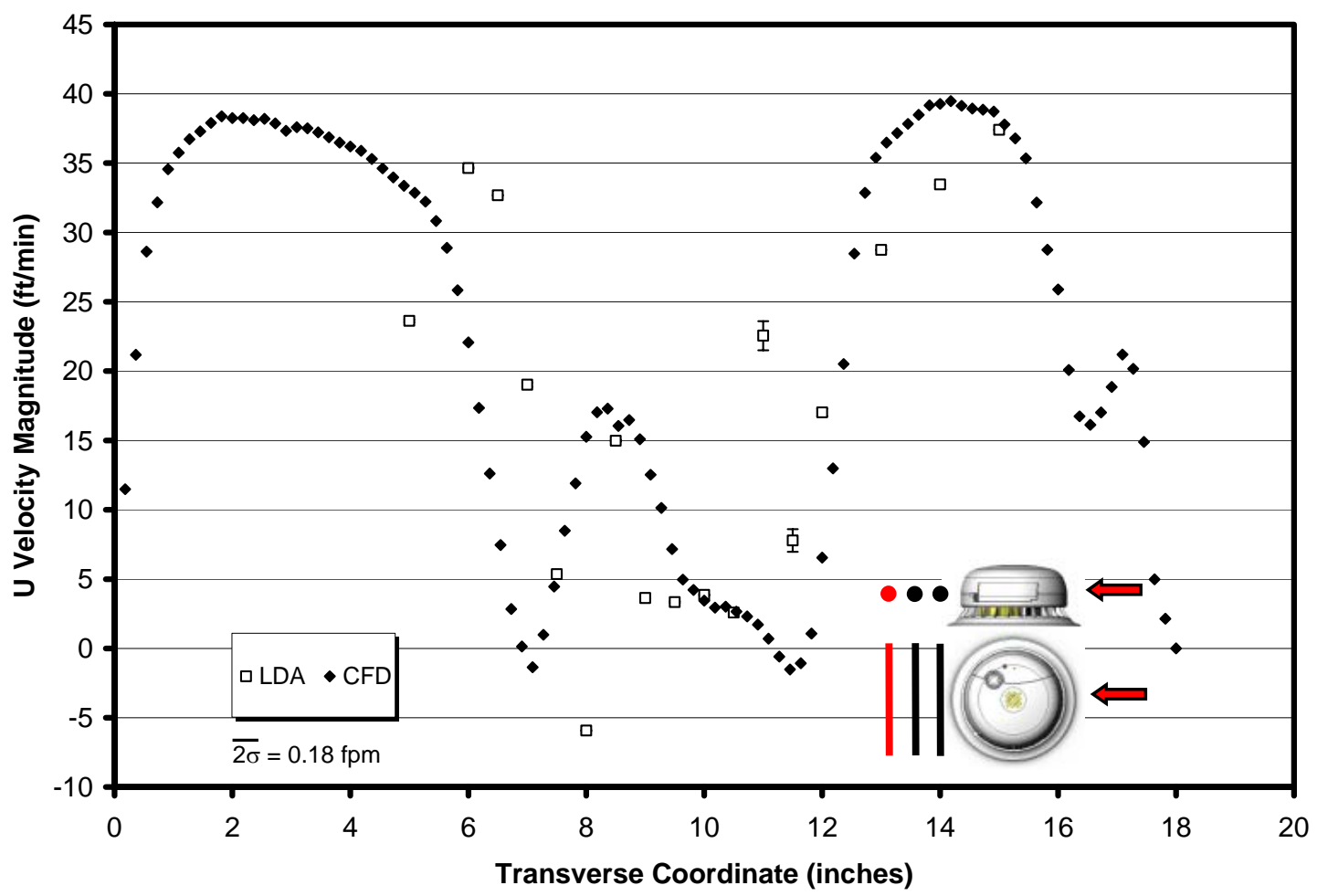

Figure 6.7.28: UL Smoke Box $u$ velocity comparisons between LDA and CFD at the $x=23$ in, $y$ $=1$ in position spanning the transverse, $z$ direction. 


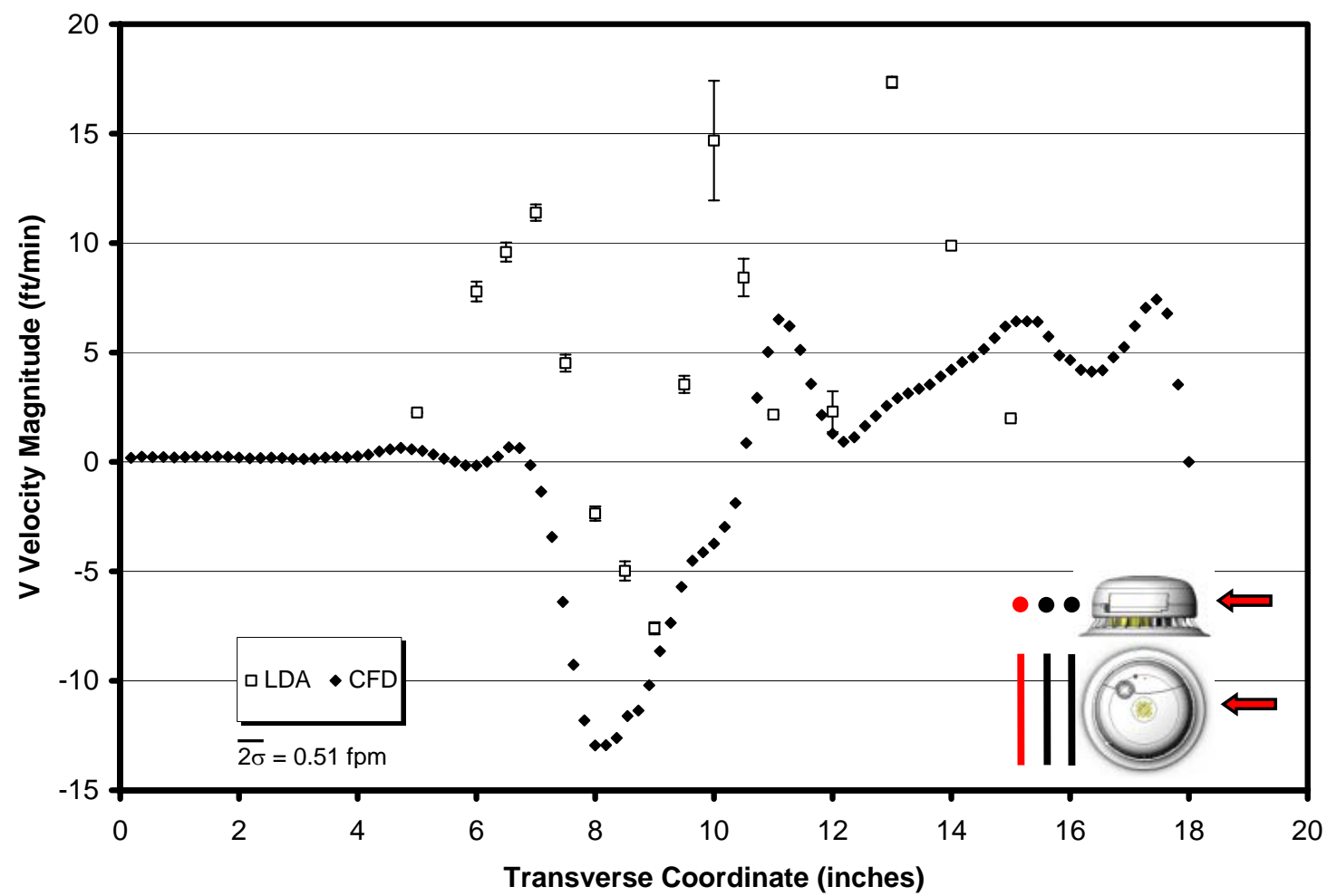

Figure 6.7.29: UL Smoke Box $v$ velocity comparisons between LDA and CFD at the $x=23$ in, $y$ $=1$ in position spanning the transverse, $z$ direction.

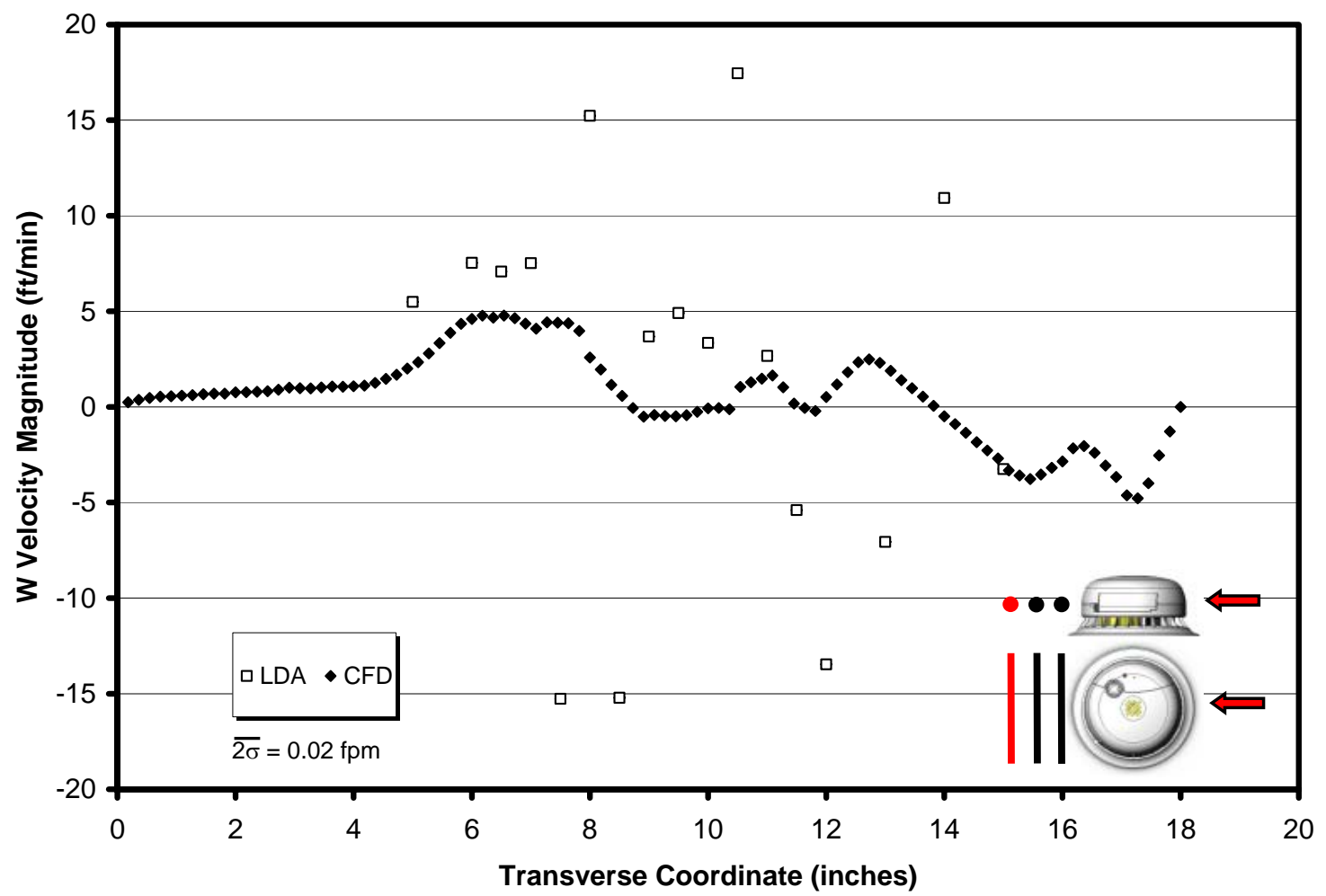

Figure 6.7.30: UL Smoke Box $v$ velocity comparisons between LDA and CFD at the $x=23$ in, $y$ $=1$ in position spanning the transverse, $z$ direction. 


\subsection{SA302 LDA VS. CFD VELOCITY COMPARISONS}

One of the primary objectives of this study lies in modeling the internal flow characteristics of the SA302. In order to do this, rakes of LDA data were selected where enough data points were available for a couple of detector orientations to compare to CFD results. Often, due to detector orientation and laser blockage issues, several points along a grid line are not taken. Figures 6.8.1 through 6.8.6 depict comparisons using the $0^{\circ}$ orientation for 3 rakes reporting their respective $u$ and $w$ component velocities inside both the photoelectric and ionization sensor. Recall the LDA data has a much coarser grid of $0.25 \mathrm{in}$, with fully populated rakes having data spanning about 8 to 10 discrete points. The CFD data is reported in 100 data samples per rake. The main purpose of these plots is to show that there is considerable agreement between the LDA and CFD data, even at such low velocities ranging from 2 to $14 \mathrm{ft} / \mathrm{min}$. From Figure 6.8.1, it is clearly evident, with reference to the visual legend, where the $x$, axial flow is moving much faster outside of the confines of the sensing chamber, and as it passes through the labyrinth of the photoelectric chamber, velocities drop significantly, and then slightly recover on the order of $4 \mathrm{ft} / \mathrm{min}$. Figure 6.8.2 shows the influence of the main flow deflector has on the transverse, $w$ velocity components, as the flow field is directed towards the photoelectric sensor creating large magnitudes. In the photoelectric sensing chamber where conditions are fairly settled, transverse velocity components taper off to a magnitude on the order of 1 to $2 \mathrm{ft} / \mathrm{min}$. Here, one can deduce that the grid spacing was somewhat misaligned in recording LDA data because the velocity points appear to be shifted 0.25 in to the right. Figures 6.8.3 through 6.8.6 present other rakes undergoing similar circumstances with regard to entry resistance produced by the ionization sensor - reporting velocities inside on the order of 1 to $2 \mathrm{ft} / \mathrm{min}$. Recall that velocities inside the sensing chambers are designed to be very small in comparison to the freestream flow field because the particle cloud for each sensor (but more so for the ionization sensor) must be very settled in order for the detectors to alarm properly given their respective operating principles.

Figures 6.8.7 through 6.8.12 show results reported for the $90^{\circ}$ orientation, depicting very similar flow patterns that make physical sense with regard to the position of the rake. In Figures like 6.8.10, the LDA data surrounding the photoelectric sensor cannot be recorded due to laser blockage. In these cases, where experimental and computational data has been 
agreeing well, CFD can be used to predict the velocities in these particular zones that are not measurable.

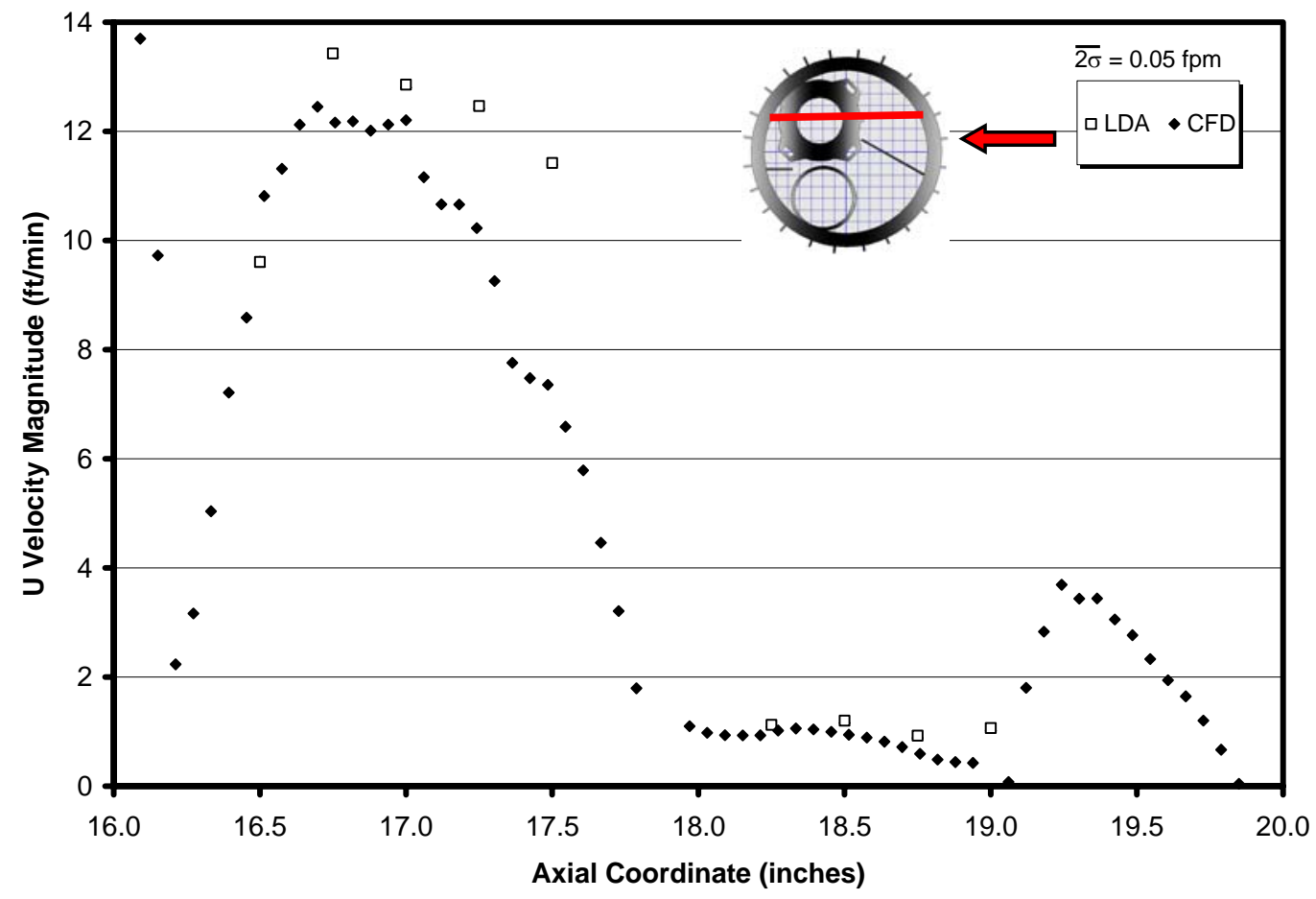

Figure 6.8.1: SA302 smoke detector $u$ velocity comparisons between LDA and CFD at $z=9.75$ in, spanning the $x$, axial direction ( $0^{\circ}$ orientation). 


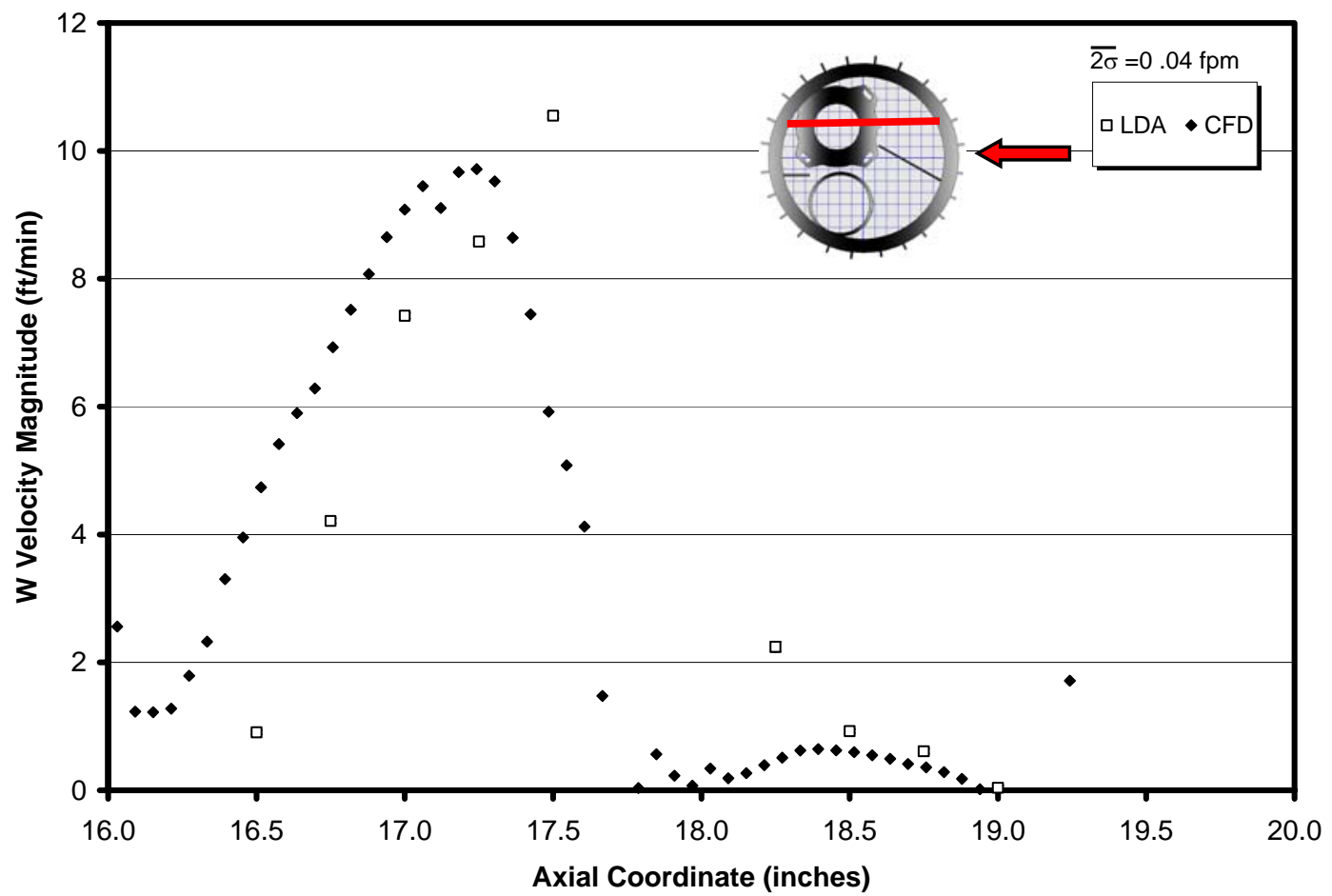

Figure 6.8.2: SA302 smoke detector $w$ velocity comparisons between LDA and CFD at $z=9.75$ in, spanning the $x$, axial direction ( $0^{\circ}$ orientation).

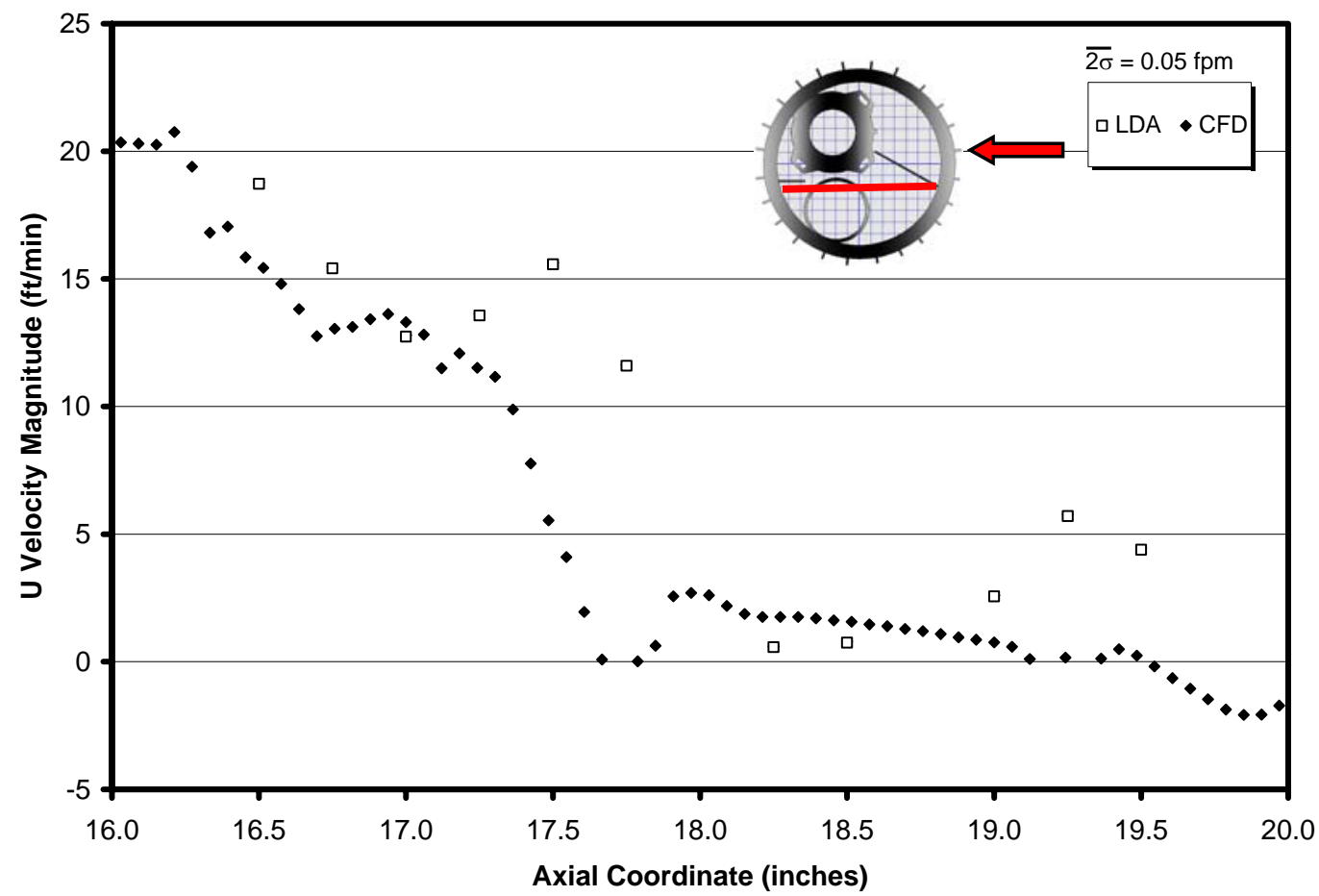

Figure 6.8.3: SA302 smoke detector $u$ velocity comparisons between LDA and CFD at $z=8.50$ in, spanning the $x$, axial direction ( $\left(0^{\circ}\right.$ orientation). 


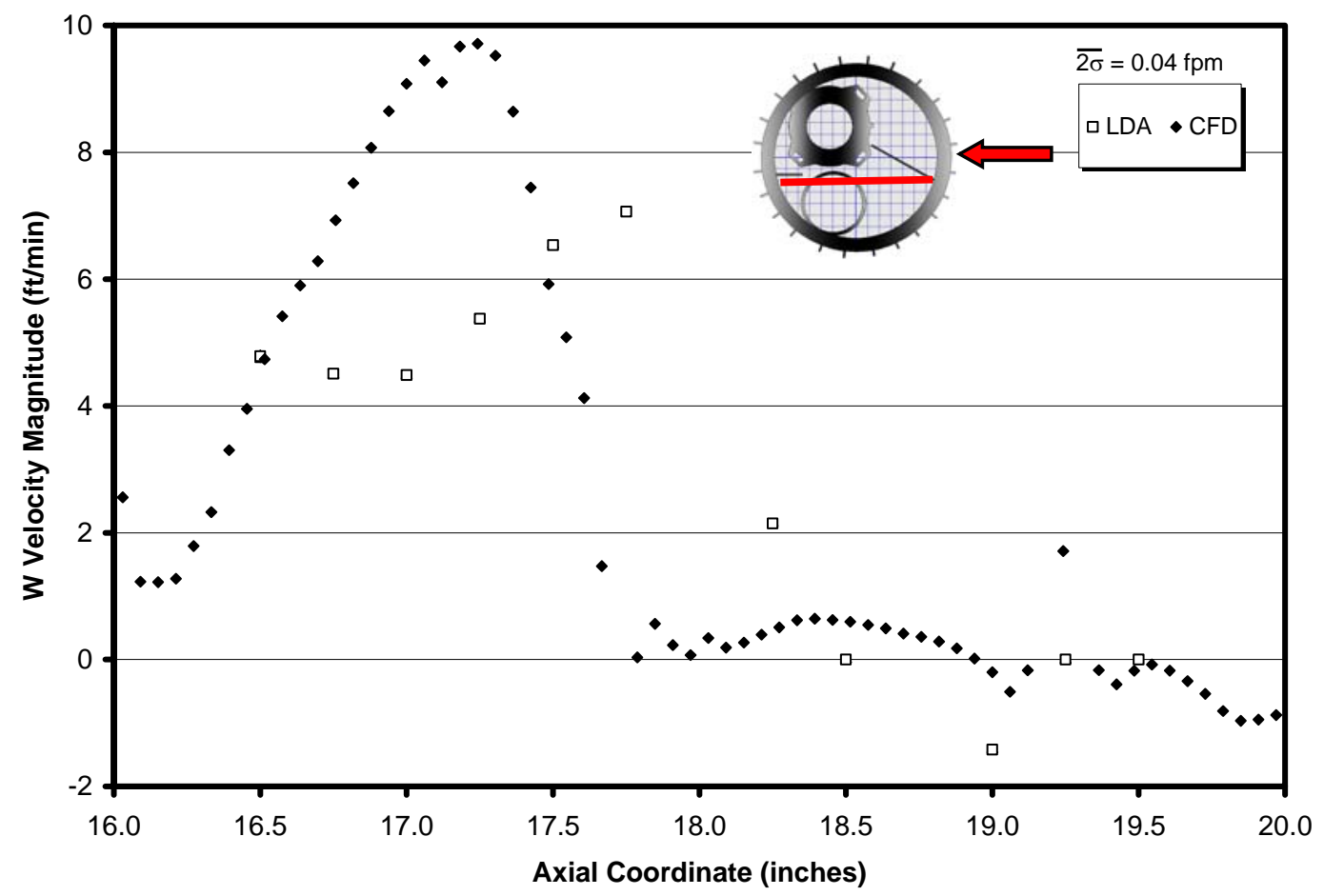

Figure 6.8.4: SA302 smoke detector $w$ velocity comparisons between LDA and CFD at $z=8.50$ in, spanning the $x$, axial direction ( $0^{\circ}$ orientation).

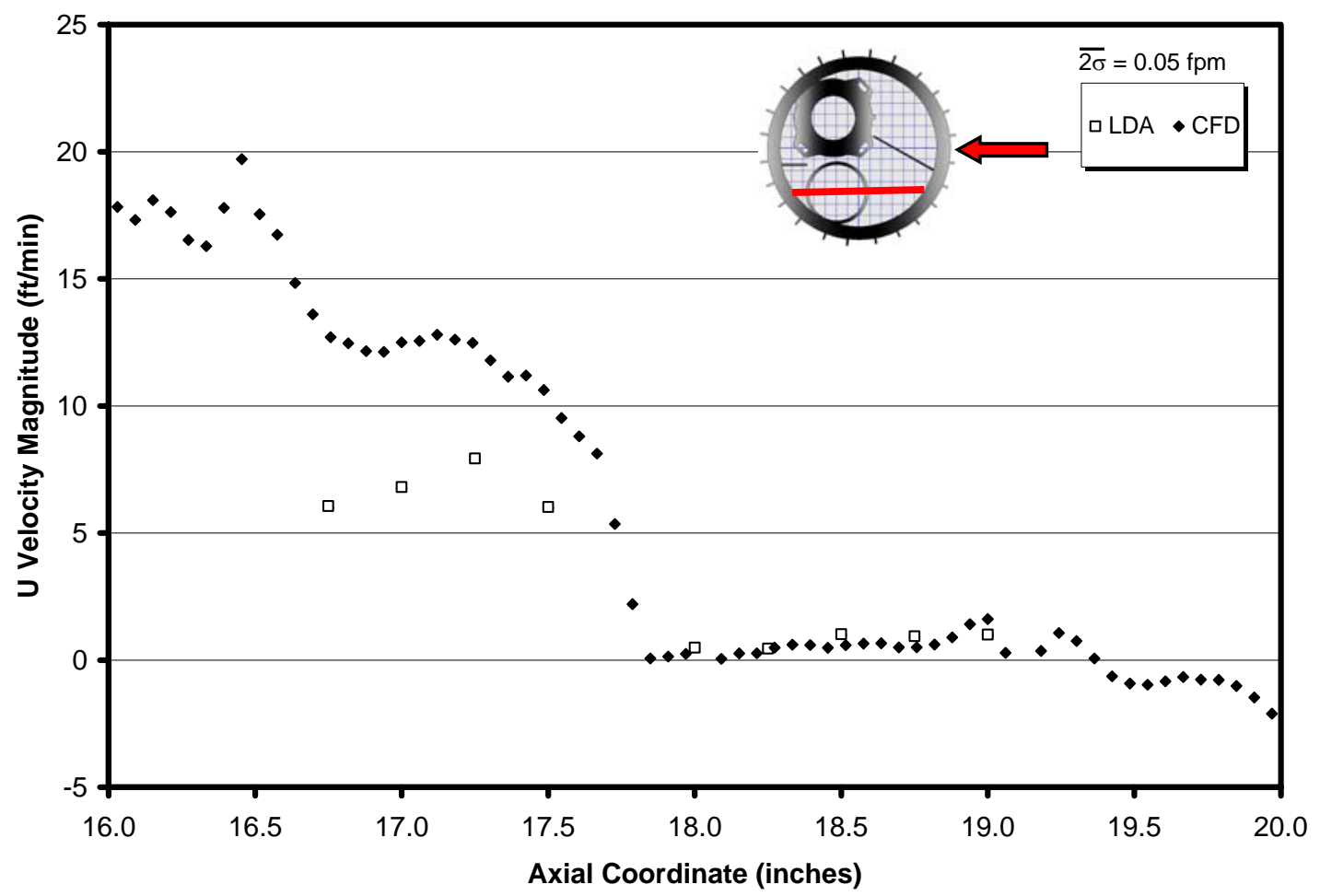

Figure 6.8.5: SA302 smoke detector $u$ velocity comparisons between LDA and CFD at $z=8.00$ in, spanning the $x$, axial direction ( $0^{\circ}$ orientation). 


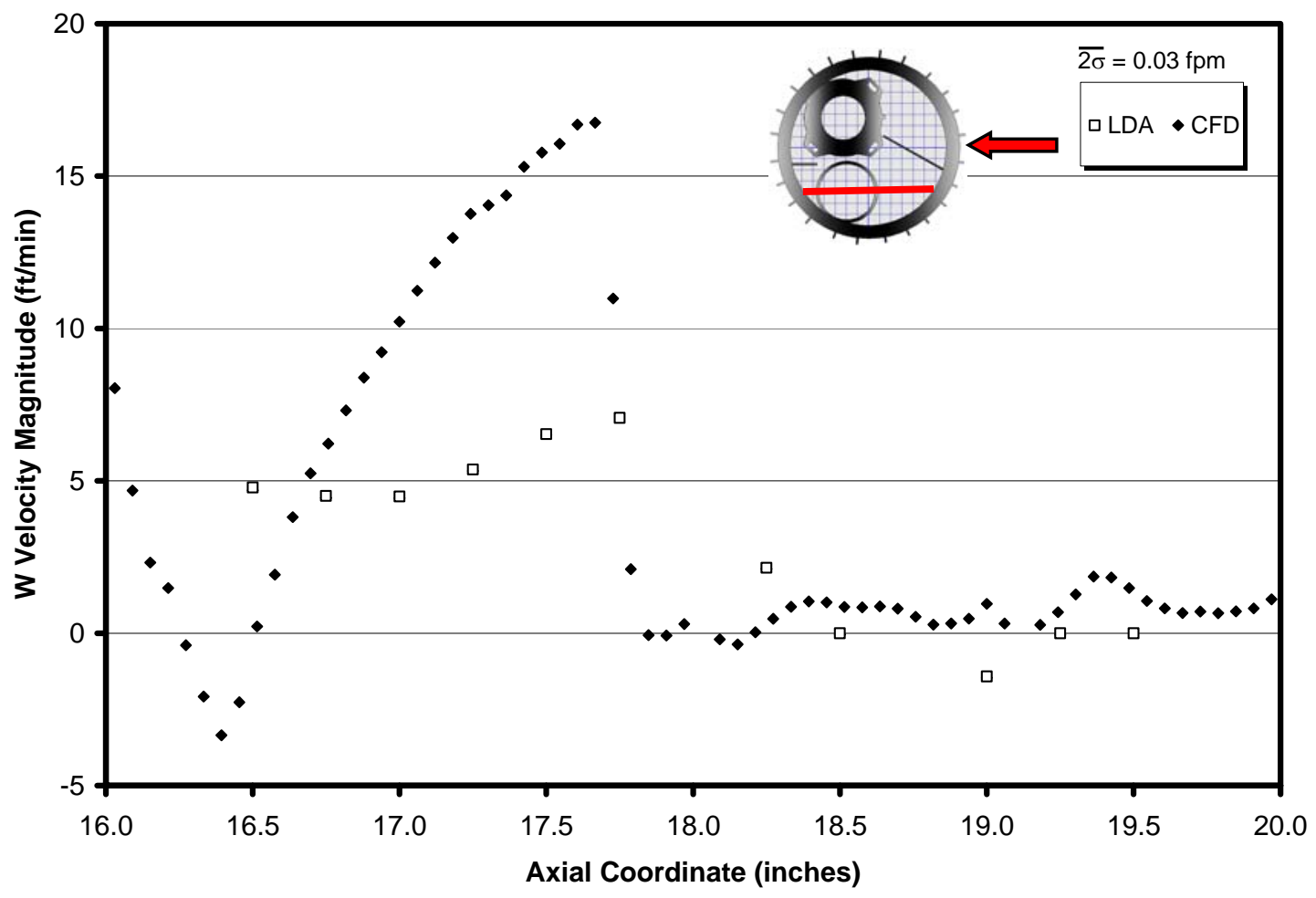

Figure 6.8.6: SA302 smoke detector $w$ velocity comparisons between LDA and CFD at $z=8.00$ in, spanning the $x$, axial direction $\left(0^{\circ}\right.$ orientation).

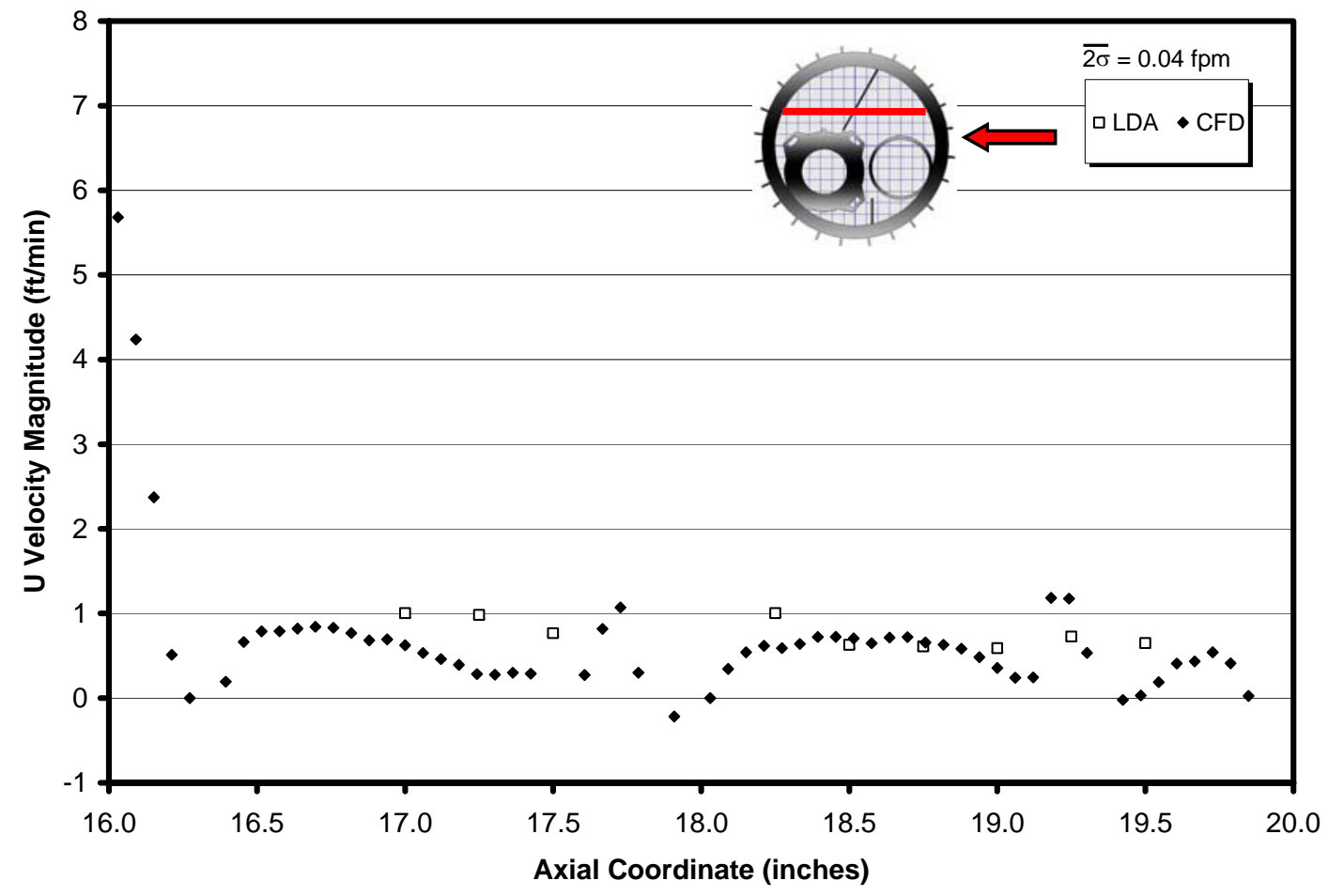

Figure 6.8.7: SA302 smoke detector $u$ velocity comparisons between LDA and CFD at $z=9.75$ in, spanning the $x$, axial direction $\left(90^{\circ}\right.$ orientation). 


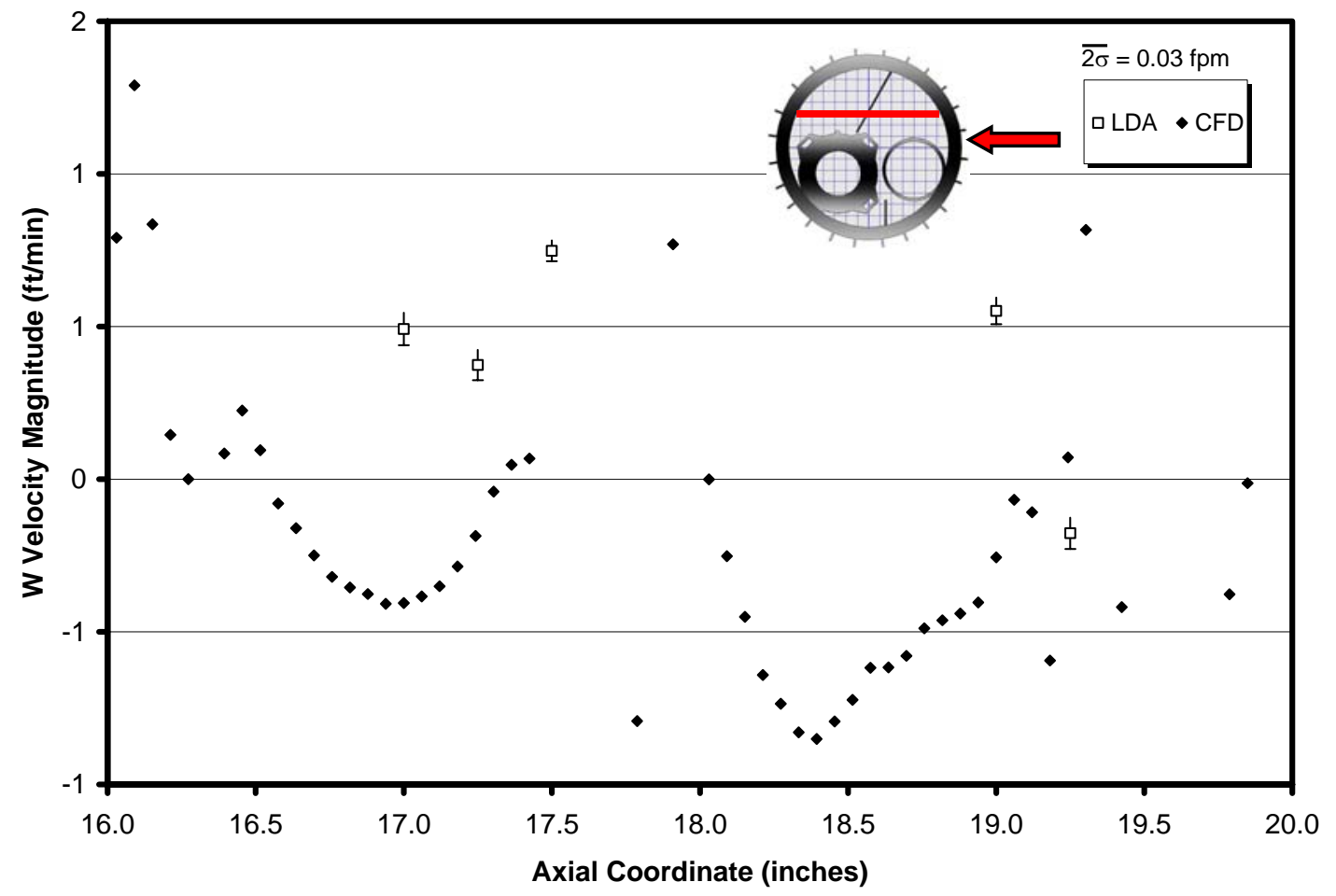

Figure 6.8.8: SA302 smoke detector $w$ velocity comparisons between LDA and CFD at $z=9.75$ in, spanning the $x$, axial direction $\left(90^{\circ}\right.$ orientation).

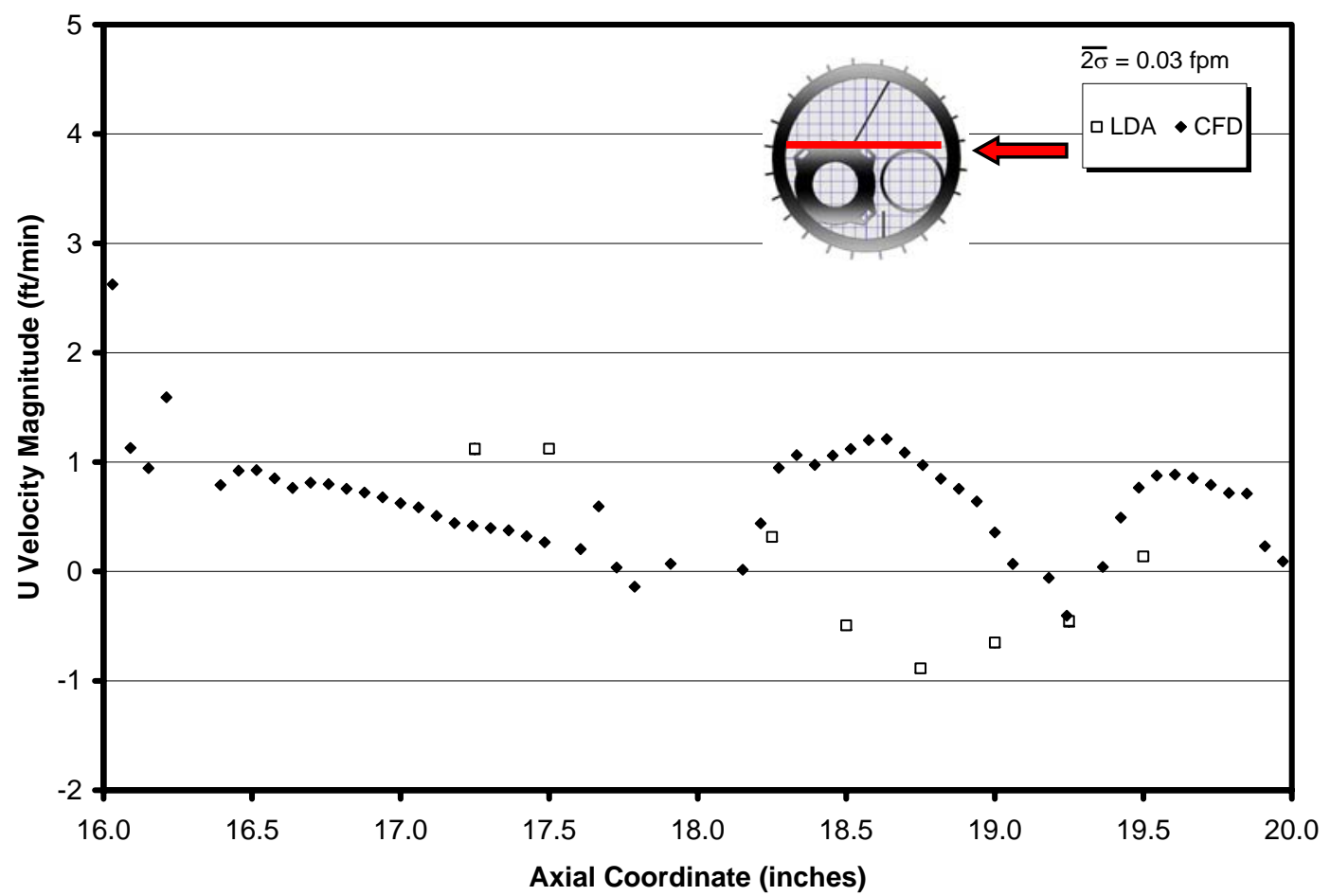

Figure 6.8.9: SA302 smoke detector $u$ velocity comparisons between LDA and CFD at $z=9.25$ in, spanning the $x$, axial direction $\left(90^{\circ}\right.$ orientation). 


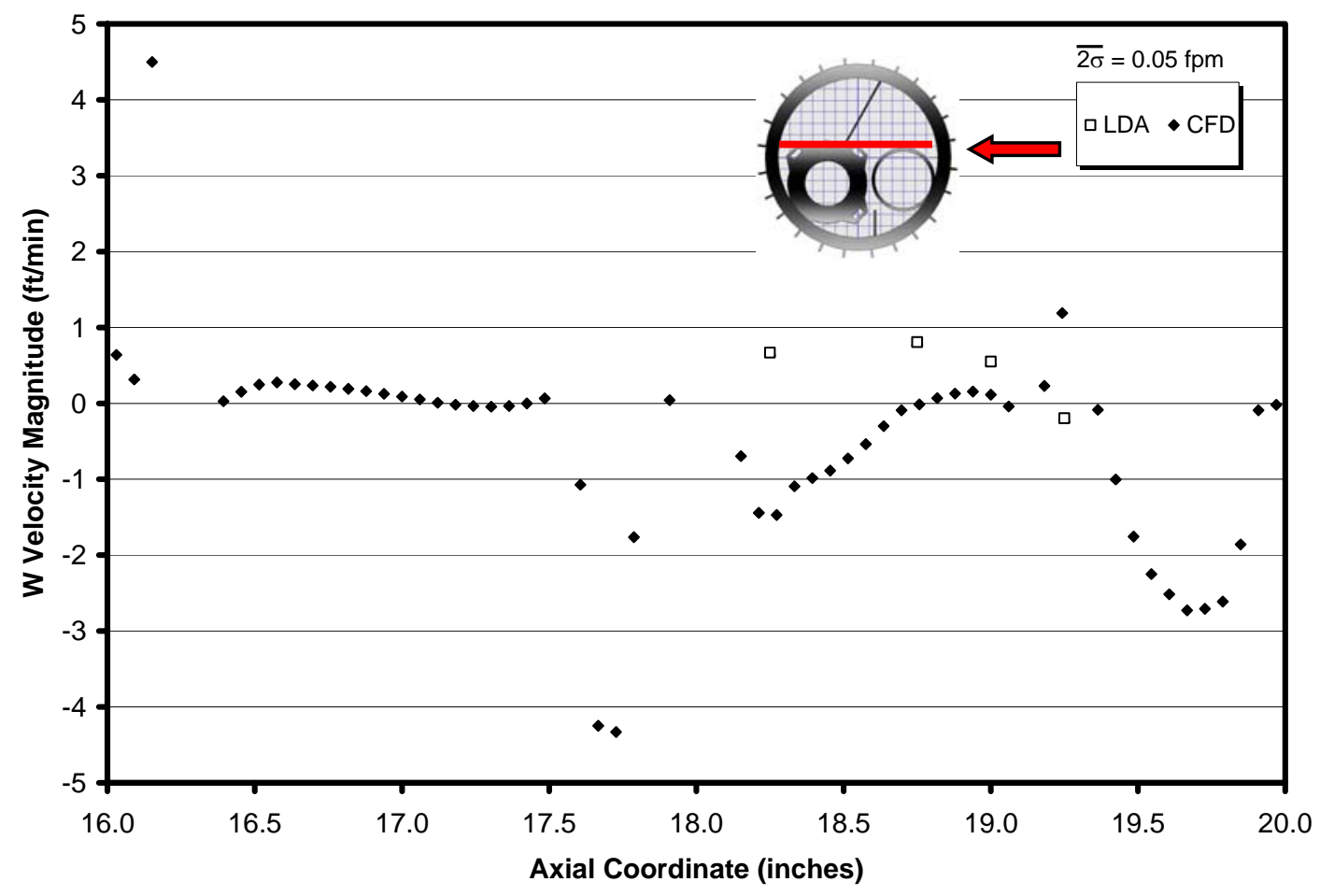

Figure 6.8.10: SA302 smoke detector $w$ velocity comparisons between LDA and CFD at $z=$ 9.25 in, spanning the $x$, axial direction $\left(90^{\circ}\right.$ orientation).

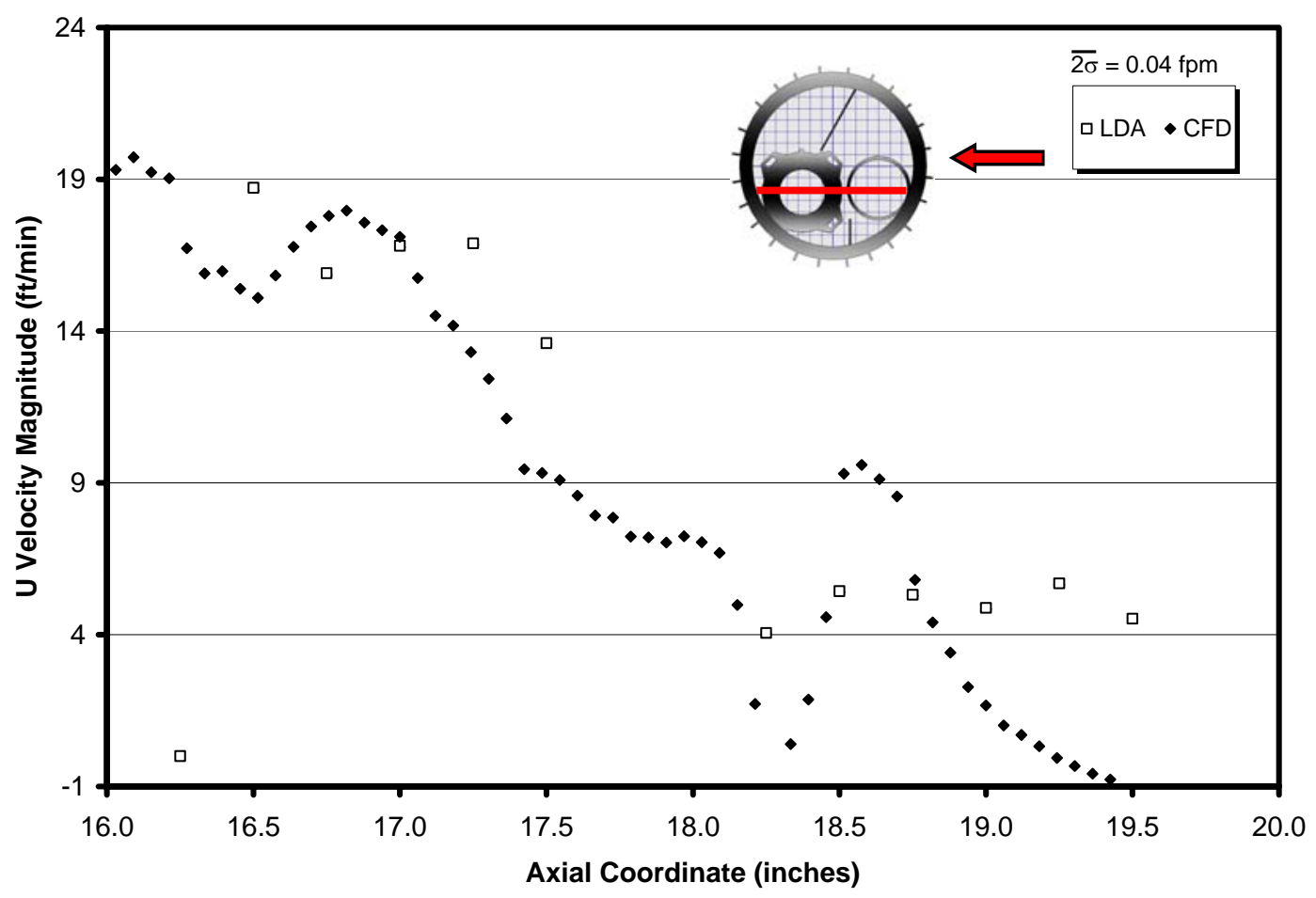

Figure 6.8.11: SA302 smoke detector $u$ velocity comparisons between LDA and CFD at $z=$ 8.50 in, spanning the $x$, axial direction ( $90^{\circ}$ orientation). 


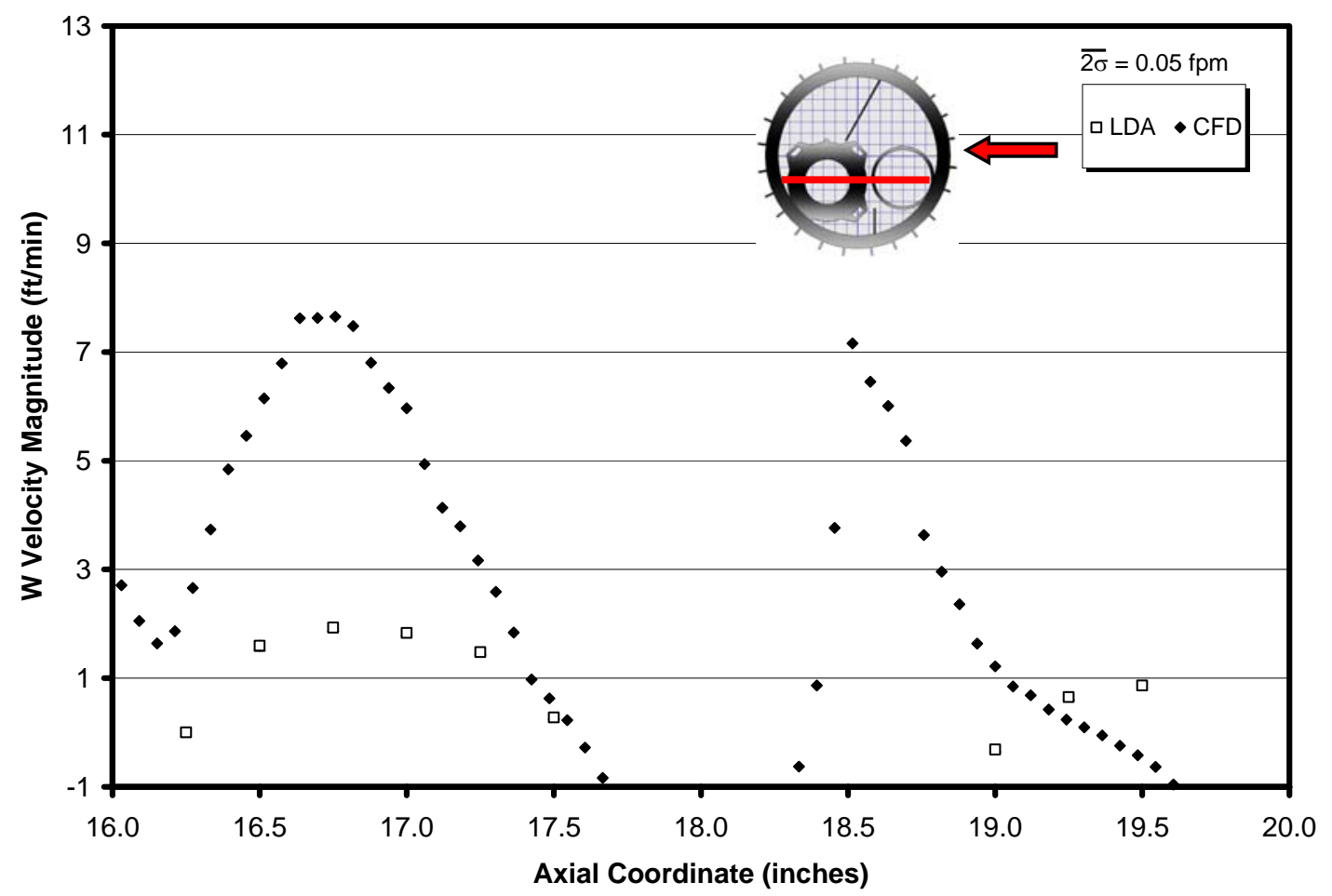

Figure 6.8.12: SA302 smoke detector $u$ velocity comparisons between LDA and CFD at $z=$ 8.50 in, spanning the $x$, axial direction $\left(90^{\circ}\right.$ orientation).

Figures 6.8.13 through 6.8.17 show comparisons between LDA and CFD by comparing velocity vectors inside each detector orientation side by side to one another, providing direct qualitative assessment of the computational flow model. The comparisons show excellent comparative results to back up the quantitative results depicted in Figure 6.8.1 through 6.8.12 earlier in this section. The photoelectric chamber is sketched differently in each figure because although the CFD model was able to report flow data through the sensor's labyrinth, the LDA laser was blocked from these areas due to the enlarged platform making up the photoelectric sensor's ceiling. Hence, no data was available in these areas for LDA. 


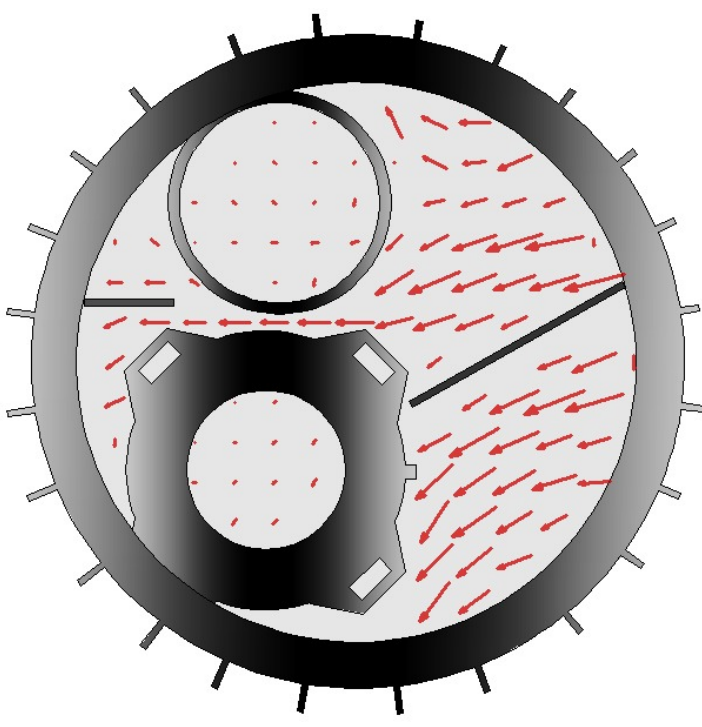

Reference Vector $32 \mathrm{fpm}$

(a.) LDA

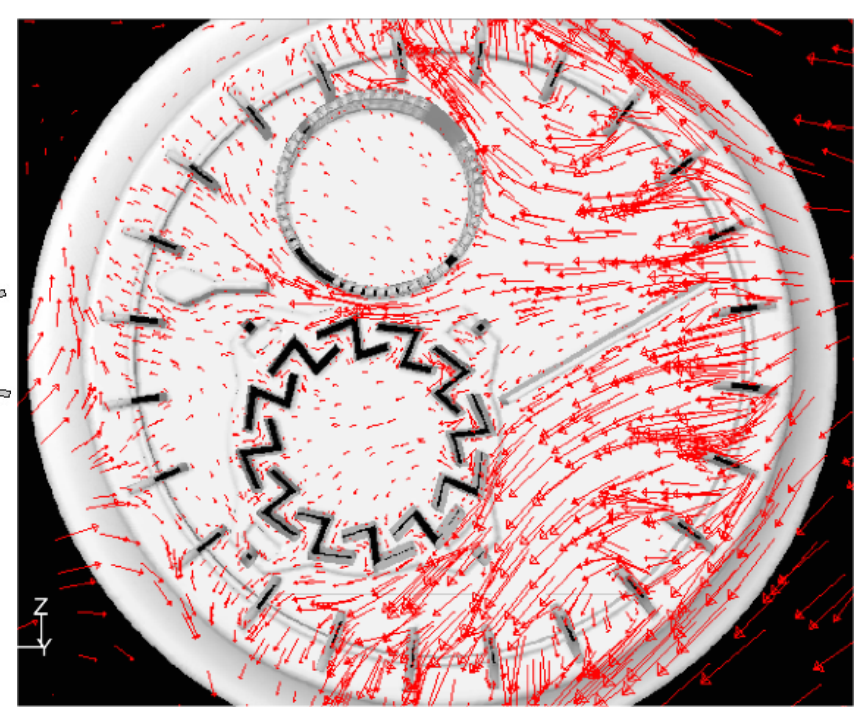

Reference Vector $32 \mathrm{fpm}$

Figure 6.8.13: A velocity vector comparison between (a.) LDA and (b.) CFD (0 $0^{\circ}$ Orientation).

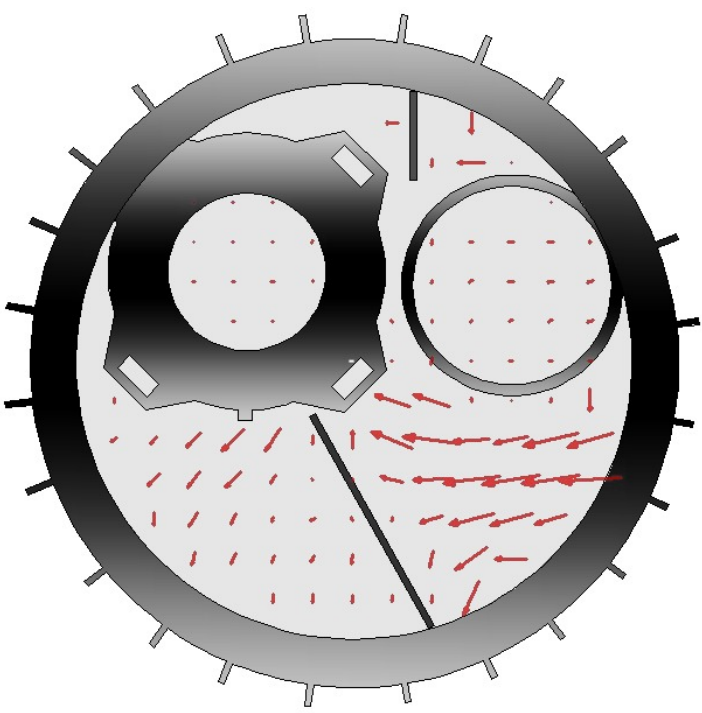

Reference Vector $32 \mathrm{fpm}$ (a.) LDA

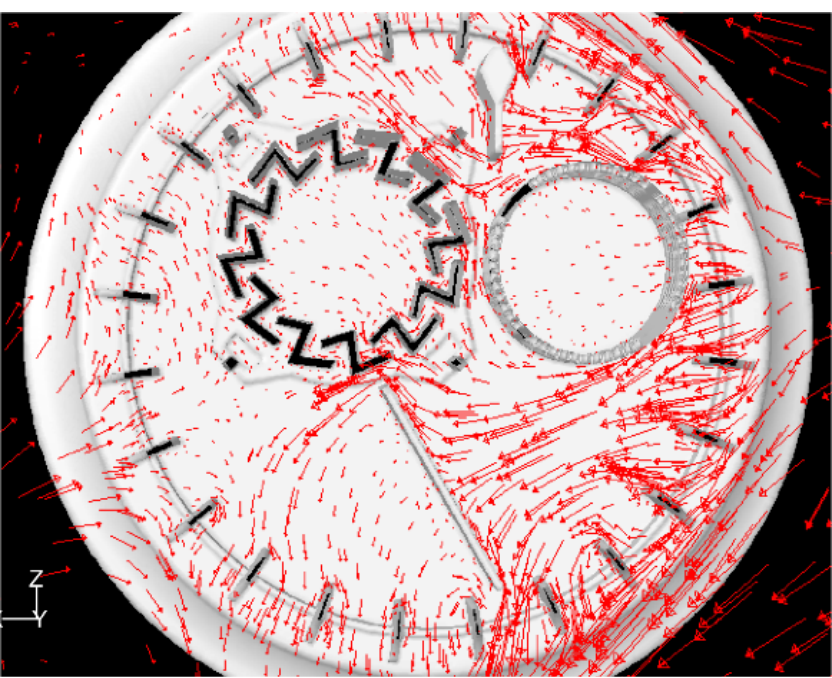

Reference Vector $32 \mathrm{fpm}$

Figure 6.8.14: A velocity vector comparison between (a.) LDA and (b.) CFD (90 Orientation). 


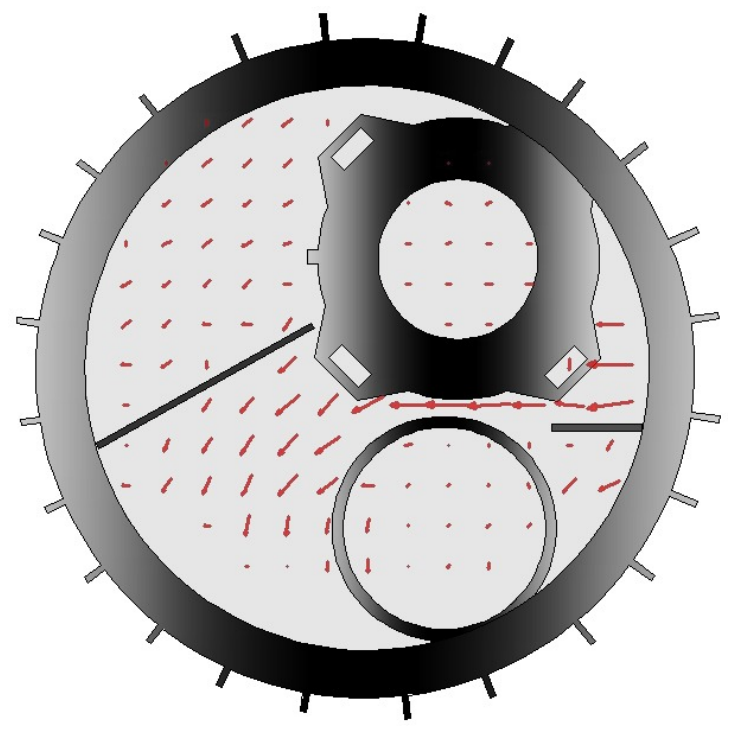

Reference Vector $32 \mathrm{fpm}$

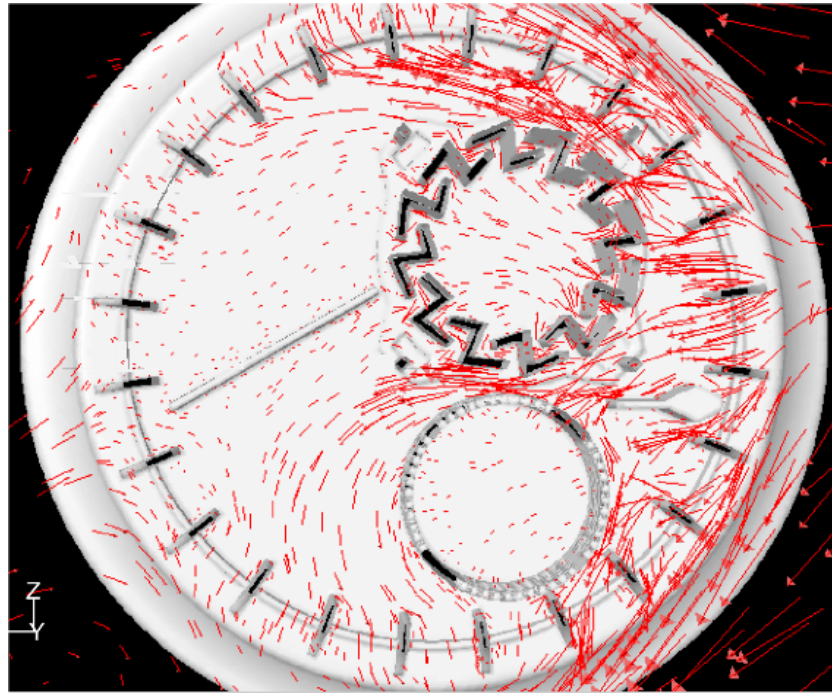

Reference Vector $32 \mathrm{fpm}$

(a.) LDA

(b.) CFD

Figure 6.8.15: A velocity vector comparison between (a.) LDA and (b.) CFD (180 ${ }^{\circ}$ Orientation).

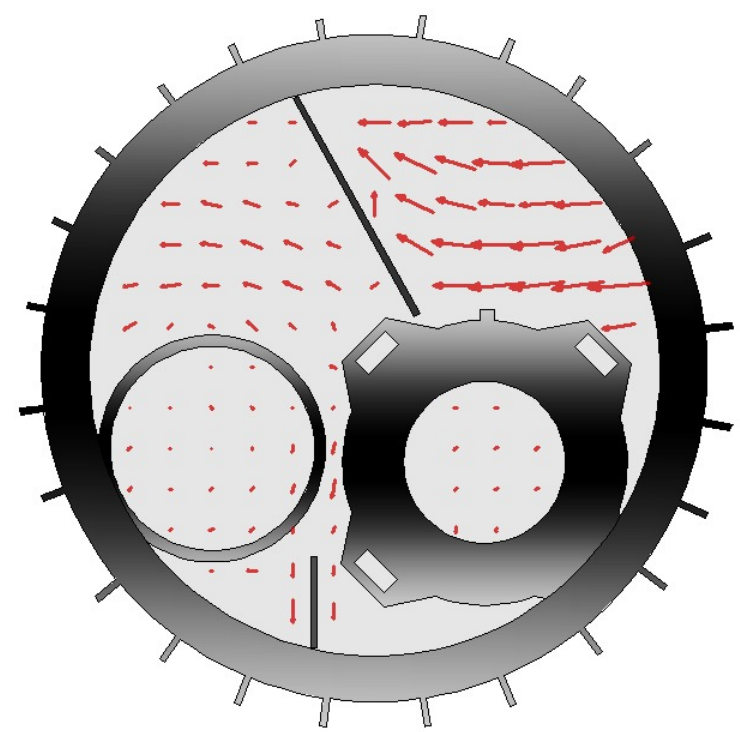

Reference Vector $32 \mathrm{fpm}$

(a.) LDA

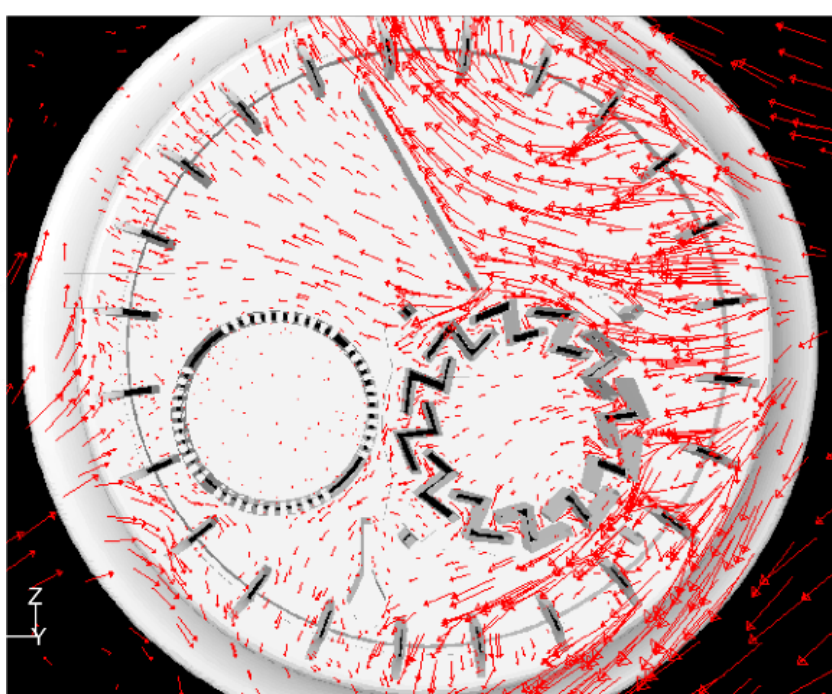

Reference Vector $32 \mathrm{fpm}$

(b.) CFD

Figure 6.8.16: A velocity vector comparison between (a.) LDA and (b.) CFD (270 Orientation). 


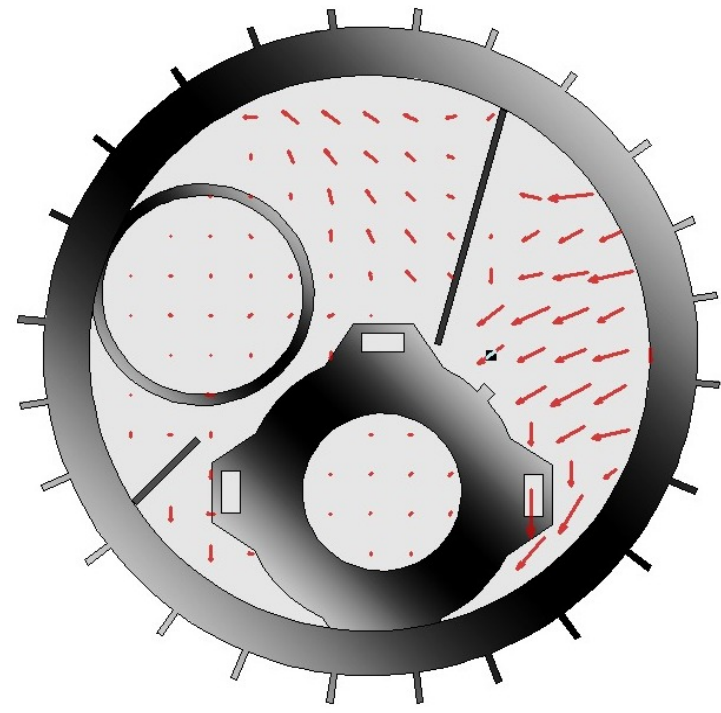

Reference Vector $32 \mathrm{fpm}$

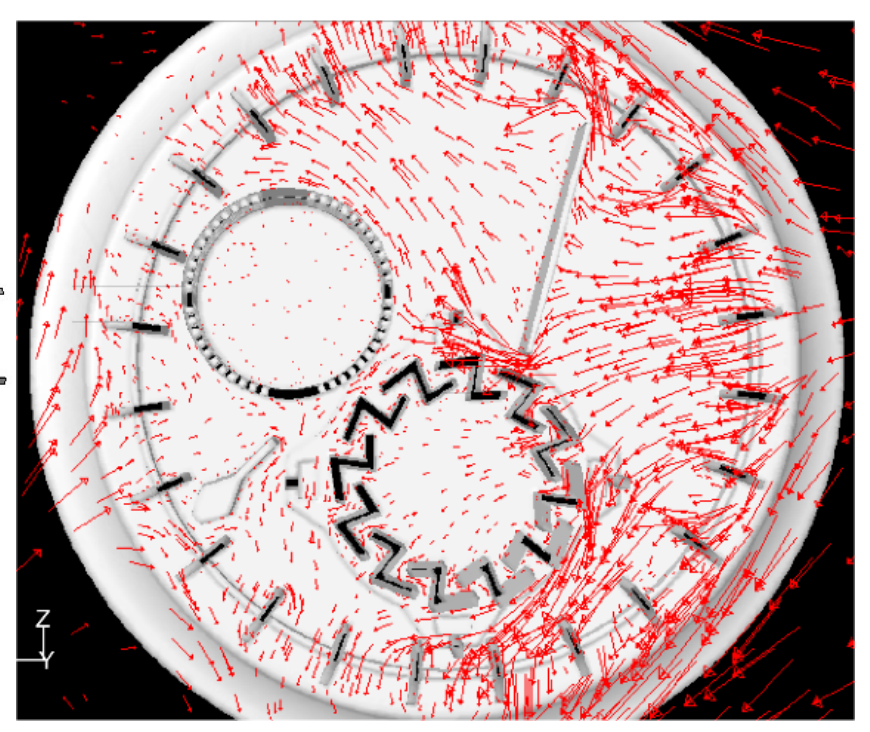

Reference Vector $32 \mathrm{fpm}$

(a.) LDA

(b.) CFD

Figure 6.8.17: A velocity vector comparison between (a.) LDA and (b.) CFD (315 ${ }^{\circ}$ Orientation). 


\section{CHAPTER 7: CONCLUSIONS}

\subsection{GENERAL OVERVIEW}

From a generalized computational modeling standpoint, with the advent of highpowered CAD packages such as Solidworks ${ }^{\circledR 20}$, it has been shown that highly complex geometries can be reduced to create a mesh able to reproduce some of the most complicated types of flow phenomena in very low speed, entry resistant flow conditions. The CFD

models that could be potentially constructed inside of solid modelers like Solidworks ${ }^{\circledR 20}$ are virtually limitless when combined with advanced, unstructured meshing algorithms such as Fluent $^{\circledR}$ 's TGrid ${ }^{\circledR 18}$ used in this particular study.

In addressing the primary objectives of this report, the goals set forth have been sufficiently achieved in characterizing the velocity flow fields present not only in the UL Smoke Box test section, but in the interior of the SA302 smoke detector as well both experimentally and computationally. Although there was noticeable error between the laser Doppler anemometry and laminar flow models upstream between the detector and flow straightener, predominantly in the $v$ and $w$ component velocities, it was clearly evident that the computational flow field recovered sufficiently very near the exterior and interior of the detector to warrant good comparability to that of the experimental measurements. Discrepancies in the $v$ and $w$ could be the result of assuming they were negligible just beyond that of the flow straightener. In all, acceptable validation of the CFD code was achieved in the critical regions within the UL Smoke Box and detector. Sensor chamber velocity measurements were not only of the same order of magnitude as those measured in LDA, but also depending upon experimental grid accuracy, very comparable often overlapping when plotted against one another to within a few percent. LDA data reporting low order turbulence intensities coupled with extensive flow visualization provided constant validity checks as to the global certainty of the experimental results reported.

The detailed internal sensor velocity results help to better understand the entry resistance associated with the not only detector housing geometry, but the flow impedance induced by the sensing chamber geometries themselves on the flow they are trying to sample. Generally speaking, the ionization sensor settling chamber is designed to allow enough smoke laden flow into the chamber for the smoke cloud to be settled enough as to not 
provide false alarms. This ionization sensor property is heavily documented in the very low velocity measurements ( 1 to $2 \mathrm{ft} / \mathrm{min}$ ) in nearly all detector orientations. The photoelectric sensor, on the other hand, normally experiences velocities two to three times greater than the ionization sensor, but based on its own operating principles, responds in relative time accordance with that of the ionization sensor for the size particles its designed to sense. In comparing the maximum velocities encountered by each sensor in each respective orientation, it was found that the ionization sensor had equally minimum flow impedance in the $0^{\circ}, 90^{\circ}$, and $180^{\circ}$ orientations with speeds maximized at $1.8 \mathrm{ft} / \mathrm{min}(0.03 \mathrm{ft} / \mathrm{sec})$. With regard to the photoelectric sensor, minimum flow impedance was achieved in the $315^{\circ}$ orientation with flow velocities as great as $9 \mathrm{ft} / \mathrm{min}(0.15 \mathrm{ft} / \mathrm{sec})$. In all, detailed inspection of the flow fields in each sensor for all five orientations examined provided an excellent foundation for future work in designing sensor chamber designs. In the future, mass concentration studies based upon this flow model could provide more detail in the sensing regions of interest.

In conclusion, due to the principal successes reached in this research venture, it is apparent that given a spot-type smoke detector of similar size and geometric design, a computational model composed using the same methodology followed here should be able to adequately predict its comprehensive flow patterns to within reason. As a preliminary design tool, computational modeling using Fluent ${ }^{\mathbb{1 1 8}}$ has the verified ability to provide predictable results able to help enhance the design evolution of spot-type smoke detectors.

\subsection{RECOMMENDATIONS}

There are several constructive recommendations that can be made from this study. Future work culminating from this design technique by actually modeling smoke particles computationally is imperative to keep this sort of innovative work alive in the fire protection industry. Also, certain limitations were experienced in what could be modeled due to the performance restrictions of the hardware at hand. By meshing the model with a size constraint smaller than that of the ionization chamber holes, for instance, one would create too many nodes for the computer to solve independently. With parallel processing beginning to take the helm during this phase of the information age, computer networks such as Beowulf clusters that share large calculations with multiple processors could be employed to 
solve more refined models than the ones presented here. From this, a true grid independent study could be carried out.

Also, the geometries of both the ionization and photoelectric sensing chambers could be adapted as well in an attempt to reduce the lag time associated with the entry resistance induced by the presence of the detector itself. Changing the distance between the baffle design in the photoelectric chamber, for instance, may direct flow patterns in such a way as to make the sensor alarm more reliably by reducing particle residence time within the detector. A unique balance must be constantly maintained by minimizing the time it takes for particles to reach the actual sensing chambers, while still maintaining a settled flow field as to measure the amount of particles accurately.

\subsection{NOTES ON MODELING SMOKE DETECTOR RESPONSE}

A natural extension to the work presented is to go beyond just characterizing the velocity field present by introducing Fluent ${ }^{\circledR 18}$, s discrete phase modeling (DPM) capabilities in predicting particle motion inside the UL Smoke Box test section, and more importantly, inside the detector. Fluent ${ }^{\circledR 18}$ provides two methodologies for reporting the properties of various particles propagating through a continuous fluid medium using either the Lagrangian (post-processing) solver, or the discrete phase domain coupled with the Eulerian (main flow) solver. By knowing some extrinsic (as well as intrinsic) properties of the smoke particles entering the detector such as mass flow rate, diameter, and size distribution discussed in Chapter 2, the work of researchers such as Lee and Mulholland ${ }^{3}$ can be applied using present day technology to model smoke detector response. Using the particle residence time function

in Fluent ${ }^{\circledR 18}$, s Lagrangian DPM solver, designers could get a rough estimate as to the entry resistance associated with how long it takes smoke particles of a certain mass, size, etc., to reach an alarm condition inside a new detector design.

The coupled Eulerian DPM solver could provide even more telling results by calculating the mass concentration of particles for a given flow field given an surface injection point and mass flow rate. In short, the multiphase capabilities are far reaching even moving to the realm of full combustion processes, which could eventually model the presence of fire growth, and how a detector dynamically responds to a presence of smoke. 


\section{VITA}

Christopher P. Menchini, the only son of Mr. and Mrs. Ernst C. Menchini, was born in Charleston, WV, on July 25 ${ }^{\text {th }}$, 1980. Growing up in Michigan, Alabama, as well as West Virginia, he concluded his secondary education in the Cabell County Public School District, graduating from Cabell Midland High School with very high honors in May of 1998. He received two Bachelor of Science Degrees from West Virginia University in August of 2002 with majors in Aerospace as well as Mechanical Engineering.

In August of 2002, he entered the graduate program at West Virginia University and is a candidate for the Masters of Science Degree in Aerospace in December 2003. Upon graduation, he has accepted a position with the title of Research Scientist from a research and development firm known as Combustion Research and Flow Technology, Inc. 


\section{WORKS CITED}

1. Pucci, W. E., "Modeling Spot-Type Smoke Detectors: Currently Available Methods and Their Merits for Everyday Use.” Fire Protection Engineering: Unique Smoke Management Designs. Summer 2000: Issue No. 7.

2. Oldweiler, A., "Investigation of the Smoke Detector L-Number in the UL Smoke Box." MS Master’s Thesis, Worcester Polytechnic Institute. Worcester, MA 1995.

3. Lee, T. and Mulholland, G.W., "Physical Properties of Smokes Pertinent to Smoke Detector Technology.” National Bureau of Standards, NBSIR 77-1312, 1977.

4. Mulholland, G.W., "Smoke Production and Properties.” SFPE Handbook of Fire Protection Engineering, $1^{\text {st }}$ Edition, Philip J. Dinennio ed., Society of Fire Protection Engineers, Boston, Massachusetts, 1988.

5. UL 217: "Standard for Single and Multiple Station Smoke Detectors.” Underwriters Laboratories, Inc., $5^{\text {th }}$ Edition, Northbrook, IL, 1997.

6. Schifiliti, R.P. and Pucci, W.E. "Fire Detection Modeling: State of the Art." The Fire Detection Institute, Bloomfield, CT., 1996.

7. Geimann, J.A. and Gotuk, D.T. “Alarm Thresholds for Smoke Detector Modeling.” $7^{\text {th }}$ International Symposium for Smoke Detector Modeling. Worcester Polytechnic Institute. Worcester, MA. 16-21 June 2002.

8. Cleary, T. and Grosshandler, W. "Smoke Detector Response to Nuisance Alarms." AUBE '99 Proceedings of the $11^{\text {th }}$ International Conference on Automatic Fire Detection, National Institute of Standards and Technology, Gaithersburg, Maryland, pp. 32-41, 16-18 March 1999.

9. Schifiliti, R.P. "Fire Detection Modeling - The Research Application Gap.” AUBE '01 Proceedings of the $12^{\text {th }}$ International Conference on Automatic Fire Detection, National Institute of Standards and Technology, Gaithersburg, Maryland, pp. 529560, March 2001.

10. Litton, C.D., “Optimizing Ionization-Type Smoke Detectors.” Fire Technology. National Fire Protection Agency, Vol. 15, No. 1, National Fire Protection Agency, February 1979. pp. 25-42.

11. Litton, C.D., “A Mathematical Model for Ionization-Type Smoke Detectors and the Reduced Source Approximation.” Fire Technology, Vol. 13, No. 4, National Fire Protection Agency, November 1974. 
12. Cleary, T. and Chernovsky et al. "Particulate Entry Lag in Spot-Type Smoke Detectors.” Sixth International Symposium on Fire Safety Science, Univ. of Poitiers, France, July 5-9, 1999.

13. Conte, Frederic. "CFD Simulations of Smoke Detection in Rooms with High Ceilings." SP Swedish National Testing and Research Institute. SP AR 2002:30, Brandeknik, Boras, 2002.

14. Ierardi, J.A. and Barnett, J.R. "Characterizing Entry Resistance of Smoke Detectors: Flow Distributions Within an Ionization Smoke Detector.” Fire Supression and Detection Research Application Symposium Proceedings, pp. 397-405, Fire Protection Research Foundation. Orlando, FL, 23-25 February 2000.

15. Wood, C.B., "Flow Simulation Through a Simplified Smoke Detector Using Computational Fluid Dynamics.” MS Master's Thesis. Worcester Polytechnic Institute. Worcester, MA, 1995.

16. Ierardi, J.A. and Barnett, J.R. "A Methodology for Predicting Smoke Detector Response.” Worcester Polytechnic Institute for Fire Safety Studies. pp. 235-239, Worcester, MA, 2001.

17. Gobeau, N. et al. "Guidance for HSE Inspectors: Smoke Movement in Complex Enclosed Spaces - Assessment of Computational Fluid Dynamics."Health \& Safety Laboratory. HSL-2000-29. Harpur Hill, Buxton, SK17 9JN.

18. Fluent, Inc. "Fluent 6 Product Information." http://www.fluentusers.com. 8 November 2003.

19. Live Easy Products, Inc. "First Alert: SA302.” http://www.leproducts.com. 5 November 2003.

20. Solidworks ${ }^{\circledR}$ Corporation. "Solidworks ${ }^{\circledR}$." http://www.solidworks.com. 5 November 2003.

21. Amtec ${ }^{\circledR}$ Engineering, Inc. “Tecplot ${ }^{\circledR}$ : Enjoy the View.” http://www.amtec.com. 5 November 2003. 


\section{APPENDIX A: UL SENSITIVITY CURVES}

Figures A.1, A.2, and A.3 show how changing the environmental conditions change the response conditions for running a test according to UL standards. Figure A.1 shows a standard UL curve given a standard day. The graph plots beam operation in percent obscuration per foot as a function of the current flow in $p A$ from the MIC. For a test to be valid, the response of both the MIC and photometer must fall within these bounds. Figure A.2 shows how the standard changes with respect to a $0^{\circ} \mathrm{C}$ temperature. Figure A.3 shows a similar comparison at $49^{\circ} \mathrm{C}$ with $30-50 \%$ relative humidity.

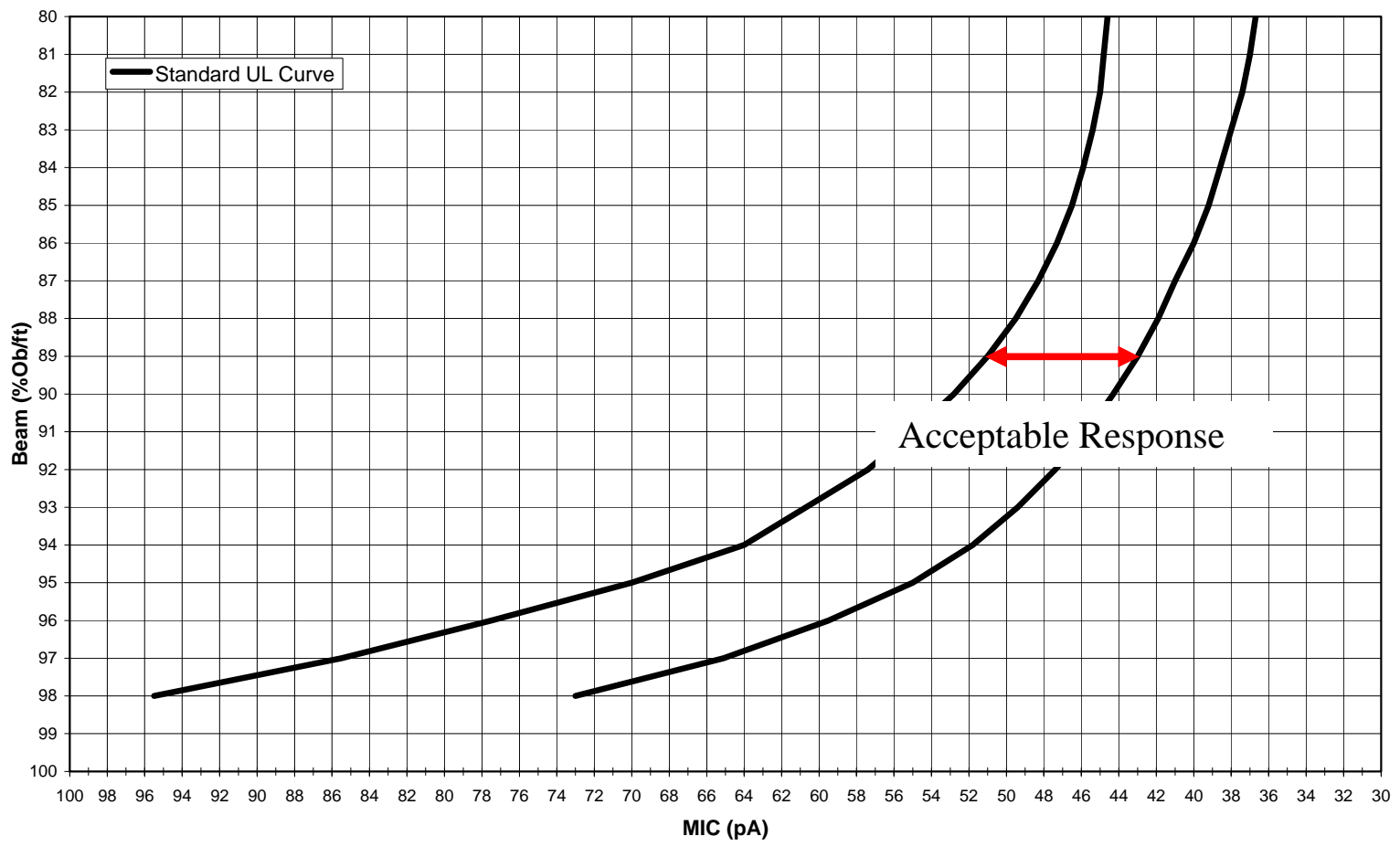

Figure A.1: A standard UL response curve under standard conditions. 


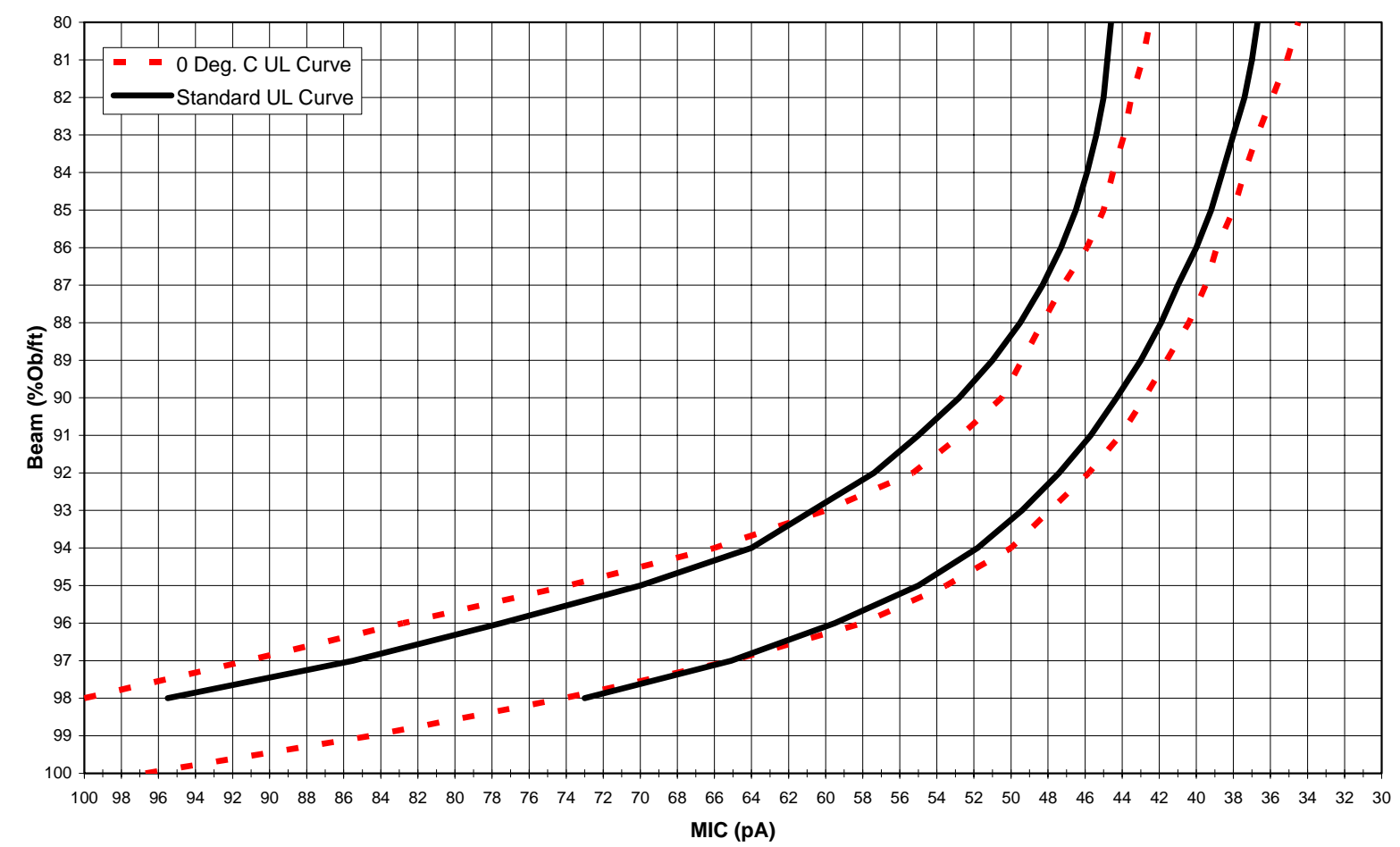

Figure A.2: A standard UL response curve compared to a curve taken at $0^{\circ} \mathrm{C}^{5}$

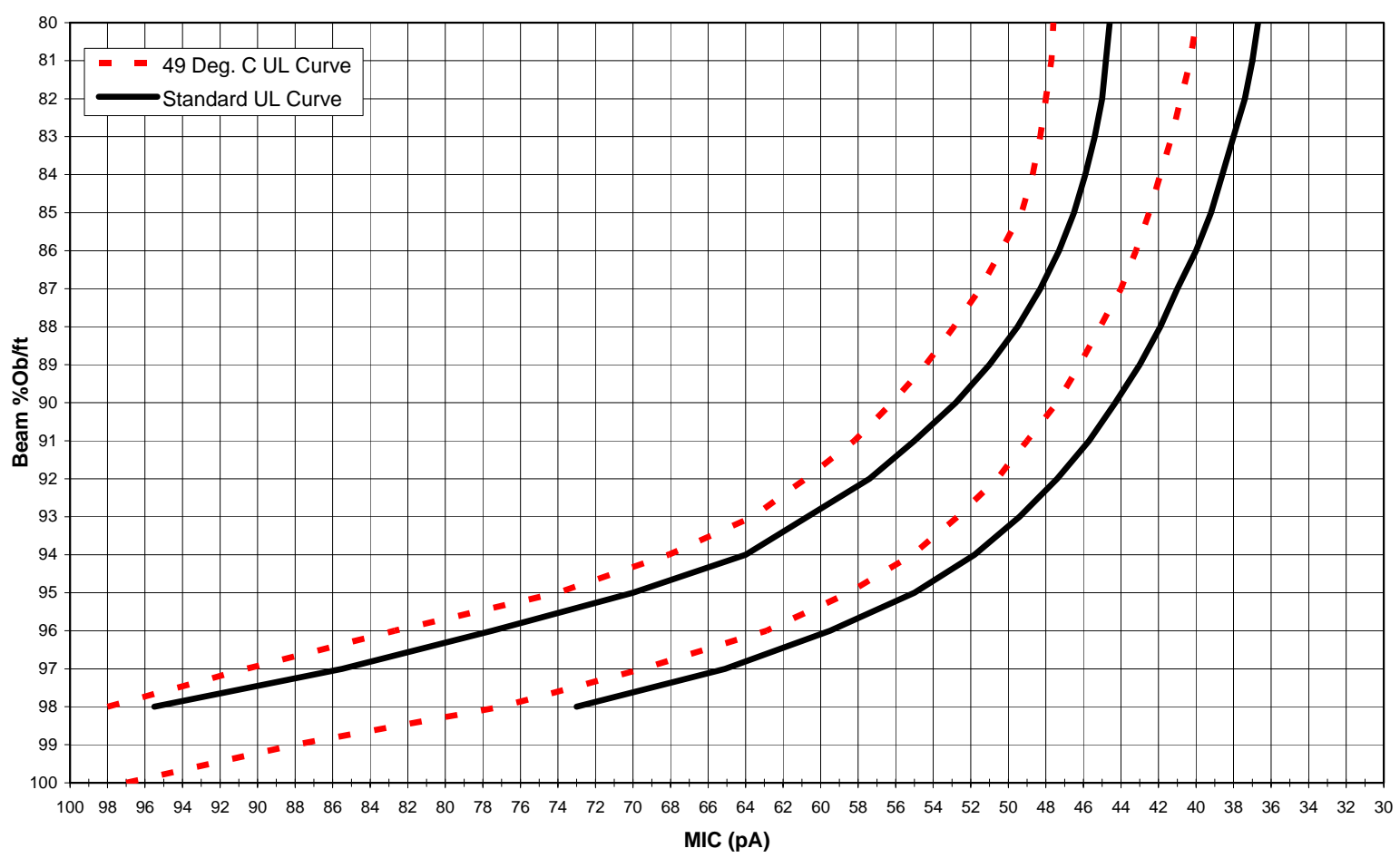

Figure A.3: A standard UL response curve compared to a curve taken at $49^{\circ} \mathrm{C}$ with relative humidity in the range of 30 to $50 \%{ }^{5}$ 


\section{APPENDIX B: PRESSURE LOSS MEASUREMENTS ACROSS THE INSECT SCREEN}

There are three main factors that must be set within Fluent ${ }^{\circledR 18}$ to fully define a porous wall that follows the basic philosophy of Darcy's Law for porous media. Darcy's law can be best described as a calculation of pressure drop across a porous surface given certain flow and porosity characteristics. Most parameters such as fluid velocity and density can be easily inputted from the calculated flow field itself. Other constant parameters, however, must be defined as porous conditions for each particular scenario. The first unknown parameter, called permeability, is fluid dependent, and carries the basic units of length squared. Normally these terms are very small (on the order of $10^{-5}$ to $10^{-13}$ ), with values getting smaller the less permeable a substance is. Another value, called the pressure-jump coefficient, is a multiplying constant that is controlled mostly by the incoming flow velocity.

In addressing these issues, a simple experiment was set-up to measure the pressure drop across the insect screen. By measuring the pressure drop, Darcy's Law can be calculated backwards in an iterative fashion to derive values for permeability and the pressure-jump coefficient. The procedure entailed taking a pipe of similar diameter to the width of the screen, and attaching a piece of the screen to the end of the pipe, letting the screened pipe end be open to the atmosphere. By placing a pressure tap slightly upstream of the screen, a pressure differential could be measured between the upstream pressure just before the screen, and the atmosphere just beyond. Unfortunately, because of slow flow speeds and screen geometry, the pressure drop was nearly un-measurable using a micromanometer manometer accurate to a $1 / 2000^{\text {th }}$ in of water, especially when taking into account instrumental error. However, because pressure drop is additive over a series of screens, 12 of them were placed in series about 1 in apart inside the pipe in an attempt to measure a bulk pressure drop. This bulk pressure drop, in theory, could then be divided by the number of screens in order to find the pressure loss across one individual screen. Various pressure differentials were recorded by varying the flow rate in the pipe. Because experimental velocities are on the order of 10 to $15 \mathrm{ft} / \mathrm{min}$ entering the photoelectric sensor, a curve fit had to be applied to the measured data because velocities that low could not be measured accurately. Slightly extrapolating this trend line down to the required velocity resulted in nearly zero pressure drop. Figure B.1 depicts these results. When taking this into 
account, it seems as if the insect screen does not significantly affect the flow entering the sensor. Thus, measures to enforce a porous zone surrounding the photoelectric sensor were abandoned. Figure B.2 depicts the basic set-up and philosophy used in the experimental procedure.

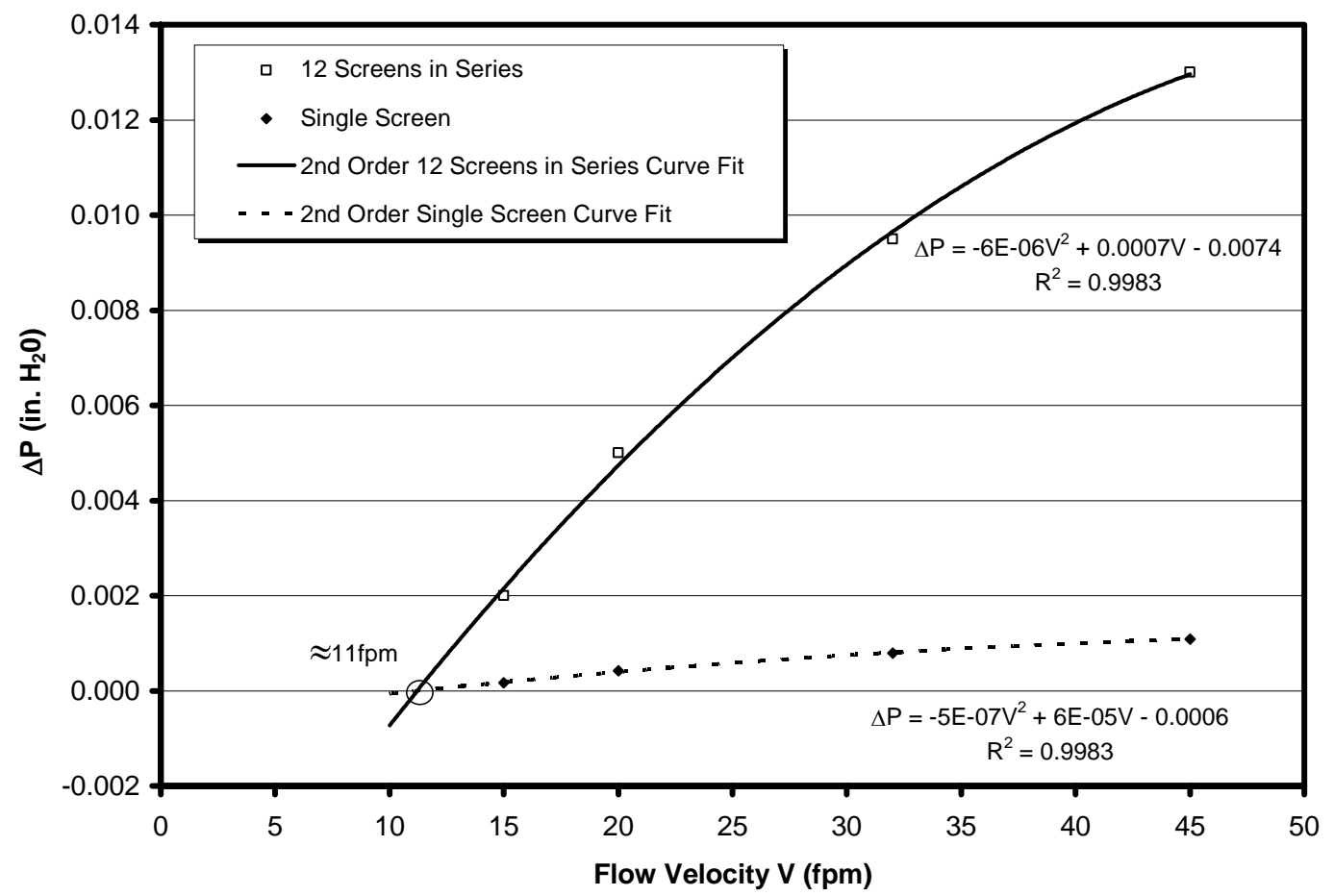

Figure B.1: Pressure loss across an insect screen as a function of constant flow velocity through a 0.475 in pipe.

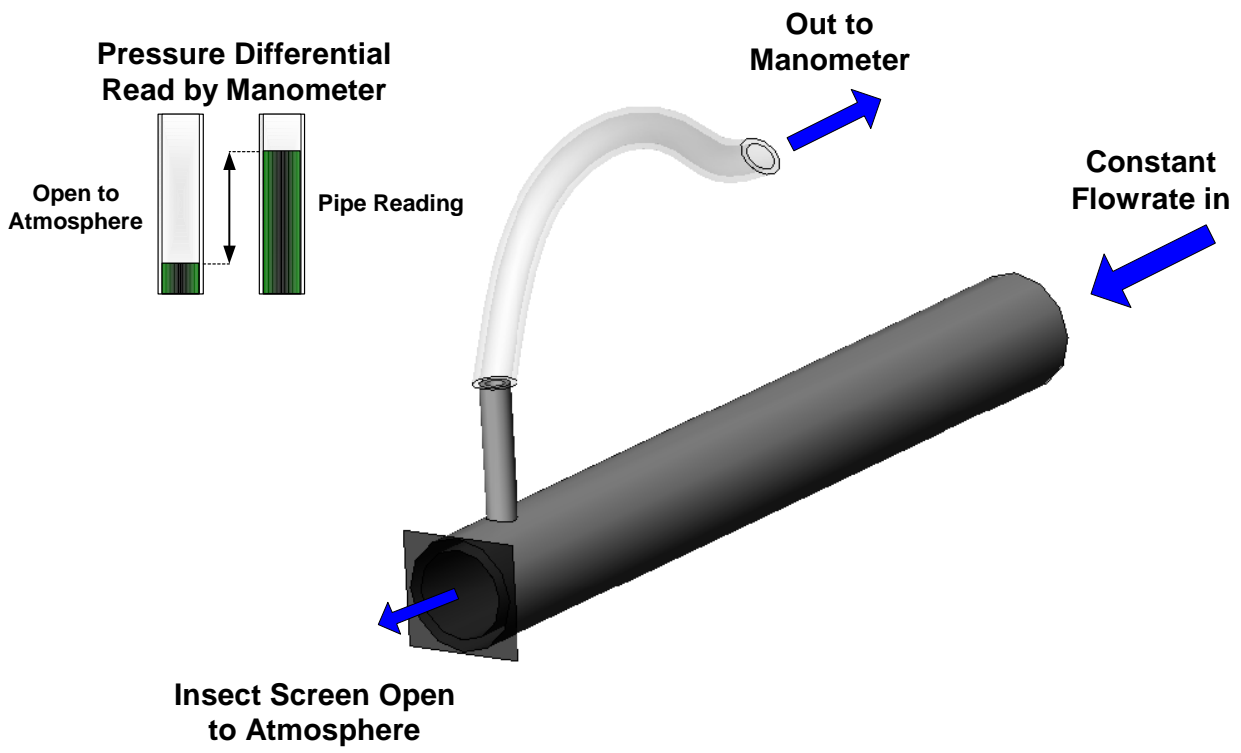

Figure B.2: The screen porosity experiment in its basic form. The actual layout of the experiment consisted of 12 screens in series at various stations within the pipe. 


\section{APPENDIX C: MULTI-SAMPLE DATA ERROR ANALYSIS}

The method used to define the uncertainty in the experimental LDA measurements is called multi-sample data error analysis. For this study, a range of 3000 to 5000 data samples $n$ were collected for each particular sample. From these samples, an average mean velocity $\bar{U}$ along with a turbulence intensity, $T I$, was recorded. Turbulence Intensity, as a fractional value, is defined as follows:

$$
T I=\frac{\sigma}{\bar{U}}
$$

where $\sigma$ is defined as the standard deviation of the set of random velocity fluctuations, $u_{k}^{\prime}$,

$$
\sigma=\sqrt{\frac{\sum_{k=1}^{n}\left(u_{k}^{\prime}\right)^{2}}{n-1}}
$$

Here, $k$ is once again an iteration parameter summed over the number of data samples $n$. By recording both the mean velocity as well as the turbulence intensity, one can then solve for the summation over the number of samples $n$ taken of the velocity fluctuations squared:

$$
\sum_{k=1}^{n}\left(u_{k}^{\prime}\right)^{2}
$$

The error bars plotted in the figures in Section 6.7 and 6.8 are graphed using 2 standard deviations, or $2 \sigma$. Two standard deviations means that, according to certain prescribed statistical calculations, $95.5 \%$ of all data in the population of the sample having a Gaussian distribution will range in between $\pm 2 \sigma$ of the true value of $\bar{U}$. Because the turbulence intensities for the most part encountered were of relatively low order of magnitude ( $\approx 2$ to $15 \% \mathrm{TI}$ ), two standard deviations normally dictated an error bound around $0.05 \mathrm{ft} / \mathrm{min}$ when the number of samples $n$ was between 3,000 and 5,000 samples.

The error analysis conducted assumes the data fall within a fairly uniform Gaussian distribution, which was clearly evident by monitoring of data during the data acquisition. Although statistical analysis tools are available that attempt to describe the uncertainty between the measured and true velocities, sample differences calculated in this particular analysis are negligible in comparison to the random error induced by other means that are commonly associated with LDA measurements (i.e. flare, surface reflections, absolute positioning error, etc.). 